\title{
Efficient Z-Selective Olefin-Acrylamide Cross-Metathesis Enabled by Sterically Demanding Cyclometalated Ruthenium Catalysts
}

Yan $\mathrm{Xu},{ }^{\dagger}$ Jonathan J. Wong, ${ }^{\dagger}$ Adrian E. Samkian, ${ }^{\dagger}$ Jeong Hoon Ko, ${ }^{\dagger}$ Shuming Chen, ${ }^{\ddagger}$ K. N. Houk $^{*}, \sharp$ and Robert H. Grubbs ${ }^{*}, \dagger$

$\dagger$ Arnold and Mabel Beckman Laboratory of Chemical Synthesis, Division of Chemistry and Chemical Engineering, California Institute of Technology, Pasadena, California 91125, United States

+ Department of Chemistry and Biochemistry, University of California, Los Angeles, California 90095-1569, United States

1. General consideration

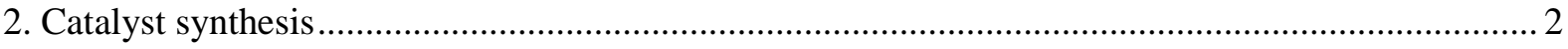

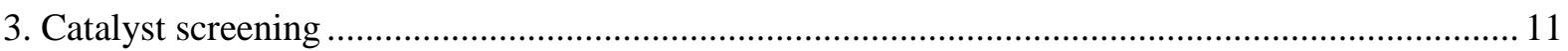

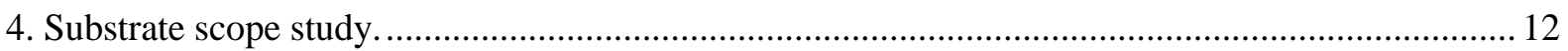

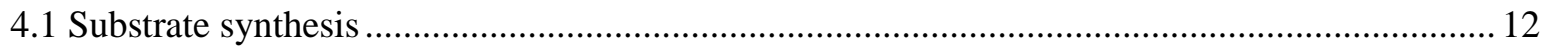

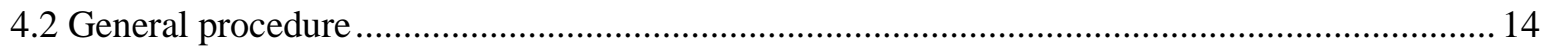

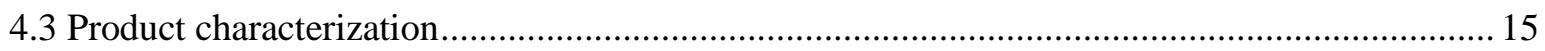

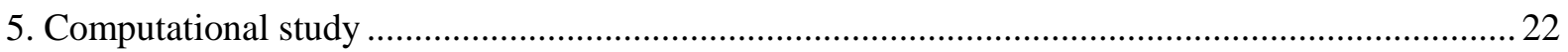

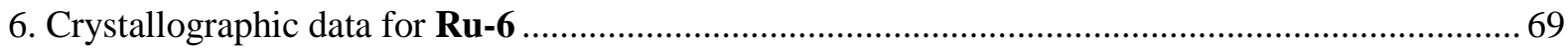

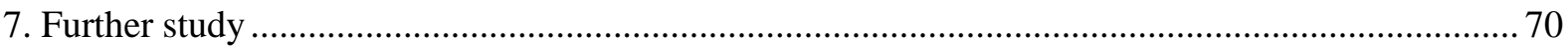

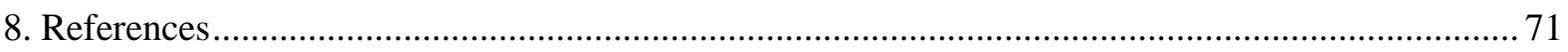

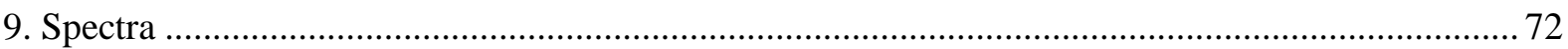




\section{General consideration}

All reactions were carried out in dry glassware under an argon atmosphere using standard Schlenk techniques or in a Vacuum Atmospheres Glovebox under a nitrogen atmosphere, unless otherwise specified. All solvents were purified by passing through solvent purification columns and further degassed by bubbling nitrogen. NMR solvents were dried over pre-baked $3 \AA$ or $4 \AA$ molecule sieves and subsequently degassed with bubbling nitrogen. $\mathrm{CDCl}_{3}$ was used as received. Liquid olefin substrates purchased from commercial sources were filtered through a plug of neutral alumina prior to use. The retention factor $\left(\mathrm{R}_{f}\right)$ of thin-layer chromatography (TLC) was recorded by using E. Merck silica gel 60 F254 precoated plates $(0.25 \mathrm{~mm})$ and visualized by UV fluorescence quenching or potassium permanganate staining. ${ }^{1} \mathrm{H}$ and ${ }^{13} \mathrm{C}$ NMR spectra were recorded on either a Bruker Ascend 400 spectrometer $(400 \mathrm{MHz}$ and $101 \mathrm{MHz}$, respectively) or a Varian Inova $500 \mathrm{MHz}\left(500 \mathrm{MHz}\right.$ and $126 \mathrm{MHz}$, respectively). For $\mathrm{CDCl}_{3}$ solutions, the chemical shifts are reported as parts per million (ppm) referenced to residual protium or carbon of the solvents; $\mathrm{CHCl}_{3} \delta \mathrm{H}(7.26$ $\mathrm{ppm})$ and $\mathrm{CDCl}_{3} \delta \mathrm{C}(77.16 \mathrm{ppm})$. For $\mathrm{C}_{6} \mathrm{D}_{6}$ solutions, the chemical shifts are reported as parts per million $(\mathrm{ppm})$ referenced to residual protium or carbon of the solvents; $\mathrm{C}_{6} \mathrm{D}_{5} \mathrm{H} \delta \mathrm{H}(7.16 \mathrm{ppm})$ and $\mathrm{C}_{6} \mathrm{D}_{6} \delta \mathrm{C}(128.06$ $\mathrm{ppm})$. Coupling constants are reported in Hertz $(\mathrm{Hz})$. Data for ${ }^{1} \mathrm{H}$ NMR spectra are reported as follows: chemical shift ( $\mathrm{ppm}$, referenced to protium: $\mathrm{s}=$ singlet, $\mathrm{d}=$ doublet, $\mathrm{t}=$ triplet, $\mathrm{q}=$ quartet, $\mathrm{p}=$ pentet (quintet), $\mathrm{h}=$ heptet, $\mathrm{dd}=$ doublet of doublets, $\mathrm{td}=$ triplet of doublets, $\mathrm{ddd}=$ doublet of doublet of doublets, $\mathrm{m}=$ multiplet, the coupling constant (Hz), and integration). High-resolution mass spectra (HRMS) were provided by the California Institute of Technology Mass Spectrometry Facility using a JEOL JMS-600H HighResolution Mass Spectrometer. Infrared (IR) spectra were recorded on a Perkin Elmer Paragon 1000 spectrometer using neat samples on ATR diamond, and are reported in frequency of absorption $\left(\mathrm{cm}^{-1}\right)$.

\section{Catalyst synthesis}

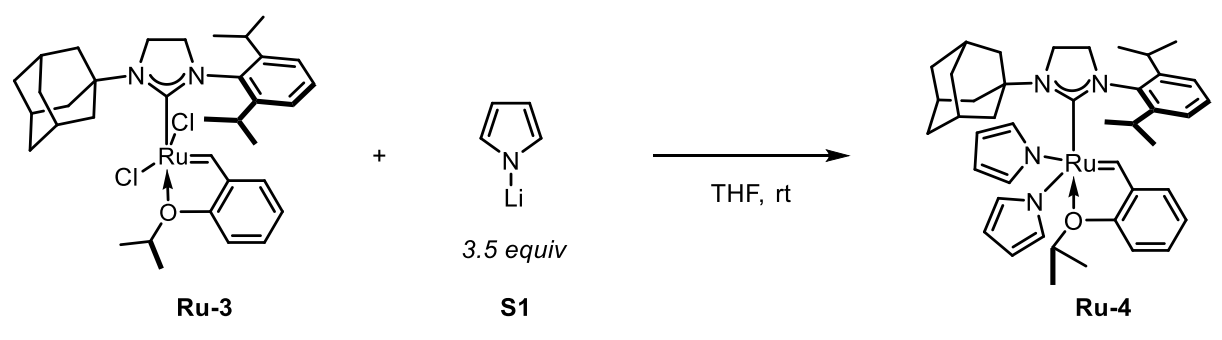

Ru-3 was synthesized according to the literature procedure. ${ }^{1}$

S1 was prepared according to the following procedure: An oven-dried flask was fitted with a stir bar and a rubber septum and cycled under an argon atmosphere. To this flask was added dry hexanes $(6 \mathrm{~mL})$ and pyrrole ( $248 \mathrm{mg}, 3.7 \mathrm{mmol}, 1.0$ equiv) through the septum. The solution was cooled to $-78{ }^{\circ} \mathrm{C}$ with a dry ice/acetone bath, and $n$-BuLi (1.5 mL, 2.45 M in hexane, $3.6 \mathrm{mmol}, 0.98$ equiv) was added dropwise. The reaction was left to stir for 15 minutes and the cold bath was removed. After stirring for additional 4 hours, the solution was transferred into a glass centrifuge tube in a glovebox, and pentane $(10 \mathrm{~mL})$ was added. The tube was centrifuged and the supernatant was removed. This rinse was repeated three more times and the residual solvent was removed using a high vacuum to yield an off-white powder in $>90 \%$ yield.

Ru-4: A $8 \mathrm{~mL}$ vial was charged with Ru-3 (130 mg, $0.19 \mathrm{mmol}, 1$ equiv) in THF (2.5 mL, solution A). A 4 $\mathrm{mL}$ vial was charged with lithium pyrrolide ( $50 \mathrm{mg}, 0.68 \mathrm{mmol}, 3.5$ equiv) in THF ( $2.25 \mathrm{~mL}$, solution $B)$. Solution $A$ was chilled in a glovebox freezer for 2 3 minutes. Solution $A$ was then taken out of the freezer, and Solution $B$ was transferred into solution $A$ using a syringe over one minute. The resulting mixture was stirred for 24 hours at room temperature and monitored by NMR to ensure full conversion. Over a period of 
3 minutes and with vigorous stirring, the reaction solution was dropwise added to a $60 \mathrm{~mL}$ vial pre-charged with pentane $(40 \mathrm{~mL})$, which led to the generation of a large amount of precipitate. The reaction vessel (the $8 \mathrm{~mL}$ vial) was rinsed with benzene $(2 \mathrm{~mL})$, and the resulting solution was transferred to the $60 \mathrm{~mL}$ vial as well. The precipitate was then collected in a glass funnel through filtration. Benzene $(6 \mathrm{~mL}+6 \mathrm{~mL}+2 \mathrm{~mL})$ was added to the glass funnel, and the resulting suspension was filtered to give a clear dark-green solution. Removal of the solvent furnished Ru-4 as a dark green solid (124 mg, 88\% yield). ${ }^{1} \mathrm{H}$ NMR (400 MHz, THF- $\left.d_{8}\right) \delta 16.04(\mathrm{~s}, 1 \mathrm{H}), 7.55(\mathrm{ddd}, J=8.7,7.4,1.7 \mathrm{~Hz}, 1 \mathrm{H}), 7.45(\mathrm{t}, J=7.7 \mathrm{~Hz}, 1 \mathrm{H}), 7.32-7.25(\mathrm{~m}, 1 \mathrm{H})$, $7.20(\mathrm{~d}, J=7.8 \mathrm{~Hz}, 2 \mathrm{H}), 6.87(\mathrm{t}, J=7.4 \mathrm{~Hz}, 1 \mathrm{H}), 6.59(\mathrm{dd}, J=7.5,1.7 \mathrm{~Hz}, 1 \mathrm{H}), 6.22(\mathrm{t}, J=1.9 \mathrm{~Hz}, 4 \mathrm{H})$, $5.72(\mathrm{t}, J=1.8 \mathrm{~Hz}, 4 \mathrm{H}), 5.17$ (hept, $J=6.0 \mathrm{~Hz}, 1 \mathrm{H}), 4.19$ (dd, $J=10.8,8.4 \mathrm{~Hz}, 2 \mathrm{H}), 3.94$ (dd, $J=10.6,8.5$ $\mathrm{Hz}, 2 \mathrm{H}), 3.58-2.18(\mathrm{~m}, 8 \mathrm{H}), 2.13(\mathrm{br}, 3 \mathrm{H}), 1.65(\mathrm{br}, 6 \mathrm{H}), 1.53(\mathrm{~d}, J=6.0 \mathrm{~Hz}, 6 \mathrm{H}), 1.08(\mathrm{~d}, J=6.7 \mathrm{~Hz}, 6 \mathrm{H})$, $0.12(\mathrm{~d}, J=6.4 \mathrm{~Hz}, 6 \mathrm{H}) .{ }^{13} \mathrm{C}$ NMR $\left(101 \mathrm{MHz}, \mathrm{THF}-d_{8}\right) \delta 296.3,214.1,153.4,149.3,144.3,140.9,130.4$, 130.0, 129.9, 125.8, 124.4, 124.0, 114.7, 107.1, 76.2, 68.0, 67.8, 67.6, 67.4, 67.1, 59.1, 56.3, 45.5, 40.3, 37.0, 31.0, 29.8, 25.9, 25.9, 25.7, 25.5, 25.3, 25.1, 23.5, 23.0. HRMS: Chemical fomular ([M]): $\mathrm{C}_{43} \mathrm{H}_{56} \mathrm{~N}_{4} \mathrm{ORu}$, calcd. $[\mathrm{M}]^{+} 746.3498$ Found: 746.3496.

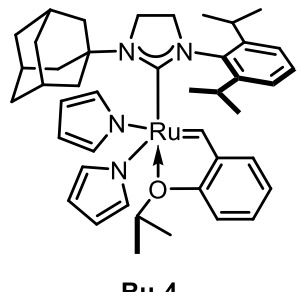

Ru-4

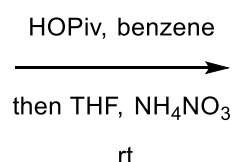

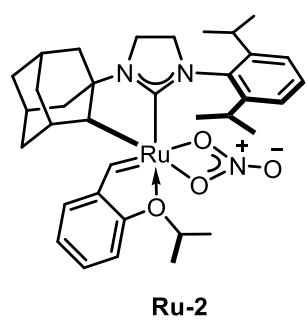

A $8 \mathrm{~mL}$ vial was charged with Ru-4 (36 mg, $0.048 \mathrm{mmol}, 1$ equiv), pivalic acid ( $22 \mathrm{mg}, 0.218 \mathrm{mmol}, 4.5$ equiv) and benzene $(2 \mathrm{~mL})$, and stirred at room temperature for 12 hours. Upon solvent removal, THF (1.4 $\mathrm{mL}$ ) was added to dissolve the residue, and the solution was stirred at room temperature for 3 hours. To this was added $\mathrm{NH}_{4} \mathrm{NO}_{3}$ (52 mg, $0.65 \mathrm{mmol}, 13.5$ equiv), and the resulting suspension was stirred at room temperature for additional 3 hours. The reaction mixture was then concentrated in vacuo, triturated with pentane $(2 \mathrm{~mL})$, and stirred at room temperature for 5 minutes. The suspension was transferred onto a short Celite column. The column was flushed with pentane $(1 \mathrm{~mL} \times 3)$ to remove a yellow band and then benzene $(2 \mathrm{~mL})$ to obtain the product as a purple solution. Removal of the solvent furnished Ru-2 as a purple solid (22.2 mg, 68\% yield). ${ }^{1} \mathrm{H}$ NMR (400 MHz, $\left.\mathrm{C}_{6} \mathrm{D}_{6}\right) \delta 15.21(\mathrm{~s}, 1 \mathrm{H}), 7.45$ (dd, $\left.J=7.5,1.7 \mathrm{~Hz}, 1 \mathrm{H}\right), 7.22-7.17$ (m, 3H), $7.00(\mathrm{dd}, J=6.6,2.7 \mathrm{~Hz}, 1 \mathrm{H}), 6.85(\mathrm{td}, J=7.4,0.9 \mathrm{~Hz}, 1 \mathrm{H}), 6.48(\mathrm{~d}, J=8.4 \mathrm{~Hz}, 1 \mathrm{H}), 4.54$ (hept, $J$ $=6.3 \mathrm{~Hz}, 1 \mathrm{H}), 4.10(\mathrm{~s}, 1 \mathrm{H}), 3.87-3.72(\mathrm{~m}, 2 \mathrm{H}), 3.59(\mathrm{ddd}, J=11.6,10.1,8.1 \mathrm{~Hz}, 1 \mathrm{H}), 3.37$ (ddd, $J=10.9$, 9.6, $8.0 \mathrm{~Hz}, 1 \mathrm{H}), 3.29-3.14(\mathrm{~m}, 2 \mathrm{H}), 2.26(\mathrm{q}, J=2.9 \mathrm{~Hz}, 1 \mathrm{H}), 2.06(\mathrm{p}, J=3.1 \mathrm{~Hz}, 1 \mathrm{H}), 1.98-1.90(\mathrm{~m}, 2 \mathrm{H})$, $1.82-1.72(\mathrm{~m}, 5 \mathrm{H}), 1.67-1.61(\mathrm{~m}, 1 \mathrm{H}), 1.49(\mathrm{~d}, J=7.6 \mathrm{~Hz}, 2 \mathrm{H}), 1.45-1.40(\mathrm{~m}, 4 \mathrm{H}), 1.20(\mathrm{~d}, J=6.9 \mathrm{~Hz}$, $3 \mathrm{H}), 1.18-1.09(\mathrm{~m}, 8 \mathrm{H}), 0.97(\mathrm{~d}, J=6.2 \mathrm{~Hz}, 3 \mathrm{H}), 0.58(\mathrm{~d}, J=11.7 \mathrm{~Hz}, 1 \mathrm{H}) \cdot{ }^{13} \mathrm{C}$ NMR $\left(101 \mathrm{MHz}, \mathrm{C}_{6} \mathrm{D}_{6}\right) \delta$ 267.4, 211.9, 154.8, 147.5, 147.4, 143.4, 135.6, 129.2, 126.9, 124.8, 124.2, 123.4, 123.4, 113.2, 74.5, 66.4, $63.2,54.1,43.0,41.6,40.3,38.0,37.8,37.8,33.3,30.9,29.8,29.0,28.7,27.9,26.8,23.6,23.1,21.1,20.3$. The spectra match reported literature. ${ }^{2}$

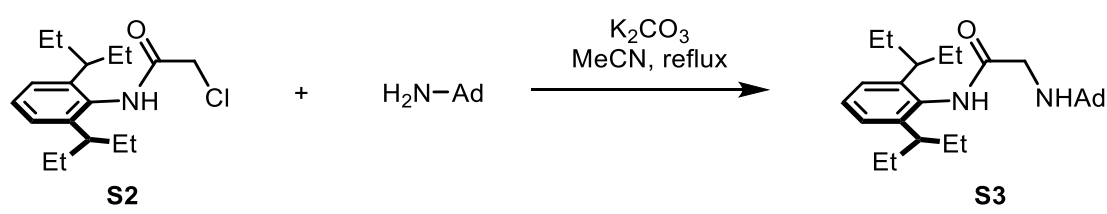

S2 was synthesized according to the literature procedure. ${ }^{3}$

S3: A $100 \mathrm{~mL}$ round bottom flask equipped with a reflux condenser was charged with $\mathbf{S 2}(3.1 \mathrm{~g}, 10 \mathrm{mmol}, 1$ equiv), 1-adamantylamine ( $3.1 \mathrm{~g}, 20 \mathrm{mmol}, 2$ equiv), $\mathrm{K}_{2} \mathrm{CO}_{3}$ (2.8 g, $20 \mathrm{mmol}, 2$ equiv), and $\mathrm{MeCN}(60 \mathrm{~mL})$. 
The flask was placed into an oil bath pre-heated to $100{ }^{\circ} \mathrm{C}$ and stirred overnight. After cooled to room temperature, the reaction mixture was filtered and concentrated in vacuo. The residue was further purified by column chromatography over silica gel (hexane/acetone, $6: 1$ to $4: 1$ ) to give $\mathbf{S 3}$ as an off-white solid (3.4 g, 80\% yield). $\mathrm{R}_{f}=0.4$ (hexane/acetone $\left.=3: 1\right) .{ }^{1} \mathrm{H} \mathrm{NMR}\left(400 \mathrm{MHz}, \mathrm{CDCl}_{3}\right) \delta 8.92(\mathrm{br}, 1 \mathrm{H}), 7.23(\mathrm{~d}, J=7.7$ $\mathrm{Hz}, 1 \mathrm{H}), 7.05(\mathrm{~d}, J=7.7 \mathrm{~Hz}, 2 \mathrm{H}), 3.43(\mathrm{br}, 2 \mathrm{H}), 2.57$ (ddd, $J=14.3,8.4,6.0 \mathrm{~Hz}, 2 \mathrm{H}), 2.11(\mathrm{~s}, 3 \mathrm{H}), 1.79-$ $1.42(\mathrm{~m}, 21 \mathrm{H}), 0.77(\mathrm{t}, J=7.4 \mathrm{~Hz}, 12 \mathrm{H}) .{ }^{13} \mathrm{C} \mathrm{NMR}\left(101 \mathrm{MHz}, \mathrm{CDCl}_{3}\right) \delta 171.4,143.2,134.6,127.6,124.0$, 51.3, 44.1, 43.1, 43.0, 36.6, 29.6, 29.0, 12.3. IR: 3305, 2958, 2904, 2849, 1677, 1499, 1461, 1143, $1097 \mathrm{~cm}^{-}$ 1. HRMS: Chemical fomular ([M]): $\mathrm{C}_{28} \mathrm{H}_{44} \mathrm{~N}_{2} \mathrm{O}$, calcd. [M+H] ${ }^{+} 425.3532$ Found: 425.3519 .

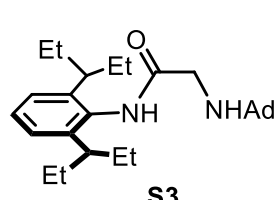

S3
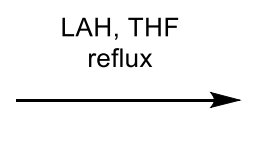

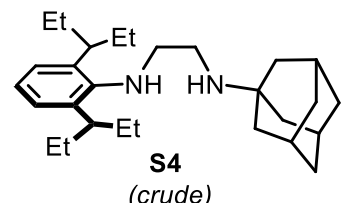

(crude)

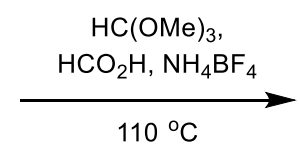

$110^{\circ} \mathrm{C}$
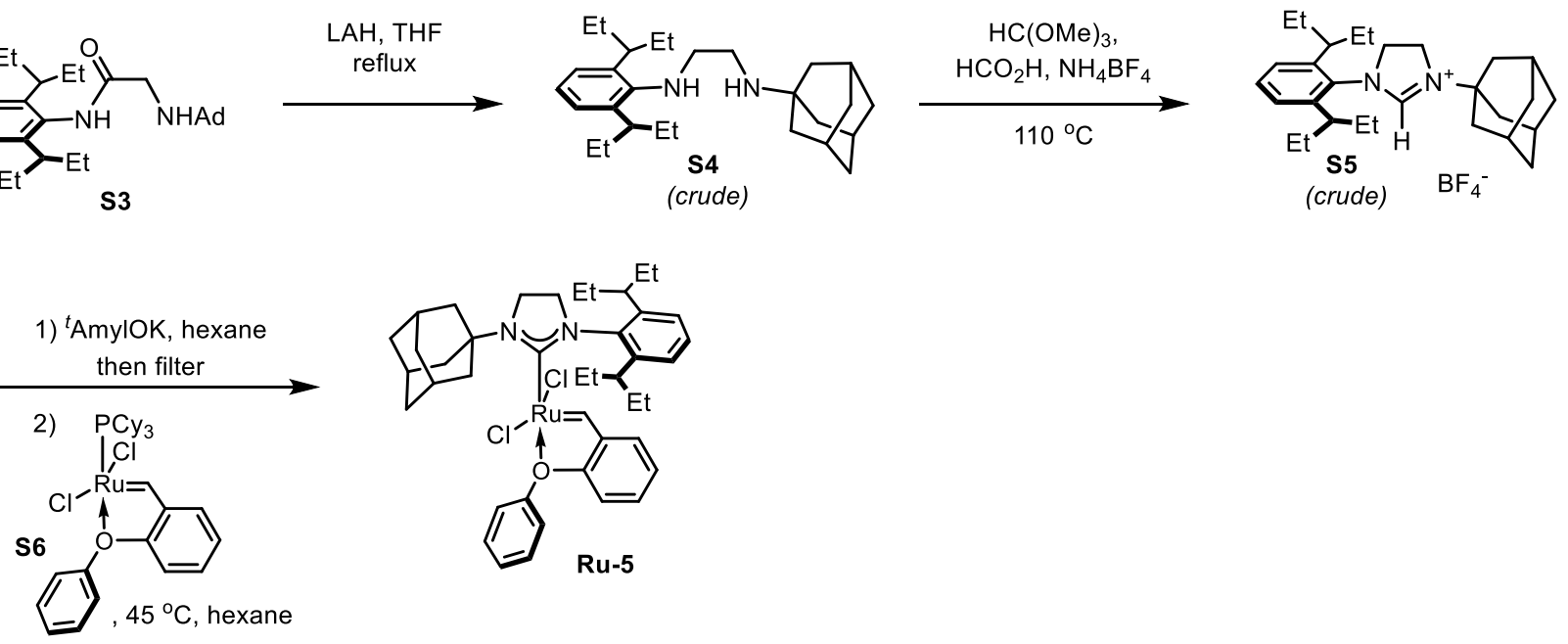

S4: A $100 \mathrm{~mL}$ round bottom flask equipped with a reflux condenser and a nitrogen bubbler was charged with S3 (2.55 g, 6 mmol, 1 equiv), lithium aluminum hydride $(1.9 \mathrm{~g}, 50 \mathrm{mmol})$ under a nitrogen atmosphere. To this was added THF $(53 \mathrm{~mL})$. The flask was placed in an oil bath and the bath temperature was then set to $95{ }^{\circ} \mathrm{C}$ (to ensure reflux). The reaction mixture was stirred at this temperature for 6 days and monitored by TLC until full conversion. The reaction mixture was then cooled with an ice bath, and sequentially charged with $\mathrm{H}_{2} \mathrm{O}(1 \mathrm{~mL}), \mathrm{NaOH}$ (aq., $\left.3 \mathrm{M}, 2 \mathrm{~mL}\right), \mathrm{H}_{2} \mathrm{O}(2 \mathrm{~mL})$, and $\mathrm{Et}_{2} \mathrm{O}(30 \mathrm{~mL})$. The resulting suspension was filtered, dried over $\mathrm{Na}_{2} \mathrm{SO}_{4}$, and concentrated in vacuo. The residue was further purified by column chromatography over silica gel (DCM/MeOH, 40:1, with $0.5 \% \mathrm{v} / \mathrm{v} \mathrm{NEt}_{3}$ ) to give crude $\mathbf{S} 4$ as a colorless oil ( $1.8 \mathrm{~g}, 73 \%$ yield, mixed with a small amount of $\mathrm{Et}_{2} \mathrm{O}$ and other minor impurities). $\mathrm{R}_{f}=0.5(\mathrm{DCM} / \mathrm{MeOH}=$ $10: 1$, with $0.5 \% \mathrm{v} / \mathrm{v} \mathrm{NEt}_{3}$ ). Crude $\mathbf{S} 4$ was directly used in the next step without further purification (in our hands, this compound appears to slowly decompose during storage and is recommended to be used immediately after preparation). A crude spectrum of $\mathbf{S} \mathbf{4}$ was recorded: ${ }^{1} \mathrm{H} \mathrm{NMR}\left(400 \mathrm{MHz}, \mathrm{CDCl}_{3}\right) \delta 7.02$ (dd, $J=8.6,6.4 \mathrm{~Hz}, 1 \mathrm{H}), 6.99-6.90(\mathrm{~m}, 2 \mathrm{H}), 3.01-3.80(\mathrm{~m}, 6 \mathrm{H}), 2.09(\mathrm{~s}, 3 \mathrm{H}), 1.82-1.56(\mathrm{~m}, 17 \mathrm{H}), 1.50$ (ddt, $J=13.5,8.6,7.3 \mathrm{~Hz}, 4 \mathrm{H}), 0.81$ (t, $J=7.4 \mathrm{~Hz}, 12 \mathrm{H}$ ).

S5: A $20 \mathrm{~mL}$ vial that was charged with crude $\mathbf{S 4}\left(740 \mathrm{mg}, 1.8 \mathrm{mmol}, 1\right.$ equiv), $\mathrm{NH}_{4} \mathrm{BF}_{4}(198 \mathrm{mg}, 1.9 \mathrm{mmol}$, 1.05 equiv), trimethyl orthoformate $(10 \mathrm{~mL})$, and a small drop of formic acid under air atmosphere. The vial was sealed and stirred at $110^{\circ} \mathrm{C}$ for 4.5 hours and monitored by TLC to ensure full conversion. After cooled to room temperature, the reaction mixture was filtered through a short plug of Celite and concentrated in vacuo. The orange-brown residue was then purified by flash column chromatography (DCM/MeOH, 50:1) to give the crude $\mathrm{NHC}$ salt as a yellow oil. The crude product was then triturated with $\mathrm{Et}_{2} \mathrm{O}$. After filtration and $\mathrm{Et}_{2} \mathrm{O}$ wash, $\mathbf{S 5}$ was collected as an off-white solid (350 mg, 38\% yield, ca. 92 95\% purity). $\mathrm{R}_{f}=0.35$ $(\mathrm{DCM} /$ Acetone $=5: 1)$. A crude spectrum of $\mathbf{S 5}$ was recorded: ${ }^{1} \mathrm{H}$ NMR $\left(400 \mathrm{MHz}, \mathrm{CDCl}_{3}\right) \delta 7.46(\mathrm{t}, J=7.8$ $\mathrm{Hz}, 1 \mathrm{H}), 7.39$ (s, 1H), 7.17 (d, $J=7.8 \mathrm{~Hz}, 2 \mathrm{H}), 4.48$ (dd, $J=12.7,9.2 \mathrm{~Hz}, 2 \mathrm{H}), 4.28$ (dd, $J=12.5,9.2 \mathrm{~Hz}$, $2 \mathrm{H}), 2.47-2.31(\mathrm{~m}, 2 \mathrm{H}), 2.26(\mathrm{~s}, 3 \mathrm{H}), 2.00(\mathrm{br}, 6 \mathrm{H}), 1.85-1.45(\mathrm{~m}, 15 \mathrm{H}), 0.85-0.66(\mathrm{~m}, 12 \mathrm{H})$. 
S6 was prepared according to the following procedure: Grubbs first generation catalyst M1a (C823) (1.5 g, $1.82 \mathrm{mmol}, 1$ equiv), phenoxylvinylbenzene ( $0.376 \mathrm{~g}, 1.91 \mathrm{mmol}, 1.05$ equiv), Amberlyst-15 (1.5 g), and DCM $(15 \mathrm{~mL})$ were added to a $20 \mathrm{~mL}$ vial in a glovebox and stirred vigorously at room temperature for 18 hours. The reaction was filtered and the filtrate concentrated in vacuo. The residue was triturated with pentane $(10 \mathrm{~mL})$ until it solidified. The resulting powder was filtered and rinsed until the eluent was clear to yield $\mathbf{S 6}$ (941 mg, 82\% yield). ${ }^{1} \mathrm{H}$ NMR (400 MHz, $\left.{ }_{6} \mathrm{D}_{6}\right) \delta 17.41$ (d, $\left.J=4.6 \mathrm{~Hz}, 1 \mathrm{H}\right), 7.79$ (d, $J=7.9 \mathrm{~Hz}$, 2H), $7.41(\mathrm{~d}, J=7.6 \mathrm{~Hz}, 1 \mathrm{H}), 7.00(\mathrm{t}, J=7.8 \mathrm{~Hz}, 3 \mathrm{H}), 6.86(\mathrm{t}, J=7.4 \mathrm{~Hz}, 1 \mathrm{H}), 6.73(\mathrm{t}, J=7.5 \mathrm{~Hz}, 1 \mathrm{H}), 6.57$ (d, $J=8.3 \mathrm{~Hz}, 1 \mathrm{H}), 2.40(\mathrm{q}, J=12.1 \mathrm{~Hz}, 3 \mathrm{H}), 2.17(\mathrm{~d}, J=12.4 \mathrm{~Hz}, 6 \mathrm{H}), 1.88(\mathrm{q}, J=12.6 \mathrm{~Hz}, 6 \mathrm{H}), 1.67$ (d, $J=11.9 \mathrm{~Hz}, 6 \mathrm{H}), 1.53(\mathrm{~d}, J=10.8 \mathrm{~Hz}, 3 \mathrm{H}), 1.28-1.01(\mathrm{~m}, 9 \mathrm{H}) .{ }^{13} \mathrm{C} \mathrm{NMR}\left(101 \mathrm{MHz}, \mathrm{C}_{6} \mathrm{D}_{6}\right) \delta 271.2,155.4$, 153.6, 142.9, 130.1, 129.2, 127.0, 124.2, 124.1, 122.4, 114.4, 36.3, 36.1, 30.4, 28.1, 28.0, 26.5. HRMS: Chemical fomular ([M]): $\mathrm{C}_{31} \mathrm{H}_{43} \mathrm{Cl}_{2} \mathrm{OPRu}$, calcd. [M] $]^{+} 634.1473$ Found: 634.1460.

Ru-5: A $20 \mathrm{~mL}$ vial was charged with $\mathbf{S 5}\left(244 \mathrm{mg}, 0.48 \mathrm{mmol}, 1\right.$ equiv) and hexane $(9 \mathrm{~mL})$ in a $\mathrm{N}_{2}$ glovebox. To this was added potassium tert-pentoxide $(0.9 \mathrm{M}$ solution in cyclohexane, $0.55 \mathrm{~mL}, 0.50 \mathrm{mmol}, 1.03$ equiv). The vial was sealed and stirred at room temperature for 1 hour. The reaction mixture was then filtered through a short plug of Celite into a $40 \mathrm{~mL}$ vial containing $\mathbf{S 6}(290 \mathrm{mg}, 0.46 \mathrm{mmol}, 0.95$ equiv). The $40 \mathrm{~mL}$ vial was sealed and stirred at $45{ }^{\circ} \mathrm{C}$ for 4 hours. After cooled to room temperature, the reaction mixture was concentrated in vacuo. The residue was further purified by flash column chromatography over silica in a glovebox (hexane/benzene/ $\mathrm{Et}_{2} \mathrm{O}, 6: 3: 0.5$ to $\left.6: 3: 1\right)$ to give $\mathbf{R u}-\mathbf{5}$ as a green solid $(230 \mathrm{mg}, 62 \%$ yield, containing ca. $2 \%$ unknown impurity). ${ }^{1} \mathrm{H}$ NMR $\left(400 \mathrm{MHz}, \mathrm{C}_{6} \mathrm{D}_{6}\right) \delta 17.14(\mathrm{~s}, 1 \mathrm{H}), 7.74(\mathrm{~d}, J=7.4 \mathrm{~Hz}, 2 \mathrm{H})$, $7.44(\mathrm{t}, J=7.7 \mathrm{~Hz}, 1 \mathrm{H}), 7.28(\mathrm{~d}, J=7.8 \mathrm{~Hz}, 2 \mathrm{H}), 7.21(\mathrm{dd}, J=7.6,1.6 \mathrm{~Hz}, 1 \mathrm{H}), 7.04-6.85(\mathrm{~m}, 4 \mathrm{H}), 6.66$ $(\mathrm{td}, J=7.4,1.0 \mathrm{~Hz}, 1 \mathrm{H}), 6.43(\mathrm{~d}, J=8.3 \mathrm{~Hz}, 1 \mathrm{H}), 3.68(\mathrm{dd}, J=10.6,8.4 \mathrm{~Hz}, 2 \mathrm{H}), 3.36(\mathrm{dd}, J=10.6,8.4 \mathrm{~Hz}$, $2 \mathrm{H}), 3.16(\mathrm{dq}, J=8.2,6.2 \mathrm{~Hz}, 2 \mathrm{H}), 2.82(\mathrm{br}, 6 \mathrm{H}), 2.01(\mathrm{br}, 3 \mathrm{H}), 1.86-1.17(\mathrm{~m}, 18 \mathrm{H}), 1.02(\mathrm{t}, J=7.2 \mathrm{~Hz}$, $6 \mathrm{H}), 0.97-0.71(\mathrm{~m}, 8 \mathrm{H}) .{ }^{13} \mathrm{C}$ NMR $\left(101 \mathrm{MHz}, \mathrm{C}_{6} \mathrm{D}_{6}\right) \delta 303.5,211.9,155.0,154.6,146.8,144.0,142.0$, 129.8, 129.7, 128.5, 126.6, 126.3, 124.2, 123.5, 123.2, 114.0, 57.2, 54.9, 44.2, 42.0, 40.9, 36.3, 30.5, 28.7, 28.3, 12.6, 11.9. HRMS: Chemical fomular ([M]): $\mathrm{C}_{42} \mathrm{H}_{54} \mathrm{Cl}_{2} \mathrm{~N}_{2} \mathrm{ORu}$, calcd. [M] 774.2657 Found 774.2682.

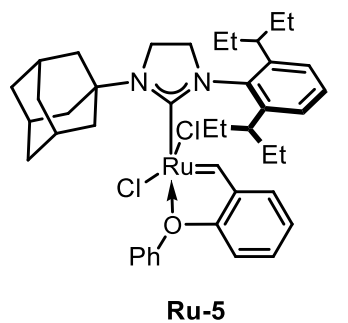

Ru-5

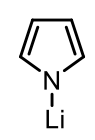

THF, rt

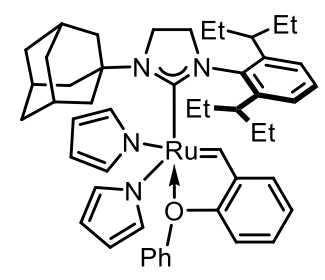

S7

A $40 \mathrm{~mL}$ vial was charged with Ru-5 (565 mg, $0.73 \mathrm{mmol}$, 1 equiv) in THF (11 mL, solution A). A $20 \mathrm{~mL}$ vial was charged with lithium pyrrolate $(270 \mathrm{mg}, 3.78 \mathrm{mmol}, 5$ equiv) in THF ( $9 \mathrm{~mL}$, solution B). Solution $A$ was chilled in a glovebox freezer for 2 3 minutes. Solution $A$ was then taken out of the freezer, and Solution $B$ was transferred into solution $A$ using a syringe over two minutes. The resulting mixture was stirred for 6 hours at room temperature, monitored by NMR to ensure full conversion, and then concentrated in vacuo. Benzene $(6 \sim 7 \mathrm{~mL})$ was added to the residue to dissolve most of the solid, resulting in a green suspension. Over a period of 3 minutes and with vigorous stirring, the suspension was dropwise added to a $100 \mathrm{~mL}$ flask pre-charged with pentane $(50 \mathrm{~mL})$, which led to a green solution and the generation of white precipitate. The white solid was removed through filtration and the filtrate was concentrated in vacuo. The residue was transferred onto a short Celite column. The column was flushed with hexamethyldisiloxane $(3 \mathrm{~mL} \times 3)$ and a hexamethyldisiloxane/pentane solution $(1: 1,2 \mathrm{~mL})$ to remove the impurity as a brown band. The Celite column was then flushed with a benzene/pentane solution $(2: 3,3 \mathrm{~mL} \times 5)$ to generate a green solution. Removal of the solvent furnished $\mathbf{S} 7$ as a green solid $\left(550 \mathrm{mg}, 90 \%\right.$ yield). ${ }^{1} \mathrm{H}$ NMR (400 MHz, $\left.\mathrm{C}_{6} \mathrm{D}_{6}\right) \delta$ $16.74(\mathrm{~s}, 1 \mathrm{H}), 7.58(\mathrm{~d}, J=8.0 \mathrm{~Hz}, 2 \mathrm{H}), 7.32$ (t, $J=7.7 \mathrm{~Hz}, 1 \mathrm{H}), 7.13$ (d, $J=7.7 \mathrm{~Hz}, 2 \mathrm{H}), 7.01-6.91$ (m, 
2H), $6.86(\mathrm{dt}, J=8.3,6.8 \mathrm{~Hz}, 3 \mathrm{H}), 6.80-6.75(\mathrm{~m}, 1 \mathrm{H}), 6.72(\mathrm{br}, 4 \mathrm{H}), 6.66(\mathrm{~d}, J=7.3 \mathrm{~Hz}, 1 \mathrm{H}), 6.49(\mathrm{br}, 4 \mathrm{H})$, 3.64 (dd, $J=10.0,8.4 \mathrm{~Hz}, 2 \mathrm{H}), 3.39$ (dd, $J=10.0,8.4 \mathrm{~Hz}, 2 \mathrm{H}), 3.21$ (dp, $J=9.6,4.9 \mathrm{~Hz}, 2 \mathrm{H}), 3.10-1.94$ (m, 4H), 1.91 (br, 3H), $1.75-1.19(\mathrm{~m}, 16 \mathrm{H}), 1.01(\mathrm{t}, J=7.4 \mathrm{~Hz}, 6 \mathrm{H}), 0.12(\mathrm{t}, J=7.3 \mathrm{~Hz}, 6 \mathrm{H}) .{ }^{13} \mathrm{C}$ NMR $\left(101 \mathrm{MHz}, \mathrm{C}_{6} \mathrm{D}_{6}\right)$ carbon signals were observed at $\delta 295.5,214.8,154.2,153.5,145.8,142.7,141.8,130.1$, 129.7, 129.0, 126.5, 126.5, 125.0, 124.4, 122.7, 113.4, 108.0, 58.4, 56.4, 44.4, 40.1, 36.2, 31.2, 30.0, 26.4, 12.0, 11.0. HRMS: Chemical fomular ([M]): $\mathrm{C}_{50} \mathrm{H}_{62} \mathrm{~N}_{4} \mathrm{ORu}$, calcd. [M] $]^{+} 836.3968$ Found: 836.3992.

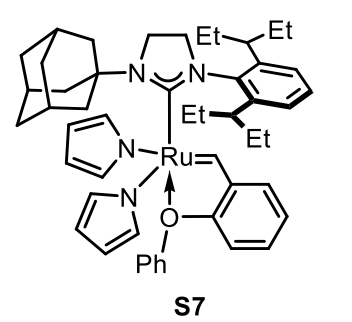

S7

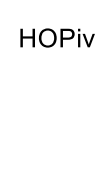

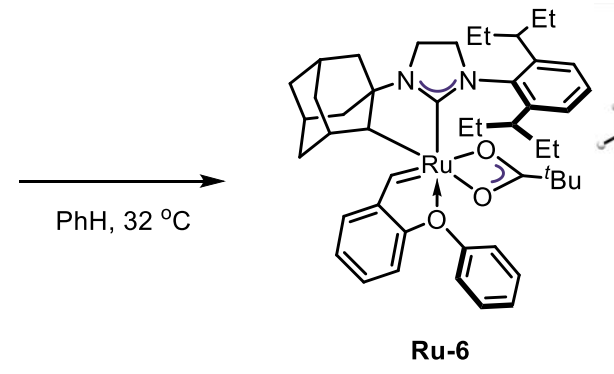

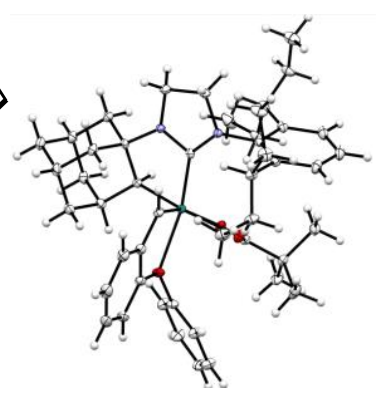

A $40 \mathrm{~mL}$ vial was charged with $\mathbf{S} 7$ (580 $\mathrm{mg}, 0.693 \mathrm{mmol}, 1$ equiv). To this, a benzene ( $20 \mathrm{~mL})$ solution of pivalic acid (290 mg, $2.84 \mathrm{mmol}, 4$ equiv.) was added at room temperature. The reaction mixture was stirred at $32{ }^{\circ} \mathrm{C}$ for 24 hours, monitored by NMR to ensure full conversion, and then concentrated in vacuo. Hexamethyldisiloxane $(5 \mathrm{~mL})$ was added to the residue, and the resulting mixture was stirred at room temperature for 5 minutes before concentrated in vacuo (During this process, formation of a purple precipitate is expected --- if not, such a "trituration" procedure should be repeated). The residue was transferred onto a short Celite column. The column was flushed with hexamethyldisiloxane $(3 \mathrm{~mL} \times 3)$ and a hexamethyldisiloxane/pentane solution $(1: 1,3 \mathrm{~mL} \times 2)$ to remove the impurity as a brown band. The Celite column was then flushed with benzene $(5 \mathrm{~mL})$ to generate a purple solution. Removal of the solvent furnished Ru-6 as a purple solid (390 mg, 70\% yield). ${ }^{1} \mathrm{H}$ NMR $\left(400 \mathrm{MHz}, \mathrm{C}_{6} \mathrm{D}_{6}\right) \delta 14.84(\mathrm{~s}, 1 \mathrm{H}), 7.53$ (dd, $J=7.5,1.6 \mathrm{~Hz}, 1 \mathrm{H}), 7.25(\mathrm{~d}, J=7.6 \mathrm{~Hz}, 2 \mathrm{H}), 7.14(\mathrm{~d}, J=7.7 \mathrm{~Hz}, 1 \mathrm{H}), 7.05$ (dd, $J=7.8,1.6 \mathrm{~Hz}, 1 \mathrm{H}), 7.00$ (ddd, $J=8.2,7.3,1.6 \mathrm{~Hz}, 1 \mathrm{H}), 6.98-6.91(\mathrm{~m}, 3 \mathrm{H}), 6.87(\mathrm{td}, J=7.4,1.1 \mathrm{~Hz}, 1 \mathrm{H}), 6.84-6.76(\mathrm{~m}, 1 \mathrm{H}), 6.60$ $(\mathrm{dt}, J=8.2,0.9 \mathrm{~Hz}, 1 \mathrm{H}), 4.11(\mathrm{~s}, 1 \mathrm{H}), 3.99(\mathrm{dt}, J=11.7,10.3 \mathrm{~Hz}, 1 \mathrm{H}), 3.63(\mathrm{td}, J=10.5,4.8 \mathrm{~Hz}, 1 \mathrm{H}), 3.59$ $-3.43(\mathrm{~m}, 2 \mathrm{H}), 3.24$ (ddd, $J=12.1,9.6,4.8 \mathrm{~Hz}, 1 \mathrm{H}), 2.79(\mathrm{q}, J=2.9 \mathrm{~Hz}, 1 \mathrm{H}), 2.31-2.13(\mathrm{~m}, 5 \mathrm{H}), 2.10$ (d, $J=12.1 \mathrm{~Hz}, 1 \mathrm{H}), 2.04-1.77(\mathrm{~m}, 4 \mathrm{H}), 1.74-1.64(\mathrm{~m}, 1 \mathrm{H}), 1.64-1.31(\mathrm{~m}, 7 \mathrm{H}), 1.30-1.20(\mathrm{~m}, 4 \mathrm{H}), 1.12$ $-1.01(\mathrm{~m}, 4 \mathrm{H}), 0.80(\mathrm{td}, J=7.4,6.2 \mathrm{~Hz}, 6 \mathrm{H}), 0.70(\mathrm{~s}, 9 \mathrm{H}), 0.68-0.63(\mathrm{~m}, 1 \mathrm{H}) .{ }^{13} \mathrm{C} \mathrm{NMR}\left(101 \mathrm{MHz}, \mathrm{C}_{6} \mathrm{D}_{6}\right)$ $\delta 256.8,213.9,188.3,157.1,154.7,147.2,145.3,142.9,140.5,129.6,128.6,125.7,125.0,124.9,124.6$, 124.4, 123.4, 122.2, 115.5, 68.4, 63.0, 55.7, 43.9, 43.5, 42.6, 41.1, 40.9, 39.6, 38.3, 38.2, 36.4, 33.8, 31.4, 31.1, 30.1, 28.1, 28.0, 28.0, 26.4, 14.2, 13.2, 13.1, 12.6. HRMS: Chemical fomular ([M]): $\mathrm{C}_{47} \mathrm{H}_{62} \mathrm{~N}_{2} \mathrm{O}_{3} \mathrm{Ru}$, calcd. [M-H] $]^{+}$803.3726 Found: 803.3737. A single-crystal of Ru-6 for X-ray diffraction analysis was prepared through slow evaporation of a benzene solution in a glovebox (see Section 6).
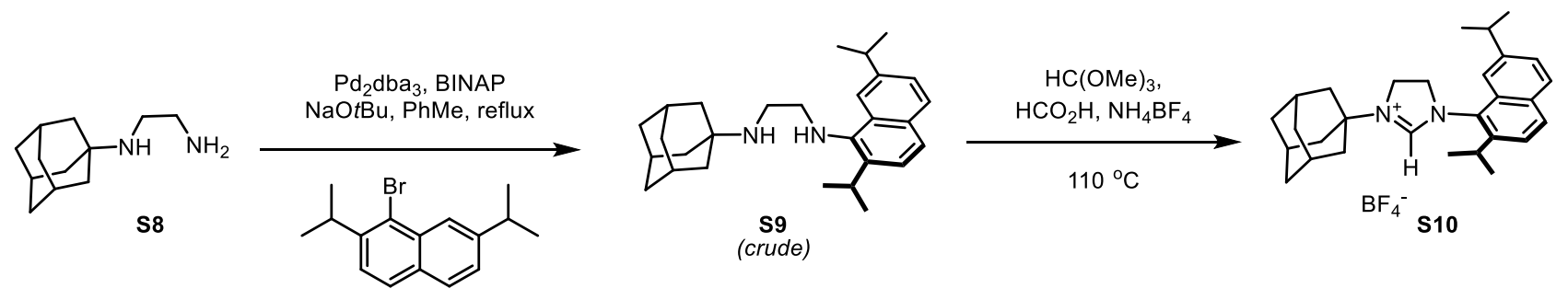

S8 was prepared according to the literature procedure. ${ }^{4}$

S9: A $20 \mathrm{~mL}$ vial was first charged with $\mathrm{Pd}_{2}(\mathrm{dba})_{3}(23 \mathrm{mg}, 0.025 \mathrm{mmol}, 0.04$ equiv), BINAP (37 mg, 0.06 mmol, 0.10 equiv), 1-bromo-2,7-diisopropylnaphthalene ${ }^{5}(174 \mathrm{mg}, 0.6 \mathrm{mmol}, 1$ equiv) and $\mathbf{S 8}$ (116 mg, 0.6 
mmol, 1 equiv) under air atmosphere, and then with $\mathrm{NaO}^{t} \mathrm{Bu}(87 \mathrm{mg}, 0.9 \mathrm{mmol}, 1.5$ equiv) and toluene (6 $\mathrm{mL}$ ) in a $\mathrm{N}_{2}$ glovebox. The vial was sealed and stirred at $110{ }^{\circ} \mathrm{C}$ for 18 hours. After cooled to room temperature, the reaction mixture was filtered through a short plug of Celite and concentrated in vacuo. The residue was purified by flash column chromatography (DCM/MeOH, 40:1 to $20: 1$, with $0.5 \% \mathrm{v} / \mathrm{v} \mathrm{NEt}$ ) to give the crude coupling products $\mathbf{S 9}$ as a yellowish oil (160 mg, ca. $90 \%$ purity). $\mathrm{R}_{f}=0.2(\mathrm{DCM} / \mathrm{MeOH}=$ 12:1). A crude NMR spectrum is recorded for $\mathbf{S 9}:{ }^{1} \mathrm{H} \mathrm{NMR}\left(400 \mathrm{MHz}, \mathrm{CDCl}_{3}\right.$, crude) $\delta 8.02$ (d, $J=1.6 \mathrm{~Hz}$, $1 \mathrm{H}), 7.71(\mathrm{~d}, J=8.4 \mathrm{~Hz}, 1 \mathrm{H}), 7.50(\mathrm{~d}, J=8.5 \mathrm{~Hz}, 1 \mathrm{H}), 7.35-7.28(\mathrm{~m}, 2 \mathrm{H}), 3.54(\mathrm{p}, J=6.8 \mathrm{~Hz}, 1 \mathrm{H}), 3.33$ (br, 2H), 3.10 (hept, $J=7.0 \mathrm{~Hz}, 1 \mathrm{H}$ ), 2.97 (t, $J=5.7 \mathrm{~Hz}, 2 \mathrm{H}), 2.09$ (s, 3H), 1.80 (br, 6H), $1.73-1.55$ (m, $7 \mathrm{H}), 1.34(\mathrm{~d}, J=7.0 \mathrm{~Hz}, 6 \mathrm{H}), 1.29(\mathrm{~d}, J=6.9 \mathrm{~Hz}, 6 \mathrm{H})$. The crude $\mathbf{S 9}$ was directly carried out to the next step without further purification.

S10: A $4 \mathrm{~mL}$ vial that was charged with crude $\mathbf{S 9}(160 \mathrm{mg}), \mathrm{NH}_{4} \mathrm{BF}_{4}(37 \mathrm{mg}, 0.35 \mathrm{mmol})$, trimethyl orthoformate $(2 \mathrm{~mL})$, and a small drop of formic acid under air atmosphere. The vial was sealed and heated at $110{ }^{\circ} \mathrm{C}$ with stirring for 3 hours and followed by TLC to ensure full conversion. After cooled to room temperature, the reaction mixture was filtered through a short plug of Celite and concentrated in vacuo. The orange-brown residue was then purified by flash column chromatography (DCM/MeOH, 40:1) to give the crude NHC salt as a yellowish solid. We also noticed that the crude NHC salt sometimes remains a thick oil instead of a solid. In such cases, the following procedure could be applied to further purify the NHC salt: To an $8 \mathrm{~mL}$ vial containing $160 \mathrm{mg}$ of crude NHC was added toluene $(0.6 \mathrm{~mL})$ and hexane $(1.2 \mathrm{~mL})$. After stirring for 1 minute, a small amount of DCM $(0.3-0.5 \mathrm{~mL})$ was then added, during which process the solid particles of the NHC salt were usually observed. More hexane $(0.8 \mathrm{~mL})$ was added dropwise in order to precipitate most of the NHC salt, which was collected through filtration as a white solid (120 mg, 40\% yield over two steps). $\mathrm{R}_{f}=0.35(\mathrm{DCM} / \mathrm{MeOH}=12: 1) .{ }^{1} \mathrm{H} \mathrm{NMR}\left(400 \mathrm{MHz}, \mathrm{CDCl}_{3}\right) \delta 7.87(\mathrm{~s}, 1 \mathrm{H}), 7.86(\mathrm{~d}, J=$ $8.8 \mathrm{~Hz}, 1 \mathrm{H}), 7.79(\mathrm{~d}, J=8.4 \mathrm{~Hz}, 1 \mathrm{H}), 7.43(\mathrm{dd}, J=8.5,1.6 \mathrm{~Hz}, 1 \mathrm{H}), 7.39(\mathrm{~d}, J=8.7 \mathrm{~Hz}, 1 \mathrm{H}), 7.37(\mathrm{~s}, 1 \mathrm{H})$, $4.53-4.18(\mathrm{~m}, 4 \mathrm{H}), 3.24-3.06(\mathrm{~m}, 2 \mathrm{H}), 2.22(\mathrm{~s}, 3 \mathrm{H}), 2.05(\mathrm{~d}, J=2.9 \mathrm{~Hz}, 6 \mathrm{H}), 1.70(\mathrm{t}, J=3.1 \mathrm{~Hz}, 6 \mathrm{H})$, $1.33-1.24(\mathrm{~m}, 12 \mathrm{H}) .{ }^{13} \mathrm{C} \mathrm{NMR}\left(101 \mathrm{MHz}, \mathrm{CDCl}_{3}\right) \delta 155.3,149.9,145.1,131.6,131.0,130.1,129.0,127.3$, 126.3, 123.4, 117.4, 58.4, 52.8, 45.5, 41.1, 35.5, 34.6, 29.3, 28.9, 24.3, 24.1, 24.0, 23.9. IR: 2970, 2913, 2855, 1624, 1263, 1048, $910 \mathrm{~cm}^{-1}$. HRMS: Chemical fomular of the cation $\left(\left[\mathrm{M}^{+}\right]\right): \mathrm{C}_{29} \mathrm{H}_{39} \mathrm{~N}_{2}{ }^{+}$, calcd. $\left[\mathrm{M}^{+}\right]$ 415.3113 Found: 415.3108.

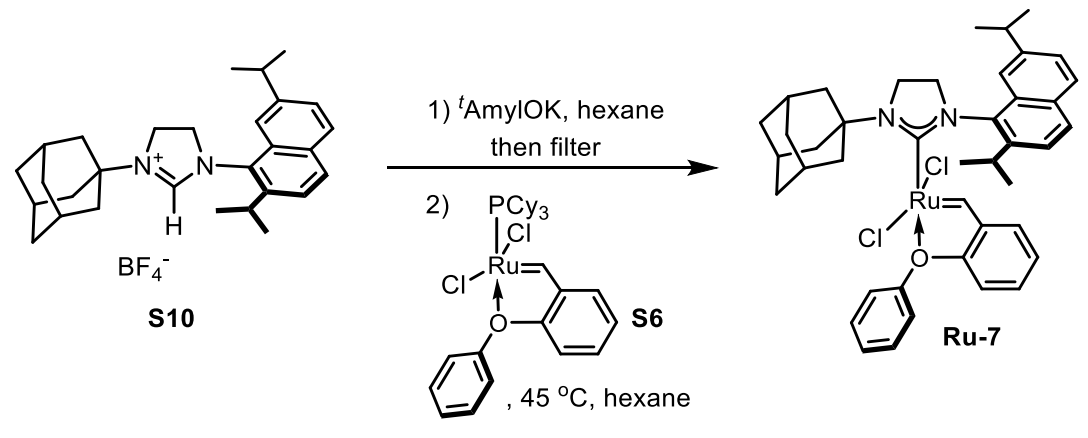

Ru-7: A $20 \mathrm{~mL}$ vial was charged with the NHC salt $\mathbf{S 1 0}$ (364 mg, $0.72 \mathrm{mmol}, 1.14$ equiv) and hexane (18 $\mathrm{mL})$ in a $\mathrm{N}_{2}$ glovebox. To this was added potassium tert-pentoxide $(0.9 \mathrm{M}$ solution in cyclohexane, $0.8 \mathrm{~mL}$, $0.72 \mathrm{mmol}, 1.14$ equiv). The vial was sealed and stirred at room temperature for 1 hour. The reaction mixture was then filtered through a short plug of Celite into a $40 \mathrm{~mL}$ vial pre-charged with $\mathbf{S 6}$ ( $400 \mathrm{mg}, 0.63 \mathrm{mmol}$, 1 equiv). The $40 \mathrm{~mL}$ vial was sealed and stirred at $45^{\circ} \mathrm{C}$ for 2 hours. After cooled to room temperature, Ru7 , as a green solid, was collected through filtration and further purified by pentane wash $(2 \mathrm{~mL} \times 3)(386 \mathrm{mg}$, $80 \%$ yield). ${ }^{1} \mathrm{H}$ NMR $\left(400 \mathrm{MHz}, \mathrm{C}_{6} \mathrm{D}_{6}\right) \delta 16.84(\mathrm{~d}, J=1.0 \mathrm{~Hz}, 1 \mathrm{H}), 8.07(\mathrm{dd}, J=1.7,0.8 \mathrm{~Hz}, 1 \mathrm{H}), 7.95(\mathrm{~d}$, $J=8.6 \mathrm{~Hz}, 1 \mathrm{H}), 7.78(\mathrm{~d}, J=8.4 \mathrm{~Hz}, 1 \mathrm{H}), 7.74-7.70(\mathrm{~m}, 1 \mathrm{H}), 7.70(\mathrm{dd}, J=2.1,0.9 \mathrm{~Hz}, 1 \mathrm{H}), 7.52(\mathrm{~d}, J=$ $8.6 \mathrm{~Hz}, 1 \mathrm{H}), 7.31(\mathrm{dd}, J=8.4,1.7 \mathrm{~Hz}, 1 \mathrm{H}), 7.03-6.98(\mathrm{~m}, 2 \mathrm{H}), 6.93-6.87(\mathrm{~m}, 1 \mathrm{H}), 6.83(\mathrm{ddd}, J=8.7,7.4$, $1.6 \mathrm{~Hz}, 1 \mathrm{H}), 6.78(\mathrm{dd}, J=7.6,1.6 \mathrm{~Hz}, 1 \mathrm{H}), 6.39(\mathrm{td}, J=7.4,0.9 \mathrm{~Hz}, 1 \mathrm{H}), 6.34(\mathrm{~d}, J=8.3 \mathrm{~Hz}, 1 \mathrm{H}), 3.78$ 
(hept, $J=6.8 \mathrm{~Hz}, 1 \mathrm{H}), 3.67-3.46(\mathrm{~m}, 2 \mathrm{H}), 3.45-3.23(\mathrm{~m}, 2 \mathrm{H}), 3.15$ (hept, $J=7.0 \mathrm{~Hz}, 1 \mathrm{H}), 2.89$ (br, $6 \mathrm{H}$ ), $2.01(\mathrm{~s}, 3 \mathrm{H}), 1.72(\mathrm{~d}, J=12.3 \mathrm{~Hz}, 3 \mathrm{H}), 1.53(\mathrm{~d}, J=12.4 \mathrm{~Hz}, 3 \mathrm{H}), 1.39(\mathrm{t}, J=6.9 \mathrm{~Hz}, 6 \mathrm{H}), 1.30(\mathrm{~d}, J=6.6$ $\mathrm{Hz}, 3 \mathrm{H}), 1.25(\mathrm{~d}, J=6.9 \mathrm{~Hz}, 3 \mathrm{H}) .{ }^{13} \mathrm{C} \mathrm{NMR}\left(101 \mathrm{MHz}, \mathrm{C}_{6} \mathrm{D}_{6}\right) \delta 305.5,211.6,154.6,154.6,148.0,145.5$, 144.5, 137.0, 132.5, 131.8, 129.8, 129.4, 129.2, 126.6, 126.5, 124.1, 123.9, 123.5, 122.9, 122.2, 113.6, 57.1, 53.7, 44.3, 41.7, 36.3, 35.1, 30.4, 28.5, 24.9, 24.2, 23.9, 23.8. HRMS: Chemical fomular ([M]): $\mathrm{C}_{42} \mathrm{H}_{48} \mathrm{Cl}_{2} \mathrm{~N}_{2} \mathrm{ORu}$, calcd. [M] ${ }^{+}$768.2188 Found: 768.2177 .
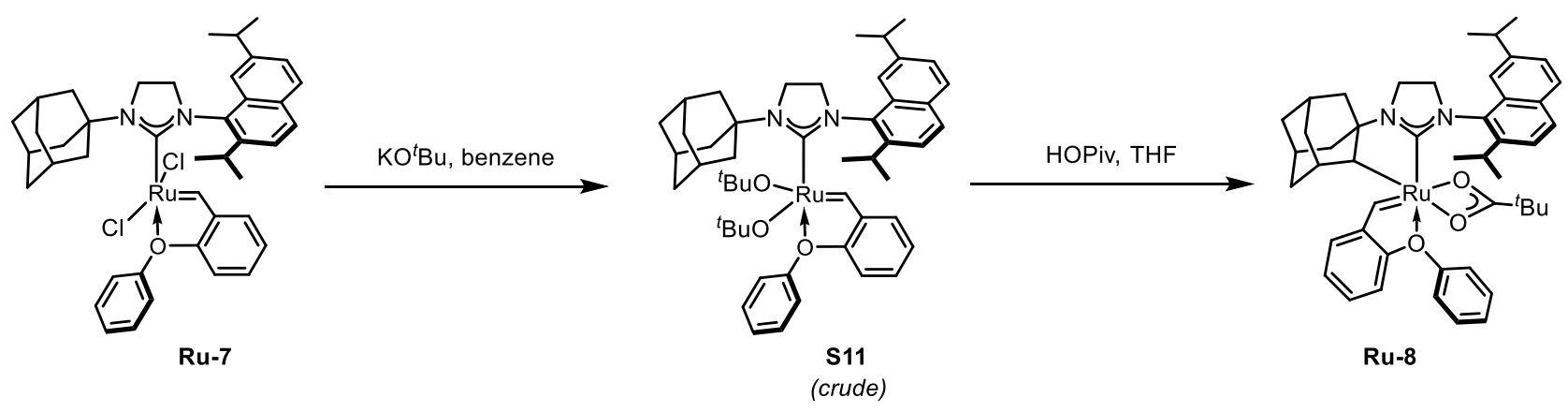

S11: A $20 \mathrm{~mL}$ vial was charged with Ru-7 (347 mg, $0.46 \mathrm{mmol}, 1$ equiv), potassium tert-butoxide (157 mg, $1.4 \mathrm{mmol}, 3$ equiv), and benzene $(13 \mathrm{~mL})$ in a $\mathrm{N}_{2}$ glovebox. The vial was sealed and stirred at room temperature for 18 hours. The reaction mixture was then filtered through a short plug of Celite and concentrated in vacuo. The residue was transferred onto a short Celite column. The column was flushed with pentane $(3 \mathrm{~mL})$ to remove the dark brown band and then a pentane/benzene solution $(8: 1,20 \mathrm{~mL})$ to generate a brown solution. Removal of the solvent furnished crude $\mathbf{S 1 1}$ as a brown solid (310 mg). A ${ }^{1} \mathrm{H}$ NMR spectrum was recorded: ${ }^{1} \mathrm{H}$ NMR $\left(400 \mathrm{MHz}, \mathrm{C}_{6} \mathrm{D}_{6}\right) \delta 16.16(\mathrm{~s}, 1 \mathrm{H}), 7.94-7.55(\mathrm{~m}, 5 \mathrm{H}), 7.54(\mathrm{~d}, J=8.6 \mathrm{~Hz}$, $1 \mathrm{H}), 7.30$ (dd, $J=8.5,1.7 \mathrm{~Hz}, 1 \mathrm{H}), 7.12(\mathrm{dd}, J=8.6,7.3 \mathrm{~Hz}, 3 \mathrm{H}), 6.94-6.89(\mathrm{~m}, 1 \mathrm{H}), 6.84$ (ddd, $J=8.6$, 7.3, $1.6 \mathrm{~Hz}, 1 \mathrm{H}), 6.70(\mathrm{~d}, J=8.2 \mathrm{~Hz}, 1 \mathrm{H}), 6.65(\mathrm{t}, J=7.4 \mathrm{~Hz}, 1 \mathrm{H}), 4.20-4.04(\mathrm{~m}, 1 \mathrm{H}), 3.49-3.31(\mathrm{~m}, 3 \mathrm{H})$, $3.31-3.23(\mathrm{~m}, 1 \mathrm{H}), 3.19$ (p, $J=6.9 \mathrm{~Hz}, 1 \mathrm{H}), 3.12-2.98(\mathrm{~m}, 6 \mathrm{H}), 2.33(\mathrm{~s}, 3 \mathrm{H}), 1.97(\mathrm{~d}, J=12.1 \mathrm{~Hz}, 2 \mathrm{H})$, $1.74(\mathrm{~d}, J=12.3 \mathrm{~Hz}, 2 \mathrm{H}), 1.51(\mathrm{~d}, J=6.6 \mathrm{~Hz}, 3 \mathrm{H}), 1.35-1.32(\mathrm{~m}, 6 \mathrm{H}), 1.24(\mathrm{~d}, J=6.8 \mathrm{~Hz}, 3 \mathrm{H}), 1.11(\mathrm{~s}$, 9H), $0.84(\mathrm{~s}, 9 \mathrm{H})$.

Ru-8: A $20 \mathrm{~mL}$ vial was charged with S11 (280 mg, $0.33 \mathrm{mmol}, 1$ equiv) in THF ( $5 \mathrm{~mL}$, solution A). A $8 \mathrm{~mL}$ vial was charged with PivOH ( $86 \mathrm{mg}, 0.84 \mathrm{mmol}, 2.5$ equiv) in THF $(5 \mathrm{~mL}$, solution $B)$. Both vials were put in the glovebox freezer $\left(-20^{\circ} \mathrm{C}\right)$ for 10 minutes. Solution $B$ was quickly transferred into solution $A$ by using a $6 \mathrm{~mL}$ syringe, and the resulting mixture was put back into the freezer and sat without stirring for 20 minutes. The mixture was then taken out of the freezer and stirred for 30 minutes at room temperature before it was concentrated in vacuo. The residue was transferred onto a short Celite column. The Celite column was flushed with pentane $(3.5 \mathrm{~mL} \times 2)$ to remove the brown band and then benzene $(7 \mathrm{~mL})$ to generate a purple solution. Removal of the solvent furnished Ru-8 as a purple solid (195 mg, 75\% yield). ${ }^{1} \mathrm{H}$ NMR (400 MHz, $\left.\mathrm{C}_{6} \mathrm{D}_{6}\right) \delta 14.87(\mathrm{~s}, 1 \mathrm{H}), 7.72-7.59(\mathrm{~m}, 3 \mathrm{H}), 7.52(\mathrm{dd}, J=7.5,1.7 \mathrm{~Hz}, 1 \mathrm{H}), 7.34(\mathrm{~d}, J=8.6 \mathrm{~Hz}, 1 \mathrm{H}), 7.24-$ $7.18(\mathrm{~m}, 3 \mathrm{H}), 7.06-6.98(\mathrm{~m}, 1 \mathrm{H}), 6.90(\mathrm{t}, J=7.7 \mathrm{~Hz}, 3 \mathrm{H}), 6.77(\mathrm{t}, J=7.4 \mathrm{~Hz}, 1 \mathrm{H}), 6.61(\mathrm{~d}, J=8.1 \mathrm{~Hz}, 1 \mathrm{H})$, $4.45-4.26(\mathrm{~m}, 2 \mathrm{H}), 3.75(\mathrm{q}, J=9.6,8.6 \mathrm{~Hz}, 1 \mathrm{H}), 3.56-3.38(\mathrm{~m}, 2 \mathrm{H}), 3.31(\mathrm{dt}, J=11.3,8.6 \mathrm{~Hz}, 1 \mathrm{H}), 3.00$ (hept, $J=6.9 \mathrm{~Hz}, 1 \mathrm{H}), 2.66(\mathrm{~s}, 1 \mathrm{H}), 2.25-2.01(\mathrm{~m}, 4 \mathrm{H}), 1.81(\mathrm{~d}, J=12.1 \mathrm{~Hz}, 1 \mathrm{H}), 1.71(\mathrm{~s}, 1 \mathrm{H}), 1.64-1.47$ $(\mathrm{m}, 3 \mathrm{H}), 1.47-1.40(\mathrm{~m}, 6 \mathrm{H}), 1.40-1.32(\mathrm{~m}, 6 \mathrm{H}), 1.24-1.14(\mathrm{~m}, 2 \mathrm{H}), 0.71(\mathrm{~d}, J=12.2 \mathrm{~Hz}, 1 \mathrm{H}), 0.30$ (br, 9H). ${ }^{13} \mathrm{C}$ NMR (101 MHz, $\left.\mathrm{C}_{6} \mathrm{D}_{6}\right) \delta 257.5,216.9,156.7,154.6,147.0,144.8,143.3,135.0,132.7,132.4$, 129.6, 128.8, 128.6, 125.6, 125.4, 125.1, 124.9, 123.9, 123.0, 122.3, 120.7, 115.2, 69.0, 62.7, 54.2, 43.8, 41.4, 40.6, 39.0, 38.3, 38.1, 37.0, 34.6, 33.7, 31.2, 30.1, 28.8, 27.2, 25.2, 24.3, 24.1, 23.6. HRMS: Chemical fomular ([M]): $\mathrm{C}_{47} \mathrm{H}_{56} \mathrm{~N}_{2} \mathrm{O}_{3} \mathrm{Ru}$, calcd. [M-H] $]^{+}$797.3257 Found: 797.3243. 


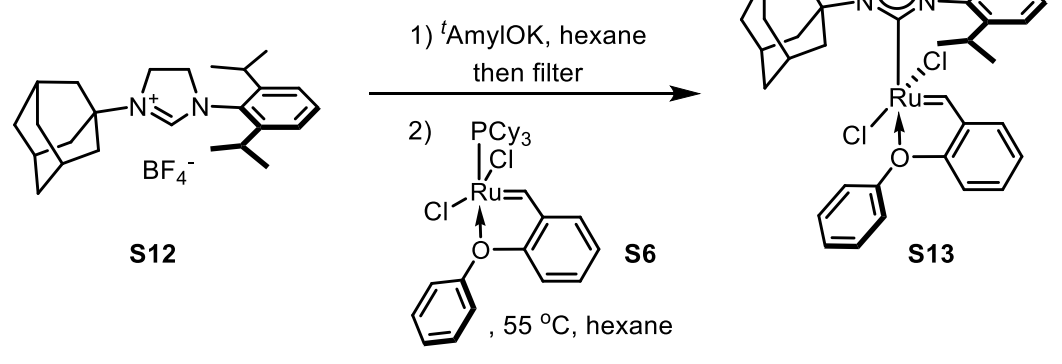

S12 was synthesized according to the literature procedure. ${ }^{1}$

S13: A $40 \mathrm{~mL}$ vial was charged with $\mathbf{S 1 2}$ (452 $\mathrm{mg}, 1 \mathrm{mmol}, 1.47$ equiv) and hexane ( $25 \mathrm{~mL}$ ) in a $\mathrm{N}_{2}$ glovebox. To this was added potassium tert-pentoxide $(0.9 \mathrm{M}$ solution in cyclohexane, $1.1 \mathrm{~mL}, 1 \mathrm{mmol}, 1.47$ equiv). The vial was sealed and stirred at room temperature for 1 hour. The reaction mixture was then filtered through a short plug of Celite into a $40 \mathrm{~mL}$ vial containing $\mathbf{S 6}$ (431 $\mathrm{mg}, 0.68 \mathrm{mmol}, 1$ equiv). The $40 \mathrm{~mL}$ vial was sealed and stirred at $55^{\circ} \mathrm{C}$ for 1 hour. After cooled to room temperature, S13, as a green solid, was collected through filtration and further purified by pentane wash $(3 \mathrm{~mL} \times 3)(422 \mathrm{mg}, 86 \%$ yield $) .{ }^{1} \mathrm{H}$ NMR $(400 \mathrm{MHz}$, $\left.\mathrm{C}_{6} \mathrm{D}_{6}\right) \delta 17.09(\mathrm{~d}, J=1.0 \mathrm{~Hz}, 1 \mathrm{H}), 7.88-7.70(\mathrm{~m}, 2 \mathrm{H}), 7.44(\mathrm{dd}, J=8.3,7.1 \mathrm{~Hz}, 1 \mathrm{H}), 7.32(\mathrm{~d}, J=7.6 \mathrm{~Hz}$, $2 \mathrm{H}), 7.21-7.18(\mathrm{~m}, 1 \mathrm{H}), 7.04-6.95(\mathrm{~m}, 3 \mathrm{H}), 6.94-6.82(\mathrm{~m}, 1 \mathrm{H}), 6.64(\mathrm{t}, J=7.4 \mathrm{~Hz}, 1 \mathrm{H}), 6.45(\mathrm{~d}, J=8.3$ $\mathrm{Hz}, 1 \mathrm{H}), 3.76-3.41$ (m, 4H), 3.34 (dd, $J=11.1,8.3 \mathrm{~Hz}, 2 \mathrm{H}), 2.83$ (br, 6H), 1.99 (br, 3H), 1.70 (d, $J=12.4$ $\mathrm{Hz}, 3 \mathrm{H}), 1.51(\mathrm{~d}, J=12.4 \mathrm{~Hz}, 3 \mathrm{H}), 1.24(\mathrm{~d}, J=6.7 \mathrm{~Hz}, 6 \mathrm{H}), 1.19(\mathrm{~d}, J=6.9 \mathrm{~Hz}, 6 \mathrm{H}) .{ }^{13} \mathrm{C} \mathrm{NMR}(101 \mathrm{MHz}$, $\left.\mathrm{C}_{6} \mathrm{D}_{6}\right) \delta 303.7,210.7,154.9,154.6,149.0,144.7,139.9,129.9,129.7,129.6,126.6,125.2,124.3,123.5$, 123.0, 114.0, 57.1, 54.3, 44.1, 41.8, 36.3, 30.4, 28.3, 26.1, 24.6. HRMS: Chemical fomular ([M]): $\mathrm{C}_{38} \mathrm{H}_{46} \mathrm{Cl}_{2} \mathrm{~N}_{2} \mathrm{ORu}$, calcd. [M] ${ }^{+}$718.2031 Found: 718.2017 .

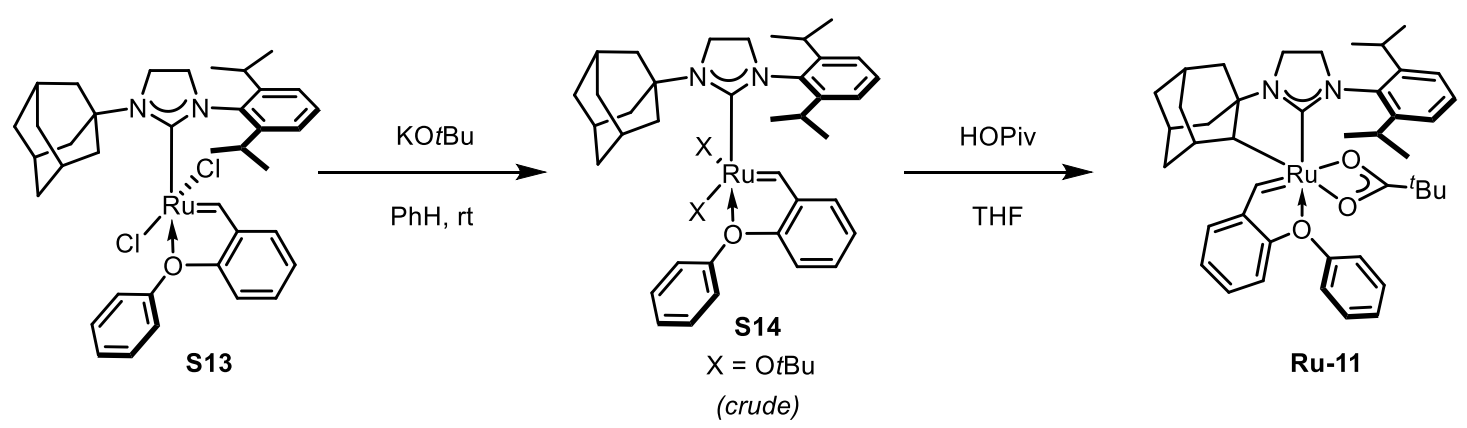

S14: A $20 \mathrm{~mL}$ vial was charged with $\mathbf{S 1 3}(360 \mathrm{mg}, 0.5 \mathrm{mmol}, 1$ equiv), potassium tert-butoxide (170 $\mathrm{mg}$, $1.5 \mathrm{mmol}, 3$ equiv), and benzene $(10 \mathrm{~mL})$ in a $\mathrm{N}_{2}$ glovebox. The vial was sealed and stirred at room temperature for 15 hours. The reaction mixture was then filtered through a short plug of Celite and concentrated in vacuo. The residue was transferred onto a short Celite column. The Celite column was flushed with a small amount of pentane $(4 \mathrm{~mL})$ to remove the impurity and then a large amount of pentane $(120 \mathrm{~mL})$ to obtain the product as a brown solution. Removal of the solvent furnished crude $\mathbf{S 1 4}$ as a brownred solid (355 mg, ca. 90\% purity). A crude NMR spectrum was recorded for S14: ${ }^{1} \mathrm{H}$ NMR (500 MHz, $\mathrm{C}_{6} \mathrm{D}_{6}$ ) $\delta 16.28(\mathrm{~s}, 1 \mathrm{H}), 7.66(\mathrm{br}, 2 \mathrm{H}), 7.29(\mathrm{br}, 3 \mathrm{H}), 7.15-7.08(\mathrm{~m}, 3 \mathrm{H}), 6.95-6.86(\mathrm{~m}, 2 \mathrm{H}), 6.76(\mathrm{t}, J=6.7 \mathrm{~Hz}$, $2 \mathrm{H}), 3.73$ (br, 2H), 3.45 (dd, $J=10.7,8.2 \mathrm{~Hz}, 2 \mathrm{H}), 3.22$ (dd, $J=10.7,8.2 \mathrm{~Hz}, 2 \mathrm{H}), 2.99(\mathrm{~s}, 6 \mathrm{H}), 2.31(\mathrm{~s}, 3 \mathrm{H})$, $1.97(\mathrm{~d}, J=12.2 \mathrm{~Hz}, 3 \mathrm{H}), 1.73(\mathrm{~d}, J=12.2 \mathrm{~Hz}, 3 \mathrm{H}), 1.36(\mathrm{~d}, J=6.6 \mathrm{~Hz}, 6 \mathrm{H}), 1.14(\mathrm{~d}, J=6.8 \mathrm{~Hz}, 6 \mathrm{H}), 1.09$ (s, $18 \mathrm{H}$ ). The crude $\mathbf{S 1 4}$ was directly carried out to the next step without further purification.

Ru-11: A $20 \mathrm{~mL}$ vial was charged with $\mathbf{S 1 4}$ (320 mg, $0.4 \mathrm{mmol}, 1$ equiv) in THF (6.5 mL, solution A). A 8 $\mathrm{mL}$ vial was charged with PivOH ( $82 \mathrm{mg}, 0.8 \mathrm{mmol}, 2$ equiv) in THF $(6.5 \mathrm{~mL}$, solution $B)$. Both vials were put in the glovebox freezer $\left(-20^{\circ} \mathrm{C}\right)$ for 10 minutes. Solution $B$ was quickly transferred into solution $A$ by 
using a $12 \mathrm{~mL}$ syringe, and the resulting mixture was put back into the freezer and sat without stirring for 2.5 hours. The mixture was then taken out of the freezer and stirred for 10 minutes at room temperature before it was concentrated in vacuo. The residue was transferred onto a short Celite column. The column was flushed with pentane $(1.5 \mathrm{~mL} \times 2)$ to remove the brown band and then benzene $(3.5 \mathrm{~mL})$ to obtain the product as a purple solution. Removal of the solvent furnished Ru-11 as a purple solid (200 $\mathrm{mg}, 59 \%$ yield over two steps). ${ }^{1} \mathrm{H}$ NMR $\left(400 \mathrm{MHz}, \mathrm{C}_{6} \mathrm{D}_{6}\right) \delta 14.95(\mathrm{~s}, 1 \mathrm{H}), 7.52(\mathrm{dd}, J=7.5,1.6 \mathrm{~Hz}, 1 \mathrm{H}), 7.28(\mathrm{~d}, J=7.6$ $\mathrm{Hz}, 2 \mathrm{H}), 7.15-7.09$ (m, 2H), $7.05-6.94(\mathrm{~m}, 4 \mathrm{H}), 6.88$ (td, $J=7.4,1.0 \mathrm{~Hz}, 1 \mathrm{H}), 6.81(\mathrm{t}, J=7.4 \mathrm{~Hz}, 1 \mathrm{H})$, $6.67(\mathrm{~d}, J=8.2 \mathrm{~Hz}, 1 \mathrm{H}), 4.23-4.04(\mathrm{~m}, 2 \mathrm{H}), 3.75(\mathrm{dt}, J=11.8,9.6 \mathrm{~Hz}, 1 \mathrm{H}), 3.53(\mathrm{td}, J=10.5,6.1 \mathrm{~Hz}, 1 \mathrm{H})$, $3.48-3.35$ (m, 1H), 3.18 (ddd, $J=12.1,9.6,6.1 \mathrm{~Hz}, 1 \mathrm{H}), 2.95(\mathrm{p}, J=6.9 \mathrm{~Hz}, 1 \mathrm{H}), 2.48(\mathrm{~s}, 1 \mathrm{H}), 2.13-2.00$ (m, 2H), $1.96(\mathrm{~d}, J=12.3 \mathrm{~Hz}, 1 \mathrm{H}), 1.88(\mathrm{~d}, J=10.8 \mathrm{~Hz}, 1 \mathrm{H}), 1.74(\mathrm{dd}, J=12.1,2.3 \mathrm{~Hz}, 1 \mathrm{H}), 1.66(\mathrm{br}, 1 \mathrm{H})$, $1.60-1.45(\mathrm{~m}, 5 \mathrm{H}), 1.45-1.30(\mathrm{~m}, 7 \mathrm{H}), 1.21(\mathrm{~d}, J=6.9 \mathrm{~Hz}, 3 \mathrm{H}), 1.16(\mathrm{~d}, J=9.7 \mathrm{~Hz}, 1 \mathrm{H}), 1.10(\mathrm{~d}, J=11.3$ $\mathrm{Hz}, 1 \mathrm{H}), 0.78(\mathrm{~s}, 9 \mathrm{H}), 0.66(\mathrm{~d}, J=12.4 \mathrm{~Hz}, 1 \mathrm{H}) .{ }^{13} \mathrm{C} \mathrm{NMR}\left(101 \mathrm{MHz}, \mathrm{C}_{6} \mathrm{D}_{6}\right) \delta 258.2,213.3,187.1,156.1$, 155.0, 148.9, 146.8, 143.5, 137.7, 129.6, 128.6, 125.4, 125.3, 125.1, 124.7, 124.1, 122.6, 122.3, 115.8, 68.4, 63.0, 55.0, 43.7, 41.1, 40.6, 39.4, 38.3, 37.6, 36.6, 33.8, 31.0, 30.1, 28.9, 28.8, 28.0, 26.6, 25.2, 23.5. HRMS: Chemical fomular ([M]): $\mathrm{C}_{43} \mathrm{H}_{54} \mathrm{~N}_{2} \mathrm{O}_{3} \mathrm{Ru}$, calcd. $[\mathrm{M}-\mathrm{H}]^{+} 747.3100$ Found: 747.3109 .

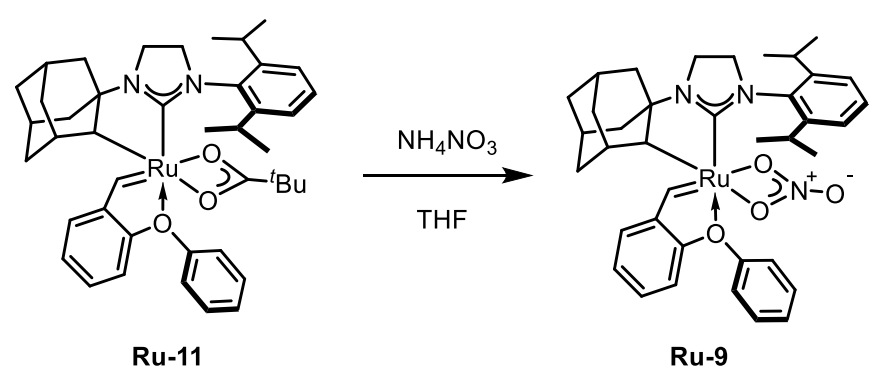

Ru-9: An 8 mL vial was charged with Ru-11 ( $80 \mathrm{mg}, 0.11$ mmol, 1 equiv), $\mathrm{NH}_{4} \mathrm{NO}_{3}$ (104 mg), and THF (3 $\mathrm{mL}$ ) in a $\mathrm{N}_{2}$ glovebox. The vial was sealed and stirred at room temperature for 2.5 hours. The reaction mixture was then filtered through a short plug of Celite and concentrated in vacuo. The residue was transferred onto a short Celite column. The column was flushed with pentane $(3 \mathrm{~mL})$ to remove the impurity and then a benzene/DCM solution $(5: 2 \mathrm{v} / \mathrm{v}, 2 \mathrm{~mL})$ to obtain the product as a purple solution. Removal of the solvent furnished Ru-9 as a purple solid (53 mg, 70\% yield). ${ }^{1} \mathrm{H}$ NMR (400 MHz, $\left.\mathrm{C}_{6} \mathrm{D}_{6}\right) \delta 15.06(\mathrm{~s}, 1 \mathrm{H})$, $7.46(\mathrm{~d}, J=7.4 \mathrm{~Hz}, 1 \mathrm{H}), 7.20(\mathrm{t}, J=7.7 \mathrm{~Hz}, 1 \mathrm{H}), 7.12(\mathrm{dd}, J=7.8,1.7 \mathrm{~Hz}, 1 \mathrm{H}), 7.06(\mathrm{~d}, J=7.3 \mathrm{~Hz}, 3 \mathrm{H})$, $6.97(\mathrm{td}, J=7.8,1.6 \mathrm{~Hz}, 1 \mathrm{H}), 6.90(\mathrm{t}, J=7.8 \mathrm{~Hz}, 2 \mathrm{H}), 6.83(\mathrm{t}, J=7.4 \mathrm{~Hz}, 1 \mathrm{H}), 6.78(\mathrm{t}, J=7.4 \mathrm{~Hz}, 1 \mathrm{H}), 6.41$ $(\mathrm{d}, J=8.2 \mathrm{~Hz}, 1 \mathrm{H}), 4.26(\mathrm{~s}, 1 \mathrm{H}), 3.84(\mathrm{p}, J=6.9 \mathrm{~Hz}, 1 \mathrm{H}), 3.74(\mathrm{td}, J=10.5,8.3 \mathrm{~Hz}, 1 \mathrm{H}), 3.63(\mathrm{td}, J=10.8$, $10.3,7.6 \mathrm{~Hz}, 1 \mathrm{H}), 3.36(\mathrm{td}, J=10.3,7.7 \mathrm{~Hz}, 1 \mathrm{H}), 3.25(\mathrm{dt}, J=11.1,8.6 \mathrm{~Hz}, 1 \mathrm{H}), 3.08$ (hept, $J=6.6 \mathrm{~Hz}, 1 \mathrm{H}$ ), $2.55(\mathrm{~d}, J=3.5 \mathrm{~Hz}, 1 \mathrm{H}), 2.03(\mathrm{~s}, 1 \mathrm{H}), 2.00-1.86(\mathrm{~m}, 2 \mathrm{H}), 1.76(\mathrm{t}, J=10.1 \mathrm{~Hz}, 2 \mathrm{H}), 1.70(\mathrm{~s}, 1 \mathrm{H}), 1.61-$ $1.47(\mathrm{~m}, 5 \mathrm{H}), 1.45(\mathrm{~d}, J=11.9 \mathrm{~Hz}, 1 \mathrm{H}), 1.27(\mathrm{t}, J=3.3 \mathrm{~Hz}, 6 \mathrm{H}), 1.24-1.13(\mathrm{~m}, 2 \mathrm{H}), 1.07(\mathrm{~d}, J=6.8 \mathrm{~Hz}$, $3 \mathrm{H}), 0.76(\mathrm{~d}, J=12.2 \mathrm{~Hz}, 1 \mathrm{H}) .{ }^{13} \mathrm{C}$ NMR $\left(101 \mathrm{MHz}, \mathrm{C}_{6} \mathrm{D}_{6}\right) \delta 264.2,213.0,157.3,154.1,147.8,147.3,142.7$, 135.8, 130.4, 129.2, 126.6, 126.6, 125.1, 124.7, 124.2, 122.7, 122.1, 115.0, 67.3, 63.1, 54.1, 42.9, 41.7, 40.2, 38.1, 37.8, 37.5, 33.4, 30.9, 30.0, 28.9, 28.7, 27.5, 26.7, 23.8, 22.9. HRMS: Chemical fomular ([M]): $\mathrm{C}_{38} \mathrm{H}_{45} \mathrm{~N}_{3} \mathrm{O}_{4} \mathrm{Ru}$, calcd. [M-H] $]^{+} 708.2376$ Found: 708.2364 .
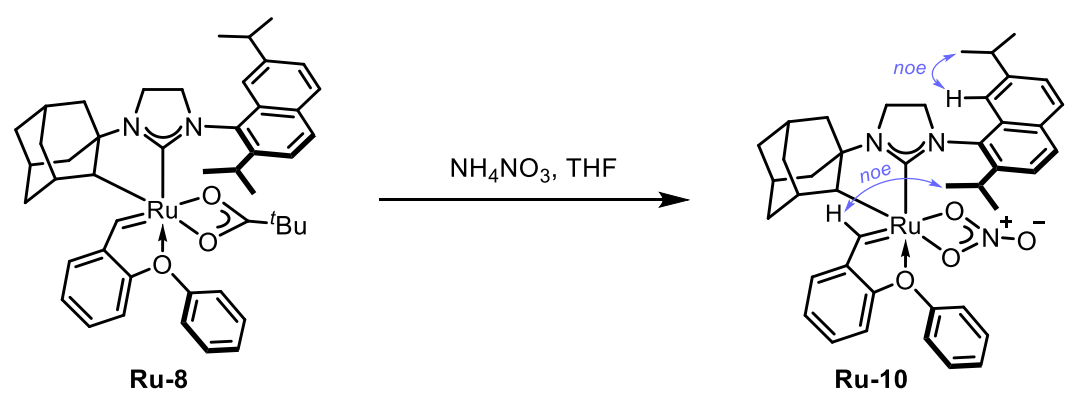
Ru-10: A $20 \mathrm{~mL}$ vial was charged with Ru-8 ( $80 \mathrm{mg}, 0.1 \mathrm{mmol}, 1$ equiv), $\mathrm{NH}_{4} \mathrm{NO}_{3}$ ( $320 \mathrm{mg}$ ) and THF (3 $\mathrm{mL}$, solution $A$ ). After stirring overnight at room temperature, all the salts were removed through filtration, and a new batch of $\mathrm{NH}_{4} \mathrm{NO}_{3}(640 \mathrm{mg})$ was re-added. The resulting suspension was stirred overnight at room temperature, filtered, and concentrated in vacuo. The residue was transferred onto a short Celite column. The column was flushed with a pentane/benzene solution to remove the impurity and then a benzene/DCM solution to obtain the product as a purple solution. Removal of the solvent furnished Ru-10 as a purple solid (60 mg, 79\% yield). ${ }^{1} \mathrm{H}$ NMR (400 MHz, $\left.\mathrm{C}_{6} \mathrm{D}_{6}\right) \delta 14.99(\mathrm{~s}, 1 \mathrm{H}), 7.68$ (dd, $\left.J=8.5,6.5 \mathrm{~Hz}, 2 \mathrm{H}\right), 7.57$ (dd, $J$ $=1.7,0.9 \mathrm{~Hz}, 1 \mathrm{H}), 7.47(\mathrm{dd}, J=7.5,1.6 \mathrm{~Hz}, 1 \mathrm{H}), 7.32(\mathrm{dd}, J=8.4,1.7 \mathrm{~Hz}, 1 \mathrm{H}), 7.22(\mathrm{~d}, J=8.7 \mathrm{~Hz}, 1 \mathrm{H})$, $6.99-6.87(\mathrm{~m}, 3 \mathrm{H}), 6.87-6.75(\mathrm{~m}, 3 \mathrm{H}), 6.72-6.65(\mathrm{~m}, 1 \mathrm{H}), 6.37(\mathrm{dd}, J=8.2,1.0 \mathrm{~Hz}, 1 \mathrm{H}), 4.36(\mathrm{~s}, 1 \mathrm{H})$, 4.06 (hept, $J=6.8 \mathrm{~Hz}, 1 \mathrm{H}), 3.72(\mathrm{td}, J=11.4,10.1 \mathrm{~Hz}, 1 \mathrm{H}), 3.52$ (ddd, $J=9.9,8.5,6.2 \mathrm{~Hz}, 1 \mathrm{H}), 3.37-3.23$ (m, 2H), 3.11 (hept, $J=6.8 \mathrm{~Hz}, 1 \mathrm{H}), 2.55(\mathrm{~d}, J=3.0 \mathrm{~Hz}, 1 \mathrm{H}), 2.16-2.05(\mathrm{~m}, 2 \mathrm{H}), 2.04-1.92(\mathrm{~m}, 2 \mathrm{H}), 1.87$ $-1.77(\mathrm{~m}, 1 \mathrm{H}), 1.73(\mathrm{~s}, 1 \mathrm{H}), 1.58(\mathrm{~d}, J=12.5 \mathrm{~Hz}, 1 \mathrm{H}), 1.54-1.46(\mathrm{~m}, 8 \mathrm{H}), 1.35(\mathrm{~d}, J=2.8 \mathrm{~Hz}, 3 \mathrm{H}), 1.34$ $(\mathrm{d}, J=2.8 \mathrm{~Hz}, 3 \mathrm{H}), 1.26(\mathrm{~d}, J=12.4 \mathrm{~Hz}, 1 \mathrm{H}), 1.16(\mathrm{~d}, J=11.3 \mathrm{~Hz}, 1 \mathrm{H}), 0.77(\mathrm{~d}, J=12.3 \mathrm{~Hz}, 1 \mathrm{H}) .{ }^{13} \mathrm{C}$ NMR $\left(101 \mathrm{MHz}, \mathrm{C}_{6} \mathrm{D}_{6}\right) \delta 263.1,217.6,157.3,154.0,147.7,143.3,142.6,133.5,133.0,132.4,130.2,129.1,128.8$, $128.6,126.5,126.4,125.7,125.0,122.8,122.5,122.0,119.0,114.9,67.9,62.9,53.8,43.4,42.0,40.3,38.0$, $37.8,37.5,35.1,33.6,31.0,30.0,28.7,25.2,24.1,24.0,23.6$. HRMS: Chemical fomular ([M]): $\mathrm{C}_{42} \mathrm{H}_{47} \mathrm{~N}_{3} \mathrm{O}_{4} \mathrm{Ru}$, calcd. [M-H] $]^{+} 758.2573$ Found: 758.2546 . The stereochemistry of the side-arm with respect to the metal center was further determined through 2D NMR analysis.

\section{Catalyst screening}

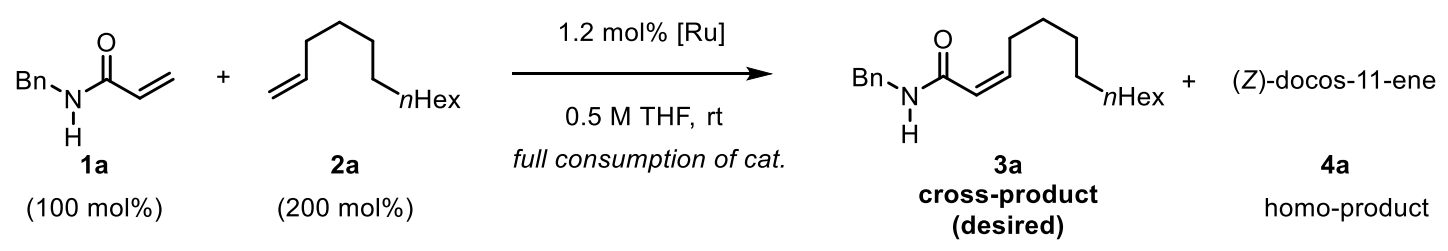

$[R u]$ catalyst tested: $\mathbf{R u - 2}, \mathbf{R u}-\mathbf{6}, \mathbf{R u}-\mathbf{8}, \mathbf{R u}-\mathbf{9}, \mathbf{R u}-\mathbf{1 0}$, and $\mathbf{R u}-\mathbf{1 1}$

The general procedure for catalyst screening: A $4 \mathrm{~mL}$ vial was charged with a stir bar, $1 \mathrm{a}(0.25 \mathrm{mmol}, 1$ equiv, $40.3 \mathrm{mg})$, 2a ( $0.5 \mathrm{mmol}, 2$ equiv, $84 \mathrm{mg}$ ), and anthracene (as the internal standard) in a nitrogen glovebox. To this was added a stock solution of the ruthenium catalyst $(0.003 \mathrm{mmol}, 0.012$ equiv $)$ in $d_{8}$-THF $(0.4 \mathrm{~mL})$. The vial was loosely capped with a screw thread hole cap, and a 20 -gauge needle was used to puncture the cap and left on (Figure S1). The reaction mixture was allowed to stir at room temperature in the glovebox for $4-48$ hours until its color changed from purple to bright yellow (which indicated the complete consumption of the catalyst). The reaction can also be monitored by ${ }^{1} \mathrm{H}$ NMR to determine the quantity of the remaining ruthenium catalyst. Upon full consumption of the catalyst, the reaction was transferred to an NMR tube and removed from the glovebox. The yields and the $Z / E$ ratio of the metathesis products were further determined by ${ }^{1} \mathrm{H}$ NMR analysis of the crude reaction mixture.

Note 1: After 24 hours of reaction, a small amount of $d_{8}$-THF $(0.15 \mathrm{~mL})$ was added to compensate for the loss of the solvent (due to slow evaporation) if the reaction hadn't reached full catalyst consumption.

Note 2: In the model reaction where 1a and 2a has an initial feed ratio of 1:2, we noticed that the ratio between 3a (cross-product) and 4a (homo-product) changes along the course of the reaction. Especially for catalysts Ru-6, Ru-8, and Ru-11 that favor cross-metathesis over homo-dimerization, the ratio between 3a and $4 \mathbf{a}$ decreases as the conversion increases. This can be easily explained: At a high conversion, the realtime ratio between the unreacted $\mathbf{2 a}$ and $\mathbf{1 a}$ would be much larger than 2:1, as most of the 1a has already been consumed at that point. Because of this, and given the fact that the conversion of each reaction varies 
drastically from $23 \%$ to $80 \%$ in the model study, we decided to simply report the NMR yields of crossproduct 3a and homo-product 4a in Scheme 3 of the manuscript, instead of a formal "selectivity ratio" that is calculated based on the final distribution of the products for a certain catalyst.
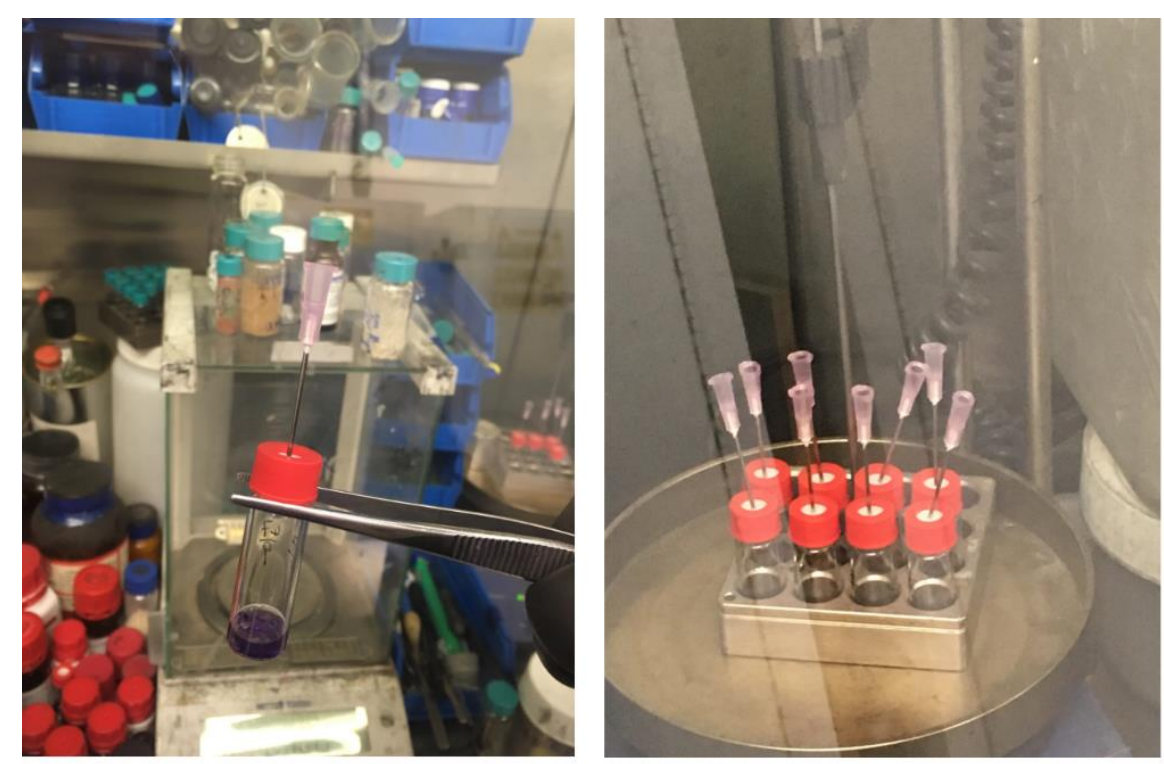

Figure S1 | Reaction setup

When Ru-6 was used as the catalyst, the reaction was further purified by flash column chromatography over silica gel, furnishing pure $Z$-product 3a in 75\% isolated yield as a colorless oil (56.2 mg). Z:E ratio $>20: 1$ (determined by ${ }^{1} \mathrm{H}$ NMR analysis of the crude reaction mixture). $\mathrm{R}_{f}=0.5$ (hexane/ethyl acetate/dichloromethane $=10: 1: 3)$. Chromatography condition: hexane/ethyl acetate/dichloromethane, 20:1:5 to 10:1:3. ${ }^{1} \mathrm{H}$ NMR (400 MHz, $\left.\mathrm{CDCl}_{3}\right) \delta 7.42-7.25(\mathrm{~m}, 5 \mathrm{H}), 6.01(\mathrm{dt}, J=11.4,7.5 \mathrm{~Hz}, 1 \mathrm{H}), 5.81$ (br, 1H), 5.69 (dt, $J=11.4,1.8 \mathrm{~Hz}, 1 \mathrm{H}), 4.47$ (d, $J=5.5 \mathrm{~Hz}, 2 \mathrm{H}), 2.67$ (qd, $J=7.4,1.7 \mathrm{~Hz}, 2 \mathrm{H}), 1.42$ (p, $J=$ $7.4 \mathrm{~Hz}, 2 \mathrm{H}), 1.35-1.23(\mathrm{~m}, 14 \mathrm{H}), 0.88(\mathrm{t}, J=6.9 \mathrm{~Hz}, 3 \mathrm{H}) .{ }^{13} \mathrm{C} \mathrm{NMR}\left(101 \mathrm{MHz}, \mathrm{CDCl}_{3}\right)$ carbon signals were observed at $\delta 166.5,146.5,138.5,128.8,128.0,127.6,121.9,43.5,32.0,29.7,29.6,29.5,29.5,29.5$, 29.0, 22.8, 14.3. IR: 3292, 2919, 2852, 1657, 1631, 1538, 1454, $1237 \mathrm{~cm}^{-1}$. HRMS: Chemical fomular ([M]): $\mathrm{C}_{20} \mathrm{H}_{31} \mathrm{NO}$, calcd. $[\mathrm{M}+\mathrm{H}]^{+} 302.2484$ Found: 302.2492 .

\section{Substrate scope study}

\subsection{Substrate synthesis}

As shown in Figure S2, 2d $,{ }^{6} \mathbf{2 e},{ }^{7} \mathbf{2} \mathbf{p},{ }^{8} \mathbf{1 t},{ }^{9}$ and $\mathbf{1} \mathbf{v}^{10}$ were synthesized according to the literature procedure.

$\mathbf{2 n}, \mathbf{1} \mathbf{u}$, and $\mathbf{1} \mathbf{w}$ were synthesized according to the following procedure (vide infra).

Other substrates were commercially available. 
<smiles>C=CCCCCCOS(C)(=O)=O</smiles>

2d<smiles>C=CC(=O)Nc1ccc(OC)cc1OC</smiles>

$1 t$<smiles>C=CCCC(=O)OCc1ccccc1</smiles>

2e<smiles>C=CC(=O)NC(C)C(F)(F)F</smiles>

1u<smiles>C=CCCC(=O)O[C@H]1CC(C)CC[C@H]1C(=C)C</smiles>

$2 n$<smiles>C=CC(=O)NC(CC(C)C)(CC(C)(C)C)C(=O)OC(C)(C)C</smiles>

1v<smiles>C=CCCC(=O)N1CCc2cc(OC)c(OC)cc2C1c1ccccc1</smiles>

$2 p$<smiles>C=CC(=O)NC1CCC(C(=O)OC)CC1</smiles>

$1 w$

Figure S2 | Substrates synthesized for this study<smiles>[M]C1CC[C@@H](C(=C)C)[C@H](O)C1</smiles>

S16<smiles>C=CCCC(=O)Cl</smiles>

S17<smiles>C=CCCC(=O)O[C@H]1CC(C)CC[C@@H]1C(=C)C</smiles>

2n

2n: A $50 \mathrm{~mL}$ round bottom flask was charged with (-)-isopulegol (S16) (1.46 g, $9.5 \mathrm{mmol}, 1.05$ equiv), pyridine $(1.45 \mathrm{~mL})$, and DCM $(10 \mathrm{~mL})$. The flask was placed into an ice bath. To this was added a DCM (5 $\mathrm{mL}$ ) solution of pent-4-enoyl chloride (S17) $(1.06 \mathrm{~g}, 9 \mathrm{mmol}, 1$ equiv) over 5 minutes. The reaction was then stirred overnight at room temperature. Upon full conversion, the reaction mixture was washed with $1 \mathrm{M} \mathrm{HCl}$ and then saturated aqueous $\mathrm{NaHCO}_{3}$, dried over $\mathrm{Na}_{2} \mathrm{SO}_{4}$, and concentrated in vacuo. The residue was further purified by column chromatography over silica gel (hexane/ethyl acetate, 40:1 to 20:1) to give $\mathbf{2 n}$ as a colorless oil $\left(1.9 \mathrm{~g}, 90 \%\right.$ yield). $\mathrm{R}_{f}=0.5$ (hexane/ethyl acetate $\left.=20: 1\right) .{ }^{1} \mathrm{H}$ NMR $\left(400 \mathrm{MHz}, \mathrm{CDCl}_{3}\right) \delta 5.90$ $-5.68(\mathrm{~m}, 1 \mathrm{H}), 5.03(\mathrm{dd}, J=16.9,1.7 \mathrm{~Hz}, 1 \mathrm{H}), 4.98(\mathrm{dd}, J=10.0,1.7 \mathrm{~Hz}, 1 \mathrm{H}), 4.80(\mathrm{td}, J=10.9,4.4 \mathrm{~Hz}$, $1 \mathrm{H}), 4.72(\mathrm{t}, J=1.4 \mathrm{~Hz}, 2 \mathrm{H}), 2.42-2.30(\mathrm{~m}, 4 \mathrm{H}), 2.10(\mathrm{ddd}, J=12.5,10.7,3.7 \mathrm{~Hz}, 1 \mathrm{H}), 2.02-1.94(\mathrm{~m}$, $1 \mathrm{H}), 1.75-1.63(\mathrm{~m}, 5 \mathrm{H}), 1.62-1.48(\mathrm{~m}, 1 \mathrm{H}), 1.46-1.31(\mathrm{~m}, 1 \mathrm{H}), 1.06-0.87(\mathrm{~m}, 5 \mathrm{H}) .{ }^{13} \mathrm{C}$ NMR $(101$ $\left.\mathrm{MHz}, \mathrm{CDCl}_{3}\right) \delta 172.6,146.4,137.0,115.4,111.9,73.6,50.9,40.6,34.3,33.9,31.5,30.6,29.2,22.2,19.6$. IR: 3076, 2954, 2925, 1733, 1453, 1252, 1170, $1129 \mathrm{~cm}^{-1}$. HRMS: Chemical fomular ([M]): $\mathrm{C}_{15} \mathrm{H}_{24} \mathrm{O}_{2}$, calcd. $[\mathrm{M}+\mathrm{H}]^{+} 237.1855$ Found: 237.1836 .

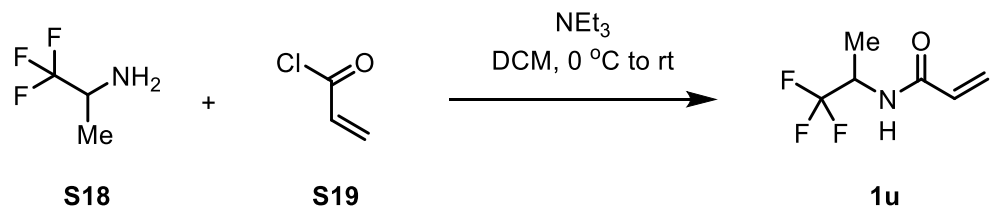

1u: To a $40 \mathrm{~mL}$ vial under an argon atmosphere was added 1,1,1-trifluoropropan-2-amine (S18) $(800 \mathrm{mg}$, $7.1 \mathrm{mmol}, 1.0$ equiv), triethylamine (790 $\mathrm{mg}, 7.8 \mathrm{mmol}, 1.1$ equiv), and DCM (15 mL). The solution was cooled to $0{ }^{\circ} \mathrm{C}$ and a solution of acryloyl chloride ( $706 \mathrm{mg}, 7.8 \mathrm{mmol}, 1.1$ equiv) in DCM (15 mL) was added dropwise with stirring. The solution was stirred for 5 min further and then removed from the ice bath and allowed to warm to room temperature, where it was stirred for $48 \mathrm{~h}$ and monitored by TLC analysis. The reaction was filtered, rinsed with DCM $(5 \mathrm{~mL})$ and the filtrate was concentrated under reduced pressure. The residue was further purified by column chromatography over silica gel (dichloromethane/ethyl acetate = 20:1) to give $1 \mathbf{u}$ as a white solid (785 mg, $67 \%) .{ }^{1} \mathrm{H}$ NMR (400 MHz, $\left.\mathrm{CDCl}_{3}\right) \delta 6.36(\mathrm{dd}, J=17.0,1.2 \mathrm{~Hz}$, $1 \mathrm{H}), 6.12$ (dd, $J=17.0,10.3 \mathrm{~Hz}, 1 \mathrm{H}), 5.85-5.65(\mathrm{~m}, 2 \mathrm{H}), 4.80$ (dhept, $J=9.4,7.1 \mathrm{~Hz}, 1 \mathrm{H}), 1.35$ (d, $J=6.8$ $\mathrm{Hz}, 3 \mathrm{H}) .{ }^{13} \mathrm{C} \mathrm{NMR}\left(101 \mathrm{MHz}, \mathrm{CDCl}_{3}\right) \delta 165.0,129.9,128.4,125.4(\mathrm{q}, J=280.9 \mathrm{~Hz}), 46.5(\mathrm{q}, J=31.7 \mathrm{~Hz})$, 
$14.6(\mathrm{q}, J=2.1 \mathrm{~Hz}) .{ }^{19} \mathrm{~F}$ NMR $\left(282 \mathrm{MHz}, \mathrm{CDCl}_{3}\right) \delta-77.51(\mathrm{~d}, J=7.4 \mathrm{~Hz}, 3 \mathrm{~F})$. IR: $3274,3066,3000,1663$, 1544, 1274, 1159, $1139 \mathrm{~cm}^{-1}$. HRMS: Chemical fomular ([M]): $\mathrm{C}_{6} \mathrm{H}_{8} \mathrm{~F}_{3} \mathrm{NO}$, calcd. $[\mathrm{M}+\mathrm{H}]^{+} 168.0636$ Found: 168.0655 .

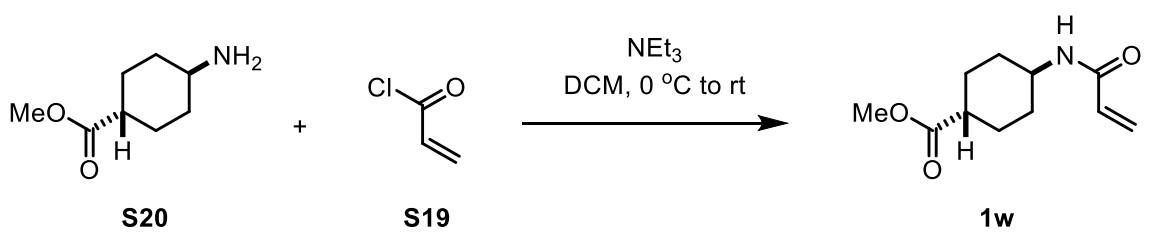

1w: A $100 \mathrm{~mL}$ round bottom flask was charged with $\mathbf{S 2 0}$ (1.05 g, $6.7 \mathrm{mmol}, 1$ equiv), triethylamine $(0.81 \mathrm{~g}$, $8 \mathrm{mmol}, 1.2$ equiv) and DCM $(20 \mathrm{~mL})$. The flask was placed into an ice bath. To this was added a DCM (10 $\mathrm{mL}$ ) solution of acryloyl chloride ( $1.36 \mathrm{~g}, 15 \mathrm{mmol}, 2.4$ equiv) over 5 minutes. The reaction was then stirred overnight at room temperature. Upon full conversion, the reaction mixture was filtered through a short pad of $\mathrm{Na}_{2} \mathrm{SO}_{4}$ and concentrated in vacuo. The residue was further purified by column chromatography over silica gel (hexane/acetone, $4: 1$ to $2: 1$ ) to give $1 \mathbf{w}$ as a white solid (1.05 g, $74 \%$ yield). $\mathrm{R}_{f}=0.3$ (hexane/acetone = 3:2). ${ }^{1} \mathrm{H}$ NMR $\left(400 \mathrm{MHz}, \mathrm{CDCl}_{3}\right) \delta 6.25(\mathrm{dd}, J=16.9,1.5 \mathrm{~Hz}, 1 \mathrm{H}), 6.05(\mathrm{dd}, J=17.0$, $10.2 \mathrm{~Hz}, 1 \mathrm{H}), 5.72-5.47(\mathrm{~m}, 2 \mathrm{H}), 3.82(\mathrm{dtd}, J=11.7,7.8,4.0 \mathrm{~Hz}, 1 \mathrm{H}), 3.65(\mathrm{~s}, 3 \mathrm{H}), 2.23(\mathrm{tt}, J=12.1,3.5$ $\mathrm{Hz}, 1 \mathrm{H}), 2.12-1.95(\mathrm{~m}, 4 \mathrm{H}), 1.55(\mathrm{qd}, J=13.1,3.0 \mathrm{~Hz}, 2 \mathrm{H}), 1.26-1.09(\mathrm{~m}, 2 \mathrm{H}) .{ }^{13} \mathrm{C} \mathrm{NMR}(101 \mathrm{MHz}$, $\left.\mathrm{CDCl}_{3}\right) \delta 175.8,164.8,131.1,126.5,51.8,47.9,42.5,32.2$, 27.8. IR: 3249, 3067, 2930, 2860, 1724, 1617, 1560, 1433, 1253, 1196, $1149 \mathrm{~cm}^{-1}$. HRMS: Chemical fomular ([M]): $\mathrm{C}_{11} \mathrm{H}_{17} \mathrm{NO}_{3}$, calcd. $[\mathrm{M}+\mathrm{H}]^{+} 212.1287$ Found: 212.1265 .

\subsection{General procedure}

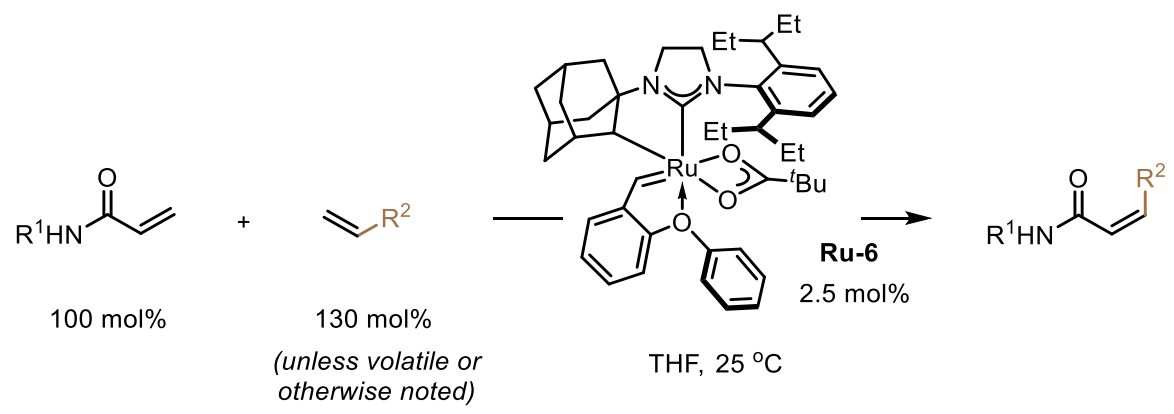

A $4 \mathrm{~mL}$ vial was charged with a stir bar, an acrylamide substrate $(0.25 \mathrm{mmol}, 1$ equiv), and an olefin substrate ( $0.325 \mathrm{mmol}, 1.3$ equiv) in a nitrogen glovebox. To this was added a stock solution of Ru-6 (5.0 mg, 0.00625 mmol, 0.025 equiv) in THF $(0.4 \mathrm{~mL})$. The vial was loosely capped with a screw thread hole cap, and a 20gauge needle was used to puncture the cap and left on (see Figure S1). The reaction mixture was allowed to stir at room temperature in the glovebox for $12-40$ hours until its color changed from purple to bright yellow (which indicates the complete consumption of the catalyst). The reaction was then removed from the glovebox, concentrated, and further purified by flash column chromatography over silica. The reported yield was the isolated yield of the $Z$-metathesis product. Unless otherwise noted, the $Z / E$ ratio of the metathesis product was determined by ${ }^{1} \mathrm{H}$ NMR analysis of the crude reaction mixture using $d_{8}$-THF or $\mathrm{CDCl}_{3}$ as the NMR solvent.

Note: In some cases, the metathesis reaction was also conducted in $d_{8}$-THF (instead of THF). No difference in the reaction outcome was observed. 


\subsection{Product characterization}<smiles>O=CCCCCCCCC=CC(=O)NCc1ccccc1</smiles>

3b: White solid (53.3 mg, 71\% yield). M.p. $=54-56^{\circ} \mathrm{C}$. Reaction time $=24$ hours. $Z: E$ ratio $>20: 1$ (determined by ${ }^{1} \mathrm{H}$ NMR analysis of the crude reaction mixture). $\mathrm{R}_{f}=0.3$ (hexane/ethyl acetate/dichloromethane $=5: 1: 2)$. Chromatography condition: hexane/ethyl acetate/dichloromethane, 10:1:3. ${ }^{1} \mathrm{H} \mathrm{NMR}\left(400 \mathrm{MHz}, \mathrm{CDCl}_{3}\right) \delta 9.73(\mathrm{t}, J=1.9 \mathrm{~Hz}, 1 \mathrm{H}), 7.35-7.25(\mathrm{~m}, 5 \mathrm{H}), 6.00(\mathrm{dt}, J=11.5,7.4 \mathrm{~Hz}, 1 \mathrm{H})$, $5.89(\mathrm{br}, 1 \mathrm{H}), 5.69(\mathrm{dt}, J=11.5,1.7 \mathrm{~Hz}, 1 \mathrm{H}), 4.45(\mathrm{~d}, J=5.6 \mathrm{~Hz}, 2 \mathrm{H}), 2.66(\mathrm{qd}, J=7.4,1.7 \mathrm{~Hz}, 2 \mathrm{H}), 2.40$ $(\operatorname{td}, J=7.4,1.9 \mathrm{~Hz}, 2 \mathrm{H}), 1.60(\mathrm{p}, J=7.0 \mathrm{~Hz}, 2 \mathrm{H}), 1.47-1.36(\mathrm{~m}, 2 \mathrm{H}), 1.36-1.23(\mathrm{~m}, 8 \mathrm{H}) .{ }^{13} \mathrm{C}$ NMR $(101$ $\left.\mathrm{MHz}, \mathrm{CDCl}_{3}\right) \delta 203.2,166.5,146.4,138.5,128.8,128.0,127.6,122.0,44.0,43.4,29.4,29.4,29.3,29.3$, 29.2, 28.9, 22.2. IR: 3296, 2924, 2853, 1721, 1657, 1631, 1533, 1454, $1234 \mathrm{~cm}^{-1}$. HRMS: Chemical fomular ([M]): $\mathrm{C}_{19} \mathrm{H}_{27} \mathrm{NO}_{2}$, calcd. [M+H] $]^{+} 302.2120$ Found: 302.2106 .

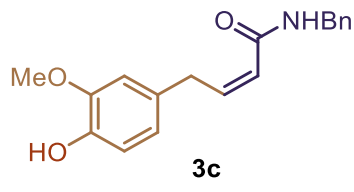

3c: Colorless oil $(55.5 \mathrm{mg}, 75 \%$ yield). Reaction time $=24$ hours. $Z: E$ ratio $>20: 1$ (determined by chromatography isolation of the crude reaction mixture). $\mathrm{R}_{f}=0.25$ (hexane/ethyl acetate/dichloromethane $=$ 3:1:1). Chromatography condition: hexane/ethyl acetate/dichloromethane, 5:1:2 to 3:1:1. ${ }^{1} \mathrm{H}$ NMR (400 $\left.\mathrm{MHz}, \mathrm{CDCl}_{3}\right) \delta 7.37-7.27(\mathrm{~m}, 5 \mathrm{H}), 6.83(\mathrm{~d}, J=8.0 \mathrm{~Hz}, 1 \mathrm{H}), 6.76(\mathrm{~d}, J=1.9 \mathrm{~Hz}, 1 \mathrm{H}), 6.71(\mathrm{dd}, J=8.0,2.0$ $\mathrm{Hz}, 1 \mathrm{H}), 6.15(\mathrm{dt}, J=11.3,7.6 \mathrm{~Hz}, 1 \mathrm{H}), 5.92(\mathrm{br}, 1 \mathrm{H}), 5.76(\mathrm{dt}, J=11.3,1.7 \mathrm{~Hz}, 1 \mathrm{H}), 4.50(\mathrm{~d}, J=5.6 \mathrm{~Hz}$, $2 \mathrm{H}), 3.99(\mathrm{dd}, J=7.6,1.7 \mathrm{~Hz}, 2 \mathrm{H}), 3.84(\mathrm{~s}, 3 \mathrm{H}) .{ }^{13} \mathrm{C} \mathrm{NMR}\left(101 \mathrm{MHz}, \mathrm{CDCl}_{3}\right) \delta 166.3,146.7,144.8,144.1$, 138.3, 132.0, 128.8, 128.0, 127.7, 121.8, 121.2, 114.5, 111.4, 56.0, 43.5, 34.7. IR: 3296, 3029, 2935, 1656, 1514, 1268, 1232, $1150 \mathrm{~cm}^{-1}$. HRMS: Chemical fomular ([M]): $\mathrm{C}_{18} \mathrm{H}_{19} \mathrm{NO}_{3}$, calcd. $[\mathrm{M}+\mathrm{H}]^{+} 298.1443$ Found: 298.1427 .<smiles>CS(=O)(=O)OCCCC/C=C\C(=O)NCBr</smiles>

3d: White solid ( $60.3 \mathrm{mg}, 78 \%$ yield). Reaction time $=15$ hours. $Z: E$ ratio $>20: 1$ (determined by ${ }^{1} \mathrm{H}$ NMR analysis of the crude reaction mixture). $\mathrm{R}_{f}=0.15$ (hexane/ethyl acetate/dichloromethane $=2: 1: 1$ ). Chromatography condition: hexane/ethyl acetate/dichloromethane, 3:1:1 to 2:1:1. ${ }^{1} \mathrm{H}$ NMR $(400 \mathrm{MHz}$, $\left.\mathrm{CDCl}_{3}\right) \delta 7.36-7.24(\mathrm{~m}, 5 \mathrm{H}), 6.17-5.81(\mathrm{~m}, 2 \mathrm{H}), 5.73(\mathrm{dt}, J=11.4,1.6 \mathrm{~Hz}, 1 \mathrm{H}), 4.44(\mathrm{~d}, J=5.4 \mathrm{~Hz}, 2 \mathrm{H})$, $4.22(\mathrm{t}, J=6.4 \mathrm{~Hz}, 2 \mathrm{H}), 2.97$ (s, 3H), $2.74(\mathrm{qd}, J=7.5,1.6 \mathrm{~Hz}, 2 \mathrm{H}), 1.78(\mathrm{dq}, J=8.3,6.3 \mathrm{~Hz}, 2 \mathrm{H}), 1.56$ (p, $J=7.5 \mathrm{~Hz}, 2 \mathrm{H}) .{ }^{13} \mathrm{C}$ NMR $\left(101 \mathrm{MHz}, \mathrm{CDCl}_{3}\right) \delta 166.2,145.3,138.4,128.8,127.9,127.6,122.7,70.1,43.4$, 37.3, 28.6, 27.8, 25.0. IR: 3304, 3028, 2935, 1660, 1632, 1526, 1347, 1176, $972 \mathrm{~cm}^{-1}$. HRMS: Chemical fomular ([M]): $\mathrm{C}_{15} \mathrm{H}_{21} \mathrm{NO}_{4} \mathrm{~S}$, calcd. $[\mathrm{M}+\mathrm{H}]^{+} 312.1270$ Found: 312.1290 .<smiles>O=C(/C=C\CCC(=O)OCc1ccccc1)NCc1ccccc1</smiles> 
3e: Colorless oil $\left(57 \mathrm{mg}, 71 \%\right.$ yield). Reaction time $=15$ hours. $Z: E$ ratio $>20: 1$ (determined by ${ }^{1} \mathrm{H}$ NMR analysis of the crude reaction mixture). $\mathrm{R}_{f}=0.3$ (hexane/ethyl acetate/dichloromethane $=3: 1: 1$ ). Chromatography condition: hexane/ethyl acetate/dichloromethane, 10:1:3 to 3:1:1. ${ }^{1} \mathrm{H}$ NMR (400 MHz, $\left.\mathrm{CDCl}_{3}\right) \delta 7.41-7.23(\mathrm{~m}, 10 \mathrm{H}), 6.52(\mathrm{br}, 1 \mathrm{H}), 5.94(\mathrm{dt}, J=11.5,7.6 \mathrm{~Hz}, 1 \mathrm{H}), 5.77(\mathrm{dt}, J=11.5,1.5 \mathrm{~Hz}, 1 \mathrm{H})$, $5.10(\mathrm{~s}, 2 \mathrm{H}), 4.48(\mathrm{~d}, J=5.7 \mathrm{~Hz}, 2 \mathrm{H}), 2.91(\mathrm{qd}, J=7.1,1.5 \mathrm{~Hz}, 2 \mathrm{H}), 2.55(\mathrm{t}, J=6.9 \mathrm{~Hz}, 2 \mathrm{H}) .{ }^{13} \mathrm{C}$ NMR $(101$ $\left.\mathrm{MHz}, \mathrm{CDCl}_{3}\right) \delta 173.2,166.2,141.6,138.4,135.9,128.8,128.7,128.4,128.0,127.5,124.1,66.6,43.5,33.4$, 24.2. The spectra match the reported literature. ${ }^{11}$

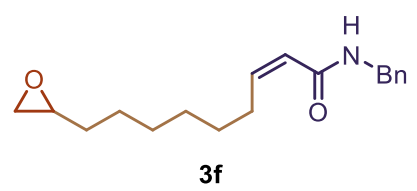

3f: Colorless oil (52.1 mg, 73\% yield). Reaction time $=20$ hours. $Z: E$ ratio $>20: 1$ (determined by ${ }^{1} \mathrm{H}$ NMR analysis of the crude reaction mixture). $\mathrm{R}_{f}=0.35$ (hexane/ethyl acetate/dichloromethane $=3: 1: 1$ ). Chromatography condition: hexane/ethyl acetate/dichloromethane, 10:1:3 to 3:1:1. ${ }^{1} \mathrm{H}$ NMR $(400 \mathrm{MHz}$, $\left.\mathrm{CDCl}_{3}\right) \delta 7.37-7.22(\mathrm{~m}, 5 \mathrm{H}), 6.00(\mathrm{dt}, J=11.5,7.5 \mathrm{~Hz}, 1 \mathrm{H}), 5.87(\mathrm{br}, 1 \mathrm{H}), 5.69(\mathrm{dt}, J=11.5,1.7 \mathrm{~Hz}, 1 \mathrm{H})$, $4.45(\mathrm{~d}, J=5.7 \mathrm{~Hz}, 2 \mathrm{H}), 2.88(\mathrm{qd}, J=5.4,3.3 \mathrm{~Hz}, 1 \mathrm{H}), 2.72(\mathrm{dd}, J=5.0,4.0 \mathrm{~Hz}, 1 \mathrm{H}), 2.67(\mathrm{td}, J=7.5,1.7$ $\mathrm{Hz}, 2 \mathrm{H}), 2.44(\mathrm{dd}, J=5.0,2.8 \mathrm{~Hz}, 1 \mathrm{H}), 1.56-1.25(\mathrm{~m}, 10 \mathrm{H}) .{ }^{13} \mathrm{C} \mathrm{NMR}\left(101 \mathrm{MHz}, \mathrm{CDCl}_{3}\right) \delta 166.5,146.4$, 138.5, 128.8, 128.0, 127.6, 122.0, 52.5, 47.2, 43.4, 32.5, 29.3, 29.3, 29.3, 28.8, 26.0. IR: 3301, 3029, 2925, 28540, 1658, 1632, 1533, $1236 \mathrm{~cm}^{-1}$. HRMS: Chemical fomular ([M]): $\mathrm{C}_{18} \mathrm{H}_{25} \mathrm{NO}_{2}$, calcd. $[\mathrm{M}+\mathrm{H}]^{+} 288.1964$ Found: 288.1957 .<smiles>O=C(NCc1ccccc1)NCC1CCCCC1</smiles>

3g: Following a modified General procedure, the reaction was conducted with the use of 1.6 equivalent (instead of 1.3 equiv.) of allylcyclohexane (b.p. $=150^{\circ} \mathrm{C}, 50 \mathrm{mg}$ ). The titled compound was isolated as a white soild $\left(57.7 \mathrm{mg}, 90 \%\right.$ yield). M.p. $=77-79^{\circ} \mathrm{C}$. Reaction time $=36$ hours. $Z: E$ ratio $>20: 1$ (determined by ${ }^{1} \mathrm{H}$ NMR analysis of the crude reaction mixture). $\mathrm{R}_{f}=0.3$ (hexane/ethyl acetate/dichloromethane $=10: 1: 3$ ). Chromatography condition: hexane/ethyl acetate/dichloromethane, 20:1:5 to 10:1:3. ${ }^{1} \mathrm{H}$ NMR $(400 \mathrm{MHz}$, $\left.\mathrm{CDCl}_{3}\right) \delta 7.37-7.26(\mathrm{~m}, 5 \mathrm{H}), 6.04(\mathrm{dt}, J=11.5,7.6 \mathrm{~Hz}, 1 \mathrm{H}), 5.83(\mathrm{br}, 1 \mathrm{H}), 5.73(\mathrm{dt}, J=11.5,1.7 \mathrm{~Hz}, 1 \mathrm{H})$, $4.46(\mathrm{~d}, J=5.5 \mathrm{~Hz}, 2 \mathrm{H}), 2.57(\mathrm{td}, J=7.3,1.7 \mathrm{~Hz}, 2 \mathrm{H}), 1.75-1.57(\mathrm{~m}, 5 \mathrm{H}), 1.38(\mathrm{tdq}, J=14.2,6.9,3.5 \mathrm{~Hz}$, $1 \mathrm{H}), 1.32-1.08(\mathrm{~m}, 3 \mathrm{H}), 1.08-0.89(\mathrm{~m}, 2 \mathrm{H}) .{ }^{13} \mathrm{C} \mathrm{NMR}\left(101 \mathrm{MHz}, \mathrm{CDCl}_{3}\right) \delta 166.6,145.1,138.5,128.8$, 128.0, 127.6, 122.6, 43.4, 38.3, 36.4, 33.2, 26.6, 26.4. IR: 3294, 2920, 2849, 1653, 1625, 1536, 1447, 1239 $\mathrm{cm}^{-1}$. HRMS: Chemical fomular ([M]): $\mathrm{C}_{17} \mathrm{H}_{23} \mathrm{NO}$, calcd. [M+H] $]^{+} 258.1858$ Found: 258.1834 .<smiles>CC(C)(C)C1(C)OB(CCC/C=C\C(=O)NCc2ccccc2)OC1(C)C</smiles>

3h: Colorless oil ( $49 \mathrm{mg}, 60 \%$ yield). Reaction time $=24$ hours. $Z: E$ ratio $>20: 1$ (determined by ${ }^{1} \mathrm{H}$ NMR analysis of the crude reaction mixture). $\mathrm{R}_{f}=0.4$ (hexane/ethyl acetate/dichloromethane $=3: 1: 1$ ). Chromatography condition: hexane/acetone/dichloromethane, 10:1:3. ${ }^{1} \mathrm{H}$ NMR (400 MHz, $\left.\mathrm{C}_{6} \mathrm{D}_{6}\right) \delta 7.13-$ $6.99(\mathrm{~m}, 5 \mathrm{H}), 5.83(\mathrm{dt}, J=11.4,7.5 \mathrm{~Hz}, 1 \mathrm{H}), 5.57-4.99(\mathrm{~m}, 2 \mathrm{H}), 4.26$ (d, $J=6.0 \mathrm{~Hz}, 2 \mathrm{H}), 2.98$ (qd, $J=7.5$, $1.7 \mathrm{~Hz}, 2 \mathrm{H}), 1.72(\mathrm{p}, J=7.7 \mathrm{~Hz}, 2 \mathrm{H}), 1.07(\mathrm{~s}, 12 \mathrm{H}), 0.99(\mathrm{~d}, J=7.7 \mathrm{~Hz}, 2 \mathrm{H}) .{ }^{13} \mathrm{C}$ NMR $\left(101 \mathrm{MHz}, \mathrm{C}_{6} \mathrm{D}_{6}\right) \delta$ 
165.8, 145.9, 139.7, 128.7, 128.1, 127.2, 122.8, 82.9, 43.2, 31.4, 25.0, 24.5, 11.34. IR: 3296, 2977, 2928, 1657, 1537, 1371, 1315, $1142 \mathrm{~cm}^{-1}$. HRMS: Chemical fomular ([M]): $\mathrm{C}_{19} \mathrm{H}_{28} \mathrm{BNO}_{3}$, calcd. $[\mathrm{M}+\mathrm{H}]^{+} 330.2241$ Found: 330.2253 .<smiles>O=C(/C=C\CCO)NCBr</smiles>

3i: Following a modified General procedure, the reaction was conducted with the use of 1.6 equivalent (instead of 1.3 equiv.) of pent-4-en-1-ol (b.p. $=133{ }^{\circ} \mathrm{C}, 34.5 \mathrm{mg}$ ). The titled compound was isolated as a colorless oil $\left(43.6 \mathrm{mg}, 80 \%\right.$ yield). Reaction time $=15$ hours. $Z: E$ ratio $>20: 1$ (determined by ${ }^{1} \mathrm{H}$ NMR analysis of the crude reaction mixture). $\mathrm{R}_{f}=0.15$ (hexane/ethyl acetate/dichloromethane $=3: 2: 2$ ). Chromatography condition: hexane/ethyl acetate/dichloromethane, 3:1:1 to $3: 2: 2 .{ }^{1} \mathrm{H}$ NMR $(400 \mathrm{MHz}$, $\left.\mathrm{CDCl}_{3}\right) \delta 7.38-7.26(\mathrm{~m}, 5 \mathrm{H}), 6.21(\mathrm{br}, 1 \mathrm{H}), 5.99(\mathrm{dt}, J=11.5,8.6 \mathrm{~Hz}, 1 \mathrm{H}), 5.84(\mathrm{~d}, J=11.5 \mathrm{~Hz}, 1 \mathrm{H}), 4.47$ $(\mathrm{d}, J=5.5 \mathrm{~Hz}, 2 \mathrm{H}), 3.58(\mathrm{t}, J=5.6 \mathrm{~Hz}, 2 \mathrm{H}), 3.16(\mathrm{br}, 1 \mathrm{H}), 2.73(\mathrm{q}, J=7.8 \mathrm{~Hz}, 2 \mathrm{H}), 1.76-1.59(\mathrm{~m}, 2 \mathrm{H}) .{ }^{13} \mathrm{C}$ $\operatorname{NMR}\left(101 \mathrm{MHz}, \mathrm{CDCl}_{3}\right) \delta 167.0,144.9,138.0,128.9,128.1,127.7,123.5,60.0,43.7,30.5,24.7$. The spectra match the reported literature. ${ }^{11}$<smiles>COCCCC/C=C\C(=O)NCc1ccccc1</smiles>

3j: Colorless oil (48 mg, 70\% yield). Reaction time $=15$ hours. $Z: E$ ratio $>20: 1$ (determined by ${ }^{1} \mathrm{H}$ NMR analysis of the crude reaction mixture). $\mathrm{R}_{f}=0.6$ (hexane/acetone/dichloromethane $=3: 1: 1$ ). Chromatography condition: hexane/acetone/dichloromethane, 10:1:3 to $5: 1: 2 .{ }^{1} \mathrm{H}$ NMR $\left(400 \mathrm{MHz}, \mathrm{CDCl}_{3}\right) \delta 7.36-7.24(\mathrm{~m}$, $5 \mathrm{H}), 5.99(\mathrm{dt}, J=11.5,7.5 \mathrm{~Hz}, 1 \mathrm{H}), 5.91(\mathrm{br}, 1 \mathrm{H}), 5.71(\mathrm{dt}, J=11.4,1.7 \mathrm{~Hz}, 1 \mathrm{H}), 4.45(\mathrm{~d}, J=5.7 \mathrm{~Hz}, 2 \mathrm{H})$, $4.05(\mathrm{t}, J=6.6 \mathrm{~Hz}, 2 \mathrm{H}), 2.72(\mathrm{qd}, J=7.5,1.7 \mathrm{~Hz}, 2 \mathrm{H}), 2.02(\mathrm{~s}, 3 \mathrm{H}), 1.71-1.59(\mathrm{~m}, 2 \mathrm{H}), 1.55-1.44(\mathrm{~m}$, $2 \mathrm{H}) .{ }^{13} \mathrm{C} \mathrm{NMR}\left(101 \mathrm{MHz}, \mathrm{CDCl}_{3}\right) \delta 171.3,166.3,145.7,138.4,128.8,128.0,127.6,122.4,64.5,43.4,28.4$, 28.4, 25.8, 21.1. IR: 3304, 2935, 1735, 1659, 1532, 1249, $1030 \mathrm{~cm}^{-1}$. HRMS: Chemical fomular ([M]): $\mathrm{C}_{16} \mathrm{H}_{21} \mathrm{NO}_{3}$, calcd. $[\mathrm{M}+\mathrm{H}]^{+} 276.1600$ Found: 276.1629 .<smiles>O=C(/C=C\CCCCCCCCBr)NCBr</smiles>

3k: Colorless oil $\left(73 \mathrm{mg}, 80 \%\right.$ yield). Reaction time $=24$ hours. $Z: E$ ratio $>20: 1$ (determined by ${ }^{1} \mathrm{H}$ NMR analysis of the crude reaction mixture). $\mathrm{R}_{f}=0.3$ (hexane/ethyl acetate/dichloromethane $=10: 1: 3$ ). Chromatography condition: hexane/ethyl acetate/dichloromethane, 20:1:5 to 10:1:3. ${ }^{1} \mathrm{H}$ NMR $(400 \mathrm{MHz}$, $\left.\mathrm{CDCl}_{3}\right) \delta 7.39-7.20(\mathrm{~m}, 5 \mathrm{H}), 6.00(\mathrm{dt}, J=11.4,7.5 \mathrm{~Hz}, 1 \mathrm{H}), 5.94(\mathrm{br}, 1 \mathrm{H}), 5.69(\mathrm{dt}, J=11.5,1.7 \mathrm{~Hz}, 1 \mathrm{H})$, $4.44(\mathrm{~d}, J=5.7 \mathrm{~Hz}, 2 \mathrm{H}), 3.39(\mathrm{t}, J=6.9 \mathrm{~Hz}, 2 \mathrm{H}), 2.66(\mathrm{qd}, J=7.4,1.7 \mathrm{~Hz}, 2 \mathrm{H}), 1.92-1.76(\mathrm{~m}, 2 \mathrm{H}), 1.50-$ $1.36(\mathrm{~m}, 4 \mathrm{H}), 1.36-1.24(\mathrm{~m}, 8 \mathrm{H}) .{ }^{13} \mathrm{C} \mathrm{NMR}\left(101 \mathrm{MHz}, \mathrm{CDCl}_{3}\right) \delta 166.5,146.4,138.5,128.7,127.9,127.5$, 121.9, 43.3, 34.2, 32.9, 29.4, 29.4, 29.4, 29.3, 28.9, 28.8, 28.2. IR: 3293, 3032, 2923, 2852, 1656, 1631, 1538, 1454, $1240 \mathrm{~cm}^{-1}$. HRMS: Chemical fomular ([M]): $\mathrm{C}_{19} \mathrm{H}_{28} \mathrm{BrNO}$, calcd. $[\mathrm{M}+\mathrm{H}]^{+} 366.1432$ Found: 366.1431 .<smiles>COc1ccc(CC/C=C\C(=O)Nc2ccccc2)cc1OC</smiles> 
31: White solid (55 mg, 71\% yield). M.p. $=77-79^{\circ} \mathrm{C}$. Reaction time $=20$ hours. $Z: E$ ratio $>20: 1$ (determined by ${ }^{1} \mathrm{H}$ NMR analysis of the crude reaction mixture). $\mathrm{R}_{f}=0.25$ (hexane/ethyl acetate/dichloromethane $=3: 1: 1$ ). Chromatography condition: hexane/ethyl acetate/dichloromethane, 20:1:5 to 5:1:2. ${ }^{1} \mathrm{H}$ NMR (400 MHz, $\left.\mathrm{CDCl}_{3}\right) \delta 7.38-7.27(\mathrm{~m}, 5 \mathrm{H}), 6.85-6.71(\mathrm{~m}, 3 \mathrm{H}), 6.17(\mathrm{dt}, J=11.3,7.6 \mathrm{~Hz}, 1 \mathrm{H}), 5.85(\mathrm{br}, 1 \mathrm{H}), 5.76(\mathrm{dt}, J$ $=11.3,1.8 \mathrm{~Hz}, 1 \mathrm{H}), 4.51(\mathrm{~d}, J=5.7 \mathrm{~Hz}, 2 \mathrm{H}), 4.01(\mathrm{dd}, J=7.6,1.8 \mathrm{~Hz}, 2 \mathrm{H}), 3.85(\mathrm{~s}, 6 \mathrm{H}) .{ }^{13} \mathrm{C}$ NMR $(101$ $\left.\mathrm{MHz}, \mathrm{CDCl}_{3}\right) \delta 166.2,149.1,147.6,144.7,138.3,132.7,128.9,128.0,127.7,121.9,120.5,112.1,111.4$, $56.1,56.0,43.6,34.6$. The spectra match the reported literature. ${ }^{11}$

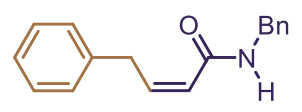

$3 \mathrm{~m}$

3m: White solid ( $42.4 \mathrm{mg}, 68 \%$ yield). M.p. $=74-75^{\circ} \mathrm{C}$. Reaction time $=20$ hours. $Z: E$ ratio $>20: 1$ (determined by ${ }^{1} \mathrm{H}$ NMR analysis of the crude reaction mixture). $\mathrm{R}_{f}=0.3$ (hexane/ethyl acetate/dichloromethane $=10: 1: 3)$. Chromatography condition: hexane/ethyl acetate/dichloromethane, 20:1:5 to 10:1:3. ${ }^{1} \mathrm{H}$ NMR (400 MHz, $\left.\mathrm{CDCl}_{3}\right) \delta 7.32-7.11(\mathrm{~m}, 10 \mathrm{H}), 6.09(\mathrm{dt}, J=11.3,7.6 \mathrm{~Hz}, 1 \mathrm{H}), 5.86$ (br, $1 \mathrm{H}), 5.70$ (dt, $J=11.3,1.7 \mathrm{~Hz}, 1 \mathrm{H}), 4.41$ (d, $J=5.0 \mathrm{~Hz}, 2 \mathrm{H}), 3.99$ (dd, $J=7.5,1.7 \mathrm{~Hz}, 2 \mathrm{H}) .{ }^{13} \mathrm{C}$ NMR $\left(101 \mathrm{MHz}, \mathrm{CDCl}_{3}\right) \delta 166.2,144.3,140.1,138.3,128.8,128.7,128.7,128.0,127.7,126.3,122.2,43.5,35.0$. IR: 3291, 3067, 3028, 1654, 1625, 1545, 1494, 1261, 1236, $1029 \mathrm{~cm}^{-1}$. HRMS: Chemical fomular ([M]): $\mathrm{C}_{17} \mathrm{H}_{17} \mathrm{NO}$, calcd. [M+H] ${ }^{+} 252.1388$ Found: 252.1379 .<smiles>C=C(C)[C@H]1CCC(C)CC1OC(=O)CC/C=C\C(=O)NCc1ccccc1</smiles>

$3 n$

3n: Colorless oil ( $57.8 \mathrm{mg}, 63 \%$ yield). Reaction time $=40$ hours. $Z: E$ ratio $>20: 1$ (determined by ${ }^{1} \mathrm{H}$ NMR analysis of the crude reaction mixture). $\mathrm{R}_{f}=0.0 .4$ (hexane/ethyl acetate/dichloromethane $=3: 1: 1$ ). Chromatography condition: hexane/ethyl acetate/dichloromethane, 10:1:3 to 5:1:2. ${ }^{1} \mathrm{H}$ NMR (400 MHz, $\left.\mathrm{CDCl}_{3}\right) \delta 7.35-7.23(\mathrm{~m}, 5 \mathrm{H}), 6.74(\mathrm{br}, 1 \mathrm{H}), 5.88(\mathrm{dt}, J=11.6,7.5 \mathrm{~Hz}, 1 \mathrm{H}), 5.76(\mathrm{dt}, J=11.5,1.4 \mathrm{~Hz}, 1 \mathrm{H})$, $4.79(\mathrm{td}, J=10.9,4.4 \mathrm{~Hz}, 1 \mathrm{H}), 4.72-4.63(\mathrm{~m}, 2 \mathrm{H}), 4.48(\mathrm{~d}, J=5.7 \mathrm{~Hz}, 2 \mathrm{H}), 2.92-2.72(\mathrm{~m}, 2 \mathrm{H}), 2.40(\mathrm{dd}$, $J=7.2,6.5 \mathrm{~Hz}, 2 \mathrm{H}), 2.08(\mathrm{ddd}, J=12.5,10.7,3.7 \mathrm{~Hz}, 1 \mathrm{H}), 1.94(\mathrm{dddd}, J=10.4,3.5,2.6,1.6 \mathrm{~Hz}, 1 \mathrm{H}), 1.73$ $-1.64(\mathrm{~m}, 2 \mathrm{H}), 1.62(\mathrm{t}, J=1.2 \mathrm{~Hz}, 3 \mathrm{H}), 1.58-1.46(\mathrm{~m}, 1 \mathrm{H}), 1.42-1.29(\mathrm{~m}, 1 \mathrm{H}), 1.06-0.97(\mathrm{~m}, 1 \mathrm{H}), 0.97$ $-0.86(\mathrm{~m}, 4 \mathrm{H}) .{ }^{13} \mathrm{C}$ NMR $\left(101 \mathrm{MHz}, \mathrm{CDCl}_{3}\right) \delta 172.9,166.4,146.2,141.4,138.5,128.7,128.0,127.4,124.0$, 112.0, 73.9, 50.8, 43.4, 40.5, 34.1, 33.5, 31.5, 30.4, 24.3, 22.1, 19.6. IR: 3304, 2950, 2925, 2868, 1727, 1660 , 1537, 1454, 1236, $1164 \mathrm{~cm}^{-1}$. HRMS: Chemical fomular ([M]): $\mathrm{C}_{23} \mathrm{H}_{31} \mathrm{NO}_{3}$, calcd. $[\mathrm{M}+\mathrm{H}]^{+} 370.2382$ Found: 370.2402 .

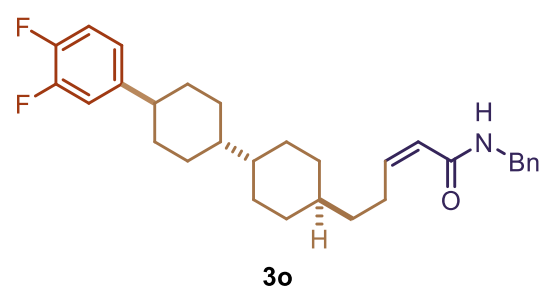

3o: White solid ( $85.8 \mathrm{mg}, 74 \%$ yield). M.p. $=145-147{ }^{\circ} \mathrm{C}$. Reaction time $=20$ hours. $Z: E$ ratio $>20: 1$ (determined by ${ }^{1} \mathrm{H}$ NMR analysis of the crude reaction mixture). $\mathrm{R}_{f}=0.65$ (hexane/ethyl acetate/dichloromethane $=3: 1: 1)$. Chromatography condition: hexane/ethyl acetate/dichloromethane, 20:1:5 to 10:1:3. ${ }^{1} \mathrm{H}$ NMR $\left(400 \mathrm{MHz}, \mathrm{CDCl}_{3}\right) \delta 7.27-7.15(\mathrm{~m}, 5 \mathrm{H}), 7.00-6.86(\mathrm{~m}, 2 \mathrm{H}), 6.81$ (ddd, $J=8.6,4.2$, 
$1.9 \mathrm{~Hz}, 1 \mathrm{H}), 5.92(\mathrm{dt}, J=11.5,7.4 \mathrm{~Hz}, 1 \mathrm{H}), 5.84(\mathrm{t}, J=5.4 \mathrm{~Hz}, 1 \mathrm{H}), 5.61(\mathrm{dt}, J=11.4,1.7 \mathrm{~Hz}, 1 \mathrm{H}), 4.37$ (d, $J=5.7 \mathrm{~Hz}, 2 \mathrm{H}), 2.62(\mathrm{qd}, J=7.6,1.7 \mathrm{~Hz}, 2 \mathrm{H}), 2.39-2.23(\mathrm{~m}, 1 \mathrm{H}), 1.87-1.68(\mathrm{~m}, 6 \mathrm{H}), 1.65(\mathrm{~d}, J=10.3$ $\mathrm{Hz}, 2 \mathrm{H}), 1.34-1.17(\mathrm{~m}, 4 \mathrm{H}), 1.15-0.73(\mathrm{~m}, 9 \mathrm{H}) .{ }^{13} \mathrm{C} \mathrm{NMR}\left(101 \mathrm{MHz}, \mathrm{CDCl}_{3}\right) \delta 166.5,150.6(\mathrm{dd}, J=$ $166.7,12.6 \mathrm{~Hz}), 148.2(\mathrm{dd}, J=165.0,12.6 \mathrm{~Hz}), 146.8,145.0(\mathrm{dd}, J=4.9,3.8 \mathrm{~Hz}), 138.5,128.8,127.9,127.5$, $122.6(\mathrm{dd}, J=5.9,3.3 \mathrm{~Hz}), 121.8,116.8(\mathrm{~d}, J=16.6 \mathrm{~Hz}), 115.5(\mathrm{~d}, J=16.6 \mathrm{~Hz}), 43.9$ (d, $J=1.3 \mathrm{~Hz}), 43.4$, $43.3,42.8,37.8,37.0,34.7,33.5,30.3,30.1,26.6 .{ }^{19} \mathrm{~F}$ NMR $\left(282 \mathrm{MHz}, \mathrm{CDCl}_{3}\right) \delta-138.76$ (ddd, $J=20.7$, 12.0, $8.2 \mathrm{~Hz}, 1 \mathrm{~F}$ ), -142.67 (dddd, $J=21.9,11.7,7.9,4.2 \mathrm{~Hz}, 1 \mathrm{~F}$ ). IR: 2919, 2850, 1657, 1515, 1450, 1212 , $905 \mathrm{~cm}^{-1}$. HRMS: Chemical fomular ([M]): $\mathrm{C}_{30} \mathrm{H}_{37} \mathrm{~F}_{2} \mathrm{NO}$, calcd. [M+H] 466.2922 Found: 466.2938 .<smiles>COc1cc2c(cc1OC)C(c1ccccc1)N(C(=O)CC/C=C\C(=O)NCc1ccccc1)CC2</smiles>

3p: Colorless wax ( $86 \mathrm{mg}, 71 \%$ yield). Reaction time $=40$ hours. $Z: E$ ratio $>20: 1$ (determined by chromatography isolation of the crude reaction mixture). $\mathrm{R}_{f}=0.15$ (hexane/acetone/dichloromethane $=$ 3:1:1). Chromatography condition: hexane/acetone/dichloromethane, 6:1:2 to 3:1:1. ${ }^{1} \mathrm{H}$ NMR $(400 \mathrm{MHz}$, $\left.\mathrm{CDCl}_{3}\right)$ (mixture of amide rotamers, ca. 5:1) $\delta 8.04(\mathrm{~s}, 0.83 \mathrm{H}), 7.82(\mathrm{~s}, 0.17 \mathrm{H}), 7.42-7.27(\mathrm{~m}, 5 \mathrm{H}), 7.26-$ $7.22(\mathrm{~m}, 3 \mathrm{H}), 7.18-7.13(\mathrm{~m}, 2 \mathrm{H}), 6.81(\mathrm{~s}, 0.83 \mathrm{H}), 6.66(\mathrm{~s}, 0.17 \mathrm{H}), 6.65(\mathrm{~s}, 1 \mathrm{H}), 6.50(\mathrm{~s}, 0.83 \mathrm{H}), 6.02(\mathrm{~s}$, $0.17 \mathrm{H}), 5.93-5.76(\mathrm{~m}, 2 \mathrm{H}), 4.61-4.42(\mathrm{~m}, 2 \mathrm{H}), 4.27(\mathrm{dt}, J=13.0,5.3 \mathrm{~Hz}, 0.17 \mathrm{H}), 3.88(\mathrm{~s}, 3 \mathrm{H}), 3.82-$ $3.72(\mathrm{~m}, 3.83 \mathrm{H}), 3.35$ (ddd, $J=13.8,11.6,4.4 \mathrm{~Hz}, 0.83 \mathrm{H}), 3.18(\mathrm{ddd}, J=13.7,9.4,5.1 \mathrm{~Hz}, 0.17 \mathrm{H}), 2.97-$ $2.51(\mathrm{~m}, 6 \mathrm{H}) .{ }^{13} \mathrm{C}$ NMR $\left(101 \mathrm{MHz}, \mathrm{CDCl}_{3}\right)$ major rotamer: $\delta 170.7,166.8,148.2,147.8,142.4,140.0,138.7$, $128.8,128.6,128.4,128.0,127.5,127.3,126.9,126.4,124.9,111.3,111.1,56.1,56.0,54.9,43.5,39.4,32.3$, 28.6, 24.7. for the minor rotamer, carbon signals were observed at: $\delta 171.4,166.7,148.5,147.5,141.4,140.3$, 138.7, 128.7, 128.6, 127.9, 127.8, 127.5, 127.3, 126.9, 124.8, 111.5, 111.1, 59.4, 56.1, 43.3, 37.6, 32.8, 27.4, 24.9. IR: $3293,3028,2935,1622,1514,1452,1249,1117 \mathrm{~cm}^{-1}$. HRMS: Chemical fomular ([M]): $\mathrm{C}_{30} \mathrm{H}_{32} \mathrm{~N}_{2} \mathrm{O}_{4}$, calcd. $[\mathrm{M}+\mathrm{H}]^{+} 485.2440$ Found: 485.2453 .

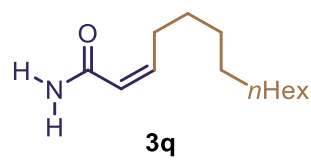

3q: Following a modified General procedure, the reaction was conducted with the use of 2.0 equivalent (instead of 1.3 equiv.) of 1-dodecene ( $84 \mathrm{mg})$. The titled compound was isolated as a white solid $(41.1 \mathrm{mg}$, $78 \%$ yield). M.p. $=62-64{ }^{\circ} \mathrm{C}$. Reaction time $=24$ hours. $Z: E$ ratio $>20: 1$ (determined by ${ }^{1} \mathrm{H}$ NMR analysis of the crude reaction mixture). $\mathrm{R}_{f}=0.2$ (hexane/ethyl acetate/dichloromethane $=3: 2: 1$ ). Chromatography condition: hexane/ethyl acetate/dichloromethane, $3: 1: 1$ to $3: 2: 1 .{ }^{1} \mathrm{H} \mathrm{NMR}\left(400 \mathrm{MHz}, \mathrm{CDCl}_{3}\right) \delta 6.05$ (dt, $J=$ 11.5, 7.4 Hz, 1H), $5.91(\mathrm{br}, 1 \mathrm{H}), 5.73(\mathrm{dt}, J=11.5,1.7 \mathrm{~Hz}, 1 \mathrm{H}), 5.63(\mathrm{br}, 1 \mathrm{H}), 2.62(\mathrm{qd}, J=7.4,1.8 \mathrm{~Hz}, 2 \mathrm{H})$, $1.41(\mathrm{p}, J=7.3 \mathrm{~Hz}, 2 \mathrm{H}), 1.36-1.08(\mathrm{~m}, 14 \mathrm{H}), 0.92-0.80(\mathrm{~m}, 3 \mathrm{H}) .{ }^{13} \mathrm{C} \mathrm{NMR}\left(101 \mathrm{MHz}, \mathrm{CDCl}_{3}\right) \delta 169.0$, 147.5, 121.2, 32.0, 29.7, 29.7, 29.6, 29.5, 29.4, 29.4, 29.0, 22.8, 14.2. IR: 3330, 3183, 2924, 2854, 1669, 1631, $1312 \mathrm{~cm}^{-1}$. HRMS: Chemical fomular ([M]): $\mathrm{C}_{13} \mathrm{H}_{25} \mathrm{NO}$, calcd. [M+H] 212.2014 Found: 212.2024 .

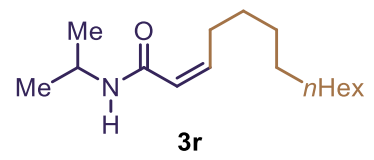

3r: White solid ( $46.7 \mathrm{mg}, 74 \%$ yield). M.p. $=45-47^{\circ} \mathrm{C}$. Reaction time $=18$ hours. $Z: E$ ratio $>20: 1$ (determined by ${ }^{1} \mathrm{H}$ NMR analysis of the crude reaction mixture). $\mathrm{R}_{f}=0.5$ (hexane/ethyl acetate/dichloromethane $=10: 1: 3)$. Chromatography condition: hexane/ethyl acetate/dichloromethane, 
20:1:5 to 10:1:3. ${ }^{1} \mathrm{H}$ NMR (400 MHz, $\left.\mathrm{CDCl}_{3}\right) \delta 5.93(\mathrm{dt}, J=11.5,7.4 \mathrm{~Hz}, 1 \mathrm{H}), 5.62(\mathrm{dt}, J=11.5,1.7 \mathrm{~Hz}$, $1 \mathrm{H}), 5.37$ (br, 1H), 4.10 (h, $J=6.6 \mathrm{~Hz}, 1 \mathrm{H}), 2.61(\mathrm{qd}, J=7.4,1.7 \mathrm{~Hz}, 2 \mathrm{H}), 1.40$ (p, $J=6.7 \mathrm{~Hz}, 2 \mathrm{H}), 1.35-$ $1.20(\mathrm{~m}, 14 \mathrm{H}), 1.15(\mathrm{~d}, J=6.6 \mathrm{~Hz}, 6 \mathrm{H}), 0.86(\mathrm{~d}, J=6.9 \mathrm{~Hz}, 3 \mathrm{H}) .{ }^{13} \mathrm{C}$ NMR $\left(101 \mathrm{MHz}, \mathrm{CDCl}_{3}\right) \delta 165.9$, 145.4, 122.6, 41.1, 32.0, 29.7, 29.7, 29.6, 29.5, 29.5, 29.5, 28.9, 23.0, 22.8, 14.2. IR: 3258, 2960, 2917, 2850, 1653, 1624, 1546, 1466, $1242 \mathrm{~cm}^{-1}$. HRMS: Chemical fomular ([M]): $\mathrm{C}_{16} \mathrm{H}_{31} \mathrm{NO}$, calcd. $[\mathrm{M}+\mathrm{H}]^{+} 254.2484$ Found: 254.2454 .<smiles>COCCCC/C=C\C(=O)NC(C)(C)C</smiles>

3s: White solid $\left(50.0 \mathrm{mg}, 72 \%\right.$ yield). M.p. $=50-53{ }^{\circ} \mathrm{C}$. Reaction time $=20$ hours. $Z: E$ ratio $=15: 1$ (determined by ${ }^{1} \mathrm{H}$ NMR analysis of the crude reaction mixture). $\mathrm{R}_{f}=0.15$ (hexane/ethyl acetate/dichloromethane $=3: 1: 1)$. Chromatography condition: hexane/ethyl acetate/dichloromethane, 5:1:2 to $3: 1: 1 .{ }^{1} \mathrm{H}$ NMR $\left(400 \mathrm{MHz}, \mathrm{CDCl}_{3}\right) \delta 5.86(\mathrm{dt}, J=11.4,7.5 \mathrm{~Hz}, 1 \mathrm{H}), 5.62(\mathrm{dt}, J=11.5,1.7 \mathrm{~Hz}, 1 \mathrm{H}), 5.34$ (br, 1H), $4.23(\mathrm{t}, J=6.4 \mathrm{~Hz}, 2 \mathrm{H}), 2.99(\mathrm{~s}, 3 \mathrm{H}), 2.69(\mathrm{qd}, J=7.5,1.7 \mathrm{~Hz}, 2 \mathrm{H}), 1.77(\mathrm{dq}, J=8.3,6.5 \mathrm{~Hz}, 2 \mathrm{H})$, $1.54(\mathrm{tt}, J=9.6,6.5 \mathrm{~Hz}, 2 \mathrm{H}), 1.35(\mathrm{~s}, 9 \mathrm{H}) .{ }^{13} \mathrm{C} \mathrm{NMR}\left(101 \mathrm{MHz}, \mathrm{CDCl}_{3}\right) \delta 166.0,143.7,124.2,70.1,51.3$, 37.4, 28.9, 28.6, 27.6, 25.1. IR: 2968, 1661, 1525, 1350, 1171, $909 \mathrm{~cm}^{-1}$. HRMS: Chemical fomular ([M]): $\mathrm{C}_{12} \mathrm{H}_{23} \mathrm{NO}_{4} \mathrm{~S}$, calcd. $[\mathrm{M}+\mathrm{H}]^{+} 278.1426$ Found: 278.1419 .<smiles>CCCCC/C=C\C(=O)Nc1ccc(OC)cc1OC</smiles>

3t: Following a modified General procedure, the reaction was conducted with the use of 2.0 equivalent (instead of 1.3 equiv.) of 1-dodecene ( $84 \mathrm{mg}$ ). The titled compound was isolated as a colorless oil $(34.4 \mathrm{mg}$, $40 \%$ yield). Reaction time $=24$ hours. $Z: E$ ratio $>20: 1$ (determined by chromatography isolation of the crude reaction mixture). $\mathrm{R}_{f}=0.4$ (hexane/ethyl acetate/dichloromethane $=10: 1: 3$ ). Chromatography condition: hexane/ethyl acetate/dichloromethane, 20:1:5 to 10:1:3. ${ }^{1} \mathrm{H}$ NMR $\left(400 \mathrm{MHz}, \mathrm{CDCl}_{3}\right) \delta 8.32$ (d, $J=9.6 \mathrm{~Hz}$, $1 \mathrm{H}), 7.58(\mathrm{br}, 1 \mathrm{H}), 6.53-6.40(\mathrm{~m}, 2 \mathrm{H}), 6.08(\mathrm{dt}, J=11.4,7.4 \mathrm{~Hz}, 1 \mathrm{H}), 5.84(\mathrm{~d}, J=11.5 \mathrm{~Hz}, 1 \mathrm{H}), 3.84$ (s, $3 \mathrm{H}), 3.79(\mathrm{~s}, 3 \mathrm{H}), 2.71(\mathrm{qd}, J=7.4,1.6 \mathrm{~Hz}, 2 \mathrm{H}), 1.45$ (p, $J=7.4 \mathrm{~Hz}, 2 \mathrm{H}), 1.40-1.22(\mathrm{~m}, 14 \mathrm{H}), 0.87$ (t, $J=$ $6.7 \mathrm{~Hz}, 3 \mathrm{H}) .{ }^{13} \mathrm{C}$ NMR $\left(101 \mathrm{MHz}, \mathrm{CDCl}_{3}\right)$ carbon signals were observed at $\delta 164.4,156.4,149.2,146.9$, 123.0, 121.5, 120.6, 103.8, 98.7, 55.8, 55.7, 32.1, 29.8, 29.7, 29.6, 29.5, 29.5, 29.0, 22.8, 14.3. IR: 2923, 2853, 1674, 1521, 1463, 1299, 1280, 1207, $1035 \mathrm{~cm}^{-1}$. HRMS: Chemical fomular ([M]): $\mathrm{C}_{21} \mathrm{H}_{33} \mathrm{NO}_{3}$, calcd. $[\mathrm{M}+\mathrm{H}]^{+} 348.2539$ Found: 348.2547 .<smiles>[M]C(NC(=O)/C=C\CCCCO)C(F)(F)F</smiles>

3u: Following a modified General procedure, the reaction was conducted with the use of 2.0 equivalent (instead of 1.3 equiv.) of 1-dodecene ( $84 \mathrm{mg}$ ). The titled compound was isolated as a white solid $(65 \mathrm{mg}, 85 \%$ yield). M.p. $=47-49^{\circ} \mathrm{C}$. Reaction time $=24$ hours. $Z: E$ ratio $>20: 1$ (determined by ${ }^{1} \mathrm{H}$ NMR analysis of the crude reaction mixture) $\mathrm{R}_{f}=0.5$ (hexane/ethyl acetate/dichloromethane $=10: 1: 3$ ). Chromatography condition: hexane/ethyl acetate/dichloromethane, 20:1:5. ${ }^{1} \mathrm{H}$ NMR $\left(400 \mathrm{MHz}, \mathrm{CDCl}_{3}\right) \delta 6.08(\mathrm{dt}, J=11.4$, $7.5 \mathrm{~Hz}, 1 \mathrm{H}), 5.74(\mathrm{~d}, J=9.8 \mathrm{~Hz}, 1 \mathrm{H}), 5.69$ (dt, $J=11.5,1.7 \mathrm{~Hz}, 1 \mathrm{H}), 4.74$ (qq, $J=9.6,7.2 \mathrm{~Hz}, 1 \mathrm{H}), 2.62$ (qd, $J=7.5,1.8 \mathrm{~Hz}, 2 \mathrm{H}), 1.41(\mathrm{p}, J=7.4 \mathrm{~Hz}, 2 \mathrm{H}), 1.36-1.14(\mathrm{~m}, 17 \mathrm{H}), 0.86(\mathrm{t}, J=6.8 \mathrm{~Hz}, 3 \mathrm{H}) .{ }^{13} \mathrm{C}$ NMR $\left(101 \mathrm{MHz}, \mathrm{CDCl}_{3}\right) \delta 165.9,148.1,125.5(\mathrm{q}, J=281.0 \mathrm{~Hz}), 121.1,45.9(\mathrm{q}, J=31.5 \mathrm{~Hz}), 32.0,29.7,29.6$, 29.5, 29.5, 29.3, 29.1, 22.8, 14.5 (q, $J=2.0 \mathrm{~Hz}), 14.2 .{ }^{19} \mathrm{~F} \mathrm{NMR}\left(282 \mathrm{MHz}, \mathrm{CDCl}_{3}\right) \delta-77.53(\mathrm{~d}, J=7.4 \mathrm{~Hz}$, 
3F). IR: 3284, 2924, 2854, 1660, 1540, 1459, 1230, $1137 \mathrm{~cm}^{-1}$. HRMS: Chemical fomular ([M]): $\mathrm{C}_{16} \mathrm{H}_{28} \mathrm{~F}_{3} \mathrm{NO}$, calcd. [M+H] 308.2201 Found: 308.2213 .<smiles>CC(C)CC(NC(=O)C=CCCCO)C(=O)OC(C)C</smiles>

3v: Following a modified General procedure, the reaction was conducted with the use of 1.8 equivalent (instead of 1.3 equiv.) of pent-4-en-1-ol (b.p. $=133{ }^{\circ} \mathrm{C}, 38.8 \mathrm{mg}$ ). The titled compound was isolated as a colorless oil ( $64 \mathrm{mg}, 86 \%$ yield). Reaction time $=24$ hours. $Z: E$ ratio $>20: 1$ (determined by ${ }^{1} \mathrm{H}$ NMR analysis of the crude reaction mixture) $\mathrm{R}_{f}=0.15$ (hexane/ethyl acetate/dichloromethane $=3: 1: 1$ ). Chromatography condition: hexane/ethyl acetate/dichloromethane, 3:1:1 to 2:1:1. ${ }^{1} \mathrm{H}$ NMR $\left(400 \mathrm{MHz}, \mathrm{CDCl}_{3}\right) \delta 6.42(\mathrm{~d}, J=$ $8.4 \mathrm{~Hz}, 1 \mathrm{H}), 5.96(\mathrm{dt}, J=11.4,8.4 \mathrm{~Hz}, 1 \mathrm{H}), 5.84(\mathrm{~d}, J=11.5 \mathrm{~Hz}, 1 \mathrm{H}), 4.53(\mathrm{td}, J=8.5,5.3 \mathrm{~Hz}, 1 \mathrm{H}), 3.70$ (br, 1H), 3.55 (t, $J=5.6 \mathrm{~Hz}, 2 \mathrm{H}), 2.69$ (qp, $J=13.8,7.0,6.1 \mathrm{~Hz}, 2 \mathrm{H}), 1.73-1.49$ (m, 5H), $1.49-1.38$ (m, 9H), $0.92(\mathrm{~d}, J=6.3 \mathrm{~Hz}, 6 \mathrm{H}) .{ }^{13} \mathrm{C} \mathrm{NMR}\left(101 \mathrm{MHz}, \mathrm{CDCl}_{3}\right) \delta 172.6,166.8,144.6,123.6,82.2,60.0,51.3$, 42.0, 30.5, 28.1, 25.1, 24.7, 22.9, 22.2. IR: 3285, 2957, 2871, 1732, 1658, 1537, 1368, 1249, $1149 \mathrm{~cm}^{-1}$. HRMS: Chemical fomular ([M]): $\mathrm{C}_{16} \mathrm{H}_{29} \mathrm{NO}_{4}$, calcd. [M+H] 300.2175 Found: 300.2195 .<smiles>COC(=O)C1CCC(NC(=O)/C=C\CCCCCC2CO2)CC1</smiles>

3w: White solid $\left(51.2 \mathrm{mg}, 61 \%\right.$ yield). M.p. $=40-41^{\circ} \mathrm{C}$. Reaction time $=24$ hours. $Z: E$ ratio $>20: 1$ (determined by ${ }^{1} \mathrm{H}$ NMR analysis of the crude reaction mixture). $\mathrm{R}_{f}=0.15$ (hexane/ethyl acetate/dichloromethane $=3: 1: 1)$. Chromatography condition: hexane/ethyl acetate/dichloromethane, 3:1:1 to 2:1:2. ${ }^{1} \mathrm{H}$ NMR $\left(400 \mathrm{MHz}, \mathrm{CDCl}_{3}\right) \delta 5.94(\mathrm{dt}, J=11.5,7.5 \mathrm{~Hz}, 1 \mathrm{H}), 5.61(\mathrm{dt}, J=11.5,1.7 \mathrm{~Hz}, 1 \mathrm{H}), 5.42$ (d, $J=8.2 \mathrm{~Hz}, 1 \mathrm{H}), 3.77$ (tdq, $J=12.0,8.1,4.0 \mathrm{~Hz}, 1 \mathrm{H}$ ), 3.64 (s, 3H), 2.87 (tdd, $J=5.3,3.9,2.7 \mathrm{~Hz}, 1 \mathrm{H}$ ), $2.71(\mathrm{dd}, J=5.0,4.0 \mathrm{~Hz}, 1 \mathrm{H}), 2.61(\mathrm{qd}, J=7.4,1.7 \mathrm{~Hz}, 2 \mathrm{H}), 2.43(\mathrm{dd}, J=5.0,2.7 \mathrm{~Hz}, 1 \mathrm{H}), 2.21(\mathrm{tt}, J=$ 12.1, $3.5 \mathrm{~Hz}, 1 \mathrm{H}), 2.10-1.93(\mathrm{~m}, 4 \mathrm{H}), 1.62-1.30(\mathrm{~m}, 12 \mathrm{H}), 1.19-1.06(\mathrm{~m}, 2 \mathrm{H}) .{ }^{13} \mathrm{C} \mathrm{NMR}(101 \mathrm{MHz}$, $\left.\mathrm{CDCl}_{3}\right) \delta 175.8,165.9,145.8,122.4,52.5,51.8,47.5,47.2,42.5,32.5,32.3,29.3,29.3,28.7,27.9,26.0$. IR: 3294, 2930, 2856, 1733, 1657, 1628, 1532, 1453, 1254, $1174 \mathrm{~cm}^{-1}$. HRMS: Chemical fomular ([M]): $\mathrm{C}_{19} \mathrm{H}_{31} \mathrm{NO}_{4}$, calcd. $[\mathrm{M}+\mathrm{H}]^{+} 338.2331$ Found: 338.2338 .<smiles>CCCCCC/C=C\C(=O)NC(C)(C)CC(C)=O</smiles>

3x: Following a modified General procedure, the reaction was conducted with the use of 2.0 equivalent (instead of 1.3 equiv.) of 1-dodecene $(84 \mathrm{mg})$. The titled compound was isolated as a white solid $(46.1 \mathrm{mg}$, $60 \%$ yield). M.p. $=49-50^{\circ} \mathrm{C}$. Reaction time $=24$ hours. $Z: E$ ratio $=17: 1$ (determined by chromatography isolation of the crude reaction mixture; trans-product: $2.7 \mathrm{mg}$ ). $\mathrm{R}_{f}=0.35$ (hexane/ethyl acetate/dichloromethane $=5: 1: 2)$. Chromatography condition: hexane/ethyl acetate/dichloromethane, 10:1:3 ${ }^{1} \mathrm{H}$ NMR $\left(400 \mathrm{MHz}, \mathrm{CDCl}_{3}\right) \delta 5.89(\mathrm{dt}, J=11.5,7.4 \mathrm{~Hz}, 1 \mathrm{H}), 5.77(\mathrm{br}, 1 \mathrm{H}), 5.60(\mathrm{dt}, J=11.5,1.7 \mathrm{~Hz}, 1 \mathrm{H})$, $2.96(\mathrm{~s}, 2 \mathrm{H}), 2.57$ (qd, $J=7.3,1.7 \mathrm{~Hz}, 2 \mathrm{H}), 2.11(\mathrm{~s}, 3 \mathrm{H}), 1.50-1.35(\mathrm{~m}, 8 \mathrm{H}), 1.34-1.18(\mathrm{~m}, 14 \mathrm{H}), 0.86(\mathrm{t}$, $J=6.9,3 \mathrm{H}) .{ }^{13} \mathrm{C}$ NMR $\left(101 \mathrm{MHz}, \mathrm{CDCl}_{3}\right)$ carbon signals were observed at $\delta 208.1,166.7,145.2,123.2$, 
52.3, 51.2, 32.0, 31.9, 29.7, 29.6, 29.5, 29.5, 29.4, 28.7, 27.7, 22.8, 14.3. IR: 3319, 2923, 2853, 1708, 1658, 1532, 1456, $1361 \mathrm{~cm}^{-1}$. HRMS: Chemical fomular ([M]): $\mathrm{C}_{19} \mathrm{H}_{35} \mathrm{NO}_{2}$, calcd. $[\mathrm{M}+\mathrm{H}]^{+} 310.2746$ Found: 310.2718 .

\section{Computational study}

5.1 Detailed energy profiles for "olefin-first" and "acrylamide-first" mechanisms 
Scheme S1 | Calculated energies (kcal/mol) for "olefin-first" pathways with both [Ru]-OPiv (top) and [Ru]$\mathrm{NO}_{3}$ (bottom).

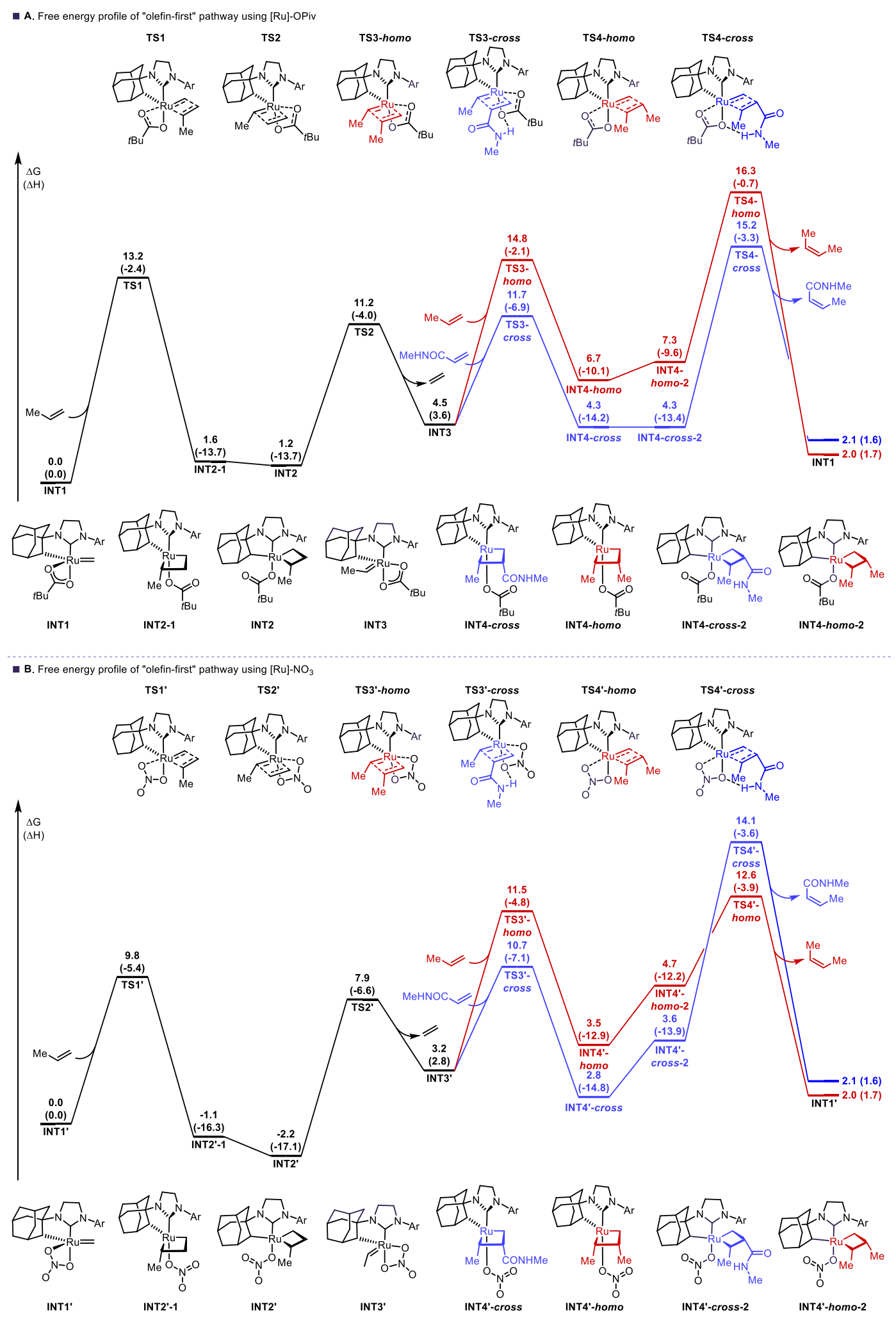


Scheme S2 | Calculated energies ( $\mathrm{kcal} / \mathrm{mol}$ ) for "acrylamide-first" pathways with both [Ru]-OPiv (top) and $[\mathrm{Ru}]-\mathrm{NO}_{3}$ (bottom). No barrier could be located for the extrusion of ethylene.

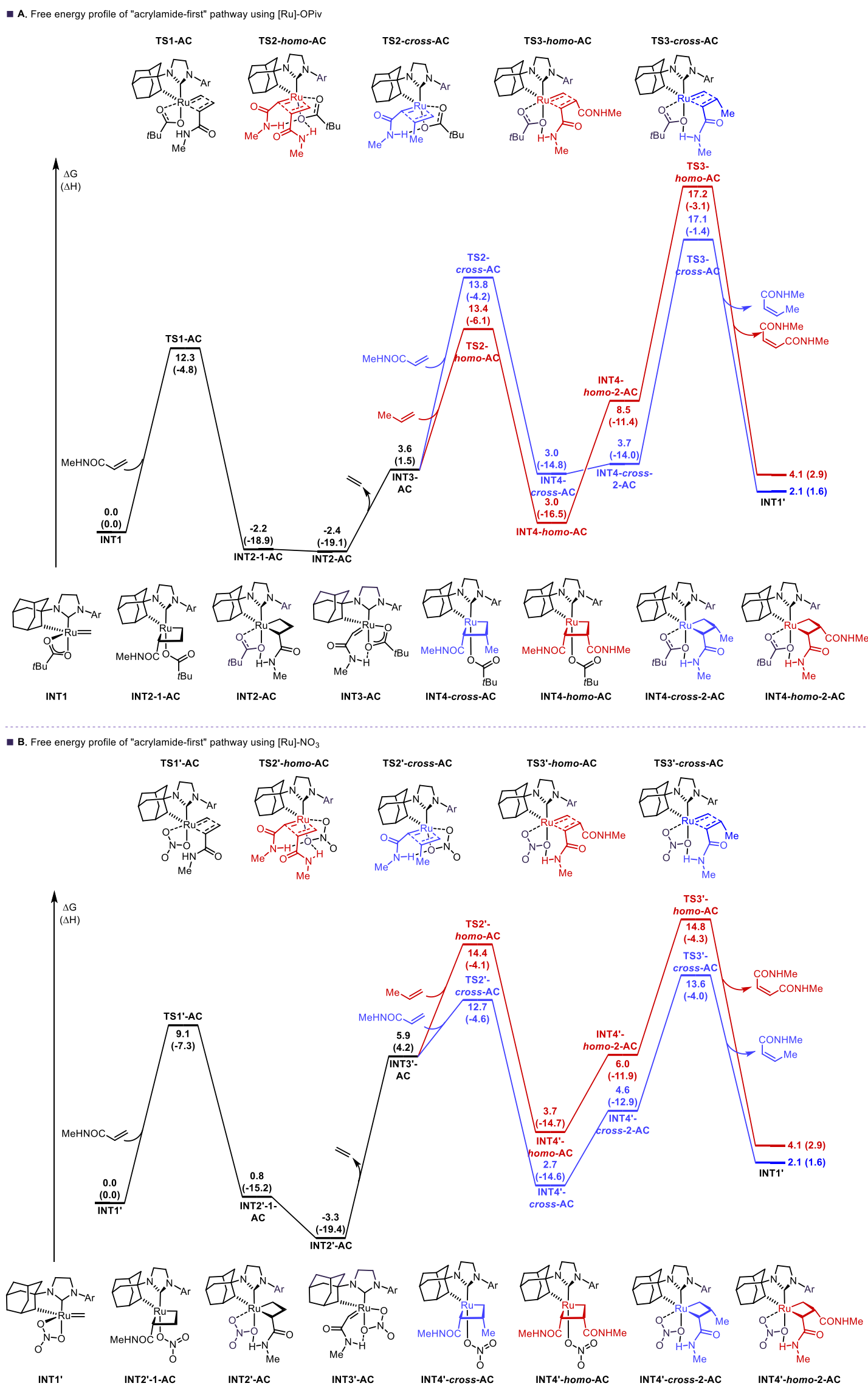




\subsection{Relative stabilities of olefin isomers}

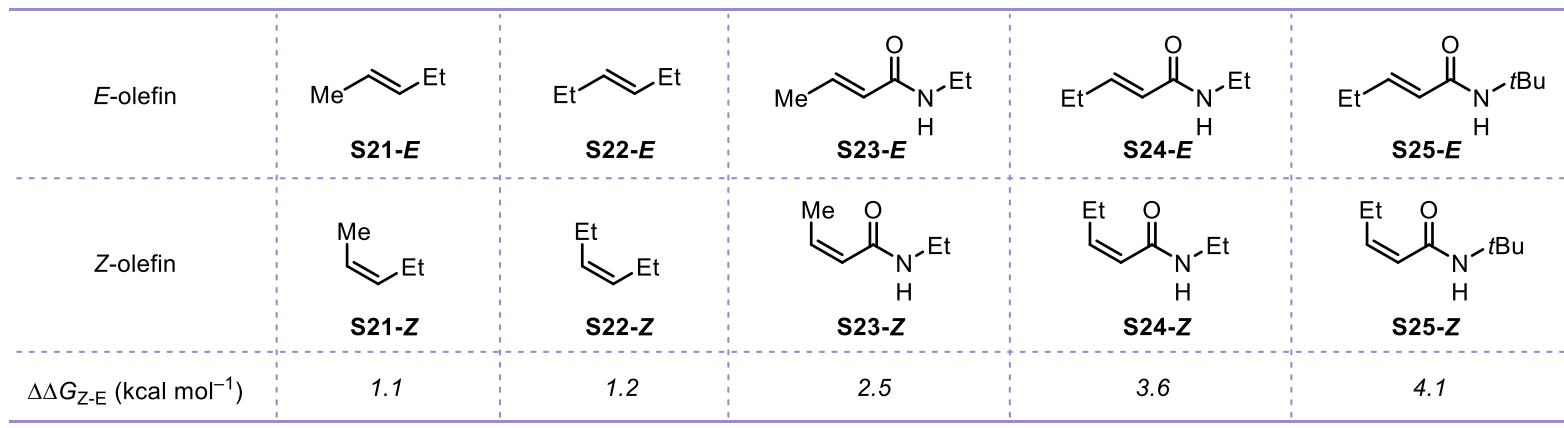

\subsection{Computational methods}

All calculations were carried out with the Gaussian $16^{12}$ software package. Ground state and transition state geometries were optimized in the gas phase using the $\mathrm{B} 3 \mathrm{LYP}^{13}$ functional with the $\mathrm{D} 3$ version of Grimme's empirical dispersion correction. ${ }^{14}$ The $\mathrm{SDD}^{15}$ basis set was used for $\mathrm{Ru}$ and the $6-31 \mathrm{G}(\mathrm{d})$ basis set for all other atoms. Frequency calculations were carried out at the same level of theory to ensure that stationary points were truly minima or saddle points on the potential energy surface. Thermal corrections to free energies were calculated using Grimme's quasi-rigid rotor-harmonic oscillator approximation at $298 \mathrm{~K} .{ }^{16}$ Intrinsic reaction coordinate calculations were also carried out at this level of theory in order to confirm that all transition states connected reactants and products. Single-point and CM5 charge calculations were performed at with the M06 ${ }^{17}$ functional with the SDD basis set for Ru and 6-311++G(d,p) basis set for all other atoms. Solvation effects were incorporated with the $\mathrm{SMD}^{18}$ model in THF. Conformational searches were carried out using the CREST conformer-rotamer ensemble sampling too ${ }^{19}$, version 2.7.1 with XTB version 6.2 RC2 $(\mathrm{SAW} 190805)^{20}$. Images of molecular structures were rendered in CYLview. ${ }^{21}$

\subsection{Computed energies}

\begin{tabular}{|c|c|c|c|c|c|c|}
\hline Structure & $\mathbf{E}(\mathrm{THF})$ & $\mathbf{\Delta G}$ & $\mathbf{G}(\mathrm{THF})$ & $\boldsymbol{\Delta} \mathbf{H}$ & $\mathbf{H}(\mathrm{THF})$ & $\begin{array}{c}\text { Imaginary } \\
\text { Frequency }\end{array}$ \\
\hline $\begin{array}{c}\boldsymbol{N}- \\
\text { methylacrylamide }\end{array}$ & -286.5029417 & 0.076517 & -286.4264247 & 0.115155 & -286.3877867 & \\
\hline propene & -117.8372324 & 0.055064 & -117.7821684 & 0.085077 & -117.7521554 & \\
\hline ethylene & -78.53966431 & 0.029686 & -78.50997831 & 0.055199 & -78.48446531 & \\
\hline INT1 & -1563.215087 & 0.657167 & -1562.55792 & 0.75511 & -1562.459977 & \\
\hline TS1 & -1681.059482 & 0.740378 & -1680.319104 & 0.843491 & -1680.215991 & -174.207 \\
\hline INT2-1 & -1681.079695 & 0.742179 & -1680.337516 & 0.84571 & -1680.233985 & \\
\hline INT2 & -1681.079033 & 0.740798 & -1680.338235 & 0.845062 & -1680.233971 & \\
\hline TS2 & -1681.061332 & 0.739102 & -1680.32223 & 0.84287 & -1680.218462 & -85.457 \\
\hline INT3 & -1602.506962 & 0.684052 & -1601.82291 & 0.784983 & -1601.721979 & \\
\hline TS3-cross & -1889.029597 & 0.791762 & -1888.237835 & 0.903199 & -1888.126398 & -147.311 \\
\hline TS3-homo & -1720.355847 & 0.767109 & -1719.588738 & 0.872675 & -1719.483172 & -189.132 \\
\hline INT4-cross & -1889.04313 & 0.793492 & -1888.249638 & 0.905073 & -1888.138057 & \\
\hline INT4-homo & -1720.370612 & 0.769052 & -1719.60156 & 0.874649 & -1719.495963 & \\
\hline INT4-cross-2 & -1889.0423 & 0.792681 & -1888.249619 & 0.905431 & -1888.136869 & \\
\hline INT4-homo-2 & -1720.370631 & 0.77001 & -1719.600621 & 0.875445 & -1719.495186 & \\
\hline TS4-cross & -1889.024016 & 0.791649 & -1888.232367 & 0.90332 & -1888.120696 & -181.9 \\
\hline TS4-homo & -1720.35421 & 0.767919 & -1719.586291 & 0.87322 & -1719.48099 & -196.89 \\
\hline INT1' & -1497.16598 & 0.542247 & -1496.623733 & 0.630328 & -1496.535652 & \\
\hline TS1' & -1615.014866 & 0.62452 & -1614.390346 & 0.718401 & -1614.296465 & -167.841 \\
\hline
\end{tabular}




\begin{tabular}{|c|c|c|c|c|c|c|}
\hline INT2'-1 & -1615.034662 & 0.627023 & -1614.407639 & 0.720923 & -1614.313739 & \\
\hline INT2' & -1615.035252 & 0.625916 & -1614.409336 & 0.720198 & -1614.315054 & \\
\hline TS2' & -1615.016256 & 0.622985 & -1614.393271 & 0.717906 & -1614.29835 & -91.457 \\
\hline INT3' & -1536.458818 & 0.56799 & -1535.890828 & 0.659983 & -1535.798835 & \\
\hline TS3'-cross & -1822.980355 & 0.675067 & -1822.305288 & 0.777969 & -1822.202386 & -152.524 \\
\hline TS3'-homo & -1654.310846 & 0.651076 & -1653.65977 & 0.747735 & -1653.563111 & -193.156 \\
\hline INT4'-cross & -1822.994489 & 0.676607 & -1822.317882 & 0.779774 & -1822.214715 & \\
\hline INT4'-homo & -1654.325969 & 0.653467 & -1653.672502 & 0.749885 & -1653.576084 & \\
\hline INT4'-cross-2 & -1822.993675 & 0.677142 & -1822.316533 & 0.780356 & -1822.213319 & \\
\hline INT4'-homo-2 & -1654.325656 & 0.654999 & -1653.670657 & 0.750786 & -1653.57487 & \\
\hline TS4'-cross & -1822.975001 & 0.675057 & -1822.299944 & 0.778108 & -1822.196893 & -178.016 \\
\hline TS4'-homo & -1654.309904 & 0.651854 & -1653.65805 & 0.748135 & -1653.561769 & -197.958 \\
\hline TS1-AC & -1849.728742 & 0.764052 & -1848.96469 & 0.873292 & -1848.85545 & -109.727 \\
\hline INT2-1-AC & -1849.753786 & 0.765973 & -1848.987813 & 0.875834 & -1848.877952 & \\
\hline INT2-AC & -1849.753605 & 0.765486 & -1848.988119 & 0.875438 & -1848.878167 & \\
\hline INT3-AC & -1771.176444 & 0.707834 & -1770.46861 & 0.81555 & -1770.360894 & \\
\hline TS2-cross-AC & -1889.025347 & 0.790829 & -1888.234518 & 0.903156 & -1888.122191 & -179.59 \\
\hline TS2-homo-AC & -2057.694046 & 0.814589 & -2056.879457 & 0.933198 & -2056.760848 & -150.201 \\
\hline INT4-cross-AC & -1889.043843 & 0.792153 & -1888.25169 & 0.904831 & -1888.139012 & \\
\hline INT4-homo-AC & -2057.712369 & 0.816402 & -2056.895967 & 0.935022 & -2056.777347 & \\
\hline INT4-cross-2-AC & -1889.042731 & 0.79206 & -1888.250671 & 0.904937 & -1888.137794 & \\
\hline INT4-homo-2-AC & -2057.705122 & 0.817953 & -2056.887169 & 0.935899 & -2056.769223 & \\
\hline TS3-cross-AC & -1889.021096 & 0.791815 & -1888.229281 & 0.90346 & -1888.117636 & -156.865 \\
\hline TS3-homo-AC & -2057.689438 & 0.816072 & -2056.873366 & 0.933423 & -2056.756015 & -84.641 \\
\hline TS1'-AC & -1783.68323 & 0.647618 & -1783.035612 & 0.748128 & -1782.935102 & -81.634 \\
\hline INT2'-1-AC & -1783.698131 & 0.649233 & -1783.048898 & 0.750412 & -1782.947719 & \\
\hline INT2'-AC & -1783.704869 & 0.649457 & -1783.055412 & 0.750426 & -1782.954443 & \\
\hline INT3'-AC & -1705.122935 & 0.592093 & -1704.530842 & 0.690661 & -1704.432274 & \\
\hline TS2'-cross-AC & -1822.97652 & 0.67449 & -1822.30203 & 0.778099 & -1822.198421 & -193.455 \\
\hline TS2'-homo-AC & -1991.641265 & 0.697577 & -1990.943688 & 0.807933 & -1990.833332 & -149.366 \\
\hline INT4'-cross-AC & -1822.994286 & 0.676219 & -1822.318067 & 0.779844 & -1822.214442 & \\
\hline INT4'-homo-AC & -1991.659687 & 0.699018 & -1990.960669 & 0.809532 & -1990.850155 & \\
\hline INT4'-cross-2-AC & -1822.991274 & 0.676254 & -1822.31502 & 0.779626 & -1822.211648 & \\
\hline $\begin{array}{c}\text { INT4'-homo-2- } \\
\text { AC }\end{array}$ & -1991.65532 & 0.698319 & -1990.957001 & 0.809569 & -1990.845751 & \\
\hline TS3'-cross-AC & -1822.975344 & 0.674714 & -1822.30063 & 0.777818 & -1822.197526 & -152.852 \\
\hline TS3'-homo-AC & -1991.641411 & 0.69835 & -1990.943061 & 0.807794 & -1990.833617 & -121.789 \\
\hline $\begin{array}{c}\text { (Z)- } N \text {-methyl-2- } \\
\text { butenamide }\end{array}$ & -325.7975671 & 0.102234 & -325.6953331 & 0.144666 & -325.6529011 & \\
\hline $\begin{array}{c}N, N \text { '-Dimethyl- } \\
\text { ethen-1,2- } \\
\text { dicarboxamid }\end{array}$ & -494.4613585 & 0.12496 & -494.3363985 & 0.174849 & -494.2865095 & \\
\hline (Z)-2-butene & -157.1319831 & 0.080762 & -157.0512211 & 0.114879 & -157.0171041 & \\
\hline S21-E & -196.424489 & 0.107505 & -196.316984 & 0.144717 & -196.279772 & \\
\hline S21-Z & -196.422758 & 0.107487 & -196.315271 & 0.144729 & -196.278029 & \\
\hline S22-E & -235.715458 & 0.134067 & -235.581391 & 0.174548 & -235.54091 & \\
\hline S22-Z & -235.713605 & 0.134183 & -235.579422 & 0.174563 & -235.539042 & \\
\hline S23-E & -365.095935 & 0.130053 & -364.965882 & 0.174649 & -364.921286 & \\
\hline S23-Z & -365.0917 & 0.129873 & -364.961827 & 0.174556 & -364.917144 & \\
\hline S24-E & -404.386657 & 0.156721 & -404.229936 & 0.204412 & -404.182245 & \\
\hline S24-Z & -404.381303 & 0.157094 & -404.224209 & 0.204532 & -404.176771 & \\
\hline S25-E & -482.974531 & 0.210501 & -482.76403 & 0.263149 & -482.711382 & \\
\hline S25-Z & -482.969167 & 0.21161 & -482.757557 & 0.263453 & -482.705714 & \\
\hline
\end{tabular}

5.5. Cartesian coordinates of computed structuresV. Cartesian Coordinates of Computed Structure

$\mathrm{N}$-methylacrylamide

$\begin{array}{lrrr}\mathrm{C} & -2.517904 & -0.043126 & 0.000097 \\ \mathrm{H} & -3.454615 & -0.592425 & 0.000147 \\ \mathrm{H} & -2.558802 & 1.042565 & 0.000107 \\ \mathrm{C} & -1.335767 & -0.660517 & 0.000007 \\ \mathrm{H} & -1.272177 & -1.748439 & -0.000026\end{array}$

C $\quad-0.064791$

O -0.044861

$\mathrm{N} \quad 1.073416$

$\mathrm{H} \quad 0.984267$

C 2.404950

H 2.967278
0.130285

1.357833

$-0.634077$

$-1.639803$

$-0.058672$

$-0.359298$
$-0.000076$

$-0.000051$

$-0.000305$

0.000412

0.000155

0.892675 
$\begin{array}{cccc}\mathrm{H} & 2.968517 & -0.360944 & -0.891002 \\ \mathrm{H} & 2.291583 & 1.026397 & -0.000868\end{array}$

\section{Propene}

$\begin{array}{lrrr}\mathrm{C} & -1.282937 & -0.220582 & 0.000002 \\ \mathrm{H} & -1.303362 & -1.309002 & -0.000005 \\ \mathrm{H} & -2.245988 & 0.283216 & -0.000011 \\ \mathrm{C} & -0.134056 & 0.456138 & 0.000004 \\ \mathrm{H} & -0.163130 & 1.546985 & -0.000007 \\ \mathrm{C} & 1.234870 & -0.163109 & -0.000001 \\ \mathrm{H} & 1.182098 & -1.257324 & -0.000014 \\ \mathrm{H} & 1.811568 & 0.150733 & -0.880727 \\ \mathrm{H} & 1.811557 & 0.150710 & 0.880740\end{array}$

\section{Ethene}

$\begin{array}{lrc}\mathrm{C} & 0.000000 & 0.665588 \\ \mathrm{H} & 0.923860 & 1.239753 \\ \mathrm{H} & -0.923823 & 1.239796 \\ \mathrm{C} & -0.000000 & -0.665588 \\ \mathrm{H} & -0.923860 & -1.239753 \\ \mathrm{H} & 0.923823 & -1.239796\end{array}$

\section{INT1}

$\mathrm{Ru}$

$\mathrm{O}$

(

(

c

然

\section{(a)}

C

\section{(a)}

$\mathrm{H} \quad 0.298707$

C $\quad-2.832843$

C -3.485973

$\mathrm{H}-2.728753$

$\mathrm{H} \quad-3.856124$

C $\quad-4.646659$

$\mathrm{H} \quad-5.099030$

C $\quad-5.700283$

H -6.105212

H $\quad-6.543200$

C $\quad-5.054033$

H $\quad-5.801344$

C $\quad-3.890814$

$\mathrm{H} \quad-3.432850$

H $\quad-4.274480$

C $\quad-4.532140$

$\mathrm{H} \quad-4.099436$

H $\quad-5.370898$

C $\quad-3.462521$

$\mathrm{H} \quad-3.083605$

C $\quad-2.312999$

$\mathrm{H} \quad-1.986925$

C -4.096532

$\mathrm{H} \quad-3.344237$

H $\quad-4.907765$

C $\quad 1.894085$
0.792580

1.559478

3.022861

$-1.622570$

$-2.065546$

$-1.087363$

$-3.082368$

$-3.454052$

$-3.543459$

$-3.330289$

$-4.177274$

$-3.492538$

$-0.808085$

$-1.160197$

$-1.096490$

$-2.194975$

$-0.192479$

$-0.449934$

$-0.323619$

$-1.345401$

0.353656

0.019173

$-0.075192$

$-0.963798$

$-0.749469$

$-1.994385$

1.472663

1.756058

2.156927

1.608358

2.637120

0.650581

0.877192

1.247954

1.335933

1.951279

$-1.804067$

0.000000
0.000000
-0.000000
-0.000000
0.000000
-0.000000

0.343963

$-0.278968$

0.689998

$-0.593293$

$-0.197060$

$-0.174227$

$-0.684200$

$-1.572192$

0.202172

$-0.730143$

$-0.115978$

$-1.755150$

$-0.371400$

0.986923

1.773850

0.959884

1.278297

2.245062

0.160114

0.141650

0.352611

$-1.198478$

$-1.996962$

$-1.482801$

$-2.457445$

$-1.518133$

$-1.145558$

$-2.114772$

$-0.955222$

$-0.030169$

$-0.001709$

$-0.369122$

$-1.412822$

1.326426

2.119194

1.559068

$-0.165174$

\begin{tabular}{|c|c|c|c|}
\hline $\mathrm{C}$ & 2.588441 & -1.974780 & 1.046854 \\
\hline $\mathrm{C}$ & 3.966644 & -1.729263 & 1.057110 \\
\hline $\mathrm{H}$ & 4.524926 & -1.856730 & 1.980254 \\
\hline $\mathrm{C}$ & 4.630381 & -1.322194 & -0.096983 \\
\hline $\mathrm{C}$ & 3.922985 & -1.152516 & 1.284534 \\
\hline $\mathrm{H}$ & 4.448002 & -0.828833 & -2.178767 \\
\hline $\mathrm{C}$ & 2.544892 & -1.385135 & -1.344389 \\
\hline $\mathrm{C}$ & 1.884219 & -2.418831 & 2.320425 \\
\hline $\mathrm{H}$ & 0.809164 & -2.423758 & 2.116818 \\
\hline $\mathrm{C}$ & 1.807104 & -1.199393 & -2.666032 \\
\hline $\mathrm{H}$ & 0.734223 & -1.312345 & -2.482618 \\
\hline $\mathrm{C}$ & -0.602293 & 0.428654 & 2.107347 \\
\hline $\mathrm{H}$ & -0.576765 & -0.545366 & 2.608643 \\
\hline $\mathrm{H}$ & -0.705651 & 1.294798 & 2.778538 \\
\hline $\mathrm{H}$ & 5.700866 & -1.135258 & -0.071001 \\
\hline $\mathrm{C}$ & 2.295868 & -3.851998 & 2.707512 \\
\hline $\mathrm{H}$ & 3.369919 & -3.908844 & 2.921607 \\
\hline $\mathrm{H}$ & 1.756005 & -4.181711 & 3.603427 \\
\hline $\mathrm{H}$ & 2.081526 & -4.560044 & 1.898081 \\
\hline $\mathrm{C}$ & 2.129742 & -1.437231 & 3.480200 \\
\hline $\mathrm{H}$ & 1.532843 & -1.724277 & 4.354416 \\
\hline $\mathrm{H}$ & 3.182301 & -1.430482 & 3.786633 \\
\hline $\mathrm{H}$ & 1.851931 & -0.418852 & 3.190916 \\
\hline $\mathrm{C}$ & 2.227326 & -2.279081 & -3.683369 \\
\hline $\mathrm{H}$ & 2.066260 & -3.290488 & -3.292059 \\
\hline $\mathrm{H}$ & 1.656539 & -2.175651 & -4.614014 \\
\hline $\mathrm{H}$ & 3.291764 & -2.188266 & -3.930226 \\
\hline $\mathrm{C}$ & 2.007453 & 0.210801 & -3.251227 \\
\hline $\mathrm{H}$ & 3.052311 & 0.380589 & -3.537661 \\
\hline $\mathrm{H}$ & 1.395401 & 0.333442 & -4.153373 \\
\hline $\mathrm{H}$ & 1.728687 & 0.972745 & -2.520018 \\
\hline $\mathrm{C}$ & 1.284336 & 2.753013 & 0.171813 \\
\hline $\mathrm{C}$ & 2.420168 & 3.769104 & 0.073378 \\
\hline $\mathrm{C}$ & 1.961944 & 5.141115 & 0.585785 \\
\hline $\mathrm{H}$ & 1.124204 & 5.523164 & -0.006329 \\
\hline $\mathrm{H}$ & 2.788723 & 5.858817 & 0.522221 \\
\hline $\mathrm{H}$ & 1.630451 & 5.081192 & 1.626641 \\
\hline $\mathrm{C}$ & 3.588656 & 3.231520 & 0.930982 \\
\hline $\mathrm{H}$ & 4.444143 & 3.914805 & 0.866239 \\
\hline $\mathrm{H}$ & 3.898870 & 2.241034 & 0.584824 \\
\hline $\mathrm{H}$ & 3.296687 & 3.152065 & 1.984940 \\
\hline $\mathrm{C}$ & 2.865678 & 3.861233 & -1.401223 \\
\hline $\mathrm{H}$ & 3.701606 & 4.564717 & -1.495259 \\
\hline $\mathrm{H}$ & 2.048671 & 4.220350 & -2.038824 \\
\hline $\mathrm{H}$ & 3.186940 & 2.883902 & -1.772123 \\
\hline
\end{tabular}

\section{TS1}

$\begin{array}{lccc}\mathrm{Ru} & 0.480811 & 0.317323 & -0.643165 \\ \mathrm{O} & 2.122507 & 1.650040 & -1.320335 \\ \mathrm{O} & 1.559528 & 1.854263 & 0.801482 \\ \mathrm{~N} & -0.186842 & -2.135336 & 0.722171 \\ \mathrm{~N} & -2.114782 & -1.151458 & 0.384623 \\ \mathrm{C} & -0.756068 & -1.006634 & 0.215329 \\ \mathrm{C} & -1.151990 & -3.182837 & 1.041648 \\ \mathrm{H} & -0.888765 & -3.708900 & 1.963157 \\ \mathrm{H} & -1.211077 & -3.919101 & 0.226837 \\ \mathrm{C} & -2.440026 & -2.365366 & 1.163539 \\ \mathrm{H} & -3.316545 & -2.869871 & 0.751885 \\ \mathrm{H} & -2.653481 & -2.092329 & 2.205956 \\ \mathrm{C} & 1.233483 & -2.363628 & 0.463743\end{array}$




\begin{tabular}{|c|c|c|c|}
\hline $\mathrm{C}$ & 1.431620 & -3.176197 & -0.839336 \\
\hline $\mathrm{H}$ & 0.888720 & -2.689801 & -1.655374 \\
\hline $\mathrm{H}$ & 1.010501 & -4.184532 & -0.715231 \\
\hline $\mathrm{C}$ & 2.931483 & -3.267020 & -1.171461 \\
\hline $\mathrm{H}$ & 3.064362 & -3.829659 & -2.105344 \\
\hline $\mathrm{C}$ & 3.653301 & -3.996179 & -0.019877 \\
\hline $\mathrm{H}$ & 3.262768 & -5.018919 & 0.081693 \\
\hline $\mathrm{H}$ & 4.726288 & -4.081064 & -0.238983 \\
\hline $\mathrm{C}$ & 3.448378 & -3.212823 & 1.293764 \\
\hline $\mathrm{H}$ & 3.954056 & -3.734699 & 2.116862 \\
\hline $\mathrm{C}$ & 1.935445 & -3.123729 & 1.612015 \\
\hline $\mathrm{H}$ & 1.770333 & -2.590333 & 2.557217 \\
\hline $\mathrm{H}$ & 1.523500 & -4.137850 & 1.726756 \\
\hline $\mathrm{C}$ & 4.046703 & -1.799294 & 1.124230 \\
\hline $\mathrm{H}$ & 3.950829 & -1.230695 & 2.059012 \\
\hline $\mathrm{H}$ & 5.121037 & -1.880903 & 0.904035 \\
\hline $\mathrm{C}$ & 3.313587 & -1.053930 & -0.020106 \\
\hline $\mathrm{H}$ & 3.741460 & -0.057139 & -0.136835 \\
\hline $\mathrm{C}$ & 1.831763 & -0.945851 & 0.369575 \\
\hline $\mathrm{H}$ & 1.777904 & -0.513083 & 1.378810 \\
\hline $\mathrm{C}$ & 3.495307 & -1.839721 & -1.332338 \\
\hline $\mathrm{H}$ & 2.979063 & -1.317475 & -2.146974 \\
\hline $\mathrm{H}$ & 4.560976 & -1.888896 & -1.596567 \\
\hline $\mathrm{C}$ & -3.067601 & -0.080823 & 0.413930 \\
\hline $\mathrm{C}$ & -4.056945 & -0.024405 & -0.592245 \\
\hline $\mathrm{C}$ & -4.989595 & 1.018149 & -0.544768 \\
\hline $\mathrm{H}$ & -5.756841 & 1.085760 & -1.310338 \\
\hline $\mathrm{C}$ & -4.952729 & 1.967294 & 0.473993 \\
\hline $\mathrm{C}$ & -3.994698 & 1.872616 & 1.479714 \\
\hline $\mathrm{H}$ & -3.986941 & 2.604829 & 2.282011 \\
\hline $\mathrm{C}$ & -3.041878 & 0.845695 & 1.480976 \\
\hline $\mathrm{C}$ & -4.163913 & -1.100266 & -1.668203 \\
\hline $\mathrm{H}$ & -3.223769 & -1.662411 & -1.663130 \\
\hline $\mathrm{C}$ & -2.053880 & 0.744866 & 2.640984 \\
\hline $\mathrm{H}$ & -1.366304 & -0.081810 & 2.445574 \\
\hline $\mathrm{C}$ & 0.150404 & -0.388469 & -2.345708 \\
\hline $\mathrm{H}$ & -0.616479 & -1.093010 & -2.695714 \\
\hline $\mathrm{C}$ & -0.913658 & 2.114302 & -0.925658 \\
\hline $\mathrm{H}$ & -1.620045 & 1.981745 & -0.115335 \\
\hline $\mathrm{C}$ & -1.144246 & 1.344300 & -2.081129 \\
\hline $\mathrm{H}$ & -2.023220 & 0.715786 & -2.120722 \\
\hline $\mathrm{H}$ & 0.829961 & -0.070871 & -3.149988 \\
\hline $\mathrm{C}$ & -4.369909 & -0.540788 & -3.087864 \\
\hline $\mathrm{H}$ & -4.356050 & -1.359427 & -3.816582 \\
\hline $\mathrm{H}$ & -5.335788 & -0.032599 & -3.187241 \\
\hline $\mathrm{H}$ & -3.588865 & 0.172863 & -3.367341 \\
\hline $\mathrm{C}$ & -5.309716 & -2.076689 & -1.326550 \\
\hline $\mathrm{H}$ & -5.349884 & -2.897700 & -2.052316 \\
\hline $\mathrm{H}$ & -5.192769 & -2.508031 & -0.326864 \\
\hline $\mathrm{H}$ & -6.275420 & -1.557324 & -1.348176 \\
\hline $\mathrm{C}$ & -2.805273 & 0.426593 & 3.950184 \\
\hline $\mathrm{H}$ & -3.472264 & 1.249149 & 4.234314 \\
\hline $\mathrm{H}$ & -3.418142 & -0.477630 & 3.855192 \\
\hline $\mathrm{H}$ & -2.093398 & 0.274604 & 4.769814 \\
\hline $\mathrm{C}$ & -1.186875 & 2.007931 & 2.800296 \\
\hline $\mathrm{H}$ & -0.545313 & 2.166752 & 1.931302 \\
\hline $\mathrm{H}$ & -1.802730 & 2.901352 & 2.959551 \\
\hline $\mathrm{H}$ & -0.530270 & 1.897958 & 3.671199 \\
\hline $\mathrm{H}$ & -5.681305 & 2.773532 & 0.491795 \\
\hline $\mathrm{H}$ & -0.790699 & 1.731074 & -3.035516 \\
\hline
\end{tabular}

$\begin{array}{lrrr}\mathrm{C} & -0.303896 & 3.492730 & -1.012195 \\ \mathrm{H} & -1.098220 & 4.225924 & -1.212632 \\ \mathrm{H} & 0.174514 & 3.772246 & -0.070352 \\ \mathrm{H} & 0.436644 & 3.557789 & -1.812723 \\ \mathrm{C} & 3.406569 & 3.236571 & 0.003793 \\ \mathrm{C} & 3.441374 & 4.167822 & -1.222500 \\ \mathrm{H} & 4.267384 & 4.883502 & -1.128384 \\ \mathrm{H} & 3.575838 & 3.593105 & -2.142687 \\ \mathrm{H} & 2.509953 & 4.738596 & -1.310785 \\ \mathrm{C} & 3.168375 & 4.050575 & 1.285271 \\ \mathrm{H} & 3.131318 & 3.399791 & 2.162944 \\ \mathrm{H} & 3.974778 & 4.781898 & 1.421044 \\ \mathrm{H} & 2.218618 & 4.594749 & 1.235839 \\ \mathrm{C} & 4.750244 & 2.481446 & 0.108478 \\ \mathrm{H} & 4.938013 & 1.893119 & -0.795732 \\ \mathrm{H} & 5.573345 & 3.196073 & 0.232485 \\ \mathrm{H} & 4.755971 & 1.804206 & 0.970223 \\ \mathrm{C} & 2.294165 & 2.190523 & -0.177375\end{array}$

\section{INT2-1}

$\mathrm{Ru} \quad-0.494493$

O $\quad-2.154144$

$-0.410849$

$-1.630092$

$-0.392762$

O $\quad-2.210766$

$-1.743466$

$\mathrm{N} \quad 0.406400$

2.211894

1.099105

0.976249

3.268807

4.003804

3.792788

2.453971

2.801436

2.442261

2.420891

2.849026

2.119765

3.823638

2.935464

3.235864

3.985843

4.974995

4.068954

3.574039

4.321717

$-1.038576$

1.179239

0.407658

0.082358

0.110399

0.338665

1.137571

$-0.626385$

0.472248

$-0.180802$

1.505090

0.235784

$-1.220030$

$-1.915089$

$-1.432051$

$-1.409888$

$-2.442761$

$-0.431848$

$-0.651765$

$-0.556616$

-4.533852
-3.116599

1.018543

C $\quad-1.584105 \quad 3.490821$

1.198732

3.219298

2.231661

4.471803

0.991769

2.197157

1.305151

C $\quad-3.748496$

$\mathrm{H} \quad-3.548341$

1.894046

2.341451

2.259783

$-4.840158$

$-3.178626$

1.136484

1.191862

0.174902

0.328016

$\mathrm{H} \quad-3.636705$

C $\quad-1.660959$

$\mathrm{H} \quad-1.466175$

1.051608

0.808059

0.556300

0.556249

1.610931

C $\quad-3.501949$

$\mathrm{H} \quad-3.113280$

1.554358

$-1.119382$

0.800403

$-1.814122$

H $\quad-4.590531$

1.597734

$-1.262583$

0.023720

0.255948

$\begin{array}{ll}\text { C } & 3.190967 \\ \text { C } & 4.015951\end{array}$

$-0.345949$

$-0.826479$

C $\quad 4.899375$

$-1.417141$

$-0.644179$

H $\quad 5.540891$

$-1.463930$ 


$\begin{array}{lrrr}\mathrm{C} & 4.975239 & -2.084310 & 0.576311 \\ \mathrm{C} & 4.191870 & -1.667039 & 1.650055 \\ \mathrm{H} & 4.275784 & -2.178127 & 2.604993 \\ \mathrm{C} & 3.295635 & -0.599507 & 1.519738 \\ \mathrm{C} & 4.022645 & 0.443154 & -2.130981 \\ \mathrm{H} & 3.147285 & 1.100478 & -2.121541 \\ \mathrm{C} & 2.492441 & -0.137053 & 2.734237 \\ \mathrm{H} & 1.866267 & 0.708298 & 2.438253 \\ \mathrm{C} & 0.246367 & -0.506419 & -2.227009 \\ \mathrm{H} & 1.034498 & 0.162238 & -2.579133 \\ \mathrm{C} & 0.663224 & -2.055436 & -0.088025 \\ \mathrm{H} & 1.598362 & -1.844299 & 0.418257 \\ \mathrm{C} & 0.811227 & -1.874707 & -1.642515 \\ \mathrm{H} & 1.885517 & -1.887237 & -1.839129 \\ \mathrm{H} & -0.523171 & -0.677932 & -2.985913 \\ \mathrm{C} & 3.931063 & -0.436161 & -3.390017 \\ \mathrm{H} & 3.907095 & 0.195391 & -4.285623 \\ \mathrm{H} & 4.795416 & -1.103417 & -3.483690 \\ \mathrm{H} & 3.027086 & -1.051518 & -3.389475 \\ \mathrm{C} & 5.283944 & 1.330599 & -2.196110 \\ \mathrm{H} & 5.267250 & 1.962461 & -3.092091 \\ \mathrm{H} & 5.365968 & 1.981127 & -1.318265 \\ \mathrm{H} & 6.189886 & 0.713729 & -2.233521 \\ \mathrm{C} & 3.440680 & 0.358996 & 3.844449 \\ \mathrm{H} & 4.060648 & -0.456443 & 4.234767 \\ \mathrm{H} & 4.115220 & 1.140604 & 3.475287 \\ \mathrm{H} & 2.864253 & 0.769501 & 4.681608 \\ \mathrm{C} & 1.536095 & -1.220131 & 3.268434 \\ \mathrm{H} & 0.759720 & -1.458129 & 2.537692 \\ \mathrm{H} & 2.072190 & -2.142716 & 3.520541 \\ \mathrm{H} & 1.039197 & -0.863147 & 4.178145 \\ \mathrm{H} & 5.660509 & -2.919168 & 0.696636 \\ \mathrm{H} & 0.306701 & -2.704784 & -2.141473 \\ \mathrm{C} & 0.106045 & -3.390499 & 0.362267 \\ \mathrm{H} & 0.895014 & -4.154901 & 0.286056 \\ \mathrm{H} & -0.224584 & -3.344112 & 1.402826 \\ \mathrm{H} & -0.739233 & -3.712340 & -0.248123 \\ \mathrm{C} & -3.974478 & -2.878134 & -0.068310 \\ \mathrm{C} & -3.611904 & -4.182107 & -0.809305 \\ \mathrm{H} & -4.508036 & -4.798257 & -0.954939 \\ \mathrm{H} & -3.176169 & -3.961798 & -1.788601 \\ \mathrm{H} & -2.888990 & -4.773367 & -0.234266 \\ \mathrm{C} & -4.544501 & -3.200435 & 1.319775 \\ \mathrm{H} & -4.798833 & -2.283999 & 1.861879 \\ \mathrm{H} & -5.450769 & -3.811742 & 1.224041 \\ \mathrm{H} & -3.815441 & -3.746785 & 1.925570 \\ \mathrm{C} & -5.007577 & -2.090246 & -0.899698 \\ \mathrm{H} & -4.598359 & -1.830907 & -1.880050 \\ \mathrm{H} & -5.915665 & -2.689456 & -1.042535 \\ \mathrm{H} & -5.295966 & -1.160474 & -0.394900 \\ \mathrm{C} & -2.695306 & -2.028261 & 0.061648\end{array}$

$\begin{array}{lccc}\text { INT2 } & & & \\ \mathrm{Ru} & -0.478446 & -0.470758 & 0.153708 \\ \mathrm{C} & 0.625002 & -1.670276 & 1.310403 \\ \mathrm{C} & -0.014669 & -2.395940 & 2.481770 \\ \mathrm{H} & -1.028794 & -2.723414 & 2.248879 \\ \mathrm{H} & -0.054996 & -1.740800 & 3.360800 \\ \mathrm{H} & 0.592787 & -3.271686 & 2.758073 \\ \mathrm{H} & 1.623222 & -1.319985 & 1.557813\end{array}$

\begin{tabular}{|c|c|c|c|}
\hline $\mathrm{C}$ & 0.657288 & -2.476631 & -0.065331 \\
\hline $\mathrm{C}$ & 0.087022 & -1.698984 & -1.308571 \\
\hline $\mathrm{H}$ & 0.849059 & -1.361530 & -2.008612 \\
\hline $\mathrm{H}$ & -0.753570 & -2.192338 & -1.792274 \\
\hline $\mathrm{H}$ & 0.078597 & -3.392446 & 0.072733 \\
\hline $\mathrm{H}$ & 1.712864 & -2.687980 & -0.255411 \\
\hline $\mathrm{C}$ & 0.954714 & 0.892015 & -0.271714 \\
\hline $\mathrm{N}$ & 0.518991 & 2.167039 & -0.441112 \\
\hline $\mathrm{C}$ & -0.914023 & 2.435772 & -0.342612 \\
\hline $\mathrm{C}$ & -1.490617 & 1.285869 & 0.502480 \\
\hline $\mathrm{C}$ & -3.018159 & 1.422045 & 0.596281 \\
\hline $\mathrm{C}$ & -3.676354 & 1.458868 & -0.797771 \\
\hline $\mathrm{C}$ & -3.097913 & 2.631761 & -1.610105 \\
\hline $\mathrm{C}$ & -1.575530 & 2.435229 & -1.744617 \\
\hline $\mathrm{H}$ & -1.357551 & 1.480864 & -2.241231 \\
\hline $\mathrm{H}$ & -1.143043 & 3.239435 & -2.356677 \\
\hline $\mathrm{C}$ & -3.381421 & 3.965285 & -0.890373 \\
\hline $\mathrm{C}$ & -2.733606 & 3.942446 & 0.509683 \\
\hline $\mathrm{C}$ & -3.311882 & 2.762565 & 1.322657 \\
\hline $\mathrm{H}$ & -4.396640 & 2.892596 & 1.440364 \\
\hline $\mathrm{H}$ & -2.877360 & 2.751898 & 2.332157 \\
\hline $\mathrm{C}$ & -1.204152 & 3.782699 & 0.354434 \\
\hline $\mathrm{H}$ & -0.708050 & 3.797765 & 1.334075 \\
\hline $\mathrm{H}$ & -0.801935 & 4.620812 & -0.233698 \\
\hline $\mathrm{H}$ & -2.941516 & 4.886506 & 1.029941 \\
\hline $\mathrm{H}$ & -4.465098 & 4.116386 & -0.798165 \\
\hline $\mathrm{H}$ & -2.986472 & 4.808938 & -1.474618 \\
\hline $\mathrm{H}$ & -3.546849 & 2.647506 & -2.611462 \\
\hline $\mathrm{H}$ & -3.505666 & 0.514304 & -1.318888 \\
\hline $\mathrm{H}$ & -4.762794 & 1.583389 & -0.685527 \\
\hline $\mathrm{H}$ & -3.420713 & 0.586843 & 1.177011 \\
\hline $\mathrm{H}$ & -1.069654 & 1.354728 & 1.530506 \\
\hline $\mathrm{C}$ & 1.479799 & 3.033737 & -1.122180 \\
\hline $\mathrm{C}$ & 2.779636 & 2.229441 & -0.970485 \\
\hline $\mathrm{N}$ & 2.288519 & 0.884242 & -0.598915 \\
\hline $\mathrm{C}$ & 3.245051 & -0.056340 & -0.085846 \\
\hline $\mathrm{C}$ & 3.623463 & 0.019540 & 1.275538 \\
\hline $\mathrm{C}$ & 4.543705 & -0.919772 & 1.755435 \\
\hline $\mathrm{C}$ & 5.092896 & -1.884545 & 0.914368 \\
\hline $\mathrm{C}$ & 4.754624 & -1.904041 & -0.436028 \\
\hline $\mathrm{C}$ & 3.836269 & -0.987490 & -0.964674 \\
\hline $\mathrm{C}$ & 3.564191 & -0.959587 & -2.463395 \\
\hline $\mathrm{C}$ & 3.289964 & -2.350628 & -3.061779 \\
\hline $\mathrm{H}$ & 4.180128 & -2.989084 & -3.025742 \\
\hline $\mathrm{H}$ & 3.000025 & -2.254877 & -4.114250 \\
\hline $\mathrm{H}$ & 2.482798 & -2.866781 & -2.533927 \\
\hline $\mathrm{H}$ & 2.675900 & -0.340822 & -2.625049 \\
\hline $\mathrm{C}$ & 4.744798 & -0.289060 & -3.197315 \\
\hline $\mathrm{H}$ & 4.943394 & 0.716019 & -2.808835 \\
\hline $\mathrm{H}$ & 5.661922 & -0.877512 & -3.073620 \\
\hline $\mathrm{H}$ & 4.536131 & -0.207513 & -4.270592 \\
\hline $\mathrm{H}$ & 5.216654 & -2.635961 & -1.091560 \\
\hline $\mathrm{H}$ & 5.802139 & -2.607979 & 1.307557 \\
\hline $\mathrm{H}$ & 4.839801 & -0.890262 & 2.800194 \\
\hline $\mathrm{C}$ & 3.125184 & 1.121954 & 2.210733 \\
\hline $\mathrm{C}$ & 2.458882 & 0.589181 & 3.492515 \\
\hline $\mathrm{H}$ & 1.565389 & 0.004258 & 3.263749 \\
\hline $\mathrm{H}$ & 3.140949 & -0.041149 & 4.074278 \\
\hline 11 & 2.158580 & 1.428142 & 4.131220 \\
\hline $\mathrm{C}$ & 4.290093 & 2.068 & 2.570 \\
\hline
\end{tabular}




$\begin{array}{lrrr} & & & \\ \mathrm{H} & 5.053553 & 1.544795 & 3.157462 \\ \mathrm{H} & 3.927749 & 2.913505 & 3.167742 \\ \mathrm{H} & 4.780917 & 2.466228 & 1.674942 \\ \mathrm{H} & 2.366334 & 1.709415 & 1.687699 \\ \mathrm{H} & 3.420231 & 2.630335 & -0.176128 \\ \mathrm{H} & 3.367042 & 2.183827 & -1.891353 \\ \mathrm{H} & 1.531208 & 4.022252 & -0.657105 \\ \mathrm{H} & 1.201156 & 3.163673 & -2.176278 \\ \mathrm{O} & -2.171287 & -1.716957 & 0.542187 \\ \mathrm{C} & -3.067286 & -1.980356 & -0.357622 \\ \mathrm{O} & -3.026099 & -1.611501 & -1.540981 \\ \mathrm{C} & -4.262198 & -2.814905 & 0.169507 \\ \mathrm{C} & -5.249869 & -3.099391 & -0.970244 \\ \mathrm{H} & -4.766971 & -3.660578 & -1.776192 \\ \mathrm{H} & -5.624744 & -2.167067 & -1.403041 \\ \mathrm{H} & -6.102154 & -3.682738 & -0.598517 \\ \mathrm{C} & -4.960390 & -2.006404 & 1.282169 \\ \mathrm{H} & -4.262666 & -1.787342 & 2.096151 \\ \mathrm{H} & -5.810969 & -2.567962 & 1.689584 \\ \mathrm{H} & -5.341884 & -1.054312 & 0.892381 \\ \mathrm{C} & -3.729147 & -4.138719 & 0.751239 \\ \mathrm{H} & -3.028251 & -3.950384 & 1.569714 \\ \mathrm{H} & -3.208258 & -4.723114 & -0.017783 \\ \mathrm{H} & -4.555955 & -4.750333 & 1.134896\end{array}$

TS2

$\begin{array}{lrrr}\mathrm{Ru} & 0.337267 & -0.576449 & -0.690959 \\ \mathrm{O} & 1.753137 & -1.341141 & 1.033538 \\ \mathrm{O} & 1.906216 & -2.127760 & -1.020865 \\ \mathrm{~N} & -0.242179 & 2.210864 & -0.221568 \\ \mathrm{~N} & -2.157976 & 1.172167 & 0.005124 \\ \mathrm{C} & -0.819287 & 0.978699 & -0.224555 \\ \mathrm{C} & -1.129819 & 3.277895 & 0.238090 \\ \mathrm{H} & -1.051972 & 4.162771 & -0.399286 \\ \mathrm{H} & -0.884258 & 3.575535 & 1.267117 \\ \mathrm{C} & -2.506600 & 2.600201 & 0.143716 \\ \mathrm{H} & -3.124969 & 2.758765 & 1.032427 \\ \mathrm{H} & -3.073101 & 2.936102 & -0.733777 \\ \mathrm{C} & 1.218800 & 2.282837 & -0.166547 \\ \mathrm{C} & 1.695357 & 2.253786 & 1.308200 \\ \mathrm{H} & 1.303873 & 1.347674 & 1.785558 \\ \mathrm{H} & 1.296269 & 3.124265 & 1.849089 \\ \mathrm{C} & 3.235086 & 2.261603 & 1.365026 \\ \mathrm{H} & 3.558184 & 2.264180 & 2.414684 \\ \mathrm{C} & 3.775880 & 3.515699 & 0.651652 \\ \mathrm{H} & 3.420888 & 4.426834 & 1.154842 \\ \mathrm{H} & 4.873254 & 3.528325 & 0.698016 \\ \mathrm{C} & 3.309958 & 3.507205 & -0.818852 \\ \mathrm{H} & 3.704508 & 4.391479 & -1.336727 \\ \mathrm{C} & 1.764872 & 3.553424 & -0.850141 \\ \mathrm{H} & 1.396301 & 3.596090 & -1.883854 \\ \mathrm{H} & 1.415619 & 4.462848 & -0.337437 \\ \mathrm{C} & 3.828447 & 2.225173 & -1.511995 \\ \mathrm{H} & 3.536118 & 2.226262 & -2.571076 \\ \mathrm{H} & 4.927689 & 2.214515 & -1.481718 \\ \mathrm{C} & 3.257385 & 0.968630 & -0.798838 \\ \mathrm{H} & 3.621927 & 0.064852 & -1.300048 \\ \mathrm{C} & 1.719415 & 1.038538 & -0.924265 \\ \mathrm{H} & 1.523219 & 1.234722 & -1.988484 \\ \mathrm{C} & 3.754518 & 0.994097 & 0.662519\end{array}$

\begin{tabular}{lrrr} 
& & & \\
$\mathrm{H}$ & 3.404454 & 0.114249 & 1.200720 \\
$\mathrm{H}$ & 4.854508 & 0.985219 & 0.676799 \\
$\mathrm{C}$ & -3.064660 & 0.180018 & 0.489492 \\
$\mathrm{C}$ & -2.982131 & -0.205081 & 1.846409 \\
$\mathrm{C}$ & -3.861887 & -1.195956 & 2.299476 \\
$\mathrm{H}$ & -3.804111 & -1.528465 & 3.332179 \\
$\mathrm{C}$ & -4.809084 & -1.760531 & 1.447746 \\
$\mathrm{C}$ & -4.915180 & -1.320795 & 0.129847 \\
$\mathrm{H}$ & -5.682369 & -1.741273 & -0.513826 \\
$\mathrm{C}$ & -4.053149 & -0.337962 & -0.371761 \\
$\mathrm{C}$ & -2.000653 & 0.442102 & 2.821048 \\
$\mathrm{H}$ & -1.464531 & 1.234533 & 2.292123 \\
$\mathrm{C}$ & -4.245054 & 0.222952 & -1.775935 \\
$\mathrm{H}$ & -3.346455 & 0.797515 & -2.023639 \\
$\mathrm{C}$ & -0.836690 & -2.310652 & 0.212485 \\
$\mathrm{H}$ & -1.546834 & -1.950049 & 0.942004 \\
$\mathrm{C}$ & -0.449737 & -0.625862 & -2.395200 \\
$\mathrm{H}$ & -1.416909 & -0.172011 & -2.658241 \\
$\mathrm{C}$ & -1.208863 & -2.401778 & -1.125977 \\
$\mathrm{H}$ & -2.207404 & -2.100649 & -1.421463 \\
$\mathrm{H}$ & -0.050414 & -2.953486 & 0.590523 \\
$\mathrm{C}$ & -5.444019 & 1.194770 & -1.785254 \\
$\mathrm{H}$ & -5.556332 & 1.664019 & -2.770015 \\
$\mathrm{H}$ & -6.374712 & 0.662678 & -1.554106 \\
$\mathrm{H}$ & -5.322153 & 1.987242 & -1.038766 \\
$\mathrm{C}$ & -4.423916 & -0.851394 & -2.861580 \\
$\mathrm{H}$ & -5.327600 & -1.449680 & -2.699581 \\
$\mathrm{H}$ & -4.518237 & -0.376608 & -3.845059 \\
$\mathrm{H}$ & 5.0237278 & -3.278073 & -0.009214 \\
$\mathrm{H}$ & 4.498889 & -2.675598 & -1.308385 \\
$\mathrm{H}$ & -3.570281 & -1.535001 & -2.901093 \\
$\mathrm{C}$ & -2.757576 & 1.106477 & 3.988263 \\
$\mathrm{H}$ & -3.513479 & 1.812826 & 3.624882 \\
$\mathrm{H}$ & -3.269145 & 0.363662 & 4.61170 \\
$\mathrm{H}$ & -2.057670 & 1.652627 & 4.631297 \\
$\mathrm{C}$ & -0.932586 & -0.539983 & 3.339847 \\
$\mathrm{H}$ & -0.308865 & -0.046053 & 4.094580 \\
$\mathrm{H}$ & -1.393810 & -1.416931 & 3.810598 \\
$\mathrm{H}$ & -0.273247 & -0.879183 & 2.536939 \\
$\mathrm{H}$ & -5.479632 & -2.531701 & 1.817530 \\
$\mathrm{H}$ & -0.689239 & -3.089131 & -1.787376 \\
$\mathrm{C}$ & 0.231088 & -1.242826 & -3.584562 \\
$\mathrm{H}$ & -0.450175 & -1.868436 & -4.180544 \\
$\mathrm{H}$ & 0.552920 & -0.417016 & -4.240483 \\
$\mathrm{C}$ & 1.115362 & -1.821323 & -3.304584 \\
$\mathrm{C}$ & 2.314066 & -2.103542 & 0.188500 \\
$\mathrm{C}$ & 3.478957 & -3.016860 & 0.592419 \\
$\mathrm{H}$ & 2.881255 & -4.468820 & 0.253284 \\
$\mathrm{H}$ & 3.900970 & -4.567699 & -0.811097 \\
\hline & -5.154130 & 0.501485 \\
\hline & -4.778627 & 0.826460 \\
\hline
\end{tabular}

\section{INT3}

$\begin{array}{llll}\mathrm{Ru} & -0.502902 & 0.233337 & -0.797113\end{array}$ 


\begin{tabular}{|c|c|c|c|}
\hline $\mathrm{C}$ & 0.341379 & -0.158742 & -2.357829 \\
\hline $\mathrm{C}$ & 0.034474 & 0.542028 & -3.654418 \\
\hline $\mathrm{H}$ & -0.778457 & 1.267283 & -3.559737 \\
\hline $\mathrm{H}$ & -0.244370 & -0.203625 & -4.416639 \\
\hline $\mathrm{H}$ & 0.926130 & 1.064266 & -4.034979 \\
\hline $\mathrm{H}$ & 1.153137 & -0.893939 & -2.430372 \\
\hline $\mathrm{C}$ & 0.397461 & -1.210926 & 0.158770 \\
\hline $\mathrm{N}$ & 1.663529 & -1.424756 & 0.601988 \\
\hline $\mathrm{C}$ & 2.753310 & -0.499998 & 0.490759 \\
\hline $\mathrm{C}$ & 2.816309 & 0.588741 & 1.392428 \\
\hline $\mathrm{C}$ & 3.909517 & 1.455516 & 1.287747 \\
\hline $\mathrm{C}$ & 4.909578 & 1.249060 & 0.339137 \\
\hline $\mathrm{C}$ & 4.828427 & 0.171799 & -0.536371 \\
\hline $\mathrm{C}$ & 3.746115 & -0.715735 & -0.485084 \\
\hline $\mathrm{C}$ & 3.684484 & -1.891422 & -1.451942 \\
\hline $\mathrm{C}$ & 4.685442 & -2.989321 & -1.038732 \\
\hline $\mathrm{H}$ & 4.513174 & -3.326743 & -0.010767 \\
\hline $\mathrm{H}$ & 5.713763 & -2.612247 & -1.093443 \\
\hline $\mathrm{H}$ & 4.607628 & -3.857919 & -1.703738 \\
\hline $\mathrm{C}$ & 3.926452 & -1.469040 & -2.912892 \\
\hline $\mathrm{H}$ & 3.750446 & -2.317558 & -3.584469 \\
\hline $\mathrm{H}$ & 3.259442 & -0.652747 & -3.207224 \\
\hline $\mathrm{H}$ & 4.957698 & -1.134097 & -3.071846 \\
\hline $\mathrm{H}$ & 2.675472 & -2.314283 & -1.401025 \\
\hline $\mathrm{H}$ & 5.611635 & 0.019388 & -1.273229 \\
\hline $\mathrm{H}$ & 5.752589 & 1.932971 & 0.283135 \\
\hline $\mathrm{H}$ & 3.985277 & 2.305030 & 1.957633 \\
\hline $\mathrm{C}$ & 1.761957 & 0.796054 & 2.476921 \\
\hline $\mathrm{C}$ & 2.078155 & -0.065725 & 3.716613 \\
\hline $\mathrm{H}$ & 2.120963 & -1.132188 & 3.476845 \\
\hline $\mathrm{H}$ & 3.045805 & 0.218449 & 4.147845 \\
\hline $\mathrm{H}$ & 1.309581 & 0.074761 & 4.486431 \\
\hline $\mathrm{C}$ & 1.588218 & 2.264694 & 2.893097 \\
\hline $\mathrm{H}$ & 1.421709 & 2.895466 & 2.018029 \\
\hline $\mathrm{H}$ & 2.453312 & 2.640499 & 3.453416 \\
\hline $\mathrm{H}$ & 0.713612 & 2.356758 & 3.547432 \\
\hline $\mathrm{H}$ & 0.800470 & 0.472172 & 2.071520 \\
\hline $\mathrm{C}$ & 1.788793 & -2.750110 & 1.241252 \\
\hline $\mathrm{H}$ & 2.440183 & -2.705605 & 2.116102 \\
\hline $\mathrm{C}$ & 0.323050 & -3.076696 & 1.571265 \\
\hline $\mathrm{N}$ & -0.363397 & -2.277200 & 0.556732 \\
\hline $\mathrm{C}$ & -1.809786 & -2.054425 & 0.453232 \\
\hline $\mathrm{C}$ & -2.631096 & -3.353800 & 0.398554 \\
\hline $\mathrm{C}$ & -4.118679 & -2.985519 & 0.171161 \\
\hline $\mathrm{C}$ & -4.623499 & -2.128580 & 1.351074 \\
\hline $\mathrm{C}$ & -3.797638 & -0.828602 & 1.435784 \\
\hline $\mathrm{C}$ & -2.317318 & -1.195194 & 1.643533 \\
\hline $\mathrm{H}$ & -2.189860 & -1.749347 & 2.584590 \\
\hline $\mathrm{H}$ & -1.707994 & -0.283339 & 1.727260 \\
\hline $\mathrm{C}$ & -3.939462 & -0.026468 & 0.125542 \\
\hline $\mathrm{C}$ & -3.442232 & -0.875958 & -1.062527 \\
\hline $\mathrm{C}$ & -1.969293 & -1.284449 & -0.884639 \\
\hline $\mathrm{H}$ & -1.715092 & -1.990060 & -1.682362 \\
\hline $\mathrm{H}$ & -3.553993 & -0.305735 & -1.992553 \\
\hline $\mathrm{C}$ & -4.282646 & -2.177201 & -1.136369 \\
\hline $\mathrm{H}$ & -3.962561 & -2.780130 & -1.996746 \\
\hline $\mathrm{H}$ & -5.345285 & -1.938517 & -1.286999 \\
\hline $\mathrm{H}$ & -4.990670 & 0.254003 & -0.027658 \\
\hline $\mathrm{H}$ & -3.365918 & 0.907844 & 0.182670 \\
\hline $\mathrm{H}$ & -4.145747 & -0.223459 & 2.283326 \\
\hline
\end{tabular}

$\begin{array}{lrcc}\mathrm{H} & -5.686541 & -1.890918 & 1.211174 \\ \mathrm{H} & -4.539630 & -2.690333 & 2.292183 \\ \mathrm{H} & -4.707869 & -3.909628 & 0.109321 \\ \mathrm{H} & -2.260366 & -3.986647 & -0.418193 \\ \mathrm{H} & -2.528976 & -3.925553 & 1.333529 \\ \mathrm{H} & 0.092403 & -4.140634 & 1.476060 \\ \mathrm{H} & 0.054031 & -2.745620 & 2.586299 \\ \mathrm{H} & 2.206028 & -3.476504 & 0.531767 \\ \mathrm{O} & -0.154055 & 2.098086 & 0.241199 \\ \mathrm{C} & -0.996755 & 2.761172 & -0.480072 \\ \mathrm{O} & -1.598904 & 2.179389 & -1.422918 \\ \mathrm{C} & -1.236249 & 4.232909 & -0.144484 \\ \mathrm{C} & 0.103985 & 4.982227 & -0.308170 \\ \mathrm{H} & -0.024407 & 6.039635 & -0.047345 \\ \mathrm{H} & 0.459501 & 4.928674 & -1.344054 \\ \mathrm{H} & 0.873931 & 4.553173 & 0.339381 \\ \mathrm{C} & -2.299125 & 4.829739 & -1.077536 \\ \mathrm{H} & -1.987559 & 4.760626 & -2.124161 \\ \mathrm{H} & -3.250911 & 4.298161 & -0.980803 \\ \mathrm{H} & -2.462441 & 5.885707 & -0.830583 \\ \mathrm{C} & -1.702080 & 4.316515 & 1.325256 \\ \mathrm{H} & -0.956218 & 3.881184 & 1.996060 \\ \mathrm{H} & -1.862151 & 5.363699 & 1.608608 \\ \mathrm{H} & -2.647971 & 3.779406 & 1.467612\end{array}$

\section{TS3-cross}

$\begin{array}{lccc}\mathrm{Ru} & -0.358285 & 0.424118 & -0.398666 \\ \mathrm{O} & -1.819454 & 0.677933 & 1.483214 \\ \mathrm{O} & -1.845149 & 2.101176 & -0.200040 \\ \mathrm{~N} & 0.050865 & -2.380053 & -0.918618 \\ \mathrm{~N} & 2.018643 & -1.606804 & -0.328867 \\ \mathrm{C} & 0.698584 & -1.268846 & -0.479962 \\ \mathrm{C} & 0.870610 & -3.591050 & -0.887514 \\ \mathrm{H} & 0.755051 & -4.171984 & -1.806244 \\ \mathrm{H} & 0.590035 & -4.231196 & -0.039728 \\ \mathrm{C} & 2.282965 & -3.004375 & -0.732890 \\ \mathrm{H} & 2.879177 & -3.514752 & 0.028755 \\ \mathrm{H} & 2.840450 & -3.020243 & -1.676827 \\ \mathrm{C} & -1.412306 & -2.382636 & -0.883044 \\ \mathrm{C} & -1.905615 & -2.874132 & 0.501923 \\ \mathrm{H} & -1.467140 & -2.234130 & 1.276785 \\ \mathrm{H} & -1.564448 & -3.904344 & 0.678375 \\ \mathrm{C} & -3.443576 & -2.812166 & 0.566717 \\ \mathrm{H} & -3.779110 & -3.184897 & 1.543629 \\ \mathrm{C} & -4.046174 & -3.679415 & -0.555545 \\ \mathrm{H} & -3.750919 & -4.730869 & -0.428100 \\ \mathrm{H} & -5.142835 & -3.644319 & -0.507074 \\ \mathrm{C} & -3.561616 & -3.155063 & -1.922999 \\ \mathrm{H} & -3.999380 & -3.760427 & -2.727484 \\ \mathrm{C} & -2.021612 & -3.274029 & -1.985068 \\ \mathrm{H} & -1.644074 & -2.950148 & -2.964170 \\ \mathrm{H} & -1.728903 & -4.326328 & -1.850124 \\ \mathrm{C} & -3.996415 & -1.680191 & -2.088103 \\ \mathrm{H} & -3.689374 & -1.305519 & -3.074131 \\ \mathrm{H} & -5.093257 & -1.616100 & -2.046756 \\ \mathrm{C} & -3.365558 & -0.812298 & -0.964409 \\ \mathrm{H} & -3.670367 & 0.231758 & -1.095157 \\ \mathrm{C} & -1.834989 & -0.921831 & -1.114972 \\ \mathrm{H} & -1.619144 & -0.699131 & -2.173158 \\ \mathrm{C} & -3.880678 & -1.346827 & 0.389302\end{array}$




\begin{tabular}{|c|c|c|c|}
\hline $\mathrm{H}$ & -3.488657 & -0.746507 & 1.210502 \\
\hline $\mathrm{H}$ & -4.978320 & -1.280664 & 0.415919 \\
\hline $\mathrm{C}$ & 2.944268 & -0.951692 & 0.546557 \\
\hline $\mathrm{C}$ & 2.770980 & -1.099507 & 1.941482 \\
\hline $\mathrm{C}$ & 3.666971 & -0.431877 & 2.785654 \\
\hline $\mathrm{H}$ & 3.542758 & -0.508900 & 3.862253 \\
\hline $\mathrm{C}$ & 4.716085 & 0.323643 & 2.266992 \\
\hline $\mathrm{C}$ & 4.905992 & 0.404157 & 0.889285 \\
\hline $\mathrm{H}$ & 5.741394 & 0.975482 & 0.496968 \\
\hline $\mathrm{C}$ & 4.030979 & -0.235115 & 0.002844 \\
\hline $\mathrm{C}$ & 1.684115 & -1.986800 & 2.546064 \\
\hline $\mathrm{H}$ & 1.133654 & -2.463548 & 1.731218 \\
\hline $\mathrm{C}$ & 4.291423 & -0.206851 & -1.499114 \\
\hline $\mathrm{H}$ & 3.364892 & -0.515134 & -1.995826 \\
\hline $\mathrm{C}$ & 0.833414 & 1.579886 & 1.008443 \\
\hline $\mathrm{H}$ & 1.409109 & 0.929260 & 1.652742 \\
\hline $\mathrm{C}$ & 0.569563 & 1.024167 & -1.940096 \\
\hline $\mathrm{H}$ & 1.517085 & 0.606016 & -2.301311 \\
\hline $\mathrm{H}$ & 0.178192 & 2.269475 & 1.533350 \\
\hline $\mathrm{C}$ & 5.379415 & -1.243255 & -1.853339 \\
\hline $\mathrm{H}$ & 5.533859 & -1.286384 & -2.938068 \\
\hline $\mathrm{H}$ & 6.333673 & -0.972556 & -1.385598 \\
\hline $\mathrm{H}$ & 5.112715 & -2.246761 & -1.503469 \\
\hline $\mathrm{C}$ & 4.682154 & 1.179249 & -2.045449 \\
\hline $\mathrm{H}$ & 5.656202 & 1.501668 & -1.659270 \\
\hline $\mathrm{H}$ & 4.770702 & 1.129430 & -3.137150 \\
\hline $\mathrm{H}$ & 3.952788 & 1.958829 & -1.802593 \\
\hline $\mathrm{C}$ & 2.317028 & -3.116031 & 3.383685 \\
\hline $\mathrm{H}$ & 3.045979 & -3.688004 & 2.797376 \\
\hline $\mathrm{H}$ & 2.836331 & -2.720693 & 4.264162 \\
\hline $\mathrm{H}$ & 1.542156 & -3.806416 & 3.736958 \\
\hline $\mathrm{C}$ & 0.649396 & -1.198131 & 3.371231 \\
\hline $\mathrm{H}$ & -0.057707 & -1.891685 & 3.842175 \\
\hline $\mathrm{H}$ & 1.132025 & -0.621660 & 4.169739 \\
\hline $\mathrm{H}$ & 0.072010 & -0.512812 & 2.745441 \\
\hline $\mathrm{H}$ & 5.397595 & 0.838575 & 2.938799 \\
\hline $\mathrm{C}$ & -0.025505 & 1.998723 & -2.918514 \\
\hline $\mathrm{H}$ & 0.691042 & 2.778288 & -3.211148 \\
\hline $\mathrm{H}$ & -0.267253 & 1.423240 & -3.826487 \\
\hline $\mathrm{H}$ & -0.945179 & 2.460792 & -2.551546 \\
\hline $\mathrm{C}$ & 1.438599 & 2.011658 & -0.205458 \\
\hline $\mathrm{H}$ & 2.404885 & 1.584753 & -0.432463 \\
\hline $\mathrm{C}$ & 1.340427 & 3.422055 & -0.729257 \\
\hline $\mathrm{O}$ & 2.309147 & 3.911171 & -1.316096 \\
\hline $\mathrm{N}$ & 0.180852 & 4.091100 & -0.504049 \\
\hline $\mathrm{H}$ & -0.642986 & 3.538194 & -0.267433 \\
\hline $\mathrm{C}$ & -0.027401 & 5.407316 & -1.080106 \\
\hline $\mathrm{H}$ & -0.861340 & 5.893133 & -0.566120 \\
\hline $\mathrm{H}$ & 0.877077 & 6.005676 & -0.952930 \\
\hline $\mathrm{H}$ & -0.252325 & 5.355655 & -2.154995 \\
\hline $\mathrm{C}$ & -2.294702 & 1.710239 & 0.938752 \\
\hline $\mathrm{C}$ & -3.410660 & 2.526301 & 1.601949 \\
\hline $\mathrm{C}$ & -3.673930 & 2.018948 & 3.027202 \\
\hline $\mathrm{H}$ & -4.475898 & 2.605492 & 3.491530 \\
\hline $\mathrm{H}$ & -3.971642 & 0.966456 & 3.021151 \\
\hline $\mathrm{H}$ & -2.776796 & 2.105183 & 3.648920 \\
\hline $\mathrm{C}$ & -2.980032 & 4.007442 & 1.632953 \\
\hline $\mathrm{H}$ & -2.825221 & 4.390216 & 0.619581 \\
\hline $\mathrm{H}$ & -3.754087 & 4.615139 & 2.116328 \\
\hline $\mathrm{H}$ & -2.047638 & 4.138929 & 2.195878 \\
\hline
\end{tabular}

$\begin{array}{llll}\mathrm{C} & -4.683780 & 2.377472 & 0.740586 \\ \mathrm{H} & -5.020397 & 1.335324 & 0.713730 \\ \mathrm{H} & -5.494012 & 2.985796 & 1.160596 \\ \mathrm{H} & -4.497262 & 2.705409 & -0.286783\end{array}$

\section{TS3-homo}

\begin{tabular}{|c|c|c|c|}
\hline $\mathrm{Ru}$ & 0.355749 & -0.587501 & -0.571758 \\
\hline $\mathrm{O}$ & 1.825822 & -1.142846 & 1.232311 \\
\hline $\mathrm{O}$ & 1.922662 & -2.163318 & -0.721388 \\
\hline $\mathrm{N}$ & -0.213655 & 2.241506 & -0.499407 \\
\hline $\mathrm{N}$ & -2.140137 & 1.259213 & -0.144163 \\
\hline $\mathrm{C}$ & -0.799149 & 1.026326 & -0.326876 \\
\hline $\mathrm{C}$ & -1.099794 & 3.372613 & -0.230140 \\
\hline $\mathrm{H}$ & -1.009073 & 4.141237 & -1.002384 \\
\hline $\mathrm{H}$ & -0.863059 & 3.832898 & 0.739184 \\
\hline $\mathrm{C}$ & -2.479340 & 2.694551 & -0.230156 \\
\hline $\mathrm{H}$ & -3.102603 & 2.991109 & 0.618796 \\
\hline $\mathrm{H}$ & -3.037934 & 2.893772 & -1.153028 \\
\hline $\mathrm{C}$ & 1.246011 & 2.317839 & -0.441490 \\
\hline $\mathrm{C}$ & 1.707295 & 2.528763 & 1.023410 \\
\hline $\mathrm{H}$ & 1.304391 & 1.714338 & 1.637144 \\
\hline $\mathrm{H}$ & 1.307956 & 3.477574 & 1.410385 \\
\hline $\mathrm{C}$ & 3.246410 & 2.538975 & 1.095113 \\
\hline $\mathrm{H}$ & 3.558308 & 2.711937 & 2.133832 \\
\hline $\mathrm{C}$ & 3.800481 & 3.658888 & 0.193083 \\
\hline $\mathrm{H}$ & 3.445308 & 4.640820 & 0.538087 \\
\hline $\mathrm{H}$ & 4.897362 & 3.675113 & 0.248246 \\
\hline $\mathrm{C}$ & 3.349295 & 3.413952 & -1.261628 \\
\hline $\mathrm{H}$ & 3.753239 & 4.201360 & -1.911663 \\
\hline $\mathrm{C}$ & 1.805196 & 3.457544 & -1.318476 \\
\hline $\mathrm{H}$ & 1.448951 & 3.330003 & -2.349554 \\
\hline $\mathrm{H}$ & 1.452682 & 4.439049 & -0.966291 \\
\hline $\mathrm{C}$ & 3.866738 & 2.034964 & -1.732560 \\
\hline $\mathrm{H}$ & 3.581768 & 1.864477 & -2.779911 \\
\hline $\mathrm{H}$ & 4.965590 & 2.024245 & -1.692087 \\
\hline $\mathrm{C}$ & 3.285077 & 0.913200 & -0.828947 \\
\hline $\mathrm{H}$ & 3.648579 & -0.059981 & -1.175832 \\
\hline $\mathrm{C}$ & 1.750458 & 0.964949 & -0.969848 \\
\hline $\mathrm{H}$ & 1.545472 & 0.956179 & -2.054308 \\
\hline $\mathrm{C}$ & 3.767132 & 1.172222 & 0.614437 \\
\hline $\mathrm{H}$ & 3.406350 & 0.388375 & 1.280305 \\
\hline $\mathrm{H}$ & 4.866823 & 1.162486 & 0.642273 \\
\hline $\mathrm{C}$ & -3.049023 & 0.372573 & 0.512673 \\
\hline $\mathrm{C}$ & -2.940879 & 0.208849 & 1.912297 \\
\hline $\mathrm{C}$ & -3.820037 & -0.685027 & 2.535646 \\
\hline $\mathrm{H}$ & -3.741900 & -0.849588 & 3.606708 \\
\hline $\mathrm{C}$ & -4.793711 & -1.363283 & 1.805342 \\
\hline $\mathrm{C}$ & -4.926639 & -1.135884 & 0.437227 \\
\hline $\mathrm{H}$ & -5.713830 & -1.641123 & -0.114777 \\
\hline $\mathrm{C}$ & -4.064267 & -0.259774 & -0.233296 \\
\hline $\mathrm{C}$ & -1.935584 & 0.993945 & 2.752984 \\
\hline $\mathrm{H}$ & -1.400780 & 1.683001 & 2.094301 \\
\hline $\mathrm{C}$ & -4.280359 & 0.067722 & -1.706224 \\
\hline $\mathrm{H}$ & -3.381092 & 0.581285 & -2.061955 \\
\hline $\mathrm{C}$ & -0.788032 & -2.053293 & 0.545356 \\
\hline $\mathrm{H}$ & -1.480517 & -1.582314 & 1.227977 \\
\hline $\mathrm{C}$ & -0.525603 & -0.936531 & -2.207090 \\
\hline $\mathrm{H}$ & -1.497577 & -0.516440 & -2.497027 \\
\hline $\mathrm{C}$ & -1.248928 & -2.397771 & -0.758159 \\
\hline $\mathrm{H}$ & -2.259473 & -2.073191 & -0.986395 \\
\hline
\end{tabular}




\begin{tabular}{|c|c|c|c|c|c|c|c|}
\hline $\mathrm{H}$ & -0.074861 & -2.726285 & 1.015820 & $\mathrm{C}$ & -3.974856 & -3.821149 & -0.522687 \\
\hline$C$ & -5.468340 & 1.042007 & -1.851529 & $\mathrm{H}$ & -3.661880 & -4.872633 & -0.452267 \\
\hline $\mathrm{H}$ & -5.597856 & 1.343362 & -2.897930 & $\mathrm{H}$ & -5.070497 & -3.805598 & -0.450927 \\
\hline $\mathrm{H}$ & -6.399422 & 0.569348 & -1.516260 & $\mathrm{C}$ & -3.527708 & -3.225905 & -1.873942 \\
\hline $\mathrm{H}$ & -5.320548 & 1.945345 & -1.249558 & $\mathrm{H}$ & -3.972221 & -3.799859 & -2.697186 \\
\hline $\mathrm{C}$ & -4.493782 & -1.167721 & -2.597299 & $\mathrm{C}$ & -1.989156 & -3.306187 & -1.977309 \\
\hline $\mathrm{H}$ & -5.403271 & -1.715050 & -2.324963 & $\mathrm{H}$ & -1.641735 & -2.917882 & -2.943876 \\
\hline $\mathrm{H}$ & -4.598930 & -0.859462 & -3.643943 & $\mathrm{H}$ & -1.663922 & -4.354529 & -1.906653 \\
\hline $\mathrm{H}$ & -3.651206 & -1.863320 & -2.536098 & $\mathrm{C}$ & -3.983089 & -1.752776 & -1.962731 \\
\hline $\mathrm{C}$ & -2.667758 & 1.850234 & 3.805558 & $\mathrm{H}$ & -3.692535 & -1.325615 & -2.932317 \\
\hline $\mathrm{H}$ & -3.422349 & 2.496367 & 3.341338 & $\mathrm{H}$ & -5.078914 & -1.698517 & -1.901867 \\
\hline $\mathrm{H}$ & -3.176929 & 1.226091 & 4.548965 & $\mathrm{C}$ & -3.353038 & -0.931661 & -0.803554 \\
\hline $\mathrm{H}$ & -1.952650 & 2.486124 & 4.340303 & $\mathrm{H}$ & -3.671834 & 0.111096 & -0.883968 \\
\hline $\mathrm{C}$ & -0.867943 & 0.099011 & 3.411714 & $\mathrm{C}$ & -1.831314 & -1.013677 & -0.960826 \\
\hline $\mathrm{H}$ & -0.229439 & 0.703961 & 4.066720 & $\mathrm{H}$ & -1.567220 & -0.641546 & -1.982932 \\
\hline $\mathrm{H}$ & -1.329051 & -0.683840 & 4.026287 & $\mathrm{C}$ & -3.825434 & -1.535254 & 0.535217 \\
\hline $\mathrm{H}$ & -0.223184 & -0.375076 & 2.667353 & $\mathrm{H}$ & -3.421031 & -0.953406 & 1.365299 \\
\hline $\mathrm{H}$ & -5.464229 & -2.055562 & 2.307622 & $\mathrm{H}$ & -4.922420 & -1.486173 & 0.590477 \\
\hline $\mathrm{C}$ & 0.179914 & -1.606868 & -3.355137 & $\mathrm{C}$ & 2.952158 & -0.930674 & 0.544469 \\
\hline $\mathrm{H}$ & 0.536836 & -0.800831 & -4.017344 & $\mathrm{C}$ & 2.769529 & -1.070045 & 1.939008 \\
\hline $\mathrm{H}$ & 1.051022 & -2.182540 & -3.032727 & $\mathrm{C}$ & 3.645944 & -0.379548 & 2.784785 \\
\hline $\mathrm{H}$ & -0.481597 & -2.243119 & -3.959769 & $\mathrm{H}$ & 3.512577 & -0.448238 & 3.860771 \\
\hline $\mathrm{C}$ & -0.822886 & -3.714551 & -1.374282 & $\mathrm{C}$ & 4.684082 & 0.392578 & 2.268663 \\
\hline $\mathrm{H}$ & -1.319604 & -4.531436 & -0.833948 & $\mathrm{C}$ & 4.884019 & 0.465163 & 0.892133 \\
\hline $\mathrm{H}$ & -1.102659 & -3.798481 & -2.428021 & $\mathrm{H}$ & 5.710600 & 1.050304 & 0.501762 \\
\hline $\mathrm{H}$ & 0.257932 & -3.848444 & -1.282254 & $\mathrm{C}$ & 4.029730 & -0.199071 & 0.003544 \\
\hline $\mathrm{C}$ & 3.520106 & -2.880989 & 0.950548 & $\mathrm{C}$ & 1.686795 & -1.961406 & 2.543776 \\
\hline $\mathrm{C}$ & 4.751265 & -2.565799 & 0.074869 & $\mathrm{H}$ & 1.155971 & -2.461468 & 1.729707 \\
\hline $\mathrm{H}$ & 5.066776 & -1.524034 & 0.202946 & $\mathrm{C}$ & 4.307520 & -0.178881 & -1.494954 \\
\hline $\mathrm{H}$ & 5.591487 & -3.211968 & 0.357749 & $\mathrm{H}$ & 3.393598 & -0.507382 & -2.001063 \\
\hline $\mathrm{H}$ & 4.524432 & -2.728054 & -0.983173 & $\mathrm{C}$ & 0.722361 & 1.450850 & 0.876431 \\
\hline $\mathrm{C}$ & 3.837593 & -2.605262 & 2.427304 & $\mathrm{H}$ & 1.447078 & 0.975719 & 1.528329 \\
\hline $\mathrm{H}$ & 2.967418 & -2.802477 & 3.061416 & $\mathrm{C}$ & 0.781871 & 1.180410 & -1.782411 \\
\hline $\mathrm{H}$ & 4.663292 & -3.246889 & 2.759250 & $\mathrm{H}$ & 1.604776 & 0.597082 & -2.200999 \\
\hline $\mathrm{H}$ & 4.127450 & -1.561421 & 2.580379 & $\mathrm{H}$ & 0.134955 & 2.197396 & 1.410269 \\
\hline $\mathrm{C}$ & 3.113789 & -4.356860 & 0.759298 & $\mathrm{C}$ & 5.418624 & -1.197652 & -1.827919 \\
\hline $\mathrm{H}$ & 2.879147 & -4.560729 & -0.289418 & $\mathrm{H}$ & 5.590184 & -1.242673 & -2.910019 \\
\hline $\mathrm{H}$ & 3.930137 & -5.019236 & 1.072445 & $\mathrm{H}$ & 6.360885 & -0.908656 & -1.347041 \\
\hline $\mathrm{H}$ & 2.230323 & -4.602497 & 1.361993 & $\mathrm{H}$ & 5.164136 & -2.204335 & -1.477706 \\
\hline $\mathrm{C}$ & 2.355675 & -2.003207 & 0.470905 & $\mathrm{C}$ & 4.680163 & 1.210496 & -2.045773 \\
\hline & & & & $\mathrm{H}$ & 5.654898 & 1.541484 & -1.668613 \\
\hline \multicolumn{4}{|c|}{ INT4-cross } & $\mathrm{H}$ & 4.758305 & 1.162141 & -3.138204 \\
\hline $\mathrm{Ru}$ & -0.397995 & 0.359910 & -0.412648 & $\mathrm{H}$ & 3.947175 & 1.982592 & -1.791645 \\
\hline $\mathrm{O}$ & -2.014479 & 0.753575 & 1.499936 & $\mathrm{C}$ & 2.319517 & -3.066826 & 3.412547 \\
\hline $\mathrm{O}$ & -1.852977 & 2.042023 & -0.291337 & $\mathrm{H}$ & 3.063447 & -3.641987 & 2.848526 \\
\hline $\mathrm{N}$ & 0.090814 & -2.430709 & -0.914072 & $\mathrm{H}$ & 2.820912 & -2.649041 & 4.292956 \\
\hline $\mathrm{N}$ & 2.041240 & -1.608309 & -0.331759 & $\mathrm{H}$ & 1.546944 & -3.758810 & 3.767927 \\
\hline $\mathrm{C}$ & 0.716888 & -1.300453 & -0.484829 & $\mathrm{C}$ & 0.630528 & -1.168849 & 3.337422 \\
\hline $\mathrm{C}$ & 0.940562 & -3.621386 & -0.888776 & $\mathrm{H}$ & 1.094025 & -0.583667 & 4.140776 \\
\hline $\mathrm{H}$ & 0.838056 & -4.202983 & -1.808838 & $\mathrm{H}$ & 0.068088 & -0.488849 & 2.692553 \\
\hline $\mathrm{H}$ & 0.679725 & -4.270041 & -0.041585 & $\mathrm{H}$ & -0.084733 & -1.860337 & 3.799432 \\
\hline $\mathrm{C}$ & 2.338347 & -2.999395 & -0.734966 & $\mathrm{H}$ & 5.348925 & 0.927377 & 2.941602 \\
\hline $\mathrm{H}$ & 2.946604 & -3.494536 & 0.027075 & $\mathrm{C}$ & 0.130380 & 2.028689 & -2.866389 \\
\hline $\mathrm{H}$ & 2.895805 & -3.002389 & -1.678873 & $\mathrm{H}$ & 0.828029 & 2.788223 & -3.247487 \\
\hline $\mathrm{C}$ & -1.367329 & -2.468771 & -0.839066 & $\mathrm{H}$ & -0.145262 & 1.378604 & -3.706313 \\
\hline $\mathrm{C}$ & -1.824756 & -3.039113 & 0.529150 & $\mathrm{H}$ & -0.773955 & 2.535251 & -2.519849 \\
\hline $\mathrm{H}$ & -1.374371 & -2.436310 & 1.326821 & $\mathrm{C}$ & 1.351696 & 1.945605 & -0.456239 \\
\hline $\mathrm{H}$ & -1.468024 & -4.072316 & 0.641209 & $\mathrm{H}$ & 2.398149 & 1.656933 & -0.419358 \\
\hline $\mathrm{C}$ & -3.361907 & -3.000187 & 0.629272 & $\mathrm{C}$ & 1.331890 & 3.458944 & -0.680160 \\
\hline $\mathrm{H}$ & -3.669717 & -3.426691 & 1.592850 & $\mathrm{O}$ & 2.357735 & 4.020965 & -1.063660 \\
\hline
\end{tabular}




$\begin{array}{crcc}\mathrm{N} & 0.158551 & 4.099844 & -0.455909 \\ \mathrm{H} & -0.677517 & 3.530440 & -0.327360 \\ \mathrm{C} & 0.000948 & 5.501784 & -0.797185 \\ \mathrm{H} & -0.850902 & 5.910718 & -0.247252 \\ \mathrm{H} & 0.908353 & 6.043126 & -0.521088 \\ \mathrm{H} & -0.167178 & 5.646447 & -1.873721 \\ \mathrm{C} & -2.380030 & 1.761963 & 0.853601 \\ \mathrm{C} & -3.490815 & 2.685956 & 1.378237 \\ \mathrm{C} & -3.703027 & 2.456470 & 2.882266 \\ \mathrm{H} & -4.515453 & 3.095041 & 3.250057 \\ \mathrm{H} & -3.955916 & 1.412646 & 3.086339 \\ \mathrm{H} & -2.795436 & 2.693582 & 3.448098 \\ \mathrm{C} & -3.111935 & 4.154728 & 1.110372 \\ \mathrm{H} & -2.997289 & 4.339921 & 0.038305 \\ \mathrm{H} & -3.891686 & 4.823037 & 1.494807 \\ \mathrm{H} & -2.168994 & 4.416134 & 1.606370 \\ \mathrm{C} & -4.781720 & 2.334865 & 0.605367 \\ \mathrm{H} & -5.076608 & 1.295082 & 0.787654 \\ \mathrm{H} & -5.604235 & 2.983345 & 0.931063 \\ \mathrm{H} & -4.640020 & 2.470608 & -0.472101\end{array}$

\section{INT4-homo}

$\begin{array}{lccc}\mathrm{Ru} & 0.403892 & -0.562873 & -0.525656 \\ \mathrm{O} & 2.045562 & -1.108261 & 1.318132 \\ \mathrm{O} & 1.955574 & -2.116991 & -0.650519 \\ \mathrm{~N} & -0.278077 & 2.234592 & -0.658599 \\ \mathrm{~N} & -2.170941 & 1.215643 & -0.228436 \\ \mathrm{C} & -0.826816 & 1.013960 & -0.398374 \\ \mathrm{C} & -1.205021 & 3.352551 & -0.486352 \\ \mathrm{H} & -1.142199 & 4.054500 & -1.322533 \\ \mathrm{H} & -0.987852 & 3.902875 & 0.439051 \\ \mathrm{C} & -2.559581 & 2.627522 & -0.423426 \\ \mathrm{H} & -3.188196 & 2.967023 & 0.405136 \\ \mathrm{H} & -3.129383 & 2.735252 & -1.354162 \\ \mathrm{C} & 1.171793 & 2.360984 & -0.546756 \\ \mathrm{C} & 1.570125 & 2.769565 & 0.896032 \\ \mathrm{H} & 1.152887 & 2.033679 & 1.594006 \\ \mathrm{H} & 1.139358 & 3.751009 & 1.138381 \\ \mathrm{C} & 3.104459 & 2.822746 & 1.025459 \\ \mathrm{H} & 3.368200 & 3.135374 & 2.044309 \\ \mathrm{C} & 3.673423 & 3.831879 & 0.008210 \\ \mathrm{H} & 3.286504 & 4.840750 & 0.211481 \\ \mathrm{H} & 4.766436 & 3.880934 & 0.102167 \\ \mathrm{C} & 3.287475 & 3.396086 & -1.420602 \\ \mathrm{H} & 3.701944 & 4.104945 & -2.149130 \\ \mathrm{C} & 1.748659 & 3.385556 & -1.547275 \\ \mathrm{H} & 1.442155 & 3.109513 & -2.564963 \\ \mathrm{H} & 1.349482 & 4.390134 & -1.343435 \\ \mathrm{C} & 3.844749 & 1.982382 & -1.695792 \\ \mathrm{H} & 3.596916 & 1.671800 & -2.720110 \\ \mathrm{H} & 4.940882 & 1.993801 & -1.618791 \\ \mathrm{C} & 3.259179 & 0.971673 & -0.670893 \\ \mathrm{H} & 3.651560 & -0.026730 & -0.882735 \\ \mathrm{C} & 1.737057 & 0.970630 & -0.850963 \\ \mathrm{H} & 1.510762 & 0.716831 & -1.918502 \\ \mathrm{C} & 3.670401 & 1.419131 & 0.746905 \\ \mathrm{H} & 3.296188 & 0.702450 & 1.479765 \\ \mathrm{H} & 4.767313 & 1.436719 & 0.819640 \\ \mathrm{C} & -3.048664 & 0.352426 & 0.501410 \\ \mathrm{C} & -2.907615 & 0.279765 & 1.905634\end{array}$

\begin{tabular}{|c|c|c|c|}
\hline$C$ & -3.752042 & -0.592867 & 2.602357 \\
\hline $\mathrm{H}$ & -3.647525 & -0.688523 & 3.679470 \\
\hline $\mathrm{C}$ & -4.724157 & -1.337066 & 1.937358 \\
\hline $\mathrm{C}$ & -4.891694 & -1.197342 & 0.561389 \\
\hline $\mathrm{H}$ & -5.677644 & -1.753567 & 0.059099 \\
\hline $\mathrm{C}$ & -4.064530 & -0.346004 & -0.181553 \\
\hline $\mathrm{C}$ & -1.907906 & 1.139587 & 2.676377 \\
\hline $\mathrm{H}$ & -1.397348 & 1.794422 & 1.965463 \\
\hline $\mathrm{C}$ & -4.320110 & -0.120230 & -1.666978 \\
\hline $\mathrm{H}$ & -3.442133 & 0.389023 & -2.077501 \\
\hline $\mathrm{C}$ & -0.642500 & -1.882145 & 0.591745 \\
\hline $\mathrm{H}$ & -1.428658 & -1.552446 & 1.261626 \\
\hline $\mathrm{C}$ & -0.711579 & -1.316924 & -1.993838 \\
\hline $\mathrm{H}$ & -1.583603 & -0.749976 & -2.330351 \\
\hline $\mathrm{C}$ & -1.164029 & -2.330647 & -0.812010 \\
\hline $\mathrm{H}$ & -2.248124 & -2.204667 & -0.763294 \\
\hline $\mathrm{H}$ & 0.008065 & -2.618281 & 1.066865 \\
\hline $\mathrm{C}$ & -5.535668 & 0.813243 & -1.847772 \\
\hline $\mathrm{H}$ & -5.695897 & 1.040765 & -2.908475 \\
\hline $\mathrm{H}$ & -6.446633 & 0.341284 & -1.460383 \\
\hline $\mathrm{H}$ & -5.399051 & 1.758261 & -1.310317 \\
\hline $\mathrm{C}$ & -4.519394 & -1.417652 & -2.468708 \\
\hline $\mathrm{H}$ & -5.415000 & -1.961403 & -2.147222 \\
\hline $\mathrm{H}$ & -4.643777 & -1.183446 & -3.532249 \\
\hline $\mathrm{H}$ & -3.661673 & -2.089132 & -2.369449 \\
\hline $\mathrm{C}$ & -2.644631 & 2.050280 & 3.679221 \\
\hline $\mathrm{H}$ & -3.417950 & 2.649011 & 3.183258 \\
\hline $\mathrm{H}$ & -3.131895 & 1.467189 & 4.469097 \\
\hline $\mathrm{H}$ & -1.936311 & 2.734397 & 4.161026 \\
\hline $\mathrm{C}$ & -0.813432 & 0.313634 & 3.379159 \\
\hline $\mathrm{H}$ & -0.173267 & 0.976058 & 3.974540 \\
\hline $\mathrm{H}$ & -1.249845 & -0.427245 & 4.059990 \\
\hline $\mathrm{H}$ & -0.177009 & -0.207340 & 2.659524 \\
\hline $\mathrm{H}$ & -5.367009 & -2.012074 & 2.496128 \\
\hline $\mathrm{C}$ & 0.035285 & -1.905433 & -3.182950 \\
\hline $\mathrm{H}$ & -0.595898 & -2.597981 & -3.759847 \\
\hline $\mathrm{H}$ & 0.326793 & -1.094207 & -3.862861 \\
\hline $\mathrm{H}$ & 0.942161 & -2.435967 & -2.881668 \\
\hline $\mathrm{C}$ & -0.800828 & -3.793302 & -1.094958 \\
\hline $\mathrm{H}$ & -1.195914 & -4.127099 & -2.061010 \\
\hline $\mathrm{H}$ & 0.284365 & -3.923502 & -1.095591 \\
\hline $\mathrm{H}$ & -1.223665 & -4.437008 & -0.315151 \\
\hline $\mathrm{C}$ & 3.620686 & -2.924392 & 0.902566 \\
\hline $\mathrm{C}$ & 4.726480 & -2.823337 & -0.167182 \\
\hline $\mathrm{H}$ & 5.153967 & -1.813430 & -0.194726 \\
\hline $\mathrm{H}$ & 5.538561 & -3.525774 & 0.058192 \\
\hline $\mathrm{H}$ & 4.327299 & -3.052287 & -1.159325 \\
\hline $\mathrm{C}$ & 4.182393 & -2.554191 & 2.282353 \\
\hline $\mathrm{H}$ & 3.405922 & -2.610070 & 3.051030 \\
\hline $\mathrm{H}$ & 4.995944 & -3.238222 & 2.554561 \\
\hline $\mathrm{H}$ & 4.573919 & -1.531688 & 2.284111 \\
\hline $\mathrm{C}$ & 3.052112 & -4.358954 & 0.924567 \\
\hline $\mathrm{H}$ & 2.646868 & -4.629763 & -0.055123 \\
\hline $\mathrm{H}$ & 3.838597 & -5.076685 & 1.189056 \\
\hline $\mathrm{H}$ & 2.247958 & -4.450525 & 1.665197 \\
\hline $\mathrm{C}$ & 2.473461 & -1.976808 & 0.516297 \\
\hline
\end{tabular}

\section{INT4-cross-2}

$\begin{array}{lccc}\mathrm{Ru} & 0.498044 & 0.146672 & -0.167850 \\ \mathrm{O} & 2.172791 & 1.551013 & -0.329389\end{array}$




\begin{tabular}{|c|c|c|c|}
\hline $\mathrm{O}$ & 2.068946 & 0.915480 & 1.790432 \\
\hline $\mathrm{N}$ & -0.366952 & -2.590245 & -0.290058 \\
\hline $\mathrm{N}$ & -2.237467 & -1.451443 & -0.189112 \\
\hline $\mathrm{C}$ & -0.869710 & -1.337670 & -0.142895 \\
\hline $\mathrm{C}$ & -1.366041 & -3.576964 & -0.692651 \\
\hline $\mathrm{H}$ & -1.219349 & -4.532944 & -0.183076 \\
\hline $\mathrm{H}$ & -1.322408 & -3.751321 & -1.776995 \\
\hline $\mathrm{C}$ & -2.661034 & -2.867548 & -0.280180 \\
\hline $\mathrm{H}$ & -3.468478 & -2.984080 & -1.005585 \\
\hline $\mathrm{H}$ & -3.022357 & -3.210202 & 0.698569 \\
\hline $\mathrm{C}$ & 1.073285 & -2.718053 & -0.493913 \\
\hline $\mathrm{C}$ & 1.428956 & -2.644966 & -2.002304 \\
\hline $\mathrm{H}$ & 0.990885 & -1.737147 & -2.433468 \\
\hline $\mathrm{H}$ & 0.988405 & -3.504880 & -2.526817 \\
\hline $\mathrm{C}$ & 2.958717 & -2.643700 & -2.177956 \\
\hline $\mathrm{H}$ & 3.200813 & -2.585363 & -3.247216 \\
\hline $\mathrm{C}$ & 3.529976 & -3.948834 & -1.586459 \\
\hline $\mathrm{H}$ & 3.125914 & -4.816523 & -2.126950 \\
\hline $\mathrm{H}$ & 4.621028 & -3.972447 & -1.709031 \\
\hline $\mathrm{C}$ & 3.170713 & -4.038924 & -0.088406 \\
\hline $\mathrm{H}$ & 3.573810 & -4.968920 & 0.332942 \\
\hline $\mathrm{C}$ & 1.633521 & -4.038256 & 0.077244 \\
\hline $\mathrm{H}$ & 1.358192 & -4.123873 & 1.136574 \\
\hline $\mathrm{H}$ & 1.202489 & -4.902832 & -0.449120 \\
\hline $\mathrm{C}$ & 3.776554 & -2.825397 & 0.645764 \\
\hline $\mathrm{H}$ & 3.557749 & -2.881716 & 1.720423 \\
\hline $\mathrm{H}$ & 4.870449 & -2.835993 & 0.537554 \\
\hline $\mathrm{C}$ & 3.200269 & -1.510148 & 0.060530 \\
\hline $\mathrm{H}$ & 3.637245 & -0.668466 & 0.596640 \\
\hline $\mathrm{C}$ & 1.680238 & -1.526517 & 0.277719 \\
\hline $\mathrm{H}$ & 1.469515 & -1.657922 & 1.348817 \\
\hline $\mathrm{C}$ & 3.549177 & -1.425592 & -1.438063 \\
\hline $\mathrm{H}$ & 3.153333 & -0.491822 & -1.855499 \\
\hline $\mathrm{H}$ & 4.640117 & -1.404047 & -1.567597 \\
\hline $\mathrm{C}$ & -3.154855 & -0.537581 & 0.436426 \\
\hline $\mathrm{C}$ & -4.040131 & 0.212476 & -0.367802 \\
\hline $\mathrm{C}$ & -4.909312 & 1.105676 & 0.270450 \\
\hline $\mathrm{H}$ & -5.587890 & 1.707573 & -0.325263 \\
\hline $\mathrm{C}$ & -4.911080 & 1.241227 & 1.656251 \\
\hline $\mathrm{C}$ & -4.057242 & 0.463919 & 2.434547 \\
\hline $\mathrm{H}$ & -4.073428 & 0.569098 & 3.515623 \\
\hline $\mathrm{C}$ & -3.171417 & -0.447134 & 1.847385 \\
\hline $\mathrm{C}$ & -4.100078 & 0.036235 & -1.881506 \\
\hline $\mathrm{H}$ & -3.164918 & -0.442221 & -2.191609 \\
\hline $\mathrm{C}$ & -2.268752 & -1.292833 & 2.744281 \\
\hline $\mathrm{H}$ & -1.679172 & -1.966767 & 2.118315 \\
\hline $\mathrm{C}$ & -0.222963 & 0.731790 & -1.909863 \\
\hline $\mathrm{H}$ & -0.959758 & 0.161408 & -2.479407 \\
\hline $\mathrm{C}$ & -0.713587 & 1.599692 & 0.590332 \\
\hline $\mathrm{H}$ & -1.625092 & 1.208232 & 1.027954 \\
\hline $\mathrm{H}$ & 0.529431 & 1.185635 & -2.561570 \\
\hline $\mathrm{C}$ & -4.241473 & 1.359237 & -2.660284 \\
\hline $\mathrm{H}$ & -4.140169 & 1.163761 & -3.734123 \\
\hline $\mathrm{H}$ & -5.230150 & 1.807961 & -2.508162 \\
\hline $\mathrm{H}$ & -3.492892 & 2.106710 & -2.379030 \\
\hline $\mathrm{C}$ & -5.262219 & -0.909531 & -2.254340 \\
\hline $\mathrm{H}$ & -5.277680 & -1.095437 & -3.334823 \\
\hline $\mathrm{H}$ & -5.187558 & -1.874338 & -1.741300 \\
\hline $\mathrm{H}$ & -6.223401 & -0.462236 & -1.973705 \\
\hline $\mathrm{C}$ & -3.110044 & -2.171400 & 3.691271 \\
\hline
\end{tabular}

$\begin{array}{lrcc}\mathrm{H} & -3.682581 & -1.563515 & 4.401136 \\ \mathrm{H} & -3.823324 & -2.790586 & 3.134410 \\ \mathrm{H} & -2.458863 & -2.835048 & 4.272026 \\ \mathrm{C} & -1.258797 & -0.437601 & 3.533913 \\ \mathrm{H} & -0.563104 & 0.076467 & 2.865932 \\ \mathrm{H} & -1.766502 & 0.312326 & 4.151960 \\ \mathrm{H} & -0.667760 & -1.076428 & 4.200897 \\ \mathrm{H} & -5.585220 & 1.949104 & 2.130910 \\ \mathrm{C} & -0.228651 & 2.766232 & 1.427662 \\ \mathrm{H} & -1.029978 & 3.517491 & 1.486792 \\ \mathrm{H} & -0.005722 & 2.420891 & 2.440331 \\ \mathrm{H} & 0.658604 & 3.258004 & 1.032485 \\ \mathrm{C} & -0.943125 & 1.808167 & -0.962059 \\ \mathrm{H} & -2.005937 & 1.624187 & -1.088038 \\ \mathrm{C} & -0.689310 & 3.241443 & -1.438730 \\ \mathrm{O} & -1.651030 & 3.955163 & -1.721428 \\ \mathrm{~N} & 0.606112 & 3.626881 & -1.540730 \\ \mathrm{H} & 1.326487 & 2.995804 & -1.187229 \\ \mathrm{C} & 0.971235 & 4.984139 & -1.900423 \\ \mathrm{H} & 1.127202 & 5.613060 & -1.013143 \\ \mathrm{H} & 1.894207 & 4.976035 & -2.488200 \\ \mathrm{H} & 0.163767 & 5.417474 & -2.493348 \\ \mathrm{C} & 2.604853 & 1.593785 & 0.889600 \\ \mathrm{C} & 3.822390 & 2.492924 & 1.172671 \\ \mathrm{C} & 3.971172 & 2.715928 & 2.684790 \\ \mathrm{H} & 4.069743 & 1.762926 & 3.211152 \\ \mathrm{H} & 4.857458 & 3.328353 & 2.891427 \\ \mathrm{H} & 3.094093 & 3.229460 & 3.093489 \\ \mathrm{C} & 5.073690 & 1.777543 & 0.616705 \\ \mathrm{H} & 4.952905 & 1.550454 & -0.447617 \\ \mathrm{H} & 5.957246 & 2.416190 & 0.738450 \\ \mathrm{H} & 5.261930 & 0.839419 & 1.150960 \\ \mathrm{C} & 3.650446 & 3.843508 & 0.450826 \\ \mathrm{H} & 2.744996 & 4.363488 & 0.785966 \\ \mathrm{H} & 4.506738 & 4.494919 & 0.663017 \\ \mathrm{H} & 3.585280 & 3.705847 & -0.632238\end{array}$

\section{INT4-homo-2}

$\begin{array}{lccc}\mathrm{Ru} & -0.503614 & -0.369980 & -0.323111 \\ \mathrm{O} & -2.161462 & -1.647028 & -0.879669 \\ \mathrm{O} & -2.167077 & -1.569623 & 1.337426 \\ \mathrm{~N} & 0.370730 & 2.314305 & 0.273376 \\ \mathrm{~N} & 2.236128 & 1.194618 & 0.055181 \\ \mathrm{C} & 0.869170 & 1.061725 & 0.083923 \\ \mathrm{C} & 1.368924 & 3.370409 & 0.121896 \\ \mathrm{H} & 1.227519 & 4.169867 & 0.853956 \\ \mathrm{H} & 1.320002 & 3.809330 & -0.884926 \\ \mathrm{C} & 2.664258 & 2.581508 & 0.334848 \\ \mathrm{H} & 3.472325 & 2.880760 & -0.337056 \\ \mathrm{H} & 3.027703 & 2.658882 & 1.368720 \\ \mathrm{C} & -1.064306 & 2.497042 & 0.080293 \\ \mathrm{C} & -1.390760 & 2.804393 & -1.405819 \\ \mathrm{H} & -0.948352 & 2.026545 & -2.039960 \\ \mathrm{H} & -0.936690 & 3.763416 & -1.693705 \\ \mathrm{C} & -2.916695 & 2.861342 & -1.604392 \\ \mathrm{H} & -3.137474 & 3.075217 & -2.658491 \\ \mathrm{C} & -3.494108 & 3.982049 & -0.715277 \\ \mathrm{H} & -3.075989 & 4.954049 & -1.013556 \\ \mathrm{H} & -4.582461 & 4.045013 & -0.848044\end{array}$




\begin{tabular}{|c|c|c|c|}
\hline $\mathrm{C}$ & -3.164535 & 3.691519 & 0.764129 \\
\hline $\mathrm{H}$ & -3.574014 & 4.489299 & 1.397431 \\
\hline $\mathrm{C}$ & -1.631869 & 3.636522 & 0.952870 \\
\hline $\mathrm{H}$ & -1.377586 & 3.451539 & 2.004749 \\
\hline $\mathrm{H}$ & -1.186609 & 4.601568 & 0.668144 \\
\hline $\mathrm{C}$ & -3.785145 & 2.336699 & 1.159865 \\
\hline $\mathrm{H}$ & -3.585072 & 2.120211 & 2.217701 \\
\hline $\mathrm{H}$ & -4.877051 & 2.380169 & 1.039820 \\
\hline $\mathrm{C}$ & -3.203073 & 1.205778 & 0.272713 \\
\hline $\mathrm{H}$ & -3.654247 & 0.261885 & 0.576665 \\
\hline $\mathrm{C}$ & -1.685417 & 1.153492 & 0.511232 \\
\hline $\mathrm{H}$ & -1.492932 & 1.001123 & 1.582782 \\
\hline $\mathrm{C}$ & -3.526900 & 1.502587 & -1.204149 \\
\hline $\mathrm{H}$ & -3.130633 & 0.698664 & -1.835559 \\
\hline $\mathrm{H}$ & -4.615599 & 1.525052 & -1.351610 \\
\hline $\mathrm{C}$ & 3.176307 & 0.149950 & 0.334405 \\
\hline $\mathrm{C}$ & 4.029378 & -0.294235 & -0.697409 \\
\hline $\mathrm{C}$ & 4.921846 & -1.334516 & -0.410463 \\
\hline $\mathrm{H}$ & 5.584350 & -1.701189 & -1.189086 \\
\hline $\mathrm{C}$ & 4.978557 & -1.900436 & 0.861149 \\
\hline $\mathrm{C}$ & 4.167807 & -1.408050 & 1.881452 \\
\hline $\mathrm{H}$ & 4.237378 & -1.839100 & 2.876202 \\
\hline $\mathrm{C}$ & 3.263082 & -0.365739 & 1.647059 \\
\hline $\mathrm{C}$ & 4.053056 & 0.385151 & -2.062339 \\
\hline $\mathrm{H}$ & 3.168886 & 1.027617 & -2.124386 \\
\hline $\mathrm{C}$ & 2.432516 & 0.186214 & 2.804335 \\
\hline $\mathrm{H}$ & 1.812681 & 1.005093 & 2.430815 \\
\hline $\mathrm{C}$ & 0.262865 & -0.564684 & -2.133140 \\
\hline $\mathrm{H}$ & 1.037500 & 0.095787 & -2.530094 \\
\hline $\mathrm{C}$ & 0.680940 & -1.976765 & 0.089412 \\
\hline $\mathrm{H}$ & 1.603715 & -1.707850 & 0.591168 \\
\hline $\mathrm{C}$ & 0.876696 & -1.894216 & -1.478145 \\
\hline $\mathrm{H}$ & 1.957128 & -1.771821 & -1.583289 \\
\hline $\mathrm{H}$ & -0.495115 & -0.805465 & -2.886676 \\
\hline $\mathrm{C}$ & 3.999921 & -0.594815 & -3.247393 \\
\hline $\mathrm{H}$ & 3.988138 & -0.037921 & -4.191463 \\
\hline $\mathrm{H}$ & 4.874255 & -1.255276 & -3.268287 \\
\hline $\mathrm{H}$ & 3.103971 & -1.221081 & -3.216155 \\
\hline $\mathrm{C}$ & 5.303946 & 1.282558 & -2.175175 \\
\hline $\mathrm{H}$ & 5.297352 & 1.839529 & -3.119657 \\
\hline $\mathrm{H}$ & 5.360404 & 2.003064 & -1.351667 \\
\hline $\mathrm{H}$ & 6.218160 & 0.677593 & -2.145207 \\
\hline $\mathrm{C}$ & 3.354281 & 0.768986 & 3.894494 \\
\hline $\mathrm{H}$ & 3.964819 & -0.011798 & 4.362659 \\
\hline $\mathrm{H}$ & 4.037175 & 1.520785 & 3.481361 \\
\hline $\mathrm{H}$ & 2.757756 & 1.242566 & 4.682861 \\
\hline $\mathrm{C}$ & 1.465005 & -0.855442 & 3.397122 \\
\hline $\mathrm{H}$ & 0.703288 & -1.145936 & 2.670061 \\
\hline $\mathrm{H}$ & 1.995694 & -1.756750 & 3.726082 \\
\hline $\mathrm{H}$ & 0.950175 & -0.433622 & 4.268346 \\
\hline $\mathrm{H}$ & 5.670565 & -2.713834 & 1.062630 \\
\hline $\mathrm{C}$ & 0.141230 & -3.265553 & 0.674509 \\
\hline $\mathrm{H}$ & 0.929159 & -4.034424 & 0.650692 \\
\hline $\mathrm{H}$ & -0.149393 & -3.111708 & 1.716457 \\
\hline $\mathrm{H}$ & -0.725027 & -3.653035 & 0.140179 \\
\hline $\mathrm{C}$ & 0.386709 & -3.129512 & -2.240905 \\
\hline $\mathrm{H}$ & -0.701750 & -3.207833 & -2.175727 \\
\hline $\mathrm{H}$ & 0.663627 & -3.046257 & -3.298183 \\
\hline $\mathrm{H}$ & 0.830596 & -4.048931 & -1.843495 \\
\hline $\mathrm{C}$ & -3.943475 & -2.830298 & 0.234889 \\
\hline
\end{tabular}

$\begin{array}{lllr}\mathrm{C} & -3.576315 & -4.190546 & -0.394663 \\ \mathrm{H} & -4.465995 & -4.828594 & -0.465961 \\ \mathrm{H} & -3.164463 & -4.053909 & -1.399365 \\ \mathrm{H} & -2.833419 & -4.719289 & 0.214611 \\ \mathrm{C} & -4.480179 & -3.034388 & 1.658155 \\ \mathrm{H} & -4.736367 & -2.076213 & 2.121497 \\ \mathrm{H} & -5.379455 & -3.662811 & 1.636932 \\ \mathrm{H} & -3.731245 & -3.515373 & 2.294351 \\ \mathrm{C} & -5.004733 & -2.134233 & -0.641792 \\ \mathrm{H} & -5.905620 & -2.756701 & -0.711760 \\ \mathrm{H} & -5.297902 & -1.167215 & -0.216145 \\ \mathrm{H} & -4.618750 & -1.957944 & -1.649653 \\ \mathrm{C} & -2.674951 & -1.956635 & 0.260182\end{array}$

\section{TS4-cross}

\begin{tabular}{|c|c|c|c|}
\hline $\mathrm{Ru}$ & 0.515593 & 0.277210 & -0.119294 \\
\hline $\mathrm{O}$ & 2.301534 & 1.597838 & -0.061494 \\
\hline $\mathrm{O}$ & 1.729135 & 0.795698 & 1.913278 \\
\hline $\mathrm{N}$ & -0.341949 & -2.440775 & -0.517034 \\
\hline $\mathrm{N}$ & -2.216593 & -1.319901 & -0.339113 \\
\hline $\mathrm{C}$ & -0.851987 & -1.213716 & -0.230172 \\
\hline $\mathrm{C}$ & -1.326218 & -3.386790 & -1.038443 \\
\hline $\mathrm{H}$ & -1.211709 & -4.375936 & -0.585810 \\
\hline $\mathrm{H}$ & -1.217092 & -3.492847 & -2.125876 \\
\hline $\mathrm{C}$ & -2.644784 & -2.700924 & -0.660587 \\
\hline $\mathrm{H}$ & -3.374667 & -2.695773 & -1.472999 \\
\hline $\mathrm{H}$ & -3.114533 & -3.160561 & 0.218003 \\
\hline $\mathrm{C}$ & 1.101955 & -2.582445 & -0.700852 \\
\hline $\mathrm{C}$ & 1.485988 & -2.483856 & -2.198421 \\
\hline $\mathrm{H}$ & 1.061425 & -1.568781 & -2.623702 \\
\hline $\mathrm{H}$ & 1.057576 & -3.333573 & -2.748693 \\
\hline $\mathrm{C}$ & 3.018501 & -2.483787 & -2.342483 \\
\hline $\mathrm{H}$ & 3.282405 & -2.394113 & -3.404600 \\
\hline $\mathrm{C}$ & 3.569356 & -3.810789 & -1.781411 \\
\hline $\mathrm{H}$ & 3.170259 & -4.657692 & -2.357558 \\
\hline $\mathrm{H}$ & 4.662698 & -3.839556 & -1.881722 \\
\hline $\mathrm{C}$ & 3.177198 & -3.943138 & -0.294899 \\
\hline $\mathrm{H}$ & 3.560362 & -4.891003 & 0.105068 \\
\hline $\mathrm{C}$ & 1.635404 & -3.929806 & -0.160745 \\
\hline $\mathrm{H}$ & 1.338039 & -4.044329 & 0.889803 \\
\hline $\mathrm{H}$ & 1.208820 & -4.775528 & -0.720476 \\
\hline $\mathrm{C}$ & 3.788770 & -2.760560 & 0.485427 \\
\hline $\mathrm{H}$ & 3.554851 & -2.848079 & 1.554900 \\
\hline $\mathrm{H}$ & 4.883897 & -2.785361 & 0.390768 \\
\hline $\mathrm{C}$ & 3.231380 & -1.423685 & -0.065435 \\
\hline $\mathrm{H}$ & 3.675591 & -0.598199 & 0.496353 \\
\hline $\mathrm{C}$ & 1.707733 & -1.434013 & 0.129298 \\
\hline $\mathrm{H}$ & 1.500611 & -1.655904 & 1.187786 \\
\hline $\mathrm{C}$ & 3.600471 & -1.291163 & -1.554386 \\
\hline $\mathrm{H}$ & 3.206830 & -0.344902 & -1.944087 \\
\hline $\mathrm{H}$ & 4.692881 & -1.266901 & -1.671606 \\
\hline $\mathrm{C}$ & -3.154065 & -0.517568 & 0.394350 \\
\hline $\mathrm{C}$ & -4.018583 & 0.351618 & -0.305972 \\
\hline $\mathrm{C}$ & -4.927455 & 1.110607 & 0.441991 \\
\hline $\mathrm{H}$ & -5.595104 & 1.798445 & -0.067220 \\
\hline $\mathrm{C}$ & -5.000275 & 0.985427 & 1.827729 \\
\hline $\mathrm{C}$ & -4.178497 & 0.078475 & 2.492274 \\
\hline $\mathrm{H}$ & -4.252643 & -0.019962 & 3.571401 \\
\hline $\mathrm{C}$ & -3.245651 & -0.697383 & 1.793143 \\
\hline $\mathrm{C}$ & -4.056267 & 0.385225 & -1.831527 \\
\hline
\end{tabular}




\begin{tabular}{|c|c|c|c|}
\hline $\mathrm{H}$ & -3.119464 & -0.053117 & -2.192815 \\
\hline $\mathrm{C}$ & -2.379082 & -1.699258 & 2.555885 \\
\hline $\mathrm{H}$ & -1.827706 & -2.308284 & 1.835991 \\
\hline $\mathrm{C}$ & 0.116528 & 0.601177 & -1.921637 \\
\hline $\mathrm{H}$ & -0.759079 & 0.280204 & -2.500361 \\
\hline $\mathrm{C}$ & -0.420002 & 2.205124 & 0.620974 \\
\hline $\mathrm{H}$ & 0.448793 & 2.825749 & 0.822125 \\
\hline $\mathrm{H}$ & 0.834485 & 1.164682 & -2.530667 \\
\hline $\mathrm{C}$ & -4.190431 & 1.790700 & -2.446489 \\
\hline $\mathrm{H}$ & -4.214456 & 1.705625 & -3.539125 \\
\hline $\mathrm{H}$ & -5.125023 & 2.276212 & -2.142318 \\
\hline $\mathrm{H}$ & -3.359525 & 2.455772 & -2.195802 \\
\hline $\mathrm{C}$ & -5.219752 & -0.504304 & -2.325456 \\
\hline $\mathrm{H}$ & -5.211813 & -0.578244 & -3.419312 \\
\hline $\mathrm{H}$ & -5.167758 & -1.516884 & -1.911319 \\
\hline $\mathrm{H}$ & -6.182549 & -0.075755 & -2.021807 \\
\hline $\mathrm{C}$ & -3.245746 & -2.663842 & 3.388528 \\
\hline $\mathrm{H}$ & -3.766468 & -2.144591 & 4.200981 \\
\hline $\mathrm{H}$ & -4.004184 & -3.156740 & 2.768896 \\
\hline $\mathrm{H}$ & -2.616852 & -3.438083 & 3.843056 \\
\hline $\mathrm{C}$ & -1.317987 & -1.007602 & 3.432307 \\
\hline $\mathrm{H}$ & -0.597037 & -0.459947 & 2.820100 \\
\hline $\mathrm{H}$ & -1.780152 & -0.306417 & 4.137429 \\
\hline $\mathrm{H}$ & -0.765489 & -1.755841 & 4.013378 \\
\hline $\mathrm{H}$ & -5.709118 & 1.587939 & 2.389475 \\
\hline $\mathrm{C}$ & -1.365681 & 2.150944 & 1.789765 \\
\hline $\mathrm{H}$ & -2.270479 & 1.593351 & 1.571705 \\
\hline $\mathrm{H}$ & -0.874400 & 1.721177 & 2.665458 \\
\hline $\mathrm{H}$ & -1.656631 & 3.180343 & 2.047745 \\
\hline $\mathrm{C}$ & -0.851414 & 2.194331 & -0.725356 \\
\hline $\mathrm{H}$ & -1.873412 & 1.893774 & -0.917085 \\
\hline $\mathrm{C}$ & -0.374163 & 3.248970 & -1.702902 \\
\hline $\mathrm{O}$ & -1.184647 & 3.744047 & -2.486056 \\
\hline $\mathrm{N}$ & 0.925694 & 3.639617 & -1.609572 \\
\hline $\mathrm{H}$ & 1.568484 & 3.059686 & -1.070579 \\
\hline $\mathrm{C}$ & 1.476655 & 4.612168 & -2.536088 \\
\hline $\mathrm{H}$ & 2.349288 & 5.089643 & -2.081345 \\
\hline $\mathrm{H}$ & 1.780630 & 4.151679 & -3.486883 \\
\hline $\mathrm{H}$ & 0.719751 & 5.368712 & -2.752343 \\
\hline $\mathrm{C}$ & 2.502877 & 1.494200 & 1.204712 \\
\hline $\mathrm{C}$ & 3.654919 & 2.291385 & 1.827047 \\
\hline $\mathrm{C}$ & 3.170862 & 3.757092 & 1.920835 \\
\hline $\mathrm{H}$ & 2.283437 & 3.836666 & 2.560100 \\
\hline $\mathrm{H}$ & 3.957079 & 4.388763 & 2.351218 \\
\hline $\mathrm{H}$ & 2.917048 & 4.151356 & 0.930548 \\
\hline $\mathrm{C}$ & 3.981321 & 1.753932 & 3.228148 \\
\hline $\mathrm{H}$ & 4.306101 & 0.708822 & 3.179706 \\
\hline $\mathrm{H}$ & 4.787578 & 2.344863 & 3.679405 \\
\hline $\mathrm{H}$ & 3.104779 & 1.797957 & 3.880067 \\
\hline $\mathrm{C}$ & 4.894977 & 2.212613 & 0.917587 \\
\hline $\mathrm{H}$ & 4.666632 & 2.573915 & -0.088628 \\
\hline $\mathrm{H}$ & 5.706932 & 2.820185 & 1.335145 \\
\hline $\mathrm{H}$ & 5.254632 & 1.180748 & 0.830826 \\
\hline
\end{tabular}

\section{TS4-homo}

$\begin{array}{lccc}\mathrm{Ru} & -0.500899 & -0.447340 & -0.550968 \\ \mathrm{O} & -2.206874 & -1.799912 & -0.960108 \\ \mathrm{O} & -1.565784 & -1.734324 & 1.151857 \\ \mathrm{~N} & 0.165243 & 2.269104 & 0.101799 \\ \mathrm{~N} & 2.117540 & 1.277580 & -0.032068\end{array}$

\begin{tabular}{|c|c|c|c|}
\hline $\mathrm{C}$ & 0.755947 & 1.055722 & -0.061145 \\
\hline $\mathrm{C}$ & 1.087517 & 3.397331 & 0.025054 \\
\hline $\mathrm{H}$ & 0.870405 & 4.149127 & 0.789207 \\
\hline $\mathrm{H}$ & 1.023398 & 3.880473 & -0.959624 \\
\hline $\mathrm{C}$ & 2.436230 & 2.700618 & 0.235217 \\
\hline $\mathrm{H}$ & 3.213750 & 3.056467 & -0.443977 \\
\hline $\mathrm{H}$ & 2.801743 & 2.814825 & 1.263653 \\
\hline $\mathrm{C}$ & -1.286079 & 2.377894 & -0.037693 \\
\hline $\mathrm{C}$ & -1.679121 & 2.817736 & -1.469262 \\
\hline $\mathrm{H}$ & -1.203870 & 2.153257 & -2.197275 \\
\hline $\mathrm{H}$ & -1.309312 & 3.836020 & -1.658254 \\
\hline $\mathrm{C}$ & -3.210131 & 2.773776 & -1.622034 \\
\hline $\mathrm{H}$ & -3.481201 & 3.066770 & -2.645189 \\
\hline $\mathrm{C}$ & -3.838956 & 3.760013 & -0.616701 \\
\hline $\mathrm{H}$ & -3.501927 & 4.784078 & -0.832385 \\
\hline $\mathrm{H}$ & -4.933058 & 3.753642 & -0.713443 \\
\hline $\mathrm{C}$ & -3.438960 & 3.356790 & 0.818018 \\
\hline $\mathrm{H}$ & -3.876463 & 4.063390 & 1.535573 \\
\hline $\mathrm{C}$ & -1.897619 & 3.393957 & 0.955714 \\
\hline $\mathrm{H}$ & -1.594572 & 3.131251 & 1.977726 \\
\hline $\mathrm{H}$ & -1.535265 & 4.413156 & 0.753451 \\
\hline $\mathrm{C}$ & -3.965779 & 1.933155 & 1.097041 \\
\hline $\mathrm{H}$ & -3.727257 & 1.633397 & 2.126360 \\
\hline $\mathrm{H}$ & -5.061334 & 1.922993 & 1.002815 \\
\hline $\mathrm{C}$ & -3.328635 & 0.933771 & 0.099191 \\
\hline $\mathrm{H}$ & -3.715068 & -0.067722 & 0.305048 \\
\hline $\mathrm{C}$ & -1.806416 & 0.967551 & 0.307806 \\
\hline $\mathrm{H}$ & -1.606867 & 0.805813 & 1.377820 \\
\hline $\mathrm{C}$ & -3.703489 & 1.338909 & -1.338434 \\
\hline $\mathrm{H}$ & -3.252849 & 0.631518 & -2.044255 \\
\hline $\mathrm{H}$ & -4.793309 & 1.289146 & -1.471986 \\
\hline $\mathrm{C}$ & 3.068433 & 0.324216 & 0.467866 \\
\hline $\mathrm{C}$ & 4.067970 & -0.172222 & -0.396500 \\
\hline $\mathrm{C}$ & 5.005222 & -1.071291 & 0.126766 \\
\hline $\mathrm{H}$ & 5.779380 & -1.475057 & -0.518451 \\
\hline $\mathrm{C}$ & 4.963751 & -1.453232 & 1.465832 \\
\hline $\mathrm{C}$ & 3.993165 & -0.923672 & 2.311569 \\
\hline $\mathrm{H}$ & 3.973375 & -1.222186 & 3.355748 \\
\hline $\mathrm{C}$ & 3.034933 & -0.018375 & 1.839097 \\
\hline $\mathrm{C}$ & 4.177925 & 0.298327 & -1.842535 \\
\hline $\mathrm{H}$ & 3.205499 & 0.717470 & -2.123723 \\
\hline $\mathrm{C}$ & 2.016908 & 0.564442 & 2.818690 \\
\hline $\mathrm{H}$ & 1.419384 & 1.318721 & 2.302877 \\
\hline $\mathrm{C}$ & -0.132654 & -0.054921 & -2.341471 \\
\hline $\mathrm{H}$ & 0.684307 & 0.538638 & -2.776892 \\
\hline $\mathrm{C}$ & 0.570379 & -2.424185 & -0.627468 \\
\hline $\mathrm{H}$ & -0.255299 & -3.127661 & -0.729407 \\
\hline $\mathrm{C}$ & 1.010786 & -1.868770 & -1.852701 \\
\hline $\mathrm{H}$ & 1.991272 & -1.403109 & -1.839122 \\
\hline $\mathrm{H}$ & -0.829388 & -0.432235 & -3.101423 \\
\hline $\mathrm{C}$ & 4.520014 & -0.827105 & -2.836561 \\
\hline $\mathrm{H}$ & 4.444105 & -0.453073 & -3.863939 \\
\hline $\mathrm{H}$ & 5.545109 & -1.189854 & -2.698875 \\
\hline $\mathrm{H}$ & 3.848834 & -1.685450 & -2.736459 \\
\hline $\mathrm{C}$ & 5.232361 & 1.420021 & -1.957823 \\
\hline $\mathrm{H}$ & 5.274030 & 1.809982 & -2.98193 \\
\hline $\mathrm{H}$ & 5.016614 & 2.253845 & -1.28254 \\
\hline $\mathrm{H}$ & 6.226994 & 1.036921 & -1.69930 \\
\hline $\mathrm{C}$ & 2.724495 & 1.273956 & 3.99025 \\
\hline $\mathrm{H}$ & 3.280676 & 0.566722 & 4.6160 \\
\hline
\end{tabular}




$\begin{array}{lrrr}\mathrm{H} & 3.434031 & 2.029783 & 3.632739 \\ \mathrm{H} & 1.986654 & 1.771318 & 4.630455 \\ \mathrm{C} & 1.022626 & -0.495353 & 3.329329 \\ \mathrm{H} & 0.399092 & -0.878828 & 2.518450 \\ \mathrm{H} & 1.544309 & -1.338069 & 3.798936 \\ \mathrm{H} & 0.357548 & -0.051886 & 4.080308 \\ \mathrm{H} & 5.694491 & -2.159056 & 1.851737 \\ \mathrm{C} & 1.498201 & -2.769320 & 0.508532 \\ \mathrm{H} & 0.978625 & -2.719908 & 1.467794 \\ \mathrm{H} & 1.843519 & -3.806474 & 0.375557 \\ \mathrm{H} & 2.376249 & -2.130917 & 0.540035 \\ \mathrm{C} & 0.625752 & -2.551623 & -3.156705 \\ \mathrm{H} & -0.433515 & -2.826261 & -3.152995 \\ \mathrm{H} & 0.829395 & -1.935676 & -4.037977 \\ \mathrm{H} & 1.214110 & -3.475331 & -3.250756 \\ \mathrm{C} & -3.477953 & -3.137277 & 0.632078 \\ \mathrm{C} & -4.104054 & -2.709647 & 1.972165 \\ \mathrm{H} & -3.342803 & -2.648600 & 2.754111 \\ \mathrm{H} & -4.578901 & -1.724982 & 1.886791 \\ \mathrm{H} & -4.871694 & -3.431140 & 2.278062 \\ \mathrm{C} & -4.548916 & -3.183391 & -0.468291 \\ \mathrm{H} & -5.007627 & -2.198464 & -0.609526 \\ \mathrm{H} & -4.116759 & -3.486323 & -1.425890 \\ \mathrm{H} & -5.337939 & -3.895446 & -0.196232 \\ \mathrm{C} & -2.815620 & -4.524764 & 0.787303 \\ \mathrm{H} & -3.564839 & -5.274436 & 1.070107 \\ \mathrm{H} & -2.354095 & -4.845366 & -0.154766 \\ \mathrm{H} & -2.040601 & -4.499088 & 1.560621 \\ \mathrm{C} & -2.359858 & -2.155712 & 0.256984\end{array}$

$\begin{array}{lccc}\text { INT1' } & & & \\ \mathrm{Ru} & -0.426987 & 1.057751 & 0.447116 \\ \mathrm{O} & 1.146322 & 2.460593 & 0.022000 \\ \mathrm{O} & -0.628526 & 3.230209 & 1.021614 \\ \mathrm{~N} & -0.982422 & -1.488826 & -0.777283 \\ \mathrm{~N} & 1.175454 & -1.410190 & -0.369463 \\ \mathrm{C} & 0.015959 & -0.717744 & -0.271672 \\ \mathrm{C} & -0.547872 & -2.864159 & -1.031356 \\ \mathrm{H} & -0.951275 & -3.248888 & -1.972000 \\ \mathrm{H} & -0.872056 & -3.527821 & -0.218083 \\ \mathrm{C} & 0.985727 & -2.702247 & -1.055994 \\ \mathrm{H} & 1.514940 & -3.501122 & -0.529345 \\ \mathrm{H} & 1.374812 & -2.644235 & -2.080125 \\ \mathrm{C} & -2.351832 & -1.050738 & -0.502576 \\ \mathrm{C} & -2.885027 & -1.713589 & 0.790736 \\ \mathrm{H} & -2.171083 & -1.541714 & -1.602036 \\ \mathrm{H} & -2.965532 & -2.799368 & 0.640625 \\ \mathrm{C} & -4.261643 & -1.127699 & 1.152260 \\ \mathrm{H} & -4.626992 & -1.602875 & 2.071973 \\ \mathrm{C} & -5.243629 & -1.407149 & -0.003293 \\ \mathrm{H} & -5.361400 & -2.490822 & -0.144098 \\ \mathrm{H} & -6.236179 & -1.003806 & 0.237667 \\ \mathrm{C} & -4.714468 & -0.754853 & -1.297612 \\ \mathrm{H} & -5.410550 & -0.953380 & -2.122566 \\ \mathrm{C} & -3.331790 & -1.355439 & -1.654206 \\ \mathrm{H} & -2.949178 & -0.919577 & -2.586448 \\ \mathrm{H} & -3.425744 & -2.440094 & -1.811436 \\ \mathrm{C} & -4.598275 & 0.769364 & -1.072824 \\ \mathrm{H} & -4.258080 & 1.264394 & -1.992512 \\ \mathrm{H} & -5.587925 & 1.182059 & -0.832764\end{array}$

$\begin{array}{lrrr}\mathrm{C} & -3.602663 & 1.057795 & 0.081548 \\ \mathrm{H} & -3.512118 & 2.140401 & 0.231039 \\ \mathrm{C} & -2.241453 & 0.481624 & -0.327336 \\ \mathrm{H} & -1.983689 & 0.904991 & -1.328622 \\ \mathrm{C} & -4.115818 & 0.392090 & 1.372340 \\ \mathrm{H} & -3.414535 & 0.587164 & 2.192449 \\ \mathrm{H} & -5.084621 & 0.824896 & 1.655850 \\ \mathrm{C} & 2.478565 & -0.822612 & -0.234414 \\ \mathrm{C} & 3.178679 & -0.999724 & 0.973881 \\ \mathrm{C} & 4.461280 & -0.448798 & 1.077259 \\ \mathrm{H} & 5.023150 & -0.572710 & 1.998631 \\ \mathrm{C} & 5.024851 & 0.257683 & 0.018296 \\ \mathrm{C} & 4.312456 & 0.425820 & -1.166347 \\ \mathrm{H} & 4.758816 & 0.983314 & -1.984594 \\ \mathrm{C} & 3.027960 & -0.107337 & -1.319176 \\ \mathrm{C} & 2.584218 & -1.772667 & 2.142266 \\ \mathrm{H} & 1.544842 & -2.004958 & 1.889984 \\ \mathrm{C} & 2.287933 & 0.073601 & -2.640191 \\ \mathrm{H} & 1.268208 & -0.305817 & -2.520262 \\ \mathrm{C} & -0.530883 & 0.438011 & 2.148824 \\ \mathrm{H} & -0.233586 & -0.547743 & 2.522709 \\ \mathrm{H} & -0.879436 & 1.138941 & 2.922187 \\ \mathrm{H} & 6.020616 & 0.681612 & 0.117114 \\ \mathrm{C} & 3.324076 & -3.107308 & 2.354621 \\ \mathrm{H} & 4.376187 & -2.937139 & 2.612334 \\ \mathrm{H} & 2.865713 & -3.678791 & 3.170678 \\ \mathrm{H} & 3.300886 & -3.724457 & 1.448521 \\ \mathrm{C} & 2.569910 & -0.934792 & 3.433394 \\ \mathrm{H} & 2.050168 & -1.476459 & 4.232968 \\ \mathrm{H} & 3.585506 & -0.720466 & 3.785575 \\ \mathrm{H} & 2.058866 & 0.019778 & 3.272750 \\ \mathrm{C} & 2.964867 & -0.742562 & -3.759859 \\ \mathrm{H} & 3.039529 & -1.805054 & -3.500170 \\ \mathrm{H} & 2.400095 & -0.656076 & -4.695772 \\ \mathrm{H} & 3.981910 & -0.377952 & -3.946058 \\ \mathrm{C} & 2.166384 & 1.554659 & -3.044687 \\ \mathrm{H} & 3.147241 & 1.991160 & -3.265936 \\ \mathrm{H} & 1.553861 & 1.646208 & -3.949996 \\ & 1.710594 & 2.143827 & -2.245048 \\ \mathrm{~N} & 0.527060 & 3.498098 & 0.540934 \\ 1.025925 & 4.598021 & 0.544518\end{array}$

$\begin{array}{lccc}\text { TS1 } & & & \\ \mathrm{Ru} & 0.570893 & 0.821147 & -0.615844 \\ \mathrm{O} & 1.772924 & 2.536465 & -1.264404 \\ \mathrm{O} & 1.210373 & 2.563064 & 0.832058 \\ \mathrm{~N} & 0.638935 & -1.761782 & 0.658741 \\ \mathrm{~N} & -1.491356 & -1.356846 & 0.349907 \\ \mathrm{C} & -0.233543 & -0.827458 & 0.197407 \\ \mathrm{C} & 0.015568 & -3.052250 & 0.939939 \\ \mathrm{H} & 0.421024 & -3.508347 & 1.846809 \\ \mathrm{H} & 0.170439 & -3.747779 & 0.102567 \\ \mathrm{C} & -1.454083 & -2.643453 & 1.078358 \\ \mathrm{H} & -2.148817 & -3.361254 & 0.637687 \\ \mathrm{H} & -1.737161 & -2.483507 & 2.127452 \\ \mathrm{C} & 2.067158 & -1.553719 & 0.420731 \\ \mathrm{C} & 2.512615 & -2.217115 & -0.904792 \\ \mathrm{H} & 1.860062 & -1.880877 & -1.716543 \\ \mathrm{H} & 2.408213 & -3.309000 & -0.826368 \\ \mathrm{C} & 3.976405 & -1.844106 & -1.202708\end{array}$




\begin{tabular}{|c|c|c|c|}
\hline $\mathrm{H}$ & 4.281350 & -2.302622 & -2.152792 \\
\hline $\mathrm{C}$ & 4.867414 & -2.374810 & -0.061247 \\
\hline $\mathrm{H}$ & 4.796436 & -3.470539 & -0.006191 \\
\hline $\mathrm{H}$ & 5.919705 & -2.129320 & -0.257354 \\
\hline $\mathrm{C}$ & 4.423045 & -1.741809 & 1.273831 \\
\hline $\mathrm{H}$ & 5.049819 & -2.123959 & 2.090080 \\
\hline $\mathrm{C}$ & 2.948234 & -2.117443 & 1.558570 \\
\hline $\mathrm{H}$ & 2.620486 & -1.695556 & 2.517617 \\
\hline $\mathrm{H}$ & 2.852996 & -3.211557 & 1.627136 \\
\hline $\mathrm{C}$ & 4.577070 & -0.208931 & 1.171238 \\
\hline $\mathrm{H}$ & 4.304203 & 0.266663 & 2.122489 \\
\hline $\mathrm{H}$ & 5.629400 & 0.041171 & 0.975827 \\
\hline $\mathrm{C}$ & 3.674047 & 0.336332 & 0.034748 \\
\hline $\mathrm{H}$ & 3.796416 & 1.421722 & -0.030106 \\
\hline $\mathrm{C}$ & 2.220935 & -0.020432 & 0.387546 \\
\hline $\mathrm{H}$ & 2.024166 & 0.335632 & 1.409779 \\
\hline $\mathrm{C}$ & 4.096460 & -0.308098 & -1.299674 \\
\hline $\mathrm{H}$ & 3.461903 & 0.071678 & -2.109649 \\
\hline $\mathrm{H}$ & 5.132243 & -0.031162 & -1.538660 \\
\hline $\mathrm{C}$ & -2.707154 & -0.599086 & 0.403249 \\
\hline $\mathrm{C}$ & -3.672861 & -0.794191 & -0.608217 \\
\hline $\mathrm{C}$ & -4.860289 & -0.056396 & -0.538551 \\
\hline $\mathrm{H}$ & -5.616629 & -0.184961 & -1.307115 \\
\hline $\mathrm{C}$ & -5.089913 & 0.835691 & 0.506270 \\
\hline $\mathrm{C}$ & -4.142942 & 0.985079 & 1.516025 \\
\hline $\mathrm{H}$ & -4.340695 & 1.666570 & 2.338343 \\
\hline $\mathrm{C}$ & -2.940294 & 0.267015 & 1.495562 \\
\hline $\mathrm{C}$ & -3.474993 & -1.827355 & -1.712773 \\
\hline $\mathrm{H}$ & -2.416777 & -2.110504 & -1.710947 \\
\hline $\mathrm{C}$ & -1.962412 & 0.411916 & 2.659628 \\
\hline $\mathrm{H}$ & -1.066210 & -0.173863 & 2.439990 \\
\hline $\mathrm{C}$ & 0.432212 & 0.080047 & -2.328127 \\
\hline $\mathrm{H}$ & -0.127341 & -0.798153 & -2.677128 \\
\hline $\mathrm{C}$ & -1.263352 & 2.191986 & -0.847413 \\
\hline $\mathrm{H}$ & -1.903458 & 1.846231 & -0.044173 \\
\hline $\mathrm{C}$ & -1.291749 & 1.434271 & -2.029135 \\
\hline $\mathrm{H}$ & -1.969804 & 0.594503 & -2.094830 \\
\hline $\mathrm{H}$ & 0.988735 & 0.572618 & -3.138692 \\
\hline $\mathrm{N}$ & 1.863703 & 3.124490 & -0.118551 \\
\hline $\mathrm{O}$ & 2.521125 & 4.137523 & 0.038026 \\
\hline $\mathrm{C}$ & -3.818566 & -1.306022 & -3.120148 \\
\hline $\mathrm{H}$ & -3.586027 & -2.072411 & -3.868363 \\
\hline $\mathrm{H}$ & -4.884207 & -1.069418 & -3.216271 \\
\hline $\mathrm{H}$ & -3.253693 & -0.404210 & -3.375530 \\
\hline $\mathrm{C}$ & -4.310534 & -3.089553 & -1.409749 \\
\hline $\mathrm{H}$ & -4.119121 & -3.870164 & -2.155550 \\
\hline $\mathrm{H}$ & -4.085424 & -3.498586 & -0.419123 \\
\hline $\mathrm{H}$ & -5.381637 & -2.855264 & -1.431427 \\
\hline $\mathrm{C}$ & -2.586458 & -0.158040 & 3.950168 \\
\hline $\mathrm{H}$ & -3.463828 & 0.424038 & 4.255805 \\
\hline $\mathrm{H}$ & -2.909538 & -1.197322 & 3.817758 \\
\hline $\mathrm{H}$ & -1.860375 & -0.125727 & 4.770699 \\
\hline $\mathrm{C}$ & -1.497113 & 1.864236 & 2.874979 \\
\hline $\mathrm{H}$ & -0.814736 & 1.912444 & 3.731198 \\
\hline $\mathrm{H}$ & -0.956471 & 2.247209 & 2.006859 \\
\hline $\mathrm{H}$ & -2.341463 & 2.531194 & 3.085596 \\
\hline $\mathrm{H}$ & -6.015266 & 1.404426 & 0.541205 \\
\hline $\mathrm{H}$ & -1.041355 & 1.925099 & -2.967877 \\
\hline $\mathrm{C}$ & -1.009227 & 3.681251 & -0.866865 \\
\hline $\mathrm{H}$ & -1.956969 & 4.202710 & -1.060840 \\
\hline
\end{tabular}

$\begin{array}{lllr}\mathrm{H} & -0.630818 & 4.034284 & 0.095499 \\ \mathrm{H} & -0.297505 & 3.964608 & -1.646912\end{array}$

\section{INT2'-1}

$\begin{array}{lccc}\mathrm{Ru} & 0.619307 & 0.845551 & -0.49072 \\ \mathrm{O} & 1.861489 & 2.458482 & -1.227508 \\ \mathrm{O} & 1.739126 & 2.624231 & 0.940157 \\ \mathrm{~N} & 0.540591 & -1.837577 & 0.550609 \\ \mathrm{~N} & -1.562761 & -1.342676 & 0.213929 \\ \mathrm{C} & -0.292349 & -0.832004 & 0.171369 \\ \mathrm{C} & -0.116203 & -3.141383 & 0.622590\end{array}$

$\begin{array}{llll}\mathrm{H} & 0.235293 & -3.724597 & 1.477581\end{array}$

$\begin{array}{llll}\mathrm{H} & 0.070605 & -3.720087 & -0.293106\end{array}$

$\begin{array}{llll}\text { C } & -1.585886 & -2.723464 & 0.741622\end{array}$

$\mathrm{H} \quad-2.265497 \quad-3.349298 \quad 0.158415$

$\mathrm{H} \quad-1.929375 \quad-2.717823 \quad 1.784881$

$\begin{array}{llll}\mathrm{C} & 1.971673 & -1.636187 & 0.338062\end{array}$

$\begin{array}{llll}\text { C } & 2.388107 & -2.080641 & -1.088657\end{array}$

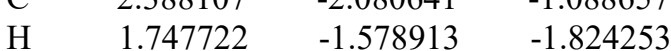

$\mathrm{H} \quad 2.231872 \quad-3.163268 \quad-1.198359$

$\begin{array}{llll}\mathrm{C} & 3.867497 & -1.730665 & -1.332367\end{array}$

$\mathrm{H} \quad 4.152266 \quad-2.044986 \quad-2.344909$

$\begin{array}{llll}\mathrm{C} & 4.734467 & -2.472960 & -0.294639\end{array}$

$\mathrm{H} \quad 4.618204 \quad-3.559629 \quad-0.413166$

$\mathrm{H} \quad 5.795674 \quad-2.242603 \quad-0.457094$

$\begin{array}{llll}\text { C } & 4.318964 & -2.044385 & 1.128403\end{array}$

$\begin{array}{llll}\mathrm{H} & 4.933998 & -2.573146 & 1.867944\end{array}$

$\begin{array}{llll}\mathrm{C} & 2.833742 & -2.402537 & 1.363378\end{array}$

$\mathrm{H} \quad 2.524258 \quad-2.126482 \quad 2.379859$

$\begin{array}{llll}\mathrm{H} & 2.690527 & -3.487994 & 1.255351\end{array}$

$\begin{array}{llll}\text { C } & 4.520712 & -0.522023 & 1.273471\end{array}$

$\mathrm{H} \quad 4.258231 \quad-0.196776 \quad 2.288774$

$\mathrm{H} \quad \begin{array}{llll}5.579527 & -0.272445 & 1.118432\end{array}$

$\begin{array}{llll}\mathrm{C} & 3.646242 & 0.232052 & 0.236982\end{array}$

$\begin{array}{llll}\mathrm{H} & 3.813455 & 1.303925 & 0.358521\end{array}$

C $\quad 2.178102 \quad-0.120141 \quad 0.529033$

$\mathrm{H} \quad 1.943535 \quad 0.133081 \quad 1.572510$

$\begin{array}{llll}\text { C } & 4.054405 & -0.206967 & -1.183739\end{array}$

$\mathrm{H} \quad 3.450353 \quad 0.329915 \quad-1.925152$

$\begin{array}{llll}\mathrm{H} & 5.103655 & 0.059937 & -1.368040\end{array}$

$\begin{array}{llll}\text { C } & -2.763971 & -0.568532 & 0.319551\end{array}$

$\begin{array}{llll}\text { C } & -3.670423 & -0.567124 & -0.760885\end{array}$

$\begin{array}{llll}\text { C } & -4.824861 & 0.217512 & -0.648407\end{array}$

$\mathrm{H} \quad-5.536722 \quad 0.242976 \quad-1.468235$

$\begin{array}{llll}\text { C } & -5.079329 & 0.957673 & 0.504050\end{array}$

$\begin{array}{llll}\text { C } & -4.198388 & 0.898029 & 1.581575\end{array}$

$\mathrm{H} \quad-4.418475 \quad 1.459520 \quad 2.485093$

$\begin{array}{lrrr}\text { C } & -3.031821 & 0.126354 & 1.520122\end{array}$

$\begin{array}{llll}\text { C } & -3.459938 & -1.456651 & -1.981240\end{array}$

$\mathrm{H} \quad-2.430886 \quad-1.828521 \quad-1.943625$

$\begin{array}{llll}\text { C } & -2.117134 & 0.043408 & 2.740829\end{array}$

$\mathrm{H} \quad-1.271335 \quad-0.606116 \quad 2.501206$

$\begin{array}{llll}\mathrm{C} & -0.109824 & 0.532412 & -2.306989\end{array}$

$\mathrm{H} \quad-0.666599 \quad-0.368475 \quad-2.572737$

$\begin{array}{llll}\mathrm{C} & -0.968511 & 2.118470 & -0.328317\end{array}$

$\begin{array}{llll}\mathrm{H} & -1.795716 & 1.704230 & 0.237271\end{array}$

$\begin{array}{llll}\mathrm{C} & -1.061544 & 1.727464 & -1.843329\end{array}$

$\begin{array}{llll}\mathrm{H} & -2.089106 & 1.393259 & -2.000683\end{array}$

$\mathrm{H} \quad 0.567241 \quad 0.845970 \quad-3.108003$

$\mathrm{N} \quad 2.220645 \quad 3.077726 \quad-0.139129$ 


$\begin{array}{lrrr}\mathrm{O} & 2.979128 & 4.033201 & -0.192187 \\ \mathrm{C} & -3.641392 & -0.725957 & -3.322831 \\ \mathrm{H} & -3.444856 & -1.414723 & -4.152498 \\ \mathrm{H} & -4.663055 & -0.349233 & -3.445956 \\ \mathrm{H} & -2.955091 & 0.119933 & -3.420220 \\ \mathrm{C} & -4.408648 & -2.672237 & -1.908691 \\ \mathrm{H} & -4.216934 & -3.363098 & -2.738213 \\ \mathrm{H} & -4.290045 & -3.222448 & -0.968618 \\ \mathrm{H} & -5.455153 & -2.350549 & -1.970782 \\ \mathrm{C} & -2.866831 & -0.596167 & 3.926929 \\ \mathrm{H} & -3.697925 & 0.035343 & 4.261605 \\ \mathrm{H} & -3.281823 & -1.574612 & 3.657531 \\ \mathrm{H} & -2.188304 & -0.732561 & 4.776924 \\ \mathrm{C} & -1.520039 & 1.407100 & 3.136549 \\ \mathrm{H} & -0.927513 & 1.303293 & 4.052971 \\ \mathrm{H} & -0.859360 & 1.792042 & 2.356174 \\ \mathrm{H} & -2.302875 & 2.150398 & 3.327693 \\ \mathrm{H} & -5.976380 & 1.567492 & 0.569935 \\ \mathrm{H} & -0.835246 & 2.608703 & -2.447737 \\ \mathrm{C} & -0.814956 & 3.599121 & -0.045353 \\ \mathrm{H} & -1.774043 & 4.101932 & -0.243267 \\ \mathrm{H} & -0.557405 & 3.772323 & 1.002710 \\ \mathrm{H} & -0.054628 & 4.072853 & -0.670974\end{array}$

$\begin{array}{lccc}\text { INT2' } & & & \\ \mathrm{Ru} & -0.491024 & 1.150985 & -0.054075 \\ \mathrm{O} & -1.855898 & 1.452297 & 2.023887 \\ \mathrm{O} & -1.608306 & 2.957798 & 0.470733 \\ \mathrm{~N} & -0.520089 & -1.529386 & -1.110911 \\ \mathrm{~N} & 1.586285 & -1.124626 & -0.659393 \\ \mathrm{C} & 0.323828 & -0.606698 & -0.574539 \\ \mathrm{C} & 0.117922 & -2.809282 & -1.419685 \\ \mathrm{H} & -0.162145 & -3.162459 & -2.415887 \\ \mathrm{H} & -0.172870 & -3.576702 & -0.690164 \\ \mathrm{C} & 1.609723 & -2.447634 & -1.317478 \\ \mathrm{H} & 2.182284 & -3.160242 & -0.716582 \\ \mathrm{H} & 2.084925 & -2.367986 & -2.302297 \\ \mathrm{C} & -1.949139 & -1.372770 & -0.855971 \\ \mathrm{C} & -2.354694 & -2.139039 & 0.430097 \\ \mathrm{H} & -1.729954 & -1.784601 & 1.259450 \\ \mathrm{H} & -2.164968 & -3.213320 & 0.298968 \\ \mathrm{C} & -3.844963 & -1.900554 & 0.738677 \\ \mathrm{H} & -4.120732 & -2.467108 & 1.637467 \\ \mathrm{C} & -4.700192 & -2.369827 & -0.455164 \\ \mathrm{H} & -4.562751 & -3.447266 & -0.625181 \\ \mathrm{H} & -5.764377 & -2.211018 & -0.236817 \\ \mathrm{C} & -4.298773 & -1.579850 & -1.718126 \\ \mathrm{H} & -4.914424 & -1.901460 & -2.567925 \\ \mathrm{C} & -2.814216 & -1.860714 & -2.037995 \\ \mathrm{H} & -2.505378 & -1.338127 & -2.953025 \\ \mathrm{H} & -2.664602 & -2.937367 & -2.206100 \\ \mathrm{C} & -4.507167 & -0.069835 & -1.468725 \\ \mathrm{H} & -4.249849 & 0.501426 & -2.371067 \\ \mathrm{H} & -5.565878 & 0.128383 & -1.253169 \\ \mathrm{C} & -3.634567 & 0.396853 & -0.269487 \\ \mathrm{H} & -3.785985 & 1.468758 & -0.108770 \\ \mathrm{C} & -2.173281 & 0.128197 & -0.647585 \\ \mathrm{H} & -1.956928 & 0.657900 & -1.610275 \\ \mathrm{C} & -4.063239 & -0.396886 & 0.982574 \\ \mathrm{H} & -3.491178 & -0.061934 & 1.849816\end{array}$

$\begin{array}{lrrr} & & & \\ \mathrm{H} & -5.124008 & -0.200861 & 1.189991 \\ \mathrm{C} & 2.695342 & -0.735777 & 0.158375 \\ \mathrm{C} & 2.680069 & -1.092427 & 1.525656 \\ \mathrm{C} & 3.756472 & -0.680051 & 2.320084 \\ \mathrm{H} & 3.759164 & -0.919349 & 3.379740 \\ \mathrm{C} & 4.823623 & 0.029031 & 1.772875 \\ \mathrm{C} & 4.850148 & 0.309793 & 0.408585 \\ \mathrm{H} & 5.705914 & 0.827757 & -0.014046 \\ \mathrm{C} & 3.793684 & -0.074512 & -0.426565 \\ \mathrm{C} & 1.565840 & -1.935883 & 2.141808 \\ \mathrm{H} & 0.847895 & -2.186060 & 1.356314 \\ \mathrm{C} & 3.884120 & 0.134957 & -1.933435 \\ \mathrm{H} & 2.885490 & -0.032501 & -2.350058 \\ \mathrm{C} & 0.900097 & 1.791728 & 1.275146 \\ \mathrm{H} & 1.631681 & 1.109078 & 1.691819 \\ \mathrm{C} & 0.693615 & 2.081108 & -1.357047 \\ \mathrm{H} & 1.384615 & 1.477021 & -1.948872 \\ \mathrm{C} & 1.394625 & 2.550713 & 0.010315 \\ \mathrm{H} & 2.461322 & 2.342670 & -0.100309 \\ \mathrm{H} & 0.467897 & 2.441039 & 2.037086 \\ \mathrm{C} & 4.832622 & -0.916905 & -2.546614 \\ \mathrm{H} & 4.860602 & -0.823714 & -3.638808 \\ \mathrm{H} & 5.853279 & -0.784496 & -2.168251 \\ \mathrm{H} & 4.517210 & -1.935962 & -2.296387 \\ \mathrm{C} & 4.324754 & 1.551592 & -2.338999 \\ \mathrm{H} & 5.343873 & 1.772516 & -2.002158 \\ \mathrm{H} & 4.313772 & 1.647971 & -3.430721 \\ \mathrm{H} & 3.659174 & 2.315488 & -1.926740 \\ \mathrm{C} & 2.134646 & -3.265935 & 2.675822 \\ \mathrm{H} & 2.682881 & -3.808358 & 1.896326 \\ \mathrm{H} & 2.823808 & -3.100266 & 3.511761 \\ \mathrm{H} & 1.323118 & -3.908824 & 3.036183 \\ \mathrm{C} & 0.778837 & -1.191752 & 3.236885 \\ \mathrm{H} & 0.033578 & -1.862410 & 3.681089 \\ \mathrm{H} & 1.439732 & -0.849313 & 4.041928 \\ \mathrm{H} & 0.250362 & -0.325463 & 2.831268 \\ \mathrm{H} & 5.647181 & 0.344948 & 2.407567 \\ \mathrm{H} & 1.213721 & 3.622327 & 0.125928 \\ \mathrm{~N} & -2.141722 & 2.616339 & 1.608405 \\ \mathrm{O} & -2.871695 & 3.386036 & 2.211391 \\ \mathrm{C} & 0.101031 & 3.213927 & -2.180694 \\ \mathrm{H} & 0.890435 & 3.891338 & -2.542147 \\ \mathrm{H} & -0.416146 & 2.814101 & -3.062152 \\ \mathrm{H} & -0.615410 & 3.806343 & -1.603047\end{array}$

$\begin{array}{lccc}\text { TS2' } & & & \\ \mathrm{Ru} & -0.445840 & 1.163954 & -0.020871 \\ \mathrm{O} & -1.566418 & 1.126775 & 1.997932 \\ \mathrm{O} & -1.635385 & 2.880417 & 0.719970 \\ \mathrm{~N} & -0.552211 & -1.527306 & -1.048448 \\ \mathrm{~N} & 1.568623 & -1.137609 & -0.660143 \\ \mathrm{C} & 0.311969 & -0.602191 & -0.557541 \\ \mathrm{C} & 0.061432 & -2.829312 & -1.308663 \\ \mathrm{H} & -0.254690 & -3.231244 & -2.274837 \\ \mathrm{H} & -0.215504 & -3.553168 & -0.529933 \\ \mathrm{C} & 1.559098 & -2.481349 & -1.273813 \\ \mathrm{H} & 2.147857 & -3.179992 & -0.672038 \\ \mathrm{H} & 1.997805 & -2.437921 & -2.278216 \\ \mathrm{C} & -1.985390 & -1.325344 & -0.824969 \\ \mathrm{C} & -2.406869 & -1.950556 & 0.529263\end{array}$




\begin{tabular}{|c|c|c|c|}
\hline $\mathrm{H}$ & -1.794254 & -1.512814 & 1.326369 \\
\hline $\mathrm{H}$ & -2.220772 & -3.034351 & 0.515200 \\
\hline $\mathrm{C}$ & -3.900572 & -1.677576 & 0.794815 \\
\hline $\mathrm{H}$ & -4.189420 & -2.147714 & 1.744009 \\
\hline $\mathrm{C}$ & -4.746113 & -2.262358 & -0.353275 \\
\hline $\mathrm{H}$ & -4.611924 & -3.352172 & -0.413547 \\
\hline $\mathrm{H}$ & -5.811918 & -2.078734 & -0.162820 \\
\hline $\mathrm{C}$ & -4.326251 & -1.603301 & -1.683234 \\
\hline $\mathrm{H}$ & -4.936674 & -2.003451 & -2.503477 \\
\hline $\mathrm{C}$ & -2.841053 & -1.936473 & -1.953820 \\
\hline $\mathrm{H}$ & -2.517180 & -1.523785 & -2.918744 \\
\hline $\mathrm{H}$ & -2.713543 & -3.028665 & -2.002129 \\
\hline $\mathrm{C}$ & -4.529808 & -0.072965 & -1.584436 \\
\hline $\mathrm{H}$ & -4.268362 & 0.403133 & -2.539462 \\
\hline $\mathrm{H}$ & -5.591586 & 0.141966 & -1.398232 \\
\hline $\mathrm{C}$ & -3.655552 & 0.506789 & -0.437215 \\
\hline $\mathrm{H}$ & -3.801464 & 1.591792 & -0.378272 \\
\hline $\mathrm{C}$ & -2.185265 & 0.200785 & -0.803558 \\
\hline $\mathrm{H}$ & -2.066089 & 0.537473 & -1.843651 \\
\hline $\mathrm{C}$ & -4.119326 & -0.155555 & 0.879175 \\
\hline $\mathrm{H}$ & -3.581092 & 0.246496 & 1.737177 \\
\hline $\mathrm{H}$ & -5.186456 & 0.056706 & 1.034181 \\
\hline $\mathrm{C}$ & 2.710510 & -0.719064 & 0.090855 \\
\hline $\mathrm{C}$ & 2.781482 & -1.064041 & 1.459214 \\
\hline $\mathrm{C}$ & 3.898127 & -0.631467 & 2.185165 \\
\hline $\mathrm{H}$ & 3.968722 & -0.864790 & 3.243907 \\
\hline $\mathrm{C}$ & 4.920205 & 0.088904 & 1.570549 \\
\hline $\mathrm{C}$ & 4.859056 & 0.364901 & 0.206166 \\
\hline $\mathrm{H}$ & 5.679565 & 0.894400 & -0.269207 \\
\hline $\mathrm{C}$ & 3.759886 & -0.040249 & -0.561036 \\
\hline $\mathrm{C}$ & 1.716655 & -1.919286 & 2.143161 \\
\hline $\mathrm{H}$ & 0.956004 & -2.179390 & 1.402353 \\
\hline $\mathrm{C}$ & 3.747806 & 0.164495 & -2.071437 \\
\hline $\mathrm{H}$ & 2.726748 & -0.024520 & -2.419213 \\
\hline $\mathrm{C}$ & 1.116391 & 1.930373 & 1.430342 \\
\hline $\mathrm{H}$ & 1.724368 & 1.115368 & 1.795384 \\
\hline $\mathrm{C}$ & 0.286181 & 1.942983 & -1.569216 \\
\hline $\mathrm{H}$ & 1.100620 & 1.508960 & -2.166551 \\
\hline $\mathrm{C}$ & 1.491506 & 2.597599 & 0.263184 \\
\hline $\mathrm{H}$ & 2.382861 & 2.278617 & -0.264559 \\
\hline $\mathrm{H}$ & 0.531134 & 2.458489 & 2.176169 \\
\hline $\mathrm{C}$ & 4.673374 & -0.872197 & -2.742528 \\
\hline $\mathrm{H}$ & 4.625579 & -0.786023 & -3.834619 \\
\hline $\mathrm{H}$ & 5.714469 & -0.717645 & -2.434641 \\
\hline $\mathrm{H}$ & 4.395150 & -1.895051 & -2.465905 \\
\hline $\mathrm{C}$ & 4.134465 & 1.586253 & -2.512631 \\
\hline $\mathrm{H}$ & 5.163693 & 1.835172 & -2.230995 \\
\hline $\mathrm{H}$ & 4.064380 & 1.671362 & -3.603155 \\
\hline $\mathrm{H}$ & 3.474362 & 2.341765 & -2.075268 \\
\hline $\mathrm{C}$ & 2.334381 & -3.239519 & 2.646275 \\
\hline $\mathrm{H}$ & 2.844303 & -3.776632 & 1.837679 \\
\hline $\mathrm{H}$ & 3.068551 & -3.061058 & 3.440366 \\
\hline $\mathrm{H}$ & 1.554482 & -3.892692 & 3.054481 \\
\hline $\mathrm{C}$ & 0.985947 & -1.182157 & 3.281462 \\
\hline $\mathrm{H}$ & 0.289086 & -1.865785 & 3.780417 \\
\hline $\mathrm{H}$ & 1.689505 & -0.813788 & 4.037661 \\
\hline $\mathrm{H}$ & 0.405111 & -0.336484 & 2.904657 \\
\hline $\mathrm{H}$ & 5.776447 & 0.419140 & 2.152521 \\
\hline $\mathrm{H}$ & 1.171974 & 3.623481 & 0.102269 \\
\hline $\mathrm{N}$ & -2.038292 & 2.305845 & 1.804147 \\
\hline
\end{tabular}

$\begin{array}{lccc}\mathrm{O} & -2.812675 & 2.839270 & 2.577043 \\ \mathrm{C} & -0.252794 & 3.207280 & -2.177780 \\ \mathrm{H} & 0.544536 & 3.903515 & -2.476981 \\ \mathrm{H} & -0.784438 & 2.922781 & -3.100537 \\ \mathrm{H} & -0.962865 & 3.722676 & -1.524766\end{array}$

\section{INT3,}

$\begin{array}{lccc}\mathrm{Ru} & 0.522053 & 0.753103 & 0.759543 \\ \mathrm{O} & -0.232309 & 2.524832 & -0.219775 \\ \mathrm{O} & 1.254311 & 2.832045 & 1.337338 \\ \mathrm{~N} & 0.760822 & -1.790758 & -0.528180\end{array}$

$\begin{array}{llll}\mathrm{N} & -1.368597 & -1.247890 & -0.593661\end{array}$

$\begin{array}{llll}\text { C } & -0.150393 & -0.843265 & -0.158948\end{array}$

$\begin{array}{llll}\text { C } & 0.202348 & -2.715787 & -1.515851\end{array}$

$\mathrm{H} \quad 0.583206 \quad-3.731162 \quad-1.382582$

H $\quad 0.430592 \quad-2.383729 \quad-2.540079$

$\begin{array}{llll}\text { C } & -1.298091 & -2.592544 & -1.202057\end{array}$

$\mathrm{H} \quad-1.940886 \quad-2.660350 \quad-2.081442$

$\mathrm{H} \quad-1.616373 \quad-3.352496 \quad-0.477033$

$\begin{array}{llll}\text { C } & 2.159095 & -1.355596 & -0.432702\end{array}$

$\begin{array}{llll}\text { C } & 2.538579 & -0.469009 & -1.649596\end{array}$

$\begin{array}{llll}\mathrm{H} & 1.803222 & 0.342503 & -1.758709\end{array}$

$\mathrm{H} \quad 2.492806 \quad-1.062751 \quad-2.573596$

$\begin{array}{llll}\mathrm{C} & 3.949733 & 0.114366 & -1.457637\end{array}$

$\begin{array}{llll}\mathrm{H} & 4.207854 & 0.736707 & -2.324212\end{array}$

C $\quad 4.954981 \quad-1.049426 \quad-1.334232$

$\mathrm{H} \quad 4.955086 \quad-1.646493 \quad-2.257012$

$\mathrm{H} \quad 5.971852 \quad-0.655731 \quad-1.205075$

C $\quad 4.577525 \quad-1.932662 \quad-0.126754$

$\begin{array}{llll}\mathrm{H} & 5.294209 & -2.759033 & -0.037022\end{array}$

$\begin{array}{llll}\text { C } & 3.159867 & -2.519885 & -0.338973\end{array}$

$\mathrm{H} \quad 2.882723 \quad-3.174093 \quad 0.497759$

$\mathrm{H} \quad 3.143584 \quad-3.128830 \quad-1.255512$

$\begin{array}{llll}\text { C } & 4.619259 & -1.068694 & 1.154334\end{array}$

$\mathrm{H} \quad 4.388160 \quad-1.684092 \quad 2.034076$

$\mathrm{H} \quad 5.635061 \quad-0.672933 \quad 1.295146$

$\begin{array}{llll}\mathrm{C} & 3.599090 & 0.094127 & 1.041317\end{array}$

$\begin{array}{llll}\mathrm{H} & 3.626510 & 0.702048 & 1.953898\end{array}$

$\begin{array}{llll}\text { C } & 2.201189 & -0.529757 & 0.879967\end{array}$

$\mathrm{H} \quad 2.054081 \quad-1.241388 \quad 1.699094$

$\begin{array}{llll}\mathrm{C} & 3.970880 & 0.969035 & -0.173584\end{array}$

$\mathrm{H} \quad 3.269200 \quad 1.809565 \quad-0.262055$

H $\quad 4.969142 \quad 1.404342 \quad-0.031386$

$\begin{array}{llll}\text { C } & -2.584264 & -0.492983 & -0.492143\end{array}$

$\begin{array}{llll}\text { C } & -2.814510 & 0.552031 & -1.417775\end{array}$

$\begin{array}{llll}\text { C } & -4.022962 & 1.250137 & -1.319994\end{array}$

$\mathrm{H} \quad-4.227957 \quad 2.063743 \quad-2.006908$

$\begin{array}{llll}\text { C } & -4.973007 & 0.921067 & -0.354975\end{array}$

$\begin{array}{llll}\mathrm{C} & -4.726023 & -0.110533 & 0.544277\end{array}$

$\begin{array}{llll}\mathrm{H} & -5.471694 & -0.358291 & 1.294008\end{array}$

$\begin{array}{llll}\text { C } & -3.524904 & -0.829482 & 0.500920\end{array}$

$\begin{array}{llll}\text { C } & -1.813496 & 0.885889 & -2.521406\end{array}$

$\begin{array}{llll}\mathrm{H} & -0.809744 & 0.698322 & -2.129726\end{array}$

$\begin{array}{llll}\text { C } & -3.282091 & -1.957899 & 1.495356\end{array}$

$\mathrm{H} \quad-2.223408 \quad-2.233450 \quad 1.439402$

$\begin{array}{llll}\text { C } & -0.237212 & 0.256090 & 2.333472\end{array}$

$\begin{array}{llll}\mathrm{H} & -0.886575 & -0.623832 & 2.424257\end{array}$

$\begin{array}{llll}\text { C } & -4.118859 & -3.199514 & 1.126335\end{array}$

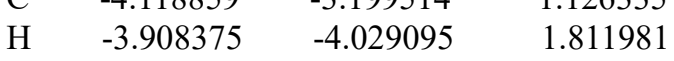

$\mathrm{H} \quad-5.190024 \quad-2.973199 \quad 1.187645$ 


$\begin{array}{lr}\mathrm{H} & -3.912182 \\ \mathrm{C} & -3.564605 \\ \mathrm{H} & -4.631371 \\ \mathrm{H} & -3.259373 \\ \mathrm{H} & -3.020429 \\ \mathrm{C} & -2.026776 \\ \mathrm{H} & -1.924322 \\ \mathrm{H} & -3.029444 \\ \mathrm{H} & -1.294642 \\ \mathrm{C} & -1.847962 \\ \mathrm{H} & -1.008343 \\ \mathrm{H} & -2.766717 \\ \mathrm{H} & -1.754912 \\ \mathrm{H} & -5.906840 \\ \mathrm{~N} & 0.495997 \\ \mathrm{O} & 0.442157 \\ \mathrm{C} & -0.067404 \\ \mathrm{H} & -1.044987 \\ \mathrm{H} & 0.358543 \\ \mathrm{H} & 0.581677\end{array}$

\section{TS3'-cross}

$\begin{array}{lrcc}\mathrm{Ru} & -0.411906 & 0.839161 & -0.038489 \\ \mathrm{O} & -1.561766 & 1.065638 & 2.005540 \\ \mathrm{O} & -1.263938 & 2.766323 & 0.688288 \\ \mathrm{~N} & -1.000346 & -1.766408 & -1.115662 \\ \mathrm{~N} & 1.152480 & -1.789611 & -0.690771 \\ \mathrm{C} & 0.014210 & -1.037198 & -0.586878 \\ \mathrm{C} & -0.631895 & -3.147923 & -1.427556 \\ \mathrm{H} & -1.001475 & -3.443214 & -2.412868 \\ \mathrm{H} & -1.049418 & -3.839335 & -0.683142 \\ \mathrm{C} & 0.903127 & -3.081142 & -1.367028 \\ \mathrm{H} & 1.346328 & -3.900978 & -0.794936 \\ \mathrm{H} & 1.355572 & -3.071648 & -2.365444 \\ \mathrm{C} & -2.374222 & -1.318618 & -0.878596 \\ \mathrm{C} & -2.906673 & -1.923442 & 0.445670 \\ \mathrm{H} & -2.225283 & -1.645339 & 1.258904 \\ \mathrm{H} & -2.920654 & -3.020608 & 0.377508 \\ \mathrm{C} & -4.325860 & -1.396438 & 0.733765 \\ \mathrm{H} & -4.698025 & -1.852053 & 1.660633 \\ \mathrm{C} & -5.260263 & -1.760529 & -0.436593 \\ \mathrm{H} & -5.326391 & -2.852187 & -0.549862 \\ \mathrm{H} & -6.275304 & -1.395608 & -0.231174 \\ \mathrm{C} & -4.724049 & -1.125136 & -1.735961 \\ \mathrm{H} & -5.394278 & -1.367941 & -2.570742 \\ \mathrm{C} & -3.322399 & -1.706155 & -2.032293 \\ \mathrm{H} & -2.926322 & -1.309610 & -2.976729 \\ \mathrm{H} & -3.391909 & -2.799379 & -2.136476 \\ \mathrm{C} & -4.647507 & 0.409726 & -1.562620 \\ \mathrm{H} & -4.300513 & 0.877023 & -2.494321 \\ \mathrm{H} & -5.652574 & 0.805163 & -1.359443 \\ \mathrm{C} & -3.687203 & 0.764925 & -0.392974 \\ \mathrm{H} & -3.633804 & 1.854333 & -0.283281 \\ \mathrm{C} & -2.298310 & 0.213468 & -0.776275 \\ \mathrm{H} & -2.092792 & 0.586354 & -1.793477 \\ \mathrm{C} & -4.261616 & 0.133584 & 0.894210 \\ \mathrm{H} & -3.651174 & 0.389130 & 1.761086 \\ \mathrm{H} & -5.270277 & 0.530766 & 1.073803 \\ \mathrm{C} & 2.315392 & -1.651102 & 0.135060 \\ \mathrm{C} & 2.226853 & -2.041011 & 1.490672\end{array}$

\begin{tabular}{|c|c|c|c|}
\hline $\mathrm{C}$ & 3.363283 & -1.878147 & 2.292558 \\
\hline $\mathrm{H}$ & 3.317367 & -2.149207 & 3.343640 \\
\hline $\mathrm{C}$ & 4.551467 & -1.380395 & 1.762275 \\
\hline $\mathrm{C}$ & 4.634459 & -1.061101 & 0.408826 \\
\hline $\mathrm{H}$ & 5.572801 & -0.698039 & 0.001534 \\
\hline $\mathrm{C}$ & 3.523728 & -1.196224 & -0.432847 \\
\hline $\mathrm{C}$ & 0.968057 & -2.671079 & 2.084518 \\
\hline $\mathrm{H}$ & 0.213087 & -2.745207 & 1.297686 \\
\hline $\mathrm{C}$ & 3.645618 & -0.921638 & -1.927523 \\
\hline $\mathrm{H}$ & 2.629228 & -0.827834 & -2.325570 \\
\hline $\mathrm{C}$ & 1.186254 & 1.234688 & 1.380219 \\
\hline $\mathrm{H}$ & 1.566512 & 0.315032 & 1.804233 \\
\hline $\mathrm{C}$ & 0.553217 & 1.461579 & -1.549482 \\
\hline $\mathrm{H}$ & 1.278829 & 0.863028 & -2.112293 \\
\hline $\mathrm{H}$ & 0.833853 & 1.955639 & 2.114373 \\
\hline $\mathrm{C}$ & 4.308954 & -2.130550 & -2.621647 \\
\hline $\mathrm{H}$ & 5.336411 & -2.263175 & -2.262149 \\
\hline $\mathrm{H}$ & 3.766006 & -3.061067 & -2.421592 \\
\hline $\mathrm{H}$ & 4.346447 & -1.980207 & -3.707080 \\
\hline $\mathrm{C}$ & 4.409397 & 0.371364 & -2.269805 \\
\hline $\mathrm{H}$ & 5.465578 & 0.299241 & -1.985310 \\
\hline $\mathrm{H}$ & 4.379890 & 0.537068 & -3.353173 \\
\hline $\mathrm{H}$ & 3.993867 & 1.259171 & -1.782153 \\
\hline $\mathrm{C}$ & 1.263795 & -4.106253 & 2.564600 \\
\hline $\mathrm{H}$ & 1.694609 & -4.715534 & 1.761242 \\
\hline $\mathrm{H}$ & 1.972501 & -4.110855 & 3.400544 \\
\hline $\mathrm{H}$ & 0.341702 & -4.590371 & 2.906758 \\
\hline $\mathrm{C}$ & 0.343523 & -1.829161 & 3.213050 \\
\hline $\mathrm{H}$ & -0.515259 & -2.358679 & 3.642283 \\
\hline $\mathrm{H}$ & 1.060955 & -1.647838 & 4.022094 \\
\hline $\mathrm{H}$ & -0.014362 & -0.864461 & 2.844245 \\
\hline $\mathrm{H}$ & 5.420719 & -1.256108 & 2.402367 \\
\hline $\mathrm{N}$ & -1.769449 & 2.305250 & 1.796211 \\
\hline $\mathrm{O}$ & -2.392574 & 3.019589 & 2.558986 \\
\hline $\mathrm{C}$ & 0.244416 & 2.764299 & -2.233672 \\
\hline $\mathrm{H}$ & 1.155652 & 3.324128 & -2.483360 \\
\hline $\mathrm{H}$ & -0.251881 & 2.508627 & -3.183390 \\
\hline $\mathrm{H}$ & -0.432016 & 3.402083 & -1.658624 \\
\hline $\mathrm{C}$ & 1.813452 & 1.720920 & 0.196624 \\
\hline $\mathrm{H}$ & 2.569706 & 1.079988 & -0.233986 \\
\hline $\mathrm{C}$ & 2.148863 & 3.171929 & -0.040167 \\
\hline $\mathrm{O}$ & 3.163243 & 3.451957 & -0.683123 \\
\hline $\mathrm{N}$ & 1.315235 & 4.105915 & 0.484666 \\
\hline $\mathrm{H}$ & 0.390573 & 3.809762 & 0.784996 \\
\hline $\mathrm{C}$ & 1.517425 & 5.515626 & 0.194380 \\
\hline $\mathrm{H}$ & 0.945594 & 6.112216 & 0.909302 \\
\hline $\mathrm{H}$ & 2.578949 & 5.754005 & 0.285751 \\
\hline $\mathrm{H}$ & 1.196744 & 5.773368 & -0.824560 \\
\hline
\end{tabular}

\section{TS3'-homo}

$\begin{array}{lccc}\mathrm{Ru} & -0.454391 & 1.085089 & -0.046200 \\ \mathrm{O} & -1.633808 & 1.189216 & 1.982766 \\ \mathrm{O} & -1.592677 & 2.865829 & 0.604207 \\ \mathrm{~N} & -0.627099 & -1.614132 & -1.047866 \\ \mathrm{~N} & 1.504367 & -1.280926 & -0.663295 \\ \mathrm{C} & 0.261195 & -0.713025 & -0.556291 \\ \mathrm{C} & -0.048033 & -2.927471 & -1.328604 \\ \mathrm{H} & -0.372782 & -3.303880 & -2.302225 \\ \mathrm{H} & -0.346852 & -3.656625 & -0.563241 \\ \mathrm{C} & 1.458146 & -2.620698 & -1.285505\end{array}$




\begin{tabular}{|c|c|c|c|}
\hline $\mathrm{H}$ & 2.024535 & -3.338914 & -0.685353 \\
\hline $\mathrm{H}$ & 1.902270 & -2.583774 & -2.287604 \\
\hline $\mathrm{C}$ & -2.053544 & -1.381843 & -0.817335 \\
\hline $\mathrm{C}$ & -2.484045 & -2.019568 & 0.528429 \\
\hline $\mathrm{H}$ & -1.855523 & -1.609170 & 1.328129 \\
\hline $\mathrm{H}$ & -2.323956 & -3.107003 & 0.495851 \\
\hline $\mathrm{C}$ & -3.968929 & -1.714411 & 0.806206 \\
\hline $\mathrm{H}$ & -4.264808 & -2.193109 & 1.748945 \\
\hline $\mathrm{C}$ & -4.833447 & -2.259763 & -0.347345 \\
\hline $\mathrm{H}$ & -4.726400 & -3.351438 & -0.425304 \\
\hline $\mathrm{H}$ & -5.893541 & -2.053067 & -0.149034 \\
\hline $\mathrm{C}$ & -4.403836 & -1.590330 & -1.669094 \\
\hline $\mathrm{H}$ & -5.027271 & -1.963029 & -2.492405 \\
\hline $\mathrm{C}$ & -2.928130 & -1.952334 & -1.953121 \\
\hline $\mathrm{H}$ & -2.599489 & -1.529065 & -2.911831 \\
\hline $\mathrm{H}$ & -2.824727 & -3.045894 & -2.021816 \\
\hline $\mathrm{C}$ & -4.569317 & -0.057630 & -1.545885 \\
\hline $\mathrm{H}$ & -4.298585 & 0.427001 & -2.494023 \\
\hline $\mathrm{H}$ & -5.624515 & 0.181492 & -1.352352 \\
\hline $\mathrm{C}$ & -3.678165 & 0.482053 & -0.392400 \\
\hline $\mathrm{H}$ & -3.797528 & 1.569014 & -0.317405 \\
\hline $\mathrm{C}$ & -2.218163 & 0.144895 & -0.760952 \\
\hline $\mathrm{H}$ & -2.070112 & 0.516434 & -1.789583 \\
\hline $\mathrm{C}$ & -4.148007 & -0.189092 & 0.916808 \\
\hline $\mathrm{H}$ & -3.586260 & 0.189461 & 1.771259 \\
\hline $\mathrm{H}$ & -5.207370 & 0.048692 & 1.086801 \\
\hline $\mathrm{C}$ & 2.649540 & -0.918271 & 0.113026 \\
\hline $\mathrm{C}$ & 2.676285 & -1.270185 & 1.481550 \\
\hline $\mathrm{C}$ & 3.794122 & -0.887648 & 2.232920 \\
\hline $\mathrm{H}$ & 3.830665 & -1.124955 & 3.292466 \\
\hline $\mathrm{C}$ & 4.860459 & -0.212384 & 1.642700 \\
\hline $\mathrm{C}$ & 4.842261 & 0.066353 & 0.277785 \\
\hline $\mathrm{H}$ & 5.695708 & 0.559093 & -0.178659 \\
\hline $\mathrm{C}$ & 3.743023 & -0.287684 & -0.514273 \\
\hline $\mathrm{C}$ & 1.561490 & -2.080528 & 2.140638 \\
\hline $\mathrm{H}$ & 0.808285 & -2.310934 & 1.382775 \\
\hline $\mathrm{C}$ & 3.774214 & -0.077777 & -2.023698 \\
\hline $\mathrm{H}$ & 2.753855 & -0.219646 & -2.394999 \\
\hline $\mathrm{C}$ & 1.071154 & 1.738113 & 1.345121 \\
\hline $\mathrm{H}$ & 1.655432 & 0.908492 & 1.716726 \\
\hline $\mathrm{C}$ & 0.407955 & 1.831857 & -1.555401 \\
\hline $\mathrm{H}$ & 1.222742 & 1.348982 & -2.108928 \\
\hline $\mathrm{C}$ & 1.544898 & 2.437785 & 0.195463 \\
\hline $\mathrm{H}$ & 2.429579 & 2.009113 & -0.265424 \\
\hline $\mathrm{H}$ & 0.578101 & 2.326897 & 2.116858 \\
\hline $\mathrm{C}$ & 4.667078 & -1.152750 & -2.678573 \\
\hline $\mathrm{H}$ & 4.648755 & -1.059774 & -3.770993 \\
\hline $\mathrm{H}$ & 5.706686 & -1.047233 & -2.345955 \\
\hline $\mathrm{H}$ & 4.336169 & -2.163071 & -2.414003 \\
\hline $\mathrm{C}$ & 4.234070 & 1.327114 & -2.449266 \\
\hline $\mathrm{H}$ & 4.187676 & 1.420873 & -3.540340 \\
\hline $\mathrm{H}$ & 3.602266 & 2.110074 & -2.018521 \\
\hline $\mathrm{H}$ & 5.268682 & 1.525915 & -2.148000 \\
\hline $\mathrm{C}$ & 2.112545 & -3.424706 & 2.657662 \\
\hline $\mathrm{H}$ & 2.619152 & -3.982560 & 1.861116 \\
\hline $\mathrm{H}$ & 2.834021 & -3.276465 & 3.469348 \\
\hline $\mathrm{H}$ & 1.296843 & -4.045107 & 3.046621 \\
\hline $\mathrm{C}$ & 0.834850 & -1.313700 & 3.261919 \\
\hline $\mathrm{H}$ & 0.097960 & -1.967858 & 3.742641 \\
\hline $\mathrm{H}$ & 1.534927 & -0.975922 & 4.03536 \\
\hline
\end{tabular}

$\begin{array}{lrrr}\mathrm{H} & 0.300471 & -0.443265 & 2.872951 \\ \mathrm{H} & 5.717314 & 0.079019 & 2.244222 \\ \mathrm{~N} & -2.037721 & 2.373774 & 1.715234 \\ \mathrm{O} & -2.789766 & 2.998014 & 2.442587 \\ \mathrm{C} & -0.157381 & 3.022252 & -2.282193 \\ \mathrm{H} & -0.836358 & 3.615726 & -1.664207 \\ \mathrm{H} & 0.614600 & 3.676981 & -2.709731 \\ \mathrm{H} & -0.740780 & 2.624047 & -3.128441 \\ \mathrm{C} & 1.445025 & 3.948962 & 0.152408 \\ \mathrm{H} & 1.685694 & 4.361215 & -0.831130 \\ \mathrm{H} & 0.444191 & 4.281989 & 0.440233 \\ \mathrm{H} & 2.162804 & 4.366325 & 0.870792\end{array}$

\section{INT4'-cross}

\begin{tabular}{|c|c|c|c|}
\hline $\mathrm{Ru}$ & -0.435307 & 0.833422 & -0.105771 \\
\hline $\mathrm{C}$ & 0.894381 & 1.416019 & -1.461177 \\
\hline $\mathrm{C}$ & 0.561099 & 2.633104 & -2.312534 \\
\hline $\mathrm{H}$ & -0.039000 & 3.375717 & -1.780404 \\
\hline $\mathrm{H}$ & -0.005812 & 2.306608 & -3.193116 \\
\hline $\mathrm{H}$ & 1.477827 & 3.124639 & -2.668068 \\
\hline $\mathrm{H}$ & 1.397643 & 0.657399 & -2.063537 \\
\hline $\mathrm{C}$ & 1.782594 & 1.646174 & -0.114876 \\
\hline $\mathrm{C}$ & 2.346919 & 3.069840 & -0.102962 \\
\hline $\mathrm{O}$ & 3.481775 & 3.260899 & -0.538773 \\
\hline $\mathrm{N}$ & 1.548305 & 4.054675 & 0.374621 \\
\hline $\mathrm{H}$ & 0.568545 & 3.841689 & 0.543970 \\
\hline $\mathrm{C}$ & 1.948134 & 5.448648 & 0.282790 \\
\hline $\mathrm{H}$ & 1.780613 & 5.856232 & -0.723638 \\
\hline $\mathrm{H}$ & 3.013065 & 5.530851 & 0.509570 \\
\hline $\mathrm{H}$ & 1.373342 & 6.034697 & 1.004345 \\
\hline $\mathrm{H}$ & 2.634418 & 0.985327 & -0.247421 \\
\hline $\mathrm{C}$ & 1.079510 & 1.178662 & 1.193417 \\
\hline $\mathrm{H}$ & 1.603357 & 0.360100 & 1.675198 \\
\hline $\mathrm{H}$ & 0.851812 & 1.976448 & 1.901296 \\
\hline $\mathrm{C}$ & -0.044470 & -1.075614 & -0.577818 \\
\hline $\mathrm{N}$ & -1.079329 & -1.787377 & -1.098893 \\
\hline $\mathrm{C}$ & -2.434838 & -1.304374 & -0.845093 \\
\hline $\mathrm{C}$ & -2.312352 & 0.212122 & -0.663611 \\
\hline $\mathrm{C}$ & -3.671115 & 0.813311 & -0.289176 \\
\hline $\mathrm{C}$ & -4.259235 & 0.159097 & 0.978789 \\
\hline $\mathrm{C}$ & -4.391996 & -1.358765 & 0.761247 \\
\hline $\mathrm{C}$ & -2.997754 & -1.937364 & 0.454202 \\
\hline $\mathrm{H}$ & -3.060461 & -3.028229 & 0.340202 \\
\hline $\mathrm{H}$ & -2.304648 & -1.724202 & 1.277431 \\
\hline $\mathrm{C}$ & -5.338699 & -1.640899 & -0.422336 \\
\hline $\mathrm{C}$ & -4.774890 & -0.985534 & -1.700091 \\
\hline $\mathrm{C}$ & -4.634674 & 0.536545 & -1.477523 \\
\hline $\mathrm{H}$ & -5.619690 & 0.972888 & -1.263267 \\
\hline $\mathrm{H}$ & -4.260046 & 1.019564 & -2.390217 \\
\hline $\mathrm{C}$ & -3.394595 & -1.601899 & -2.017354 \\
\hline $\mathrm{H}$ & -3.494572 & -2.686837 & -2.167100 \\
\hline $\mathrm{H}$ & -2.980112 & -1.178467 & -2.941784 \\
\hline $\mathrm{H}$ & -5.452029 & -1.173440 & -2.543021 \\
\hline $\mathrm{H}$ & -6.337363 & -1.239785 & -0.205382 \\
\hline $\mathrm{H}$ & -5.451524 & -2.723990 & -0.572403 \\
\hline $\mathrm{H}$ & -4.784423 & -1.831499 & 1.670739 \\
\hline $\mathrm{H}$ & -3.619074 & 0.366969 & 1.838612 \\
\hline $\mathrm{H}$ & -5.245220 & 0.595690 & 1.187914 \\
\hline $\mathrm{H}$ & -3.572257 & 1.894346 & -0.148024 \\
\hline $\mathrm{H}$ & -1.986470 & 0.660479 & -1.637316 \\
\hline
\end{tabular}




$\begin{array}{lrrc}\mathrm{C} & -0.749183 & -3.185272 & -1.380960 \\ \mathrm{C} & 0.786545 & -3.163987 & -1.297026 \\ \mathrm{~N} & 1.067098 & -1.866201 & -0.645528 \\ \mathrm{C} & 2.222213 & -1.746085 & 0.196896 \\ \mathrm{C} & 3.460128 & -1.367956 & -0.362961 \\ \mathrm{C} & 4.557796 & -1.242691 & 0.497322 \\ \mathrm{C} & 4.433656 & -1.499384 & 1.860710 \\ \mathrm{C} & 3.215060 & -1.925049 & 2.384328 \\ \mathrm{C} & 2.089505 & -2.074129 & 1.565100 \\ \mathrm{C} & 0.791037 & -2.613582 & 2.161516 \\ \mathrm{C} & 0.178623 & -1.673241 & 3.216549 \\ \mathrm{H} & 0.880072 & -1.484252 & 4.037689 \\ \mathrm{H} & -0.721882 & -2.128565 & 3.646069 \\ \mathrm{H} & -0.107316 & -0.712966 & 2.779801 \\ \mathrm{H} & 0.054011 & -2.706664 & 1.359660 \\ \mathrm{C} & 1.008224 & -4.026562 & 2.738886 \\ \mathrm{H} & 0.056201 & -4.446159 & 3.084533 \\ \mathrm{H} & 1.430645 & -4.704544 & 1.987807 \\ \mathrm{H} & 1.693752 & -4.009871 & 3.593671 \\ \mathrm{H} & 3.135353 & -2.146421 & 3.444896 \\ \mathrm{H} & 5.293665 & -1.382246 & 2.514432 \\ \mathrm{H} & 5.518238 & -0.935188 & 0.096208 \\ \mathrm{C} & 3.629136 & -1.164202 & -1.864004 \\ \mathrm{C} & 4.462693 & 0.075919 & -2.238197 \\ \mathrm{H} & 5.512550 & -0.046343 & -1.947313 \\ \mathrm{H} & 4.445143 & 0.214253 & -3.325537 \\ \mathrm{H} & 4.095726 & 0.995813 & -1.772244 \\ \mathrm{C} & 4.250335 & -2.430950 & -2.490759 \\ \mathrm{H} & 5.260319 & -2.597784 & -2.097666 \\ \mathrm{H} & 4.323483 & -2.326921 & -3.579820 \\ \mathrm{H} & 3.657844 & -3.325869 & -2.269533 \\ \mathrm{H} & 2.628863 & -1.039069 & -2.292292 \\ \mathrm{H} & 1.254039 & -3.191360 & -2.287886 \\ \mathrm{H} & 1.194612 & -3.983910 & -0.699596 \\ \mathrm{H} & -1.112570 & -3.487653 & -2.366636 \\ \mathrm{H} & -1.196603 & -3.850576 & -0.630689 \\ \mathrm{O} & -1.680092 & 1.497341 & 2.007112 \\ \mathrm{O} & -1.679546 & 2.683020 & 1.579045 \\ \mathrm{O} & -2.156596 & 3.629620 & 2.182845 \\ & -1.118708 & 2.866180 & 0.407480\end{array}$

\begin{tabular}{|c|c|c|c|}
\hline $\mathrm{C}$ & -4.816290 & -2.321844 & -0.406220 \\
\hline $\mathrm{H}$ & -4.714739 & -3.405928 & -0.558135 \\
\hline $\mathrm{H}$ & -5.873996 & -2.124572 & -0.187757 \\
\hline $\mathrm{C}$ & -4.393442 & -1.567047 & -1.683621 \\
\hline $\mathrm{H}$ & -5.022089 & -1.882675 & -2.526120 \\
\hline $\mathrm{C}$ & -2.919912 & -1.901033 & -2.002914 \\
\hline $\mathrm{H}$ & -2.597399 & -1.403741 & -2.927270 \\
\hline $\mathrm{H}$ & -2.805505 & -2.984517 & -2.153617 \\
\hline $\mathrm{C}$ & -4.551390 & -0.047153 & -1.459340 \\
\hline $\mathrm{H}$ & -4.277974 & 0.500140 & -2.371753 \\
\hline $\mathrm{H}$ & -5.602289 & 0.189682 & -1.244557 \\
\hline $\mathrm{C}$ & -3.660386 & 0.410890 & -0.270294 \\
\hline $\mathrm{H}$ & -3.775796 & 1.489708 & -0.128076 \\
\hline $\mathrm{C}$ & -2.210119 & 0.087765 & -0.646023 \\
\hline $\mathrm{H}$ & -1.974953 & 0.597989 & -1.616067 \\
\hline $\mathrm{C}$ & -4.110187 & -0.346902 & 0.996496 \\
\hline $\mathrm{H}$ & -3.523513 & -0.016541 & 1.855842 \\
\hline $\mathrm{H}$ & -5.162992 & -0.112384 & 1.204428 \\
\hline $\mathrm{C}$ & 2.630681 & -0.934408 & 0.167857 \\
\hline $\mathrm{C}$ & 2.600606 & -1.265005 & 1.541378 \\
\hline $\mathrm{C}$ & 3.695020 & -0.886623 & 2.328161 \\
\hline $\mathrm{H}$ & 3.687808 & -1.106572 & 3.391988 \\
\hline $\mathrm{C}$ & 4.793323 & -0.237938 & 1.767845 \\
\hline $\mathrm{C}$ & 4.831470 & 0.016120 & 0.398623 \\
\hline $\mathrm{H}$ & 5.708918 & 0.487898 & -0.033506 \\
\hline $\mathrm{C}$ & 3.757266 & -0.333572 & -0.428897 \\
\hline $\mathrm{C}$ & 1.452794 & -2.051020 & 2.171780 \\
\hline $\mathrm{H}$ & 0.722198 & -2.280231 & 1.391621 \\
\hline $\mathrm{C}$ & 3.851297 & -0.145666 & -1.938467 \\
\hline $\mathrm{H}$ & 2.844770 & -0.275065 & -2.349605 \\
\hline $\mathrm{C}$ & 0.921140 & 1.679086 & 1.224361 \\
\hline $\mathrm{H}$ & 1.615793 & 0.977300 & 1.672495 \\
\hline $\mathrm{C}$ & 0.716576 & 1.933821 & -1.403904 \\
\hline $\mathrm{H}$ & 1.375700 & 1.292986 & -1.994416 \\
\hline $\mathrm{C}$ & 1.486072 & 2.386801 & -0.052394 \\
\hline $\mathrm{H}$ & 2.491488 & 1.978571 & -0.176467 \\
\hline $\mathrm{H}$ & 0.519559 & 2.375729 & 1.963130 \\
\hline $\mathrm{C}$ & 4.752857 & -1.242735 & -2.542891 \\
\hline $\mathrm{H}$ & 4.780305 & -1.163313 & -3.636182 \\
\hline $\mathrm{H}$ & 5.779669 & -1.148223 & -2.169813 \\
\hline $\mathrm{H}$ & 4.396905 & -2.245038 & -2.279737 \\
\hline $\mathrm{C}$ & 4.349067 & 1.247002 & -2.361549 \\
\hline $\mathrm{H}$ & 5.377821 & 1.428909 & -2.030643 \\
\hline $\mathrm{H}$ & 4.337900 & 1.331078 & -3.454300 \\
\hline $\mathrm{H}$ & 3.717803 & 2.042821 & -1.955715 \\
\hline $\mathrm{C}$ & 1.966061 & -3.397527 & 2.720823 \\
\hline $\mathrm{H}$ & 2.487426 & -3.972834 & 1.946469 \\
\hline $\mathrm{H}$ & 2.665085 & -3.250949 & 3.552138 \\
\hline $\mathrm{H}$ & 1.129114 & -4.000369 & 3.092163 \\
\hline $\mathrm{C}$ & 0.702134 & -1.260640 & 3.259865 \\
\hline $\mathrm{H}$ & -0.069869 & -1.893331 & 3.714008 \\
\hline $\mathrm{H}$ & 1.379548 & -0.937544 & 4.059187 \\
\hline $\mathrm{H}$ & 0.210014 & -0.377316 & 2.845366 \\
\hline $\mathrm{H}$ & 5.631196 & 0.051263 & 2.396532 \\
\hline $\mathrm{N}$ & -2.091459 & 2.619821 & 1.559136 \\
\hline $\mathrm{O}$ & -2.784507 & 3.431387 & 2.151708 \\
\hline $\mathrm{C}$ & 0.109930 & 3.024560 & -2.275403 \\
\hline $\mathrm{H}$ & 0.885190 & 3.667478 & -2.717978 \\
\hline $\mathrm{H}$ & -0.436895 & 2.560608 & -3.106364 \\
\hline$H$ & -0.588300 & 3.662761 & -1.727357 \\
\hline
\end{tabular}

\begin{tabular}{|c|c|c|c|}
\hline \multicolumn{4}{|c|}{ INT4'-homo } \\
\hline $\mathrm{Ru}$ & -0.493817 & 1.066022 & -0.080730 \\
\hline $\mathrm{O}$ & -1.841188 & 1.457881 & 2.000392 \\
\hline $\mathrm{O}$ & -1.562600 & 2.914120 & 0.406337 \\
\hline $\mathrm{N}$ & -0.613249 & -1.629578 & -1.087901 \\
\hline $\mathrm{N}$ & 1.506423 & -1.291278 & -0.643803 \\
\hline $\mathrm{C}$ & 0.263001 & -0.727165 & -0.567170 \\
\hline $\mathrm{C}$ & -0.020629 & -2.936160 & -1.373987 \\
\hline $\mathrm{H}$ & -0.316293 & -3.298682 & -2.362266 \\
\hline $\mathrm{H}$ & -0.334343 & -3.679109 & -0.628753 \\
\hline $\mathrm{C}$ & 1.482422 & -2.623439 & -1.283371 \\
\hline $\mathrm{H}$ & 2.033880 & -3.346367 & -0.675213 \\
\hline $\mathrm{H}$ & 1.954903 & -2.573169 & -2.271433 \\
\hline $\mathrm{C}$ & -2.035471 & -1.422051 & -0.831560 \\
\hline $\mathrm{C}$ & -2.461688 & -2.153059 & 0.468491 \\
\hline $\mathrm{H}$ & -1.822889 & -1.805181 & 1.289839 \\
\hline $\mathrm{H}$ & -2.307531 & -3.234977 & 0.355041 \\
\hline $\mathrm{C}$ & -3.942250 & -1.860787 & 0.777174 \\
\hline $\mathrm{H}$ & -4.233353 & -2.402939 & 1.686121 \\
\hline
\end{tabular}




$\begin{array}{lllr}\mathrm{C} & 1.537656 & 3.907918 & 0.135875 \\ \mathrm{H} & 1.967199 & 4.406541 & -0.740074 \\ \mathrm{H} & 0.536664 & 4.311048 & 0.311153 \\ \mathrm{H} & 2.160513 & 4.151092 & 1.003919\end{array}$

\section{INT4'-cross-2}

\begin{tabular}{|c|c|c|c|}
\hline $\mathrm{Ru}$ & 0.614649 & 0.630755 & 0.073479 \\
\hline $\mathrm{O}$ & 1.612457 & 2.545641 & 0.310344 \\
\hline $\mathrm{O}$ & 1.662879 & 1.595175 & 2.271257 \\
\hline $\mathrm{N}$ & 0.901681 & -2.159049 & -0.523826 \\
\hline $\mathrm{N}$ & -1.268551 & -1.850484 & -0.492790 \\
\hline $\mathrm{C}$ & -0.062427 & -1.238781 & -0.273732 \\
\hline $\mathrm{C}$ & 0.395836 & -3.373492 & -1.160772 \\
\hline $\mathrm{H}$ & 0.875675 & -4.270059 & -0.759932 \\
\hline $\mathrm{H}$ & 0.570432 & -3.341357 & -2.245203 \\
\hline $\mathrm{C}$ & -1.096934 & -3.285093 & -0.818843 \\
\hline $\mathrm{H}$ & -1.748224 & -3.573943 & -1.645838 \\
\hline $\mathrm{H}$ & -1.354784 & -3.896831 & 0.055544 \\
\hline $\mathrm{C}$ & 2.286501 & -1.694590 & -0.570295 \\
\hline $\mathrm{C}$ & 2.669734 & -1.239963 & -2.003098 \\
\hline $\mathrm{H}$ & 1.936370 & -0.505993 & -2.358356 \\
\hline $\mathrm{H}$ & 2.630722 & -2.101367 & -2.684861 \\
\hline $\mathrm{C}$ & 4.083880 & -0.631044 & -1.996271 \\
\hline $\mathrm{H}$ & 4.344259 & -0.308927 & -3.012787 \\
\hline $\mathrm{C}$ & 5.086600 & -1.700601 & -1.516843 \\
\hline $\mathrm{H}$ & 5.087260 & -2.554676 & -2.208743 \\
\hline $\mathrm{H}$ & 6.104098 & -1.288073 & -1.513275 \\
\hline $\mathrm{C}$ & 4.706434 & -2.165894 & -0.095512 \\
\hline $\mathrm{H}$ & 5.417661 & -2.928710 & 0.246226 \\
\hline $\mathrm{C}$ & 3.286796 & -2.778105 & -0.114400 \\
\hline $\mathrm{H}$ & 3.006153 & -3.137876 & 0.884124 \\
\hline $\mathrm{H}$ & 3.262386 & -3.640011 & -0.797452 \\
\hline $\mathrm{C}$ & 4.744243 & -0.952890 & 0.856872 \\
\hline $\mathrm{H}$ & 4.504911 & -1.264627 & 1.882140 \\
\hline $\mathrm{H}$ & 5.757986 & -0.529590 & 0.876839 \\
\hline $\mathrm{C}$ & 3.732732 & 0.124750 & 0.384901 \\
\hline $\mathrm{H}$ & 3.779940 & 0.971863 & 1.072267 \\
\hline $\mathrm{C}$ & 2.333222 & -0.509431 & 0.418489 \\
\hline $\mathrm{H}$ & 2.131144 & -0.892006 & 1.430351 \\
\hline $\mathrm{C}$ & 4.105564 & 0.579320 & -1.040631 \\
\hline $\mathrm{H}$ & 3.402707 & 1.350012 & -1.379809 \\
\hline $\mathrm{H}$ & 5.105582 & 1.033229 & -1.036504 \\
\hline $\mathrm{C}$ & -2.507418 & -1.459001 & 0.124140 \\
\hline $\mathrm{C}$ & -3.555110 & -0.964265 & -0.682209 \\
\hline $\mathrm{C}$ & -4.742336 & -0.576646 & -0.049084 \\
\hline $\mathrm{H}$ & -5.557242 & -0.175531 & -0.642795 \\
\hline $\mathrm{C}$ & -4.891376 & -0.688793 & 1.330839 \\
\hline $\mathrm{C}$ & -3.859988 & -1.215521 & 2.103988 \\
\hline $\mathrm{H}$ & -3.990251 & -1.309866 & 3.178278 \\
\hline $\mathrm{C}$ & -2.653357 & -1.620403 & 1.521047 \\
\hline $\mathrm{C}$ & -3.437936 & -0.891285 & -2.200945 \\
\hline $\mathrm{H}$ & -2.371673 & -0.929125 & -2.448647 \\
\hline $\mathrm{C}$ & -1.559412 & -2.214522 & 2.406914 \\
\hline $\mathrm{H}$ & -0.707430 & -2.490162 & 1.780688 \\
\hline $\mathrm{C}$ & -0.180851 & 1.226688 & -1.632221 \\
\hline $\mathrm{H}$ & -0.600511 & 0.534399 & -2.364562 \\
\hline $\mathrm{C}$ & -1.090308 & 1.386765 & 0.895824 \\
\hline $\mathrm{H}$ & -1.801931 & 0.617235 & 1.175581 \\
\hline $\mathrm{H}$ & 0.366769 & 2.042128 & -2.113890 \\
\hline $\mathrm{N}$ & 1.947293 & 2.604193 & 1.578252 \\
\hline
\end{tabular}

$\begin{array}{lrcc}\mathrm{O} & 2.496282 & 3.605449 & 2.010856 \\ \mathrm{C} & -4.025448 & 0.396388 & -2.811917 \\ \mathrm{H} & -3.780562 & 0.439351 & -3.879449 \\ \mathrm{H} & -5.118774 & 0.412287 & -2.733104 \\ \mathrm{H} & -3.647975 & 1.308506 & -2.338987 \\ \mathrm{C} & -4.115472 & -2.123068 & -2.839468 \\ \mathrm{H} & -3.984035 & -2.115273 & -3.927925 \\ \mathrm{H} & -3.708603 & -3.063079 & -2.451474 \\ \mathrm{H} & -5.191487 & -2.119272 & -2.628032 \\ \mathrm{C} & -2.055693 & -3.501921 & 3.094559 \\ \mathrm{H} & -2.874605 & -3.293548 & 3.792487 \\ \mathrm{H} & -2.422277 & -4.230853 & 2.362186 \\ \mathrm{H} & -1.242678 & -3.966351 & 3.664583 \\ \mathrm{C} & -1.028813 & -1.204835 & 3.442825 \\ \mathrm{H} & -0.554509 & -0.348453 & 2.956766 \\ \mathrm{H} & -1.831905 & -0.833743 & 4.090068 \\ \mathrm{H} & -0.277758 & -1.682977 & 4.082558 \\ \mathrm{H} & -5.816954 & -0.371723 & 1.803552 \\ \mathrm{C} & -1.114720 & 2.489189 & 1.936667 \\ \mathrm{H} & -2.133624 & 2.899817 & 1.991030 \\ \mathrm{H} & -0.866087 & 2.072608 & 2.916392 \\ \mathrm{H} & -0.438181 & 3.319766 & 1.737732 \\ \mathrm{C} & -1.312286 & 1.756568 & -0.627029 \\ \mathrm{H} & -2.209838 & 1.207501 & -0.895992 \\ \mathrm{C} & -1.625057 & 3.238901 & -0.865454 \\ \mathrm{O} & -2.773772 & 3.559274 & -1.165848 \\ \mathrm{~N} & -0.595792 & 4.113285 & -0.755965 \\ \mathrm{H} & 0.298131 & 3.775735 & -0.405531 \\ \mathrm{C} & -0.800519 & 5.545723 & -0.887098 \\ \mathrm{H} & -1.068744 & 6.007978 & 0.072330 \\ \mathrm{H} & 0.116478 & 6.011192 & -1.258592 \\ \mathrm{H} & -1.614237 & 5.722345 & -1.593097\end{array}$

\section{INT4'-homo-2}

$\begin{array}{lccc}\mathrm{Ru} & 0.623999 & 0.849877 & -0.280458 \\ \mathrm{O} & 1.832465 & 2.602735 & -0.696818 \\ \mathrm{O} & 1.704182 & 2.321928 & 1.456984 \\ \mathrm{~N} & 0.609668 & -1.984832 & 0.217314 \\ \mathrm{~N} & -1.508107 & -1.476613 & 0.018349 \\ \mathrm{C} & -0.247002 & -0.941055 & 0.058284 \\ \mathrm{C} & -0.022780 & -3.290187 & 0.039351 \\ \mathrm{H} & 0.356236 & -4.024438 & 0.754861 \\ \mathrm{H} & 0.154558 & -3.671101 & -0.976489 \\ \mathrm{C} & -1.496180 & -2.934406 & 0.264848 \\ \mathrm{H} & -2.177913 & -3.448060 & -0.417021 \\ \mathrm{H} & -1.816216 & -3.140102 & 1.295352 \\ \mathrm{C} & 2.034167 & -1.715682 & 0.040120 \\ \mathrm{C} & 2.448767 & -1.861193 & -1.447802 \\ \mathrm{H} & 1.792423 & -1.240136 & -2.069074 \\ \mathrm{H} & 2.315531 & -2.904695 & -1.767160 \\ \mathrm{C} & 3.918556 & -1.435897 & -1.622023 \\ \mathrm{H} & 4.201993 & -1.536956 & -2.677771 \\ \mathrm{C} & 4.809785 & -2.348901 & -0.755160 \\ \mathrm{H} & 4.716176 & -3.392963 & -1.086230 \\ \mathrm{H} & 5.864450 & -2.066798 & -0.872446 \\ \mathrm{C} & 4.396185 & -2.220074 & 0.725900 \\ \mathrm{H} & 5.027823 & -2.871029 & 1.344043 \\ \mathrm{C} & 2.920660 & -2.650793 & 0.890000 \\ \mathrm{H} & 2.613376 & -2.588880 & 1.942136 \\ \mathrm{H} & 2.799917 & -3.696215 & 0.569231\end{array}$




\begin{tabular}{|c|c|c|c|c|c|c|c|}
\hline $\mathrm{C}$ & 4.566669 & -0.752546 & 1.169186 & $\mathrm{O}$ & 1.284392 & 1.218548 & 2.375402 \\
\hline $\mathrm{H}$ & 4.305084 & -0.640907 & 2.229675 & $\mathrm{~N}$ & 0.913935 & -2.134840 & -0.506977 \\
\hline $\mathrm{H}$ & 5.618755 & -0.453694 & 1.063161 & $\mathrm{~N}$ & -1.256500 & -1.831326 & -0.471660 \\
\hline $\mathrm{C}$ & 3.668174 & 0.172020 & 0.305643 & $\mathrm{C}$ & -0.052907 & -1.218293 & -0.242934 \\
\hline $\mathrm{H}$ & 3.813919 & 1.202112 & 0.636474 & $\mathrm{C}$ & 0.403335 & -3.350936 & -1.137264 \\
\hline $\mathrm{C}$ & 2.210486 & -0.263966 & 0.527933 & $\mathrm{H}$ & 0.890658 & -4.245735 & -0.741348 \\
\hline $\mathrm{H}$ & 1.980842 & -0.229722 & 1.601924 & $\mathrm{H}$ & 0.566104 & -3.319065 & -2.223613 \\
\hline $\mathrm{C}$ & 4.074272 & 0.032061 & -1.175226 & $\mathrm{C}$ & -1.085114 & -3.267968 & -0.780986 \\
\hline $\mathrm{H}$ & 3.453518 & 0.691522 & -1.793657 & $\mathrm{H}$ & -1.744211 & -3.570449 & -1.596700 \\
\hline $\mathrm{H}$ & 5.116312 & 0.353099 & -1.306731 & $\mathrm{H}$ & -1.330107 & -3.869163 & 0.104771 \\
\hline $\mathrm{C}$ & -2.718268 & -0.764891 & 0.305998 & $\mathrm{C}$ & 2.301395 & -1.675343 & -0.581312 \\
\hline $\mathrm{C}$ & -3.659632 & -0.575729 & -0.727282 & $\mathrm{C}$ & 2.681875 & -1.292756 & -2.032449 \\
\hline $\mathrm{C}$ & -4.821459 & 0.148226 & -0.432592 & $\mathrm{H}$ & 1.943253 & -0.588293 & -2.428356 \\
\hline $\mathrm{H}$ & -5.559592 & 0.315837 & -1.211402 & $\mathrm{H}$ & 2.660050 & -2.187828 & -2.670331 \\
\hline $\mathrm{C}$ & -5.048745 & 0.649379 & 0.847231 & $\mathrm{C}$ & 4.087307 & -0.664725 & -2.050586 \\
\hline $\mathrm{C}$ & -4.132747 & 0.402264 & 1.867317 & $\mathrm{H}$ & 4.343957 & -0.380573 & -3.079602 \\
\hline $\mathrm{H}$ & -4.331436 & 0.776053 & 2.867745 & $\mathrm{C}$ & 5.102659 & -1.702431 & -1.530100 \\
\hline $\mathrm{C}$ & -2.958176 & -0.320210 & 1.625325 & $\mathrm{H}$ & 5.111511 & -2.583146 & -2.187827 \\
\hline $\mathrm{C}$ & -3.474106 & -1.208162 & -2.102413 & $\mathrm{H}$ & 6.116150 & -1.279880 & -1.543615 \\
\hline $\mathrm{H}$ & -2.438200 & -1.556602 & -2.166759 & $\mathrm{C}$ & 4.726382 & -2.112806 & -0.091117 \\
\hline $\mathrm{C}$ & -2.004944 & -0.618604 & 2.781080 & $\mathrm{H}$ & 5.442619 & -2.857395 & 0.279606 \\
\hline $\mathrm{H}$ & -1.164976 & -1.204857 & 2.399866 & $\mathrm{C}$ & 3.309071 & -2.736287 & -0.084263 \\
\hline $\mathrm{C}$ & -0.122543 & 0.843541 & -2.109762 & $\mathrm{H}$ & 3.032652 & -3.056358 & 0.928787 \\
\hline $\mathrm{H}$ & -0.655592 & -0.008688 & -2.538087 & $\mathrm{H}$ & 3.294157 & -3.627305 & -0.729502 \\
\hline $\mathrm{C}$ & -0.994966 & 2.037027 & 0.114332 & $\mathrm{C}$ & 4.766416 & -0.858284 & 0.808176 \\
\hline $\mathrm{H}$ & -1.799512 & 1.498599 & 0.602904 & $\mathrm{H}$ & 4.539579 & -1.126508 & 1.848441 \\
\hline $\mathrm{C}$ & -1.128057 & 1.914202 & -1.452460 & $\mathrm{H}$ & 5.779531 & -0.432346 & 0.798456 \\
\hline $\mathrm{H}$ & -2.115776 & 1.466468 & -1.582810 & $\mathrm{C}$ & 3.738866 & 0.186150 & 0.300355 \\
\hline $\mathrm{H}$ & 0.533913 & 1.320392 & -2.846400 & $\mathrm{H}$ & 3.779248 & 1.070344 & 0.944193 \\
\hline $\mathrm{N}$ & 2.173954 & 2.999143 & 0.495054 & $\mathrm{C}$ & 2.348057 & -0.463510 & 0.369573 \\
\hline $\mathrm{O}$ & 2.903852 & 3.966842 & 0.642728 & $\mathrm{H}$ & 2.206482 & -0.855887 & 1.388349 \\
\hline $\mathrm{C}$ & -3.711180 & -0.237627 & -3.272615 & $\mathrm{C}$ & 4.093560 & 0.585279 & -1.145086 \\
\hline $\mathrm{H}$ & -3.531922 & -0.750683 & -4.224461 & $\mathrm{H}$ & 3.369291 & 1.322910 & -1.512318 \\
\hline $\mathrm{H}$ & -4.742032 & 0.133770 & -3.290541 & $\mathrm{H}$ & 5.084456 & 1.058460 & -1.171389 \\
\hline $\mathrm{H}$ & -3.041844 & 0.626109 & -3.226289 & $\mathrm{C}$ & -2.512076 & -1.413733 & 0.085346 \\
\hline $\mathrm{C}$ & -4.399977 & -2.435771 & -2.238850 & $\mathrm{C}$ & -3.518181 & -0.926741 & -0.777760 \\
\hline $\mathrm{H}$ & -4.223424 & -2.950420 & -3.190745 & $\mathrm{C}$ & -4.730314 & -0.520182 & -0.207320 \\
\hline $\mathrm{H}$ & -4.242999 & -3.153227 & -1.425769 & $\mathrm{H}$ & -5.515734 & -0.128169 & -0.845460 \\
\hline $\mathrm{H}$ & -5.452974 & -2.131125 & -2.208770 & $\mathrm{C}$ & -4.943676 & -0.607338 & 1.166336 \\
\hline $\mathrm{C}$ & -2.712795 & -1.473621 & 3.851359 & $\mathrm{C}$ & -3.952433 & -1.125089 & 1.995963 \\
\hline $\mathrm{H}$ & -3.533505 & -0.922988 & 4.325346 & $\mathrm{H}$ & -4.133539 & -1.201375 & 3.064328 \\
\hline $\mathrm{H}$ & -3.133899 & -2.388663 & 3.418322 & $\mathrm{C}$ & -2.722138 & -1.546801 & 1.476615 \\
\hline $\mathrm{H}$ & -2.005303 & -1.759422 & 4.638296 & $\mathrm{C}$ & -3.331873 & -0.892376 & -2.291783 \\
\hline $\mathrm{C}$ & -1.400464 & 0.654805 & 3.401926 & $\mathrm{H}$ & -2.256150 & -0.958265 & -2.490541 \\
\hline $\mathrm{H}$ & -0.763369 & 1.179637 & 2.686405 & $\mathrm{C}$ & -1.674587 & -2.136301 & 2.419025 \\
\hline $\mathrm{H}$ & -2.179931 & 1.344709 & 3.745974 & $\mathrm{H}$ & -0.788889 & -2.405267 & 1.837764 \\
\hline $\mathrm{H}$ & -0.781702 & 0.391208 & 4.267624 & $\mathrm{C}$ & 0.276545 & 1.102879 & -1.626560 \\
\hline $\mathrm{H}$ & -5.951721 & 1.217233 & 1.054882 & $\mathrm{H}$ & -0.288264 & 0.561189 & -2.395338 \\
\hline $\mathrm{C}$ & -0.866150 & 3.421940 & 0.715510 & $\mathrm{C}$ & -1.352530 & 1.582723 & 0.938892 \\
\hline $\mathrm{H}$ & -0.620129 & 3.349064 & 1.777754 & $\mathrm{H}$ & -1.916320 & 0.710936 & 1.247726 \\
\hline $\mathrm{H}$ & -0.111179 & 4.044525 & 0.233580 & $\mathrm{H}$ & 0.726040 & 2.026650 & -2.013433 \\
\hline $\mathrm{H}$ & -1.832079 & 3.943196 & 0.633262 & $\mathrm{~N}$ & 1.744283 & 2.327212 & 1.950843 \\
\hline $\mathrm{C}$ & -1.023546 & 3.248555 & -2.199400 & $\mathrm{O}$ & 2.295563 & 3.146655 & 2.661453 \\
\hline $\mathrm{H}$ & -0.015026 & 3.662009 & -2.110225 & $\mathrm{C}$ & -3.862076 & 0.389723 & -2.963167 \\
\hline $\mathrm{H}$ & -1.238463 & 3.095158 & -3.262929 & $\mathrm{H}$ & -3.605776 & 0.374657 & -4.028914 \\
\hline \multirow[t]{2}{*}{$\mathrm{H}$} & -1.736681 & 3.982932 & -1.809640 & $\mathrm{H}$ & -4.954595 & 0.452481 & -2.897907 \\
\hline & & & & $\mathrm{H}$ & -3.450033 & 1.308024 & -2.532962 \\
\hline \multicolumn{4}{|c|}{ TS4'-cross } & $\mathrm{C}$ & -4.008177 & -2.126212 & -2.928310 \\
\hline $\mathrm{Ru}$ & 0.576938 & 0.647039 & 0.165375 & $\mathrm{H}$ & -3.820856 & -2.155247 & -4.008140 \\
\hline $\mathrm{O}$ & 1.582730 & 2.535284 & 0.676006 & $\mathrm{H}$ & -3.647814 & -3.063889 & -2.491884 \\
\hline
\end{tabular}




$\begin{array}{lccc}\mathrm{H} & -5.093173 & -2.088409 & -2.773642 \\ \mathrm{C} & -2.202892 & -3.427241 & 3.075719 \\ \mathrm{H} & -3.061599 & -3.222283 & 3.725353 \\ \mathrm{H} & -2.523861 & -4.157293 & 2.323318 \\ \mathrm{H} & -1.421693 & -3.888394 & 3.691011 \\ \mathrm{C} & -1.207220 & -1.126554 & 3.484545 \\ \mathrm{H} & -0.714344 & -0.264286 & 3.029022 \\ \mathrm{H} & -2.045489 & -0.767449 & 4.093034 \\ \mathrm{H} & -0.484405 & -1.600633 & 4.158614 \\ \mathrm{H} & -5.889082 & -0.279055 & 1.589769 \\ \mathrm{C} & -1.276809 & 2.639657 & 2.019397 \\ \mathrm{H} & -2.289475 & 3.030602 & 2.190751 \\ \mathrm{H} & -0.924286 & 2.203067 & 2.956133 \\ \mathrm{H} & -0.639094 & 3.487150 & 1.770298 \\ \mathrm{C} & -1.437321 & 1.853031 & -0.449822 \\ \mathrm{H} & -2.071781 & 1.184052 & -1.012950 \\ \mathrm{C} & -1.457004 & 3.241731 & -1.051161 \\ \mathrm{O} & -2.387515 & 3.534115 & -1.803326 \\ \mathrm{~N} & -0.467806 & 4.104171 & -0.711261 \\ \mathrm{H} & 0.328074 & 3.760271 & -0.181297 \\ \mathrm{C} & -0.422320 & 5.447118 & -1.264712 \\ \mathrm{H} & 0.208745 & 6.072434 & -0.628369 \\ \mathrm{H} & -0.018113 & 5.454584 & -2.285677 \\ \mathrm{H} & -1.432807 & 5.860763 & -1.297504\end{array}$

\section{TS4'-homo}

$\begin{array}{lccc}\mathrm{Ru} & 0.573385 & 0.854310 & -0.361078 \\ \mathrm{O} & 1.718802 & 2.678582 & -0.767894 \\ \mathrm{O} & 1.285675 & 2.346331 & 1.333355 \\ \mathrm{~N} & 0.710898 & -1.913542 & 0.444810 \\ \mathrm{~N} & -1.427931 & -1.511824 & 0.207445 \\ \mathrm{C} & -0.186108 & -0.929870 & 0.165148 \\ \mathrm{C} & 0.119623 & -3.249640 & 0.470755 \\ \mathrm{H} & 0.533676 & -3.858940 & 1.278407 \\ \mathrm{H} & 0.296848 & -3.769470 & -0.481488 \\ \mathrm{C} & -1.362185 & -2.914527 & 0.668273 \\ \mathrm{H} & -2.033274 & -3.547906 & 0.084013 \\ \mathrm{H} & -1.660488 & -2.973872 & 1.723921 \\ \mathrm{C} & 2.128334 & -1.638014 & 0.207544 \\ \mathrm{C} & 2.537455 & -2.042803 & -1.229722 \\ \mathrm{H} & 1.852681 & -1.577048 & -1.945632 \\ \mathrm{H} & 2.451930 & -3.132630 & -1.347969 \\ \mathrm{C} & 3.985226 & -1.594514 & -1.500633 \\ \mathrm{H} & 4.264104 & -1.869642 & -2.526527 \\ \mathrm{C} & 4.921291 & -2.303786 & -0.500970 \\ \mathrm{H} & 4.866998 & -3.392840 & -0.641472 \\ \mathrm{H} & 5.962979 & -2.006892 & -0.682126 \\ \mathrm{C} & 4.513947 & -1.929270 & 0.939333 \\ \mathrm{H} & 5.173570 & -2.438896 & 1.653725 \\ \mathrm{C} & 3.054867 & -2.378784 & 1.197716 \\ \mathrm{H} & 2.754178 & -2.141575 & 2.226528 \\ \mathrm{H} & 2.976346 & -3.469203 & 1.071921 \\ \mathrm{C} & 4.642541 & -0.400087 & 1.109337 \\ \mathrm{H} & 4.394475 & -0.108104 & 2.138282 \\ \mathrm{H} & 5.684097 & -0.097801 & 0.930436 \\ \mathrm{C} & 3.694550 & 0.321801 & 0.116967 \\ \mathrm{H} & 3.799305 & 1.403272 & 0.245876 \\ \mathrm{C} & 2.258975 & -0.121441 & 0.443146 \\ \mathrm{H} & 2.087857 & 0.045517 & 1.516810 \\ \mathrm{C} & 4.081427 & -0.064173 & -1.323896\end{array}$

\begin{tabular}{|c|c|c|c|}
\hline $\mathrm{H}$ & 3.415292 & 0.442215 & -2.033005 \\
\hline $\mathrm{H}$ & 5.105019 & 0.270921 & -1.540089 \\
\hline $\mathrm{C}$ & -2.663730 & -0.808345 & 0.378472 \\
\hline $\mathrm{C}$ & -3.595393 & -0.802603 & -0.681849 \\
\hline $\mathrm{C}$ & -4.794095 & -0.102562 & -0.500831 \\
\hline $\mathrm{H}$ & -5.526214 & -0.077433 & -1.302566 \\
\hline $\mathrm{C}$ & -5.066043 & 0.556101 & 0.696364 \\
\hline $\mathrm{C}$ & -4.153122 & 0.501044 & 1.746688 \\
\hline $\mathrm{H}$ & -4.383291 & 1.001112 & 2.683144 \\
\hline $\mathrm{C}$ & -2.941187 & -0.188948 & 1.617492 \\
\hline $\mathrm{C}$ & -3.352482 & -1.597996 & -1.960579 \\
\hline $\mathrm{H}$ & -2.292010 & -1.871112 & -1.979860 \\
\hline $\mathrm{C}$ & -1.989186 & -0.267396 & 2.809025 \\
\hline $\mathrm{H}$ & -1.105013 & -0.837983 & 2.513705 \\
\hline $\mathrm{C}$ & 0.310358 & 0.422272 & -2.164429 \\
\hline $\mathrm{H}$ & -0.276099 & -0.391008 & -2.612510 \\
\hline $\mathrm{C}$ & -1.266068 & 2.167702 & -0.252438 \\
\hline $\mathrm{H}$ & -1.923485 & 1.603835 & 0.396552 \\
\hline $\mathrm{C}$ & -1.303628 & 1.792424 & -1.621050 \\
\hline $\mathrm{H}$ & -1.996575 & 0.991546 & -1.851964 \\
\hline $\mathrm{H}$ & 0.840944 & 1.021194 & -2.916451 \\
\hline $\mathrm{N}$ & 1.868926 & 3.071692 & 0.455243 \\
\hline $\mathrm{O}$ & 2.517203 & 4.064283 & 0.736238 \\
\hline $\mathrm{C}$ & -3.657239 & -0.816650 & -3.251287 \\
\hline $\mathrm{H}$ & -4.717657 & -0.550560 & -3.325327 \\
\hline $\mathrm{H}$ & -3.075600 & 0.107659 & -3.320150 \\
\hline $\mathrm{H}$ & -3.413371 & -1.431858 & -4.124987 \\
\hline $\mathrm{C}$ & -4.182216 & -2.899516 & -1.932280 \\
\hline $\mathrm{H}$ & -3.959216 & -3.521353 & -2.807384 \\
\hline $\mathrm{H}$ & -3.981087 & -3.489344 & -1.031600 \\
\hline $\mathrm{H}$ & -5.254974 & -2.672061 & -1.942279 \\
\hline $\mathrm{C}$ & -2.655776 & -1.020895 & 3.977669 \\
\hline $\mathrm{H}$ & -3.521986 & -0.471522 & 4.364409 \\
\hline $\mathrm{H}$ & -3.004027 & -2.013511 & 3.668368 \\
\hline $\mathrm{H}$ & -1.945379 & -1.147419 & 4.802732 \\
\hline $\mathrm{C}$ & -1.484860 & 1.116322 & 3.260342 \\
\hline $\mathrm{H}$ & -0.884101 & 1.594878 & 2.483652 \\
\hline $\mathrm{H}$ & -2.315616 & 1.782451 & 3.521788 \\
\hline $\mathrm{H}$ & -0.850286 & 1.010653 & 4.147799 \\
\hline $\mathrm{H}$ & -5.999001 & 1.100353 & 0.816239 \\
\hline $\mathrm{C}$ & -1.092494 & 3.611652 & 0.165344 \\
\hline $\mathrm{H}$ & -2.043389 & 4.143143 & 0.010232 \\
\hline $\mathrm{H}$ & -0.836674 & 3.685138 & 1.224171 \\
\hline $\mathrm{H}$ & -0.326142 & 4.135534 & -0.408894 \\
\hline $\mathrm{C}$ & -1.084043 & 2.819431 & -2.714950 \\
\hline $\mathrm{H}$ & -0.127382 & 3.333993 & -2.589876 \\
\hline $\mathrm{H}$ & -1.105664 & 2.362416 & -3.708490 \\
\hline $\mathrm{H}$ & -1.880360 & 3.575128 & -2.668944 \\
\hline
\end{tabular}

\section{TS1-AC}

$\begin{array}{lccc}\mathrm{Ru} & 0.470884 & 0.086930 & -0.587622 \\ \mathrm{O} & 2.169145 & 1.480210 & -1.022746 \\ \mathrm{O} & 1.432537 & 1.421194 & 1.054028 \\ \mathrm{~N} & -0.187779 & -2.457093 & 0.598491 \\ \mathrm{~N} & -2.108613 & -1.423106 & 0.414341 \\ \mathrm{C} & -0.762644 & -1.294702 & 0.186736 \\ \mathrm{C} & -1.155437 & -3.504886 & 0.910673 \\ \mathrm{H} & -0.856003 & -4.083637 & 1.788215 \\ \mathrm{H} & -1.268723 & -4.194501 & 0.061363 \\ \mathrm{C} & -2.418130 & -2.672104 & 1.143089\end{array}$




\begin{tabular}{|c|c|c|c|}
\hline $\mathrm{H}$ & -3.325635 & -3.135879 & 0.751545 \\
\hline $\mathrm{H}$ & -2.569744 & -2.449668 & 2.207068 \\
\hline $\mathrm{C}$ & 1.221453 & -2.684260 & 0.286991 \\
\hline $\mathrm{C}$ & 1.375678 & -3.411292 & -1.070534 \\
\hline $\mathrm{H}$ & 0.816947 & -2.868365 & -1.838591 \\
\hline $\mathrm{H}$ & 0.944629 & -4.420475 & -1.000434 \\
\hline $\mathrm{C}$ & 2.865110 & -3.495168 & -1.448617 \\
\hline $\mathrm{H}$ & 2.966754 & -3.996529 & -2.420390 \\
\hline $\mathrm{C}$ & 3.609734 & -4.303983 & -0.367030 \\
\hline $\mathrm{H}$ & 3.210763 & -5.327297 & -0.320815 \\
\hline $\mathrm{H}$ & 4.675244 & -4.384556 & -0.620963 \\
\hline $\mathrm{C}$ & 3.448841 & -3.605085 & 0.999226 \\
\hline $\mathrm{H}$ & 3.970351 & -4.183589 & 1.772862 \\
\hline $\mathrm{C}$ & 1.945686 & -3.524469 & 1.363817 \\
\hline $\mathrm{H}$ & 1.810756 & -3.053877 & 2.346299 \\
\hline $\mathrm{H}$ & 1.527147 & -4.540459 & 1.421936 \\
\hline $\mathrm{C}$ & 4.059984 & -2.189540 & 0.905171 \\
\hline $\mathrm{H}$ & 3.997577 & -1.681121 & 1.876449 \\
\hline $\mathrm{H}$ & 5.126551 & -2.268440 & 0.649677 \\
\hline $\mathrm{C}$ & 3.304994 & -1.363978 & -0.167834 \\
\hline $\mathrm{H}$ & 3.738961 & -0.363198 & -0.234845 \\
\hline $\mathrm{C}$ & 1.836283 & -1.271527 & 0.275712 \\
\hline $\mathrm{H}$ & 1.819641 & -0.916404 & 1.315224 \\
\hline $\mathrm{C}$ & 3.441003 & -2.065901 & -1.532143 \\
\hline $\mathrm{H}$ & 2.909950 & -1.488114 & -2.298529 \\
\hline $\mathrm{H}$ & 4.498335 & -2.108035 & -1.828306 \\
\hline $\mathrm{C}$ & -3.065428 & -0.356898 & 0.495186 \\
\hline $\mathrm{C}$ & -4.082672 & -0.292319 & -0.482304 \\
\hline $\mathrm{C}$ & -5.028128 & 0.734916 & -0.385144 \\
\hline $\mathrm{H}$ & -5.817004 & 0.808915 & -1.127985 \\
\hline $\mathrm{C}$ & -4.974997 & 1.660973 & 0.653478 \\
\hline $\mathrm{C}$ & -3.985907 & 1.560291 & 1.627613 \\
\hline $\mathrm{H}$ & -3.960252 & 2.281080 & 2.438718 \\
\hline $\mathrm{C}$ & -3.017232 & 0.549782 & 1.579259 \\
\hline $\mathrm{C}$ & -4.204038 & -1.339688 & -1.584980 \\
\hline $\mathrm{H}$ & -3.272395 & -1.915848 & -1.596337 \\
\hline $\mathrm{C}$ & -1.991203 & 0.441330 & 2.705138 \\
\hline $\mathrm{H}$ & -1.234205 & -0.293934 & 2.418819 \\
\hline $\mathrm{C}$ & 0.179774 & -0.504443 & -2.342123 \\
\hline $\mathrm{H}$ & -0.572216 & -1.191577 & -2.753923 \\
\hline $\mathrm{C}$ & -0.923097 & 1.886874 & -0.742309 \\
\hline $\mathrm{H}$ & -1.598318 & 1.743551 & 0.088882 \\
\hline $\mathrm{C}$ & -1.191767 & 1.171656 & -1.924579 \\
\hline $\mathrm{H}$ & -2.057385 & 0.524181 & -1.950912 \\
\hline $\mathrm{H}$ & 0.847643 & -0.108135 & -3.122441 \\
\hline $\mathrm{C}$ & -4.397344 & -0.736809 & -2.988836 \\
\hline $\mathrm{H}$ & -4.408264 & -1.534833 & -3.740279 \\
\hline $\mathrm{H}$ & -5.347691 & -0.197564 & -3.070649 \\
\hline $\mathrm{H}$ & -3.596746 & -0.037983 & -3.250609 \\
\hline $\mathrm{C}$ & -5.363528 & -2.308941 & -1.271728 \\
\hline $\mathrm{H}$ & -5.413862 & -3.109578 & -2.019322 \\
\hline $\mathrm{H}$ & -5.253669 & -2.768432 & -0.283583 \\
\hline $\mathrm{H}$ & -6.322293 & -1.776795 & -1.280111 \\
\hline $\mathrm{C}$ & -2.678870 & -0.062330 & 3.992094 \\
\hline $\mathrm{H}$ & -3.416619 & 0.666454 & 4.348624 \\
\hline $\mathrm{H}$ & -3.204748 & -1.010961 & 3.832628 \\
\hline $\mathrm{H}$ & -1.938993 & -0.209289 & 4.787596 \\
\hline $\mathrm{C}$ & -1.247805 & 1.762376 & 2.980425 \\
\hline $\mathrm{H}$ & -0.721089 & 2.130615 & 2.099171 \\
\hline $\mathrm{H}$ & -1.932040 & 2.544715 & 3.328267 \\
\hline
\end{tabular}

$\begin{array}{lrrr}\mathrm{H} & -0.500081 & 1.601237 & 3.765318 \\ \mathrm{H} & -5.711169 & 2.458236 & 0.708821 \\ \mathrm{H} & -0.891894 & 1.608781 & -2.873931 \\ \mathrm{C} & -0.412423 & 3.300930 & -0.780196 \\ \mathrm{O} & -0.889342 & 4.135453 & -0.012860 \\ \mathrm{~N} & 0.477945 & 3.629819 & -1.770034 \\ \mathrm{H} & 1.151265 & 2.908022 & -2.009368 \\ \mathrm{C} & 0.898866 & 5.014910 & -1.910258 \\ \mathrm{H} & 1.511660 & 5.362067 & -1.068740 \\ \mathrm{H} & 1.473832 & 5.116401 & -2.834840 \\ \mathrm{H} & 0.015315 & 5.655609 & -1.962296 \\ \mathrm{C} & 3.356234 & 2.825369 & 0.652696 \\ \mathrm{C} & 2.696702 & 3.946813 & 1.483110 \\ \mathrm{H} & 3.454148 & 4.673051 & 1.801916 \\ \mathrm{H} & 1.927898 & 4.474386 & 0.909158 \\ \mathrm{H} & 2.210817 & 3.530953 & 2.369774 \\ \mathrm{C} & 4.330545 & 2.031308 & 1.552314 \\ \mathrm{H} & 4.840534 & 1.241862 & 0.988238 \\ \mathrm{H} & 5.095309 & 2.703907 & 1.959245 \\ \mathrm{H} & 3.794276 & 1.567380 & 2.385735 \\ \mathrm{C} & 4.113457 & 3.404850 & -0.550716 \\ \mathrm{H} & 3.447688 & 3.977857 & -1.202932 \\ \mathrm{H} & 4.909611 & 4.074649 & -0.204304 \\ \mathrm{H} & 4.563505 & 2.609195 & -1.151985 \\ \mathrm{C} & 2.257214 & 1.860751 & 0.198982\end{array}$

\section{INT2-1-AC}

$\begin{array}{lccc}\mathrm{Ru} & 0.634559 & 0.599723 & -0.301608 \\ \mathrm{C} & 1.281422 & 0.077412 & -2.288515 \\ \mathrm{H} & 1.424029 & -0.996035 & -2.440209 \\ \mathrm{H} & 2.190630 & 0.618704 & -2.551984 \\ \mathrm{C} & 0.021875 & 0.622814 & -2.941948 \\ \mathrm{C} & -0.929706 & 0.459978 & -1.777255 \\ \mathrm{C} & -2.004797 & 1.506137 & -1.641169 \\ \mathrm{O} & -1.896607 & 2.657610 & -2.076184 \\ \mathrm{~N} & -3.147327 & 1.069219 & -1.027934 \\ \mathrm{H} & -3.102265 & 0.180266 & -0.556437 \\ \mathrm{C} & -4.225957 & 1.960138 & -0.655490 \\ \mathrm{H} & -5.179209 & 1.423002 & -0.698124 \\ \mathrm{H} & -4.245127 & 2.791193 & -1.362476 \\ \mathrm{H} & -4.093061 & 2.366164 & 0.356660 \\ \mathrm{H} & -1.347395 & -0.542020 & -1.717580 \\ \mathrm{H} & 0.124890 & 1.687017 & -3.170479 \\ \mathrm{H} & -0.285284 & 0.102124 & -3.865553 \\ \mathrm{C} & 0.365657 & -1.246090 & 0.385743 \\ \mathrm{~N} & 1.484769 & -1.892193 & 0.847012 \\ \mathrm{C} & 2.796894 & -1.351592 & 0.532162 \\ \mathrm{C} & 2.556249 & 0.077824 & 0.044822 \\ \mathrm{C} & 3.864040 & 0.719815 & -0.420903 \\ \mathrm{C} & 4.596866 & -0.131354 & -1.482002 \\ \mathrm{C} & 4.858726 & -1.542716 & -0.927631 \\ \mathrm{C} & 3.510814 & -2.190282 & -0.557481 \\ \mathrm{H} & 3.678989 & -3.208415 & -0.181400 \\ \mathrm{H} & 2.862935 & -2.265851 & -1.437311 \\ \mathrm{C} & 5.751713 & -1.453046 & 0.325392 \\ \mathrm{C} & 5.044581 & -0.599664 & 1.397623 \\ \mathrm{C} & 4.779109 & 0.814759 & 0.833938 \\ \mathrm{H} & 4.308276 & 1.447276 & 1.598220 \\ \mathrm{H} & 5.730169 & 1.292394 & 0.562356 \\ \mathrm{C} & 3.711943 & -1.279697 & 1.782643\end{array}$




\begin{tabular}{|c|c|c|c|}
\hline $\mathrm{H}$ & 3.197079 & -0.717907 & 2.572411 \\
\hline $\mathrm{H}$ & 3.904952 & -2.290369 & 2.170375 \\
\hline $\mathrm{H}$ & 5.678579 & -0.525458 & 2.290262 \\
\hline $\mathrm{H}$ & 6.718614 & -1.003307 & 0.064157 \\
\hline $\mathrm{H}$ & 5.958735 & -2.458204 & 0.718513 \\
\hline $\mathrm{H}$ & 5.353152 & -2.155687 & -1.692001 \\
\hline $\mathrm{H}$ & 5.548006 & 0.354743 & -1.737889 \\
\hline $\mathrm{H}$ & 4.012179 & -0.195921 & -2.403221 \\
\hline $\mathrm{H}$ & 3.653880 & 1.722391 & -0.808738 \\
\hline $\mathrm{H}$ & 2.182600 & 0.696585 & 0.935405 \\
\hline $\mathrm{C}$ & 1.247884 & -3.284913 & 1.215782 \\
\hline $\mathrm{C}$ & -0.268848 & -3.280498 & 1.402536 \\
\hline $\mathrm{N}$ & -0.680275 & -2.098963 & 0.617318 \\
\hline $\mathrm{C}$ & -2.090038 & -1.869312 & 0.498391 \\
\hline $\mathrm{C}$ & -2.769823 & -2.450787 & -0.598107 \\
\hline $\mathrm{C}$ & -4.164600 & -2.343466 & -0.644749 \\
\hline $\mathrm{C}$ & -4.869027 & -1.691904 & 0.365163 \\
\hline $\mathrm{C}$ & -4.184035 & -1.127011 & 1.437514 \\
\hline $\mathrm{C}$ & -2.786776 & -1.202215 & 1.530797 \\
\hline $\mathrm{C}$ & -2.086980 & -0.599976 & 2.742952 \\
\hline $\mathrm{C}$ & -2.490557 & 0.868001 & 2.976119 \\
\hline $\mathrm{H}$ & -1.910151 & 1.282144 & 3.809210 \\
\hline $\mathrm{H}$ & -3.551231 & 0.955834 & 3.240739 \\
\hline $\mathrm{H}$ & -2.285447 & 1.473343 & 2.091883 \\
\hline $\mathrm{C}$ & -2.371094 & -1.441695 & 4.004530 \\
\hline $\mathrm{H}$ & -2.088534 & -2.492408 & 3.873611 \\
\hline $\mathrm{H}$ & -1.817541 & -1.042339 & 4.862405 \\
\hline $\mathrm{H}$ & -3.438901 & -1.418109 & 4.253478 \\
\hline $\mathrm{H}$ & -1.008941 & -0.610966 & 2.555573 \\
\hline $\mathrm{H}$ & -4.740700 & -0.617152 & 2.217586 \\
\hline $\mathrm{H}$ & -5.951918 & -1.618861 & 0.312764 \\
\hline $\mathrm{H}$ & -4.705450 & -2.775309 & -1.481176 \\
\hline $\mathrm{C}$ & -2.031195 & -3.201352 & -1.701186 \\
\hline $\mathrm{C}$ & -2.248769 & -4.722186 & -1.563926 \\
\hline $\mathrm{H}$ & -1.940760 & -5.093043 & -0.580124 \\
\hline $\mathrm{H}$ & -3.307937 & -4.974899 & -1.693680 \\
\hline $\mathrm{H}$ & -1.676697 & -5.263464 & -2.326685 \\
\hline $\mathrm{C}$ & -2.435854 & -2.735791 & -3.113175 \\
\hline $\mathrm{H}$ & -3.475045 & -2.998067 & -3.342705 \\
\hline $\mathrm{H}$ & -2.327878 & -1.653575 & -3.228698 \\
\hline $\mathrm{H}$ & -1.801880 & -3.224538 & -3.861867 \\
\hline $\mathrm{H}$ & -0.962377 & -2.998587 & -1.579578 \\
\hline $\mathrm{H}$ & -0.756336 & -4.184036 & 1.031038 \\
\hline $\mathrm{H}$ & -0.549716 & -3.140842 & 2.454045 \\
\hline $\mathrm{H}$ & 1.790194 & -3.564610 & 2.123623 \\
\hline $\mathrm{H}$ & 1.554276 & -3.961669 & 0.405600 \\
\hline $\mathrm{O}$ & 0.930509 & 2.758903 & -0.675085 \\
\hline $\mathrm{C}$ & -0.014938 & 3.035359 & 0.121133 \\
\hline $\mathrm{O}$ & -0.610673 & 2.061147 & 0.722771 \\
\hline $\mathrm{C}$ & -0.405700 & 4.477785 & 0.417658 \\
\hline $\mathrm{C}$ & -1.909860 & 4.561921 & 0.726502 \\
\hline $\mathrm{H}$ & -2.182143 & 5.597615 & 0.963965 \\
\hline $\mathrm{H}$ & -2.485175 & 4.234644 & -0.142995 \\
\hline $\mathrm{H}$ & -2.176072 & 3.928247 & 1.577980 \\
\hline $\mathrm{C}$ & -0.065460 & 5.356326 & -0.798007 \\
\hline $\mathrm{H}$ & 1.003871 & 5.318183 & -1.024141 \\
\hline $\mathrm{H}$ & -0.345068 & 6.397218 & -0.593778 \\
\hline $\mathrm{H}$ & -0.612666 & 5.005192 & -1.677714 \\
\hline $\mathrm{C}$ & 0.417523 & 4.917503 & 1.650905 \\
\hline $\mathrm{H}$ & 0.186915 & 4.291275 & 2.52058 \\
\hline
\end{tabular}

$\begin{array}{llll}\mathrm{H} & 1.492958 & 4.851446 & 1.448748 \\ \mathrm{H} & 0.180736 & 5.957748 & 1.904719\end{array}$

\section{INT2-AC}

$\begin{array}{llll}\mathrm{Ru} & 0.381653 & -0.547533 & -0.085859 \\ \mathrm{O} & 1.928001 & -0.233516 & 1.819699\end{array}$

$\begin{array}{llll}\mathrm{O} & 2.018286 & -1.944298 & 0.430728\end{array}$

$\mathrm{N} \quad-0.436024 \quad 1.980354 \quad-1.201252$

$\mathrm{N} \quad-2.286605 \quad 1.060385 \quad-0.478334$

$\begin{array}{llll}\text { C } & -0.932185 & 0.901619 & -0.542140\end{array}$

$\begin{array}{llll}\text { C } & -1.426989 & 3.020269 & -1.476567\end{array}$

$\mathrm{H} \quad-1.372289 \quad 3.353890 \quad-2.516416$

$\mathrm{H} \quad-1.268454 \quad 3.891943 \quad-0.827867$

$\begin{array}{llll}\text { C } & -2.743881 & 2.289343 & -1.159475\end{array}$

$\mathrm{H} \quad-3.403795 \quad 2.863309 \quad-0.502062$

$\begin{array}{llll}\mathrm{H} & -3.301813 & 2.028847 & -2.066067\end{array}$

$\begin{array}{llll}\mathrm{C} & 1.002757 & 2.211270 & -1.156583\end{array}$

$\begin{array}{llll}\mathrm{C} & 1.365381 & 3.139940 & 0.031483\end{array}$

$\begin{array}{llll}\mathrm{H} & 0.977675 & 2.692575 & 0.954914\end{array}$

$\mathrm{H} \quad 0.882742 \quad 4.118093 \quad-0.101081$

$\begin{array}{llll}\mathrm{C} & 2.894170 & 3.312934 & 0.117471\end{array}$

$\begin{array}{llll}\mathrm{H} & 3.132901 & 3.990738 & 0.947283\end{array}$

C $\quad 3.423512 \quad 3.902283 \quad-1.205164$

$\mathrm{H} \quad 2.985587 \quad 4.894454 \quad-1.384786$

$\mathrm{H} \quad 4.511700 \quad 4.036443 \quad-1.144901$

$\begin{array}{llll}\mathrm{C} & 3.073961 & 2.952034 & -2.369263\end{array}$

$\mathrm{H} \quad 3.460863 \quad 3.361221 \quad-3.311239$

$\begin{array}{llll}\mathrm{C} & 1.539003 & 2.821079 & -2.470510\end{array}$

$\mathrm{H} \quad 1.255034 \quad 2.175088 \quad-3.311376$

$\begin{array}{llll}\mathrm{H} & 1.089914 & 3.809530 & -2.645977\end{array}$

$\begin{array}{llll}\mathrm{C} & 3.702347 & 1.565401 & -2.109990\end{array}$

$\begin{array}{llll}\mathrm{H} & 3.480148 & 0.887596 & -2.945199\end{array}$

$\begin{array}{llll}\mathrm{H} & 4.795621 & 1.657454 & -2.050614\end{array}$

$\begin{array}{llll}\mathrm{C} & 3.156122 & 0.976061 & -0.778960\end{array}$

$\mathrm{H} \quad 3.597822 \quad-0.010772 \quad-0.611970$

$\begin{array}{llll}\mathrm{C} & 1.639479 & 0.836598 & -0.940299\end{array}$

$\begin{array}{llll}\mathrm{H} & 1.437277 & 0.200330 & -1.839374\end{array}$

$\begin{array}{llll}\text { C } & 3.531129 & 1.934916 & 0.370257\end{array}$

$\mathrm{H} \quad 3.185460 \quad 1.525874 \quad 1.320941$

$\begin{array}{llll}\mathrm{H} & 4.625017 & 2.031556 & 0.420570\end{array}$

$\begin{array}{llll}\text { C } & -3.151252 & 0.426431 & 0.469998\end{array}$

$\begin{array}{llll}\text { C } & -3.085376 & 0.843301 & 1.818620\end{array}$

$\begin{array}{llll}\text { C } & -3.906409 & 0.189169 & 2.744685\end{array}$

$\mathrm{H} \quad-3.857944 \quad 0.470825 \quad 3.792810$

$\begin{array}{llll}\text { C } & -4.784274 & -0.815260 & 2.342229\end{array}$

C $\quad-4.883320 \quad-1.159799 \quad 0.996016$

$\mathrm{H} \quad-5.600209 \quad-1.915171 \quad 0.688456$

$\begin{array}{llll}\mathrm{C} & -4.078276 & -0.540279 & 0.031553\end{array}$

$\begin{array}{llll}\mathrm{C} & -2.188658 & 1.990607 & 2.280294\end{array}$

$\begin{array}{llll}\mathrm{H} & -1.687387 & 2.410364 & 1.403749\end{array}$

C $\quad-4.278498 \quad-0.840164 \quad-1.449558$

H $\quad-3.422822 \quad-0.425563 \quad-1.991795$

C $\quad-0.635199 \quad-1.469970 \quad 1.415322$

$\begin{array}{llll}\mathrm{C} & -0.635199 & -1.469970 & 1.415322 \\ \mathrm{H} & -1.472963 & -0.979747 & 1.896685\end{array}$

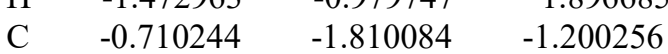

$\mathrm{H} \quad-1.602762 \quad-1.425614 \quad-1.686863$

$\begin{array}{llll}\text { C } & -1.041529 & -2.410421 & 0.249120\end{array}$

$\begin{array}{llll}\mathrm{C} & -1.041529 & -2.410421 & 0.249120 \\ \mathrm{H} & -2.128000 & -2.523875 & 0.280014\end{array}$

$\begin{array}{llll}\mathrm{H} & -2.128000 & -2.523875 & 0.280014 \\ \mathrm{H} & 0.036947 & -1.930639 & 2.139288\end{array}$

C $\quad-5.547632 \quad-0.120412 \quad-1.953423$ 


$\begin{array}{cccc}\mathrm{H} & -5.672336 & -0.269378 & -3.032371 \\ \mathrm{H} & -6.440217 & -0.511955 & -1.450755 \\ \mathrm{H} & -5.504185 & 0.956988 & -1.757431 \\ \mathrm{C} & -4.350035 & -2.340893 & -1.779179 \\ \mathrm{H} & -5.221695 & -2.817422 & -1.315918 \\ \mathrm{H} & -4.434512 & -2.479365 & -2.862424 \\ \mathrm{H} & -3.453510 & -2.872440 & -1.448721 \\ \mathrm{C} & -3.036779 & 3.120073 & 2.898775 \\ \mathrm{H} & -3.822626 & 3.453123 & 2.210473 \\ \mathrm{H} & -3.523100 & 2.795974 & 3.825950 \\ \mathrm{H} & -2.403101 & 3.981447 & 3.140296 \\ \mathrm{C} & -1.078873 & 1.538521 & 3.249025 \\ \mathrm{H} & -0.517509 & 2.411107 & 3.604753 \\ \mathrm{H} & -1.499628 & 1.033145 & 4.126743 \\ \mathrm{H} & -0.371422 & 0.861376 & 2.763921 \\ \mathrm{H} & -5.409377 & -1.315474 & 3.077161 \\ \mathrm{H} & -0.551240 & -3.380871 & 0.346687 \\ \mathrm{C} & -0.007131 & -2.690736 & -2.203381 \\ \mathrm{O} & -0.377189 & -2.690575 & -3.380357 \\ \mathrm{~N} & 1.031080 & -3.467128 & -1.764017 \\ \mathrm{H} & 1.479272 & -3.200422 & -0.891751 \\ \mathrm{C} & 1.835663 & -4.198007 & -2.727639 \\ \mathrm{H} & 2.503919 & -3.536984 & -3.298653 \\ \mathrm{H} & 2.438667 & -4.942662 & -2.200139 \\ \mathrm{H} & 1.177603 & -4.701313 & -3.439046 \\ \mathrm{C} & 2.458190 & -1.315199 & 1.463414 \\ \mathrm{C} & 3.629567 & -1.933766 & 2.236356 \\ \mathrm{C} & 3.161782 & -3.299192 & 2.783322 \\ \mathrm{H} & 2.850338 & -3.958795 & 1.967762 \\ \mathrm{H} & 3.975533 & -3.785159 & 3.335101 \\ \mathrm{H} & 2.314132 & -3.177686 & 3.468929 \\ \mathrm{C} & 4.804536 & -2.141737 & 1.258665 \\ \mathrm{H} & 5.157823 & -1.183036 & 0.860114 \\ \mathrm{H} & 5.644671 & -2.622011 & 1.774641 \\ \mathrm{H} & 4.503748 & -2.771962 & 0.416674 \\ \mathrm{C} & 4.055979 & -1.015682 & 3.390611 \\ \mathrm{H} & 3.227455 & -0.844537 & 4.084378 \\ \mathrm{H} & 4.887663 & -1.469279 & 3.943387 \\ \mathrm{H} & 4.380471 & -0.039363 & 3.016588\end{array}$

\section{INT3-AC}

$\begin{array}{lccc}\mathrm{Ru} & 0.518297 & 0.217089 & 0.394889 \\ \mathrm{C} & -0.524196 & 0.253286 & 1.893772 \\ \mathrm{C} & -0.350110 & 1.019732 & 3.174153 \\ \mathrm{O} & -1.080872 & 0.734148 & 4.132199 \\ \mathrm{~N} & 0.603275 & 1.984242 & 3.247286 \\ \mathrm{H} & 1.189517 & 2.155442 & 2.437068 \\ \mathrm{C} & 0.839177 & 2.709515 & 4.480328 \\ \mathrm{H} & -0.116095 & 2.983743 & 4.937166 \\ \mathrm{H} & 1.397352 & 2.106530 & 5.209208 \\ \mathrm{H} & 1.411156 & 3.614574 & 4.258575 \\ \mathrm{H} & -1.450101 & -0.320668 & 1.952769 \\ \mathrm{C} & -0.517719 & -1.289784 & -0.310249 \\ \mathrm{~N} & -1.801313 & -1.476993 & -0.698439 \\ \mathrm{C} & -2.832491 & -0.477960 & -0.653860 \\ \mathrm{C} & -2.858993 & 0.508264 & -1.667664 \\ \mathrm{C} & -3.870047 & 1.473476 & -1.608437 \\ \mathrm{C} & -4.825946 & 1.456580 & -0.594614 \\ \mathrm{C} & -4.786146 & 0.471743 & 0.386182 \\ \mathrm{C} & -3.786877 & -0.509743 & 0.384102\end{array}$

\begin{tabular}{|c|c|c|c|}
\hline $\mathrm{C}$ & -3.796116 & -1.596789 & 1.455126 \\
\hline $\mathrm{C}$ & -4.886715 & -2.640374 & 1.131255 \\
\hline $\mathrm{H}$ & -5.881001 & -2.180784 & 1.182506 \\
\hline $\mathrm{H}$ & -4.859103 & -3.466594 & 1.851610 \\
\hline $\mathrm{H}$ & -4.769881 & -3.056127 & 0.124028 \\
\hline $\mathrm{C}$ & -3.988686 & -1.055233 & 2.885014 \\
\hline $\mathrm{H}$ & -4.966921 & -0.576006 & 3.006049 \\
\hline $\mathrm{H}$ & -3.945957 & -1.887519 & 3.597476 \\
\hline $\mathrm{H}$ & -3.213882 & -0.336981 & 3.169947 \\
\hline $\mathrm{H}$ & -2.823798 & -2.101735 & 1.434386 \\
\hline $\mathrm{H}$ & -5.536079 & 0.467152 & 1.170963 \\
\hline $\mathrm{H}$ & -5.604030 & 2.215180 & -0.571127 \\
\hline $\mathrm{H}$ & -3.915532 & 2.249747 & -2.364403 \\
\hline $\mathrm{C}$ & -1.857244 & 0.501267 & -2.820116 \\
\hline $\mathrm{C}$ & -2.311714 & -0.474416 & -3.925106 \\
\hline $\mathrm{H}$ & -1.581985 & -0.494654 & -4.743782 \\
\hline $\mathrm{H}$ & -3.278713 & -0.164704 & -4.339661 \\
\hline $\mathrm{H}$ & -2.423328 & -1.495833 & -3.549029 \\
\hline $\mathrm{C}$ & -1.600904 & 1.890707 & -3.423054 \\
\hline $\mathrm{H}$ & -1.338933 & 2.609663 & -2.644039 \\
\hline $\mathrm{H}$ & -2.467772 & 2.264756 & -3.981452 \\
\hline $\mathrm{H}$ & -0.761195 & 1.834523 & -4.125041 \\
\hline $\mathrm{H}$ & -0.898716 & 0.151175 & -2.430033 \\
\hline $\mathrm{C}$ & -2.030214 & -2.876733 & -1.117180 \\
\hline $\mathrm{H}$ & -2.669367 & -2.921196 & -2.000905 \\
\hline $\mathrm{H}$ & -2.510809 & -3.440935 & -0.309399 \\
\hline $\mathrm{C}$ & -0.594420 & -3.374686 & -1.364094 \\
\hline $\mathrm{N}$ & 0.158850 & -2.453769 & -0.513636 \\
\hline $\mathrm{C}$ & 1.613719 & -2.363996 & -0.362134 \\
\hline $\mathrm{C}$ & 2.278510 & -3.709918 & -0.024398 \\
\hline $\mathrm{C}$ & 3.786244 & -3.463320 & 0.229958 \\
\hline $\mathrm{C}$ & 4.436242 & -2.894841 & -1.048713 \\
\hline $\mathrm{C}$ & 3.769547 & -1.552645 & -1.414264 \\
\hline $\mathrm{C}$ & 2.266558 & -1.791032 & -1.648391 \\
\hline $\mathrm{H}$ & 2.114460 & -2.489006 & -2.484106 \\
\hline $\mathrm{H}$ & 1.770050 & -0.849528 & -1.926368 \\
\hline $\mathrm{C}$ & 3.946879 & -0.545030 & -0.259388 \\
\hline $\mathrm{C}$ & 3.302863 & -1.104510 & 1.026403 \\
\hline $\mathrm{C}$ & 1.804050 & -1.386431 & 0.830606 \\
\hline $\mathrm{H}$ & 1.426349 & -1.894188 & 1.723350 \\
\hline $\mathrm{C}$ & 3.982979 & -2.452343 & 1.382979 \\
\hline $\mathrm{H}$ & 3.557654 & -2.850914 & 2.313473 \\
\hline $\mathrm{H}$ & 5.057755 & -2.301755 & 1.557517 \\
\hline $\mathrm{H}$ & 3.436839 & -0.390032 & 1.847364 \\
\hline $\mathrm{H}$ & 5.015911 & -0.357220 & -0.089973 \\
\hline $\mathrm{H}$ & 3.491692 & 0.419419 & -0.517752 \\
\hline $\mathrm{H}$ & 4.221633 & -1.151968 & -2.330982 \\
\hline $\mathrm{H}$ & 5.512361 & -2.748177 & -0.886600 \\
\hline $\mathrm{H}$ & 4.329278 & -3.607496 & -1.878578 \\
\hline $\mathrm{H}$ & 4.262055 & -4.416878 & 0.492172 \\
\hline $\mathrm{H}$ & 1.800959 & -4.140465 & 0.865121 \\
\hline $\mathrm{H}$ & 2.153516 & -4.427811 & -0.848973 \\
\hline $\mathrm{H}$ & -0.449225 & -4.415402 & -1.064533 \\
\hline $\mathrm{H}$ & -0.300227 & -3.266029 & -2.419124 \\
\hline $\mathrm{O}$ & 1.855751 & 2.120270 & 0.666120 \\
\hline $\mathrm{C}$ & 1.358787 & 2.544466 & -0.417662 \\
\hline $\mathrm{O}$ & 0.495784 & 1.816876 & -1.038194 \\
\hline $\mathrm{C}$ & 1.733983 & 3.901313 & -1.005704 \\
\hline $\mathrm{C}$ & 2.936405 & 4.495207 & -0.257676 \\
\hline $\mathrm{H}$ & 3.812950 & 3.844140 & -0.339871 \\
\hline
\end{tabular}




$\begin{array}{lrrr}\mathrm{H} & 2.716163 & 4.622697 & 0.806182 \\ \mathrm{H} & 3.190917 & 5.474002 & -0.680656 \\ \mathrm{C} & 2.060063 & 3.722677 & -2.502475 \\ \mathrm{H} & 2.928364 & 3.066811 & -2.639668 \\ \mathrm{H} & 1.212962 & 3.285178 & -3.037269 \\ \mathrm{H} & 2.296368 & 4.694769 & -2.950734 \\ \mathrm{C} & 0.498705 & 4.818202 & -0.845701 \\ \mathrm{H} & 0.236258 & 4.944049 & 0.211253 \\ \mathrm{H} & -0.368353 & 4.402189 & -1.367242 \\ \mathrm{H} & 0.715412 & 5.808342 & -1.263479\end{array}$

\section{TS2-cross-AC}

\begin{tabular}{|c|c|c|c|}
\hline u & -0.348122 & 0.501833 & -0.097843 \\
\hline $\mathrm{O}$ & -1.785566 & 0.174709 & 1.793709 \\
\hline $\mathrm{O}$ & -1.983747 & 1.922475 & 0.466496 \\
\hline $\mathrm{N}$ & 0.309229 & -2.076594 & -1.210396 \\
\hline$\Lambda$ & 2.213552 & -1.250147 & -0.513942 \\
\hline $\mathrm{C}$ & 0.864826 & -1.023819 & -0.560940 \\
\hline $\mathrm{C}$ & 1.240987 & -3.172663 & -1.474218 \\
\hline $\mathrm{H}$ & 1.153463 & -3.526807 & -2.504795 \\
\hline $\mathrm{H}$ & 1.045506 & -4.020345 & -0.803392 \\
\hline C & 2.598418 & -2.505055 & -1.192095 \\
\hline $\mathrm{H}$ & 3.243930 & -3.105228 & -0.543796 \\
\hline $\mathrm{H}$ & 3.150106 & -2.279132 & -2.112475 \\
\hline $\mathrm{C}$ & -1.145424 & -2.220103 & -1.187701 \\
\hline 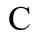 & -1.576531 & -3.051385 & 0.046990 \\
\hline 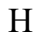 & -1.187957 & -2.564205 & 0.949384 \\
\hline $\mathrm{H}$ & -1.141444 & -4.059619 & -0.009842 \\
\hline $\mathrm{C}$ & -3.113496 & -3.143868 & 0.108874 \\
\hline $\mathrm{H}$ & -3.404032 & -3.754108 & 0.974279 \\
\hline $\mathrm{C}$ & -3.648302 & -3.788025 & -1.184909 \\
\hline $\mathrm{H}$ & -3.258479 & -4.810333 & -1.293880 \\
\hline $\mathrm{H}$ & -4.743069 & -3.863590 & -1.139864 \\
\hline $\mathrm{C}$ & -3.227999 & -2.929309 & -2.395473 \\
\hline $\mathrm{H}$ & -3.618358 & -3.375717 & -3.319336 \\
\hline $\mathrm{C}$ & -1.684582 & -2.892439 & -2.468054 \\
\hline $\mathrm{H}$ & -1.348099 & -2.324561 & -3.345541 \\
\hline $\mathrm{H}$ & -1.297133 & -3.917508 & -2.569011 \\
\hline $\mathrm{C}$ & -3.793902 & -1.499735 & -2.231119 \\
\hline $\mathrm{H}$ & -3.530759 & -0.887798 & -3.104511 \\
\hline $\mathrm{H}$ & -4.891611 & -1.543966 & -2.187158 \\
\hline $\mathrm{C}$ & -3.232336 & -0.853213 & -0.934743 \\
\hline $\mathrm{H}$ & -3.631364 & 0.161920 & -0.832663 \\
\hline $\mathrm{C}$ & -1.700514 & -0.789173 & -1.092288 \\
\hline $\mathrm{H}$ & -1.513419 & -0.312319 & -2.068031 \\
\hline $\mathrm{C}$ & -3.682822 & -1.721236 & 0.259805 \\
\hline $\mathrm{H}$ & -3.337106 & -1.287811 & 1.198094 \\
\hline $\mathrm{H}$ & -4.781580 & -1.761553 & 0.290022 \\
\hline $\mathrm{C}$ & 3.121183 & -0.652063 & 0.415432 \\
\hline $\mathrm{C}$ & 3.076872 & -1.076494 & 1.762954 \\
\hline $\mathrm{C}$ & 3.940415 & -0.454144 & 2.672325 \\
\hline $\mathrm{H}$ & 3.909877 & -0.741404 & 3.719676 \\
\hline $\mathrm{C}$ & 4.840249 & 0.523976 & 2.253146 \\
\hline $\mathrm{C}$ & 4.917740 & 0.872984 & 0.906670 \\
\hline $\mathrm{H}$ & 5.652073 & 1.605609 & 0.585157 \\
\hline $\mathrm{C}$ & 4.068620 & 0.286796 & -0.040917 \\
\hline $\mathrm{C}$ & 2.161305 & -2.205482 & 2.235406 \\
\hline $\mathrm{H}$ & 1.637896 & -2.610350 & 1.365057 \\
\hline $\mathrm{C}$ & 4.243832 & 0.586482 & -1.526239 \\
\hline $\mathrm{H}$ & 3.377655 & 0.171835 & -2.052056 \\
\hline
\end{tabular}

\begin{tabular}{|c|c|c|c|}
\hline $\mathrm{C}$ & 0.772742 & 1.421513 & 1.56052 \\
\hline $\mathrm{H}$ & 1.464521 & 0.700395 & 1.970011 \\
\hline $\mathrm{C}$ & 1.239002 & 2.313961 & 0.56683 \\
\hline $\mathrm{H}$ & 2.245636 & 2.140329 & 0.20155 \\
\hline $\mathrm{C}$ & 0.755209 & 3.745205 & 0.52387 \\
\hline $\mathrm{H}$ & 1.244285 & 4.294170 & 1.3402 \\
\hline $\mathrm{H}$ & 1.012603 & 4.241781 & -0.41504 \\
\hline $\mathrm{H}$ & -0.325775 & 3.794999 & 0.67766 \\
\hline $\mathrm{H}$ & 0.026964 & 1.793732 & 2.25809 \\
\hline $\mathrm{C}$ & 5.499813 & -0.144866 & -2.046625 \\
\hline $\mathrm{H}$ & 5.604861 & -0.007384 & -3.129135 \\
\hline $\mathrm{H}$ & 6.404536 & 0.245969 & -1.565613 \\
\hline $\mathrm{H}$ & 5.452480 & -1.220056 & -1.838763 \\
\hline $\mathrm{C}$ & 4.315238 & 2.085843 & -1.865782 \\
\hline $\mathrm{H}$ & 5.145240 & 2.581152 & -1.349106 \\
\hline $\mathrm{H}$ & 4.474402 & 2.213140 & -2.942198 \\
\hline $\mathrm{H}$ & 3.387888 & 2.610447 & -1.619683 \\
\hline $\mathrm{C}$ & 2.994252 & -3.355027 & 2.83727 \\
\hline $\mathrm{H}$ & 3.766163 & -3.697961 & 2.138275 \\
\hline $\mathrm{H}$ & 3.496344 & -3.047101 & 3.76157 \\
\hline $\mathrm{H}$ & 2.346682 & -4.205741 & 3.079393 \\
\hline $\mathrm{C}$ & 1.074094 & -1.736529 & 3.222050 \\
\hline $\mathrm{H}$ & 0.515027 & -2.601935 & 3.597759 \\
\hline $\mathrm{H}$ & 1.515037 & -1.224092 & 4.085757 \\
\hline $\mathrm{H}$ & 0.358108 & -1.062231 & 2.744980 \\
\hline $\mathrm{H}$ & 5.499716 & 0.998693 & 2.97485 \\
\hline $\mathrm{C}$ & 0.559088 & 1.506599 & -1.416774 \\
\hline $\mathrm{H}$ & 1.538583 & 1.241888 & -1.822661 \\
\hline $\mathrm{C}$ & 0.073046 & 2.714586 & -2.177677 \\
\hline $\mathrm{O}$ & 0.852730 & 3.318427 & -2.925119 \\
\hline $\mathrm{N}$ & -1.225939 & 3.081112 & -2.021732 \\
\hline $\mathrm{H}$ & -1.749238 & 2.665393 & -1.256325 \\
\hline $\mathrm{C}$ & -1.719931 & 4.303016 & -2.628220 \\
\hline $\mathrm{H}$ & -2.811137 & 4.315909 & -2.567368 \\
\hline $\mathrm{H}$ & -1.322212 & 5.197411 & -2.128056 \\
\hline $\mathrm{H}$ & -1.412462 & 4.343158 & -3.676121 \\
\hline $\mathrm{C}$ & -2.375418 & 1.245275 & 1.483257 \\
\hline $\mathrm{C}$ & -3.552916 & 1.783246 & 2.304629 \\
\hline $\mathrm{C}$ & -3.192272 & 3.208635 & 2.773481 \\
\hline $\mathrm{H}$ & -2.989712 & 3.859663 & 1.918094 \\
\hline $\mathrm{H}$ & -4.019397 & 3.636801 & 3.352372 \\
\hline $\mathrm{H}$ & -2.301963 & 3.198416 & 3.414526 \\
\hline $\mathrm{C}$ & -4.794633 & 1.837303 & 1.389467 \\
\hline $\mathrm{H}$ & -5.075464 & 0.834261 & 1.048959 \\
\hline $\mathrm{H}$ & -5.646136 & 2.261297 & 1.935305 \\
\hline $\mathrm{H}$ & -4.601253 & 2.457273 & 0.508692 \\
\hline $\mathrm{C}$ & -3.826703 & 0.879635 & 3.515442 \\
\hline $\mathrm{H}$ & -2.947176 & 0.811182 & 4.16323 \\
\hline $\mathrm{H}$ & -4.661080 & 1.283117 & 4.10193 \\
\hline $\mathrm{H}$ & -4.084920 & -0.135771 & 3.20033 \\
\hline
\end{tabular}

\section{TS2-homo-AC}

$\begin{array}{lccc}\mathrm{Ru} & -0.344078 & 0.265813 & -0.114925 \\ \mathrm{O} & -1.736689 & -0.018787 & 1.813357 \\ \mathrm{O} & -1.972578 & 1.723179 & 0.487982 \\ \mathrm{~N} & 0.284054 & -2.326788 & -1.198366 \\ \mathrm{~N} & 2.199249 & -1.509512 & -0.511757 \\ \mathrm{C} & 0.853992 & -1.275660 & -0.559960 \\ \mathrm{C} & 1.208010 & -3.431128 & -1.458127 \\ \mathrm{H} & 1.106321 & -3.798222 & -2.482571\end{array}$




\begin{tabular}{|c|c|c|c|}
\hline $\mathrm{H}$ & 1.016975 & -4.267758 & -0.772433 \\
\hline $\mathrm{C}$ & 2.569806 & -2.763354 & -1.202377 \\
\hline $\mathrm{H}$ & 3.230621 & -3.363547 & -0.570564 \\
\hline $\mathrm{H}$ & 3.097314 & -2.531553 & -2.134621 \\
\hline $\mathrm{C}$ & -1.170697 & -2.466962 & -1.145741 \\
\hline $\mathrm{C}$ & -1.575799 & -3.284862 & 0.106812 \\
\hline $\mathrm{H}$ & -1.164948 & -2.791460 & 0.995954 \\
\hline $\mathrm{H}$ & -1.145034 & -4.294641 & 0.049899 \\
\hline $\mathrm{C}$ & -3.110743 & -3.372927 & 0.204836 \\
\hline $\mathrm{H}$ & -3.382751 & -3.973702 & 1.082684 \\
\hline $\mathrm{C}$ & -3.676265 & -4.028419 & -1.070069 \\
\hline $\mathrm{H}$ & -3.291287 & -5.052591 & -1.177089 \\
\hline $\mathrm{H}$ & -4.769821 & -4.100928 & -0.999515 \\
\hline $\mathrm{C}$ & -3.281389 & -3.182932 & -2.298338 \\
\hline $\mathrm{H}$ & -3.693555 & -3.637207 & -3.208554 \\
\hline $\mathrm{C}$ & -1.739633 & -3.150391 & -2.406944 \\
\hline $\mathrm{H}$ & -1.421710 & -2.592387 & -3.297363 \\
\hline $\mathrm{H}$ & -1.356535 & -4.177097 & -2.505720 \\
\hline $\mathrm{C}$ & -3.841757 & -1.750891 & -2.135242 \\
\hline $\mathrm{H}$ & -3.598545 & -1.147843 & -3.020382 \\
\hline $\mathrm{H}$ & -4.938053 & -1.792944 & -2.064818 \\
\hline $\mathrm{C}$ & -3.249141 & -1.092291 & -0.858604 \\
\hline $\mathrm{H}$ & -3.643776 & -0.075069 & -0.757823 \\
\hline $\mathrm{C}$ & -1.722048 & -1.034576 & -1.055188 \\
\hline $\mathrm{H}$ & -1.558720 & -0.571830 & -2.041727 \\
\hline $\mathrm{C}$ & -3.672920 & -1.947493 & 0.354876 \\
\hline $\mathrm{H}$ & -3.306002 & -1.506476 & 1.281962 \\
\hline $\mathrm{H}$ & -4.770663 & -1.984827 & 0.410705 \\
\hline $\mathrm{C}$ & 3.110052 & -0.937449 & 0.434222 \\
\hline $\mathrm{C}$ & 3.035941 & -1.367932 & 1.778447 \\
\hline $\mathrm{C}$ & 3.907250 & -0.776937 & 2.701587 \\
\hline $\mathrm{H}$ & 3.856303 & -1.069976 & 3.746543 \\
\hline $\mathrm{C}$ & 4.841318 & 0.175375 & 2.298986 \\
\hline $\mathrm{C}$ & 4.941987 & 0.534328 & 0.956721 \\
\hline $\mathrm{H}$ & 5.691373 & 1.257371 & 0.650497 \\
\hline $\mathrm{C}$ & 4.085922 & -0.018281 & -0.003996 \\
\hline $\mathrm{C}$ & 2.083668 & -2.472175 & 2.235985 \\
\hline $\mathrm{H}$ & 1.551748 & -2.853950 & 1.360432 \\
\hline $\mathrm{C}$ & 4.257280 & 0.319024 & -1.481007 \\
\hline $\mathrm{H}$ & 3.348119 & -0.003202 & -1.999416 \\
\hline $\mathrm{C}$ & 0.802744 & 1.226608 & 1.501949 \\
\hline $\mathrm{H}$ & 1.449062 & 0.493517 & 1.964200 \\
\hline $\mathrm{H}$ & 0.094158 & 1.698201 & 2.176263 \\
\hline $\mathrm{C}$ & 5.435937 & -0.492787 & -2.059988 \\
\hline $\mathrm{H}$ & 5.533130 & -0.313891 & -3.137114 \\
\hline $\mathrm{H}$ & 6.377576 & -0.199020 & -1.580692 \\
\hline $\mathrm{H}$ & 5.305040 & -1.569109 & -1.899880 \\
\hline $\mathrm{C}$ & 4.454524 & 1.819523 & -1.767114 \\
\hline $\mathrm{H}$ & 5.406323 & 2.182218 & -1.361229 \\
\hline $\mathrm{H}$ & 4.478788 & 1.983544 & -2.850079 \\
\hline $\mathrm{H}$ & 3.656047 & 2.445532 & -1.356655 \\
\hline $\mathrm{C}$ & 2.875841 & -3.652983 & 2.832068 \\
\hline $\mathrm{H}$ & 3.637918 & -4.016185 & 2.132597 \\
\hline $\mathrm{H}$ & 3.385418 & -3.368132 & 3.759560 \\
\hline $\mathrm{H}$ & 2.199810 & -4.483589 & 3.066337 \\
\hline $\mathrm{C}$ & 1.008278 & -1.975170 & 3.221322 \\
\hline $\mathrm{H}$ & 0.410220 & -2.822121 & 3.578802 \\
\hline $\mathrm{H}$ & 1.462363 & -1.495128 & 4.096654 \\
\hline $\mathrm{H}$ & 0.324596 & -1.264539 & 2.749604 \\
\hline U & 5.505272 & 0.626647 & 3.031393 \\
\hline
\end{tabular}

\begin{tabular}{|c|c|c|c|}
\hline $\mathrm{C}$ & 0.514223 & 1.223450 & -1.508794 \\
\hline $\mathrm{H}$ & 1.500002 & 0.989420 & -1.915658 \\
\hline $\mathrm{C}$ & -0.125764 & 2.224268 & -2.441076 \\
\hline $\mathrm{O}$ & 0.392815 & 2.400242 & -3.546597 \\
\hline $\mathrm{N}$ & -1.284508 & 2.825841 & -2.066899 \\
\hline $\mathrm{H}$ & -1.740636 & 2.525773 & -1.212487 \\
\hline $\mathrm{C}$ & -1.980354 & 3.714274 & -2.978568 \\
\hline $\mathrm{H}$ & -2.716867 & 4.294959 & -2.416709 \\
\hline $\mathrm{H}$ & -1.261963 & 4.392228 & -3.447460 \\
\hline $\mathrm{H}$ & -2.493056 & 3.163292 & -3.778238 \\
\hline $\mathrm{C}$ & 1.325819 & 1.980028 & 0.425439 \\
\hline $\mathrm{H}$ & 2.310599 & 1.707143 & 0.075387 \\
\hline $\mathrm{C}$ & 1.058768 & 3.447442 & 0.196058 \\
\hline $\mathrm{O}$ & 1.924959 & 4.132586 & -0.345875 \\
\hline $\mathrm{N}$ & -0.119370 & 3.946317 & 0.659566 \\
\hline $\mathrm{H}$ & -0.867069 & 3.278666 & 0.828872 \\
\hline $\mathrm{C}$ & -0.506628 & 5.305358 & 0.321798 \\
\hline $\mathrm{H}$ & -0.804870 & 5.390847 & -0.731968 \\
\hline $\mathrm{H}$ & -1.342050 & 5.606526 & 0.959992 \\
\hline $\mathrm{H}$ & 0.339065 & 5.974489 & 0.491371 \\
\hline $\mathrm{C}$ & -3.495467 & 1.582417 & 2.369099 \\
\hline $\mathrm{C}$ & -3.664391 & 0.739992 & 3.641821 \\
\hline $\mathrm{H}$ & -4.496480 & 1.129749 & 4.240081 \\
\hline $\mathrm{H}$ & -3.872679 & -0.305562 & 3.397170 \\
\hline $\mathrm{H}$ & -2.756885 & 0.762532 & 4.253694 \\
\hline $\mathrm{C}$ & -3.190489 & 3.050235 & 2.735563 \\
\hline $\mathrm{H}$ & -3.108164 & 3.669508 & 1.836995 \\
\hline $\mathrm{H}$ & -3.994534 & 3.456538 & 3.360019 \\
\hline $\mathrm{H}$ & -2.252287 & 3.133906 & 3.297791 \\
\hline $\mathrm{C}$ & -4.780270 & 1.520289 & 1.514279 \\
\hline $\mathrm{H}$ & -5.028804 & 0.486440 & 1.251379 \\
\hline $\mathrm{H}$ & -5.623192 & 1.940565 & 2.075640 \\
\hline $\mathrm{H}$ & -4.659757 & 2.091341 & 0.588309 \\
\hline $\mathrm{C}$ & -2.337282 & 1.046154 & 1.52225 \\
\hline
\end{tabular}

\section{INT4-cross-AC}

$\begin{array}{lccc}\mathrm{Ru} & 0.399384 & -0.455334 & -0.089480 \\ \mathrm{O} & 2.000701 & -0.212066 & 1.820720 \\ \mathrm{O} & 2.012756 & -1.886902 & 0.382619 \\ \mathrm{~N} & -0.359457 & 2.093527 & -1.198765 \\ \mathrm{~N} & -2.233296 & 1.219308 & -0.479678 \\ \mathrm{C} & -0.882984 & 1.026573 & -0.539821 \\ \mathrm{C} & -1.323554 & 3.159139 & -1.471821 \\ \mathrm{H} & -1.259365 & 3.495128 & -2.510410 \\ \mathrm{H} & -1.143809 & 4.024537 & -0.820187 \\ \mathrm{C} & -2.658744 & 2.461013 & -1.158178 \\ \mathrm{H} & -3.304259 & 3.049855 & -0.499638 \\ \mathrm{H} & -3.223397 & 2.217276 & -2.065430 \\ \mathrm{C} & 1.084755 & 2.288798 & -1.154379 \\ \mathrm{C} & 1.471506 & 3.202029 & 0.038093 \\ \mathrm{H} & 1.075576 & 2.758218 & 0.959753 \\ \mathrm{H} & 1.011090 & 4.191761 & -0.087543 \\ \mathrm{C} & 3.004005 & 3.339243 & 0.121424 \\ \mathrm{H} & 3.260286 & 4.006099 & 0.954842 \\ \mathrm{C} & 3.543818 & 3.924597 & -1.198733 \\ \mathrm{H} & 3.128486 & 4.927674 & -1.371354 \\ \mathrm{H} & 4.634939 & 4.033236 & -1.140252 \\ \mathrm{C} & 3.169911 & 2.989899 & -2.367849 \\ \mathrm{H} & 3.563731 & 3.396236 & -3.308257 \\ \mathrm{C} & 1.632285 & 2.894633 & -2.465567\end{array}$




\begin{tabular}{|c|c|c|c|}
\hline $\mathrm{H}$ & 1.331965 & 2.260850 & -3.310234 \\
\hline $\mathrm{H}$ & 1.205586 & 3.894133 & -2.634172 \\
\hline $\mathrm{C}$ & 3.766504 & 1.587546 & -2.118855 \\
\hline $\mathrm{H}$ & 3.526470 & 0.920253 & -2.957718 \\
\hline $\mathrm{H}$ & 4.861803 & 1.653915 & -2.062087 \\
\hline $\mathrm{C}$ & 3.210120 & 1.002621 & -0.790388 \\
\hline $\mathrm{H}$ & 3.630673 & 0.005430 & -0.630819 \\
\hline $\mathrm{C}$ & 1.689619 & 0.897827 & -0.946649 \\
\hline $\mathrm{H}$ & 1.469999 & 0.270185 & -1.847675 \\
\hline $\mathrm{C}$ & 3.609875 & 1.945425 & 0.363842 \\
\hline $\mathrm{H}$ & 3.258090 & 1.537602 & 1.312756 \\
\hline $\mathrm{H}$ & 4.705828 & 2.016925 & 0.411659 \\
\hline $\mathrm{C}$ & -3.118394 & 0.604954 & 0.462995 \\
\hline $\mathrm{C}$ & -3.044710 & 1.015210 & 1.813307 \\
\hline $\mathrm{C}$ & -3.884369 & 0.379233 & 2.735195 \\
\hline $\mathrm{H}$ & -3.830966 & 0.655481 & 3.784555 \\
\hline $\mathrm{C}$ & -4.789400 & -0.598097 & 2.325996 \\
\hline $\mathrm{C}$ & -4.897041 & -0.931807 & 0.977764 \\
\hline $\mathrm{H}$ & -5.634910 & -1.664506 & 0.665189 \\
\hline $\mathrm{C}$ & -4.072464 & -0.332111 & 0.017081 \\
\hline $\mathrm{C}$ & -2.128223 & 2.146126 & 2.278314 \\
\hline $\mathrm{H}$ & -1.611285 & 2.551971 & 1.404378 \\
\hline $\mathrm{C}$ & -4.281511 & -0.620671 & -1.466039 \\
\hline $\mathrm{H}$ & -3.424842 & -0.209360 & -2.009764 \\
\hline $\mathrm{C}$ & -0.626960 & -1.332689 & 1.427821 \\
\hline $\mathrm{H}$ & -1.415984 & -0.795767 & 1.941354 \\
\hline $\mathrm{C}$ & -1.139918 & -2.258963 & 0.289569 \\
\hline $\mathrm{H}$ & -2.227836 & -2.165955 & 0.296013 \\
\hline $\mathrm{C}$ & -0.730150 & -3.720786 & 0.503318 \\
\hline $\mathrm{H}$ & -1.158467 & -4.086242 & 1.443623 \\
\hline $\mathrm{H}$ & -1.094546 & -4.354720 & -0.312503 \\
\hline $\mathrm{H}$ & 0.356849 & -3.816056 & 0.563873 \\
\hline $\mathrm{H}$ & 0.042976 & -1.834012 & 2.128384 \\
\hline $\mathrm{C}$ & -5.545508 & 0.119357 & -1.954140 \\
\hline $\mathrm{H}$ & -5.675822 & -0.012810 & -3.034575 \\
\hline $\mathrm{H}$ & -6.440436 & -0.270519 & -1.454407 \\
\hline $\mathrm{H}$ & -5.490408 & 1.193500 & -1.742408 \\
\hline $\mathrm{C}$ & -4.368795 & -2.117602 & -1.811925 \\
\hline $\mathrm{H}$ & -5.204449 & -2.605243 & -1.296918 \\
\hline $\mathrm{H}$ & -4.529643 & -2.237722 & -2.888795 \\
\hline $\mathrm{H}$ & -3.446942 & -2.650875 & -1.566627 \\
\hline $\mathrm{C}$ & -2.961852 & 3.294162 & 2.882717 \\
\hline $\mathrm{H}$ & -3.735638 & 3.636653 & 2.185440 \\
\hline $\mathrm{H}$ & -3.462242 & 2.983574 & 3.807014 \\
\hline $\mathrm{H}$ & -2.315569 & 4.145890 & 3.125150 \\
\hline $\mathrm{C}$ & -1.035036 & 1.681751 & 3.259905 \\
\hline $\mathrm{H}$ & -0.467812 & 2.548117 & 3.621434 \\
\hline $\mathrm{H}$ & -1.470622 & 1.181395 & 4.133181 \\
\hline $\mathrm{H}$ & -0.330131 & 0.996299 & 2.782688 \\
\hline $\mathrm{H}$ & -5.429793 & -1.084102 & 3.057307 \\
\hline $\mathrm{C}$ & -0.736435 & -1.682862 & -1.178606 \\
\hline $\mathrm{H}$ & -1.624371 & -1.273807 & -1.655255 \\
\hline $\mathrm{C}$ & -0.185899 & -2.707167 & -2.154514 \\
\hline $\mathrm{O}$ & -0.929297 & -3.172786 & -3.023188 \\
\hline $\mathrm{N}$ & 1.119498 & -3.072874 & -2.044131 \\
\hline $\mathrm{H}$ & 1.648793 & -2.754386 & -1.238159 \\
\hline $\mathrm{C}$ & 1.655021 & -4.146564 & -2.860378 \\
\hline $\mathrm{H}$ & 2.746719 & -4.128916 & -2.801674 \\
\hline $\mathrm{H}$ & 1.295700 & -5.131435 & -2.530554 \\
\hline $\mathrm{H}$ & 1.343837 & -4.009362 & -3.898999 \\
\hline
\end{tabular}

$\begin{array}{llll}\mathrm{C} & 2.491747 & -1.294976 & 1.421247 \\ \mathrm{C} & 3.653070 & -1.976608 & 2.156495 \\ \mathrm{C} & 3.116429 & -3.301876 & 2.739175 \\ \mathrm{H} & 2.734255 & -3.949716 & 1.944260 \\ \mathrm{H} & 3.915397 & -3.833720 & 3.269933 \\ \mathrm{H} & 2.302658 & -3.116203 & 3.450735 \\ \mathrm{C} & 4.777112 & -2.273316 & 1.143552 \\ \mathrm{H} & 5.174348 & -1.345379 & 0.714461 \\ \mathrm{H} & 5.604133 & -2.796045 & 1.639071 \\ \mathrm{H} & 4.409117 & -2.898076 & 0.324603 \\ \mathrm{C} & 4.177373 & -1.073954 & 3.282176 \\ \mathrm{H} & 3.386233 & -0.842147 & 4.001120 \\ \mathrm{H} & 4.998481 & -1.572042 & 3.812013 \\ \mathrm{H} & 4.548912 & -0.124580 & 2.882703\end{array}$

\section{INT4-homo-AC}

$\mathrm{Ru} \quad-0.389081$

O $\quad-1.959536$

O $\quad-1.957313$

$\mathrm{N} \quad 0.262383$

$\mathrm{N} \quad 2.168041$

C $\quad 0.827933$

C $\quad 1.186039$

$\mathrm{H} \quad 1.103117$

H 0.979549

C $\quad 2.545764$

$\mathrm{H} \quad 3.185704$

H 3.099072

C $\quad-1.188872$

C $\quad-1.615952$

$\mathrm{H} \quad-1.202270$

H $\quad-1.199099$

C -3.153326

H $\quad-3.439446$

C $\quad-3.715537$

$\mathrm{H} \quad-3.343018$

H $\quad-4.810339$

C $\quad-3.299869$

H $\quad-3.708772$

C $\quad-1.759182$

H $\quad-1.430239$

H $\quad-1.375260$

C -3.837722

H $\quad-3.568616$

H -4.934862

C $\quad-3.258735$

$\mathrm{H} \quad-3.635730$

C $\quad-1.736030$

H $\quad-1.488996$

C $\quad-3.700342$

H $\quad-3.333888$

$\mathrm{H} \quad-4.798421$

C $\quad 3.073135$

C $\quad 2.972511$

C $\quad 3.831389$

H $\quad 3.758768$

C $\quad 4.777337$

C 4.905423

$\begin{array}{ll}\mathrm{C} & 4.905423 \\ \mathrm{H} & 5.665105\end{array}$

C 4.064692
0.210787

0.095949

1.744675

$-2.414372$

$-1.581579$

$-1.342161$

$-3.528178$

$-3.918663$

$-4.347730$

$-2.859704$

$-3.442515$

$-2.662757$

$-2.548956$

$-3.392837$

$-2.928525$

$-4.405994$

$-3.460340$

$-4.078683$

$-4.078516$

$-5.104759$

$-4.138230$

$-3.212104$

$-3.641527$

$-3.186478$

$-2.602922$

$-4.209463$

$-1.776578$

$-1.156867$

$-1.794202$

$-1.158614$

$-0.138264$

$-1.127031$

$-0.551909$

$-2.032619$

$-1.601412$

$-2.055936$

$-0.973168$

$-1.338164$

$-0.709040$

$-0.949535$

0.218889

0.510024

1.212528

$-0.085353$
$-0.131386$

1.799566

0.332969

$-1.115341$

$-0.425536$

$-0.503668$

$-1.332649$

$-2.350334$

$-0.631422$

$-1.065209$

$-0.396663$

$-1.990324$

$-1.068120$

0.160936

1.064469

0.078848

0.244433

1.105003

$-1.051150$

$-1.178355$

$-0.991870$

$-2.258096$

$-3.181480$

$-2.354432$

$-3.224093$

$-2.478242$

$-2.072099$

$-2.938121$

$-2.015901$

$-0.768398$

$-0.654400$

$-0.923582$

$-1.852029$

0.424150

1.357346

0.471001

0.504644

1.866237

2.775099

3.832055

2.344069

0.987997

0.660202

0.038806 


\begin{tabular}{|c|c|c|c|}
\hline $\mathrm{C}$ & 1.998516 & -2.406125 & 2.361373 \\
\hline $\mathrm{H}$ & 1.482047 & -2.830238 & 1.495666 \\
\hline $\mathrm{C}$ & 4.278008 & 0.173542 & -1.448439 \\
\hline $\mathrm{H}$ & 3.388045 & -0.180743 & -1.978221 \\
\hline $\mathrm{C}$ & 0.687320 & 1.132754 & 1.324383 \\
\hline $\mathrm{H}$ & 1.457392 & 0.594074 & 1.864480 \\
\hline $\mathrm{H}$ & 0.061157 & 1.711263 & 2.002848 \\
\hline $\mathrm{C}$ & 5.477650 & -0.660152 & -1.947684 \\
\hline $\mathrm{H}$ & 5.609554 & -0.533032 & -3.028485 \\
\hline $\mathrm{H}$ & 6.402486 & -0.339971 & -1.452950 \\
\hline $\mathrm{H}$ & 5.344139 & -1.728244 & -1.740016 \\
\hline $\mathrm{C}$ & 4.475638 & 1.658619 & -1.806041 \\
\hline $\mathrm{H}$ & 5.421104 & 2.043022 & -1.405294 \\
\hline $\mathrm{H}$ & 4.511777 & 1.769946 & -2.895073 \\
\hline $\mathrm{H}$ & 3.670397 & 2.299640 & -1.435713 \\
\hline $\mathrm{C}$ & 2.763023 & -3.561134 & 3.038617 \\
\hline $\mathrm{H}$ & 3.535044 & -3.971474 & 2.377040 \\
\hline $\mathrm{H}$ & 3.256487 & -3.231266 & 3.959830 \\
\hline $\mathrm{H}$ & 2.071848 & -4.369692 & 3.304066 \\
\hline $\mathrm{C}$ & 0.908367 & -1.842927 & 3.293409 \\
\hline $\mathrm{H}$ & 0.286326 & -2.660928 & 3.676981 \\
\hline $\mathrm{H}$ & 1.350404 & -1.328341 & 4.155058 \\
\hline $\mathrm{H}$ & 0.253004 & -1.142209 & 2.769863 \\
\hline $\mathrm{H}$ & 5.429691 & 0.703109 & 3.065765 \\
\hline $\mathrm{C}$ & 0.781410 & 1.303659 & -1.323842 \\
\hline $\mathrm{H}$ & 1.644098 & 0.835626 & -1.789402 \\
\hline $\mathrm{C}$ & 0.184121 & 2.190709 & -2.401799 \\
\hline $\mathrm{O}$ & 0.736709 & 2.250906 & -3.500828 \\
\hline $\mathrm{N}$ & -0.970972 & 2.865709 & -2.138840 \\
\hline $\mathrm{H}$ & -1.498812 & 2.637482 & -1.305190 \\
\hline $\mathrm{C}$ & -1.602691 & 3.660949 & -3.175727 \\
\hline $\mathrm{H}$ & -2.381848 & 4.280006 & -2.722239 \\
\hline $\mathrm{H}$ & -0.857560 & 4.305012 & -3.649724 \\
\hline $\mathrm{H}$ & -2.052079 & 3.036344 & -3.959779 \\
\hline $\mathrm{C}$ & 1.252487 & 1.921441 & 0.113213 \\
\hline $\mathrm{H}$ & 2.324214 & 1.737779 & 0.107398 \\
\hline $\mathrm{C}$ & 1.094680 & 3.445275 & 0.129960 \\
\hline $\mathrm{O}$ & 2.006738 & 4.138488 & -0.311478 \\
\hline $\mathrm{N}$ & -0.055319 & 3.953586 & 0.647962 \\
\hline $\mathrm{H}$ & -0.833345 & 3.309830 & 0.756821 \\
\hline $\mathrm{C}$ & -0.372087 & 5.359314 & 0.462462 \\
\hline $\mathrm{H}$ & -0.684555 & 5.569492 & -0.569996 \\
\hline $\mathrm{H}$ & -1.176706 & 5.639132 & 1.147851 \\
\hline $\mathrm{H}$ & 0.514961 & 5.958089 & 0.677252 \\
\hline $\mathrm{C}$ & -3.589806 & 1.878608 & 2.123751 \\
\hline $\mathrm{C}$ & -3.779386 & 1.270395 & 3.521357 \\
\hline $\mathrm{H}$ & -4.623469 & 1.752182 & 4.029111 \\
\hline $\mathrm{H}$ & -3.974983 & 0.196495 & 3.457895 \\
\hline $\mathrm{H}$ & -2.882950 & 1.407886 & 4.135341 \\
\hline $\mathrm{C}$ & -3.294356 & 3.387269 & 2.231840 \\
\hline $\mathrm{H}$ & -3.196941 & 3.840482 & 1.240733 \\
\hline $\mathrm{H}$ & -4.108749 & 3.894033 & 2.762550 \\
\hline $\mathrm{H}$ & -2.365931 & 3.570844 & 2.786606 \\
\hline $\mathrm{C}$ & -4.862496 & 1.659528 & 1.275247 \\
\hline $\mathrm{H}$ & -5.101859 & 0.593114 & 1.194867 \\
\hline $\mathrm{H}$ & -5.716274 & 2.164741 & 1.742414 \\
\hline $\mathrm{H}$ & -4.732811 & 2.062081 & 0.265074 \\
\hline $\mathrm{C}$ & -2.430691 & 1.183140 & 1.397677 \\
\hline
\end{tabular}

\begin{tabular}{|c|c|c|}
\hline 0.487045 & 0.105534 & \\
\hline 2.215582 & 1.227943 & -0.821666 \\
\hline 3.105490 & 1.558701 & 1.21362 \\
\hline-0.586180 & -2.493753 & 0.41462 \\
\hline-2.367284 & -1.313754 & -0.045741 \\
\hline-1.006114 & -1.244303 & 0.083297 \\
\hline-1.621866 & -3.517431 & 0.279448 \\
\hline-1.588900 & -4.235762 & 1.10260 \\
\hline-1.500652 & -4.066702 & -0.664403 \\
\hline-2.889969 & -2.658399 & 0.281210 \\
\hline-3.629706 & -2.972417 & -0.458959 \\
\hline-3.373154 & -2.641369 & 1.265572 \\
\hline 0.845565 & -2.765153 & 0.35650 \\
\hline 1.251214 & -3.227298 & -1.069535 \\
\hline 0.907690 & -2.480377 & -1.797414 \\
\hline 0.748394 & -4.175300 & -1.306984 \\
\hline 2.777385 & -3.402853 & -1.153510 \\
\hline 3.049401 & -3.733244 & -2.164558 \\
\hline 3.217098 & -4.464013 & -0.123862 \\
\hline 2.753711 & -5.432899 & -0.358236 \\
\hline 4.304679 & -4.606784 & -0.173240 \\
\hline 2.813736 & -4.008982 & 4527 \\
\hline 3.127833 & -4.763042 & .027670 \\
\hline 1.279182 & -3.845825 & 1.368888 \\
\hline 0.969306 & -3.545740 & 2.378494 \\
\hline 0.79 & -4.80 & 213 \\
\hline 3.494095 & -2.660817 & 1.603143 \\
\hline 3.240664 & -2.329027 & 2.618895 \\
\hline 4.586111 & -2.778573 & 749 \\
\hline 3.051335 & -1.58 & 0.573479 \\
\hline 3.53 & -0.647503 & 0595 \\
\hline 1.527289 & -1.423810 & .691701 \\
\hline 1.26 & -1.1 & 1.7 \\
\hline 3.448915 & -2.052057 & -0.841663 \\
\hline 3.148282 & -1.295965 & -1.576237 \\
\hline 4.54 & -2.15 & -0.909371 \\
\hline-3.30 & -0.22 & 67 \\
\hline-3.950285 & 0.180398 & 1.176447 \\
\hline-4.855641 & 1.246502 & 1.100295 \\
\hline-5.36 & 1.583381 & -2.000027 \\
\hline-5.12 & 1.87 & 40 \\
\hline-4.50 & 1.432101 & 105 \\
\hline 4.734114 & 1.920610 & 221854 \\
\hline-3.59 & & \\
\hline-3.748962 & -0.556101 & -2.49 \\
\hline-2.869354 & -1.198512 & -2.384878 \\
\hline-2.998457 & -0.131145 & 2.572224 \\
\hline-2.25 & & \\
\hline-0.13 & 71 & -20 \\
\hline-0.883708 & -0.136247 & -2.558811 \\
\hline-0.7 & & \\
\hline 79 & 97 & \\
\hline-0.129541 & 3.068731 & -1.944128 \\
\hline-0.262311 & 3.093919 & -3.031717 \\
\hline-0.616818 & 3.941778 & -1.504422 \\
\hline & & 17272 \\
\hline 0.695003 & 0.744910 & -2.758683 \\
\hline-3.501680 & 0.381748 & -3.691038 \\
\hline-3.279378 & -0.206814 & -4.58863 \\
\hline-4.382 & 0.9943 & -3.912 \\
\hline
\end{tabular}




$\begin{array}{lrrr}\mathrm{H} & -2.660723 & 1.057617 & -3.512064 \\ \mathrm{C} & -4.963646 & -1.464325 & -2.781984 \\ \mathrm{H} & -4.804034 & -2.047955 & -3.696424 \\ \mathrm{H} & -5.151344 & -2.161839 & -1.958197 \\ \mathrm{H} & -5.871128 & -0.863825 & -2.917030 \\ \mathrm{C} & -4.101411 & -0.778755 & 3.436235 \\ \mathrm{H} & -4.843775 & -0.033544 & 3.744993 \\ \mathrm{H} & -4.634397 & -1.566665 & 2.891551 \\ \mathrm{H} & -3.669913 & -1.218660 & 4.342919 \\ \mathrm{C} & -2.269500 & 0.965180 & 3.372813 \\ \mathrm{H} & -1.405098 & 1.358856 & 2.833960 \\ \mathrm{H} & -2.931511 & 1.805764 & 3.606974 \\ \mathrm{H} & -1.910374 & 0.550365 & 4.322122 \\ \mathrm{H} & -5.814198 & 2.713473 & 0.148271 \\ \mathrm{C} & -0.668580 & 1.686779 & 0.218518 \\ \mathrm{H} & -1.652364 & 1.427793 & 0.589943 \\ \mathrm{C} & -0.308172 & 2.970534 & 0.955490 \\ \mathrm{O} & -1.112342 & 3.906961 & 0.897474 \\ \mathrm{~N} & 0.803048 & 2.997998 & 1.712730 \\ \mathrm{H} & 1.518152 & 2.272083 & 1.605822 \\ \mathrm{C} & 1.176733 & 4.210981 & 2.419930 \\ \mathrm{H} & 2.122370 & 4.028908 & 2.935030 \\ \mathrm{H} & 1.295195 & 5.054722 & 1.729151 \\ \mathrm{H} & 0.407458 & 4.484753 & 3.149699 \\ \mathrm{C} & 3.147992 & 1.636729 & -0.031939 \\ \mathrm{C} & 4.380021 & 2.250686 & -0.733297 \\ \mathrm{C} & 5.373842 & 2.778114 & 0.311137 \\ \mathrm{H} & 6.251143 & 3.210026 & -0.186457 \\ \mathrm{H} & 4.913127 & 3.549861 & 0.935854 \\ \mathrm{H} & 5.707272 & 1.975858 & 0.975966 \\ \mathrm{C} & 5.045455 & 1.155436 & -1.591092 \\ \mathrm{H} & 4.344676 & 0.768450 & -2.336779 \\ \mathrm{H} & 5.922130 & 1.560623 & -2.111723 \\ \mathrm{H} & 5.380093 & 0.316547 & -0.969180 \\ \mathrm{C} & 3.908413 & 3.401712 & -1.643926 \\ \mathrm{H} & 4.767723 & 3.870153 & -2.139824 \\ \mathrm{H} & 3.221892 & 3.033840 & -2.411479 \\ \mathrm{H} & 3.390442 & 4.175936 & -1.064370\end{array}$

\begin{tabular}{lccc}
\multicolumn{2}{l}{ INT4-homo-2-AC } & & \\
$\mathrm{Ru}$ & -0.483880 & 0.108095 & -0.233847 \\
$\mathrm{O}$ & -2.224007 & -1.064844 & -0.725749 \\
$\mathrm{O}$ & -2.506679 & -0.847232 & 1.470034 \\
$\mathrm{~N}$ & 0.660888 & 2.724514 & 0.085517 \\
$\mathrm{~N}$ & 2.407301 & 1.414177 & 0.015725 \\
$\mathrm{C}$ & 1.041168 & 1.428157 & 0.041067 \\
$\mathrm{C}$ & 1.758710 & 3.690047 & 0.062895 \\
$\mathrm{H}$ & 1.801249 & 4.249180 & 1.005500 \\
$\mathrm{H}$ & 1.634898 & 4.409050 & -0.753291 \\
$\mathrm{C}$ & 2.976746 & 2.773606 & -0.147185 \\
$\mathrm{H}$ & 3.401088 & 2.880772 & -1.150824 \\
$\mathrm{H}$ & 3.777225 & 2.943662 & 0.576635 \\
$\mathrm{C}$ & -0.749807 & 3.038763 & -0.106816 \\
$\mathrm{C}$ & -1.043396 & 3.267351 & -1.614154 \\
$\mathrm{H}$ & -0.687839 & 2.398273 & -2.182125 \\
$\mathrm{H}$ & -0.480940 & 4.143549 & -1.967776 \\
$\mathrm{C}$ & -2.552687 & 3.474531 & -1.831874 \\
$\mathrm{H}$ & -2.747180 & 3.636008 & -2.900048 \\
$\mathrm{C}$ & -3.007063 & 4.710081 & -1.027588 \\
$\mathrm{H}$ & -2.486821 & 5.608128 & -1.389608
\end{tabular}

\begin{tabular}{|c|c|c|c|}
\hline $\mathrm{H}$ & -4.081994 & 4.879829 & -1.173483 \\
\hline $\mathrm{C}$ & -2.712628 & 4.490839 & 0.471416 \\
\hline $\mathrm{H}$ & -3.036558 & 5.369481 & 1.043846 \\
\hline $\mathrm{C}$ & -1.193957 & 4.289779 & 0.679555 \\
\hline $\mathrm{H}$ & -0.965131 & 4.154390 & 1.744845 \\
\hline $\mathrm{H}$ & -0.646753 & 5.178874 & 0.332955 \\
\hline $\mathrm{C}$ & -3.475307 & 3.240380 & 0.954173 \\
\hline $\mathrm{H}$ & -3.303054 & 3.078339 & 2.026616 \\
\hline $\mathrm{H}$ & -4.555693 & 3.390878 & 0.820381 \\
\hline $\mathrm{C}$ & -3.016456 & 1.993430 & 0.153430 \\
\hline $\mathrm{H}$ & -3.561171 & 1.124177 & 0.518472 \\
\hline $\mathrm{C}$ & -1.514305 & 1.803664 & 0.410769 \\
\hline $\mathrm{H}$ & -1.342219 & 1.704205 & 1.494604 \\
\hline $\mathrm{C}$ & -3.302761 & 2.218380 & -1.343690 \\
\hline $\mathrm{H}$ & -2.990025 & 1.335812 & -1.915185 \\
\hline $\mathrm{H}$ & -4.382626 & 2.341712 & -1.502444 \\
\hline $\mathrm{C}$ & 3.290462 & 0.328403 & 0.331531 \\
\hline $\mathrm{C}$ & 4.103552 & -0.216972 & -0.684498 \\
\hline $\mathrm{C}$ & 5.017876 & -1.219726 & -0.335045 \\
\hline $\mathrm{H}$ & 5.647036 & -1.657687 & -1.103577 \\
\hline $\mathrm{C}$ & 5.119964 & -1.671174 & 0.974594 \\
\hline $\mathrm{C}$ & 4.305760 & -1.126063 & 1.966215 \\
\hline $\mathrm{H}$ & 4.391424 & -1.500370 & 2.980048 \\
\hline $\mathrm{C}$ & 3.381780 & -0.117168 & 1.675031 \\
\hline $\mathrm{C}$ & 4.032846 & 0.255308 & -2.132222 \\
\hline $\mathrm{H}$ & 3.142522 & 0.884512 & -2.234154 \\
\hline $\mathrm{C}$ & 2.500351 & 0.468829 & 2.779879 \\
\hline $\mathrm{H}$ & 1.515231 & 0.671389 & 2.344969 \\
\hline $\mathrm{C}$ & 0.300972 & -0.406391 & -1.964075 \\
\hline $\mathrm{H}$ & 1.130530 & 0.138237 & -2.415950 \\
\hline $\mathrm{H}$ & -0.448130 & -0.706516 & -2.699988 \\
\hline $\mathrm{C}$ & 3.898006 & -0.907131 & -3.137183 \\
\hline $\mathrm{H}$ & 3.710034 & -0.508271 & -4.140826 \\
\hline $\mathrm{H}$ & 4.820406 & -1.496398 & -3.189775 \\
\hline $\mathrm{H}$ & 3.087125 & -1.595965 & -2.883227 \\
\hline $\mathrm{C}$ & 5.267531 & 1.111880 & -2.482359 \\
\hline $\mathrm{H}$ & 5.190835 & 1.500767 & -3.504606 \\
\hline $\mathrm{H}$ & 5.385144 & 1.960060 & -1.798963 \\
\hline $\mathrm{H}$ & 6.181805 & 0.510102 & -2.418937 \\
\hline $\mathrm{C}$ & 3.059925 & 1.807051 & 3.302741 \\
\hline $\mathrm{H}$ & 4.070273 & 1.670079 & 3.705963 \\
\hline $\mathrm{H}$ & 3.109418 & 2.566816 & 2.519116 \\
\hline $\mathrm{H}$ & 2.423569 & 2.198283 & 4.105346 \\
\hline $\mathrm{C}$ & 2.282577 & -0.492309 & 3.961741 \\
\hline $\mathrm{H}$ & 1.975068 & -1.488322 & 3.628633 \\
\hline $\mathrm{H}$ & 3.188701 & -0.600426 & 4.569332 \\
\hline $\mathrm{H}$ & 1.501911 & -0.093377 & 4.620052 \\
\hline $\mathrm{H}$ & 5.828243 & -2.455597 & 1.226316 \\
\hline $\mathrm{C}$ & 0.513157 & -1.532569 & 0.439949 \\
\hline $\mathrm{H}$ & 1.423534 & -1.321799 & 0.993929 \\
\hline $\mathrm{C}$ & -0.270194 & -2.603519 & 1.168111 \\
\hline $\mathrm{O}$ & -0.789808 & -3.585463 & 0.645771 \\
\hline $\mathrm{N}$ & -0.280196 & -2.396513 & 2.526818 \\
\hline $\mathrm{H}$ & -0.158001 & -1.428691 & 2.797046 \\
\hline $\mathrm{C}$ & -1.191752 & -3.157848 & 3.368410 \\
\hline $\mathrm{H}$ & -1.222873 & -4.184589 & 3.001310 \\
\hline $\mathrm{H}$ & -0.824741 & -3.152806 & 4.399306 \\
\hline $\mathrm{H}$ & -2.201842 & -2.734474 & 3.332021 \\
\hline $\mathrm{C}$ & 0.841312 & -1.644268 & -1.089326 \\
\hline $\mathrm{H}$ & 1.924995 & -1.554221 & -1.119553 \\
\hline
\end{tabular}




$\begin{array}{lrrr}\mathrm{C} & 0.516924 & -2.985237 & -1.777525 \\ \mathrm{O} & 1.449670 & -3.748412 & -2.009859 \\ \mathrm{~N} & -0.764691 & -3.183156 & -2.171165 \\ \mathrm{H} & -1.458754 & -2.602815 & -1.702159 \\ \mathrm{C} & -1.183457 & -4.516622 & -2.569084 \\ \mathrm{H} & -1.313550 & -5.164161 & -1.692092 \\ \mathrm{H} & -2.128700 & -4.452102 & -3.114532 \\ \mathrm{H} & -0.418642 & -4.951366 & -3.215152 \\ \mathrm{C} & -4.209811 & -2.058863 & 0.214437 \\ \mathrm{C} & -5.226767 & -1.112713 & -0.461799 \\ \mathrm{H} & -4.841577 & -0.748160 & -1.419509 \\ \mathrm{H} & -6.169518 & -1.642865 & -0.644954 \\ \mathrm{H} & -5.447071 & -0.246622 & 0.173762 \\ \mathrm{C} & -3.983827 & -3.296877 & -0.673865 \\ \mathrm{H} & -3.177709 & -3.919277 & -0.272285 \\ \mathrm{H} & -4.900614 & -3.897352 & -0.721867 \\ \mathrm{H} & -3.719003 & -3.003011 & -1.693933 \\ \mathrm{C} & -4.732873 & -2.493149 & 1.590896 \\ \mathrm{H} & -5.712607 & -2.975324 & 1.486365 \\ \mathrm{H} & -4.048665 & -3.209219 & 2.057037 \\ \mathrm{H} & -4.831778 & -1.635586 & 2.262201 \\ \mathrm{C} & -2.888622 & -1.282037 & 0.365387\end{array}$

\section{TS3-cross-AC}

$\begin{array}{lccc}\mathrm{Ru} & 0.488469 & 0.072508 & -0.531608 \\ \mathrm{O} & 2.136451 & 1.543317 & -1.002287 \\ \mathrm{O} & 1.494672 & 1.404900 & 1.103117 \\ \mathrm{~N} & -0.059611 & -2.495083 & 0.664243 \\ \mathrm{~N} & -2.020889 & -1.538153 & 0.501251 \\ \mathrm{C} & -0.683242 & -1.351986 & 0.267618 \\ \mathrm{C} & -0.983481 & -3.584100 & 0.970401 \\ \mathrm{H} & -0.655614 & -4.159510 & 1.839930 \\ \mathrm{H} & -1.073854 & -4.268399 & 0.114272 \\ \mathrm{C} & -2.277384 & -2.805918 & 1.217165 \\ \mathrm{H} & -3.167483 & -3.300496 & 0.822947 \\ \mathrm{H} & -2.434921 & -2.601191 & 2.284177 \\ \mathrm{C} & 1.344375 & -2.674708 & 0.302167 \\ \mathrm{C} & 1.469747 & -3.371850 & -1.074697 \\ \mathrm{H} & 0.869241 & -2.829303 & -1.810873 \\ \mathrm{H} & 1.070335 & -4.394197 & -1.009167 \\ \mathrm{C} & 2.945873 & -3.407069 & -1.506876 \\ \mathrm{H} & 3.026261 & -3.887676 & -2.491067 \\ \mathrm{C} & 3.751448 & -4.213669 & -0.468180 \\ \mathrm{H} & 3.383197 & -5.248687 & -0.427541 \\ \mathrm{H} & 4.808978 & -4.259060 & -0.761273 \\ \mathrm{C} & 3.620624 & -3.544915 & 0.916378 \\ \mathrm{H} & 4.185744 & -4.122367 & 1.659586 \\ \mathrm{C} & 2.129971 & -3.513479 & 1.335987 \\ \mathrm{H} & 2.017802 & -3.065289 & 2.331668 \\ \mathrm{H} & 1.741548 & -4.541697 & 1.388930 \\ \mathrm{C} & 4.188193 & -2.11063 & 0.827522 \\ \mathrm{H} & 4.147888 & -1.622200 & 1.809948 \\ \mathrm{H} & 5.246303 & -2.154502 & 0.531461 \\ \mathrm{C} & 3.371029 & -1.288391 & -0.201117 \\ \mathrm{H} & 3.775031 & -0.276262 & -0.266014 \\ \mathrm{C} & 1.918060 & -1.244583 & 0.298799 \\ \mathrm{H} & 1.926467 & -0.908819 & 1.343762 \\ \mathrm{C} & 3.476471 & -1.960465 & -1.583393 \\ \mathrm{H} & 2.900619 & -1.384108 & -2.317965 \\ \mathrm{H} & 4.523143 & -1.965325 & -1.918127\end{array}$

\begin{tabular}{|c|c|c|c|}
\hline$C$ & -3.024663 & -0.517373 & 0.591492 \\
\hline $\mathrm{C}$ & -4.028519 & -0.470429 & -0.400409 \\
\hline $\mathrm{C}$ & -5.009697 & 0.522167 & -0.297721 \\
\hline $\mathrm{H}$ & -5.789842 & 0.584257 & -1.050847 \\
\hline $\mathrm{C}$ & -5.002782 & 1.429303 & 0.759252 \\
\hline $\mathrm{C}$ & -4.026778 & 1.342543 & 1.747711 \\
\hline $\mathrm{H}$ & -4.034839 & 2.050261 & 2.570598 \\
\hline $\mathrm{C}$ & -3.024618 & 0.366056 & 1.694622 \\
\hline $\mathrm{C}$ & -4.096598 & -1.502498 & -1.522118 \\
\hline $\mathrm{H}$ & -3.143104 & -2.041695 & -1.531609 \\
\hline $\mathrm{C}$ & -2.005174 & 0.273020 & 2.827504 \\
\hline $\mathrm{H}$ & -1.247173 & -0.466952 & 2.555774 \\
\hline $\mathrm{C}$ & 0.039861 & -0.474883 & -2.279339 \\
\hline $\mathrm{H}$ & -0.711897 & -1.196228 & -2.625965 \\
\hline $\mathrm{C}$ & -1.217383 & 1.121308 & -1.859388 \\
\hline $\mathrm{H}$ & -2.087369 & 0.476458 & -1.809298 \\
\hline $\mathrm{C}$ & -1.082889 & 1.848293 & -3.190928 \\
\hline $\mathrm{H}$ & -1.367237 & 1.191235 & -4.018627 \\
\hline $\mathrm{H}$ & -1.753697 & 2.716336 & -3.191632 \\
\hline $\mathrm{H}$ & -0.072321 & 2.215351 & -3.372290 \\
\hline $\mathrm{H}$ & 0.656620 & -0.092981 & -3.104235 \\
\hline $\mathrm{C}$ & -4.297131 & -0.885933 & -2.918925 \\
\hline $\mathrm{H}$ & -4.276638 & -1.673379 & -3.681294 \\
\hline $\mathrm{H}$ & -5.263138 & -0.375461 & -3.001952 \\
\hline $\mathrm{H}$ & -3.515905 & -0.159800 & -3.164196 \\
\hline $\mathrm{C}$ & -5.220126 & -2.521366 & -1.236746 \\
\hline $\mathrm{H}$ & -5.231731 & -3.311039 & -1.997508 \\
\hline $\mathrm{H}$ & -5.101130 & -2.992324 & -0.255024 \\
\hline $\mathrm{H}$ & -6.198990 & -2.027150 & -1.246081 \\
\hline $\mathrm{C}$ & -2.698345 & -0.215122 & 4.116968 \\
\hline $\mathrm{H}$ & -3.437036 & 0.516866 & 4.464550 \\
\hline $\mathrm{H}$ & -3.223323 & -1.165633 & 3.963910 \\
\hline $\mathrm{H}$ & -1.961521 & -0.354293 & 4.916678 \\
\hline $\mathrm{C}$ & -1.260599 & 1.597359 & 3.081868 \\
\hline $\mathrm{H}$ & -0.699569 & 1.925134 & 2.206134 \\
\hline $\mathrm{H}$ & -1.949605 & 2.399569 & 3.368348 \\
\hline $\mathrm{H}$ & -0.545236 & 1.460551 & 3.901291 \\
\hline $\mathrm{H}$ & -5.764965 & 2.201540 & 0.816865 \\
\hline $\mathrm{C}$ & -0.944663 & 1.755295 & -0.595029 \\
\hline $\mathrm{H}$ & -1.654805 & 1.519138 & 0.183462 \\
\hline $\mathrm{C}$ & -0.602231 & 3.220182 & -0.478867 \\
\hline $\mathrm{O}$ & -1.279815 & 3.923495 & 0.272114 \\
\hline $\mathrm{N}$ & 0.362623 & 3.745587 & -1.288013 \\
\hline $\mathrm{H}$ & 1.087294 & 3.113942 & -1.613034 \\
\hline $\mathrm{C}$ & 0.618382 & 5.175100 & -1.280428 \\
\hline $\mathrm{H}$ & 1.245129 & 5.426817 & -2.140490 \\
\hline $\mathrm{H}$ & -0.331007 & 5.711374 & -1.353667 \\
\hline $\mathrm{H}$ & 1.120287 & 5.511660 & -0.364572 \\
\hline $\mathrm{C}$ & 2.273409 & 1.882263 & 0.228781 \\
\hline $\mathrm{C}$ & 3.389208 & 2.839854 & 0.667315 \\
\hline $\mathrm{C}$ & 4.486171 & 1.993851 & 1.354338 \\
\hline $\mathrm{H}$ & 5.255395 & 2.653173 & 1.774477 \\
\hline $\mathrm{H}$ & 4.061730 & 1.394123 & 2.165834 \\
\hline $\mathrm{H}$ & 4.973976 & 1.318012 & 0.643492 \\
\hline $\mathrm{C}$ & 3.988431 & 3.582105 & -0.536369 \\
\hline $\mathrm{H}$ & 3.245230 & 4.223005 & -1.021374 \\
\hline $\mathrm{H}$ & 4.817356 & 4.220205 & -0.207287 \\
\hline $\mathrm{H}$ & 4.364089 & 2.878153 & -1.284428 \\
\hline $\mathrm{C}$ & 2.806121 & 3.834302 & 1.691197 \\
\hline $\mathrm{H}$ & 3.580 & 4.542278 & $2.010 t$ \\
\hline
\end{tabular}




\begin{tabular}{|c|c|c|c|c|c|c|c|}
\hline \multirow{3}{*}{$\begin{array}{l}\mathrm{H} \\
\mathrm{H}\end{array}$} & 1.972763 & 4.401941 & 1.265318 & $\mathrm{H}$ & -4.761009 & -3.025534 & -3.003408 \\
\hline & 2.426712 & 3.304945 & 2.568918 & $\mathrm{H}$ & -4.539343 & -3.356150 & -1.272824 \\
\hline & & & & $\mathrm{H}$ & -5.835728 & -2.279824 & -1.804580 \\
\hline \multicolumn{4}{|c|}{ TS3-homo-AC } & $\mathrm{C}$ & -2.615471 & -2.102798 & 3.948408 \\
\hline $\mathrm{Ru}$ & 0.527833 & 0.016619 & -0.352518 & $\mathrm{H}$ & -3.332203 & -1.495356 & 4.513320 \\
\hline $\mathrm{O}$ & 1.919325 & 1.703142 & -0.605390 & $\mathrm{H}$ & -3.164853 & -2.949781 & 3.520872 \\
\hline $\mathrm{O}$ & 1.339494 & 1.297609 & 1.477329 & $\mathrm{H}$ & -1.877926 & -2.494679 & 4.658333 \\
\hline $\mathrm{N}$ & 0.260804 & -2.814884 & 0.133123 & $\mathrm{C}$ & -1.125482 & -0.101602 & 3.469805 \\
\hline $\mathrm{N}$ & -1.803566 & -2.079693 & 0.031042 & $\mathrm{H}$ & -0.550968 & 0.442460 & 2.715942 \\
\hline $\mathrm{C}$ & -0.483892 & -1.687144 & 0.001181 & $\mathrm{H}$ & -1.789857 & 0.601940 & 3.988093 \\
\hline $\mathrm{C}$ & -0.517856 & -4.047157 & 0.036519 & $\mathrm{H}$ & -0.414158 & -0.482392 & 4.212015 \\
\hline $\mathrm{H}$ & -0.188540 & -4.790613 & 0.767511 & $\mathrm{H}$ & -5.682937 & 1.192184 & 1.575069 \\
\hline $\mathrm{H}$ & -0.424647 & -4.484775 & -0.967057 & $\mathrm{C}$ & -1.239064 & 1.496080 & -0.146865 \\
\hline $\mathrm{C}$ & -1.936327 & -3.528539 & 0.303415 & $\mathrm{H}$ & -1.961937 & 0.893950 & 0.381296 \\
\hline $\mathrm{H}$ & -2.689206 & -3.978762 & -0.346505 & $\mathrm{C}$ & -0.961380 & 2.807749 & 0.524552 \\
\hline $\mathrm{H}$ & -2.241177 & -3.686960 & 1.346136 & $\mathrm{O}$ & -0.256611 & 3.710351 & 0.071451 \\
\hline $\mathrm{C}$ & 1.707309 & -2.722021 & -0.064963 & $\mathrm{~N}$ & -1.638806 & 2.926476 & 1.704779 \\
\hline $\mathrm{C}$ & 2.085547 & -3.057290 & -1.527624 & $\mathrm{H}$ & -2.151186 & 2.125341 & 2.044583 \\
\hline $\mathrm{H}$ & 1.487639 & -2.443253 & -2.208592 & $\mathrm{C}$ & -1.506542 & 4.078153 & 2.574176 \\
\hline $\mathrm{H}$ & 1.847924 & -4.109607 & -1.739829 & $\mathrm{H}$ & -2.477801 & 4.330510 & 3.012570 \\
\hline $\mathrm{C}$ & 3.586273 & -2.797735 & -1.749044 & $\mathrm{H}$ & -0.784498 & 3.895986 & 3.380288 \\
\hline $\mathrm{H}$ & 3.843344 & -3.021315 & -2.792882 & $\mathrm{H}$ & -1.153852 & 4.918192 & 1.973637 \\
\hline $\mathrm{C}$ & 4.393324 & -3.715045 & -0.807678 & $\mathrm{C}$ & -1.209339 & 1.287312 & -1.534496 \\
\hline $\mathrm{H}$ & 4.189385 & -4.769304 & -1.043148 & $\mathrm{H}$ & -1.918997 & 0.560215 & -1.903425 \\
\hline $\mathrm{H}$ & 5.469864 & -3.554515 & -0.954017 & $\mathrm{C}$ & -0.941552 & 2.351502 & -2.579728 \\
\hline $\mathrm{C}$ & 4.013806 & -3.411021 & 0.656764 & $\mathrm{O}$ & -1.890127 & 2.627232 & -3.318184 \\
\hline $\mathrm{H}$ & 4.580435 & -4.068681 & 1.328763 & $\mathrm{~N}$ & 0.286917 & 2.900360 & -2.645660 \\
\hline $\mathrm{C}$ & 2.500612 & -3.669892 & 0.863377 & $\mathrm{H}$ & 0.922856 & 2.706345 & -1.872143 \\
\hline $\mathrm{H}$ & 2.215574 & -3.481734 & 1.906811 & $\mathrm{C}$ & 0.529138 & 4.089992 & -3.440801 \\
\hline $\mathrm{H}$ & 2.273514 & -4.723560 & 0.642116 & $\mathrm{H}$ & 0.296125 & 4.998932 & -2.870360 \\
\hline $\mathrm{C}$ & 4.354667 & -1.935519 & 0.959424 & $\mathrm{H}$ & 1.580213 & 4.118220 & -3.741873 \\
\hline $\mathrm{H}$ & 4.131464 & -1.699737 & 2.008586 & $\mathrm{H}$ & -0.105933 & 4.060549 & -4.328175 \\
\hline $\mathrm{H}$ & 5.431978 & -1.771612 & 0.813801 & $\mathrm{C}$ & 2.892248 & 3.142517 & 1.100888 \\
\hline $\mathrm{C}$ & 3.535692 & -1.006451 & 0.027689 & $\mathrm{C}$ & 4.258565 & 2.524804 & 1.477220 \\
\hline $\mathrm{H}$ & 3.778773 & 0.034832 & 0.247577 & $\mathrm{H}$ & 4.737592 & 2.065055 & 0.605497 \\
\hline $\mathrm{C}$ & 2.048916 & -1.265597 & 0.308752 & $\mathrm{H}$ & 4.927683 & 3.305900 & 1.858119 \\
\hline $\mathrm{H}$ & 1.889017 & -1.167942 & 1.393769 & $\mathrm{H}$ & 4.144421 & 1.760336 & 2.253870 \\
\hline $\mathrm{C}$ & 3.891079 & -1.317071 & -1.438225 & $\mathrm{C}$ & 3.071127 & 4.163341 & -0.035635 \\
\hline $\mathrm{H}$ & 3.314453 & -0.662178 & -2.102608 & $\mathrm{H}$ & 2.102594 & 4.582182 & -0.324082 \\
\hline $\mathrm{H}$ & 4.955324 & -1.111498 & -1.618327 & $\mathrm{H}$ & 3.723136 & 4.980751 & 0.296109 \\
\hline $\mathrm{C}$ & -2.874826 & -1.222519 & 0.454044 & $\mathrm{H}$ & 3.522332 & 3.696082 & -0.916105 \\
\hline $\mathrm{C}$ & -3.853080 & -0.823879 & -0.483518 & $\mathrm{C}$ & 2.274208 & 3.826349 & 2.332330 \\
\hline $\mathrm{C}$ & -4.856174 & 0.053228 & -0.052232 & $\mathrm{H}$ & 2.947642 & 4.608446 & 2.703826 \\
\hline $\mathrm{H}$ & -5.607991 & 0.393325 & -0.756577 & $\mathrm{H}$ & 1.319972 & 4.286468 & 2.063629 \\
\hline $\mathrm{C}$ & -4.900995 & 0.504572 & 1.264383 & $\mathrm{H}$ & 2.098082 & 3.103532 & 3.134160 \\
\hline $\mathrm{C}$ & -3.952380 & 0.065509 & 2.186083 & $\mathrm{C}$ & 1.986739 & 1.995996 & 0.640674 \\
\hline $\mathrm{H}$ & -4.011070 & 0.402289 & 3.217868 & & & & \\
\hline $\mathrm{C}$ & -2.925035 & -0.808275 & 1.806074 & $\mathbf{T S}$ & $\mathbf{A C}$ & & \\
\hline $\mathrm{C}$ & -3.858581 & -1.360477 & -1.911323 & $\mathrm{Ru}$ & 0.569363 & 0.598168 & -0.515087 \\
\hline $\mathrm{H}$ & -2.841780 & -1.703982 & -2.134063 & $\mathrm{O}$ & 1.664899 & 2.480624 & -0.852382 \\
\hline $\mathrm{C}$ & -1.915628 & -1.271946 & 2.854511 & $\mathrm{O}$ & 0.988834 & 2.154039 & 1.185512 \\
\hline $\mathrm{H}$ & -1.178658 & -1.918250 & 2.372781 & $\mathrm{~N}$ & 0.833361 & -2.102613 & 0.455204 \\
\hline $\mathrm{C}$ & 0.359676 & -0.285699 & -2.187726 & $\mathrm{~N}$ & -1.327074 & -1.807453 & 0.238327 \\
\hline $\mathrm{H}$ & -0.322207 & -0.954018 & -2.732142 & $\mathrm{C}$ & -0.108159 & -1.188523 & 0.110565 \\
\hline $\mathrm{H}$ & 0.999616 & 0.301630 & -2.858315 & $\mathrm{C}$ & 0.302310 & -3.453046 & 0.622076 \\
\hline $\mathrm{C}$ & -4.260040 & -0.323436 & -2.978530 & $\mathrm{H}$ & 0.757149 & -3.963560 & 1.474768 \\
\hline $\mathrm{H}$ & -4.139148 & -0.763466 & -3.974999 & $\mathrm{H}$ & 0.485589 & -4.053659 & -0.280155 \\
\hline $\mathrm{H}$ & -5.313651 & -0.036482 & -2.882051 & $\mathrm{C}$ & -1.187924 & -3.159815 & 0.823005 \\
\hline $\mathrm{H}$ & -3.660002 & 0.591486 & -2.943675 & $\mathrm{H}$ & -1.843054 & -3.871284 & 0.316656 \\
\hline $\mathrm{C}$ & -4.800019 & -2.581802 & -2.001722 & $\mathrm{H}$ & -1.459285 & -3.131973 & 1.886282 \\
\hline
\end{tabular}




\begin{tabular}{|c|c|c|c|}
\hline $\mathrm{C}$ & 2.242795 & -1.771043 & 0.246785 \\
\hline $\mathrm{C}$ & 2.732388 & -2.267917 & -1.134192 \\
\hline $\mathrm{H}$ & 2.059172 & -1.902015 & -1.915002 \\
\hline $\mathrm{H}$ & 2.703292 & -3.366754 & -1.162867 \\
\hline $\mathrm{C}$ & 4.165979 & -1.766671 & -1.385847 \\
\hline $\mathrm{H}$ & 4.500313 & -2.106441 & -2.374940 \\
\hline $\mathrm{C}$ & 5.093835 & -2.343889 & -0.297687 \\
\hline $\mathrm{H}$ & 5.100235 & -3.441865 & -0.351645 \\
\hline $\mathrm{H}$ & 6.125856 & -2.006981 & -0.462681 \\
\hline $\mathrm{C}$ & 4.607676 & -1.878657 & 1.090700 \\
\hline $\mathrm{H}$ & 5.259976 & -2.295319 & 1.869042 \\
\hline $\mathrm{C}$ & 3.163035 & -2.383057 & 1.328038 \\
\hline $\mathrm{H}$ & 2.807570 & -2.083872 & 2.322707 \\
\hline $\mathrm{H}$ & 3.145409 & -3.482331 & 1.285931 \\
\hline $\mathrm{C}$ & 4.655354 & -0.336127 & 1.140521 \\
\hline $\mathrm{H}$ & 4.352877 & 0.023511 & 2.132881 \\
\hline $\mathrm{H}$ & 5.687306 & 0.003844 & 0.975252 \\
\hline $\mathrm{C}$ & 3.714192 & 0.254797 & 0.058979 \\
\hline $\mathrm{H}$ & 3.758815 & 1.347271 & 0.100192 \\
\hline $\mathrm{C}$ & 2.291092 & -0.235424 & 0.372859 \\
\hline $\mathrm{H}$ & 2.081038 & -0.003715 & 1.427665 \\
\hline $\mathrm{C}$ & 4.176324 & -0.224134 & -1.330423 \\
\hline $\mathrm{H}$ & 3.514066 & 0.186784 & -2.102228 \\
\hline $\mathrm{H}$ & 5.188493 & 0.148609 & -1.537334 \\
\hline $\mathrm{C}$ & -2.587711 & -1.140920 & 0.402490 \\
\hline $\mathrm{C}$ & -3.581684 & -1.317860 & -0.585576 \\
\hline $\mathrm{C}$ & -4.808020 & -0.666308 & -0.413402 \\
\hline $\mathrm{H}$ & -5.584405 & -0.780066 & -1.163872 \\
\hline $\mathrm{C}$ & -5.048621 & 0.125217 & 0.706844 \\
\hline $\mathrm{C}$ & -4.074564 & 0.249016 & 1.693751 \\
\hline $\mathrm{H}$ & -4.284719 & 0.840653 & 2.579939 \\
\hline $\mathrm{C}$ & -2.833743 & -0.390691 & 1.574552 \\
\hline $\mathrm{C}$ & -3.365590 & -2.235505 & -1.785071 \\
\hline $\mathrm{H}$ & -2.293666 & -2.455419 & -1.842108 \\
\hline $\mathrm{C}$ & -1.843590 & -0.321527 & 2.734959 \\
\hline $\mathrm{H}$ & -0.905039 & -0.788947 & 2.425705 \\
\hline $\mathrm{C}$ & 0.573880 & 0.034059 & -2.293692 \\
\hline $\mathrm{H}$ & 0.132754 & -0.858159 & -2.758405 \\
\hline $\mathrm{C}$ & -1.346585 & 1.866611 & -0.714885 \\
\hline $\mathrm{H}$ & -1.885778 & 1.560297 & 0.175724 \\
\hline $\mathrm{C}$ & -1.399332 & 1.057378 & -1.848809 \\
\hline $\mathrm{H}$ & -1.960255 & 0.136853 & -1.834980 \\
\hline $\mathrm{H}$ & 1.060475 & 0.694651 & -3.025799 \\
\hline $\mathrm{N}$ & 1.647186 & 2.898078 & 0.361012 \\
\hline $\mathrm{O}$ & 2.208967 & 3.920774 & 0.712156 \\
\hline $\mathrm{C}$ & -3.787723 & -1.608360 & -3.127016 \\
\hline $\mathrm{H}$ & -3.530630 & -2.283788 & -3.950926 \\
\hline $\mathrm{H}$ & -4.869125 & -1.437460 & -3.171141 \\
\hline $\mathrm{H}$ & -3.292864 & -0.649573 & -3.308919 \\
\hline $\mathrm{C}$ & -4.118002 & -3.566655 & -1.573945 \\
\hline $\mathrm{H}$ & -3.911158 & -4.263303 & -2.394897 \\
\hline $\mathrm{H}$ & -3.834897 & -4.050546 & -0.633028 \\
\hline $\mathrm{H}$ & -5.200394 & -3.394380 & -1.540493 \\
\hline $\mathrm{C}$ & -2.390316 & -1.117222 & 3.938937 \\
\hline $\mathrm{H}$ & -3.307596 & -0.659381 & 4.327458 \\
\hline $\mathrm{H}$ & -2.628955 & -2.151752 & 3.666531 \\
\hline $\mathrm{H}$ & -1.653504 & -1.136568 & 4.750324 \\
\hline $\mathrm{C}$ & -1.502232 & 1.119245 & 3.152372 \\
\hline $\mathrm{H}$ & -1.032998 & 1.664967 & 2.332348 \\
\hline $\mathrm{H}$ & -2.391356 & 1.668619 & 3.482491 \\
\hline
\end{tabular}

$\begin{array}{lrrr}\mathrm{H} & -0.789314 & 1.109119 & 3.984358 \\ \mathrm{H} & -6.003200 & 0.631922 & 0.819280 \\ \mathrm{H} & -1.250628 & 1.546968 & -2.808148 \\ \mathrm{C} & -1.114373 & 3.341993 & -0.928359 \\ \mathrm{O} & -1.037198 & 3.832888 & -2.051846 \\ \mathrm{~N} & -1.060585 & 4.083209 & 0.218633 \\ \mathrm{H} & -0.859519 & 3.581268 & 1.073835 \\ \mathrm{C} & -0.692970 & 5.491746 & 0.176315 \\ \mathrm{H} & -1.083542 & 5.994424 & 1.065769 \\ \mathrm{H} & 0.395704 & 5.618116 & 0.136968 \\ \mathrm{H} & -1.132768 & 5.936416 & -0.717549\end{array}$

\section{INT2'-1-AC}

\begin{tabular}{|c|c|c|c|}
\hline $\mathrm{Ru}$ & 0.606755 & 0.521973 & -0.544877 \\
\hline $\mathrm{O}$ & 1.739775 & 2.244649 & -1.238195 \\
\hline $\mathrm{O}$ & 1.516976 & 2.333931 & 0.925579 \\
\hline $\mathrm{N}$ & 0.729046 & -2.113006 & 0.582028 \\
\hline $\mathrm{N}$ & -1.412159 & -1.768604 & 0.291970 \\
\hline $\mathrm{C}$ & -0.180404 & -1.185238 & 0.190237 \\
\hline $\mathrm{C}$ & 0.161656 & -3.451598 & 0.737580 \\
\hline $\mathrm{H}$ & 0.571051 & -3.965025 & 1.611240 \\
\hline $\mathrm{H}$ & 0.366083 & -4.063124 & -0.152451 \\
\hline $\mathrm{C}$ & -1.331329 & -3.125093 & 0.875562 \\
\hline $\mathrm{H}$ & -1.978721 & -3.818725 & 0.334774 \\
\hline $\mathrm{H}$ & -1.652697 & -3.097564 & 1.925478 \\
\hline $\mathrm{C}$ & 2.141523 & -1.815904 & 0.349245 \\
\hline $\mathrm{C}$ & 2.584951 & -2.278556 & -1.062484 \\
\hline $\mathrm{H}$ & 1.908138 & -1.855894 & -1.813802 \\
\hline $\mathrm{H}$ & 2.511079 & -3.373288 & -1.130714 \\
\hline $\mathrm{C}$ & 4.033255 & -1.825620 & -1.325319 \\
\hline $\mathrm{H}$ & 4.336160 & -2.148313 & -2.329756 \\
\hline $\mathrm{C}$ & 4.957362 & -2.469549 & -0.271567 \\
\hline $\mathrm{H}$ & 4.920729 & -3.564896 & -0.355861 \\
\hline $\mathrm{H}$ & 5.998026 & -2.167324 & -0.447935 \\
\hline $\mathrm{C}$ & 4.517482 & -2.027981 & 1.139956 \\
\hline $\mathrm{H}$ & 5.170231 & -2.489279 & 1.892079 \\
\hline $\mathrm{C}$ & 3.062044 & -2.483546 & 1.394202 \\
\hline $\mathrm{H}$ & 2.737019 & -2.198385 & 2.403261 \\
\hline $\mathrm{H}$ & 2.996900 & -3.579244 & 1.320954 \\
\hline $\mathrm{C}$ & 4.612370 & -0.491464 & 1.237713 \\
\hline $\mathrm{H}$ & 4.333287 & -0.154277 & 2.244669 \\
\hline $\mathrm{H}$ & 5.649943 & -0.173145 & 1.066763 \\
\hline $\mathrm{C}$ & 3.680044 & 0.165291 & 0.185916 \\
\hline $\mathrm{H}$ & 3.769777 & 1.250369 & 0.271079 \\
\hline $\mathrm{C}$ & 2.244239 & -0.281571 & 0.500511 \\
\hline $\mathrm{H}$ & 2.001606 & -0.027274 & 1.540522 \\
\hline $\mathrm{C}$ & 4.107588 & -0.287916 & -1.224583 \\
\hline $\mathrm{H}$ & 3.459269 & 0.178930 & -1.976020 \\
\hline $\mathrm{H}$ & 5.132515 & 0.049407 & -1.428411 \\
\hline $\mathrm{C}$ & -2.647969 & -1.050035 & 0.396681 \\
\hline $\mathrm{C}$ & -3.592908 & -1.162112 & -0.645094 \\
\hline $\mathrm{C}$ & -4.771227 & -0.413525 & -0.543977 \\
\hline $\mathrm{H}$ & -5.511962 & -0.471117 & -1.335765 \\
\hline $\mathrm{C}$ & -5.007670 & 0.406465 & 0.557561 \\
\hline $\mathrm{C}$ & -4.088400 & 0.455740 & 1.602675 \\
\hline $\mathrm{H}$ & -4.296327 & 1.076037 & 2.469805 \\
\hline $\mathrm{C}$ & -2.900470 & -0.284769 & 1.556101 \\
\hline $\mathrm{C}$ & -3.384618 & -2.124853 & -1.809608 \\
\hline $\mathrm{H}$ & -2.331260 & -2.425052 & -1.803394 \\
\hline $\mathrm{C}$ & -1.957824 & -0.274265 & 2.758713 \\
\hline
\end{tabular}




$\begin{array}{lrrr}\mathrm{H} & -1.124486 & -0.952420 & 2.556677 \\ \mathrm{C} & -0.028144 & 0.039372 & -2.350309 \\ \mathrm{H} & -0.445171 & -0.939707 & -2.597954 \\ \mathrm{C} & -1.059134 & 1.735673 & -0.557612 \\ \mathrm{H} & -1.823264 & 1.418070 & 0.146638 \\ \mathrm{C} & -1.183822 & 1.080782 & -1.950505 \\ \mathrm{H} & -2.121828 & 0.530148 & -1.962563 \\ \mathrm{H} & 0.608575 & 0.430982 & -3.149921 \\ \mathrm{~N} & 1.979426 & 2.875322 & -0.134030 \\ \mathrm{O} & 2.616422 & 3.915858 & -0.123851 \\ \mathrm{C} & -3.684170 & -1.514850 & -3.190280 \\ \mathrm{H} & -4.735972 & -1.223323 & -3.286918 \\ \mathrm{H} & -3.070425 & -0.631781 & -3.389352 \\ \mathrm{H} & -3.476694 & -2.251919 & -3.974424 \\ \mathrm{C} & -4.245189 & -3.388908 & -1.596641 \\ \mathrm{H} & -4.048687 & -4.128534 & -2.381764 \\ \mathrm{H} & -4.047471 & -3.856667 & -0.625821 \\ \mathrm{H} & -5.312050 & -3.137353 & -1.627595 \\ \mathrm{C} & -2.683551 & -0.805738 & 4.011152 \\ \mathrm{H} & -3.494183 & -0.136175 & 4.320146 \\ \mathrm{H} & -3.120580 & -1.794511 & 3.829038 \\ \mathrm{H} & -1.982384 & -0.888291 & 4.849606 \\ \mathrm{C} & -1.336877 & 1.109690 & 3.023789 \\ \mathrm{H} & -0.653805 & 1.385718 & 2.217555 \\ \mathrm{H} & -2.103412 & 1.887772 & 3.114347 \\ \mathrm{H} & -0.760229 & 1.092183 & 3.955904 \\ \mathrm{H} & -5.920199 & 0.993847 & 0.610049 \\ \mathrm{H} & -1.172841 & 1.896073 & -2.676464 \\ \mathrm{C} & -0.982695 & 3.258176 & -0.620972 \\ \mathrm{O} & -1.135843 & 3.877589 & -1.671425 \\ \mathrm{~N} & -0.837551 & 3.879566 & 0.588131 \\ \mathrm{H} & -0.394479 & 3.322861 & 1.307113 \\ \mathrm{C} & -0.566611 & 5.311479 & 0.639284 \\ \mathrm{H} & -0.791562 & 5.683549 & 1.642877 \\ \mathrm{H} & 0.481537 & 5.528417 & 0.397477 \\ \mathrm{H} & -1.205460 & 5.814828 & -0.087707\end{array}$

\begin{tabular}{|c|c|c|c|}
\hline $\mathrm{H}$ & 4.782131 & -1.347364 & 3.059186 \\
\hline $\mathrm{C}$ & 2.682449 & -1.412296 & 2.532868 \\
\hline $\mathrm{H}$ & 2.408266 & -0.556238 & 3.162794 \\
\hline $\mathrm{H}$ & 2.478690 & -2.326508 & 3.108942 \\
\hline $\mathrm{C}$ & 4.459072 & -0.079550 & 1.323740 \\
\hline $\mathrm{H}$ & 4.238876 & 0.813177 & 1.924209 \\
\hline $\mathrm{H}$ & 5.523843 & -0.035595 & 1.057995 \\
\hline $\mathrm{C}$ & 3.600294 & -0.080922 & 0.026348 \\
\hline $\mathrm{H}$ & 3.801858 & 0.833305 & -0.541020 \\
\hline $\mathrm{C}$ & 2.134799 & -0.104842 & 0.469277 \\
\hline $\mathrm{H}$ & 1.951411 & 0.773245 & 1.141053 \\
\hline $\mathrm{C}$ & 3.976478 & -1.324718 & -0.806435 \\
\hline $\mathrm{H}$ & 3.415037 & -1.333921 & -1.742765 \\
\hline $\mathrm{H}$ & 5.043966 & -1.279573 & -1.060894 \\
\hline $\mathrm{C}$ & -2.804957 & -0.854007 & 0.085664 \\
\hline $\mathrm{C}$ & -2.878191 & -1.691080 & -1.050414 \\
\hline $\mathrm{C}$ & -3.956322 & -1.510995 & -1.924922 \\
\hline $\mathrm{H}$ & -4.025701 & -2.123757 & -2.819267 \\
\hline $\mathrm{C}$ & -4.941604 & -0.560970 & -1.663825 \\
\hline $\mathrm{C}$ & -4.885419 & 0.203933 & -0.500806 \\
\hline $\mathrm{H}$ & -5.680257 & 0.912254 & -0.287528 \\
\hline $\mathrm{C}$ & -3.823859 & 0.066603 & 0.402699 \\
\hline $\mathrm{C}$ & -1.858604 & -2.795206 & -1.324084 \\
\hline $\mathrm{H}$ & -1.128044 & -2.800505 & -0.510370 \\
\hline $\mathrm{C}$ & -3.834084 & 0.823001 & 1.726172 \\
\hline $\mathrm{H}$ & -2.835463 & 0.738052 & 2.166537 \\
\hline $\mathrm{C}$ & -0.896592 & 0.922672 & -1.932047 \\
\hline $\mathrm{H}$ & -1.680669 & 0.184401 & -2.050543 \\
\hline $\mathrm{C}$ & -0.627122 & 2.123615 & 0.399490 \\
\hline $\mathrm{H}$ & -1.325602 & 1.806193 & 1.169039 \\
\hline $\mathrm{C}$ & -1.305959 & 2.134980 & -1.049107 \\
\hline $\mathrm{H}$ & -2.381887 & 2.064325 & -0.873108 \\
\hline $\mathrm{H}$ & -0.455418 & 1.202848 & -2.889531 \\
\hline $\mathrm{C}$ & -4.833012 & 0.154480 & 2.694603 \\
\hline $\mathrm{H}$ & -4.805610 & 0.644288 & 3.674946 \\
\hline $\mathrm{H}$ & -5.856045 & 0.227455 & 2.306564 \\
\hline $\mathrm{H}$ & -4.608236 & -0.908941 & 2.834610 \\
\hline $\mathrm{C}$ & -4.150483 & 2.321753 & 1.585179 \\
\hline $\mathrm{H}$ & -3.449384 & 2.823591 & 0.912543 \\
\hline $\mathrm{H}$ & -5.165587 & 2.488557 & 1.207011 \\
\hline $\mathrm{H}$ & -4.077942 & 2.810263 & 2.562833 \\
\hline $\mathrm{C}$ & -2.549856 & -4.173660 & -1.320668 \\
\hline $\mathrm{H}$ & -3.104538 & -4.340649 & -0.389711 \\
\hline $\mathrm{H}$ & -3.259150 & -4.268150 & -2.150782 \\
\hline $\mathrm{H}$ & -1.806220 & -4.972174 & -1.426077 \\
\hline $\mathrm{C}$ & -1.066264 & -2.578368 & -2.627209 \\
\hline $\mathrm{H}$ & -0.398025 & -3.429818 & -2.803305 \\
\hline $\mathrm{H}$ & -1.734868 & -2.496186 & -3.492286 \\
\hline $\mathrm{H}$ & -0.452093 & -1.675710 & -2.578305 \\
\hline $\mathrm{H}$ & -5.767796 & -0.432106 & -2.357872 \\
\hline $\mathrm{H}$ & -1.064558 & 3.075169 & -1.549132 \\
\hline $\mathrm{N}$ & 2.151179 & 1.205150 & -2.617761 \\
\hline $\mathrm{O}$ & 2.873735 & 1.566920 & -3.528399 \\
\hline $\mathrm{C}$ & 0.060339 & 3.363799 & 0.911664 \\
\hline $\mathrm{O}$ & -0.012319 & 3.644642 & 2.110145 \\
\hline $\mathrm{N}$ & 0.752068 & 4.141987 & 0.022331 \\
\hline $\mathrm{H}$ & 1.025090 & 3.728076 & -0.861018 \\
\hline $\mathrm{C}$ & 1.574260 & 5.237201 & 0.511360 \\
\hline $\mathrm{H}$ & 2.502669 & 4.880152 & 0.979103 \\
\hline $\mathrm{H}$ & 1.826769 & 5.896087 & -0.323705 \\
\hline
\end{tabular}

$\begin{array}{lccc}\text { INT2'-AC } & & \\ \mathrm{Ru} & 0.506885 & 0.716326 & -0.473783 \\ \mathrm{O} & 1.758654 & 0.009247 & -2.459452 \\ \mathrm{O} & 1.730936 & 2.050022 & -1.717409 \\ \mathrm{~N} & 0.401171 & -1.358193 & 1.521450 \\ \mathrm{~N} & -1.687820 & -1.005735 & 0.968878 \\ \mathrm{C} & -0.401672 & -0.653807 & 0.685871 \\ \mathrm{C} & -0.302908 & -2.357540 & 2.325395 \\ \mathrm{H} & -0.023243 & -2.283575 & 3.379795 \\ \mathrm{H} & -0.068047 & -3.372057 & 1.978302 \\ \mathrm{C} & -1.774903 & -1.980702 & 2.076638 \\ \mathrm{H} & -2.390315 & -2.835048 & 1.779342 \\ \mathrm{H} & -2.236638 & -1.510972 & 2.952105 \\ \mathrm{C} & 1.832218 & -1.389038 & 1.243258 \\ \mathrm{C} & 2.185267 & -2.618901 & 0.367482 \\ \mathrm{H} & 1.571358 & -2.589315 & -0.541419 \\ \mathrm{H} & 1.940747 & -3.542367 & 0.909977 \\ \mathrm{C} & 3.683266 & -2.597277 & 0.008038 \\ \mathrm{H} & 3.921484 & -3.485037 & -0.591630 \\ \mathrm{C} & 4.524476 & -2.599961 & 1.300216 \\ \mathrm{H} & 4.333440 & -3.514430 & 1.879438 \\ \mathrm{H} & 5.593239 & -2.594768 & 1.049278 \\ \mathrm{C} & 4.175944 & -1.356746 & 2.144556\end{array}$




\begin{tabular}{|c|c|c|c|}
\hline $\mathrm{H}$ & 1.012946 & 5.797125 & 1.261733 \\
\hline \multicolumn{4}{|c|}{ INT3'-AC } \\
\hline $\mathrm{Ru}$ & -0.560941 & 0.666504 & 0.316241 \\
\hline $\mathrm{O}$ & -0.207580 & 0.834218 & 2.434003 \\
\hline $\mathrm{O}$ & -1.525752 & 2.330036 & 1.570001 \\
\hline $\mathrm{N}$ & -0.665603 & -1.921637 & -0.849823 \\
\hline $\mathrm{N}$ & 1.450676 & -1.563116 & -0.382963 \\
\hline $\mathrm{C}$ & 0.213512 & -1.020050 & -0.341688 \\
\hline $\mathrm{C}$ & -0.074007 & -3.254863 & -0.960761 \\
\hline $\mathrm{H}$ & -0.420199 & -3.778922 & -1.854769 \\
\hline $\mathrm{H}$ & -0.316322 & -3.865115 & -0.078000 \\
\hline $\mathrm{C}$ & 1.423823 & -2.898853 & -1.019335 \\
\hline $\mathrm{H}$ & 2.061957 & -3.600193 & -0.478754 \\
\hline $\mathrm{H}$ & 1.775761 & -2.832875 & -2.054799 \\
\hline $\mathrm{C}$ & -2.082740 & -1.575202 & -0.710417 \\
\hline $\mathrm{C}$ & -2.608426 & -1.948368 & 0.700952 \\
\hline $\mathrm{H}$ & -1.952315 & -1.510702 & 1.468605 \\
\hline $\mathrm{H}$ & -2.568007 & -3.038579 & 0.835444 \\
\hline $\mathrm{C}$ & -4.049948 & -1.436184 & 0.877486 \\
\hline $\mathrm{H}$ & -4.412811 & -1.709803 & 1.876413 \\
\hline $\mathrm{C}$ & -4.945560 & -2.084182 & -0.198252 \\
\hline $\mathrm{H}$ & -4.954301 & -3.176130 & -0.074525 \\
\hline $\mathrm{H}$ & -5.981148 & -1.738484 & -0.082233 \\
\hline $\mathrm{C}$ & -4.421634 & -1.710264 & -1.600529 \\
\hline $\mathrm{H}$ & -5.059912 & -2.167999 & -2.366781 \\
\hline $\mathrm{C}$ & -2.977880 & -2.243517 & -1.768812 \\
\hline $\mathrm{H}$ & -2.593629 & -2.012881 & -2.770714 \\
\hline $\mathrm{H}$ & -2.970053 & -3.337650 & -1.653402 \\
\hline $\mathrm{C}$ & -4.450986 & -0.172298 & -1.753945 \\
\hline $\mathrm{H}$ & -4.112747 & 0.115475 & -2.758051 \\
\hline $\mathrm{H}$ & -5.483913 & 0.186781 & -1.645607 \\
\hline $\mathrm{C}$ & -3.541808 & 0.483792 & -0.681615 \\
\hline $\mathrm{H}$ & -3.559504 & 1.574332 & -0.796206 \\
\hline $\mathrm{C}$ & -2.113490 & -0.034869 & -0.915722 \\
\hline $\mathrm{H}$ & -1.839420 & 0.155658 & -1.957692 \\
\hline $\mathrm{C}$ & -4.063869 & 0.098171 & 0.718326 \\
\hline $\mathrm{H}$ & -3.447675 & 0.569225 & 1.494691 \\
\hline $\mathrm{H}$ & -5.085621 & 0.477467 & 0.852666 \\
\hline $\mathrm{C}$ & 2.658441 & -0.900508 & 0.027568 \\
\hline $\mathrm{C}$ & 2.958524 & -0.841206 & 1.408010 \\
\hline $\mathrm{C}$ & 4.137339 & -0.190727 & 1.789063 \\
\hline $\mathrm{H}$ & 4.393829 & -0.118543 & 2.840211 \\
\hline $\mathrm{C}$ & 4.992246 & 0.367397 & 0.841004 \\
\hline $\mathrm{C}$ & 4.680594 & 0.290306 & -0.512129 \\
\hline $\mathrm{H}$ & 5.353788 & 0.728812 & -1.242058 \\
\hline $\mathrm{C}$ & 3.505960 & -0.335093 & -0.948658 \\
\hline $\mathrm{C}$ & 2.065593 & -1.504897 & 2.453431 \\
\hline $\mathrm{H}$ & 1.031078 & -1.435331 & 2.106780 \\
\hline $\mathrm{C}$ & 3.214338 & -0.428929 & -2.444312 \\
\hline $\mathrm{H}$ & 2.165018 & -0.719683 & -2.569004 \\
\hline $\mathrm{C}$ & 4.097667 & -1.523000 & -3.081931 \\
\hline $\mathrm{H}$ & 3.847492 & -1.655053 & -4.141248 \\
\hline $\mathrm{H}$ & 5.155801 & -1.242949 & -3.017369 \\
\hline $\mathrm{H}$ & 3.984089 & -2.488843 & -2.576839 \\
\hline $\mathrm{C}$ & 3.400095 & 0.903245 & -3.197131 \\
\hline $\mathrm{H}$ & 4.440856 & 1.244522 & -3.161875 \\
\hline $\mathrm{H}$ & 3.141209 & 0.762537 & -4.253128 \\
\hline $\mathrm{H}$ & 2.761969 & 1.701190 & -2.805600 \\
\hline $\mathrm{C}$ & 2.419968 & -3.000327 & 2.589036 \\
\hline
\end{tabular}

$\begin{array}{lrrr}\mathrm{H} & 2.318575 & -3.533110 & 1.638251 \\ \mathrm{H} & 3.455622 & -3.120920 & 2.928891 \\ \mathrm{H} & 1.763359 & -3.486197 & 3.320799 \\ \mathrm{C} & 2.116585 & -0.825916 & 3.831130 \\ \mathrm{H} & 1.342043 & -1.252823 & 4.477533 \\ \mathrm{H} & 3.080550 & -0.983793 & 4.329834 \\ \mathrm{H} & 1.929565 & 0.247580 & 3.751291 \\ \mathrm{H} & 5.903981 & 0.865236 & 1.160521 \\ \mathrm{~N} & -0.946285 & 1.896024 & 2.630297 \\ \mathrm{O} & -1.070502 & 2.398466 & 3.720410 \\ \mathrm{C} & 0.418925 & 1.618438 & -0.894176 \\ \mathrm{H} & 1.231825 & 1.146211 & -1.447173 \\ \mathrm{C} & 0.304362 & 3.050208 & -1.335065 \\ \mathrm{O} & 0.819913 & 3.361094 & -2.416005 \\ \mathrm{~N} & -0.358321 & 3.941854 & -0.554397 \\ \mathrm{H} & -0.804513 & 3.613220 & 0.292889 \\ \mathrm{C} & -0.547019 & 5.318087 & -0.974988 \\ \mathrm{H} & -0.735218 & 5.940523 & -0.096291 \\ \mathrm{H} & 0.355003 & 5.666303 & -1.484209 \\ \mathrm{H} & -1.390167 & 5.420628 & -1.671407\end{array}$

\section{TS2'-cross-AC}

$\begin{array}{lccc}\mathrm{Ru} & -0.474581 & 0.683943 & 0.399004 \\ \mathrm{O} & -1.644295 & 0.025812 & 2.332760 \\ \mathrm{O} & -1.687568 & 2.077475 & 1.627752 \\ \mathrm{~N} & -0.538728 & -1.543367 & -1.430599 \\ \mathrm{~N} & 1.580305 & -1.243137 & -0.967028 \\ \mathrm{C} & 0.316491 & -0.812851 & -0.676898 \\ \mathrm{C} & 0.097058 & -2.649167 & -2.147583 \\ \mathrm{H} & -0.222229 & -2.677396 & -3.192627 \\ \mathrm{H} & -0.160581 & -3.611080 & -1.684504 \\ \mathrm{C} & 1.589512 & -2.305729 & -1.994468 \\ \mathrm{H} & 2.191478 & -3.154493 & -1.656962 \\ \mathrm{H} & 2.026178 & -1.922130 & -2.924073 \\ \mathrm{C} & -1.972321 & -1.453228 & -1.155258 \\ \mathrm{C} & -2.381695 & -2.521691 & -0.110401 \\ \mathrm{H} & -1.771638 & -2.383806 & 0.790587 \\ \mathrm{H} & -2.178987 & -3.527373 & -0.505765 \\ \mathrm{C} & -3.878365 & -2.380127 & 0.229947 \\ \mathrm{H} & -4.158789 & -3.158490 & 0.951424 \\ \mathrm{C} & -4.718671 & -2.532823 & -1.053068 \\ \mathrm{H} & -4.570074 & -3.529241 & -1.493440 \\ \mathrm{H} & -5.786213 & -2.442526 & -0.812535 \\ \mathrm{C} & -4.311257 & -1.441545 & -2.064338 \\ \mathrm{H} & -4.917408 & -1.534994 & -2.974689 \\ \mathrm{C} & -2.822275 & -1.634700 & -2.430768 \\ \mathrm{H} & -2.506429 & -0.901587 & -3.184816 \\ \mathrm{H} & -2.676845 & -2.636444 & -2.862061 \\ \mathrm{C} & -4.535175 & -0.047033 & -1.434477 \\ \mathrm{H} & -4.279932 & 0.738517 & -2.158617 \\ \mathrm{H} & -5.598702 & 0.076259 & -1.186656 \\ \mathrm{C} & -3.668694 & 0.101165 & -0.152329 \\ \mathrm{H} & -3.831760 & 1.094282 & 0.282811 \\ \mathrm{C} & -2.197720 & -0.039888 & -0.594639 \\ \mathrm{H} & -2.059885 & 0.659619 & -1.435476 \\ \mathrm{C} & -4.116500 & -0.989087 & 0.845990 \\ \mathrm{H} & -3.575342 & -0.903602 & 1.788665 \\ \mathrm{H} & -5.184795 & -0.859359 & 1.067791 \\ \mathrm{C} & 2.730939 & -1.065949 & -0.134920 \\ \mathrm{C} & 2.830305 & -1.839209 & 1.044223\end{array}$




\begin{tabular}{lrrr} 
& & & \\
$\mathrm{C}$ & 3.943736 & -1.634763 & 1.867766 \\
$\mathrm{H}$ & 4.035529 & -2.199811 & 2.791148 \\
$\mathrm{C}$ & 4.937585 & -0.722992 & 1.517326 \\
$\mathrm{C}$ & 4.853592 & -0.023897 & 0.315334 \\
$\mathrm{H}$ & 5.655011 & 0.652155 & 0.032830 \\
$\mathrm{C}$ & 3.756562 & -0.187731 & -0.540593 \\
$\mathrm{C}$ & 1.804969 & -2.912446 & 1.408464 \\
$\mathrm{H}$ & 1.032995 & -2.929839 & 0.634481 \\
$\mathrm{C}$ & 3.738473 & 0.487062 & -1.908444 \\
$\mathrm{H}$ & 2.731092 & 0.372217 & -2.322116 \\
$\mathrm{C}$ & 1.032394 & 0.897240 & 1.97311 \\
$\mathrm{H}$ & 1.626806 & -0.002346 & 2.039630 \\
$\mathrm{C}$ & 1.515882 & 1.963192 & 1.170025 \\
$\mathrm{H}$ & 2.409486 & 1.753421 & 0.590926 \\
$\mathrm{C}$ & 1.330147 & 3.398871 & 1.606048 \\
$\mathrm{H}$ & 2.025918 & 3.594446 & 2.433170 \\
$\mathrm{H}$ & 1.553669 & 4.106569 & 0.804305 \\
$\mathrm{H}$ & 0.313666 & 3.570612 & 1.970267 \\
$\mathrm{H}$ & 0.495553 & 1.162747 & 2.881629 \\
$\mathrm{C}$ & 4.715013 & -0.249640 & -2.850285 \\
$\mathrm{H}$ & 4.663760 & 0.169124 & -3.862042 \\
$\mathrm{H}$ & 5.747359 & -0.150575 & -2.494071 \\
$\mathrm{H}$ & 4.486114 & -1.320020 & -2.908166 \\
$\mathrm{C}$ & 4.056846 & 1.992552 & -1.874828 \\
$\mathrm{H}$ & 3.313703 & 2.562466 & -1.310165 \\
$\mathrm{H}$ & 5.046875 & 2.188684 & -1.447820 \\
$\mathrm{H}$ & 4.053750 & 2.392728 & -2.894667 \\
$\mathrm{C}$ & 2.474228 & -4.301998 & 1.416260 \\
$\mathrm{H}$ & 2.978734 & -4.508267 & 0.464918 \\
$\mathrm{H}$ & 3.223734 & -4.379663 & 2.212190 \\
$\mathrm{H}$ & 1.725485 & -5.084563 & 1.585362 \\
$\mathrm{C}$ & 1.082457 & -2.643325 & 2.742264 \\
$\mathrm{H}$ & 0.422751 & -3.484810 & 2.984319 \\
$\mathrm{H}$ & 1.793667 & -2.529937 & 3.568925 \\
$\mathrm{H}$ & 0.464252 & -1.743183 & 2.691260 \\
$\mathrm{H}$ & 5.792178 & -0.574596 & 2.171987 \\
$\mathrm{~N}$ & -2.117689 & 1.201095 & 2.484287 \\
$\mathrm{O}$ & -2.912819 & 1.49556 & 3.356498 \\
$\mathrm{C}$ & 0.420316 & 1.879687 & -0.762905 \\
$\mathrm{H}$ & 1.253755 & 1.591214 & -1.406552 \\
$\mathrm{C}$ & 0.088809 & 3.316961 & -1.076113 \\
$\mathrm{O}$ & 0.893975 & 4.004174 & -1.715245 \\
$\mathrm{~N}$ & -1.108465 & 3.799744 & -0.651875 \\
$\mathrm{H}$ & -1.654161 & 3.241823 & -0.006578 \\
$\mathrm{C}$ & -1.440754 & 5.204078 & -0.814919 \\
$\mathrm{H}$ & -1.215261 & 5.516157 & -1.837553 \\
$\mathrm{H}$ & -2.505754 & 5.343714 & -0.615405 \\
$\mathrm{H}$ & -0.860787 & 5.839630 & -0.132210 \\
& & & \\
\hline
\end{tabular}

\section{TS2'-homo-AC}

$\begin{array}{lccc}\mathrm{Ru} & -0.440721 & 0.524642 & 0.258459 \\ \mathrm{O} & -1.571708 & 0.219329 & 2.298639 \\ \mathrm{O} & -1.425339 & 2.186346 & 1.395460 \\ \mathrm{~N} & -0.856505 & -1.869446 & -1.285191 \\ \mathrm{~N} & 1.298534 & -1.806948 & -0.889792 \\ \mathrm{C} & 0.113092 & -1.181158 & -0.639055 \\ \mathrm{C} & -0.393105 & -3.126007 & -1.876096 \\ \mathrm{H} & -0.752180 & -3.234485 & -2.902567 \\ \mathrm{H} & -0.752972 & -3.984173 & -1.293009 \\ \mathrm{C} & 1.134913 & -2.962351 & -1.798426\end{array}$

\begin{tabular}{|c|c|c|c|}
\hline & 1.640507 & -3.841063 & -1.388354 \\
\hline & 1.577259 & -2.731724 & -2.774126 \\
\hline 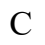 & -2.255427 & -1.566061 & -0.982495 \\
\hline & -2.746483 & -2.447463 & 0.193692 \\
\hline & -2.084129 & -2.288610 & 1.053515 \\
\hline & -2.687912 & -3.509004 & -0.085564 \\
\hline & -4.197367 & -2.077993 & 0.559776 \\
\hline & -4.539125 & -2.727043 & 1.376327 \\
\hline & -5.106471 & -2.266261 & -0.670455 \\
\hline 1 & -5.101915 & -3.316958 & -0.993833 \\
\hline & -6.142846 & -2.013537 & -0.410467 \\
\hline & -4.612659 & -1.357997 & -1.815303 \\
\hline & -5.265770 & -1.475369 & -2.689479 \\
\hline & -3.176915 & -1.780763 & -2.201627 \\
\hline & -2.806756 & -1.185078 & -3.046367 \\
\hline & -3.175854 & -2.835377 & -2.514792 \\
\hline & -4.635345 & 0.116110 & -1.347832 \\
\hline & -4.318439 & 0.776119 & -2.166520 \\
\hline $\mathrm{H}$ & -5.663358 & 0.400720 & -1.083835 \\
\hline & -3.700479 & 0.297216 & -0.118621 \\
\hline $\mathrm{H}$ & -3.718208 & 1.346287 & 0.200295 \\
\hline & -2.281395 & -0.080599 & -0.589084 \\
\hline & -2.100768 & 0.493647 & -1.511678 \\
\hline & -4.233969 & -0.607141 & 1.014247 \\
\hline $\mathrm{H}$ & -3.646227 & -0.487862 & 1.925051 \\
\hline 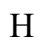 & -5.266806 & 82 & 1.251558 \\
\hline $\mathrm{C}$ & 2.481350 & -1.708559 & -0.087451 \\
\hline$S^{2}$ & 2.489659 & -2.350881 & 1.171503 \\
\hline & 3.639218 & -2.223939 & 1.960750 \\
\hline & 3.666641 & -2.688877 & 2.942293 \\
\hline & 4.749551 & -1.517403 & 1.502892 \\
\hline$x$ & 4.742737 & -0.948537 & 0.231311 \\
\hline $\mathrm{T}$ & 5.623272 & 3546 & -0.125323 \\
\hline $\mathrm{C}$ & 3.614336 & -1.038375 & -0.593499 \\
\hline 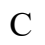 & 1.325140 & -3.210169 & 1.662524 \\
\hline & 0.548461 & -3.213218 & 0.893000 \\
\hline & 3.647671 & -0.483944 & -2.013327 \\
\hline & 2.614873 & -0.441226 & -2.374921 \\
\hline & 1.153589 & 0.759568 & 1.760074 \\
\hline & 1.589340 & -0.206430 & 1.974738 \\
\hline & 0.727264 & 1.267752 & 2.621298 \\
\hline & 4.419347 & -1.458678 & -2.928491 \\
\hline+ & 4.399450 & -1.107419 & -3.966623 \\
\hline 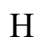 & 5.467648 & 2877 & -2.615315 \\
\hline$\Pi$ & 3.992617 & -2.467834 & -2.897726 \\
\hline & 4.243420 & 0.932419 & -2.121160 \\
\hline & & & -1.877058 \\
\hline & 4.142593 & 1.29 & -3.150534 \\
\hline$\Pi$ & 3.750452 & 1.659026 & -1.467874 \\
\hline 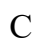 & 1.782968 & -4.670704 & 1.850437 \\
\hline & 2.239864 & -5.066694 & 0.935879 \\
\hline H & 2.522317 & -4.757834 & 2654685 \\
\hline 1 & 0.929279 & -5.306706 & 2.111956 \\
\hline & 0.668117 & -2.669880 & 2.946890 \\
\hline & -0.105981 & -3.365603 & 3.291519 \\
\hline & 1.399917 & -2.559025 & 3755704 \\
\hline$\Pi$ & 0.189238 & -1.701656 & 2.778078 \\
\hline & 5.630271 & -1.424994 & 2.132594 \\
\hline & -1.897408 & 1.446783 & 2.361988 \\
\hline & -2.590590 & 1.918750 & .241 \\
\hline
\end{tabular}




$\begin{array}{lrrr}\mathrm{C} & 0.494293 & 1.453287 & -1.106174 \\ \mathrm{H} & 1.285290 & 1.019185 & -1.719632 \\ \mathrm{C} & 0.124762 & 2.775043 & -1.736617 \\ \mathrm{O} & 0.565504 & 3.025719 & -2.860033 \\ \mathrm{~N} & -0.737810 & 3.601330 & -1.087635 \\ \mathrm{H} & -1.191074 & 3.282950 & -0.241969 \\ \mathrm{C} & -1.185990 & 4.834734 & -1.709483 \\ \mathrm{H} & -1.666055 & 5.459399 & -0.952164 \\ \mathrm{H} & -0.326456 & 5.364039 & -2.129581 \\ \mathrm{H} & -1.896693 & 4.646349 & -2.524437 \\ \mathrm{C} & 1.746659 & 1.531785 & 0.733560 \\ \mathrm{H} & 2.539976 & 1.060518 & 0.171210 \\ \mathrm{C} & 1.930218 & 3.029667 & 0.771641 \\ \mathrm{O} & 2.871479 & 3.527581 & 0.156393 \\ \mathrm{~N} & 1.063457 & 3.751555 & 1.531400 \\ \mathrm{H} & 0.189473 & 3.311658 & 1.798467 \\ \mathrm{C} & 1.093869 & 5.204333 & 1.475907 \\ \mathrm{H} & 0.495497 & 5.604608 & 2.298085 \\ \mathrm{H} & 2.125422 & 5.547307 & 1.575604 \\ \mathrm{H} & 0.699624 & 5.578516 & 0.521808\end{array}$

\begin{tabular}{|c|c|c|c|}
\hline \multicolumn{4}{|c|}{ INT4'-cross-AC } \\
\hline $\mathrm{Ru}$ & -0.516671 & 0.679948 & 0.405221 \\
\hline $\mathrm{O}$ & -1.854588 & 0.213702 & 2.453236 \\
\hline $\mathrm{O}$ & -1.653269 & 2.172923 & 1.536545 \\
\hline $\mathrm{N}$ & -0.554243 & -1.512734 & -1.463741 \\
\hline $\mathrm{N}$ & 1.556959 & -1.262674 & -0.942109 \\
\hline $\mathrm{C}$ & 0.298366 & -0.813531 & -0.673043 \\
\hline $\mathrm{C}$ & 0.078297 & -2.604762 & -2.204614 \\
\hline $\mathrm{H}$ & -0.207215 & -2.583649 & -3.259840 \\
\hline $\mathrm{H}$ & -0.214963 & -3.577780 & -1.789526 \\
\hline $\mathrm{C}$ & 1.572825 & -2.304831 & -1.990274 \\
\hline $\mathrm{H}$ & 2.138415 & -3.176099 & -1.647059 \\
\hline $\mathrm{H}$ & 2.053630 & -1.917144 & -2.895398 \\
\hline $\mathrm{C}$ & -1.983261 & -1.431212 & -1.184532 \\
\hline $\mathrm{C}$ & -2.413739 & -2.576051 & -0.231414 \\
\hline $\mathrm{H}$ & -1.798055 & -2.526028 & 0.675383 \\
\hline $\mathrm{H}$ & -2.229486 & -3.546962 & -0.711385 \\
\hline $\mathrm{C}$ & -3.906886 & -2.435006 & 0.121309 \\
\hline $\mathrm{H}$ & -4.200813 & -3.264268 & 0.777328 \\
\hline $\mathrm{C}$ & -4.748195 & -2.468888 & -1.170181 \\
\hline $\mathrm{H}$ & -4.616832 & -3.429633 & -1.687847 \\
\hline $\mathrm{H}$ & -5.814125 & -2.378916 & -0.923324 \\
\hline $\mathrm{C}$ & -4.321874 & -1.308609 & -2.093235 \\
\hline $\mathrm{H}$ & -4.927300 & -1.320677 & -3.008406 \\
\hline $\mathrm{C}$ & -2.835557 & -1.485227 & -2.471800 \\
\hline $\mathrm{H}$ & -2.508340 & -0.691949 & -3.156594 \\
\hline $\mathrm{H}$ & -2.691856 & -2.446361 & -2.986324 \\
\hline $\mathrm{C}$ & -4.520768 & 0.035237 & -1.358871 \\
\hline $\mathrm{H}$ & -4.244038 & 0.870413 & -2.016359 \\
\hline $\mathrm{H}$ & -5.580285 & 0.165357 & -1.100323 \\
\hline $\mathrm{C}$ & -3.662290 & 0.064107 & -0.062061 \\
\hline $\mathrm{H}$ & -3.807076 & 1.024247 & 0.443418 \\
\hline $\mathrm{C}$ & -2.199656 & -0.081929 & -0.494658 \\
\hline $\mathrm{H}$ & -1.961791 & 0.736954 & -1.221390 \\
\hline $\mathrm{C}$ & -4.117281 & -1.095493 & 0.849303 \\
\hline $\mathrm{H}$ & -3.557214 & -1.078722 & 1.786182 \\
\hline $\mathrm{H}$ & -5.179529 & -0.965487 & 1.096422 \\
\hline $\mathrm{C}$ & 2.693931 & -1.126558 & -0.081496 \\
\hline $\mathrm{C}$ & 2.730119 & -1.895982 & 1.103368 \\
\hline
\end{tabular}

$\begin{array}{lrrr} & & & \\ \mathrm{C} & 3.828846 & -1.731831 & 1.954886 \\ \mathrm{H} & 3.872632 & -2.293712 & 2.883638 \\ \mathrm{C} & 4.868363 & -0.864415 & 1.625434 \\ \mathrm{H} & 4.845455 & -0.171877 & 0.416995 \\ \mathrm{C} & 3.680842 & 0.468947 & 0.151904 \\ \mathrm{C} & 1.654958 & -0.295153 & -0.466665 \\ \mathrm{H} & 0.898932 & -2.926248 & 1.445901 \\ \mathrm{C} & 3.812433 & 0.374902 & -1.856053 \\ \mathrm{H} & 2.811776 & 0.308354 & -2.275909 \\ \mathrm{C} & 0.885779 & 0.847961 & 1.862822 \\ \mathrm{H} & 1.586342 & 0.042199 & 2.048539 \\ \mathrm{C} & 1.446044 & 1.958149 & 0.926099 \\ \mathrm{H} & 2.470301 & 1.660478 & 0.692049 \\ \mathrm{C} & 1.429094 & 3.340055 & 1.589739 \\ \mathrm{H} & 2.048395 & 3.318903 & 2.493306 \\ \mathrm{H} & 1.828999 & 4.103795 & 0.914005 \\ \mathrm{H} & 0.414408 & 3.624731 & 1.880104 \\ \mathrm{H} & 0.458817 & 1.235983 & 2.789881 \\ \mathrm{C} & 4.778520 & -0.408108 & -2.751311 \\ \mathrm{H} & 4.772689 & 0.010362 & -3.764460 \\ \mathrm{H} & 5.804567 & -0.354260 & -2.368225 \\ \mathrm{H} & 4.504744 & -1.467705 & -2.814144 \\ \mathrm{C} & 4.198900 & 1.864063 & -1.796704 \\ \mathrm{H} & 3.476198 & 2.463255 & -1.237269 \\ \mathrm{H} & 5.193069 & 2.013383 & -1.360221 \\ \mathrm{H} & 4.222537 & 2.265004 & -2.815774 \\ \mathrm{C} & 2.267143 & -4.341832 & 1.465254 \\ \mathrm{H} & 2.778836 & -4.569407 & 0.522566 \\ \mathrm{H} & 2.999593 & -4.448551 & 2.273554 \\ \mathrm{H} & 1.484821 & -5.093597 & 1.622317 \\ \mathrm{C} & 0.918088 & -2.627830 & 2.765105 \\ \mathrm{H} & 0.209159 & -3.434664 & 2.986100 \\ \mathrm{H} & 1.615617 & -2.559026 & 3.608097 \\ \mathrm{H} & 0.353780 & -1.693687 & 2.706656 \\ \mathrm{H} & 5.710658 & -0.746226 & 2.301822 \\ \mathrm{~N} & -2.171197 & 1.438548 & 2.483869 \\ \mathrm{O} & -2.906489 & 1.932264 & 3.320277 \\ \mathrm{C} & 0.720353 & 1.934803 & -0.529508 \\ \mathrm{H} & 1.405112 & 1.512928 & -1.261045 \\ \mathrm{C} & 0.326765 & 3.292770 & -1.083595 \\ \mathrm{O} & 1.066898 & 3.840311 & -1.904866 \\ \mathrm{~N} & -0.835116 & 3.864406 & -0.666847 \\ \mathrm{H} & -1.345430 & 3.445557 & 0.100482 \\ \mathrm{C} & -1.173757 & 5.219381 & -1.067862 \\ \mathrm{H} & -1.050589 & 5.324789 & -2.148618 \\ \mathrm{H} & -2.213367 & 5.419836 & -0.797232 \\ \mathrm{H} & -0.526452 & 5.961439 & -0.581700 \\ & & & \\ & & & \end{array}$

\begin{tabular}{lccc}
\multicolumn{2}{l}{ INT4'-homo-AC } & & \\
$\mathrm{Ru}$ & -0.461124 & 0.567993 & 0.225743 \\
$\mathrm{O}$ & -1.726751 & 0.699025 & 2.364644 \\
$\mathrm{O}$ & -1.184311 & 2.432359 & 1.171124 \\
$\mathrm{~N}$ & -1.066017 & -1.823248 & -1.257704 \\
$\mathrm{~N}$ & 1.076247 & -1.959768 & -0.820045 \\
$\mathrm{C}$ & -0.044006 & -1.216858 & -0.604984 \\
$\mathrm{C}$ & -0.715007 & -3.124337 & -1.829439 \\
$\mathrm{H}$ & -1.053079 & -3.203879 & -2.865934 \\
$\mathrm{H}$ & -1.174531 & -3.938310 & -1.253741 \\
$\mathrm{C}$ & 0.819399 & -3.114827 & -1.706926
\end{tabular}




\begin{tabular}{|c|c|c|c|}
\hline $\mathrm{H}$ & 1.219076 & -4.029417 & -1.259776 \\
\hline $\mathrm{H}$ & 1.311709 & -2.957503 & -2.672845 \\
\hline $\mathrm{C}$ & -2.428587 & -1.395940 & -0.960305 \\
\hline $\mathrm{C}$ & -3.033930 & -2.269406 & 0.168973 \\
\hline $\mathrm{H}$ & -2.369569 & -2.226376 & 1.041135 \\
\hline $\mathrm{H}$ & -3.088188 & -3.315668 & -0.161321 \\
\hline $\mathrm{C}$ & -4.439886 & -1.756570 & 0.534994 \\
\hline $\mathrm{H}$ & -4.862726 & -2.397089 & 1.319362 \\
\hline $\mathrm{C}$ & -5.344438 & -1.794226 & -0.712825 \\
\hline $\mathrm{H}$ & -5.449617 & -2.825476 & -1.078135 \\
\hline $\mathrm{H}$ & -6.350822 & -1.439562 & -0.454845 \\
\hline $\mathrm{C}$ & -4.738190 & -0.901762 & -1.815514 \\
\hline $\mathrm{H}$ & -5.385156 & -0.915219 & -2.701609 \\
\hline $\mathrm{C}$ & -3.347014 & -1.450189 & -2.201460 \\
\hline $\mathrm{H}$ & -2.900655 & -0.855529 & -3.009000 \\
\hline $\mathrm{H}$ & -3.439571 & -2.484007 & -2.564166 \\
\hline $\mathrm{C}$ & -4.607686 & 0.545751 & -1.293046 \\
\hline $\mathrm{H}$ & -4.201801 & 1.196964 & -2.078771 \\
\hline $\mathrm{H}$ & -5.599164 & 0.937623 & -1.029156 \\
\hline $\mathrm{C}$ & -3.686403 & 0.576555 & -0.039992 \\
\hline $\mathrm{H}$ & -3.593806 & 1.608626 & 0.313937 \\
\hline $\mathrm{C}$ & -2.317713 & 0.054511 & -0.485273 \\
\hline $\mathrm{H}$ & -1.958064 & 0.685316 & -1.339020 \\
\hline $\mathrm{C}$ & -4.318850 & -0.311974 & 1.052347 \\
\hline $\mathrm{H}$ & -3.712690 & -0.283286 & 1.960118 \\
\hline $\mathrm{H}$ & -5.312670 & 0.080380 & 1.306446 \\
\hline $\mathrm{C}$ & 2.254443 & -1.954434 & -0.002780 \\
\hline $\mathrm{C}$ & 2.173616 & -2.533837 & 1.283662 \\
\hline $\mathrm{C}$ & 3.316008 & -2.489805 & 2.091448 \\
\hline $\mathrm{H}$ & 3.276468 & -2.904819 & 3.094618 \\
\hline $\mathrm{C}$ & 4.501839 & -1.924443 & 1.626902 \\
\hline $\mathrm{C}$ & 4.577356 & -1.422832 & 0.329700 \\
\hline $\mathrm{H}$ & 5.514444 & -1.011779 & -0.032141 \\
\hline $\mathrm{C}$ & 3.460907 & -1.435671 & -0.516382 \\
\hline $\mathrm{C}$ & 0.914122 & -3.232199 & 1.793849 \\
\hline $\mathrm{H}$ & 0.160559 & -3.209486 & 1.001524 \\
\hline $\mathrm{C}$ & 3.587465 & -0.968368 & -1.962121 \\
\hline $\mathrm{H}$ & 2.576135 & -0.841559 & -2.361564 \\
\hline $\mathrm{C}$ & 1.046508 & 0.674571 & 1.582853 \\
\hline $\mathrm{H}$ & 1.581678 & -0.218185 & 1.885741 \\
\hline $\mathrm{H}$ & 0.792402 & 1.291406 & 2.445519 \\
\hline $\mathrm{C}$ & 4.281154 & -2.065666 & -2.797670 \\
\hline $\mathrm{H}$ & 4.327695 & -1.771830 & -3.852661 \\
\hline $\mathrm{H}$ & 5.306668 & -2.228555 & -2.445219 \\
\hline $\mathrm{H}$ & 3.752075 & -3.023389 & -2.729465 \\
\hline $\mathrm{C}$ & 4.326843 & 0.373117 & -2.125162 \\
\hline $\mathrm{H}$ & 5.386923 & 0.278325 & -1.861750 \\
\hline $\mathrm{H}$ & 4.275880 & 0.693109 & -3.171360 \\
\hline $\mathrm{H}$ & 3.901738 & 1.174620 & -1.514145 \\
\hline $\mathrm{C}$ & 1.207232 & -4.715784 & 2.094719 \\
\hline $\mathrm{H}$ & 1.640363 & -5.221846 & 1.223905 \\
\hline $\mathrm{H}$ & 1.912624 & -4.824522 & 2.926287 \\
\hline $\mathrm{H}$ & 0.283657 & -5.237333 & 2.371913 \\
\hline $\mathrm{C}$ & 0.289790 & -2.534329 & 3.017030 \\
\hline $\mathrm{H}$ & -0.571093 & -3.110219 & 3.377424 \\
\hline $\mathrm{H}$ & 1.007735 & -2.457535 & 3.842077 \\
\hline $\mathrm{H}$ & -0.060040 & -1.528618 & 2.770181 \\
\hline $\mathrm{H}$ & 5.375807 & -1.891882 & 2.271754 \\
\hline $\mathrm{N}$ & -1.764187 & 1.954028 & 2.247132 \\
\hline $\mathrm{O}$ & -2.285475 & 2.706073 & 3.050520 \\
\hline
\end{tabular}

$\begin{array}{lrrr}\mathrm{C} & 0.927522 & 1.346637 & -0.979856 \\ \mathrm{H} & 1.454280 & 0.677038 & -1.652743 \\ \mathrm{C} & 0.643640 & 2.586623 & -1.808077 \\ \mathrm{O} & 1.003705 & 2.607333 & -2.985256 \\ \mathrm{~N} & -0.033551 & 3.629007 & -1.249245 \\ \mathrm{H} & -0.473183 & 3.518468 & -0.346970 \\ \mathrm{C} & -0.370436 & 4.793835 & -2.049504 \\ \mathrm{H} & -0.757462 & 5.574564 & -1.389761 \\ \mathrm{H} & 0.523264 & 5.159983 & -2.561543 \\ \mathrm{H} & -1.123744 & 4.561181 & -2.813378 \\ \mathrm{C} & 1.756317 & 1.423295 & 0.420419 \\ \mathrm{H} & 2.650003 & 0.845472 & 0.195570 \\ \mathrm{C} & 2.248483 & 2.851089 & 0.683330 \\ \mathrm{O} & 3.294265 & 3.216278 & 0.154463 \\ \mathrm{~N} & 1.504446 & 3.644079 & 1.498304 \\ \mathrm{H} & 0.561521 & 3.343961 & 1.717797 \\ \mathrm{C} & 1.801671 & 5.063849 & 1.601229 \\ \mathrm{H} & 1.291333 & 5.474759 & 2.475858 \\ \mathrm{H} & 2.879594 & 5.196016 & 1.710813 \\ \mathrm{H} & 1.480408 & 5.607874 & 0.702461\end{array}$

\section{INT4'-cross-2-AC}

0.349214

$\begin{array}{llll}\mathrm{C} & 1.063396 & 1.618068 & -1.541777\end{array}$

$\begin{array}{llll}\mathrm{H} & 1.877583 & 1.187974 & -2.119833\end{array}$

$\begin{array}{llll}\mathrm{H} & 1.320694 & 2.654466 & -1.283945\end{array}$

$\begin{array}{llll}\text { C } & -0.304091 & 1.484577 & -2.190288\end{array}$

$\begin{array}{llll}\text { C } & -0.675605 & 2.550502 & -3.226858\end{array}$

$\mathrm{H} \quad-1.704195 \quad 2.408223 \quad-3.578605$

$\begin{array}{llll}\mathrm{H} & -0.002238 & 2.497438 & -4.091609\end{array}$

$\begin{array}{llll}\mathrm{H} & -0.603478 & 3.555394 & -2.797771\end{array}$

$\mathrm{H} \quad-0.356594 \quad 0.499320 \quad-2.674396$

$\begin{array}{llll}\text { C } & -1.128979 & 1.402224 & -0.901619\end{array}$

$\begin{array}{llll}\text { C } & -1.948682 & 2.631696 & -0.561370\end{array}$

$\begin{array}{llll}\text { O } & -3.169521 & 2.605414 & -0.738507\end{array}$

$\begin{array}{llll}\mathrm{N} & -1.306319 & 3.768269 & -0.160807\end{array}$

$\begin{array}{llll}\mathrm{H} & -0.351137 & 3.683228 & 0.157534\end{array}$

$\begin{array}{llll}\text { C } & -2.052166 & 4.946590 & 0.252350\end{array}$

$\mathrm{H} \quad-2.405817 \quad 4.862007 \quad 1.288621$

$\mathrm{H} \quad-2.919700 \quad 5.060143 \quad-0.399903$

$\mathrm{H} \quad-1.411413 \quad 5.828862 \quad 0.166943$

$\begin{array}{llll}\mathrm{H} & -1.844495 & 0.587929 & -0.888975\end{array}$

$\begin{array}{llll}\text { C } & 0.249022 & -1.061672 & -0.311616\end{array}$

$\mathrm{N} \quad 1.366053 \quad-1.776180 \quad-0.620092$

$\begin{array}{llll}\text { C } & 2.675212 & -1.165952 & -0.448502\end{array}$

$\begin{array}{llll}\text { C } & 2.422539 & 0.199243 & 0.208478\end{array}$

$\begin{array}{llll}\text { C } & 3.718517 & 1.006576 & 0.296626\end{array}$

$\begin{array}{llll}\mathrm{C} & 4.442229 & 1.132248 & -1.061001\end{array}$

$\begin{array}{llll}\text { C } & 4.728765 & -0.270946 & -1.633388\end{array}$

$\begin{array}{llll}\text { C } & 3.400147 & -1.030006 & -1.810004\end{array}$

$\mathrm{H} \quad 3.589854 \quad-2.035747 \quad-2.210550$

$\begin{array}{llll}\mathrm{H} & 2.745312 & -0.520602 & -2.522207\end{array}$

$\begin{array}{llll}\text { C } & 5.631854 & -1.055457 & -0.660765\end{array}$

$\begin{array}{llll}\mathrm{C} & 4.920300 & -1.191715 & 0.701103\end{array}$

$\begin{array}{llll}\mathrm{C} & 4.644656 & 0.218650 & 1.269008\end{array}$

$\begin{array}{llll}\mathrm{H} & 4.179149 & 0.148743 & 2.261021\end{array}$

$\begin{array}{llll}\mathrm{H} & 5.592057 & 0.759180 & 1.396246\end{array}$

$\begin{array}{llll}\text { C } & 3.596298 & -1.969319 & 0.509777\end{array}$

$\mathrm{H} \quad 3.086735 \quad-2.116771 \quad 1.471089$

$\begin{array}{llll}\mathrm{H} & 3.805329 & -2.964313 & 0.092369\end{array}$ 


\begin{tabular}{|c|c|c|c|}
\hline $\mathrm{H}$ & 5.558789 & -1.748205 & 1.398628 \\
\hline $\mathrm{H}$ & 6.589560 & -0.534671 & -0.531332 \\
\hline $\mathrm{H}$ & 5.857483 & -2.049143 & -1.071647 \\
\hline $\mathrm{H}$ & 5.224816 & -0.178853 & -2.607672 \\
\hline $\mathrm{H}$ & 5.386713 & 1.673175 & -0.914538 \\
\hline $\mathrm{H}$ & 3.845847 & 1.720515 & -1.764257 \\
\hline $\mathrm{H}$ & 3.504331 & 1.998047 & 0.710968 \\
\hline $\mathrm{H}$ & 2.087371 & 0.009343 & 1.287209 \\
\hline $\mathrm{C}$ & 1.103644 & -3.199469 & -0.831570 \\
\hline $\mathrm{C}$ & -0.416892 & -3.184803 & -1.053676 \\
\hline $\mathrm{N}$ & -0.817892 & -1.894075 & -0.454012 \\
\hline $\mathrm{C}$ & -2.139930 & -1.702285 & 0.077159 \\
\hline $\mathrm{C}$ & -3.212934 & -1.492251 & -0.812709 \\
\hline $\mathrm{C}$ & -4.500601 & -1.375119 & -0.277416 \\
\hline $\mathrm{C}$ & -4.712879 & -1.444763 & 1.094625 \\
\hline $\mathrm{C}$ & -3.638442 & -1.641130 & 1.959438 \\
\hline $\mathrm{C}$ & -2.333394 & -1.783273 & 1.476176 \\
\hline $\mathrm{C}$ & -1.184611 & -2.077072 & 2.439081 \\
\hline $\mathrm{C}$ & -1.377738 & -1.468854 & 3.837979 \\
\hline $\mathrm{H}$ & -2.170517 & -1.977966 & 4.398977 \\
\hline $\mathrm{H}$ & -0.453165 & -1.580919 & 4.416745 \\
\hline $\mathrm{H}$ & -1.617410 & -0.404964 & 3.773912 \\
\hline $\mathrm{C}$ & -0.948509 & -3.597432 & 2.552505 \\
\hline $\mathrm{H}$ & -1.840202 & -4.095066 & 2.952004 \\
\hline $\mathrm{H}$ & -0.110845 & -3.808307 & 3.228740 \\
\hline $\mathrm{H}$ & -0.721556 & -4.051941 & 1.583005 \\
\hline $\mathrm{H}$ & -0.274909 & -1.629169 & 2.028300 \\
\hline $\mathrm{H}$ & -3.821635 & -1.684173 & 3.027396 \\
\hline $\mathrm{H}$ & -5.717173 & -1.336686 & 1.495294 \\
\hline $\mathrm{H}$ & -5.341137 & -1.205650 & -0.942928 \\
\hline $\mathrm{C}$ & -3.006339 & -1.382935 & -2.318855 \\
\hline $\mathrm{C}$ & -3.736384 & -0.169161 & -2.928688 \\
\hline $\mathrm{H}$ & -3.408839 & -0.022699 & -3.965059 \\
\hline $\mathrm{H}$ & -3.546818 & 0.748108 & -2.362997 \\
\hline $\mathrm{H}$ & -4.820951 & -0.328649 & -2.951647 \\
\hline $\mathrm{C}$ & -3.441967 & -2.682413 & -3.025637 \\
\hline $\mathrm{H}$ & -3.251472 & -2.619704 & -4.103857 \\
\hline $\mathrm{H}$ & -4.515707 & -2.852956 & -2.882735 \\
\hline $\mathrm{H}$ & -2.915479 & -3.560052 & -2.633601 \\
\hline $\mathrm{H}$ & -1.934238 & -1.240690 & -2.495844 \\
\hline $\mathrm{H}$ & -0.666295 & -3.193039 & -2.121417 \\
\hline $\mathrm{H}$ & -0.937467 & -4.014048 & -0.571163 \\
\hline $\mathrm{H}$ & 1.390579 & -3.783314 & 0.054181 \\
\hline $\mathrm{H}$ & 1.651214 & -3.586922 & -1.695357 \\
\hline $\mathrm{O}$ & 0.677641 & 2.629050 & 1.606252 \\
\hline $\mathrm{N}$ & -0.318746 & 2.282346 & 2.352688 \\
\hline $\mathrm{O}$ & -0.712363 & 2.945115 & 3.284342 \\
\hline $\mathrm{O}$ & -0.866534 & 1.155485 & 2.014588 \\
\hline
\end{tabular}

$\begin{array}{lccc}\text { INT4'-homo-2-AC } & & \\ \mathrm{Ru} & 0.660688 & 0.418239 & -0.111830 \\ \mathrm{O} & 1.607477 & 2.373758 & -0.047055 \\ \mathrm{O} & 2.506362 & 2.248835 & -2.057784 \\ \mathrm{~N} & 0.971861 & -2.430861 & -0.075664 \\ \mathrm{~N} & -1.187599 & -2.158725 & -0.321918 \\ \mathrm{C} & -0.008268 & -1.497049 & -0.147749 \\ \mathrm{C} & 0.526381 & -3.777956 & -0.431825 \\ \mathrm{H} & 0.924358 & -4.527991 & 0.256803 \\ \mathrm{H} & 0.854757 & -4.033104 & -1.447915 \\ \mathrm{C} & -0.998219 & -3.627337 & -0.337915\end{array}$

\begin{tabular}{|c|c|c|c|}
\hline $\mathrm{H}$ & -1.529423 & -4.069217 & -1.183989 \\
\hline $\mathrm{H}$ & -1.395934 & -4.062140 & 0.583663 \\
\hline $\mathrm{C}$ & 2.368636 & -2.006974 & -0.019789 \\
\hline $\mathrm{C}$ & 3.015230 & -2.038213 & -1.428129 \\
\hline $\mathrm{H}$ & 2.413041 & -1.423974 & -2.110634 \\
\hline $\mathrm{H}$ & 3.009463 & -3.067503 & -1.812757 \\
\hline $\mathrm{C}$ & 4.458947 & -1.504111 & -1.356950 \\
\hline $\mathrm{H}$ & 4.907157 & -1.548161 & -2.357478 \\
\hline $\mathrm{C}$ & 5.278820 & -2.369362 & -0.379215 \\
\hline $\mathrm{H}$ & 5.323956 & -3.409084 & -0.732919 \\
\hline $\mathrm{H}$ & 6.311538 & -2.000340 & -0.330672 \\
\hline $\mathrm{C}$ & 4.634634 & -2.314277 & 1.021620 \\
\hline $\mathrm{H}$ & 5.219673 & -2.922456 & 1.723068 \\
\hline $\mathrm{C}$ & 3.201235 & -2.885888 & 0.940292 \\
\hline $\mathrm{H}$ & 2.725733 & -2.892844 & 1.929981 \\
\hline $\mathrm{H}$ & 3.237027 & -3.924809 & 0.582234 \\
\hline $\mathrm{C}$ & 4.596372 & -0.850106 & 1.512821 \\
\hline $\mathrm{H}$ & 4.162683 & -0.800853 & 2.521115 \\
\hline $\mathrm{H}$ & 5.619250 & -0.455948 & 1.582150 \\
\hline $\mathrm{C}$ & 3.766294 & 0.017354 & 0.527824 \\
\hline $\mathrm{H}$ & 3.732515 & 1.053706 & 0.880219 \\
\hline $\mathrm{C}$ & 2.345991 & -0.562168 & 0.506044 \\
\hline $\mathrm{H}$ & 1.943207 & -0.562702 & 1.546388 \\
\hline $\mathrm{C}$ & 4.424522 & -0.045493 & -0.865405 \\
\hline $\mathrm{H}$ & 3.870492 & 0.581037 & -1.567651 \\
\hline $\mathrm{H}$ & 5.446106 & 0.353322 & -0.805417 \\
\hline $\mathrm{C}$ & -2.518321 & -1.666945 & -0.066876 \\
\hline $\mathrm{C}$ & -3.353420 & -1.354348 & -1.161052 \\
\hline $\mathrm{C}$ & -4.655794 & -0.914460 & -0.893612 \\
\hline $\mathrm{H}$ & -5.309419 & -0.651445 & -1.718919 \\
\hline $\mathrm{C}$ & -5.120364 & -0.795950 & 0.412030 \\
\hline $\mathrm{C}$ & -4.293956 & -1.137884 & 1.478354 \\
\hline $\mathrm{H}$ & -4.667865 & -1.047420 & 2.493117 \\
\hline $\mathrm{C}$ & -2.985966 & -1.588624 & 1.267992 \\
\hline $\mathrm{C}$ & -2.894157 & -1.503758 & -2.605759 \\
\hline $\mathrm{H}$ & -1.804263 & -1.608265 & -2.598127 \\
\hline $\mathrm{C}$ & -2.145150 & -2.010769 & 2.472122 \\
\hline $\mathrm{H}$ & -1.103866 & -2.111673 & 2.148299 \\
\hline $\mathrm{C}$ & -0.344846 & 1.000100 & -1.723187 \\
\hline $\mathrm{H}$ & -0.771347 & 0.249083 & -2.383395 \\
\hline $\mathrm{H}$ & 0.129092 & 1.812929 & -2.272341 \\
\hline $\mathrm{N}$ & 2.264417 & 2.932722 & -1.057231 \\
\hline $\mathrm{O}$ & 2.567534 & 4.115522 & -0.922270 \\
\hline $\mathrm{C}$ & -3.256662 & -0.282829 & -3.475809 \\
\hline $\mathrm{H}$ & -2.739934 & -0.344463 & -4.440426 \\
\hline $\mathrm{H}$ & -4.331662 & -0.250410 & -3.686215 \\
\hline $\mathrm{H}$ & -2.990414 & 0.665525 & -2.999010 \\
\hline $\mathrm{C}$ & -3.479941 & -2.788704 & -3.226976 \\
\hline $\mathrm{H}$ & -3.122153 & -2.917757 & -4.255274 \\
\hline $\mathrm{H}$ & -3.204027 & -3.681116 & -2.653933 \\
\hline $\mathrm{H}$ & -4.574942 & -2.739089 & -3.252773 \\
\hline $\mathrm{C}$ & -2.620656 & -3.381794 & 3.001766 \\
\hline $\mathrm{H}$ & -3.648215 & -3.307929 & 3.376464 \\
\hline $\mathrm{H}$ & -2.612556 & -4.156262 & 2.227476 \\
\hline $\mathrm{H}$ & -1.982988 & -3.716465 & 3.828461 \\
\hline $\mathrm{C}$ & -2.172792 & -0.988616 & 3.628119 \\
\hline $\mathrm{H}$ & -1.974643 & 0.034281 & 3.300039 \\
\hline $\mathrm{H}$ & -3.147745 & -0.983547 & 4.128704 \\
\hline $\mathrm{H}$ & -1.425079 & -1.265323 & 4.380571 \\
\hline $\mathrm{H}$ & -6.128523 & -0.437631 & 0.599684 \\
\hline
\end{tabular}




$\begin{array}{lr}\mathrm{C} & -0.917196 \\ \mathrm{H} & -1.598550 \\ \mathrm{C} & -0.943946 \\ \mathrm{O} & -2.016102 \\ \mathrm{~N} & 0.199605 \\ \mathrm{H} & 0.992206 \\ \mathrm{C} & 0.157197 \\ \mathrm{H} & 1.176964 \\ \mathrm{H} & -0.306457 \\ \mathrm{H} & -0.432836 \\ \mathrm{C} & -1.373139 \\ \mathrm{H} & -2.280619 \\ \mathrm{C} & -1.766123 \\ \mathrm{O} & -2.875705 \\ \mathrm{~N} & -0.831726 \\ \mathrm{H} & 0.063227 \\ \mathrm{C} & -1.072308 \\ \mathrm{H} & -1.524288 \\ \mathrm{H} & -0.119656 \\ \mathrm{H} & -1.755945\end{array}$

\section{TS3'-cross-AC}

$\begin{array}{lccc}\mathrm{Ru} & 0.590105 & 0.647008 & -0.575606 \\ \mathrm{O} & 1.560913 & 2.571926 & -0.889178 \\ \mathrm{O} & 0.828685 & 2.227295 & 1.121935 \\ \mathrm{~N} & 1.035174 & -2.092369 & 0.178407 \\ \mathrm{~N} & -1.142691 & -1.951881 & -0.033491 \\ \mathrm{C} & 0.027502 & -1.226862 & -0.083375 \\ \mathrm{C} & 0.625901 & -3.492983 & 0.204125 \\ \mathrm{H} & 1.095554 & -4.035271 & 1.029189 \\ \mathrm{H} & 0.899693 & -3.991424 & -0.735920 \\ \mathrm{C} & -0.894802 & -3.357769 & 0.364647 \\ \mathrm{H} & -1.457839 & -4.044707 & -0.270971 \\ \mathrm{H} & -1.211575 & -3.511457 & 1.403828 \\ \mathrm{C} & 2.413585 & -1.604893 & 0.137058 \\ \mathrm{C} & 3.066476 & -1.877045 & -1.238346 \\ \mathrm{H} & 2.421725 & -1.486320 & -2.031185 \\ \mathrm{H} & 3.160251 & -2.961812 & -1.393140 \\ \mathrm{C} & 4.451355 & -1.206510 & -1.292892 \\ \mathrm{H} & 4.901915 & -1.385275 & -2.278045 \\ \mathrm{C} & 5.344600 & -1.819105 & -0.195079 \\ \mathrm{H} & 5.476940 & -2.895727 & -0.373825 \\ \mathrm{H} & 6.343072 & -1.362849 & -0.221073 \\ \mathrm{C} & 4.697420 & -1.581204 & 1.185258 \\ \mathrm{H} & 5.325103 & -2.024912 & 1.968945 \\ \mathrm{C} & 3.302795 & -2.252377 & 1.224212 \\ \mathrm{H} & 2.835458 & -2.115352 & 2.208382 \\ \mathrm{H} & 3.410316 & -3.334146 & 1.055043 \\ \mathrm{C} & 4.569514 & -0.060350 & 1.417405 \\ \mathrm{H} & 4.147313 & 0.140435 & 2.410981 \\ \mathrm{H} & 5.567540 & 0.399069 & 1.392564 \\ \mathrm{C} & 3.663098 & 0.566030 & 0.326082 \\ \mathrm{H} & 3.587887 & 1.642940 & 0.501501 \\ \mathrm{C} & 2.281355 & -0.097623 & 0.441611 \\ \mathrm{H} & 1.950446 & -0.011769 & 1.487361 \\ \mathrm{C} & 4.288095 & 0.311119 & -1.059432 \\ \mathrm{H} & 3.651934 & 0.750778 & -1.836886 \\ \mathrm{H} & 5.267876 & 0.803237 & -1.123697 \\ \mathrm{C} & -2.407567 & -1.387022 & 0.344427 \\ \mathrm{C} & -3.465267 & -1.391661 & -0.589189 \\ & & & \end{array}$

$\begin{array}{cc}1.141298 & 0.881369 \\ 0.385955 & 1.255304 \\ 2.243854 & 1.941714 \\ 2.444036 & 2.513886 \\ 2.890644 & 2.278074 \\ 2.827790 & 1.645975 \\ 3.989616 & 3.230210 \\ 4.228979 & 3.541126 \\ 4.884631 & 2.794727 \\ 3.693805 & 4.100517 \\ 1.486982 & -0.615334 \\ 0.906058 & -0.760965 \\ 2.950439 & -0.887062 \\ 3.174856 & -1.358834 \\ 3.897246 & -0.644006 \\ 3.618307 & -0.262141 \\ 5.306415 & -0.903716 \\ 5.804657 & -0.036619 \\ 5.787359 & -1.139677 \\ 5.402087 & -1.750140\end{array}$

H -4.688635

C $\quad-4.859202$

C $\quad-3.813296$

$\mathrm{H} \quad-3.964715$

C $\quad-2.573155$

C $\quad-3.298293$

$\mathrm{H} \quad-2.222458$

C $\quad-1.485157$

$\mathrm{H} \quad-0.582910$

C $\quad 0.640728$

$\mathrm{H} \quad 0.227137$

C $\quad-1.088447$

H $\quad-1.605771$

C $\quad-0.751694$

H $\quad-0.533545$

H $\quad-1.609695$

$\mathrm{H} \quad 0.089658$

$\begin{array}{ll}\mathrm{H} & 0.089658 \\ \mathrm{H} & 1.217097\end{array}$

$\mathrm{N} \quad 1.463314$

O 1.959255

C $\quad-3.915285$

$\mathrm{H} \quad-3.627024$

H $\quad-5.010472$

H $\quad-3.588003$

C $\quad-3.899454$

H -3.734338

$\mathrm{H} \quad-3.459904$

H $\quad-4.980639$

C $\quad-1.926554$

$\mathrm{H} \quad-2.796855$

H -2.199843

H $\quad-1.116265$

C $\quad-1.087473$

$\mathrm{H} \quad-0.689244$

H $\quad-1.937665$

H $\quad-0.307565$

H $\quad-5.810836$

C -1.448517

H $\quad-2.149372$

C $\quad-1.589794$

O $\quad-1.128999$

$\mathrm{N} \quad-2.327057$

$\mathrm{H} \quad-2.543861$

C $\quad-2.324774$

$\mathrm{H} \quad-1.428075$

$\mathrm{H}-2.330415$

H $\quad-3.218410$

$-0.828293$

$-0.810366$

$-0.278605$

$-0.311796$

0.095742

$-0.875706$

$-2.000507$

$-2.082154$

$-0.951343$

$-1.378623$

0.142441

$-0.751040$

1.373066

0.548931

2.540186

2.186826

3.221069

3.115993

0.768347

3.009166

4.057802

$-1.139962$

$-1.535015$

$-1.145129$

$-0.097395$

$-3.420980$

$-3.884955$

$-4.069890$

$-3.384898$

$-1.885469$

$-1.480437$

$-2.880144$

$-2.001650$

0.432272

1.077448

0.938789

0.322110

0.167455

1.521209

0.786079

2.865274

3.910150

2.824229

1.913231

3.950035

3.949080

4.872400

3.911632

\section{TS3'-homo-AC}

$\begin{array}{lccc}\mathrm{Ru} & 0.584658 & 0.570779 & -0.115605 \\ \mathrm{O} & 1.385617 & 2.592399 & 0.095691 \\ \mathrm{O} & 0.937486 & 1.587025 & 1.965304 \\ \mathrm{~N} & 1.292500 & -2.220307 & -0.250324 \\ \mathrm{~N} & -0.897339 & -2.209197 & -0.343894 \\ \mathrm{C} & 0.208479 & -1.409381 & -0.195557 \\ \mathrm{C} & 0.980336 & -3.589263 & -0.656201 \\ \mathrm{H} & 1.540549 & -4.321176 & -0.068382 \\ \mathrm{H} & 1.222128 & -3.739689 & -1.717173 \\ \mathrm{C} & -0.532062 & -3.642261 & -0.399927\end{array}$

$-0.205928$

$-0.909954$

1.061769

1.981867

2.977769

1.653103

$-1.975025$

$-2.165038$

2.723211

2.280227

$-2.388945$

$-2.875368$

$-2.180719$

$-2.666976$

$-3.087865$

$-4.100448$

$-3.123957$

$-2.703499$

$-3.081704$

0.320650

0.676551

$-3.092654$

$-4.073377$

$-3.050441$

$-3.031066$

$-2.014048$

$-2.993623$

$-1.249309$

$-1.833650$

3.868574

4.397884

3.497287

4.597553

3.269026

2.483361

3.742724

4.031227

1.337242

$-0.803982$

$-0.433069$

$-0.153506$

$-0.606203$

1.001449

1.381536

1.919377

2.553501

1.336698

2.549544

$-0.399927$ 


\begin{tabular}{|c|c|c|c|}
\hline $\mathrm{H}$ & -1.086013 & -4.152369 & -1.190508 \\
\hline $\mathrm{H}$ & -0.770618 & -4.124488 & 0.557032 \\
\hline $\mathrm{C}$ & 2.621877 & -1.610061 & -0.293518 \\
\hline $\mathrm{C}$ & 3.108219 & -1.435469 & -1.752179 \\
\hline $\mathrm{H}$ & 2.345359 & -0.905972 & -2.331466 \\
\hline $\mathrm{H}$ & 3.247449 & -2.422442 & -2.216138 \\
\hline $\mathrm{C}$ & 4.432340 & -0.650057 & -1.764977 \\
\hline $\mathrm{H}$ & 4.761840 & -0.513424 & -2.803226 \\
\hline $\mathrm{C}$ & 5.493658 & -1.451978 & -0.984561 \\
\hline $\mathrm{H}$ & 5.665308 & -2.422783 & -1.470837 \\
\hline $\mathrm{H}$ & 6.451728 & -0.915530 & -0.988684 \\
\hline $\mathrm{C}$ & 5.016400 & -1.657847 & 0.468146 \\
\hline $\mathrm{H}$ & 5.765145 & -2.236345 & 1.024534 \\
\hline $\mathrm{C}$ & 3.680517 & -2.440504 & 0.467941 \\
\hline $\mathrm{H}$ & 3.336081 & -2.617640 & 1.495298 \\
\hline $\mathrm{H}$ & 3.827387 & -3.421974 & -0.006862 \\
\hline $\mathrm{C}$ & 4.828029 & -0.275551 & 1.128883 \\
\hline $\mathrm{H}$ & 4.524833 & -0.391431 & 2.177864 \\
\hline $\mathrm{H}$ & 5.784767 & 0.265050 & 1.127889 \\
\hline $\mathrm{C}$ & 3.753730 & 0.535568 & 0.358924 \\
\hline $\mathrm{H}$ & 3.636811 & 1.513151 & 0.835148 \\
\hline $\mathrm{C}$ & 2.440319 & -0.259749 & 0.429321 \\
\hline $\mathrm{H}$ & 2.238527 & -0.489272 & 1.486532 \\
\hline $\mathrm{C}$ & 4.209950 & 0.725365 & -1.100518 \\
\hline $\mathrm{H}$ & 3.453630 & 1.297054 & -1.651923 \\
\hline $\mathrm{H}$ & 5.141557 & 1.306259 & -1.128551 \\
\hline $\mathrm{C}$ & -2.198223 & -1.861104 & 0.151752 \\
\hline $\mathrm{C}$ & -3.252122 & -1.658446 & -0.765472 \\
\hline $\mathrm{C}$ & -4.491170 & -1.247937 & -0.257334 \\
\hline $\mathrm{H}$ & -5.314271 & -1.064922 & -0.940350 \\
\hline $\mathrm{C}$ & -4.685251 & -1.070881 & 1.110440 \\
\hline $\mathrm{C}$ & -3.650003 & -1.338886 & 2.004432 \\
\hline $\mathrm{H}$ & -3.824307 & -1.236524 & 3.072254 \\
\hline $\mathrm{C}$ & -2.390638 & -1.749474 & 1.548873 \\
\hline $\mathrm{C}$ & -3.083809 & -1.956552 & -2.252573 \\
\hline $\mathrm{H}$ & -2.008529 & -1.965512 & -2.465302 \\
\hline $\mathrm{C}$ & -1.299826 & -2.086728 & 2.563944 \\
\hline $\mathrm{H}$ & -0.384684 & -2.344199 & 2.025777 \\
\hline $\mathrm{C}$ & 0.467517 & 0.639989 & -1.992017 \\
\hline $\mathrm{H}$ & 0.071124 & -0.095929 & -2.701681 \\
\hline $\mathrm{H}$ & 0.862856 & 1.535133 & -2.487238 \\
\hline $\mathrm{N}$ & 1.423615 & 2.628470 & 1.383226 \\
\hline $\mathrm{O}$ & 1.897790 & 3.562790 & 1.997117 \\
\hline $\mathrm{C}$ & -3.743469 & -0.932248 & -3.194650 \\
\hline $\mathrm{H}$ & -3.554373 & -1.225024 & -4.233864 \\
\hline $\mathrm{H}$ & -4.831361 & -0.905663 & -3.062858 \\
\hline $\mathrm{H}$ & -3.357288 & 0.083591 & -3.068325 \\
\hline $\mathrm{C}$ & -3.639594 & -3.367831 & -2.548686 \\
\hline $\mathrm{H}$ & -3.449370 & -3.645683 & -3.591861 \\
\hline $\mathrm{H}$ & -3.192046 & -4.130122 & -1.901617 \\
\hline $\mathrm{H}$ & -4.723477 & -3.393163 & -2.383751 \\
\hline $\mathrm{C}$ & -1.709129 & -3.318205 & 3.396902 \\
\hline $\mathrm{H}$ & -2.595791 & -3.108826 & 4.006530 \\
\hline $\mathrm{H}$ & -1.943913 & -4.176072 & 2.756111 \\
\hline $\mathrm{H}$ & -0.897356 & -3.606913 & 4.074548 \\
\hline $\mathrm{C}$ & -0.943339 & -0.899764 & 3.476588 \\
\hline $\mathrm{H}$ & -0.558103 & -0.053317 & 2.903109 \\
\hline $\mathrm{H}$ & -1.809541 & -0.569730 & 4.064584 \\
\hline $\mathrm{H}$ & -0.162964 & -1.193529 & 4.187861 \\
\hline $\mathrm{H}$ & -5.651589 & -0.741441 & 1.482335 \\
\hline
\end{tabular}

$\begin{array}{llll}\mathrm{C} & -1.511720 & 1.320452 & 0.221120 \\ \mathrm{H} & -2.045475 & 0.467075 & 0.616585 \\ \mathrm{C} & -1.590010 & 2.513163 & 1.122749 \\ \mathrm{O} & -1.181056 & 3.648697 & 0.859417 \\ \mathrm{~N} & -2.172552 & 2.239932 & 2.323195 \\ \mathrm{H} & -2.354667 & 1.273527 & 2.551799 \\ \mathrm{C} & -2.071574 & 3.196600 & 3.415310 \\ \mathrm{H} & -2.447825 & 4.168051 & 3.087150 \\ \mathrm{H} & -2.673194 & 2.839849 & 4.254751 \\ \mathrm{H} & -1.028776 & 3.320359 & 3.732436 \\ \mathrm{C} & -1.440423 & 1.321767 & -1.201022 \\ \mathrm{H} & -1.908318 & 0.452736 & -1.643313 \\ \mathrm{C} & -1.539769 & 2.479805 & -2.184154 \\ \mathrm{O} & -2.145292 & 2.266561 & -3.238784 \\ \mathrm{~N} & -0.929323 & 3.639171 & -1.874839 \\ \mathrm{H} & -0.660080 & 3.779135 & -0.898626 \\ \mathrm{C} & -1.032607 & 4.787810 & -2.756828 \\ \mathrm{H} & -0.230545 & 5.489662 & -2.515651 \\ \mathrm{H} & -0.933154 & 4.458564 & -3.794075 \\ \mathrm{H} & -1.998875 & 5.299323 & -2.653641\end{array}$

(Z)-N-methyl-2-butenamide

$\begin{array}{lccc}\mathrm{C} & 2.025221 & 0.702964 & 0.000033 \\ \mathrm{H} & 2.700276 & 1.560604 & 0.000103 \\ \mathrm{C} & 0.711929 & 0.981133 & 0.000205 \\ \mathrm{H} & 0.416565 & 2.030348 & 0.000348 \\ \mathrm{C} & -0.399528 & -0.013422 & 0.000112 \\ \mathrm{O} & -0.250446 & -1.235219 & 0.000273 \\ \mathrm{~N} & -1.646670 & 0.564680 & -0.000253 \\ \mathrm{H} & -1.717629 & 1.571512 & -0.000068 \\ \mathrm{C} & -2.871151 & -0.211520 & -0.000087 \\ \mathrm{H} & -3.474704 & -0.002686 & 0.891956 \\ \mathrm{H} & -3.475282 & -0.001915 & -0.891540 \\ \mathrm{H} & -2.589634 & -1.265545 & -0.000593 \\ \mathrm{C} & 2.698478 & -0.636275 & -0.000240 \\ \mathrm{H} & 1.977784 & -1.451981 & -0.000788 \\ \mathrm{H} & 3.351969 & -0.723962 & -0.879348 \\ \mathrm{H} & 3.351212 & -0.724660 & 0.879373\end{array}$

\section{$N, N$ '-Dimethyl-ethen-1,2-dicarboxamid}

$\begin{array}{lrcc}\mathrm{C} & 0.619599 & 1.348230 & 0.061719 \\ \mathrm{H} & 1.185501 & 2.279378 & 0.092100 \\ \mathrm{C} & -0.719764 & 1.485360 & 0.048633 \\ \mathrm{H} & -1.096086 & 2.505575 & 0.064175 \\ \mathrm{C} & -1.896383 & 0.545535 & -0.011435 \\ \mathrm{O} & -3.019706 & 1.047124 & -0.114669 \\ \mathrm{~N} & -1.682730 & -0.785232 & 0.059211 \\ \mathrm{H} & -0.721502 & -1.126132 & 0.100586 \\ \mathrm{C} & -2.804431 & -1.701721 & -0.011297 \\ \mathrm{H} & -3.542604 & -1.467384 & 0.763449 \\ \mathrm{H} & -3.312786 & -1.635619 & -0.981156 \\ \mathrm{H} & -2.436164 & -2.719931 & 0.133790 \\ \mathrm{C} & 1.463263 & 0.121161 & 0.036122 \\ \mathrm{~N} & 2.802054 & 0.376526 & -0.007362 \\ \mathrm{H} & 3.120022 & 1.327954 & -0.118262 \\ \mathrm{C} & 3.778753 & -0.697336 & -0.081599 \\ \mathrm{H} & 4.751804 & -0.320768 & 0.242978 \\ \mathrm{H} & 3.464193 & -1.510921 & 0.575353 \\ \mathrm{H} & 3.867532 & -1.096084 & -1.100101 \\ \mathrm{O} & 1.049531 & -1.044936 & 0.066082\end{array}$


(Z)-2-butene

$\begin{array}{lrrr}\mathrm{C} & -1.282937 & -0.220582 & 0.000002 \\ \mathrm{H} & -1.303362 & -1.309002 & -0.000005 \\ \mathrm{H} & -2.245988 & 0.283216 & -0.000011 \\ \mathrm{C} & -0.134056 & 0.456138 & 0.000004 \\ \mathrm{H} & -0.163130 & 1.546985 & -0.000007 \\ \mathrm{C} & 1.234870 & -0.163109 & -0.000001 \\ \mathrm{H} & 1.182098 & -1.257324 & -0.000014 \\ \mathrm{H} & 1.811568 & 0.150733 & -0.880727 \\ \mathrm{H} & 1.811557 & 0.150710 & 0.880740\end{array}$

\section{S21- $E$}

$\begin{array}{lr}\text { C } & -1.168882 \\ \mathrm{C} & -0.063653 \\ \mathrm{C} & 1.304201 \\ \mathrm{C} & 2.331105 \\ \mathrm{H} & 3.326503 \\ \mathrm{H} & 2.411835 \\ \mathrm{H} & 2.035460 \\ \mathrm{H} & 1.658613 \\ \mathrm{H} & 1.248410 \\ \mathrm{H} & -0.127474 \\ \mathrm{C} & -2.530208 \\ \mathrm{H} & -2.910720 \\ \mathrm{H} & -2.514893 \\ \mathrm{H} & -3.262292 \\ \mathrm{H} & -1.100818\end{array}$

0.334453

$-0.025321$

0.568265

$-0.472023$

$-0.025981$

$-1.302644$

$-0.892136$

1.003820

1.394482

$-0.828894$

$-0.271750$

$-0.702849$

$-1.065144$

0.483730

1.133875

\section{S21- $Z$}

C $\quad 0.012459$

C $\quad 1.301149$

C 2.056371

$\mathrm{H} \quad 1.430957$

H 2.491506

$\mathrm{H} \quad 2.895014$

$\mathrm{H} \quad 1.894944$

C $\quad-1.020668$

H $\quad-0.570382$

C $\quad-2.173144$

$\mathrm{H} \quad-2.940291$

$\mathrm{H} \quad-1.803854$

H $\quad-2.654398$

H $\quad-1.434268$

H $\quad-0.366228$

\section{S22- $E$}

C $\quad-0.575503$

C $\quad 0.575506$

C $\quad 1.949686$

C 2.748572

$\mathrm{H} \quad 3.756521$

H 2.243756

H 2.850376

$\mathrm{H} \quad 1.870314$

H 2.509042

H $\quad 0.543874$

C $\quad-1.949684$

C $\quad-2.748577$

$\mathrm{H} \quad-3.756524$
0.833264

0.611713

$-0.685658$

$-1.521937$

$-0.950690$

$-0.601455$

1.461903

$-0.158601$

$-1.139190$

$-0.308289$

$-0.996452$

$-0.693107$

0.657958

0.179906

1.848499

$$
\begin{array}{cc}
-0.484719 & -0.338471 \\
-0.484728 & 0.338467 \\
-0.465741 & -0.274142 \\
0.789246 & 0.118038 \\
0.768613 & -0.312323 \\
1.696198 & -0.233275 \\
0.866554 & 1.207476 \\
-0.528734 & -1.367242 \\
-1.356372 & 0.049968 \\
-0.478941 & 1.431056 \\
-0.465742 & 0.274139 \\
0.789243 & -0.118033 \\
0.768603 & 0.312330
\end{array}
$$

0.124045

$-0.160689$

$-0.070189$

0.254329

$-1.043430$

0.634554

$-0.499346$

0.588955

0.777864

$-0.419952$

$-0.046350$

$-1.377163$

$-0.614480$

1.550747

$-0.009743$
0.987532

$-0.704327$

$-0.265892$

0.985022

$\begin{array}{ll}\mathrm{H} & -2.243764 \\ \mathrm{H} & -2.850383 \\ \mathrm{H} & -1.870310 \\ \mathrm{H} & -2.509035 \\ \mathrm{H} & -0.543872\end{array}$

S22- $Z$

C $\quad 0.669066$

C $\quad-0.669280$

C $\quad-1.598226$

C $\quad-2.443777$

H $\quad-3.023429$

$\mathrm{H} \quad-3.148377$

H $\quad-1.802635$

H -1.043527

H $\quad-2.273662$

H $\quad-1.174509$

C $\quad 1.597727$

C 2.444381

$\mathrm{H} \quad 1.803991$

H 3.148702

H 3.024436

$\begin{array}{ll}\mathrm{H} & 3.024436 \\ \mathrm{H} & 2.272432\end{array}$

H 1.042689

H 1.174541

$-0.121978$

\section{S23-E}

C $\quad-2.352470$

$-0.475534$

H $\quad-2.211417$

\section{S23- $Z$}

C $\quad-2.495667$
1.696196

0.866555

$-0.528741$

$-1.356374$

$-0.478909$

$-0.946666$

$-0.946879$

0.847479

0.307086

1.407720

1.565972

0.442650

$-0.794161$

$-1.600835$

$-0.121564$

0.846877

1.565251

1.407300

0.305654

$-0.793643$

0.443888

$-1.600493$

0.283430

0.147965

1.363328

$-0.743113$

$-1.713317$

$-0.285598$

0.085907

0.431834

$-0.773331$

0.890243

$-1.075444$

0.587416

$-1.563191$

$-0.225382$

$-1.319544$

0.157767

0.124866

1.363138

0.233283

$-1.207470$

1.367239

$-0.049976$

$-1.431059$

$-0.283409$

$-0.283441$

0.567708

$-0.277382$

$-1.035673$

0.348188

$-0.800407$

1.324885

1.117564

$-0.997062$

0.567849

$-0.277288$

$-0.801399$

0.348432

$-1.034680$

1.118742

1.324162

$-0.996977$

0.082723

$-0.060130$

$-0.264309$

$-0.340540$

$-0.385509$

$-0.157821$

$-0.466545$

0.897523

0.790062

1.578744

1.345970

$-0.947265$

$-1.124480$

$-0.031518$

0.291732

0.317871

1.232681

$-0.508168$

0.040847

0.569197

0.268669

$0.991372 \quad 0.127632$

$0.146108-0.228843$

$-1.054503-0.500541$

$0.833753-0.256089$

$1.771540 \quad 0.118509$

$0.143275 \quad-0.474647$

$-0.556783 \quad 0.782256$

$-1.068344 \quad 0.571775$

$-1.299169 \quad 1.121238$

$0.161048 \quad 1.593109$

$-0.592418 \quad-1.265388$ 


$\begin{array}{rrrr} & & & \\ \mathrm{H} & 3.123593 & 0.877793 & -0.847118 \\ \mathrm{H} & -1.025485 & 2.050063 & 0.287321 \\ \mathrm{C} & -3.042201 & -0.817008 & 0.105232 \\ \mathrm{H} & -3.802380 & -0.825240 & -0.688408 \\ \mathrm{H} & -2.260898 & -1.534415 & -0.139405 \\ \mathrm{H} & -3.554687 & -1.127072 & 1.026405 \\ \mathrm{H} & -3.231769 & 1.329005 & 0.537125\end{array}$

\section{S24- $E$}

$\begin{array}{lrrr}\mathrm{C} & -1.789831 & 0.182804 & -0.342881 \\ \mathrm{C} & -0.694761 & -0.530908 & -0.061007 \\ \mathrm{C} & 0.614075 & 0.142636 & 0.178882 \\ \mathrm{O} & 0.757321 & 1.364372 & 0.162929 \\ \mathrm{~N} & 1.658252 & -0.707152 & 0.444668 \\ \mathrm{H} & 1.526158 & -1.697745 & 0.295060 \\ \mathrm{C} & 3.020984 & -0.207866 & 0.584824 \\ \mathrm{C} & 3.700252 & 0.066973 & -0.760460 \\ \mathrm{H} & 4.717043 & 0.448175 & -0.609454 \\ \mathrm{H} & 3.127022 & 0.815958 & -1.314631 \\ \mathrm{H} & 3.762983 & -0.844694 & -1.366476 \\ \mathrm{H} & 2.963672 & 0.715796 & 1.166873 \\ \mathrm{H} & 3.588879 & -0.940015 & 1.169731 \\ \mathrm{H} & -0.741335 & -1.618721 & -0.001279 \\ \mathrm{C} & -3.160203 & -0.374323 & -0.585324 \\ \mathrm{C} & -4.185918 & 0.151946 & 0.435300 \\ \mathrm{H} & -3.912941 & -0.149338 & 1.452495 \\ \mathrm{H} & -5.186983 & -0.237030 & 0.218734 \\ \mathrm{H} & -4.236137 & 1.246785 & 0.413465 \\ \mathrm{H} & -3.488562 & -0.087460 & -1.595502 \\ \mathrm{H} & -3.134917 & -1.471210 & -0.560889 \\ \mathrm{H} & -1.678804 & 1.267016 & -0.380240\end{array}$

\section{S24-Z}

C 1.943198

C $\quad 0.832925$

C $\quad-0.580692$

O $\quad-1.485501$

$\mathrm{N} \quad-0.811463$

H $\quad-0.047522$

C $\quad-2.147075$

C $\quad-2.521388$

H $\quad-2.488368$

$\mathrm{H} \quad-3.534750$

$\mathrm{H} \quad-1.826355$

$\mathrm{H} \quad-2.179013$

H $\quad-2.857795$

$\mathrm{H} \quad 0.899835$

C $\quad 2.070142$

$\mathrm{H} \quad 1.090463$

C $\quad 2.759555$

H $\quad 3.747119$

H 2.892613

$\mathrm{H} \quad 2.172356$

H 2.670439

H 2.885229

\section{S25- $E$}

$\begin{array}{ll}\mathrm{C} & 2.511895 \\ \mathrm{C} & 1.361345 \\ \mathrm{C} & 0.059476\end{array}$
$-0.895310$

$-1.555241$

$-1.062264$

$-1.765985$

0.187384

0.690591

0.775111

1.377711

0.598903

1.794984

2.177320

1.534215

$-0.015007$

$-2.607403$

0.521966

0.928504

1.441141

1.054559

2.450611

1.520819

0.513070

$-1.443665$
0.186412

$-0.172513$

$-0.133038$

0.309867

$-0.640746$

$-1.065913$

$-0.637619$

0.719263

1.486588

0.690519

1.000582

$-1.426411$

$-0.900041$

$-0.438963$

0.681674

0.951061

$-0.343871$

0.060574

$-1.267482$

1.601908

0.133270
$-0.621256$
$\mathrm{O}$

$-0.015195$

$\mathrm{H} \quad-0.872895$

C $\quad-2.436144$

C $\quad-2.609897$

$\mathrm{H} \quad-3.652910$

H $\quad-1.968217$

H $\quad-2.343304$

C $\quad-2.829079$

H $\quad-2.718199$

$\mathrm{H} \quad-3.874245$

$\mathrm{H} \quad-2.191593$

C $\quad-3.299056$

$\mathrm{H} \quad-3.030844$

$\mathrm{H} \quad-3.185631$

$\mathrm{H} \quad-4.355542$

H 1.357139

C $\quad 3.877692$

C 4.786854

$\mathrm{H} \quad 4.884838$

$\mathrm{H} \quad 5.790356$

$\mathrm{H} \quad 4.375004$

$\mathrm{H}$
$\mathrm{H}$

$\mathrm{H} \quad 4.343131$

$\mathrm{H} \quad 2.451150$

\section{S25- $Z$}

C $\quad-2.579449$

C $\quad-1.509482$

C $\quad-0.071496$

$\begin{array}{lr}\mathrm{C} & -0.071496 \\ \mathrm{O} & 0.651442\end{array}$

$\mathrm{N} \quad 0.358165$

$\mathrm{H} \quad-0.264366$

C $\quad 1.740663$

$\begin{array}{ll}\text { C } & 2.714408\end{array}$

H $\quad 3.736729$

H 2.440308

H 2.688923

$\begin{array}{ll}\mathrm{C} & 1.733658\end{array}$

$\mathrm{H} \quad 1.031347$

H 2.731391

H $\quad 1.453015$

C $\quad 2.135948$

H $\quad 3.149677$

H 2.105778

H 1.447205

$\begin{array}{lr}\mathrm{H} & -1.654302\end{array}$

$\begin{array}{ll}\text { C } & -2.623568\end{array}$

H $\quad-3.381360$

C $\quad-2.993201$

$\mathrm{H} \quad-3.948956$

$\mathrm{H} \quad-2.235471$

$\mathrm{H} \quad-3.079640$

$\mathrm{H} \quad-1.667871$

H $\quad-3.555995$
$-1.454208$

0.593204

1.590648

0.142382

$-0.696900$

$-1.018852$

$-1.579262$

$-0.106794$

$-0.678890$

$-0.076075$

$-1.000615$

$-1.560961$

1.410112

2.014708

2.027353

1.139096

1.591899

0.439389

0.006839

$-1.084341$

0.432958

0.338314

1.533925

0.125470

$-1.259923$

$-0.792073$

$-1.532470$

$-1.273610$

$-2.192031$

0.015862

0.639634

0.493380

$-0.234788$

0.129198

$-0.056082$

$-1.309878$

1.998639

2.529684

2.419146

2.191741

0.258542

0.634375

$-0.806892$

0.785826

$-2.495874$

0.513447

0.429880

1.700593

1.528976

1.859315

2.626071

0.706818

$-1.176689$
$-0.142349$

$-0.040084$

$-0.048728$

0.078511

1.357797

1.458484

1.319608

2.242018

$-1.163356$

$-2.071899$

$-1.087218$

$-1.248775$

0.162690

1.038210

$-0.737413$

0.252817

$-0.237867$

$-0.450517$

0.714241

0.752257

0.605680

1.673720

$-0.482993$

$-1.396778$

$-0.353430$

$-0.096697$

$-0.421080$

$-0.083693$

0.299475

$-0.244220$

$-0.737982$

$-0.020817$

$-0.965947$

$-0.811071$

$-2.012193$

$-0.776144$

$-0.325429$

0.328485

$-0.165552$

$-1.368953$

1.447697

1.627634

1.682968

2.117536

$-0.904220$

0.654409

1.445561

$-0.254574$

$-0.763253$

$-1.032120$

0.325389

1.150405

$-0.395971$ 


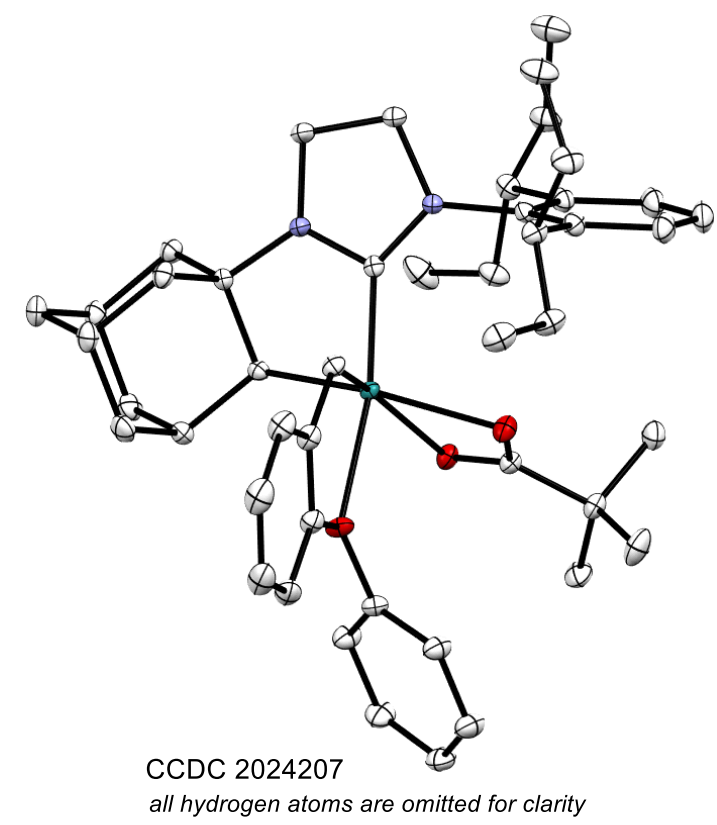

Table S1 | Crystal data and structure refinement for Ru-6.

Identification code

Empirical formula

Formula weight

Temperature/K

Crystal system

Space group

$\mathrm{a} / \AA$

$\mathrm{b} / \AA$

$\mathrm{c} / \AA$

$\alpha /{ }^{\circ}$

$\beta /{ }^{\circ}$

$\gamma /{ }^{\circ}$

Volume $/ \AA^{3}$

Z

$\rho_{\text {calc }} \mathrm{g} / \mathrm{cm}^{3}$

$\mu / \mathrm{mm}^{-1}$

$\mathrm{F}(000)$

Crystal size $/ \mathrm{mm}^{3}$

Radiation

$2 \Theta$ range for data collection/ ${ }^{\circ}$

Index ranges yx-531

$\mathrm{C}_{53} \mathrm{H}_{68} \mathrm{~N}_{2} \mathrm{O}_{3} \mathrm{Ru}$

882.16

100.0

triclinic

P-1

$11.506(8)$

12.614(6)

$17.118(16)$

$75.681(17)$

$71.28(3)$

$88.660(15)$

2276(3)

2

1.287

0.389

936.0

$0.28 \times 0.27 \times 0.07$

$\operatorname{MoK} \alpha(\lambda=0.71073)$

3.338 to 72.28

$-19 \leq \mathrm{h} \leq 19,-20 \leq \mathrm{k} \leq 20,-28 \leq 1 \leq 28$ 
Reflections collected

Independent reflections

Data/restraints/parameters

Goodness-of-fit on $\mathrm{F}^{2}$

Final $R$ indexes $[\mathrm{I}>=2 \sigma(\mathrm{I})]$

Final $\mathrm{R}$ indexes [all data]

Largest diff. peak/hole / e $\AA^{-3}$
210134

$20832\left[\mathrm{R}_{\text {int }}=0.0507, \mathrm{R}_{\text {sigma }}=0.0345\right]$

$20832 / 21 / 539$

1.043

$\mathrm{R}_{1}=0.0338, \mathrm{wR}_{2}=0.0724$

$\mathrm{R}_{1}=0.0472, \mathrm{wR}_{2}=0.0769$

$1.30 /-1.17$

\section{Further study}

\subsection{Further lowering the equivalent of the regular olefin:}

Scheme S3 | Further lowering the equivalent of the regular olefin.
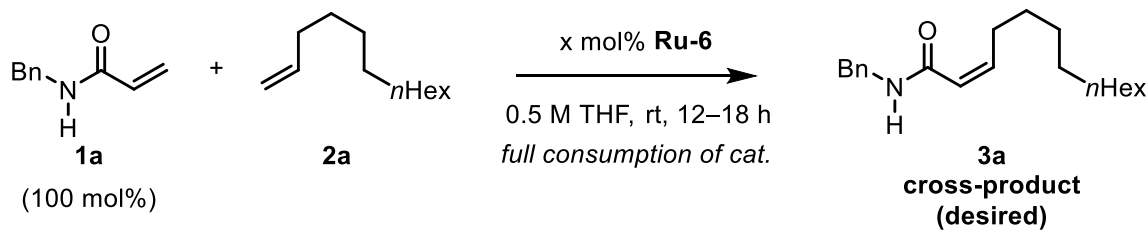

\begin{tabular}{cccc}
\hline entry & equiv. of $\mathbf{2 a}$ & loading of Ru-6 & yield of $\mathbf{3 a}$ \\
\hline 1 & 2 equiv. & 1.2 mol\% cat. & $80 \%$ yield $(75 \%$ isolated $)$ \\
\hline 2 & 1.3 equiv. & 2.5 mol\% cat. & $79 \%$ yield $(75 \%$ isolated $)$ \\
\hline 3 & 1 equiv. & 2.5 mol\% cat. & $65 \%$ yield \\
\hline
\end{tabular}

The experiment in entry 1 was conducted as described in Section 3. The experiment in entry 2 was conducted as described in Section 4.2. The experiment in entry 3 was conducted as follows:

A $4 \mathrm{~mL}$ vial was charged with a stir bar, $1 \mathbf{a}(0.25 \mathrm{mmol}, 1$ equiv, $40.3 \mathrm{mg}), \mathbf{2 a}(0.25 \mathrm{mmol}, 1$ equiv, $42 \mathrm{mg})$, and anthracene (as the internal standard) in a nitrogen glovebox. To this was added a stock solution of the ruthenium catalyst Ru-6 (0.00625 mmol, 0.025 equiv, $5.0 \mathrm{mg})$ in $d_{8}$-THF $(0.4 \mathrm{~mL})$. The vial was loosely capped with a screw thread hole cap, and a 20-gauge needle was used to puncture the cap and left on (see Figure S1). The reaction mixture was allowed to stir at room temperature in the glovebox for 22 hours. The reaction was then transferred to an NMR tube and removed from the glovebox. The yield of the metathesis product 3a was determined by ${ }^{1} \mathrm{H}$ NMR analysis of the crude reaction mixture.

7.2 Kinetic preference between cross- and homo-metathesis using acrylate S21

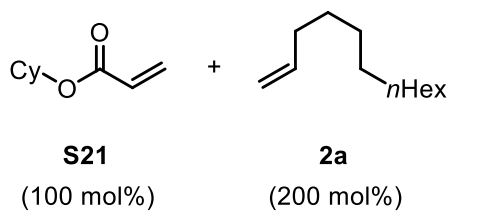

(100 mol\%)

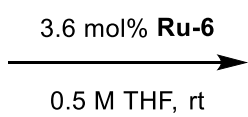

$$
0.5 \text { M THF, rt }
$$

(200 mol\%)

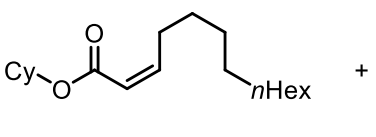

S22

cross-product

$5.5 \%$ yield
(Z)-docos-11-ene

$4 a$

homo-product

$15 \%$ yield 
A $4 \mathrm{~mL}$ vial was charged with a stir bar, $\mathbf{S 2 1}(0.25 \mathrm{mmol}, 1$ equiv, $38.6 \mathrm{mg}), \mathbf{2 a}(0.5 \mathrm{mmol}, 2$ equiv, $84 \mathrm{mg})$, and anthracene (as the internal standard) in a nitrogen glovebox. To this was added a stock solution of the ruthenium catalyst Ru-6 ( $0.009 \mathrm{mmol}, 0.036$ equiv, $7.2 \mathrm{mg})$ in $d_{8}$-THF $(0.4 \mathrm{~mL})$. The vial was loosely capped with a screw thread hole cap, and a 20 -gauge needle was used to puncture the cap and left on (see Figure S1). The reaction mixture was stir at room temperature, and then removed from the glovebox when the yield of $\mathbf{S 2 2}$ reached $\sim 5 \%$. The yields of the metathesis products were determined by ${ }^{1} \mathrm{H}$ NMR analysis of the crude reaction mixture: $\mathbf{S 2 2}, 5.5 \%$ yield; $4 \mathrm{a}$, $15 \%$ yield. This result indicates that the reaction with acrylate $\mathbf{S 2 1}$ favors homo-metathesis over cross-metathesis kinetically.

\section{References}

(1) Herbert, M. B.; Lan, Y.; Keitz, B. K.; Liu, P.; Endo, K.; Day, M. W.; Houk, K. N.; Grubbs, R. H. J. Am. Chem. Soc. 2012, 134, 7861.

(2) Rosebrugh, L. E.; Herbert, M. B.; Marx, V. M.; Keitz, B. K.; Grubbs, R. H. J. Am. Chem. Soc. 2013, 135, 1276.

(3) Małecki, P.; Gajda, K.; Gajda, R.; Woźniak, K.; Trzaskowski, B.; Kajetanowicz, A.; Grela, K. ACS Catal. 2019, $9,587$.

(4) Paczal, A.; Bényei, A. C.; Kotschy, A. J. Org. Chem. 2006, 71, 5969.

(5) Luan, X.; Mariz, R.; Gatti, M.; Costabile, C.; Poater, A.; Cavallo, L.; Linden, A.; Dorta, R. J. Am. Chem. Soc. 2008, 130, 6848.

(6) Aubineau, T.; Cossy, J. Chem. Commun. 2013, 49, 3303.

(7) Hogg, J. H.; Ollmann, I. R.; Wetterholm, A.; Andberg, M. B.; Haeggström, J.; Samuelsson, B.; Wong, C.H. Chem. Eur. J. 1998, 4, 1698.

(8) Kreituss, I.; Murakami, Y.; Binanzer, M.; Bode, J. W. Angew. Chem. Int. Ed. 2012, 51, 10660.

(9) Wang, S.; Huang, X.; Wen, Y.; Ge, Z.; Wang, X.; Li, R. Tetrahedron 2015, 71, 8117.

(10) Bentolila, A.; Vlodavsky, I.; Ishai-Michaeli, R.; Kovalchuk, O.; Haloun, C.; Domb, A. J. J. Med. Chem. 2000, 43, 2591.

(11) Liu, Z.; Xu, C.; del Pozo, J.; Torker, S.; Hoveyda, A. H. J. Am. Chem. Soc. 2019, 141, 7137.

(12) Gaussian 16, Revision C.01, Frisch, M. J.; Trucks, G. W.; Schlegel, H. B.; Scuseria, G. E.; Robb, M. A.; Cheeseman, J. R.; Scalmani, G.; Barone, V.; Petersson, G. A.; Nakatsuji, H.; Li, X.; Caricato, M.; Marenich, A. V.; Bloino, J.; Janesko, B. G.; Gomperts, R.; Mennucci, B.; Hratchian, H. P.; Ortiz, J. V.; Izmaylov, A. F.; Sonnenberg, J. L.; Williams-Young, D.; Ding, F.; Lipparini, F.; Egidi, F.; Goings, J.; Peng, B.; Petrone, A.; Henderson, T.; Ranasinghe, D.; Zakrzewski, V. G.; Gao, J.; Rega, N.; Zheng, G.; Liang, W.; Hada, M.; Ehara, M.; Toyota, K.; Fukuda, R.; Hasegawa, J.; Ishida, M.; Nakajima, T.; Honda, Y.; Kitao, O.; Nakai, H.; Vreven, T.; Throssell, K.; Montgomery, J. A., Jr.; Peralta, J. E.; Ogliaro, F.; Bearpark, M. J.; Heyd, J. J.; Brothers, E. N.; Kudin, K. N.; Staroverov, V. N.; Keith, T. A.; Kobayashi, R.; Normand, J.; Raghavachari, K.; Rendell, A. P.; Burant, J. C.; Iyengar, S. S.; Tomasi, J.; Cossi, M.; Millam, J. M.; Klene, M.; Adamo, C.; Cammi, R.; Ochterski, J. W.; Martin, R. L.; Morokuma, K.; Farkas, O.; Foresman, J. B.; Fox, D. J. Gaussian, Inc., Wallingford CT, 2016. 
(13) (a) Head-Gordon, M.; Pople, J. A.; Frisch, M. J. Chem. Phys. Lett. 1988, 153, 503. (b) Becke, A. D. J. Chem. Phys. 1993, 98, 5648. (c) Lee, C.; Yang, W.; Parr, R. G. Phys. Rev. B: Condens. Matter Mater. Phys. 1988, 37, 785. (d) Vosko, S. H.; Wilk, L.; Nusair, M. Can. J. Phys. 1980, 58, 1200. (e) Stephens, P. J.; Devlin, F. J.; Chabalowski, C. F.; Frisch, M. J. J. Phys. Chem. 1994, 98, 11623.

(14) Grimme, S.; Antony, J.; Ehrlich, S.; Krieg, H. J. Chem. Phys. 2010, 132, 154104.

(15) (a) Häussermann, U.; Dolg, M.; Stoll, H.; Preuss, H.; Schwerdtfeger, P.; Pitzer, R. M. Mol. Phys. 1993, 78, 1211. (b) Küchle, W.; Dolg, M.; Stoll, H.; Preuss, H. J. Chem. Phys. 1994, 100, 7535.

(16) Grimme, S. Supramolecular Binding Thermodynamics by Dispersion-Corrected Density Functional Theory. Chem.-Eur. J. 2012, 18, 9955.

(17) Zhao, Y.; Truhlar, D.G. Theor.Chem. Acc. 2008, 120, 215.

(18) Marenich, A.V.; Cramer, C.J.; Truhlar, D.G. J. Phys. Chem. B. 2009, 113, 6378.

(19) (a) Grimme, S.; Bannwarth, C.; Dohm, S.; Hansen, A.; Pisarek, J.; Pracht, P.; Seibert, J.; Neese, F.; Angew. Chem. Int. Ed., 2017, 56, 14763. (b) Grimme, S. J. Chem. Theory Comput., 2019, 155, 2847.

(20) (a) Grimme, S.; Bannwarth, C.; Shushkov, P.; J. Chem. Theory Comput., 2017, 13, 1989. (b) Bannwarth, C.; Ehlert, S.; Grimme S.; J. Chem. Theory Comput., 2019, 15, 1652. (c) Pracht, P.; Caldeweyher, E.; Ehlert, S.; Grimme, S. ChemRxiv, 2019, preprint. DOI: 10.26434/chemrxiv.8326202.v1

(21) Legault, C. Y. CYLview, 1.0b; Université de Sherbrooke, 2009; http://www.cylview.org.

\section{Spectra}


Figure S3 $\mid{ }^{1} \mathrm{H}$ NMR (400 MHz, THF- $d_{8}$ ) spectrum of Ru-4.

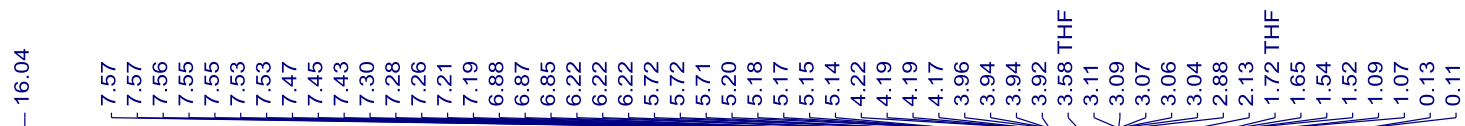

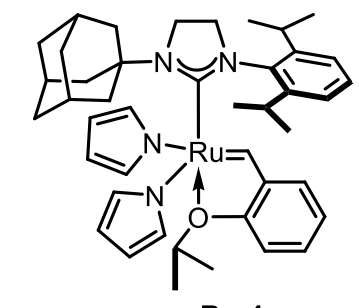

$\mathrm{Ru}-4$
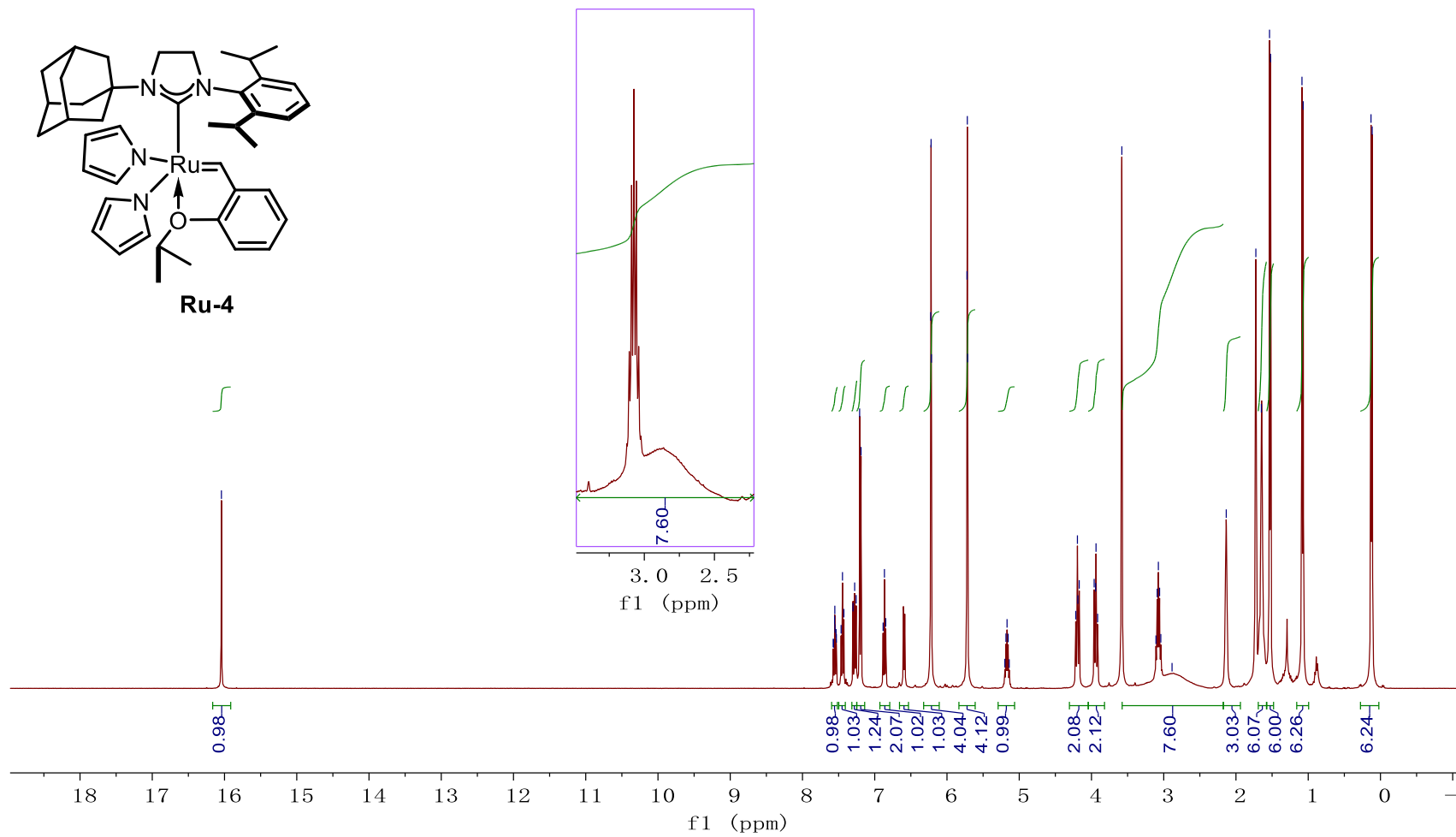

Figure S4 $\mid{ }^{13} \mathrm{C}$ NMR (101 MHz, THF- $\left.d_{8}\right)$ spectrum of Ru-4.

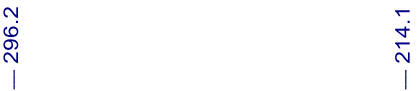

岌岌岌岌岌

岌岌岌岌岌

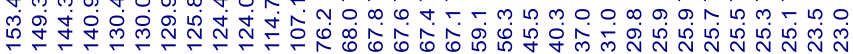

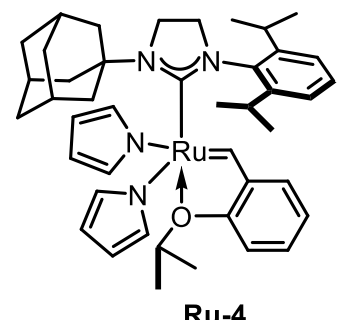

Ru-4

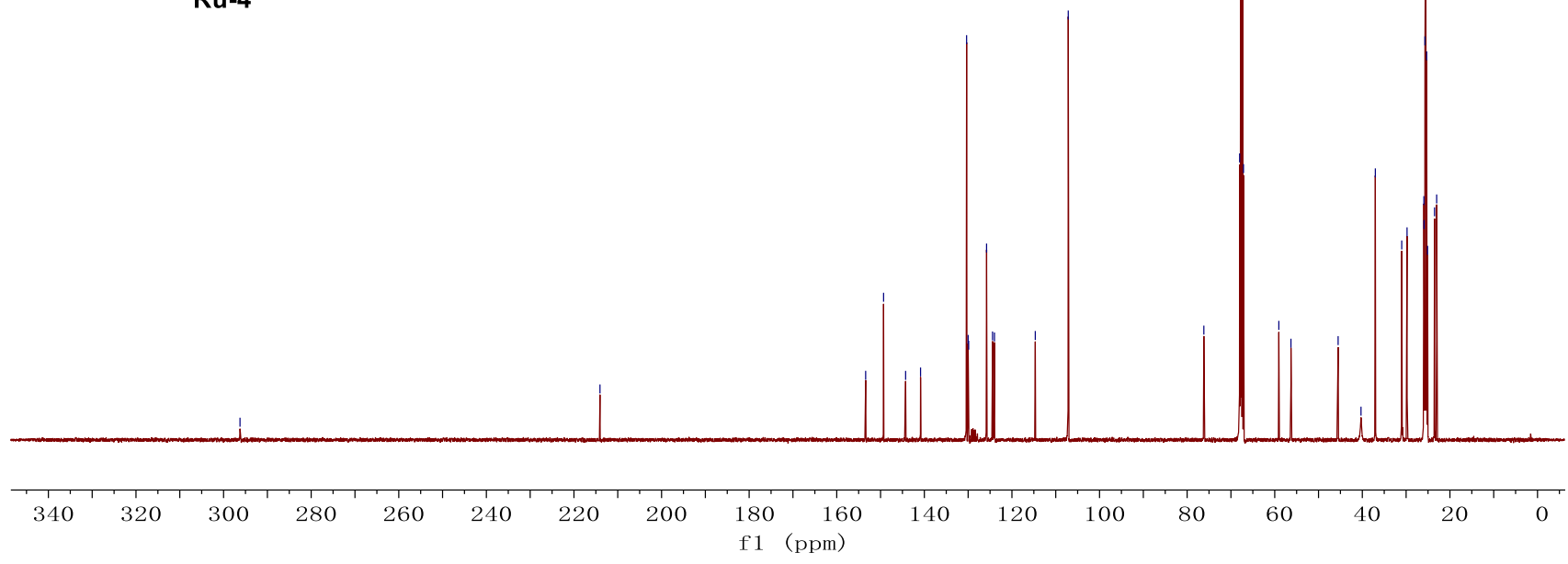


Figure S5 | ${ }^{1} \mathrm{H}$ NMR (400 MHz, $\left.\mathrm{C}_{6} \mathrm{D}_{6}\right)$ spectrum of Ru-2.

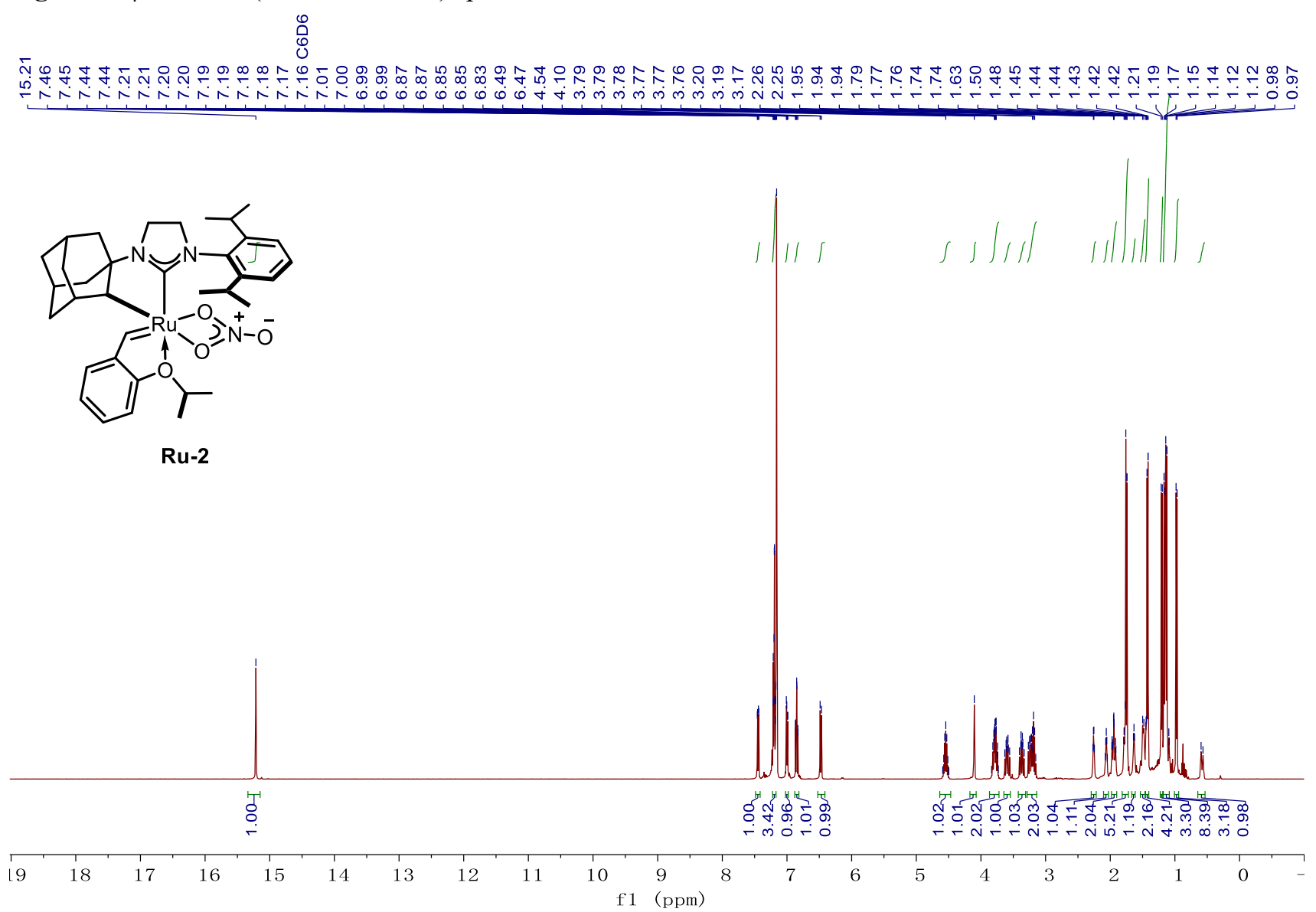

Figure S6 $\mid{ }^{13} \mathrm{C}$ NMR (101 MHz, $\left.\mathrm{C}_{6} \mathrm{D}_{6}\right)$ spectrum of Ru-2.

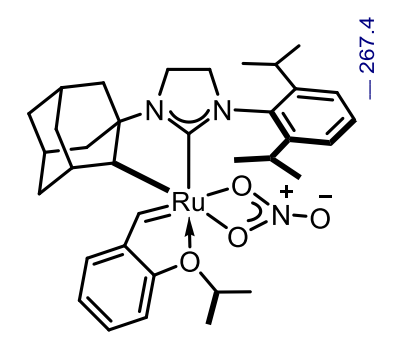

管

Ru-2

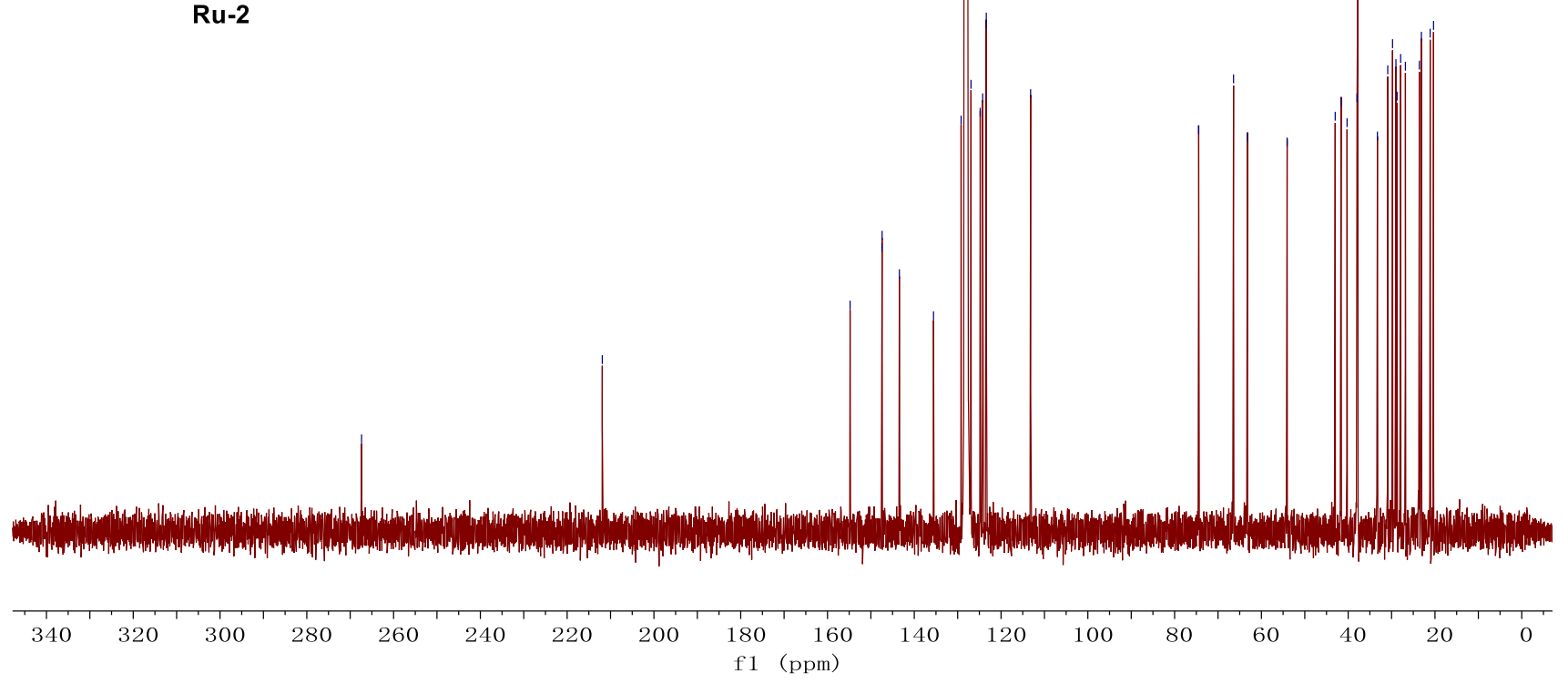


Figure S7 | ${ }^{1} \mathrm{H}$ NMR (400 MHz, $\left.\mathrm{CDCl}_{3}\right)$ spectrum of $\mathbf{S 3}$.

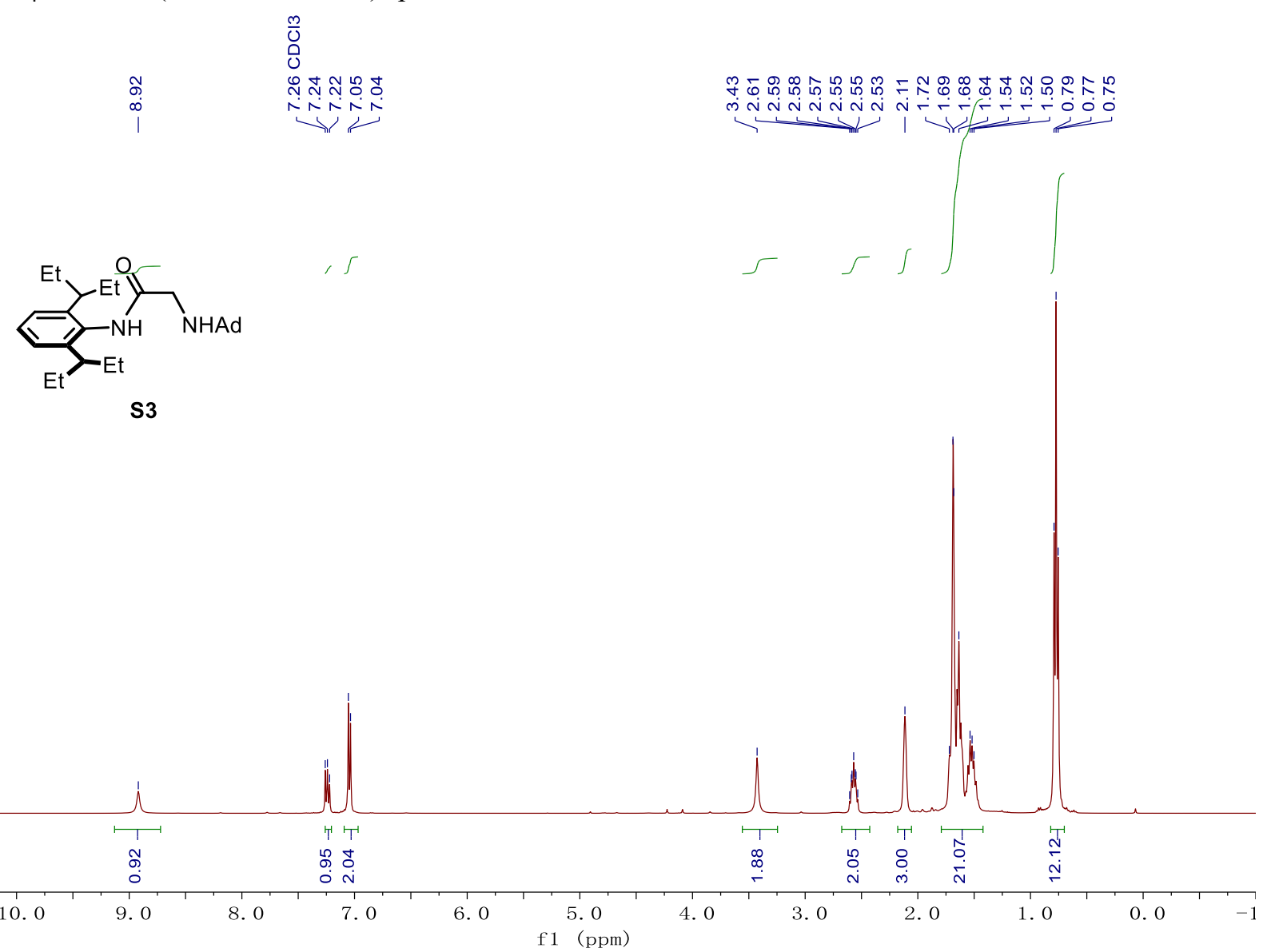

Figure S8 $\mid{ }^{13} \mathrm{C}$ NMR $\left(101 \mathrm{MHz}, \mathrm{CDCl}_{3}\right)$ spectrum of $\mathbf{S 3}$.
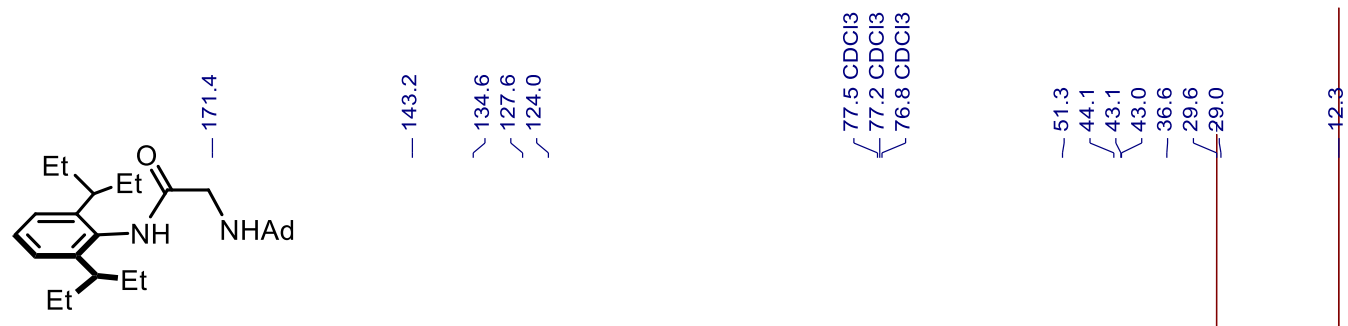

53

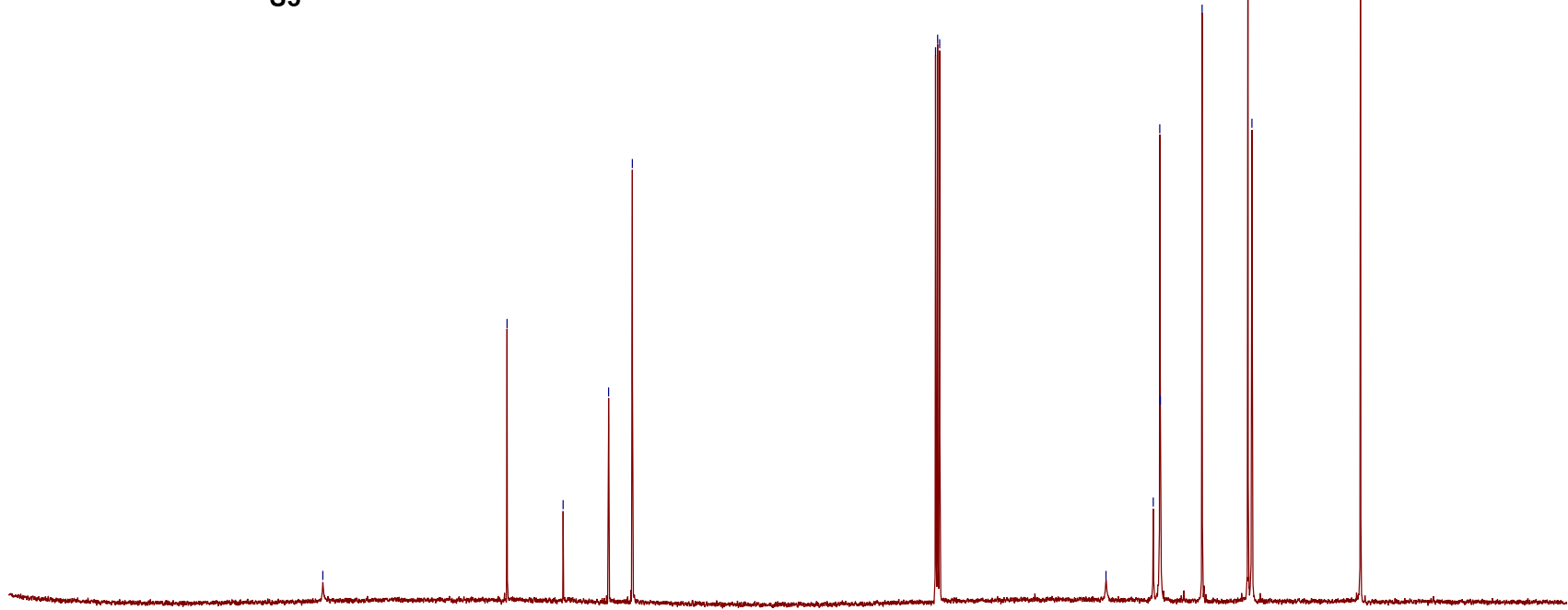

$\begin{array}{llllllllllllllllllllllllllllll}210 & 200 & 190 & 180 & 170 & 160 & 150 & 140 & 130 & 120 & 110 & 100 & 90 & 80 & 70 & 60 & 50 & 40 & 30 & 20 & 10 & 0 & -10\end{array}$ f1 (ppm) 
Figure S9 | Crude ${ }^{1} \mathrm{H}$ NMR (400 MHz, $\left.\mathrm{CDCl}_{3}\right)$ spectrum of $\mathbf{S 4}$.

学

我莫

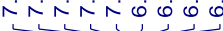

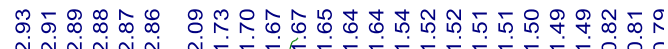

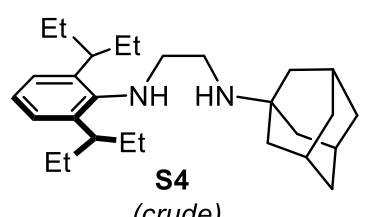

(crude)
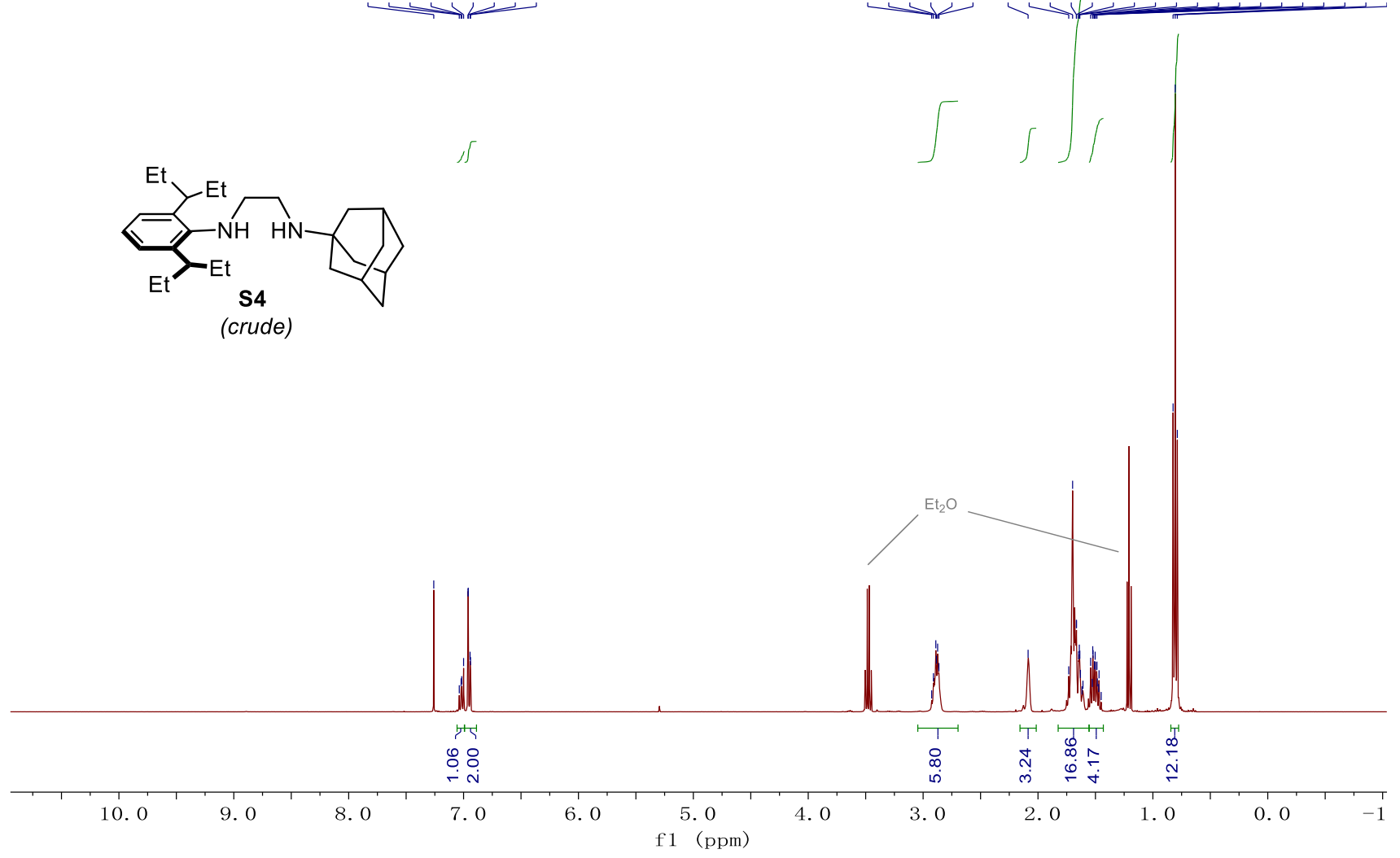

Figure S10 | Crude ${ }^{1} \mathrm{H}$ NMR (400 MHz, $\mathrm{CDCl}_{3}$ ) spectrum of $\mathbf{S 4}$.
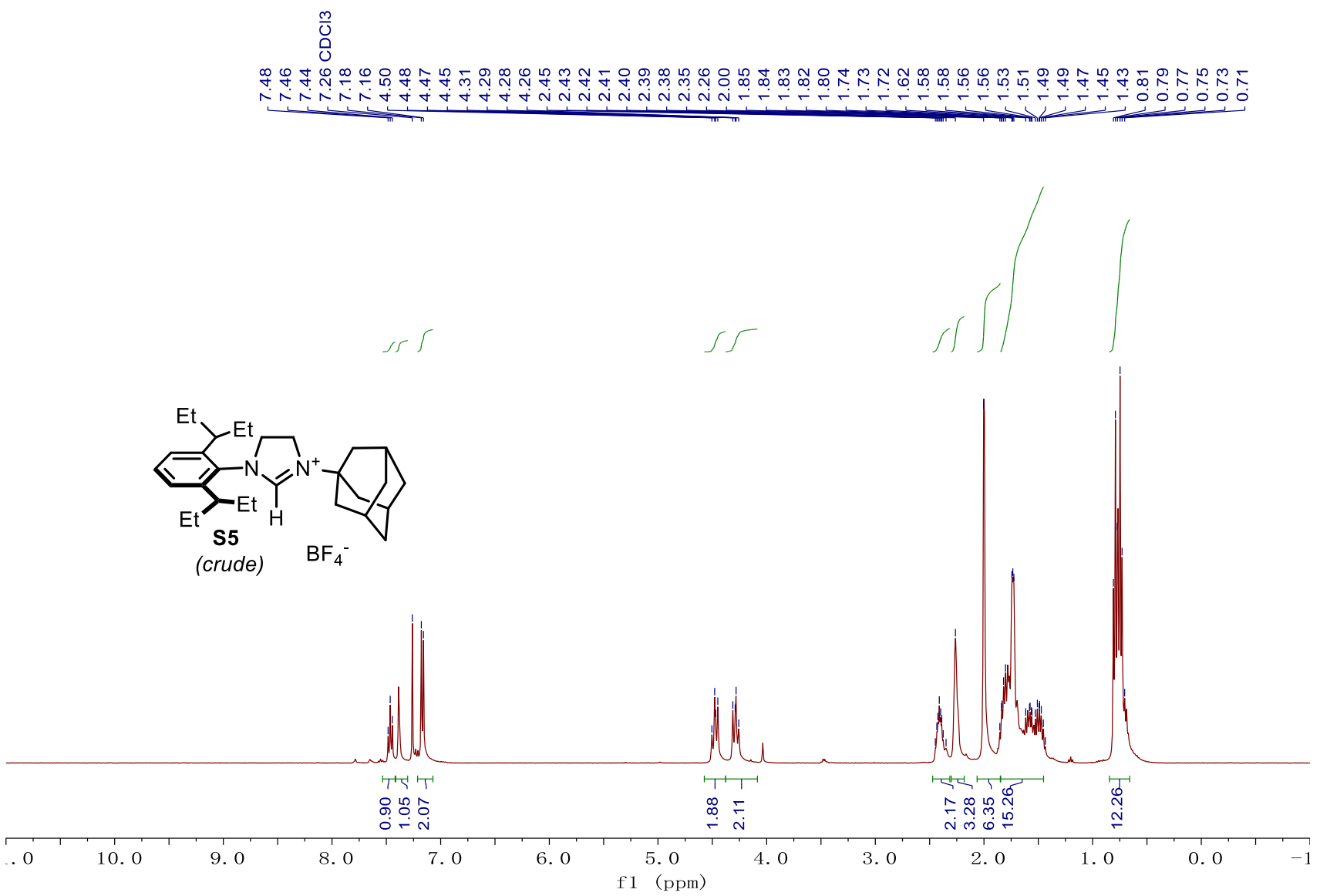
Figure S11 | ${ }^{1} \mathrm{H}$ NMR (400 MHz, $\left.\mathrm{C}_{6} \mathrm{D}_{6}\right)$ spectrum of $\mathbf{S 6}$.

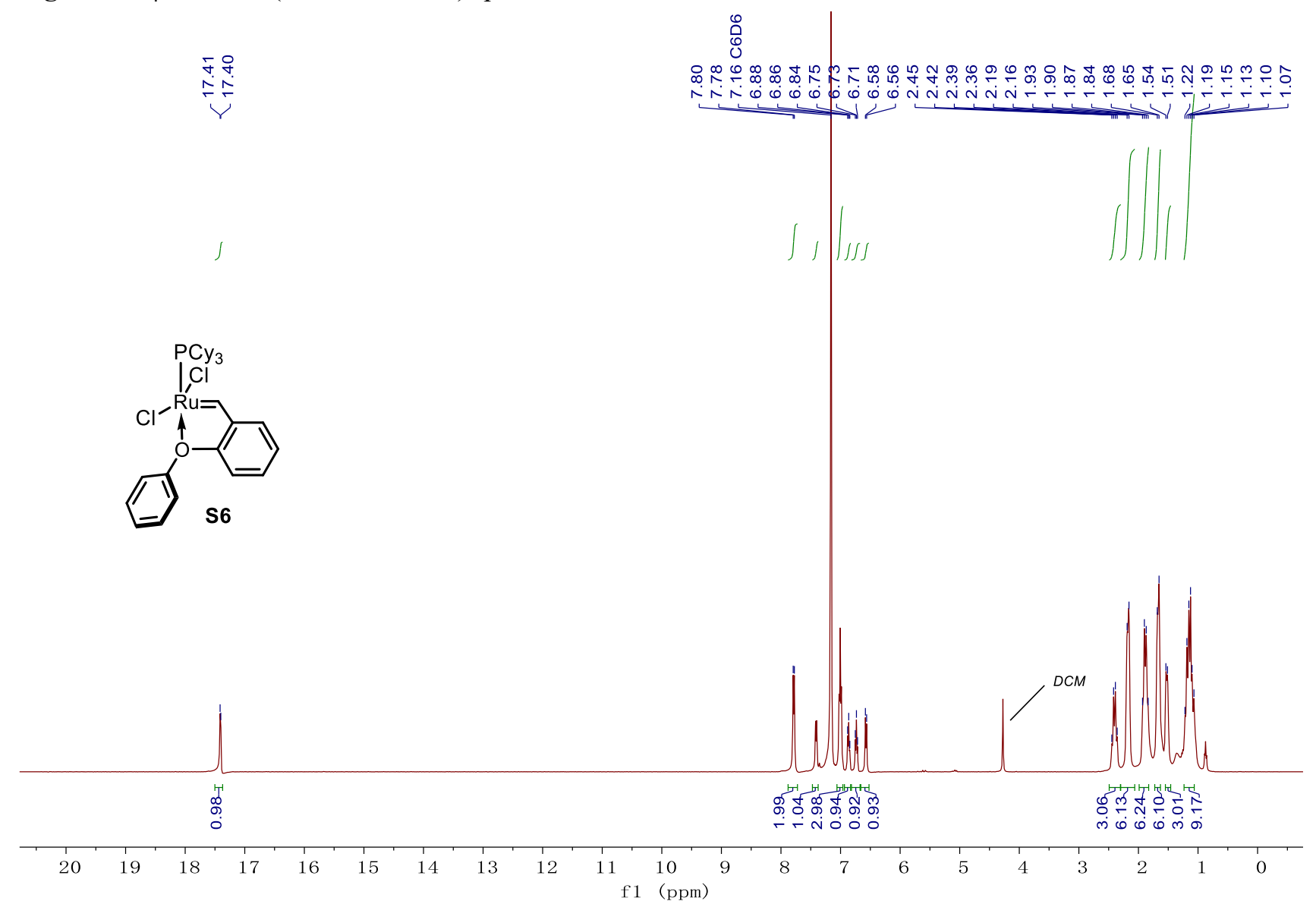

Figure S12 $\mid{ }^{13} \mathrm{C}$ NMR (101 MHz, $\left.\mathrm{C}_{6} \mathrm{D}_{6}\right)$ spectrum of $\mathbf{S 6}$.
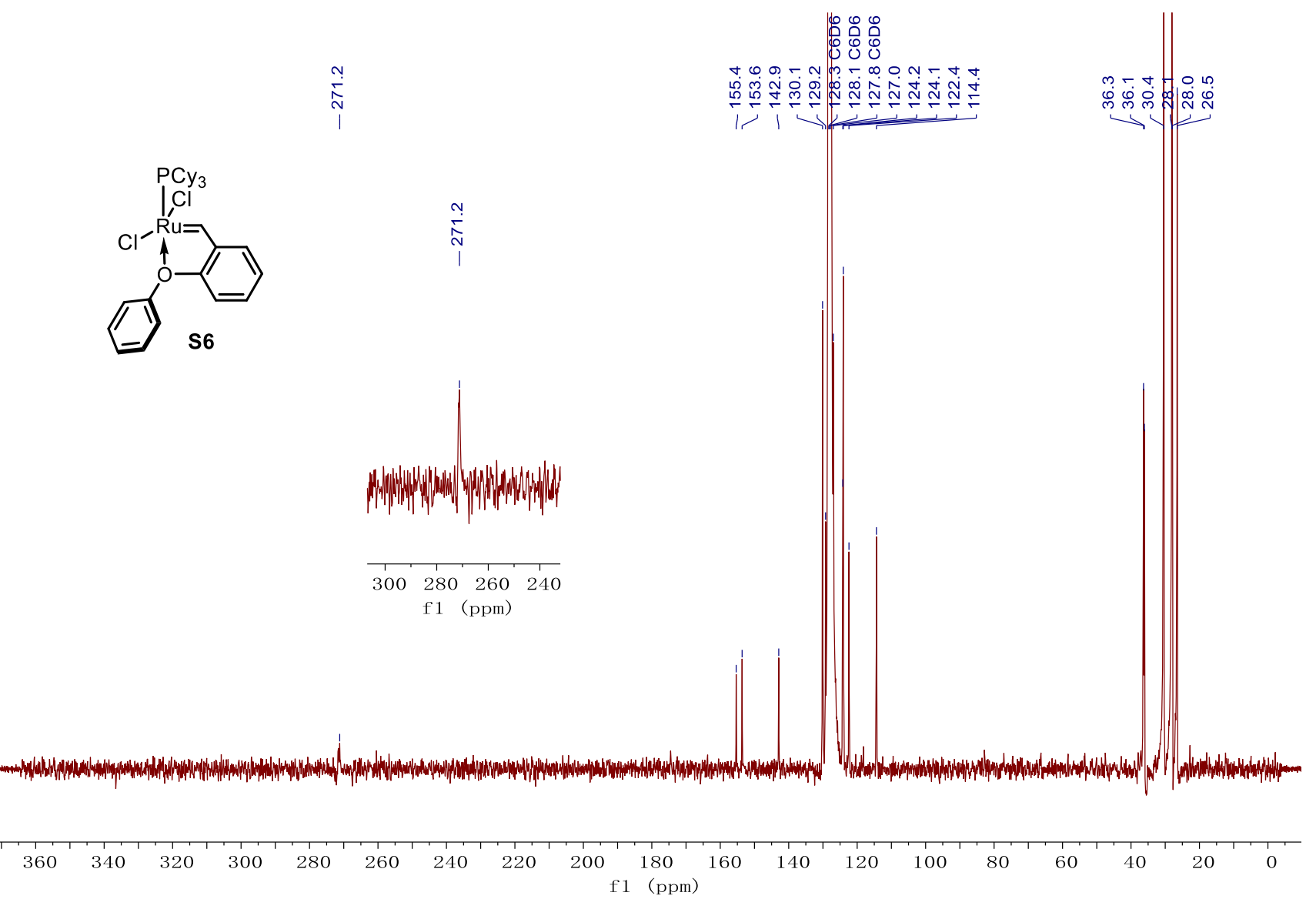
Figure S13 $\mid{ }^{1} \mathrm{H}$ NMR (400 MHz, $\left.\mathrm{C}_{6} \mathrm{D}_{6}\right)$ spectrum of Ru-5.

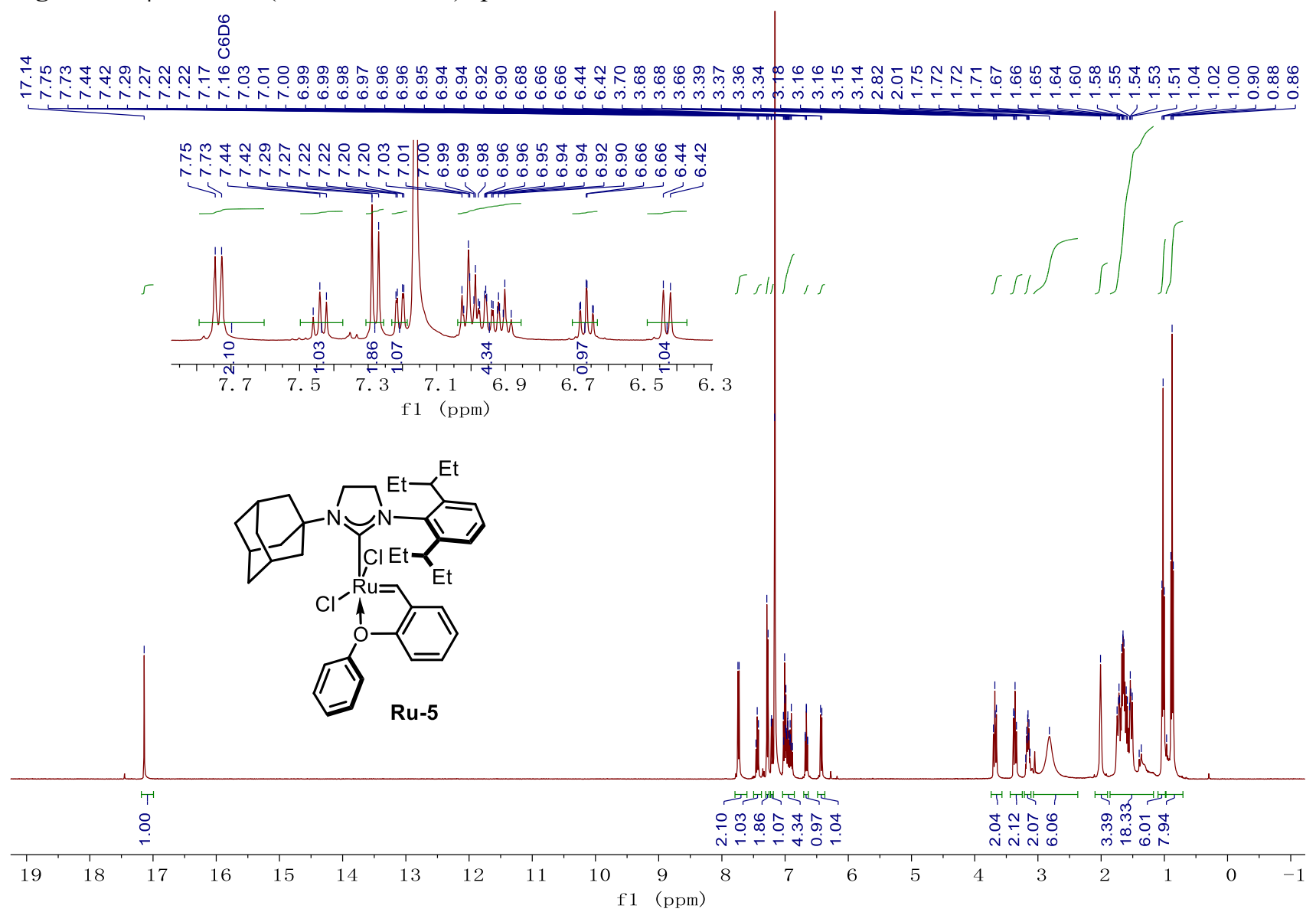

Figure S14 $\mid{ }^{13} \mathrm{C}$ NMR (101 MHz, $\left.\mathrm{C}_{6} \mathrm{D}_{6}\right)$ spectrum of Ru-5.
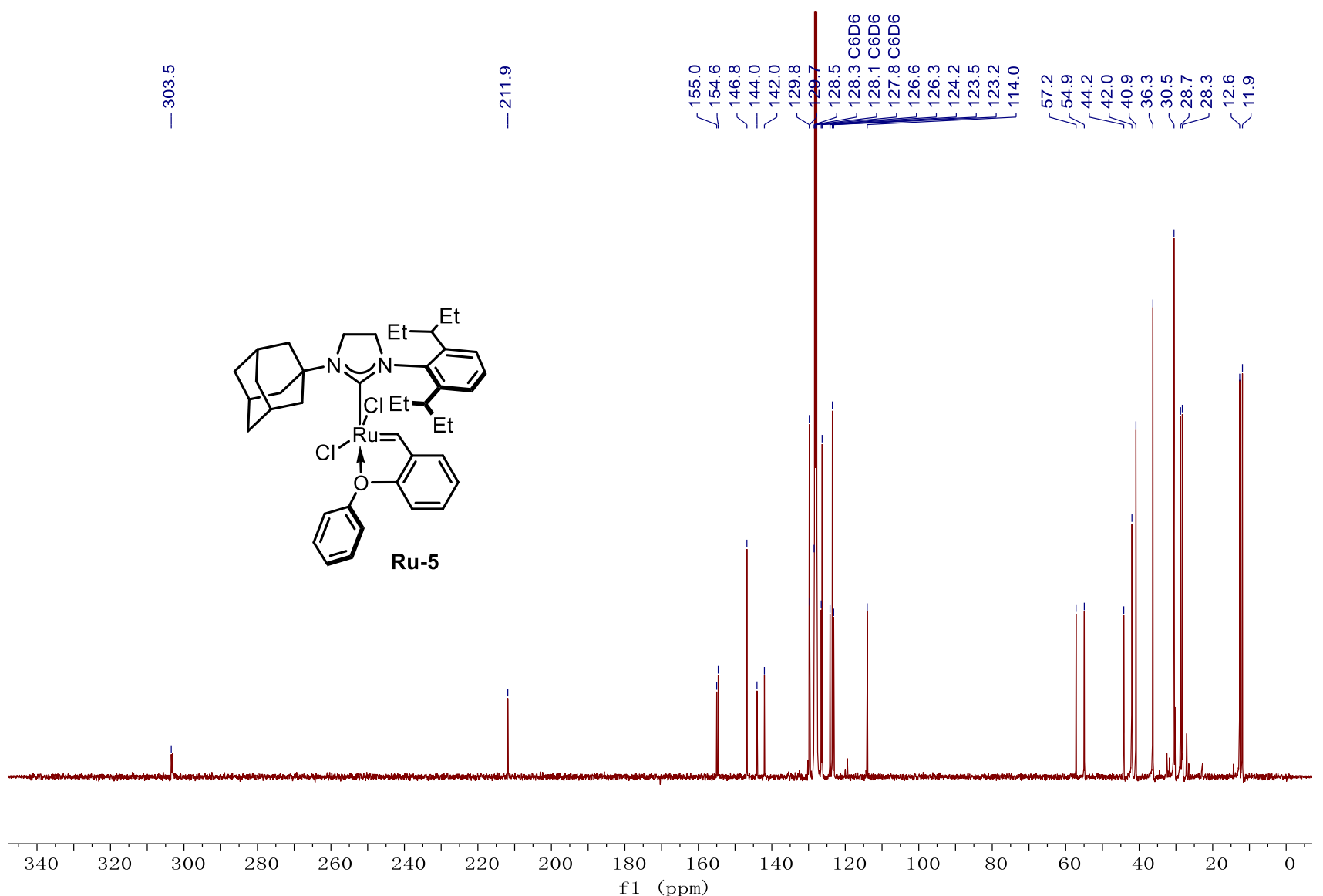
Figure $\mathbf{S 1 5} \mid{ }^{1} \mathrm{H}$ NMR (400 MHz, $\left.\mathrm{C}_{6} \mathrm{D}_{6}\right)$ spectrum of $\mathbf{S} 7$.
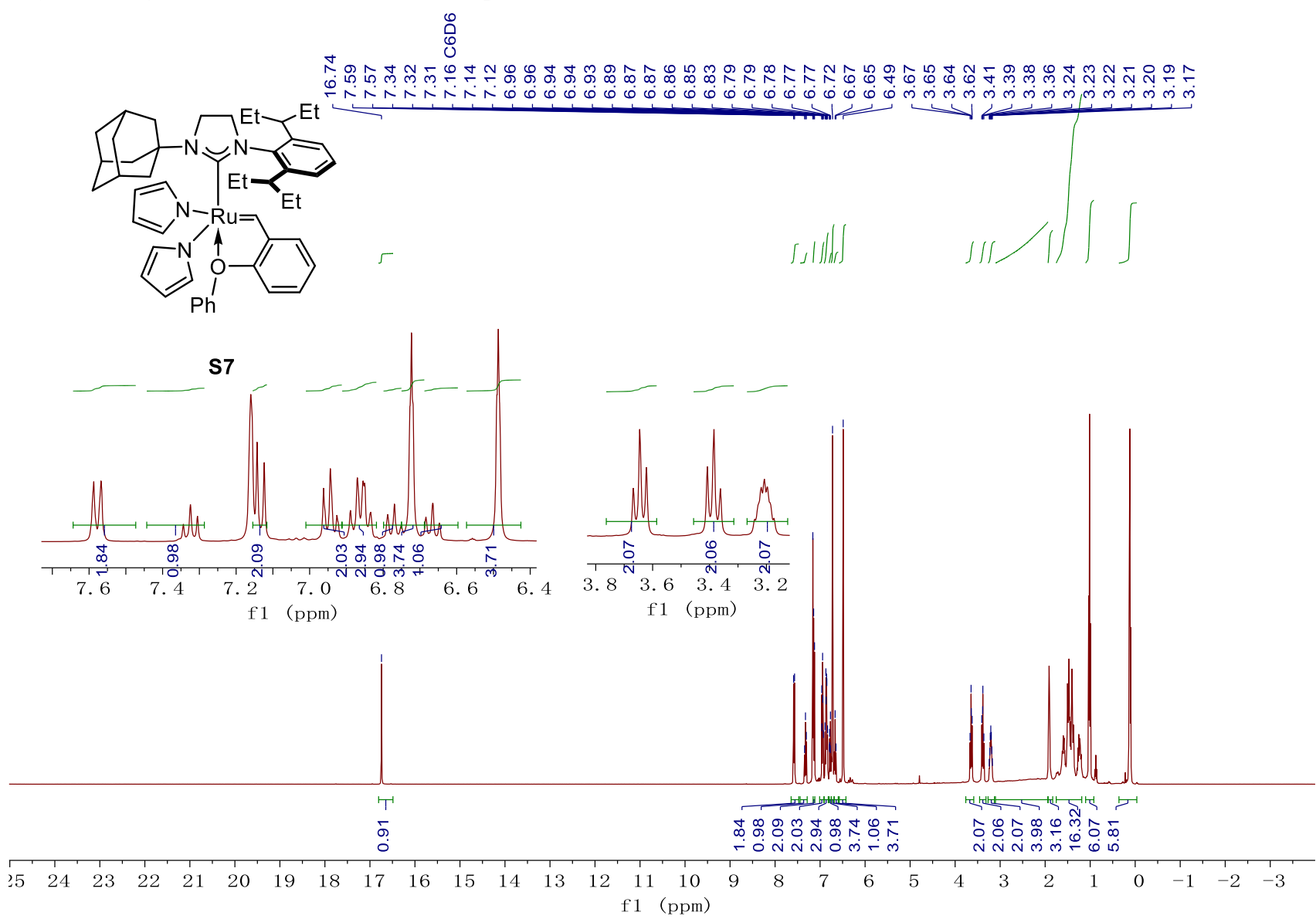

Figure $\mathbf{S 1 6} \mid{ }^{13} \mathrm{C}$ NMR $\left(101 \mathrm{MHz}, \mathrm{C}_{6} \mathrm{D}_{6}\right)$ spectrum of $\mathbf{S} 7$.

吕

$\stackrel{+}{\stackrel{+}{N}}$

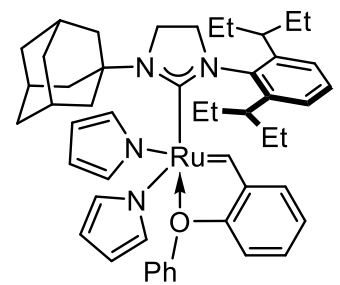

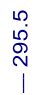

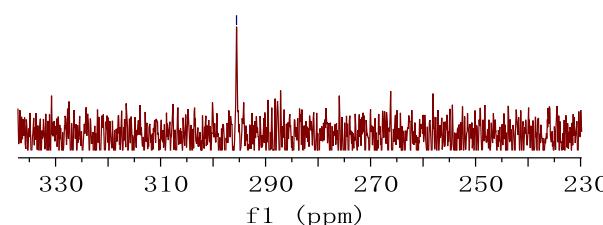

s7

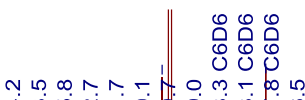

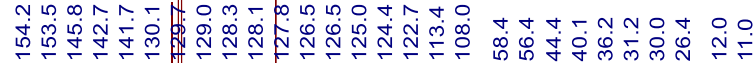

lर

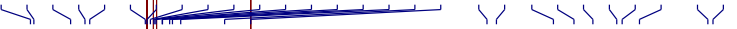


Figure S17 | ${ }^{1} \mathrm{H}$ NMR (400 MHz, $\mathrm{C}_{6} \mathrm{D}_{6}$ ) spectrum of Ru-6.

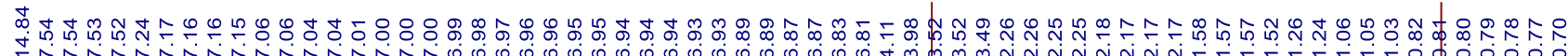

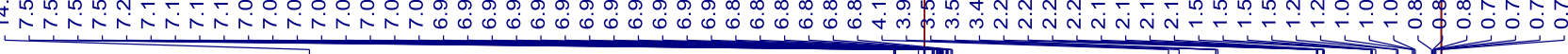

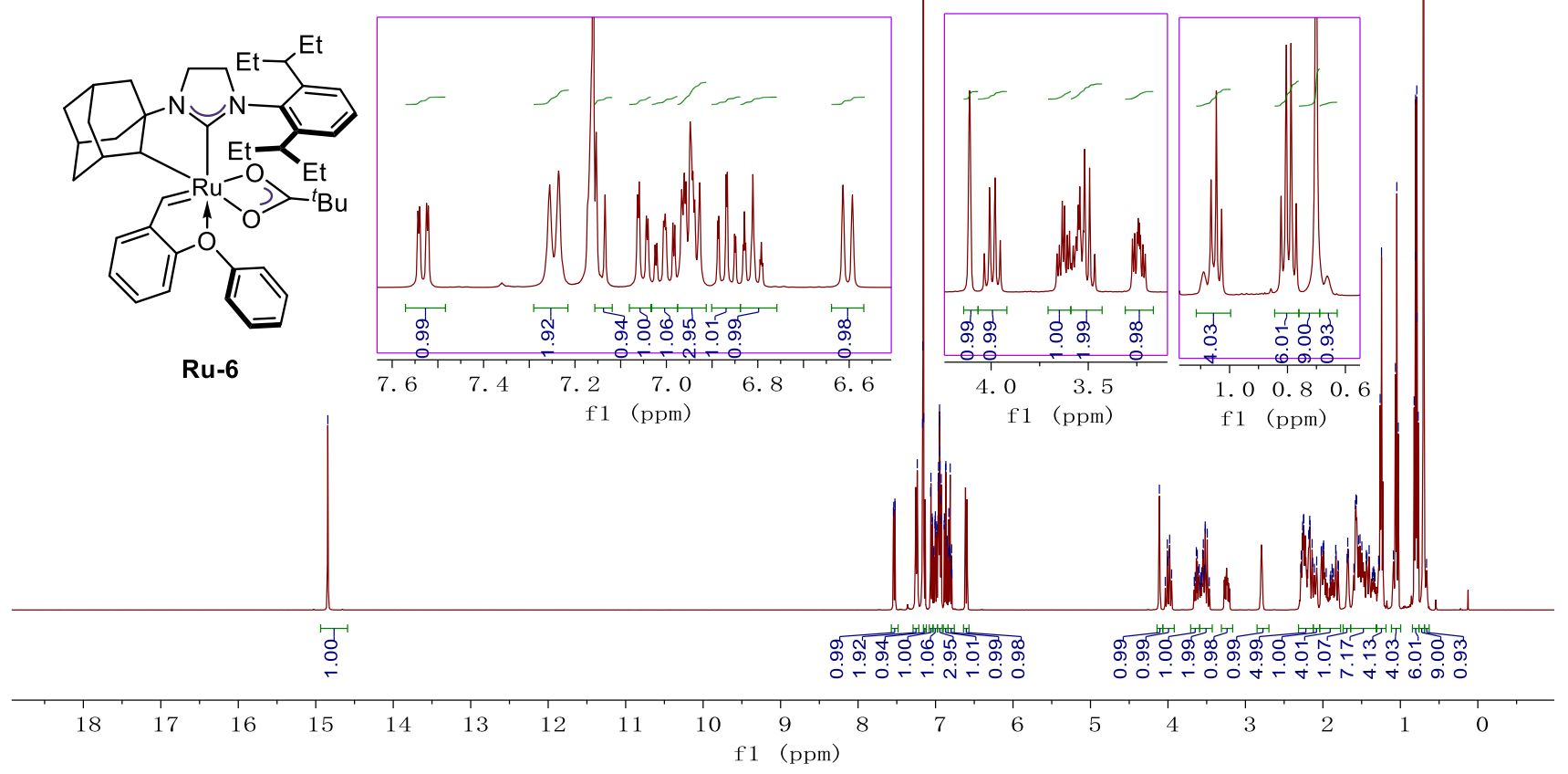

Figure S18 $\mid{ }^{13} \mathrm{C}$ NMR (101 MHz, $\left.\mathrm{C}_{6} \mathrm{D}_{6}\right)$ spectrum of Ru-6.
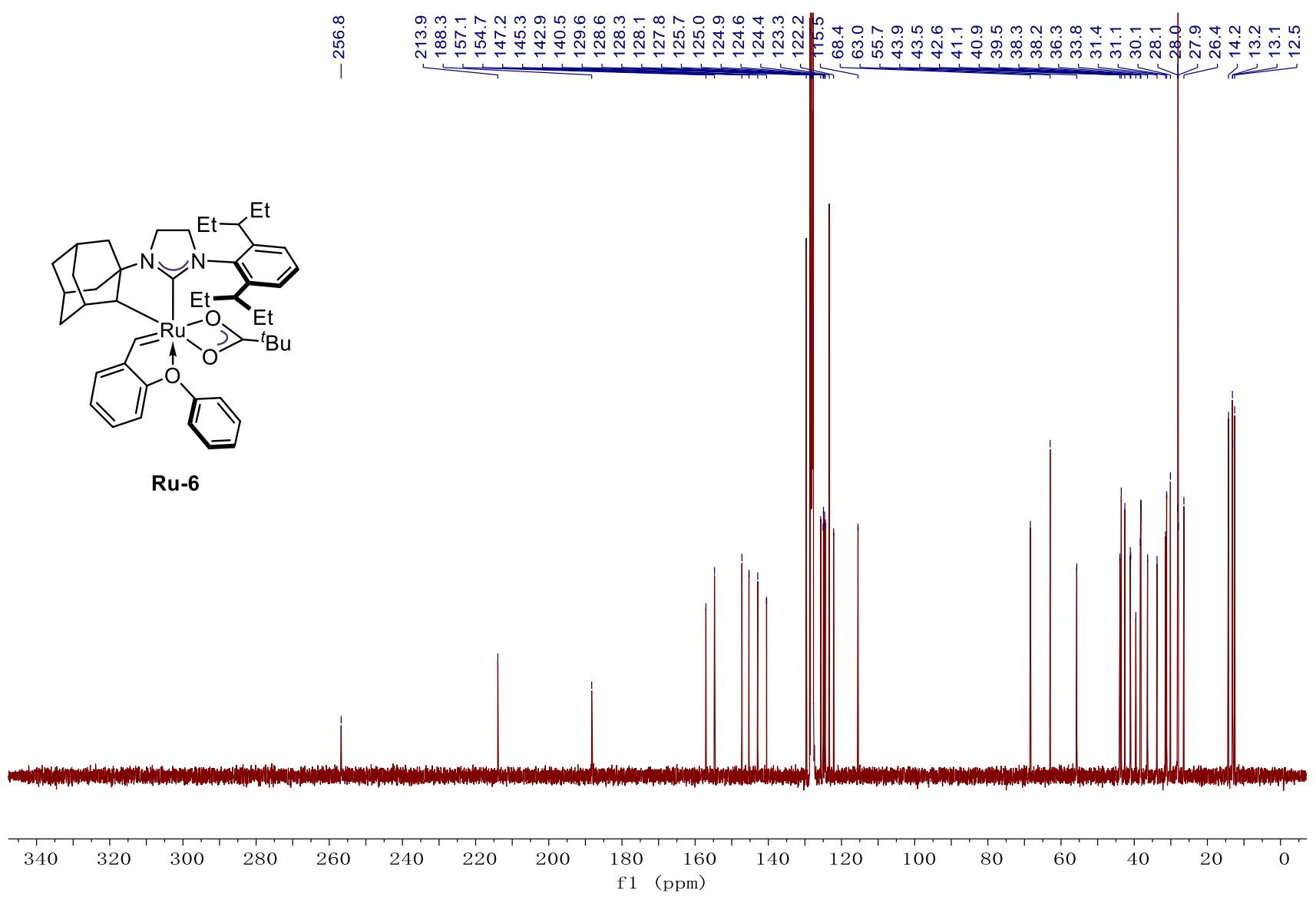
Figure $\mathbf{S 1 9}$ | Crude ${ }^{1} \mathrm{H}$ NMR (400 $\left.\mathrm{MHz}, \mathrm{CDCl}_{3}\right)$ spectrum of $\mathbf{S 9}$.

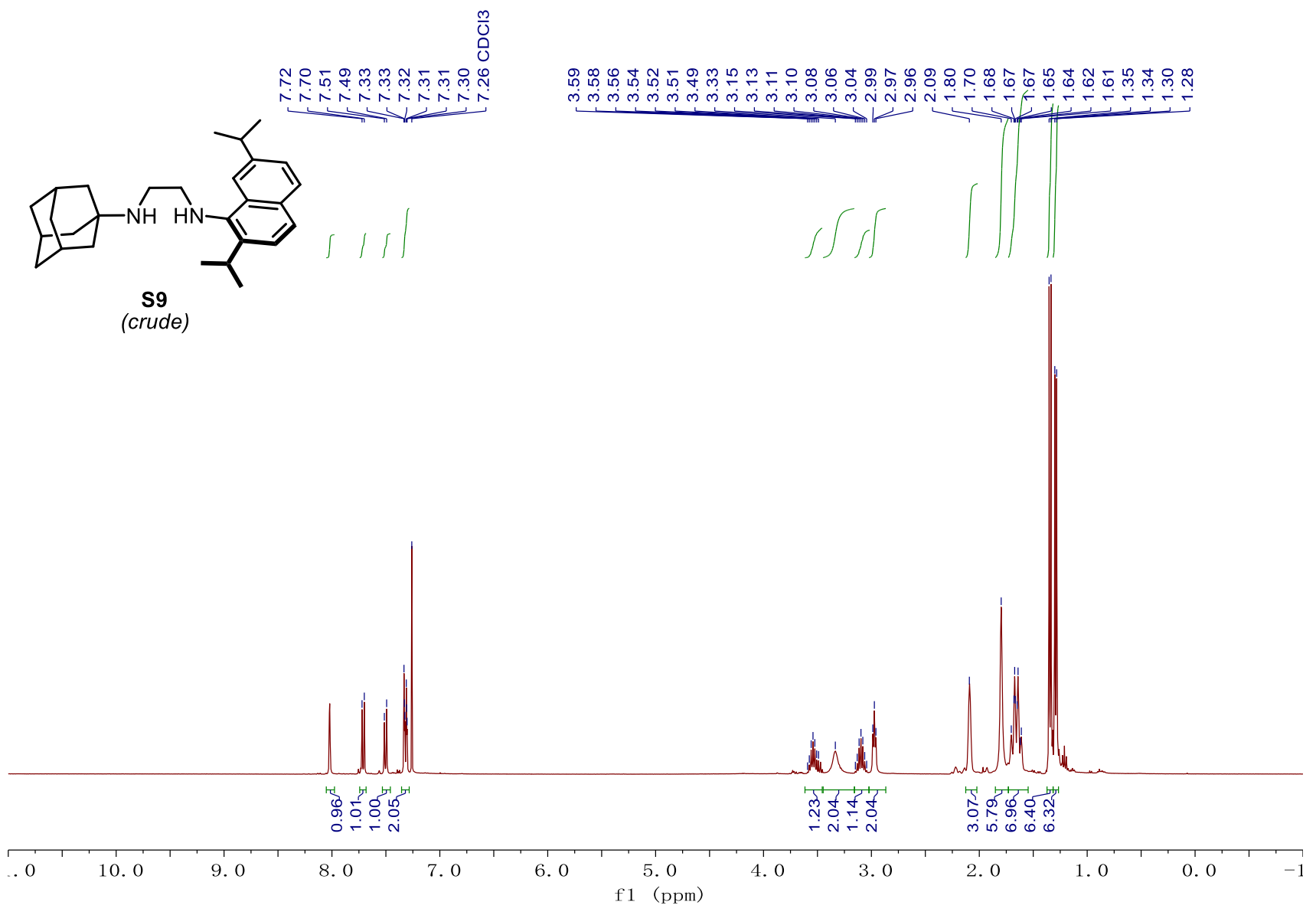


Figure S20 | ${ }^{1} \mathrm{H}$ NMR (400 MHz, $\left.\mathrm{CDCl}_{3}\right)$ spectrum of $\mathbf{S 1 0}$.

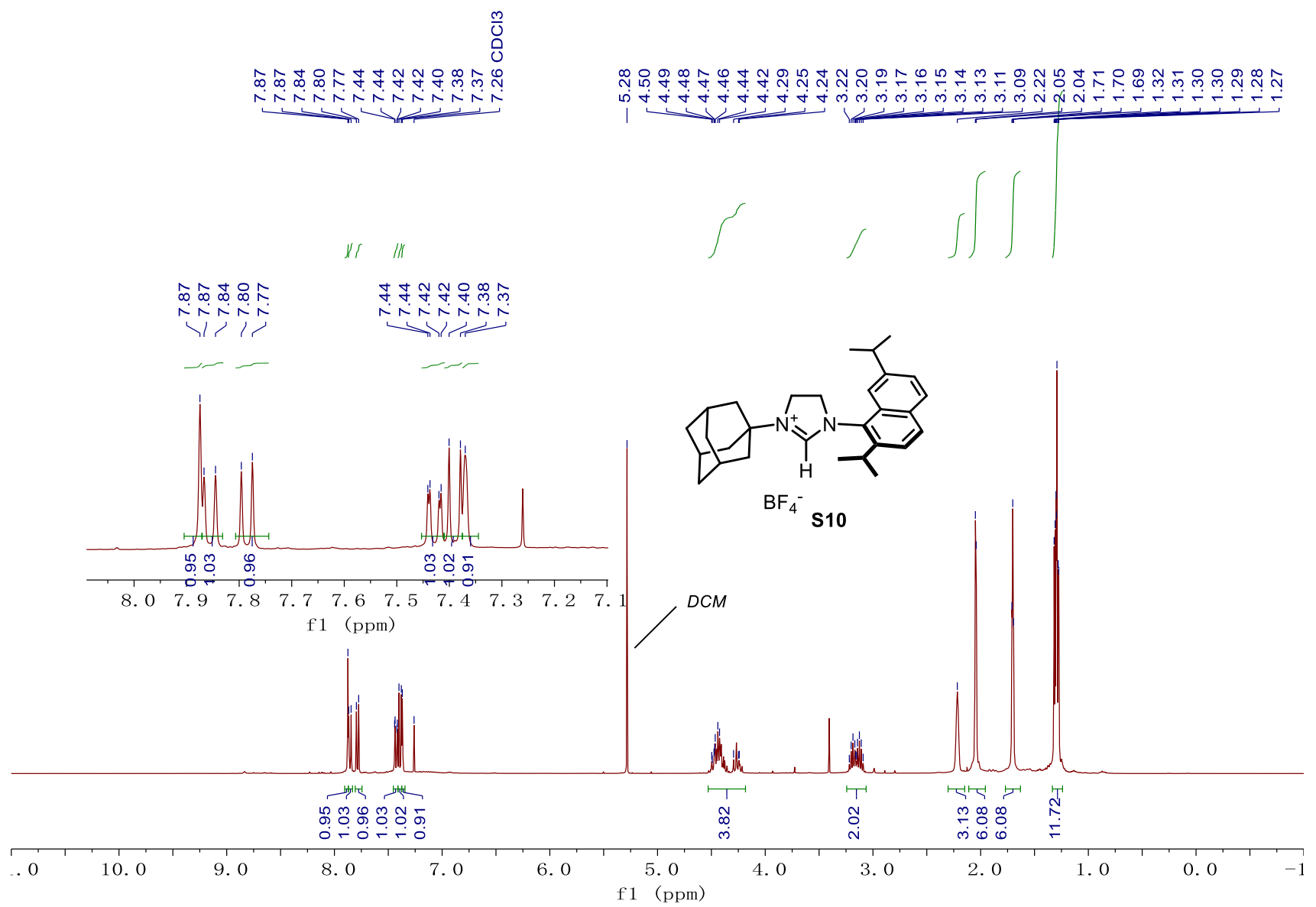

Figure S21 $\mid{ }^{13} \mathrm{C}$ NMR $\left(101 \mathrm{MHz}, \mathrm{CDCl}_{3}\right)$ spectrum of $\mathbf{S 1 0}$.
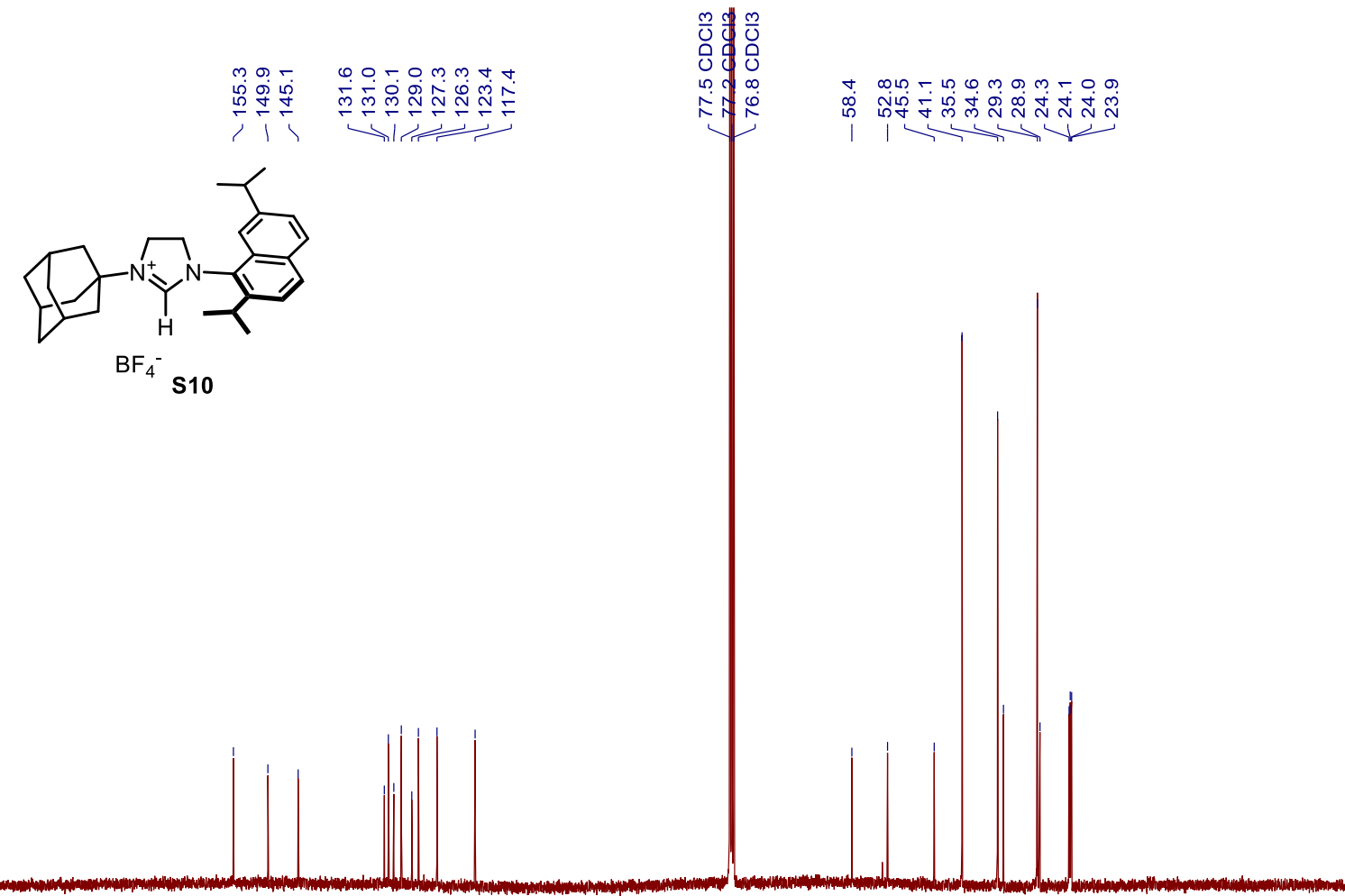

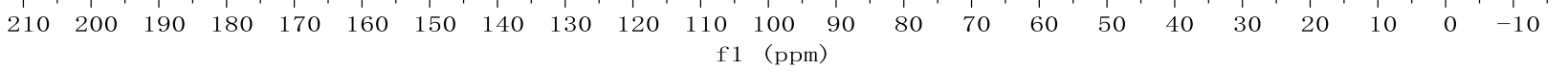


Figure S22 | ${ }^{1} \mathrm{H}$ NMR (400 MHz, $\left.\mathrm{C}_{6} \mathrm{D}_{6}\right)$ spectrum of Ru-7.

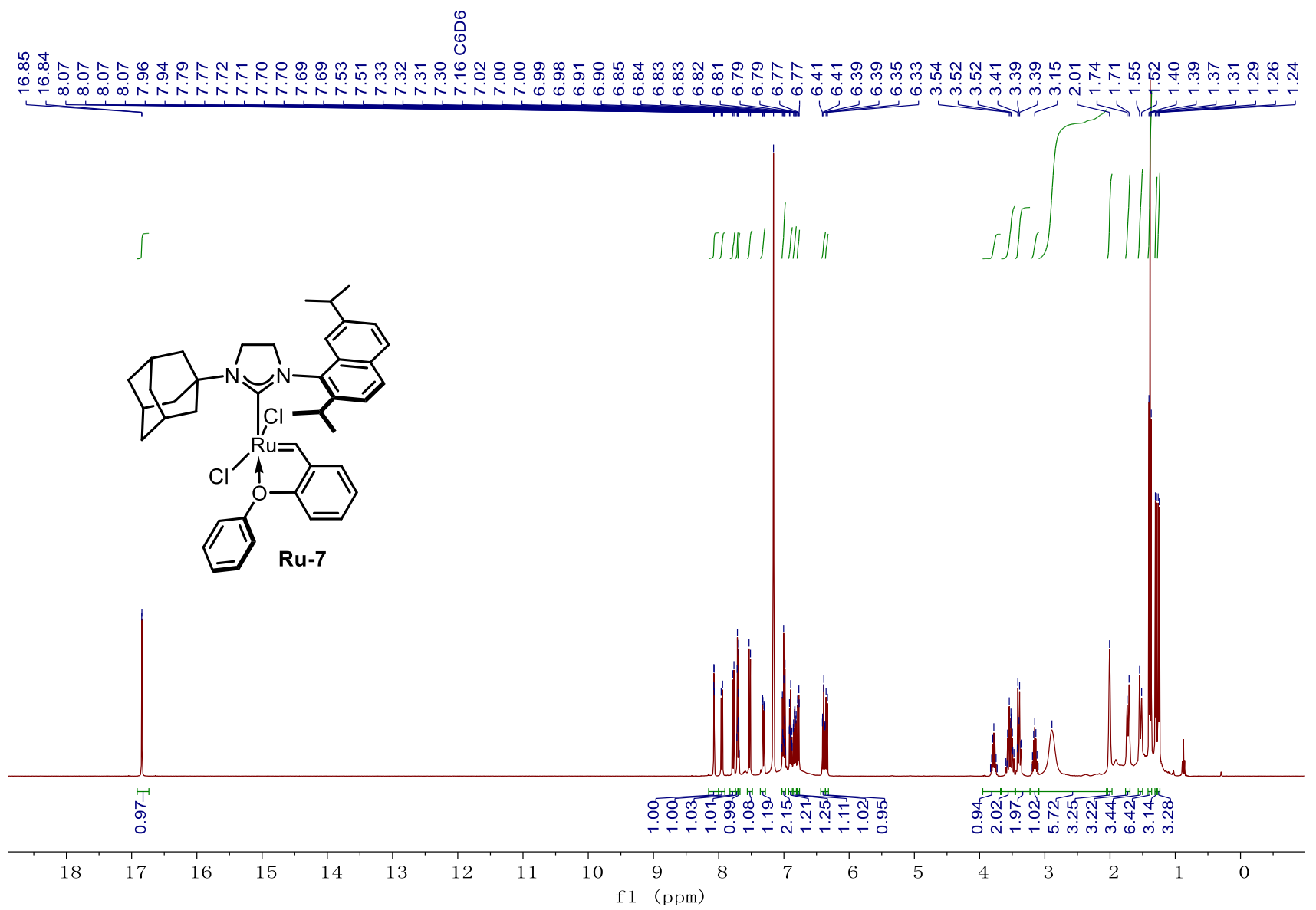

Figure S23 $\mid{ }^{13} \mathrm{C}$ NMR (101 MHz, $\left.\mathrm{C}_{6} \mathrm{D}_{6}\right)$ spectrum of Ru-7.
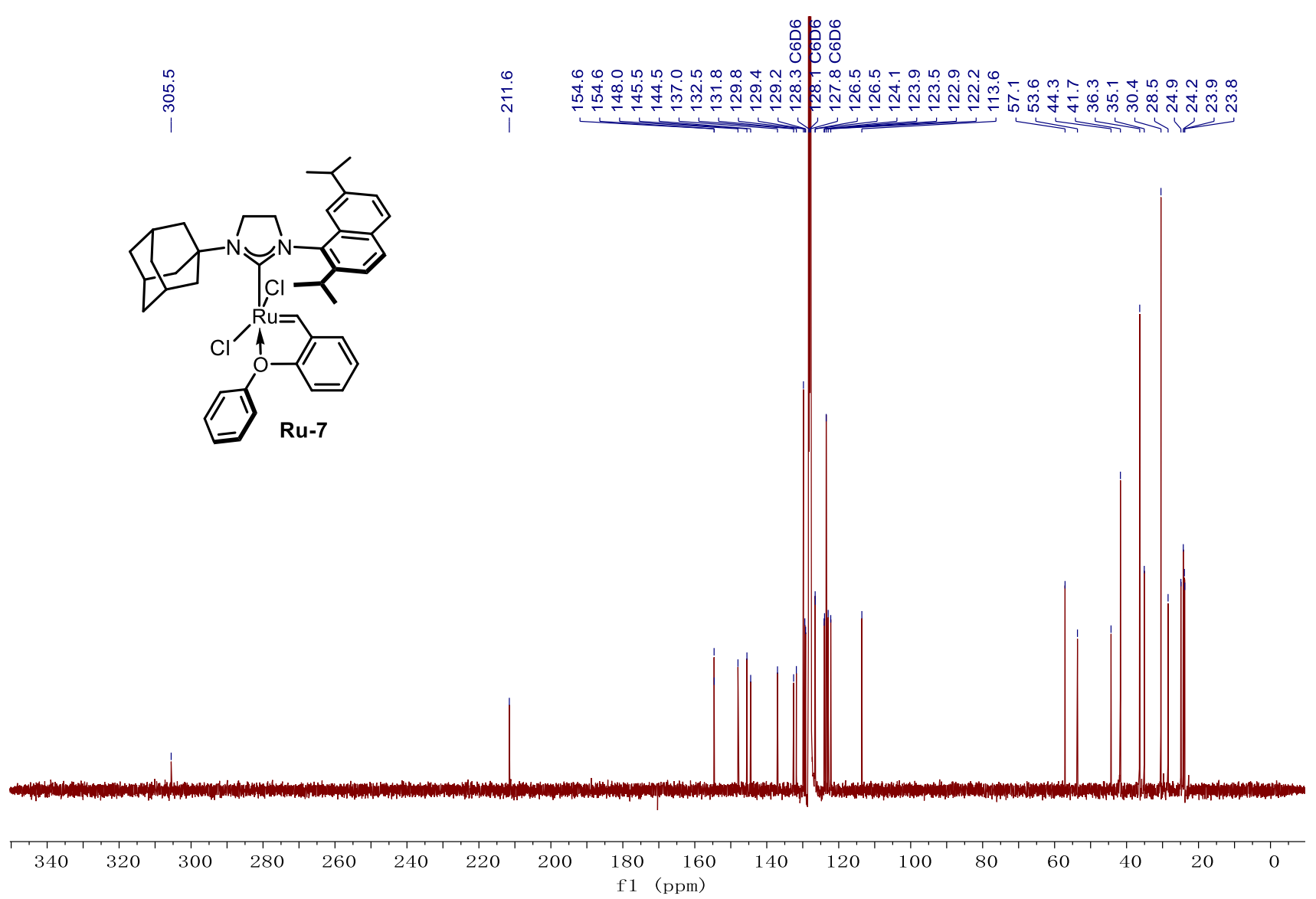
Figure S24 | Crude ${ }^{1} \mathrm{H}$ NMR (400 MHz, $\left.\mathrm{C}_{6} \mathrm{D}_{6}\right)$ spectrum of $\mathbf{S 1 1}$.

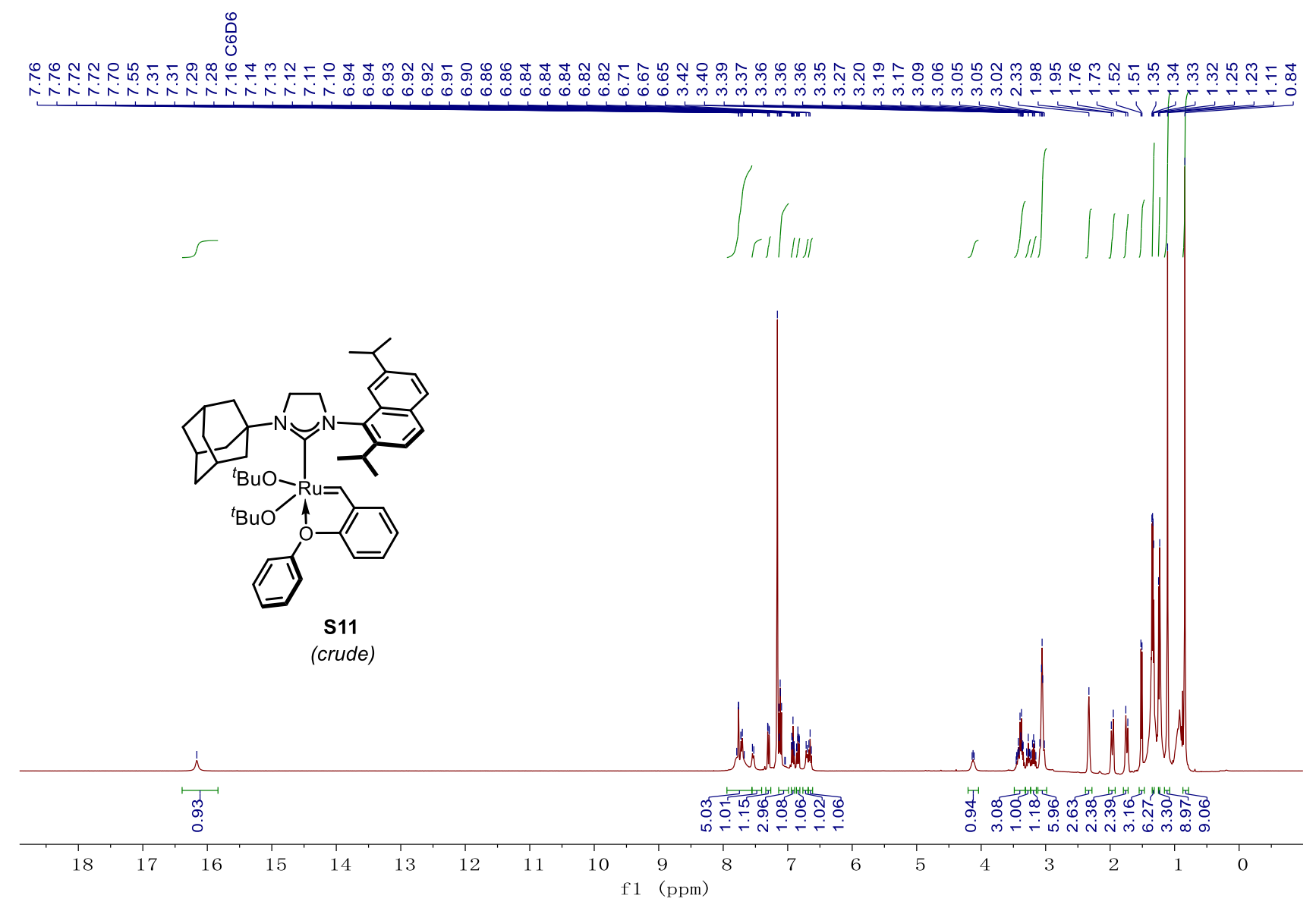


Figure S25 $\mid{ }^{1} \mathrm{H}$ NMR (400 MHz, $\left.\mathrm{C}_{6} \mathrm{D}_{6}\right)$ spectrum of Ru-8.

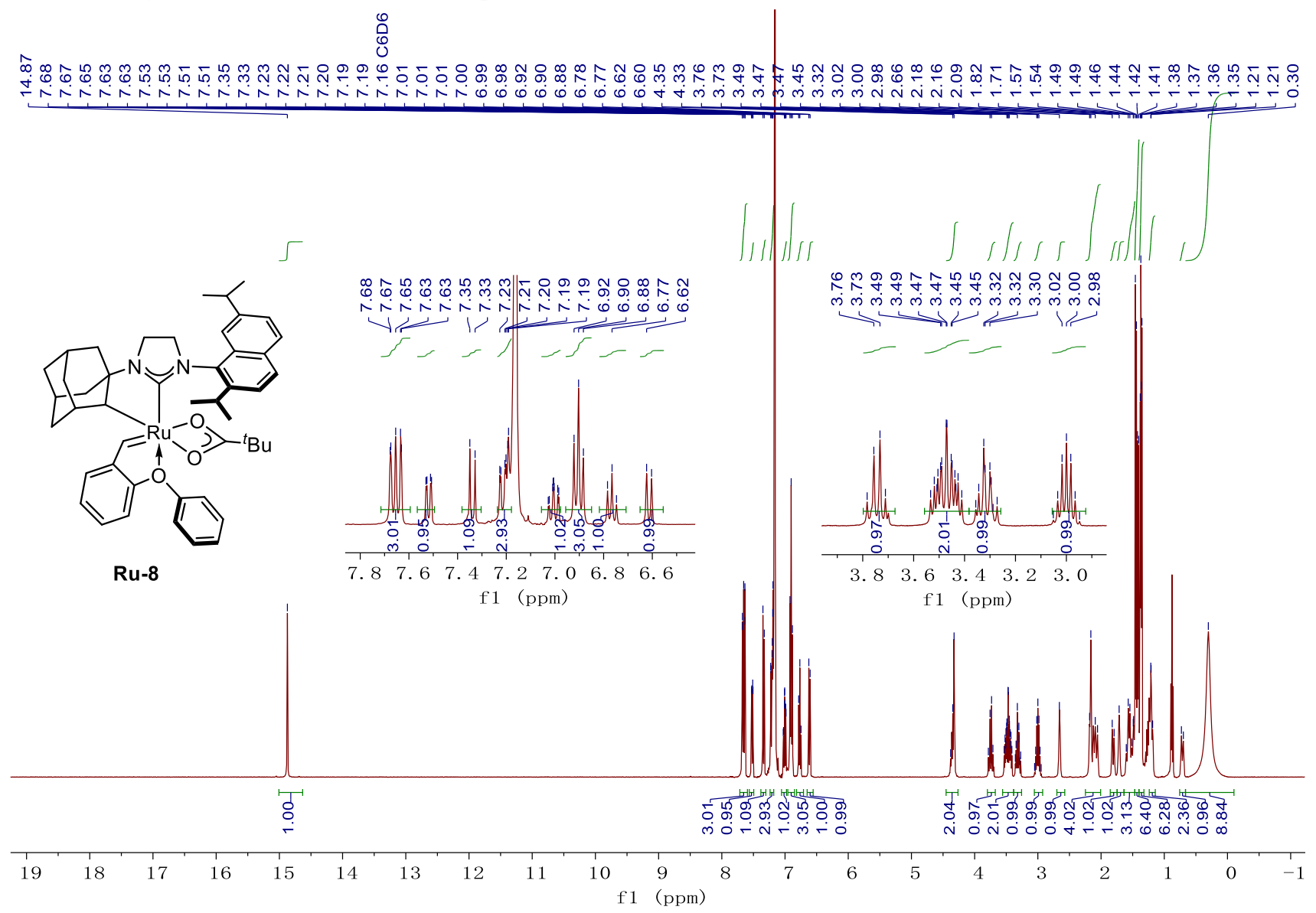

Figure S26 $\mid{ }^{13} \mathrm{C}$ NMR (101 MHz, $\left.\mathrm{C}_{6} \mathrm{D}_{6}\right)$ spectrum of Ru-8.

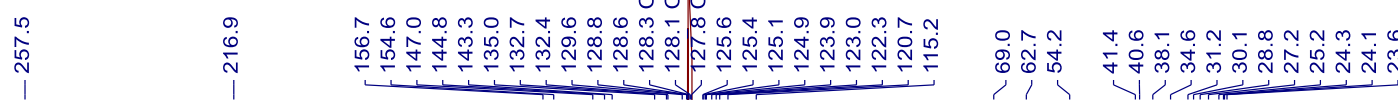

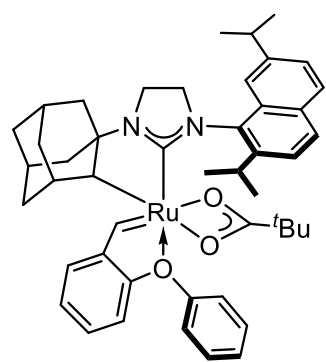

Ru-8

$300 \quad 280 \quad 260$

$240 \quad 220$

$200 \quad 180$ f 160 (ppm) 
Figure S27 | ${ }^{1} \mathrm{H}$ NMR $\left(400 \mathrm{MHz}, \mathrm{C}_{6} \mathrm{D}_{6}\right)$ spectrum of $\mathbf{S 1 3}$.

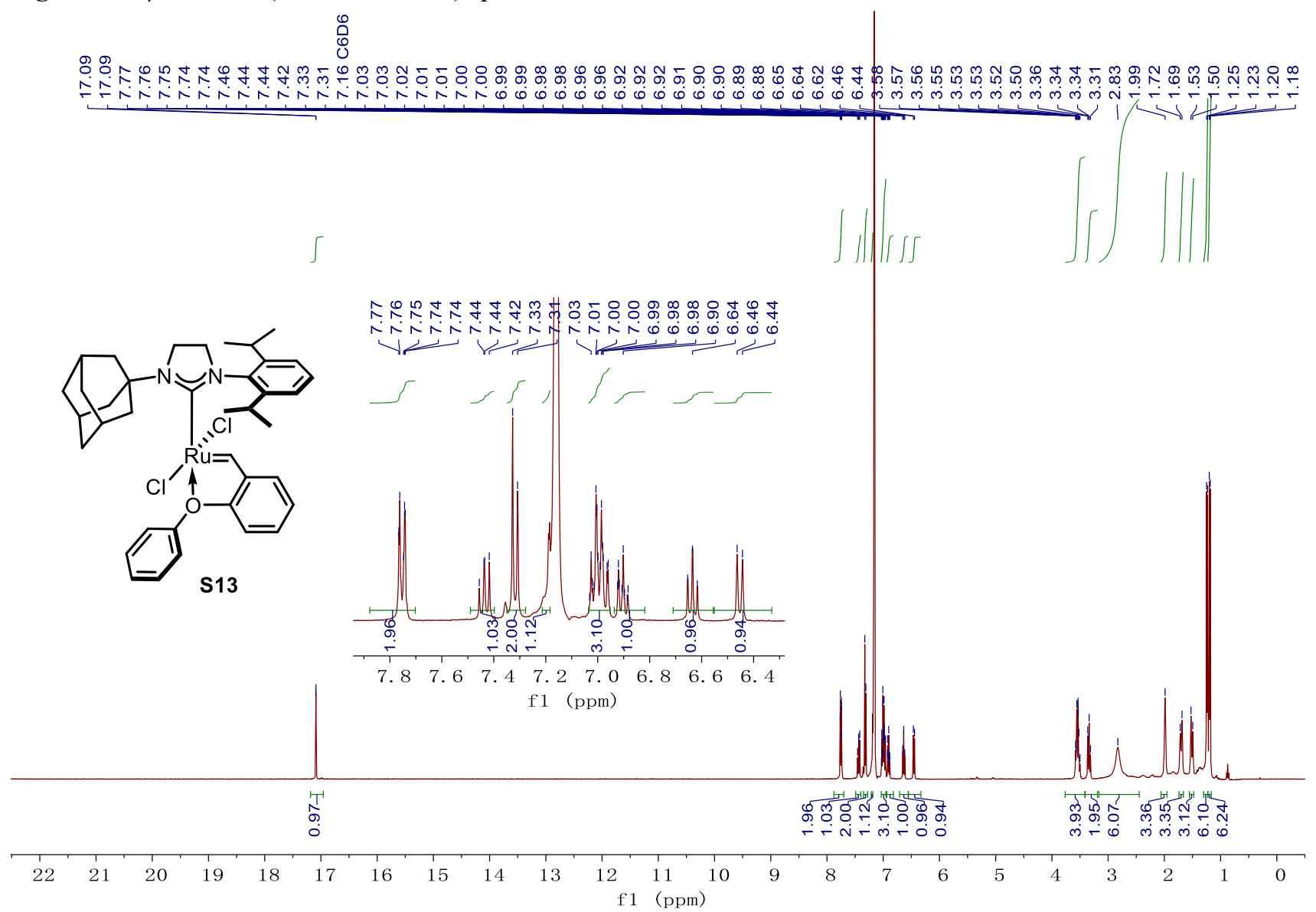

Figure $\mathbf{S 2 8} \mid{ }^{13} \mathrm{C}$ NMR $\left(101 \mathrm{MHz}, \mathrm{C}_{6} \mathrm{D}_{6}\right)$ spectrum of $\mathbf{S 1 3}$.
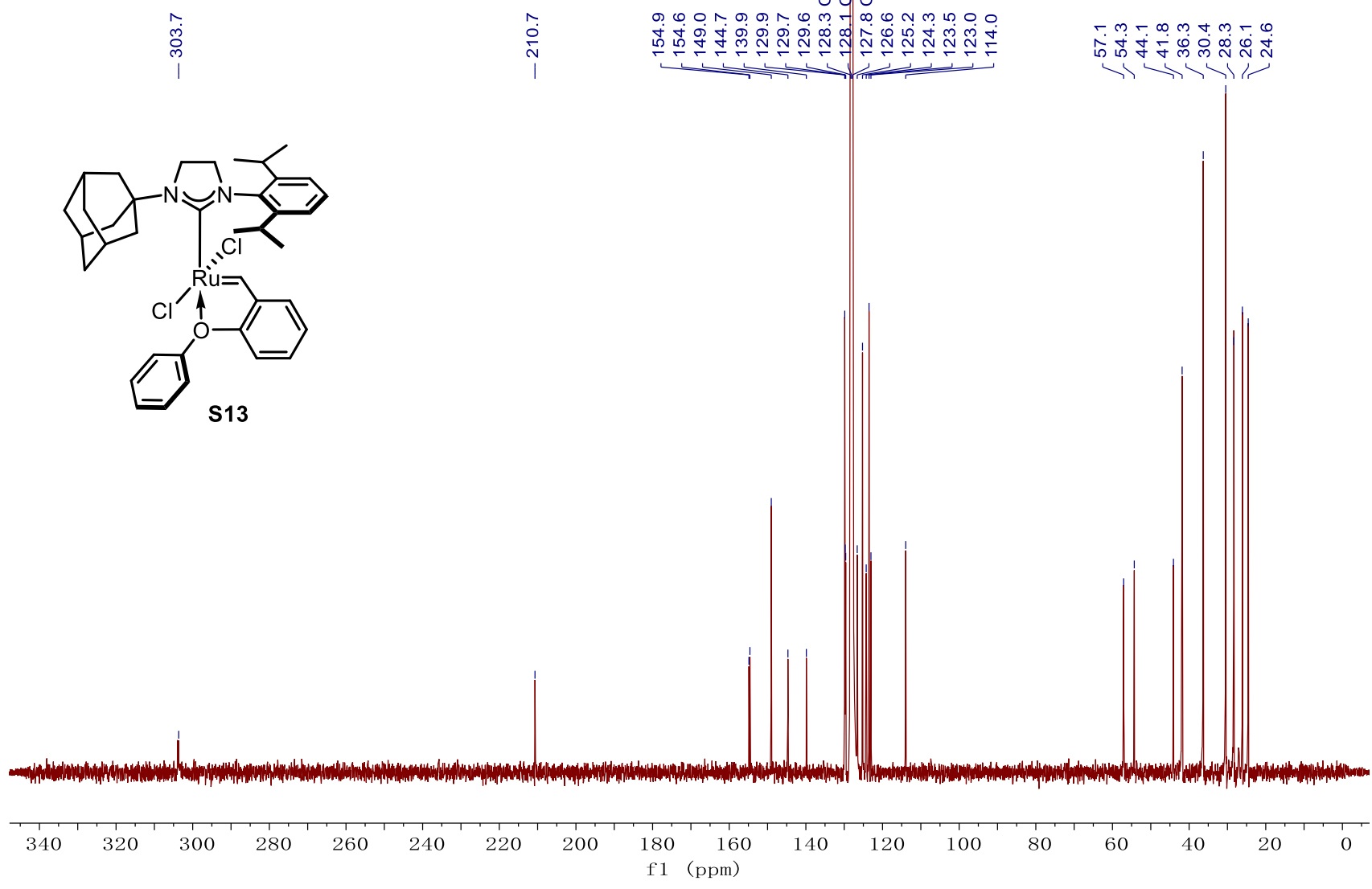
Figure S29 | Crude ${ }^{1} \mathrm{H}$ NMR (500 MHz, $\left.\mathrm{C}_{6} \mathrm{D}_{6}\right)$ spectrum of $\mathbf{S 1 4}$.

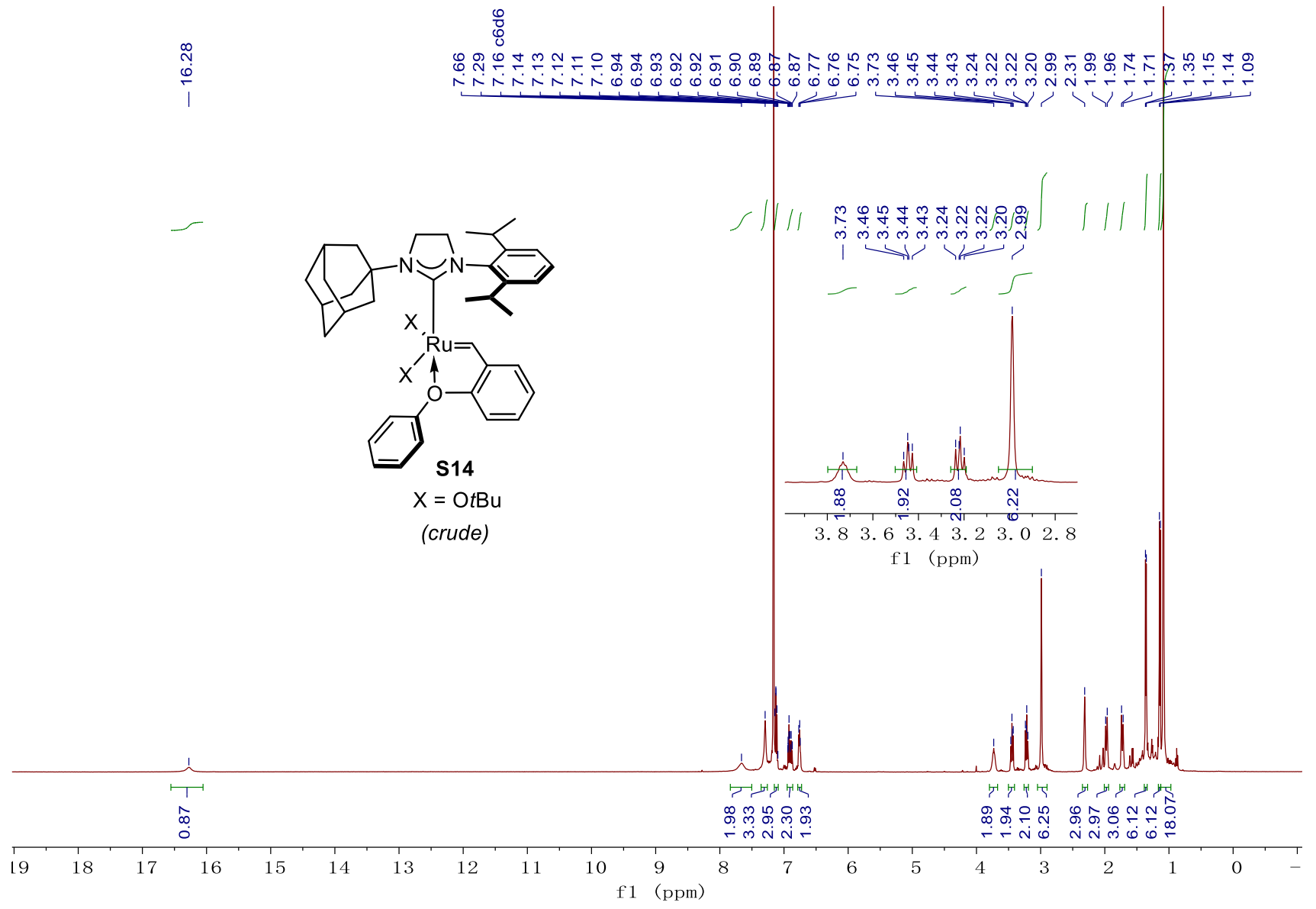


Figure S30 | ${ }^{1} \mathrm{H}$ NMR (400 MHz, $\left.\mathrm{C}_{6} \mathrm{D}_{6}\right)$ spectrum of Ru-11.

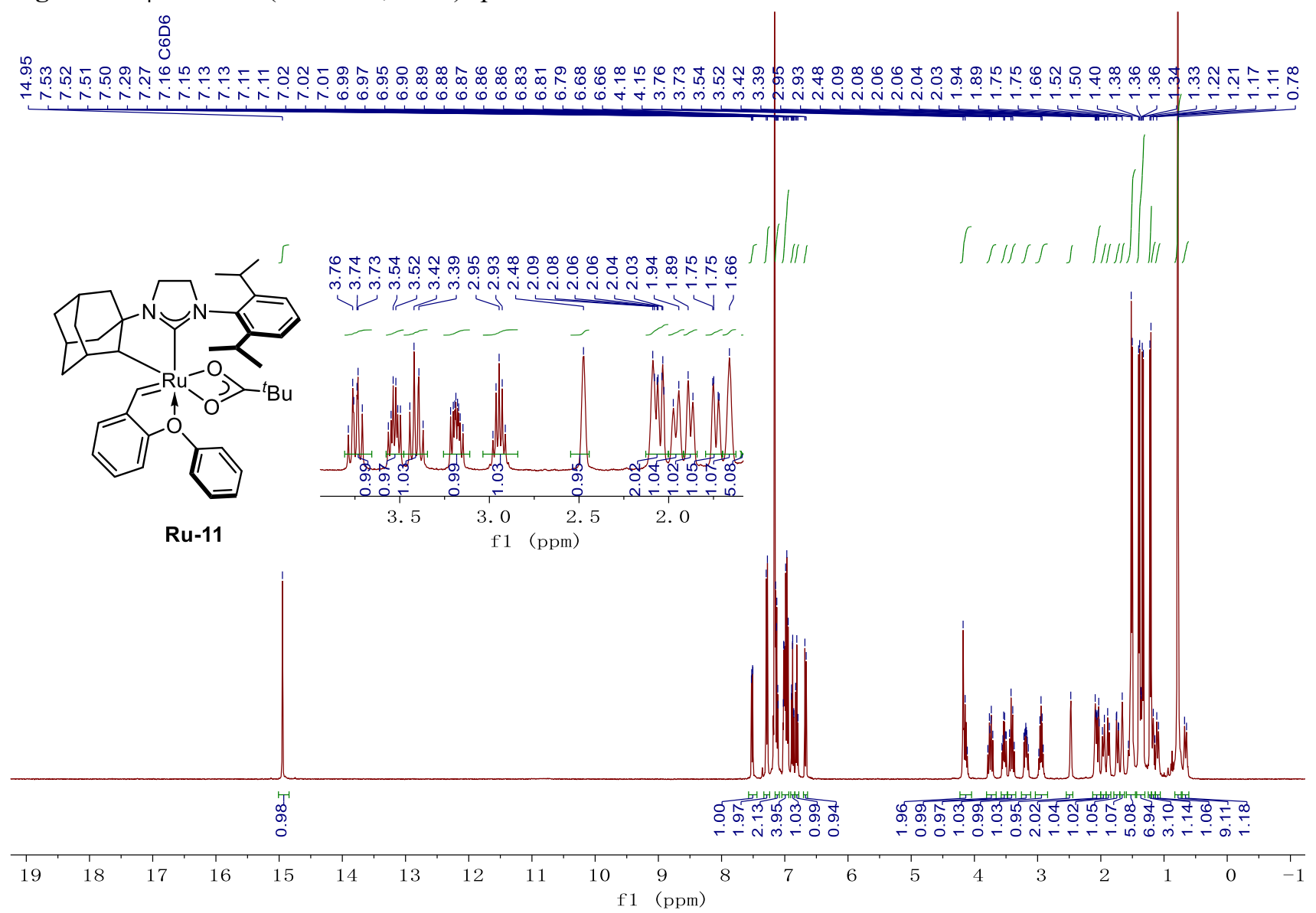

Figure S31 | ${ }^{13} \mathrm{C}$ NMR (101 MHz, $\left.\mathrm{C}_{6} \mathrm{D}_{6}\right)$ spectrum of Ru-11.
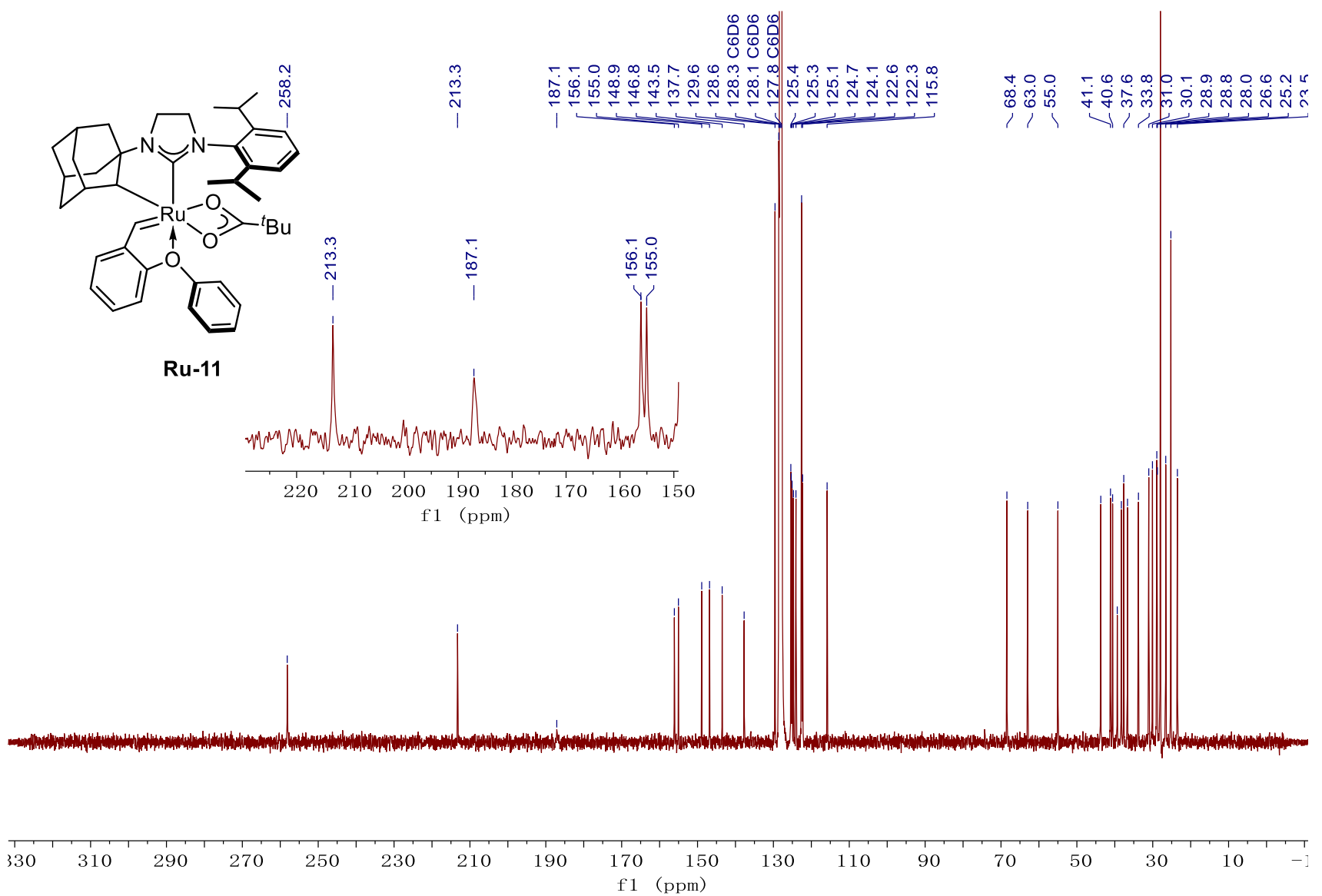
Figure S32 $\mid{ }^{1} \mathrm{H}$ NMR (400 MHz, $\mathrm{C}_{6} \mathrm{D}_{6}$ ) spectrum of Ru-9.

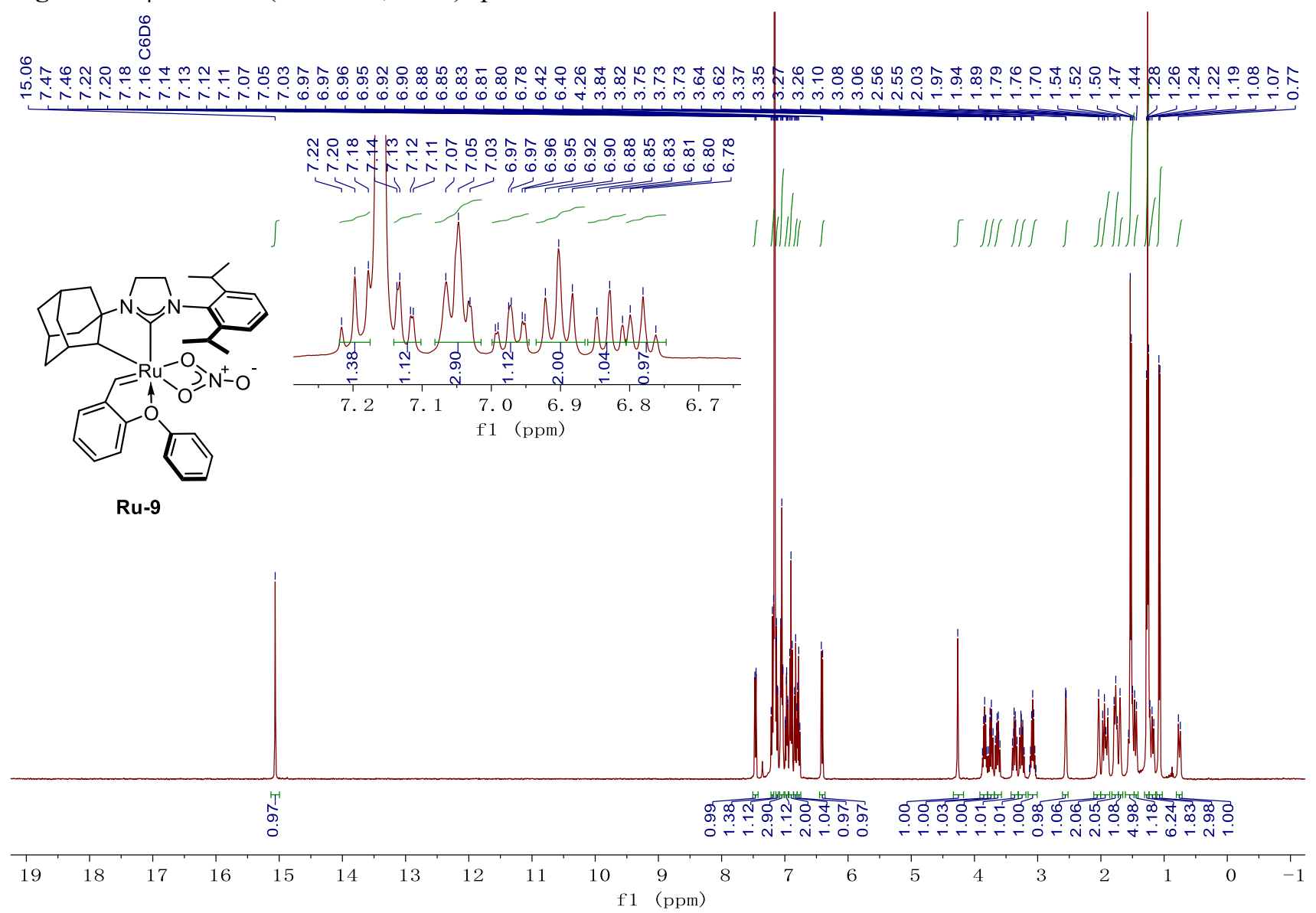

Figure S33 $\mid{ }^{13} \mathrm{C}$ NMR (101 MHz, $\left.\mathrm{C}_{6} \mathrm{D}_{6}\right)$ spectrum of Ru-9.
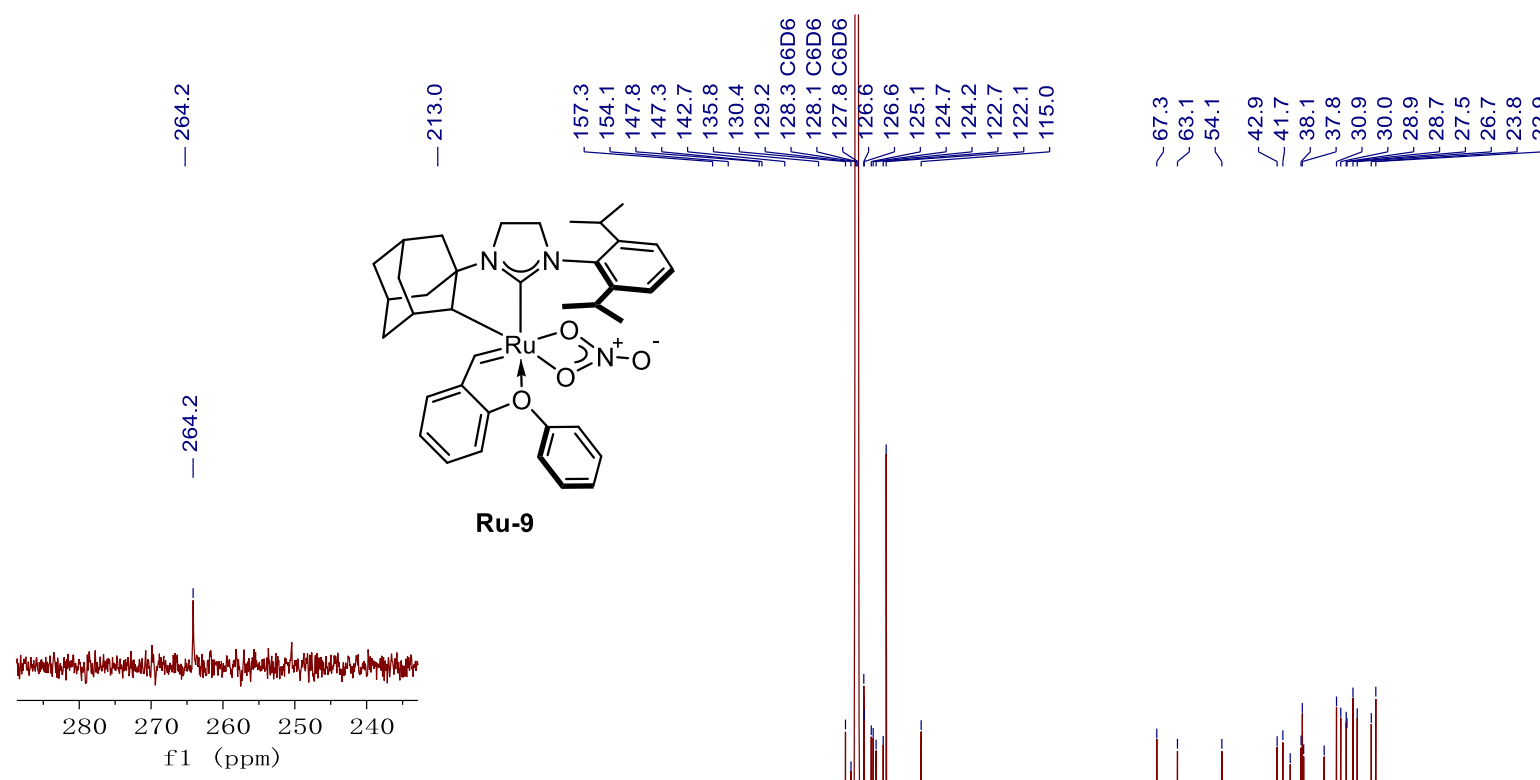

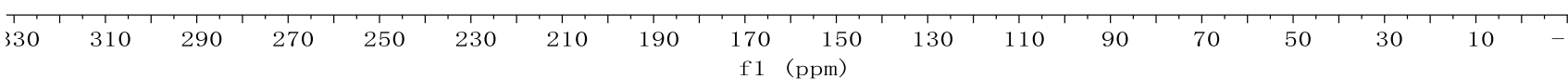


Figure S34 $\mid{ }^{1} \mathrm{H}$ NMR (400 MHz, $\left.\mathrm{C}_{6} \mathrm{D}_{6}\right)$ spectrum of Ru-10.

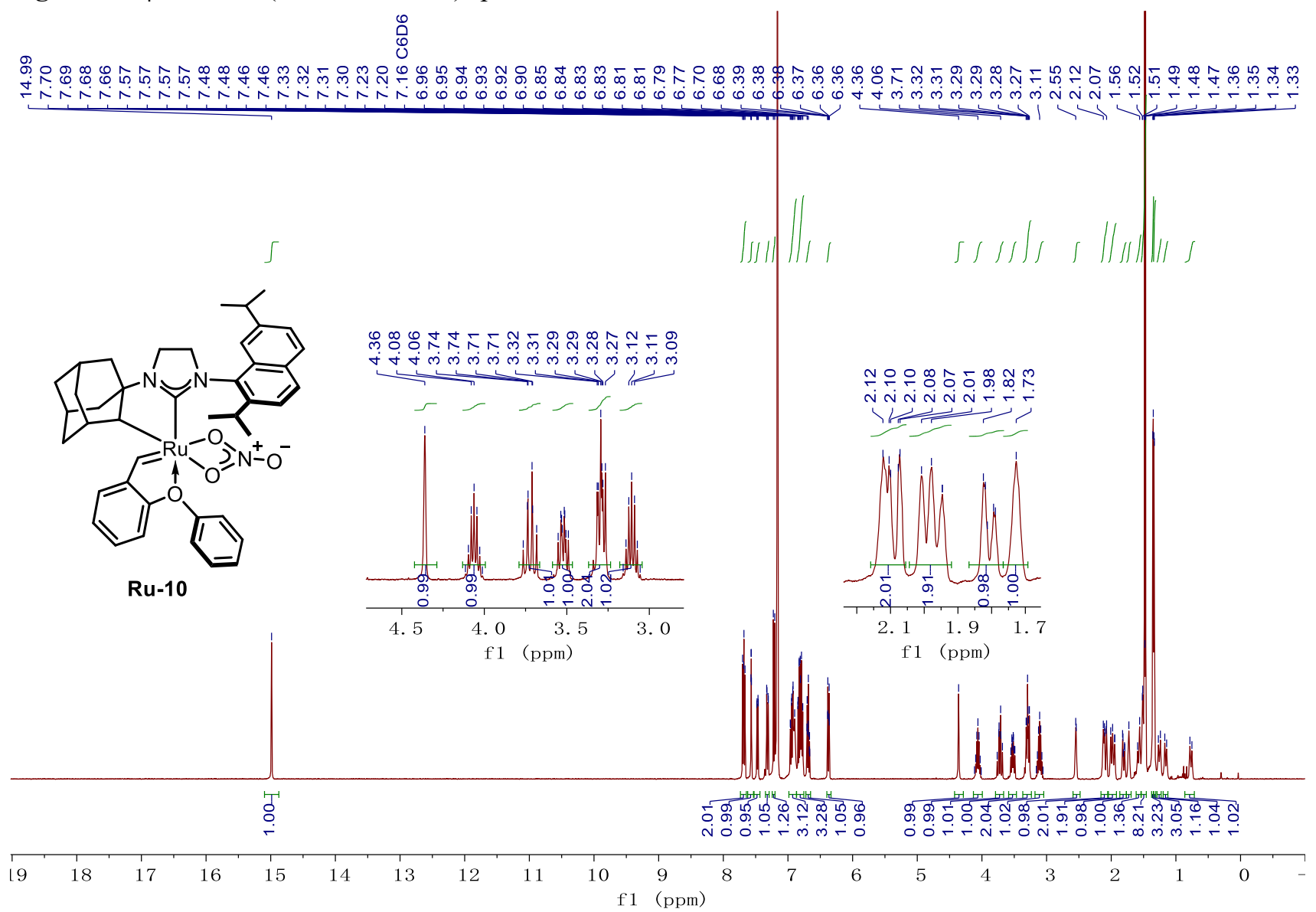

Figure S35 $\mid{ }^{13} \mathrm{C}$ NMR (101 MHz, $\left.\mathrm{C}_{6} \mathrm{D}_{6}\right)$ spectrum of Ru-10.

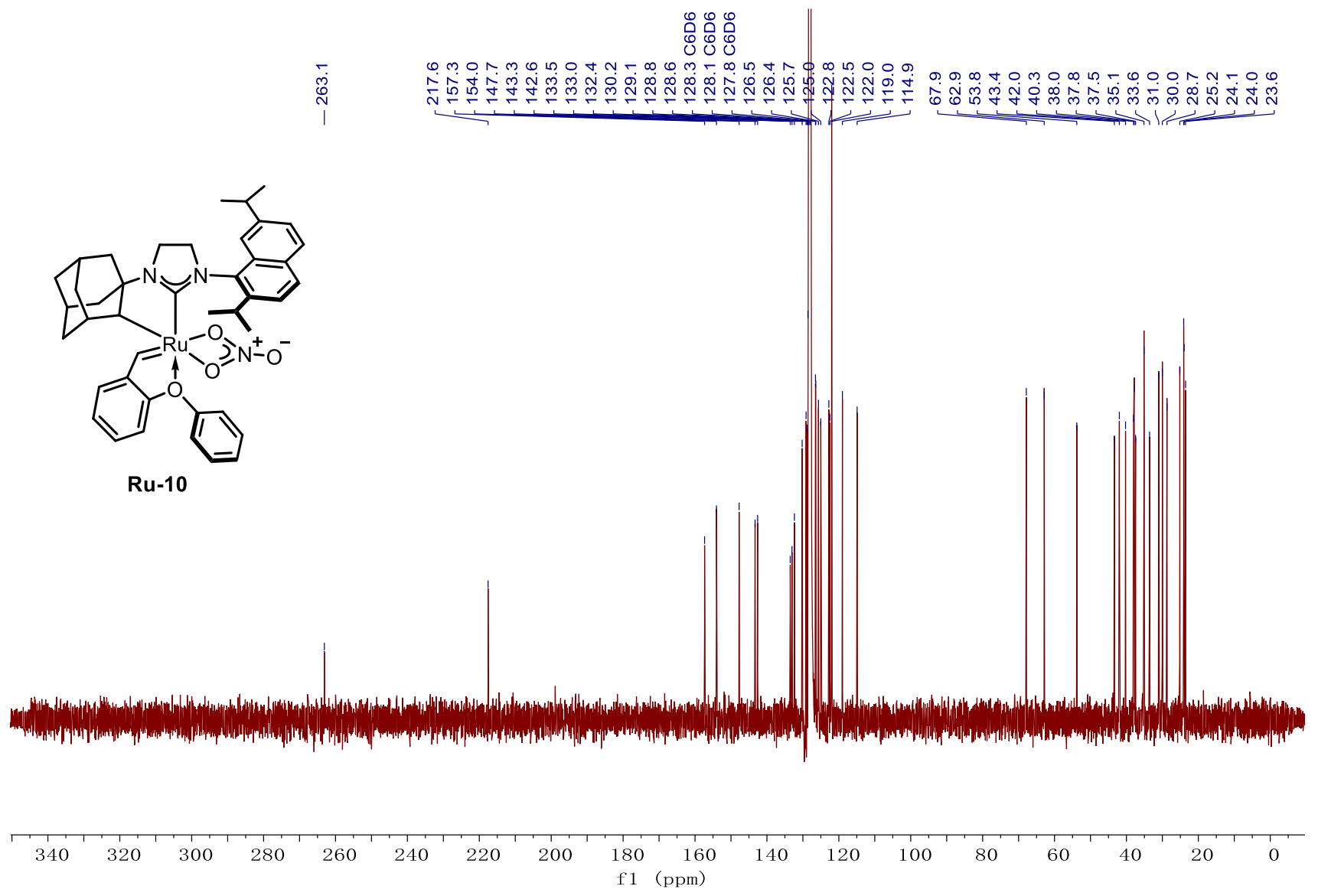


Figure $\mathbf{S 3 6} \mid{ }^{1} \mathrm{H}$ NMR (400 MHz, $\left.\mathrm{CDCl}_{3}\right)$ spectrum of $\mathbf{2 n}$.

$\frac{m}{0}$

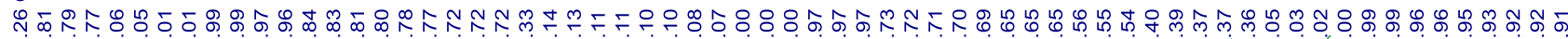

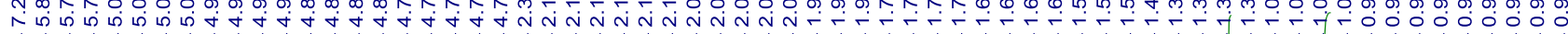<smiles>C=CCCC(=O)O[C@H]1CC(C)CC[C@H]1C(=C)C</smiles>

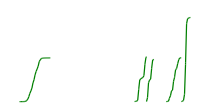

$2 n$

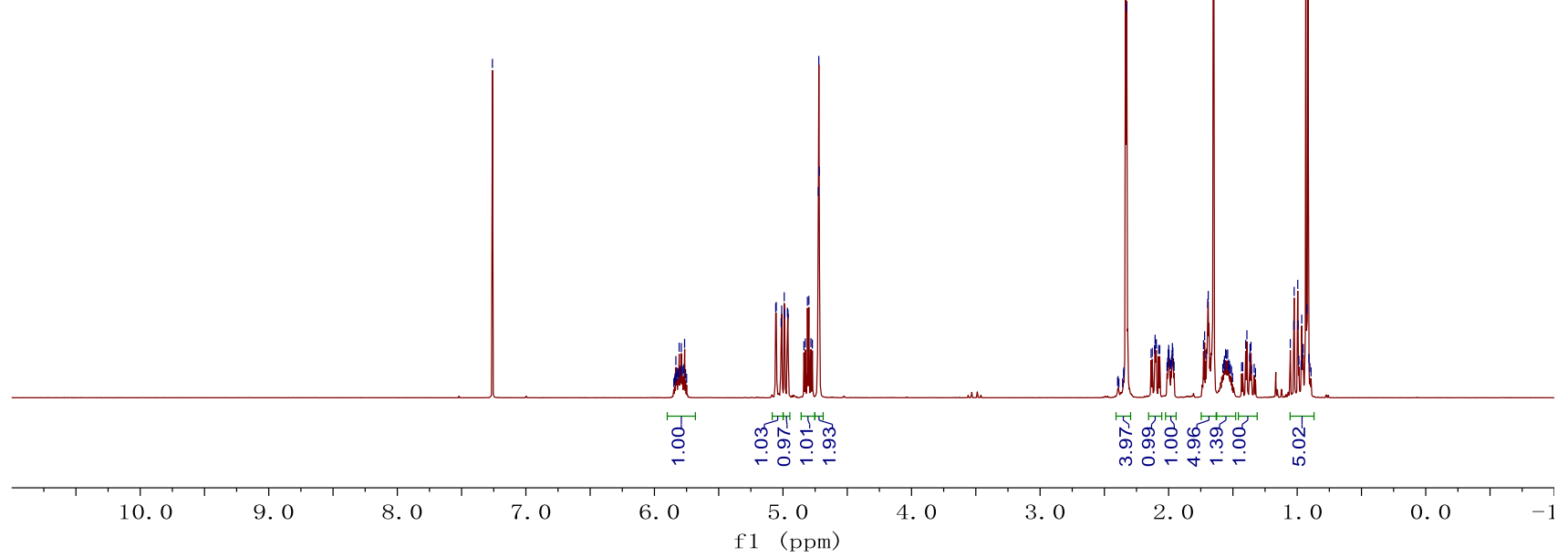

Figure S37 $\mid{ }^{13} \mathrm{C}$ NMR (101 $\left.\mathrm{MHz}, \mathrm{CDCl}_{3}\right)$ spectrum of $\mathbf{2 n}$.<smiles>C=CCCC(=O)O[C@H]1CC(C)CC[C@H]1C(=C)C</smiles>

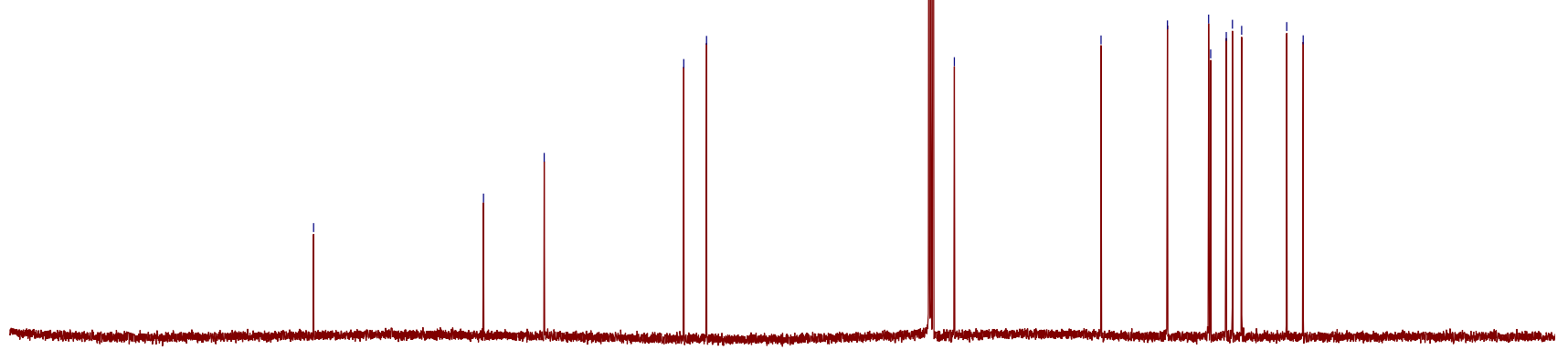

$\begin{array}{lllllllllllllllllllllll}210 & 200 & 190 & 180 & 170 & 160 & 150 & 140 & 130 & 120 & \begin{array}{c}110 \\ \mathrm{f} 1\end{array} \begin{array}{c}100 \\ (\mathrm{ppm})\end{array} & 90 & 80 & 70 & 60 & 50 & 40 & 30 & 20 & 10 & 0 & -10\end{array}$ 
Figure S38 $\mid{ }^{1} \mathrm{H}$ NMR (400 MHz, $\left.\mathrm{CDCl}_{3}\right)$ spectrum of $\mathbf{1} \mathbf{u}$.

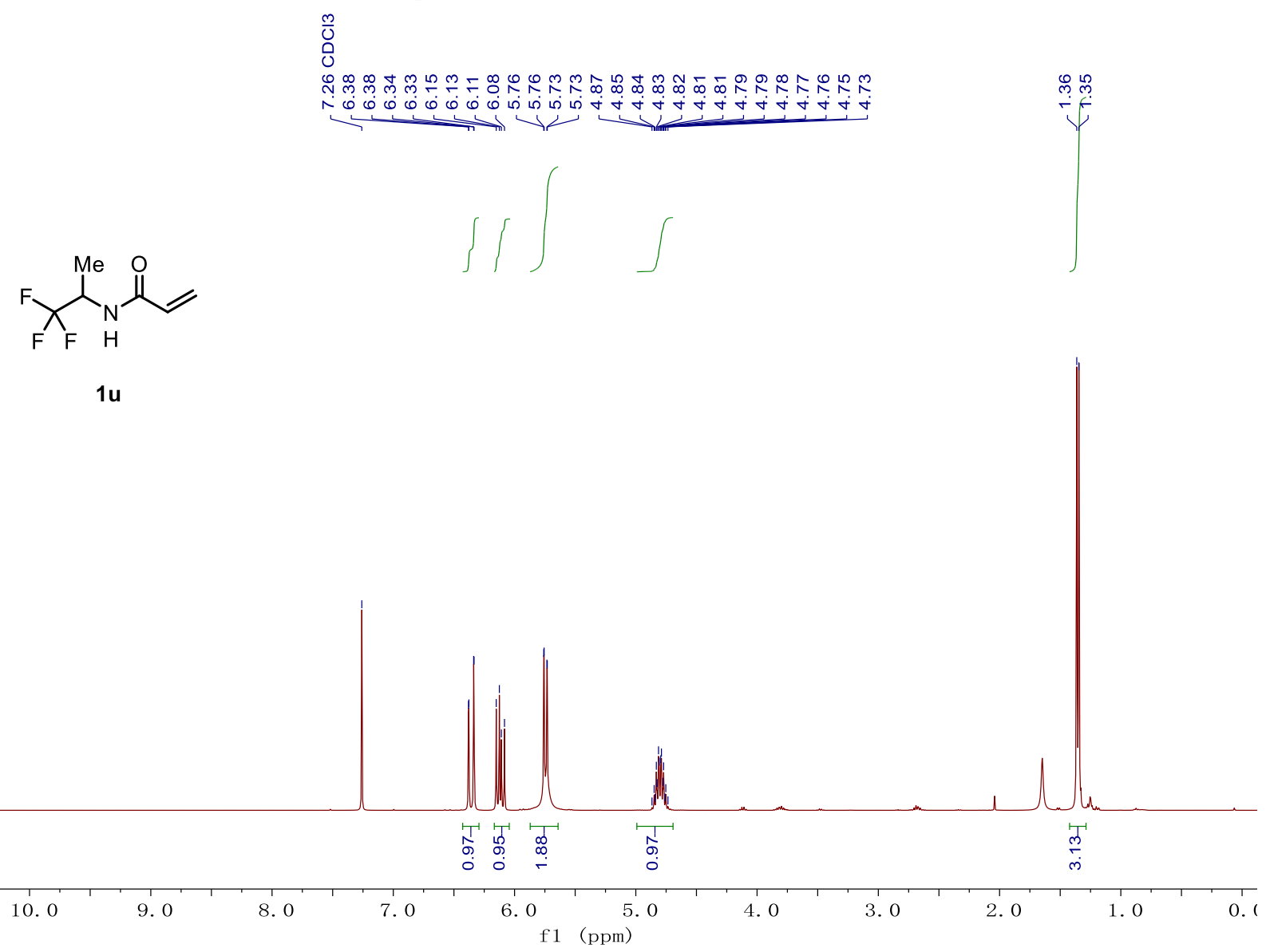

Figure S39 | ${ }^{13} \mathrm{C}$ NMR $\left(101 \mathrm{MHz}, \mathrm{CDCl}_{3}\right)$ spectrum of $\mathbf{1 u}$.
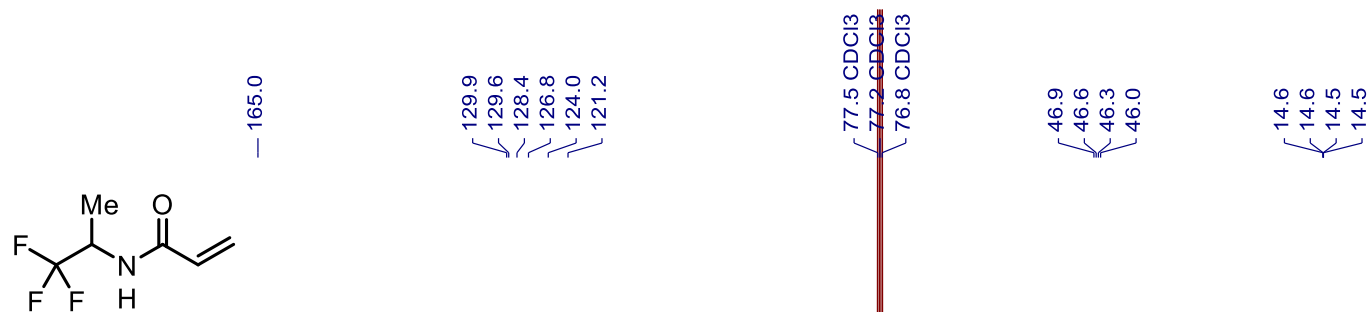

$1 \mathrm{u}$

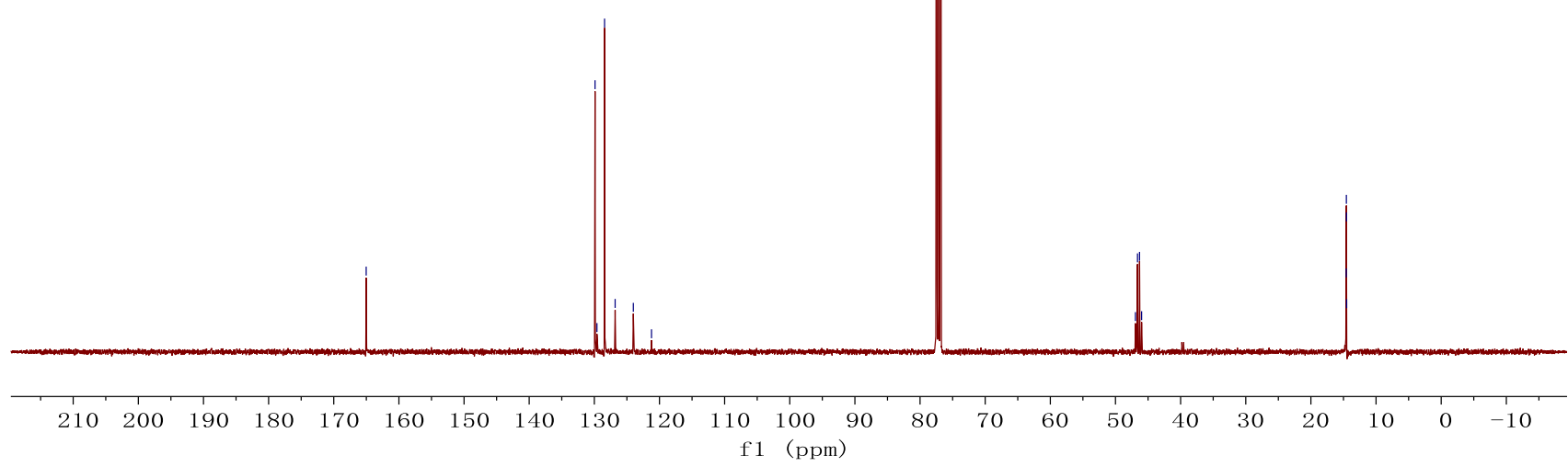


Figure $\mathbf{S 4 0} \mid{ }^{19} \mathrm{~F}$ NMR (282 $\left.\mathrm{MHz}, \mathrm{CDCl}_{3}\right)$ spectrum of $\mathbf{1 u}$.<smiles>C=CC(=O)NC([14CH3])C(F)(F)F</smiles>

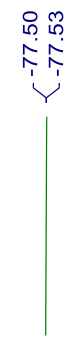

$1 \mathrm{u}$

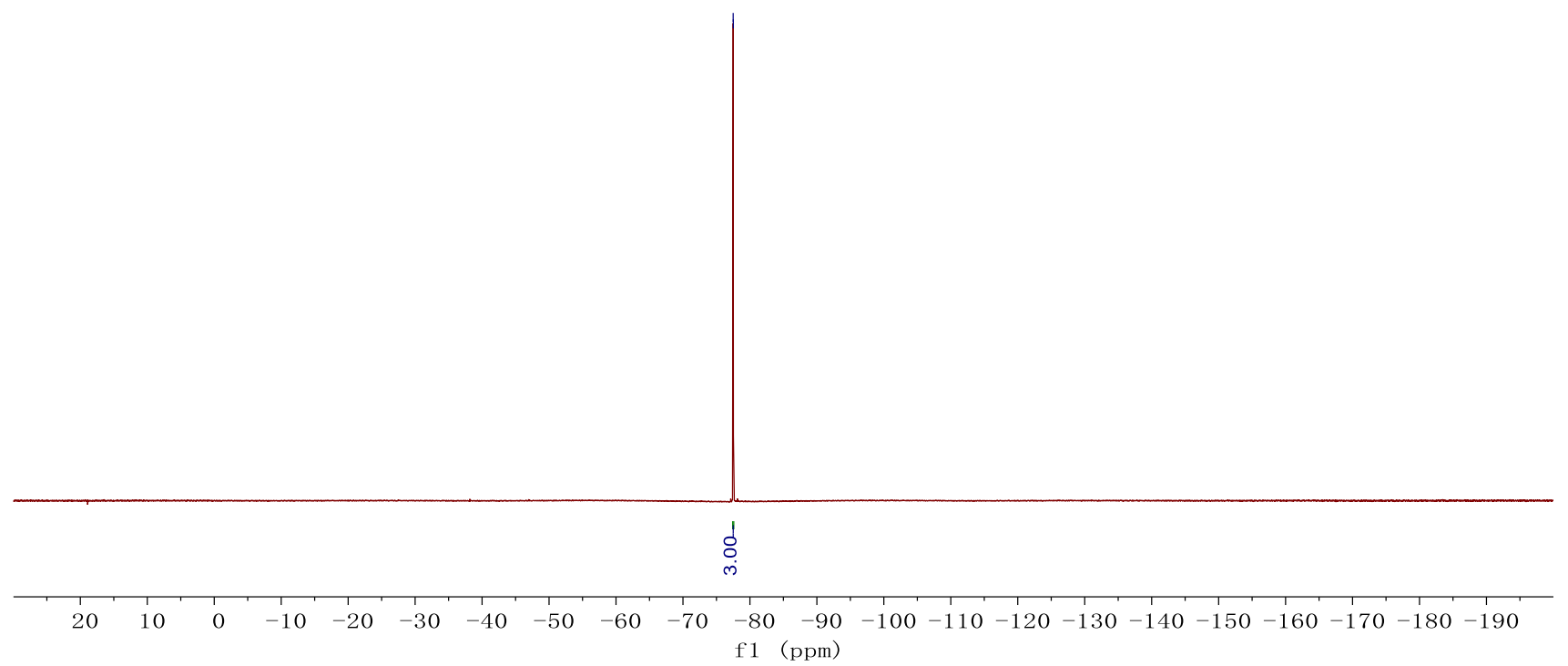


Figure S41 | ${ }^{1} \mathrm{H}$ NMR (400 MHz, $\left.\mathrm{CDCl}_{3}\right)$ spectrum of $\mathbf{1 w}$.

$\frac{m}{0}$
0

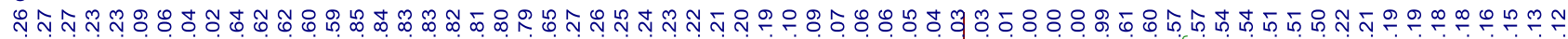

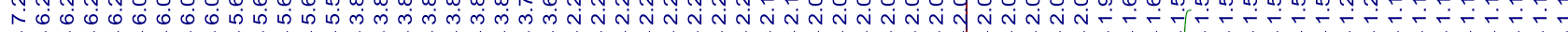<smiles>C=CC(=O)NC1CCC(C(=O)OC)CC1</smiles>

$1 w$
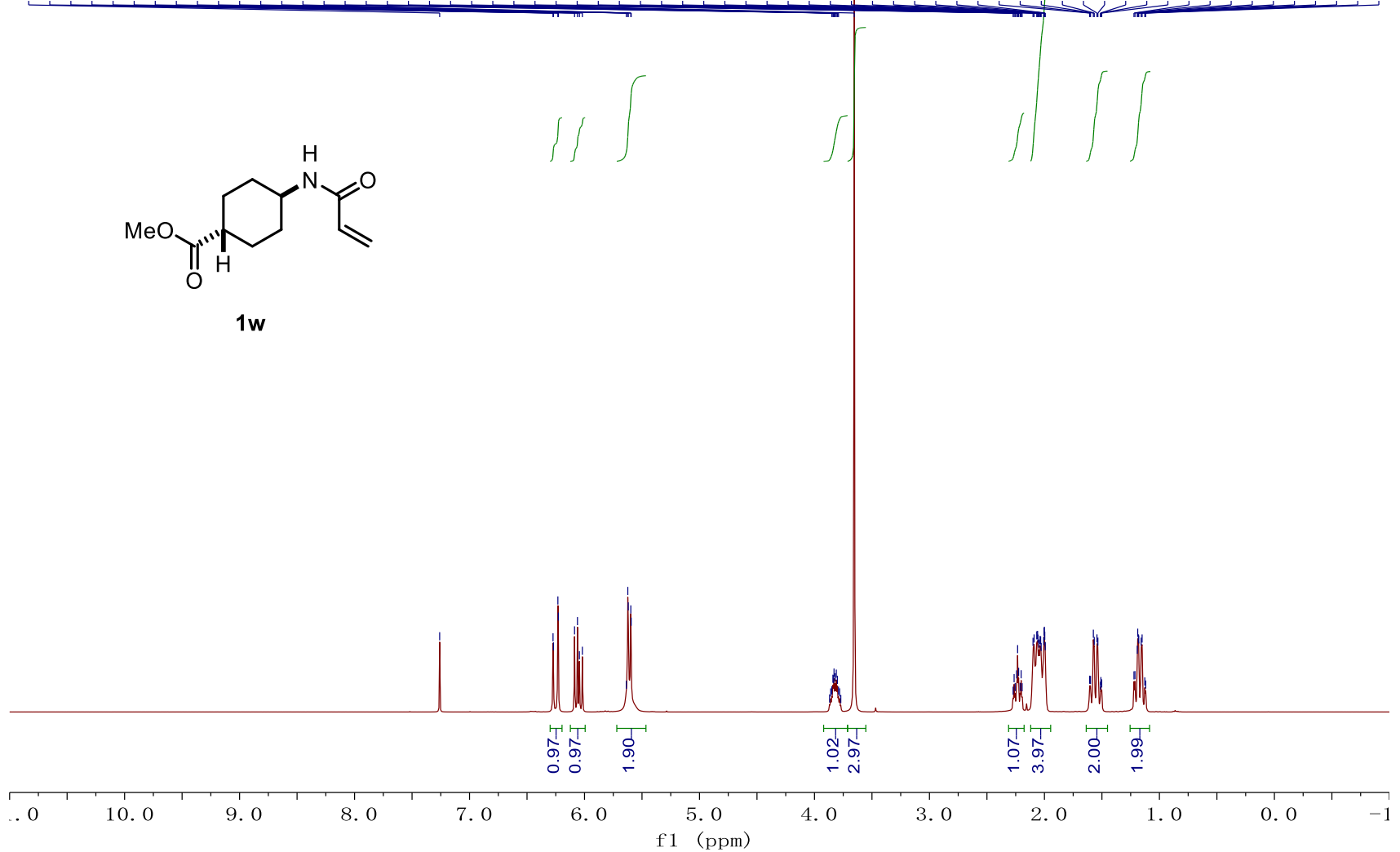

Figure $\mathbf{S 4 2} \mid{ }^{13} \mathrm{C}$ NMR $\left(101 \mathrm{MHz}, \mathrm{CDCl}_{3}\right)$ spectrum of $\mathbf{1 w}$.<smiles>C=CC(=O)NC1CCC(C(=O)O)CC1</smiles>

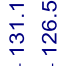

\section{$\frac{m}{0} \frac{m}{0}$
000
0}

点点点

i̊

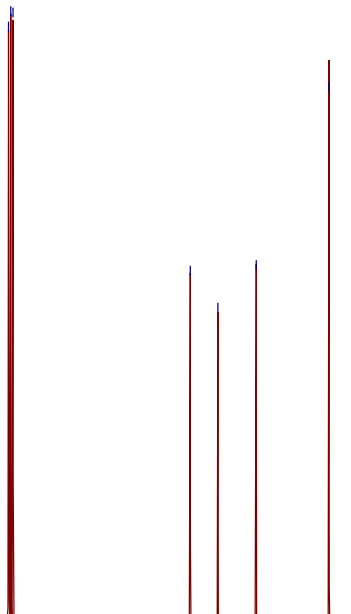

$\begin{array}{lllllllllllllllllllllllllllllll}210 & 200 & 190 & 180 & 170 & 160 & 150 & 140 & 130 & 120 & 110 & 100 & 90 & 80 & 70 & 60 & 50 & 40 & 30 & 20 & 10 & 0 & -10\end{array}$ 
Figure $\mathbf{S 4 3} \mid{ }^{1} \mathrm{H}$ NMR (400 MHz, $\left.\mathrm{CDCl}_{3}\right)$ spectrum of $\mathbf{3 a}$.

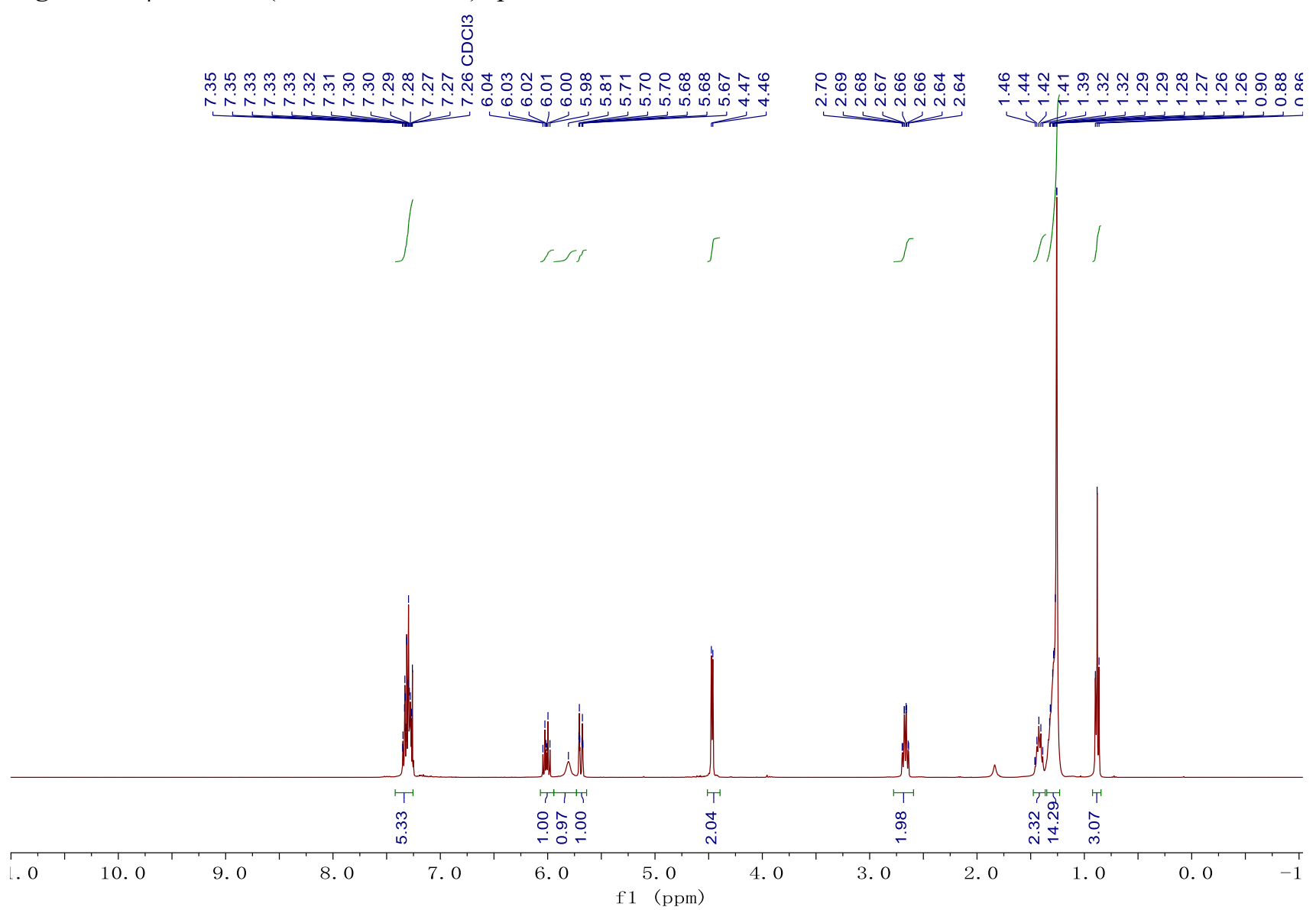

Figure $\mathbf{S 4 4} \mid{ }^{13} \mathrm{C}$ NMR $\left(101 \mathrm{MHz}, \mathrm{CDCl}_{3}\right)$ spectrum of $\mathbf{3 a}$.

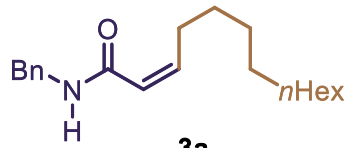

$3 a$

$\begin{array}{cccccccccccc}210 & 200 & 190 & 180 & 170 & 160 & 150 & 140 & 130 & 120 & 110 & 100 \\ \mathrm{f} 1 & (\mathrm{ppm})\end{array}$ 
Figure S45 $\mid{ }^{1} \mathrm{H}$ NMR $\left(400 \mathrm{MHz}, \mathrm{CDCl}_{3}\right)$ spectrum of $\mathbf{3 b}$.

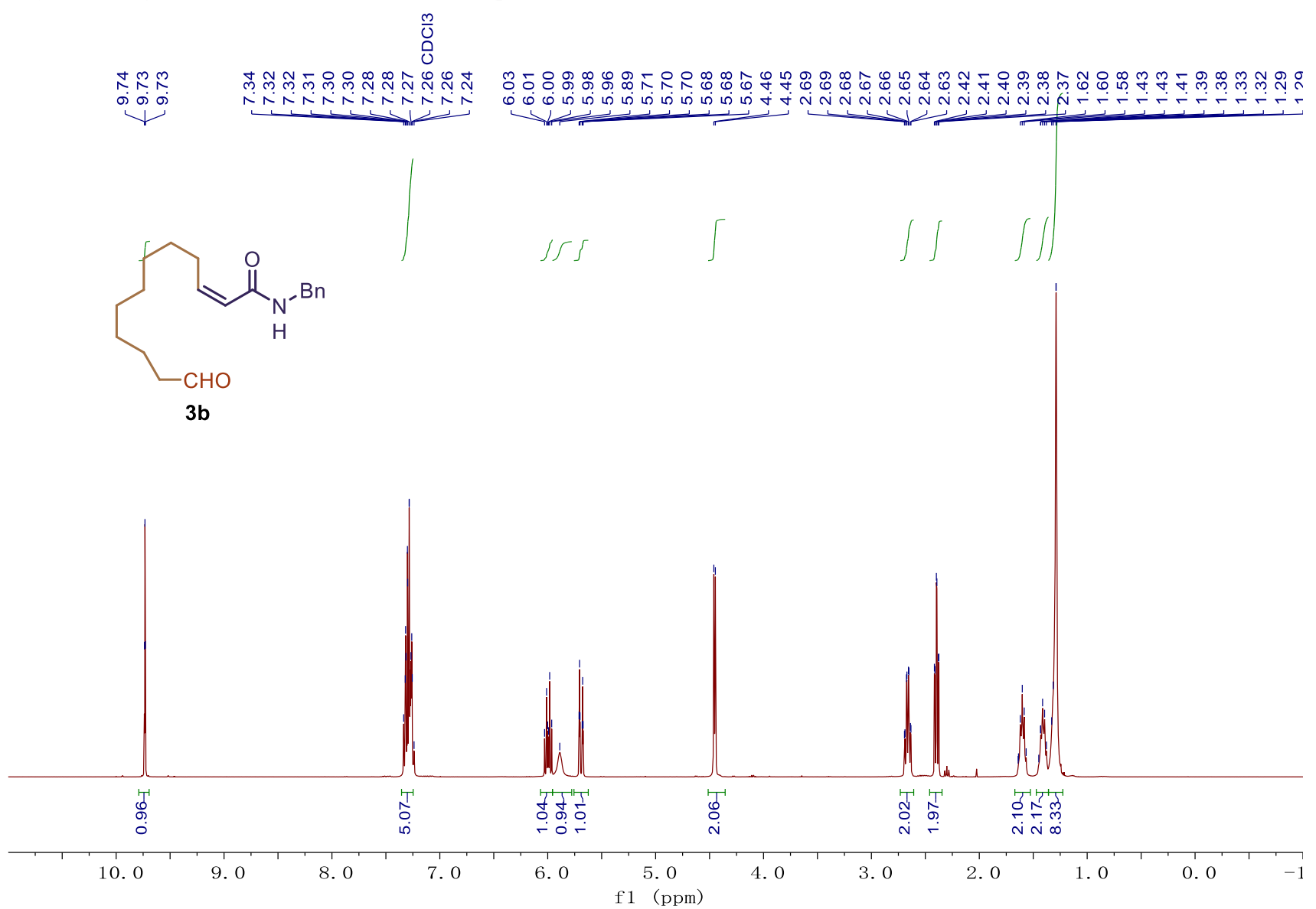

Figure $\mathbf{S 4 6} \mid{ }^{13} \mathrm{C}$ NMR (101 MHz, $\left.\mathrm{CDCl}_{3}\right)$ spectrum of $\mathbf{3 b}$.

$\stackrel{\substack{\infty \\ \text { i }}}{i}$

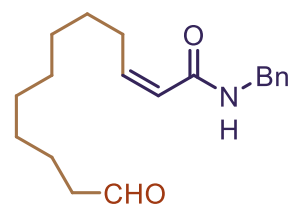

3b

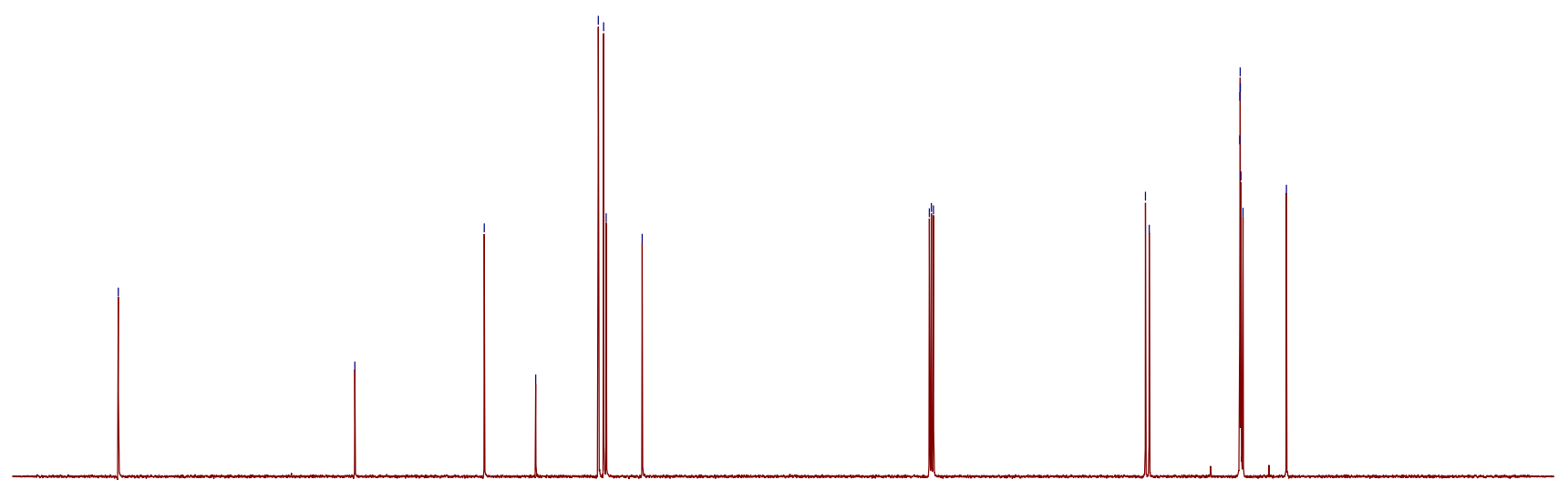

$\begin{array}{lllllllllllllllllllllll}210 & 200 & 190 & 180 & 170 & 160 & 150 & 140 & 130 & 120 & 110 & 100 & 90 & 80 & 70 & 60 & 50 & 40 & 30 & 20 & 10 & 0 & -10\end{array}$ 
Figure $\mathbf{S 4 7} \mid{ }^{1} \mathrm{H}$ NMR (400 MHz, $\left.\mathrm{CDCl}_{3}\right)$ spectrum of $\mathbf{3 c}$.

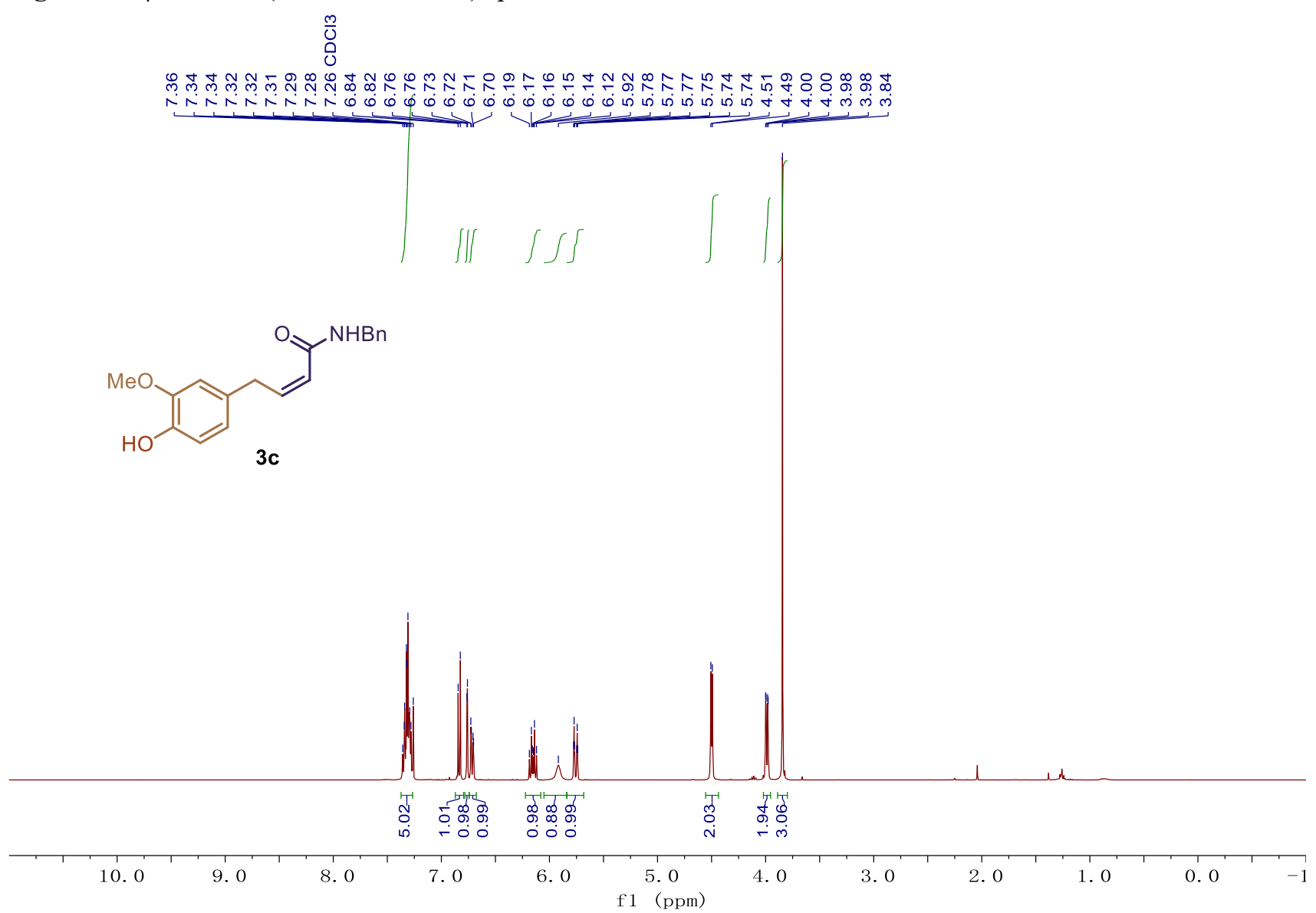

Figure S48 $\mid{ }^{13} \mathrm{C}$ NMR $\left(101 \mathrm{MHz}, \mathrm{CDCl}_{3}\right)$ spectrum of $\mathbf{3 c}$.

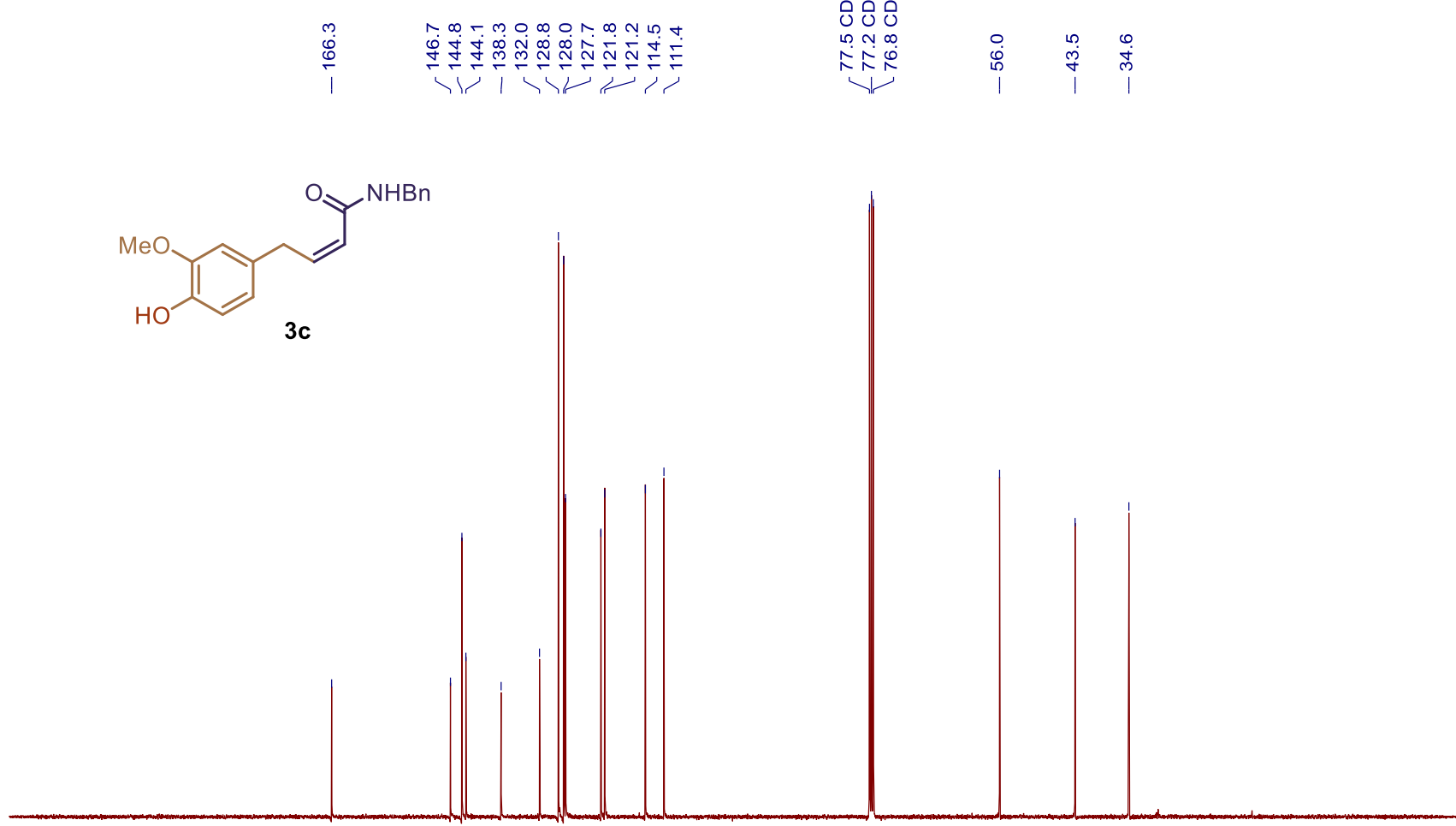

$\begin{array}{llllllllllllllllllllllllllll} & 210 & 200 & 190 & 180 & 170 & 160 & 150 & 140 & 130 & 120 & 110 & 100 & 90 & 80 & 70 & 60 & 50 & 40 & 30 & 20 & 10 & 0 & -10\end{array}$ 
Figure $\mathbf{S 4 9} \mid{ }^{1} \mathrm{H}$ NMR (400 MHz, $\left.\mathrm{CDCl}_{3}\right)$ spectrum of 3d.

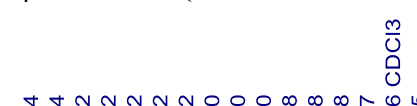

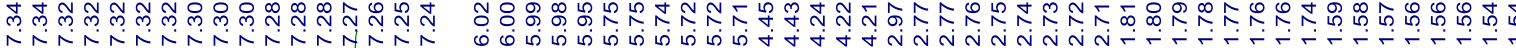<smiles>CS(=O)(=O)OCCCC/C=C\C(=O)Nc1ccccc1</smiles>

3d
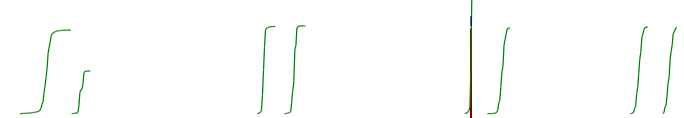

O’"O

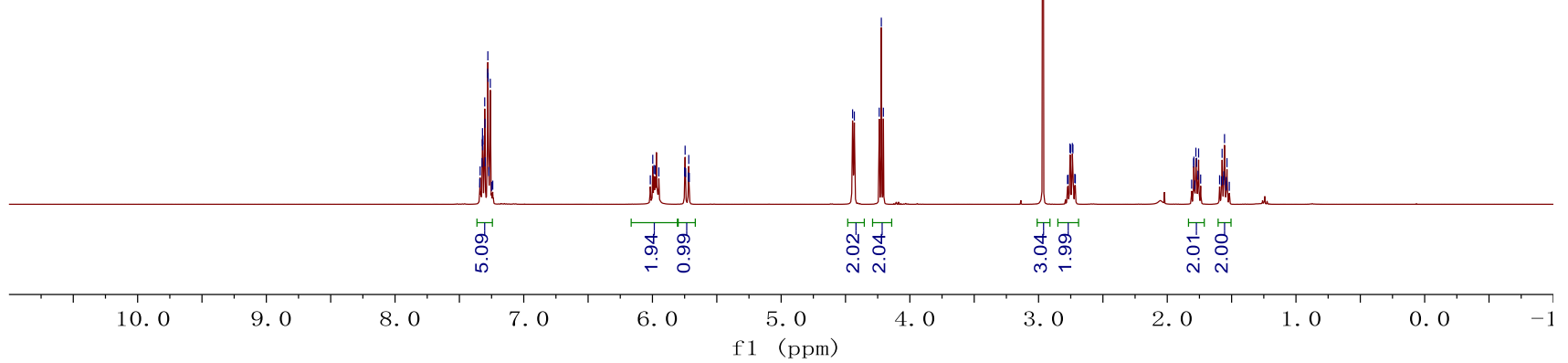

Figure S50 $\mid{ }^{13} \mathrm{C}$ NMR (101 MHz, $\left.\mathrm{CDCl}_{3}\right)$ spectrum of 3d.
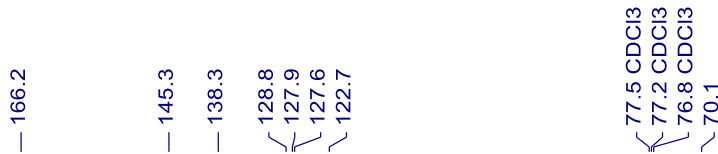

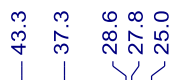<smiles>CS(=O)(=O)OCCCC/C=C\C(=O)NCc1ccccc1</smiles>

3d

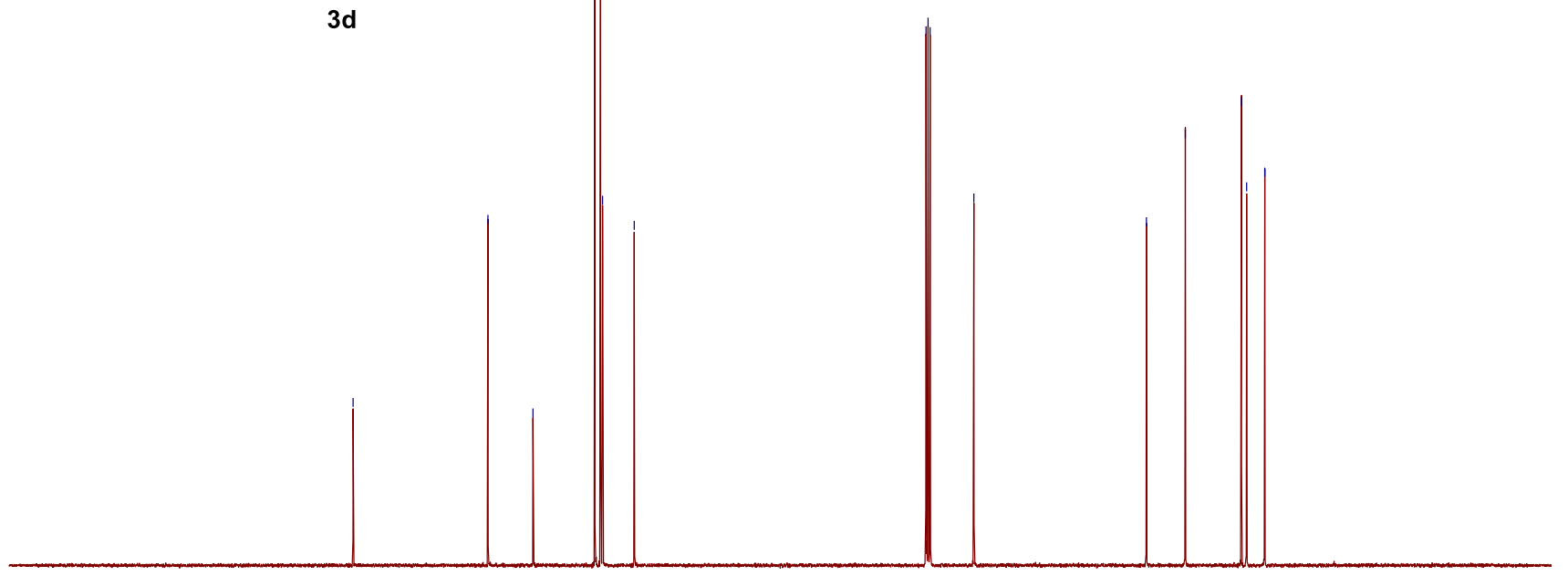

$\begin{array}{llllllllllll}210 & 200 & 190 & 180 & 170 & 160 & 150 & 140 & 130 & 120 & 110 & 100 \\ \text { f1 } & (\mathrm{pmm}) & \end{array}$ 
Figure S51 | ${ }^{1} \mathrm{H}$ NMR (400 MHz, $\left.\mathrm{CDCl}_{3}\right)$ spectrum of $3 \mathbf{e}$.

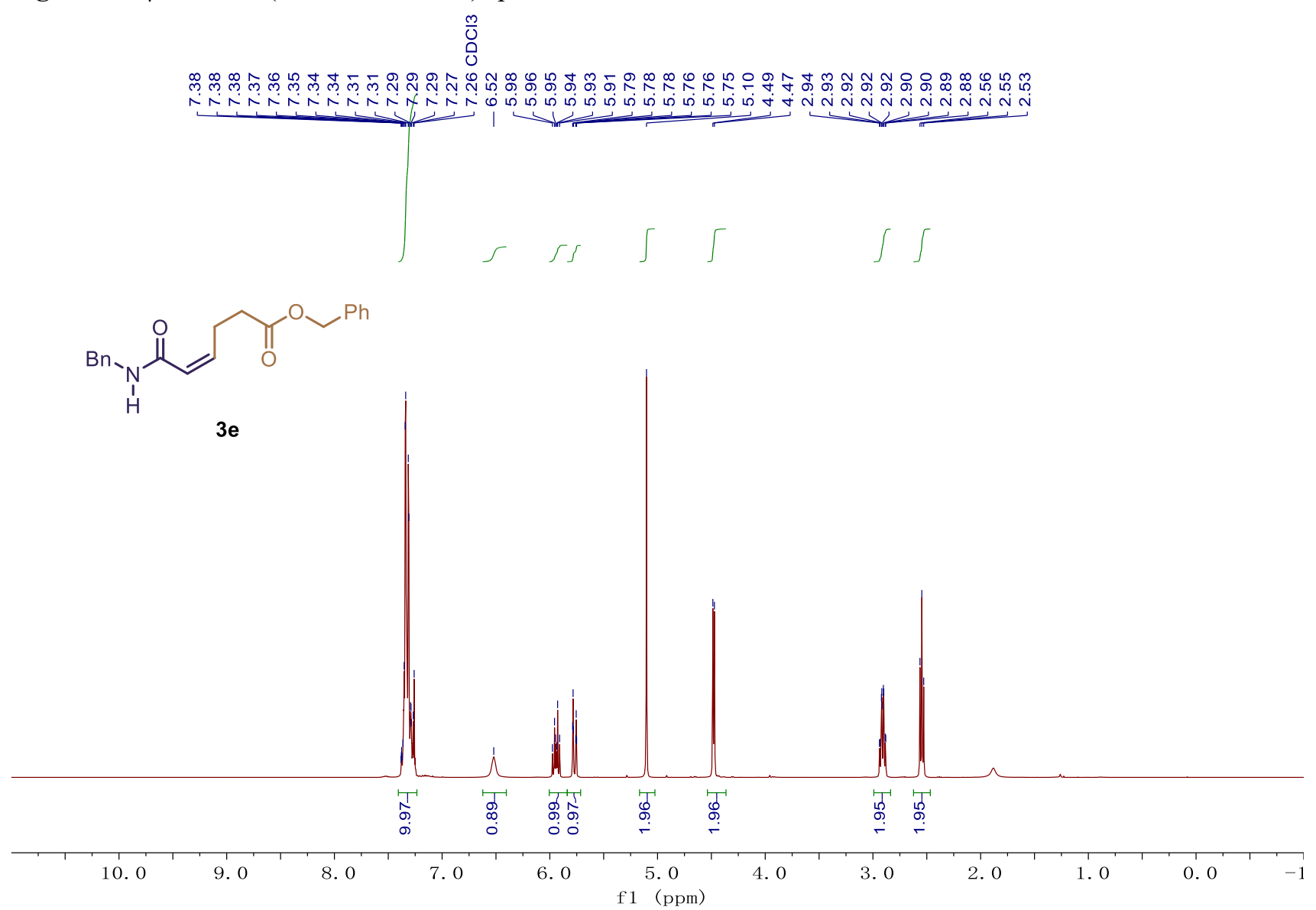

Figure S52 $\mid{ }^{13} \mathrm{C}$ NMR $\left(101 \mathrm{MHz}, \mathrm{CDCl}_{3}\right)$ spectrum of $\mathbf{3 e}$.

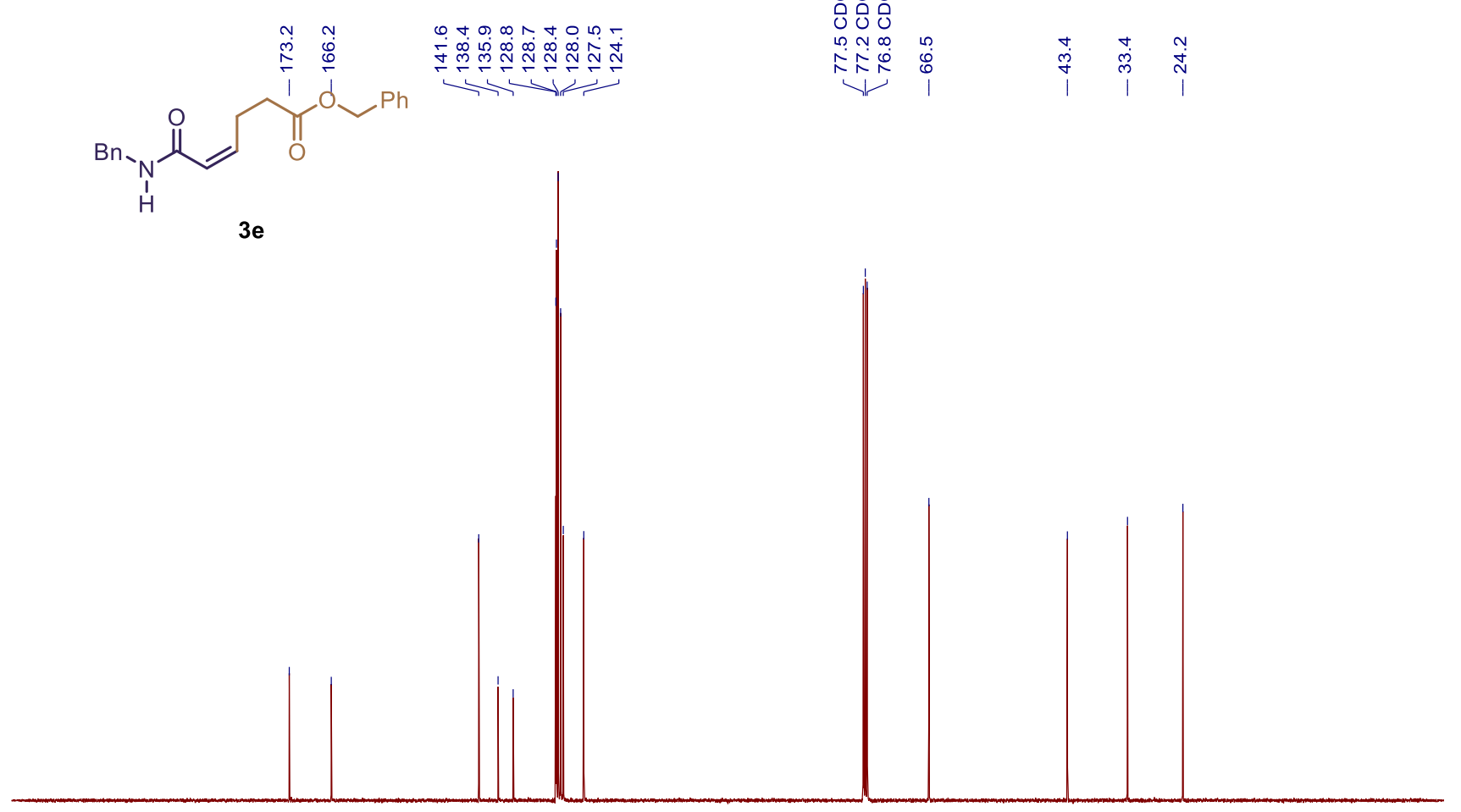

$\begin{array}{llllllllllll}210 & 200 & 190 & 180 & 170 & 160 & 150 & 140 & 130 & 120 & 110 & 100 \\ \text { f1 } & (\mathrm{ppm})\end{array}$ 
Figure S53 $\mid{ }^{1} \mathrm{H}$ NMR (400 MHz, $\left.\mathrm{CDCl}_{3}\right)$ spectrum of $\mathbf{3 f}$.

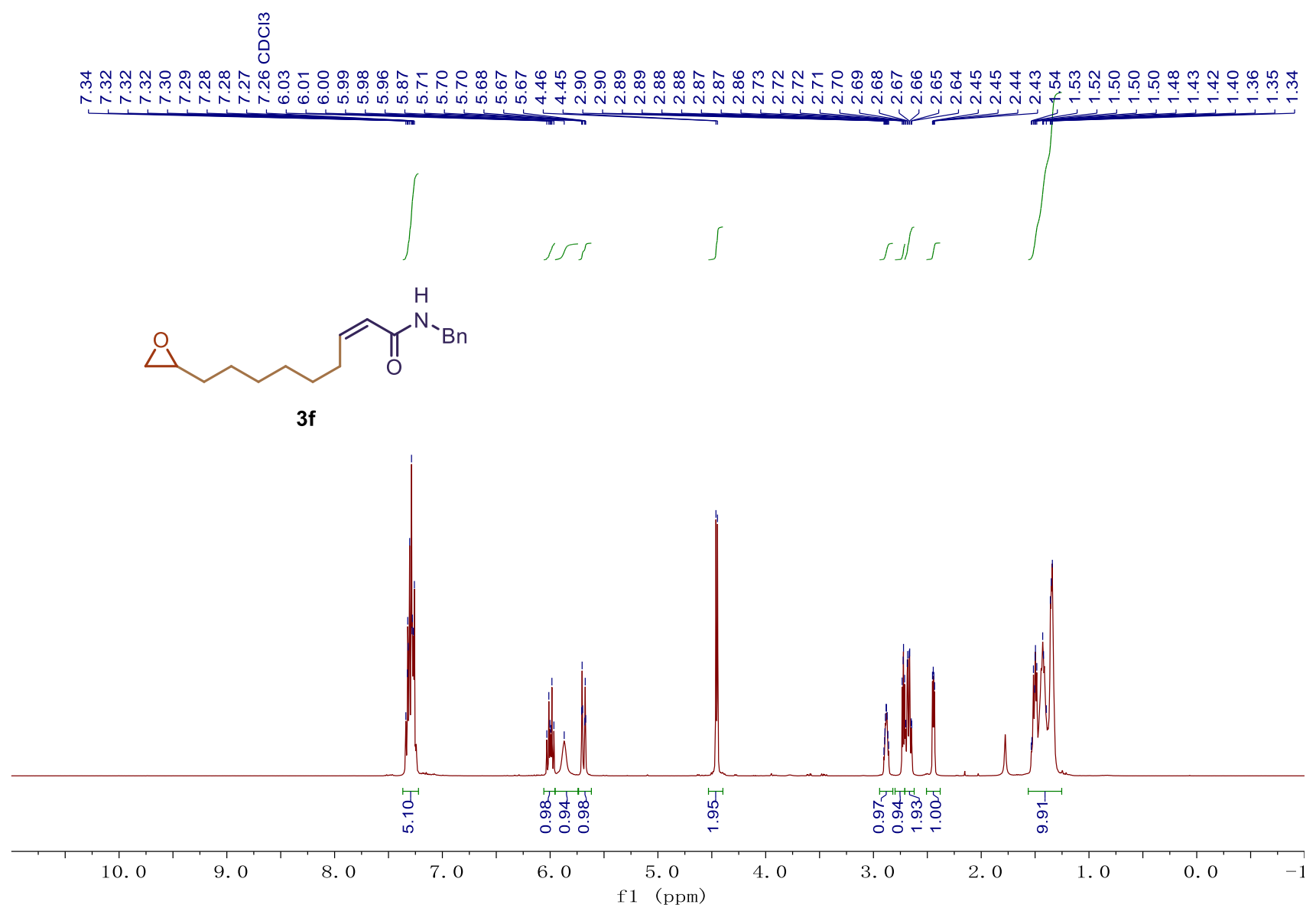

Figure S54 $\mid{ }^{13} \mathrm{C}$ NMR $\left(101 \mathrm{MHz}, \mathrm{CDCl}_{3}\right)$ spectrum of $\mathbf{3 f}$.
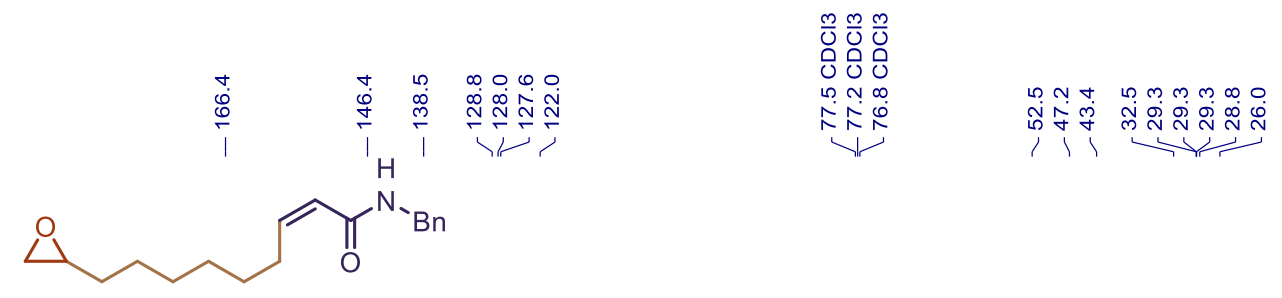

$3 f$

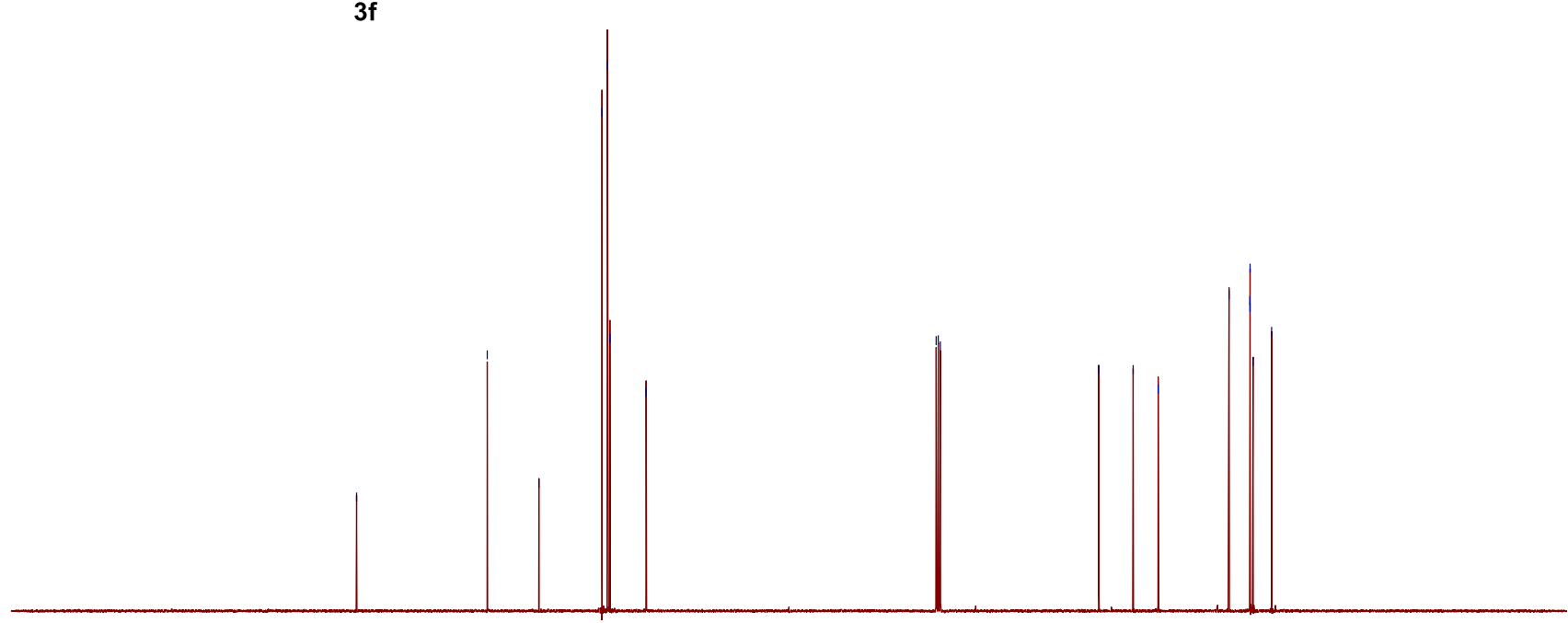

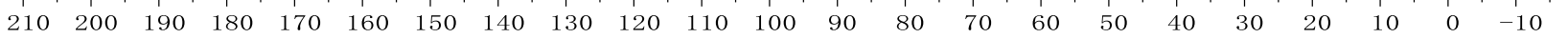


Figure S55 | ${ }^{1} \mathrm{H}$ NMR (400 MHz, $\left.\mathrm{CDCl}_{3}\right)$ spectrum of $\mathbf{3 g}$.

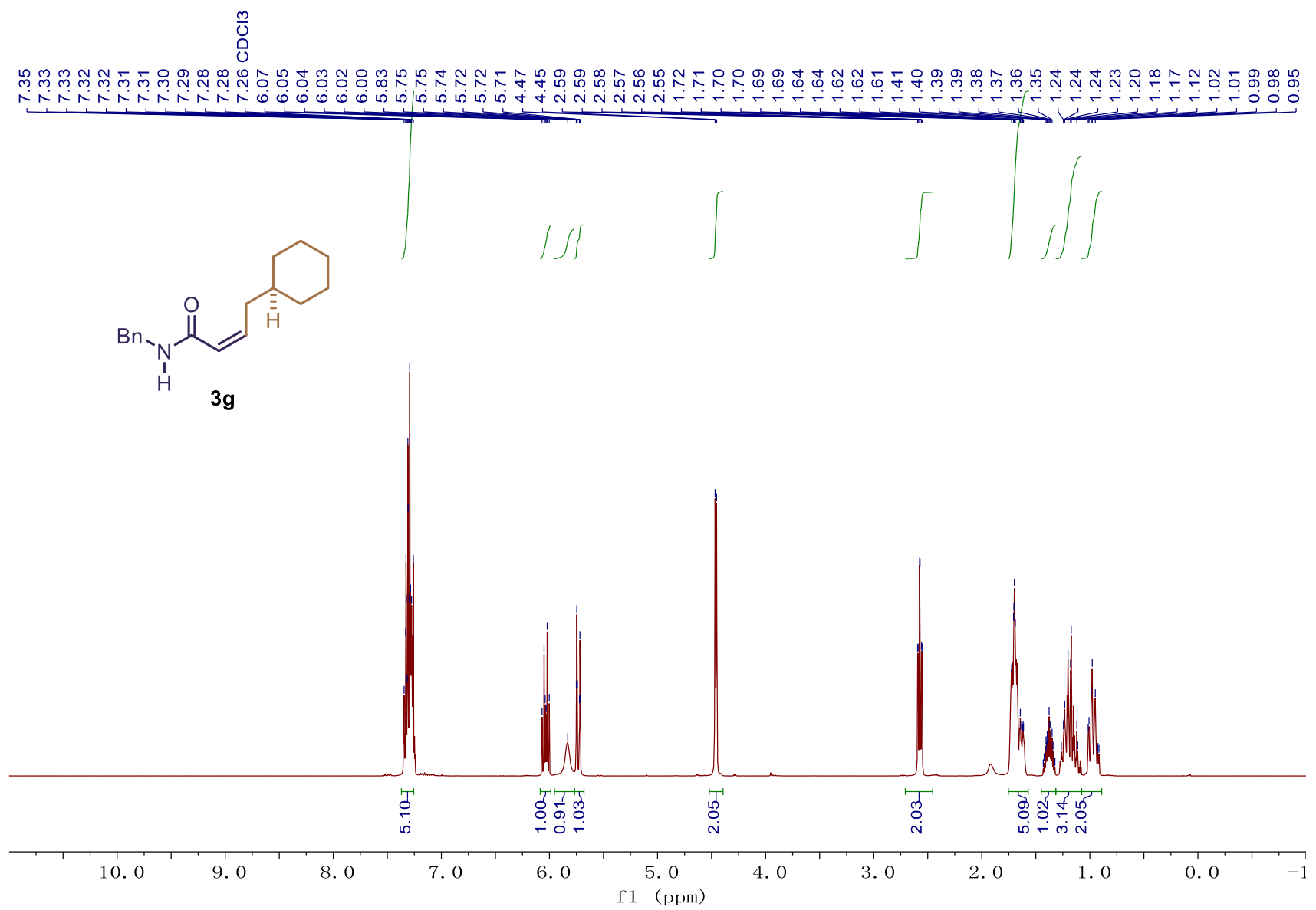

Figure S56 $\mid{ }^{13} \mathrm{C}$ NMR $\left(101 \mathrm{MHz}, \mathrm{CDCl}_{3}\right)$ spectrum of $\mathbf{3 g}$.

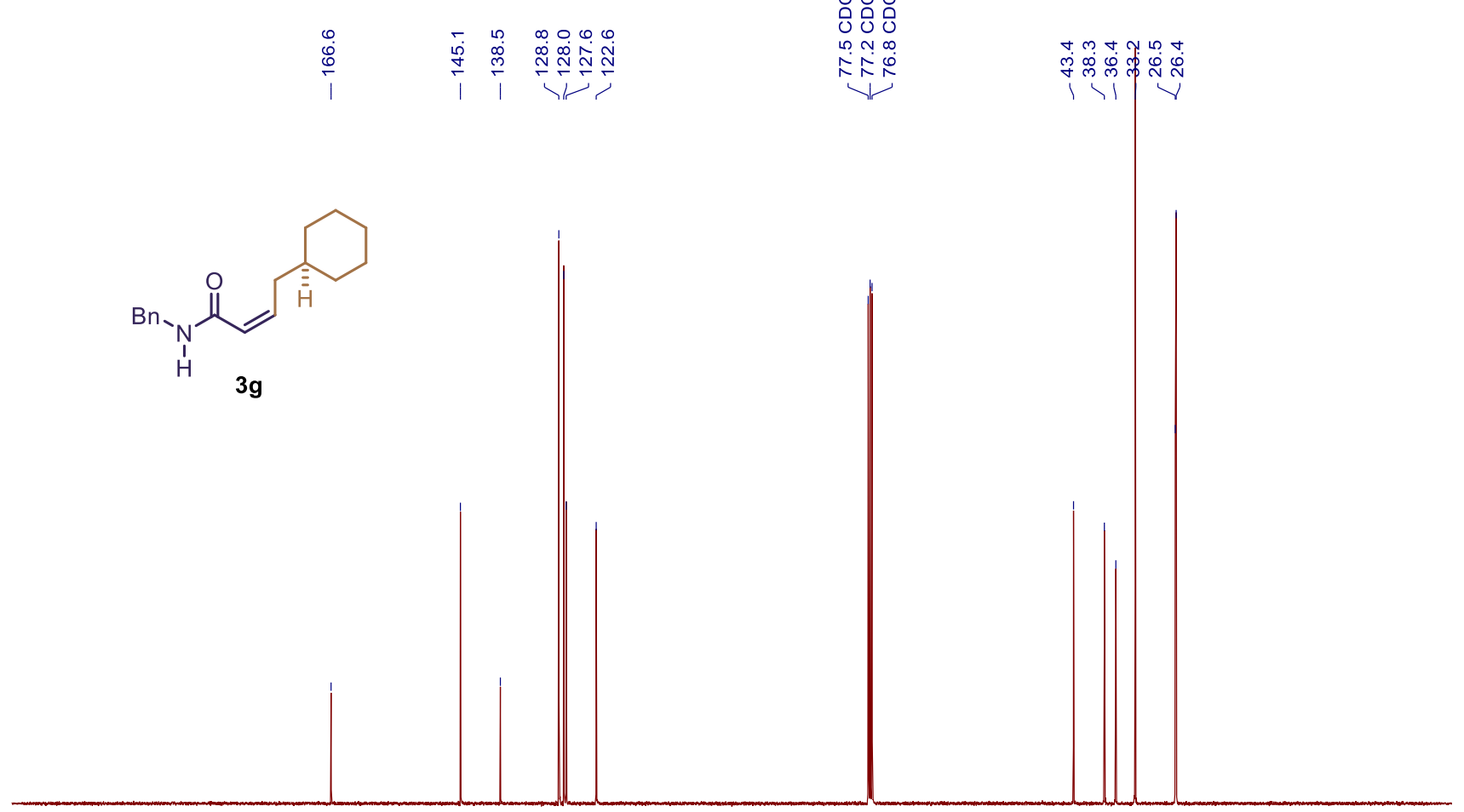

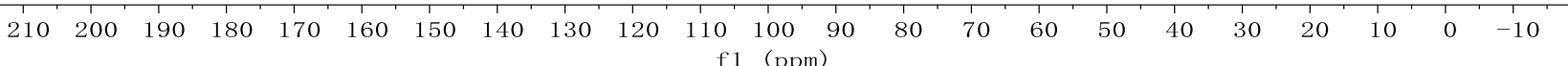


Figure S57 | ${ }^{1} \mathrm{H}$ NMR (400 MHz, $\left.\mathrm{C}_{6} \mathrm{D}_{6}\right)$ spectrum of $\mathbf{3 h}$.

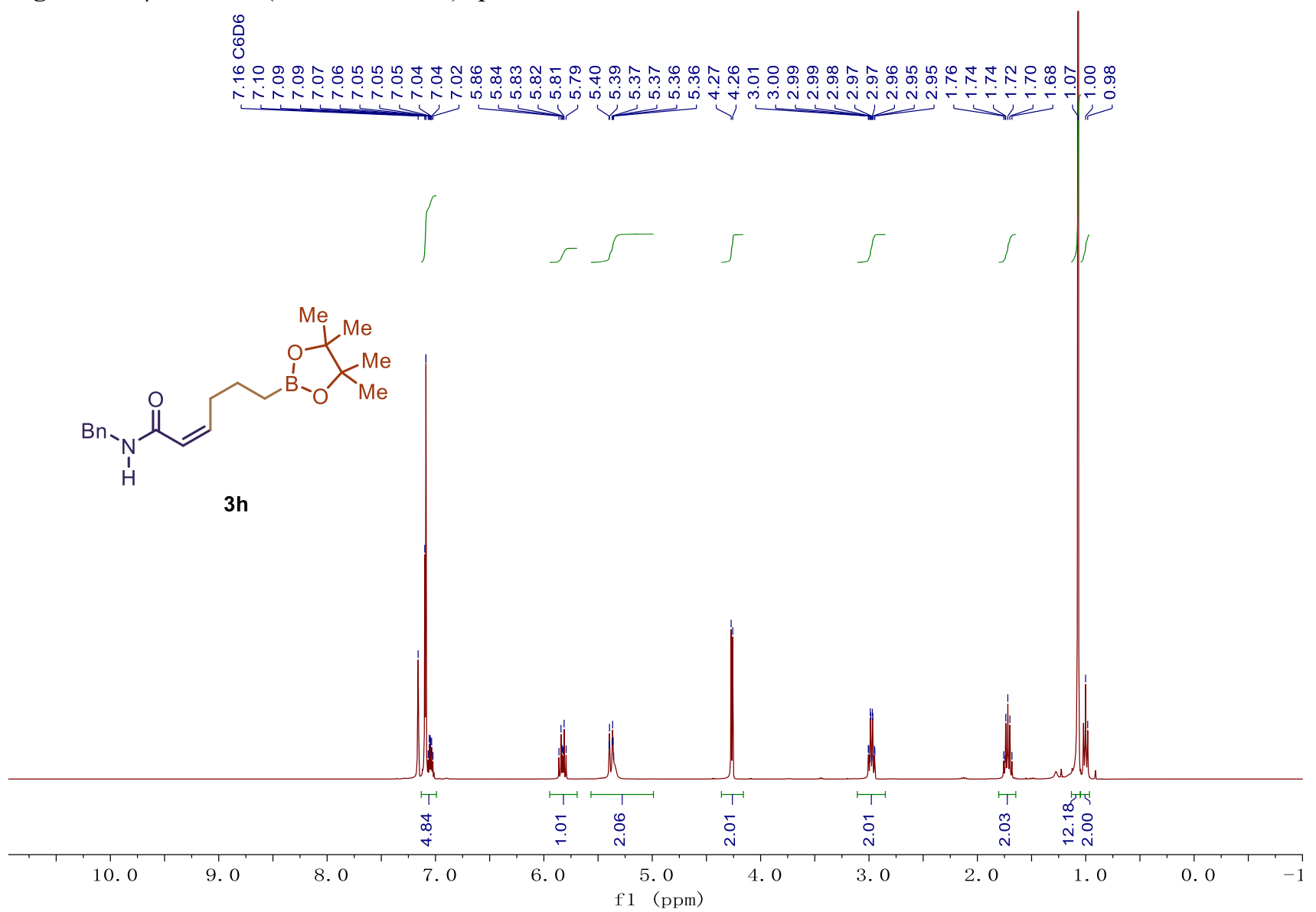

Figure S58 $\mid{ }^{13} \mathrm{C}$ NMR $\left(101 \mathrm{MHz}, \mathrm{C}_{6} \mathrm{D}_{6}\right)$ spectrum of $\mathbf{3 h}$.

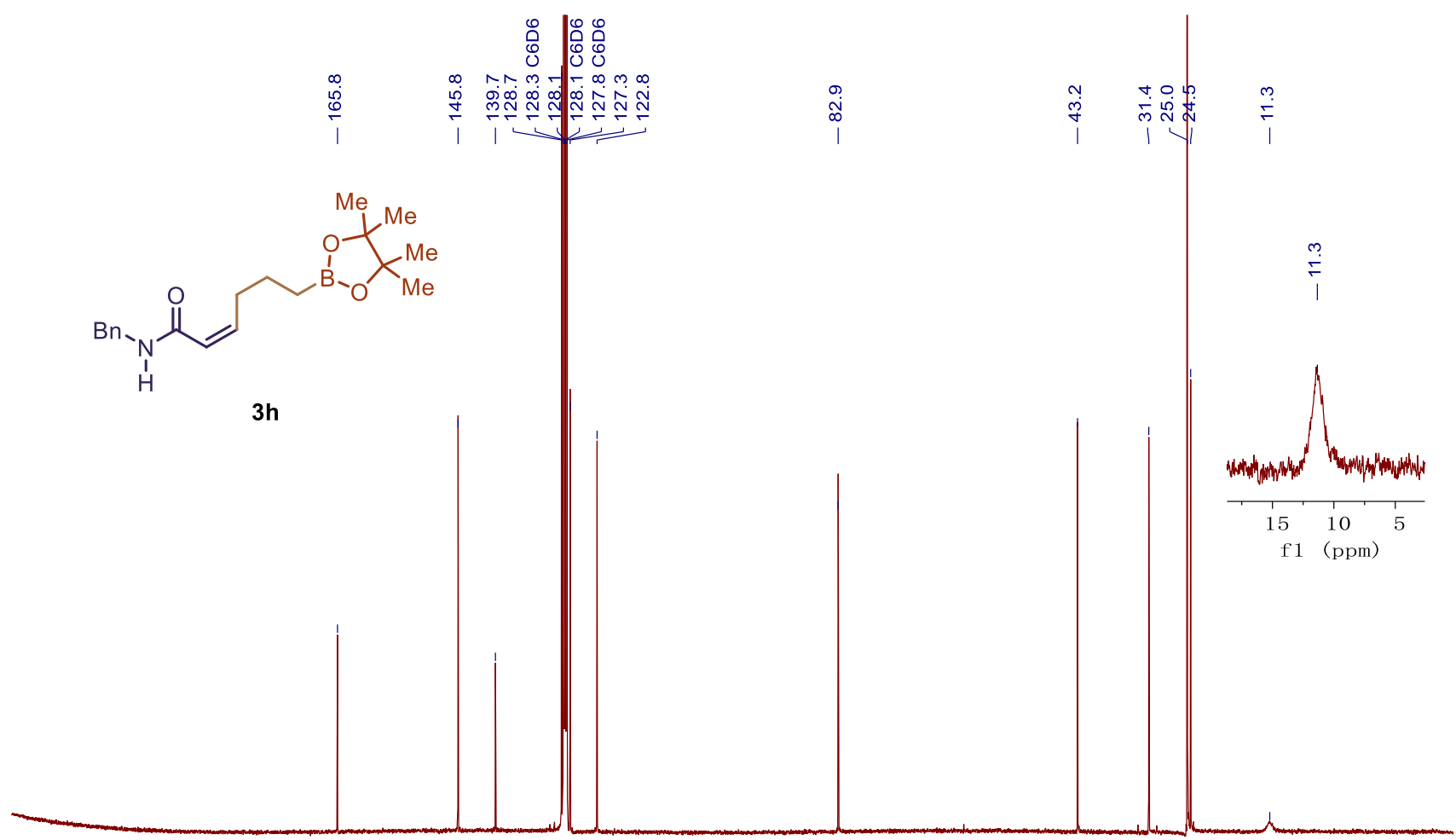

$\begin{array}{rlllllllllllllllllllllll}210 & 200 & 190 & 180 & 170 & 160 & 150 & 140 & 130 & 120 & 110 & 100 & 90 & 80 & 70 & 60 & 50 & 40 & 30 & 20 & 10 & 0 & -10\end{array}$ 
Figure S59 $\mid{ }^{1} \mathrm{H}$ NMR (400 MHz, $\left.\mathrm{CDCl}_{3}\right)$ spectrum of $\mathbf{3 i}$.

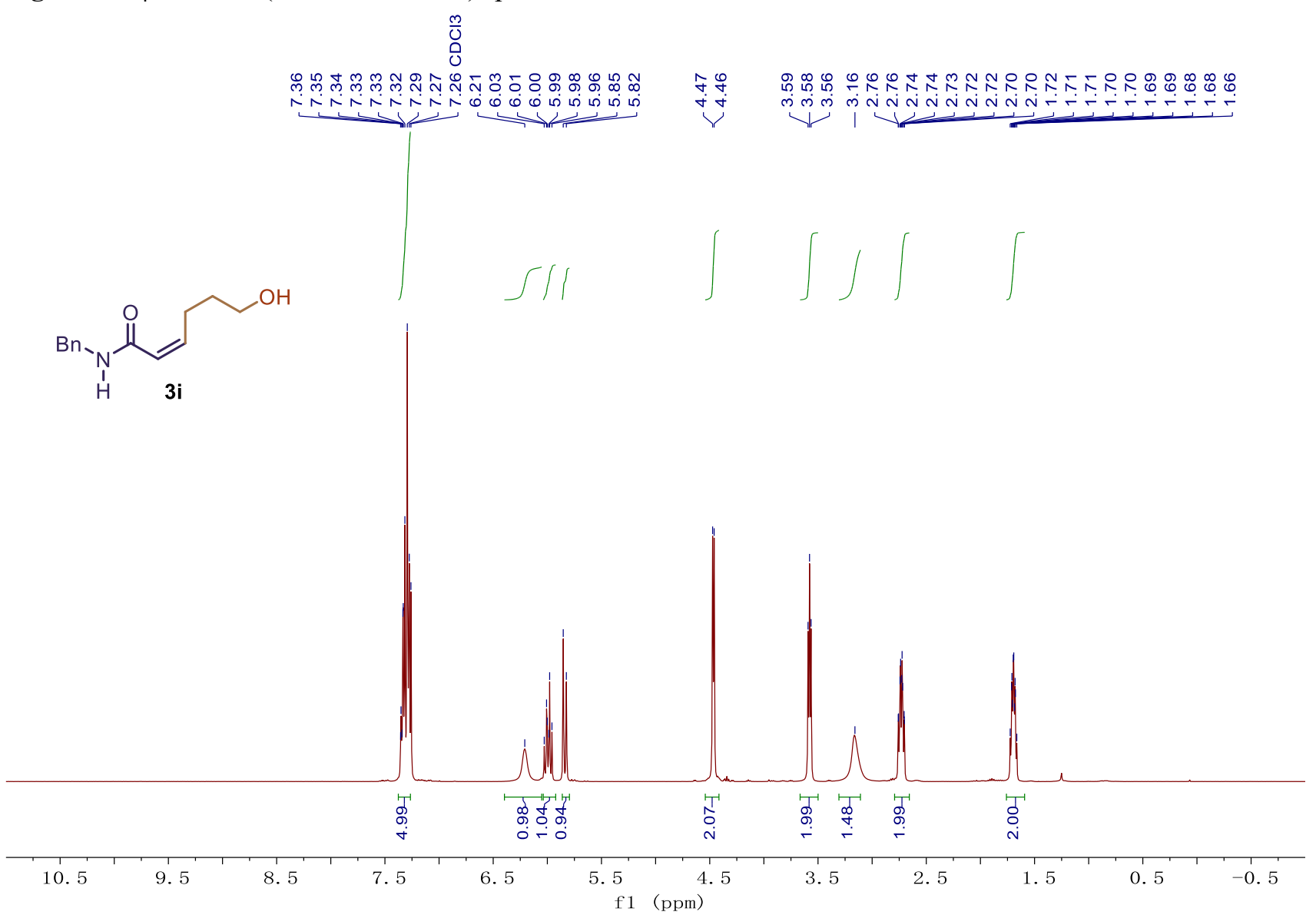

Figure S60 $\mid{ }^{13} \mathrm{C}$ NMR $\left(101 \mathrm{MHz}, \mathrm{CDCl}_{3}\right)$ spectrum of $3 \mathbf{i}$.

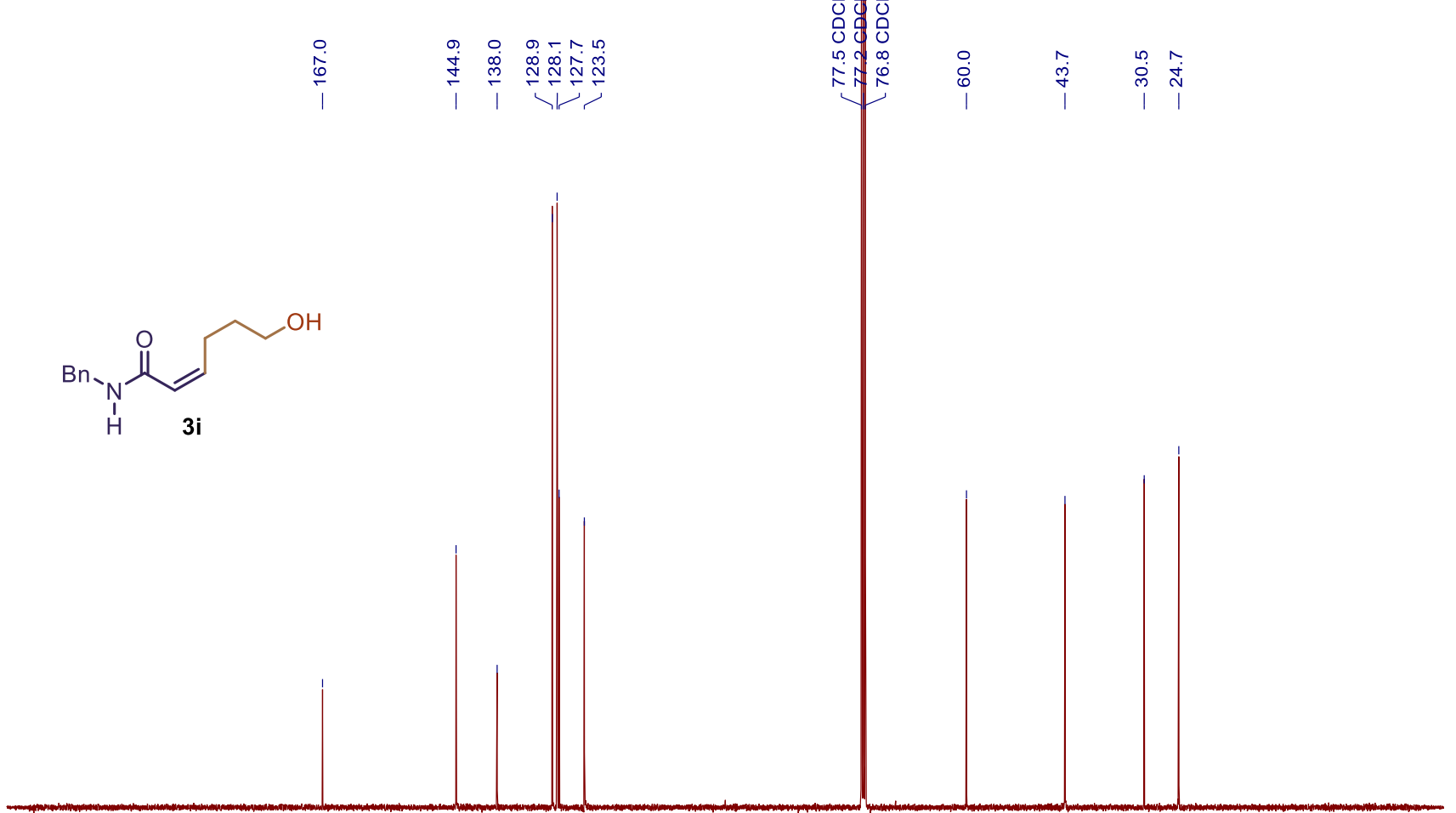

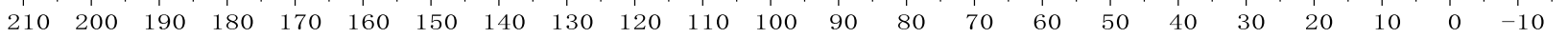


Figure S61 | ${ }^{1} \mathrm{H}$ NMR (400 MHz, $\left.\mathrm{CDCl}_{3}\right)$ spectrum of $\mathbf{3 j}$.

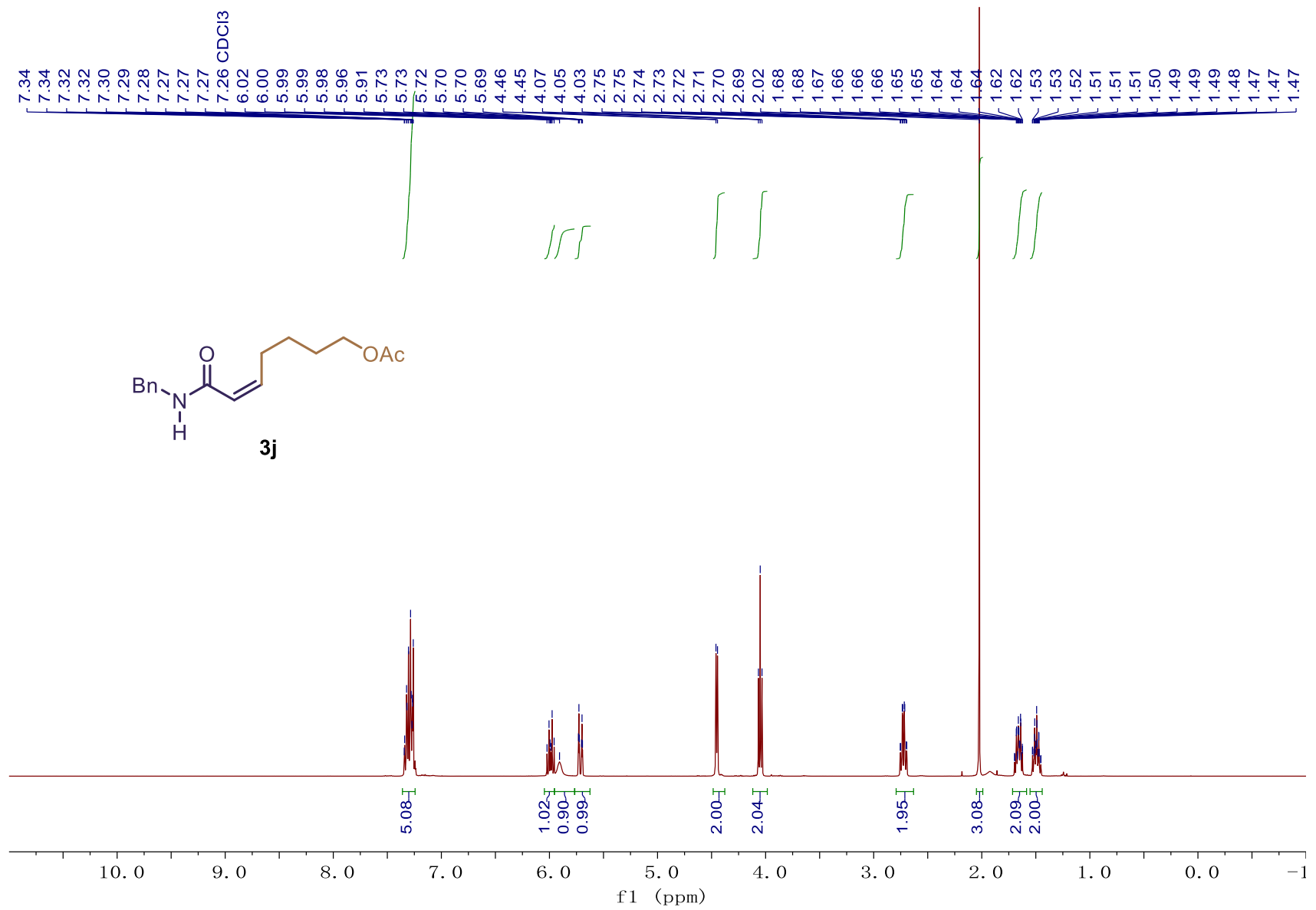

Figure S62 $\mid{ }^{13} \mathrm{C}$ NMR $\left(101 \mathrm{MHz}, \mathrm{CDCl}_{3}\right)$ spectrum of $\mathbf{3 j}$.

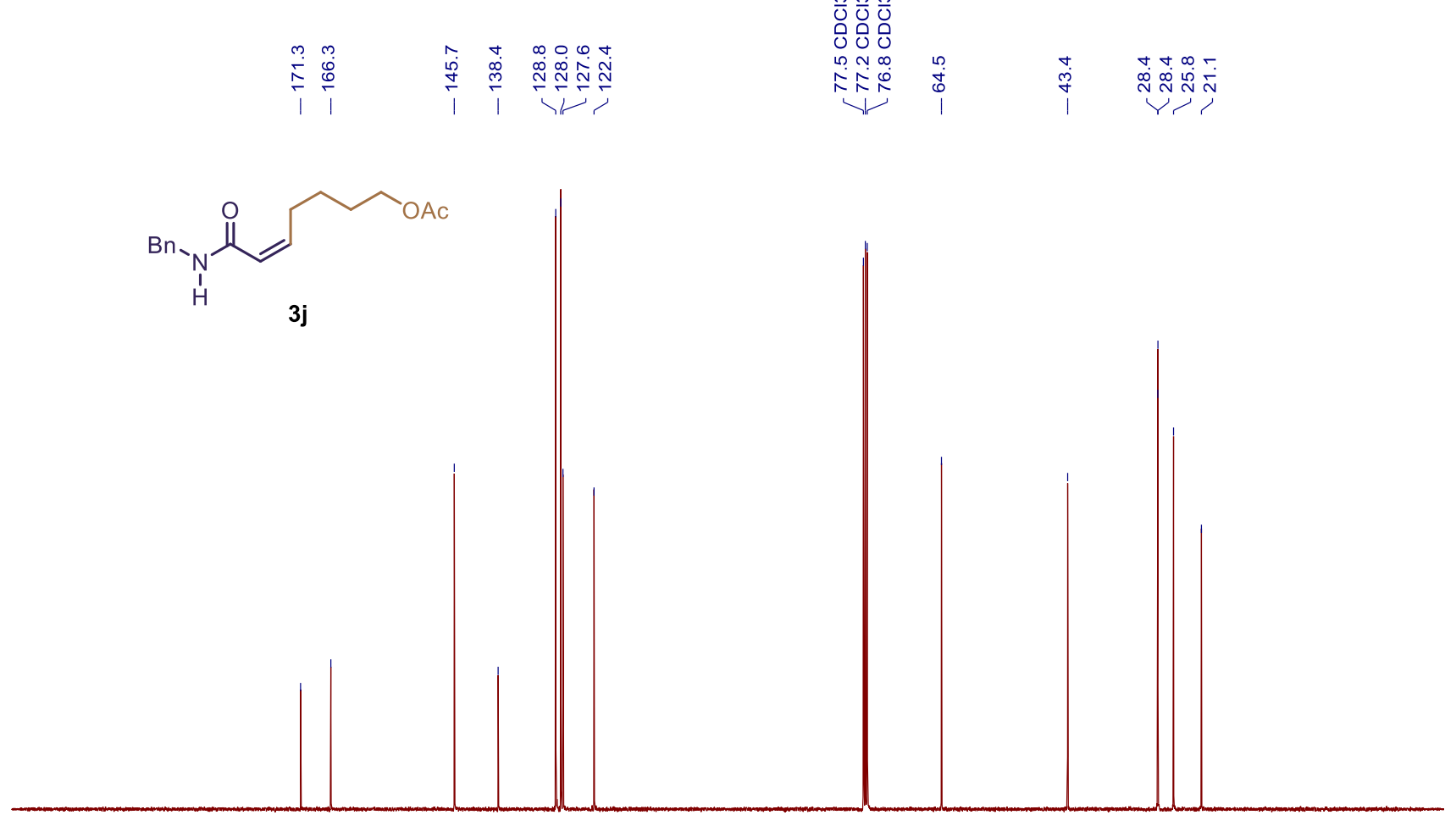

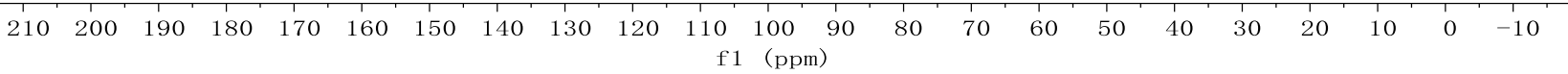


Figure S63 $\mid{ }^{1} \mathrm{H}$ NMR $\left(400 \mathrm{MHz}, \mathrm{CDCl}_{3}\right)$ spectrum of $\mathbf{3 k}$.

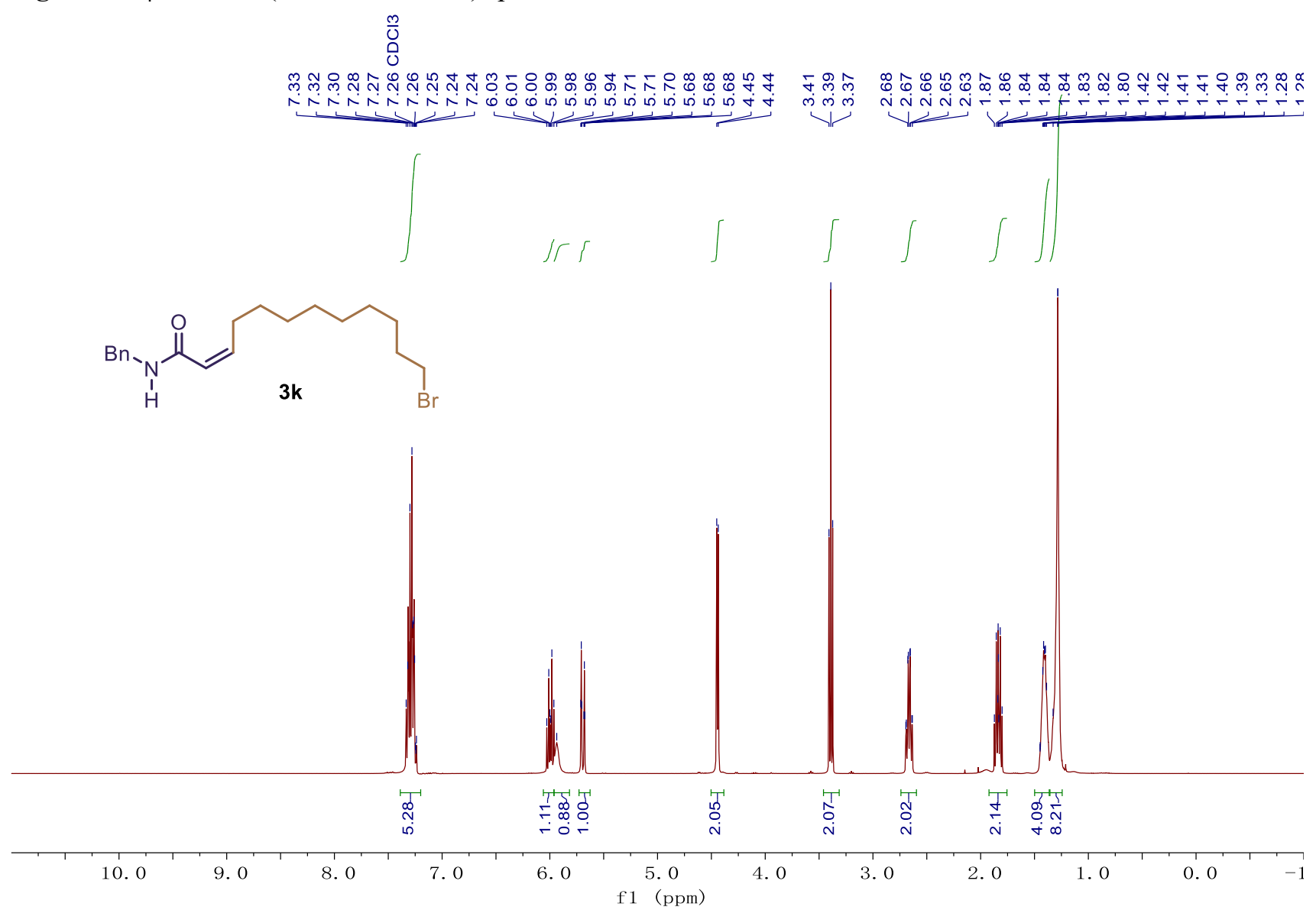

Figure S64 | ${ }^{13} \mathrm{C}$ NMR (101 MHz, $\left.\mathrm{CDCl}_{3}\right)$ spectrum of $\mathbf{3 k}$.<smiles>O=C(/C=C\CCCCCCCCBr)NCc1ccccc1</smiles>

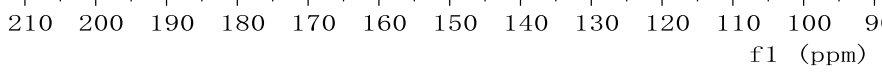

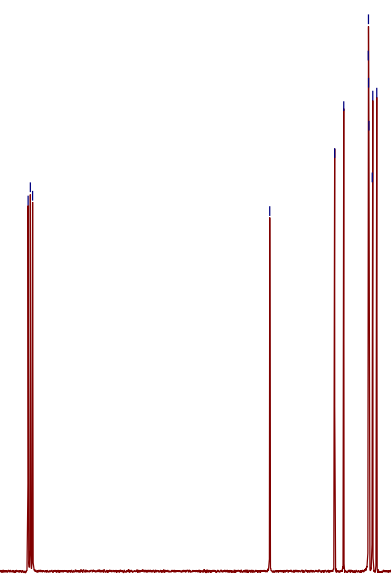


Figure $\mathbf{S 6 5} \mid{ }^{1} \mathrm{H}$ NMR (400 MHz, $\left.\mathrm{CDCl}_{3}\right)$ spectrum of 31 .

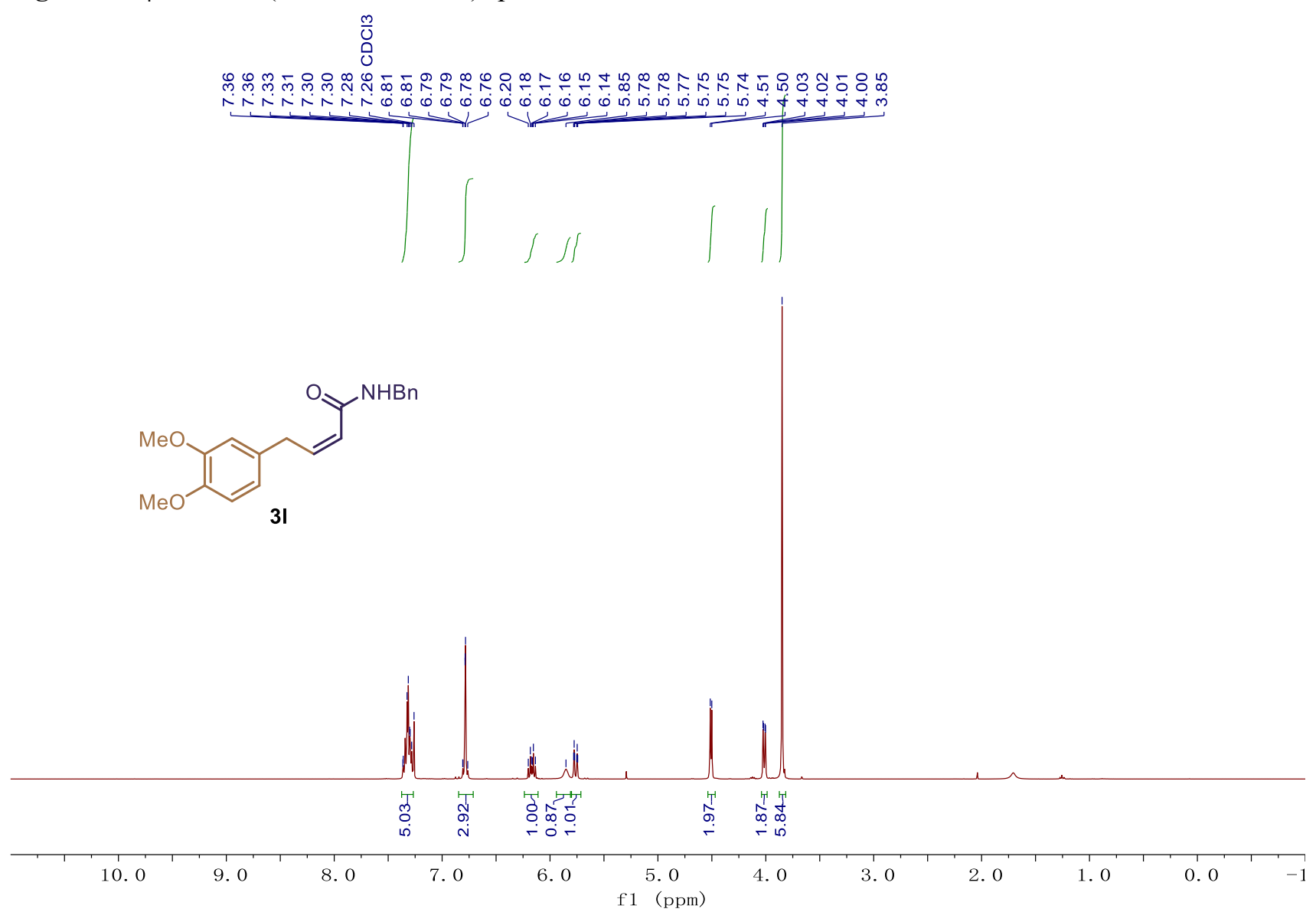

Figure S66 $\mid{ }^{13} \mathrm{C}$ NMR $\left(101 \mathrm{MHz}, \mathrm{CDCl}_{3}\right)$ spectrum of $\mathbf{3 l}$.

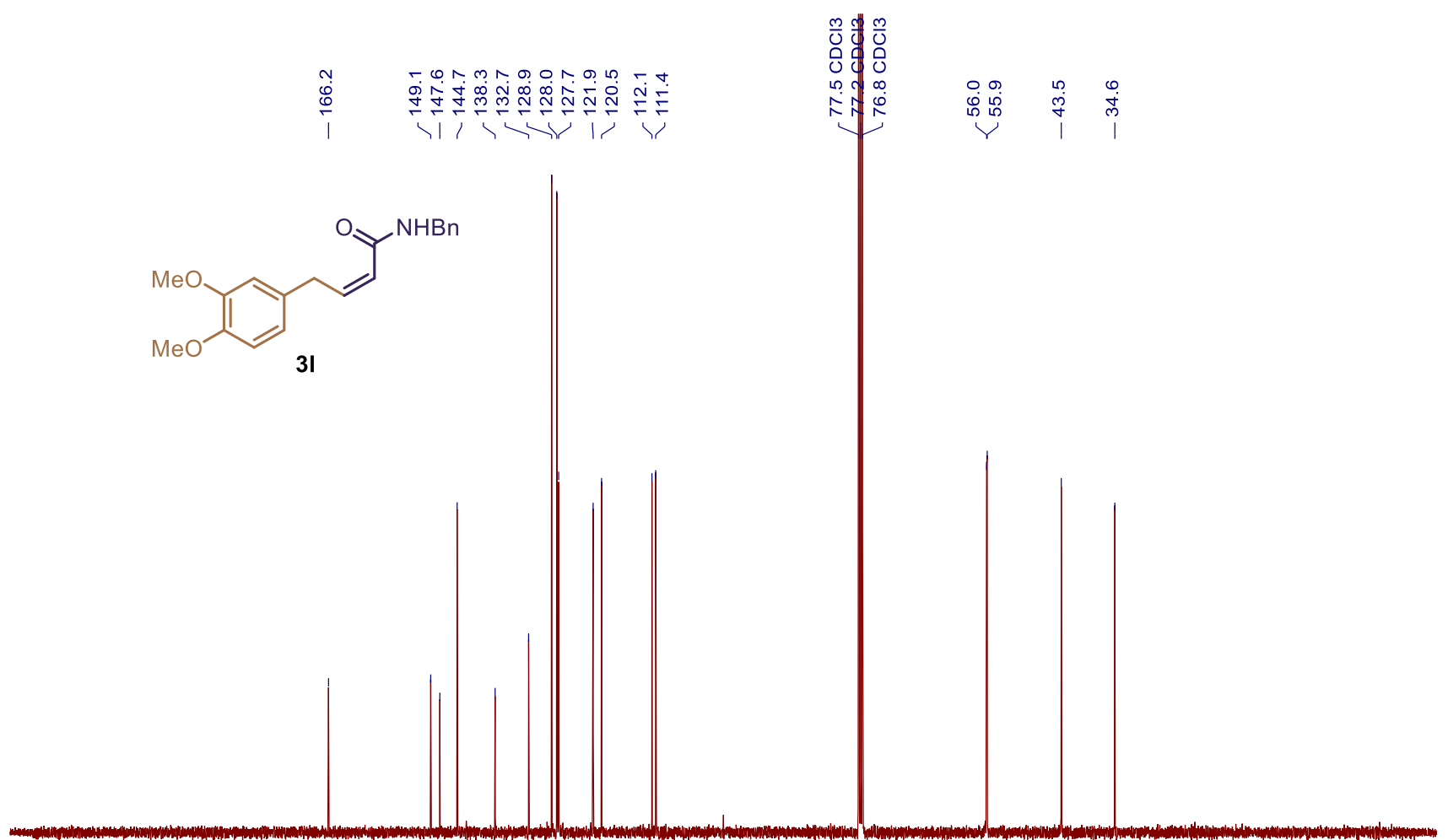

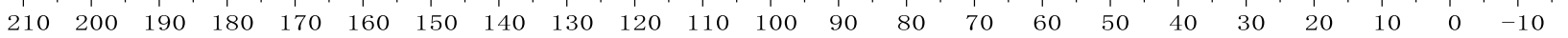


Figure $\mathbf{S 6 7} \mid{ }^{1} \mathrm{H}$ NMR (400 MHz, $\left.\mathrm{CDCl}_{3}\right)$ spectrum of $\mathbf{3 m}$.

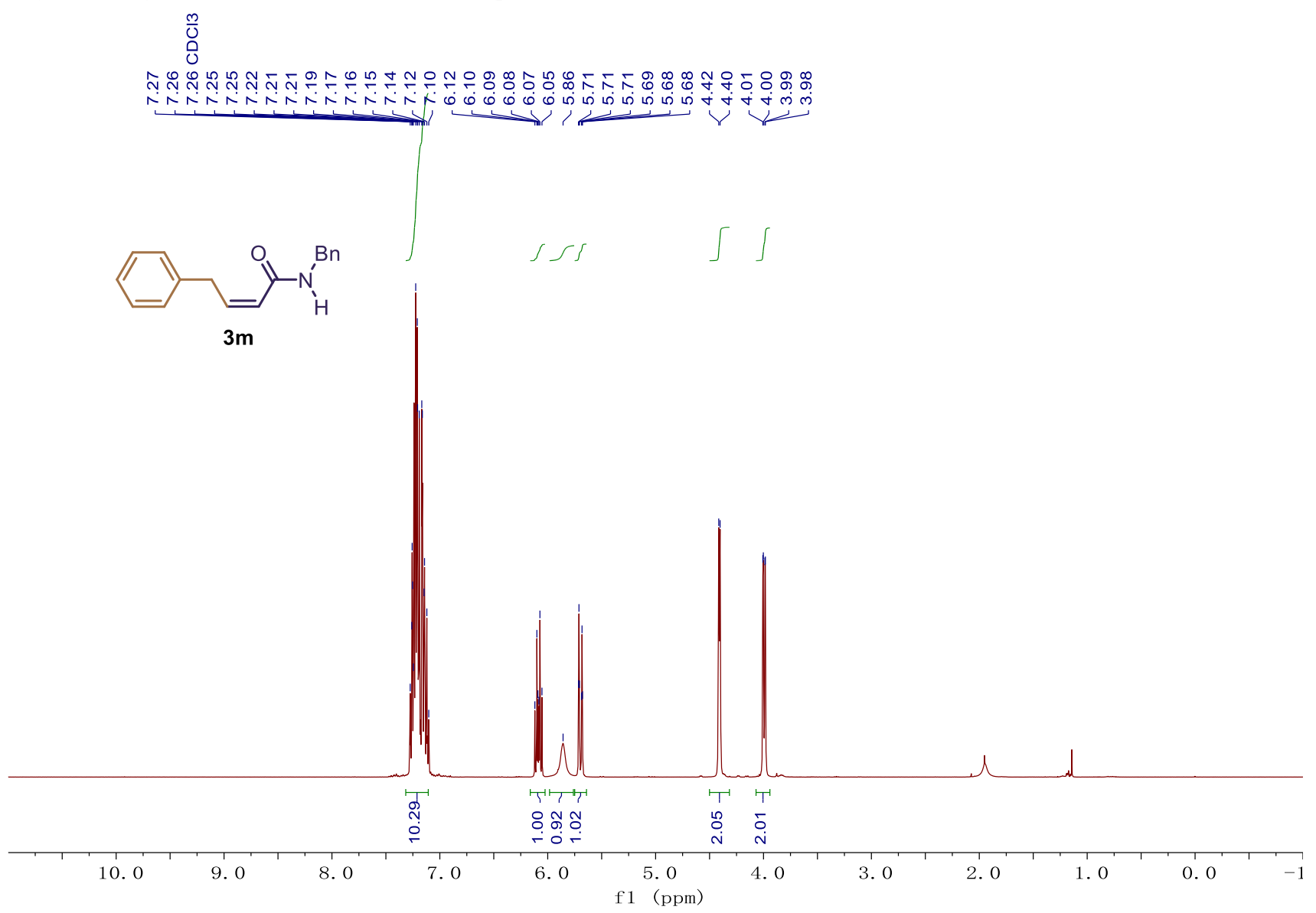

Figure S68 $\mid{ }^{13} \mathrm{C}$ NMR $\left(101 \mathrm{MHz}, \mathrm{CDCl}_{3}\right)$ spectrum of $\mathbf{3 m}$.
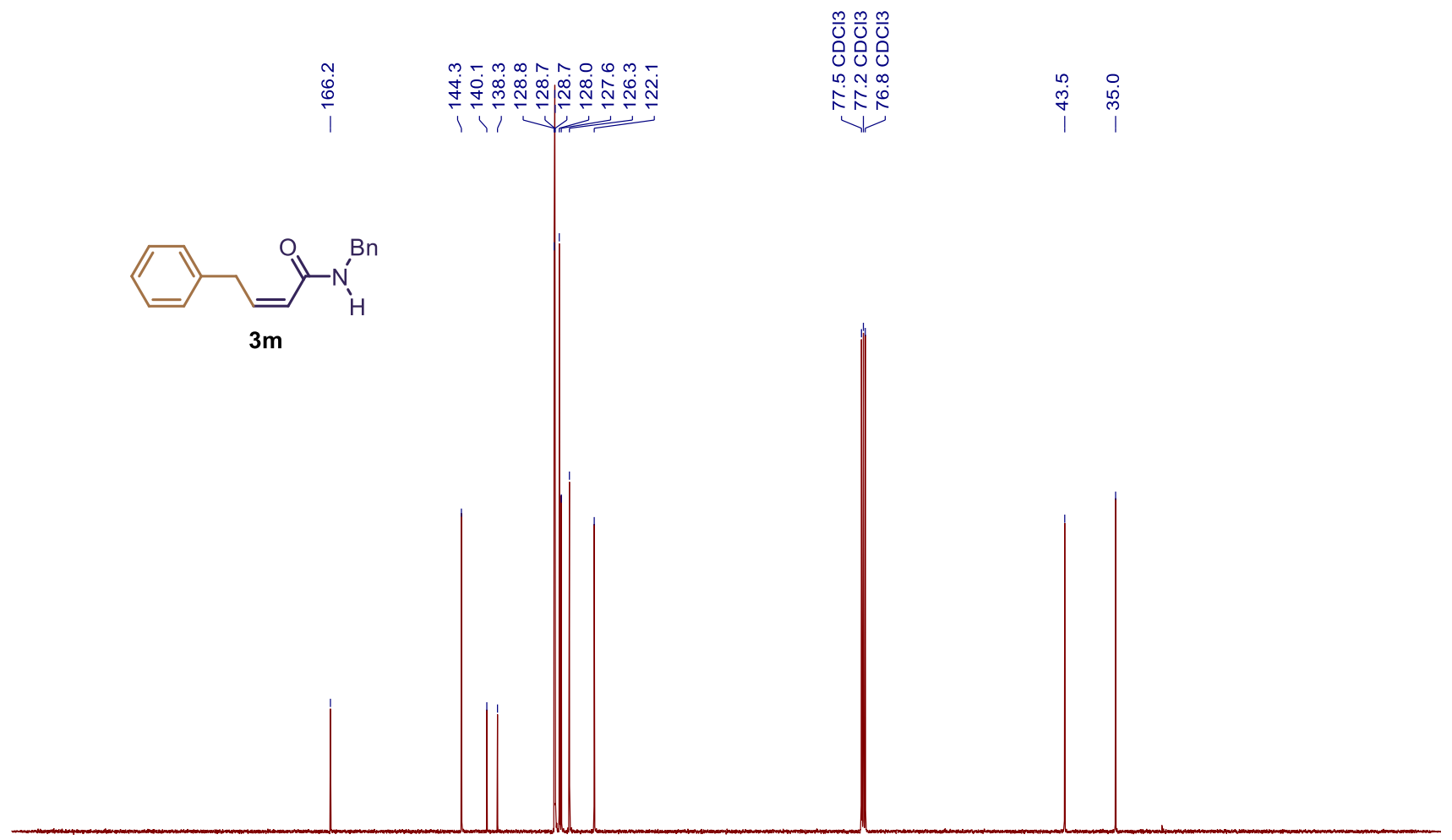

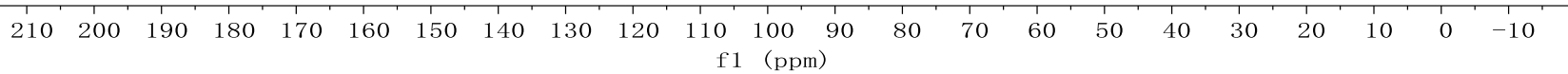


Figure S69 | ${ }^{1} \mathrm{H}$ NMR (400 MHz, $\left.\mathrm{CDCl}_{3}\right)$ spectrum of $\mathbf{3 n}$.

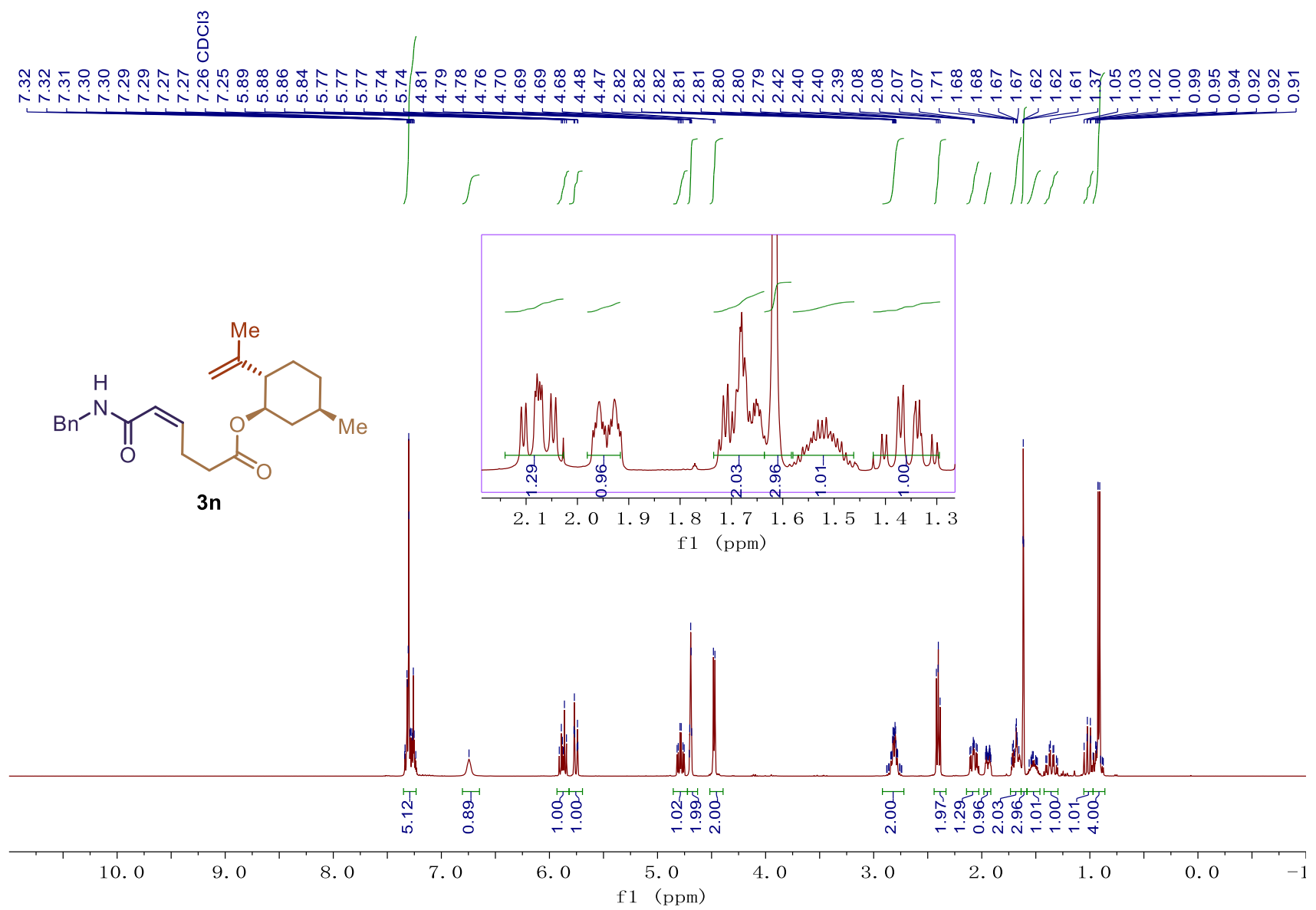

Figure S70 $\mid{ }^{13} \mathrm{C}$ NMR $\left(101 \mathrm{MHz}, \mathrm{CDCl}_{3}\right)$ spectrum of $\mathbf{3 n}$.

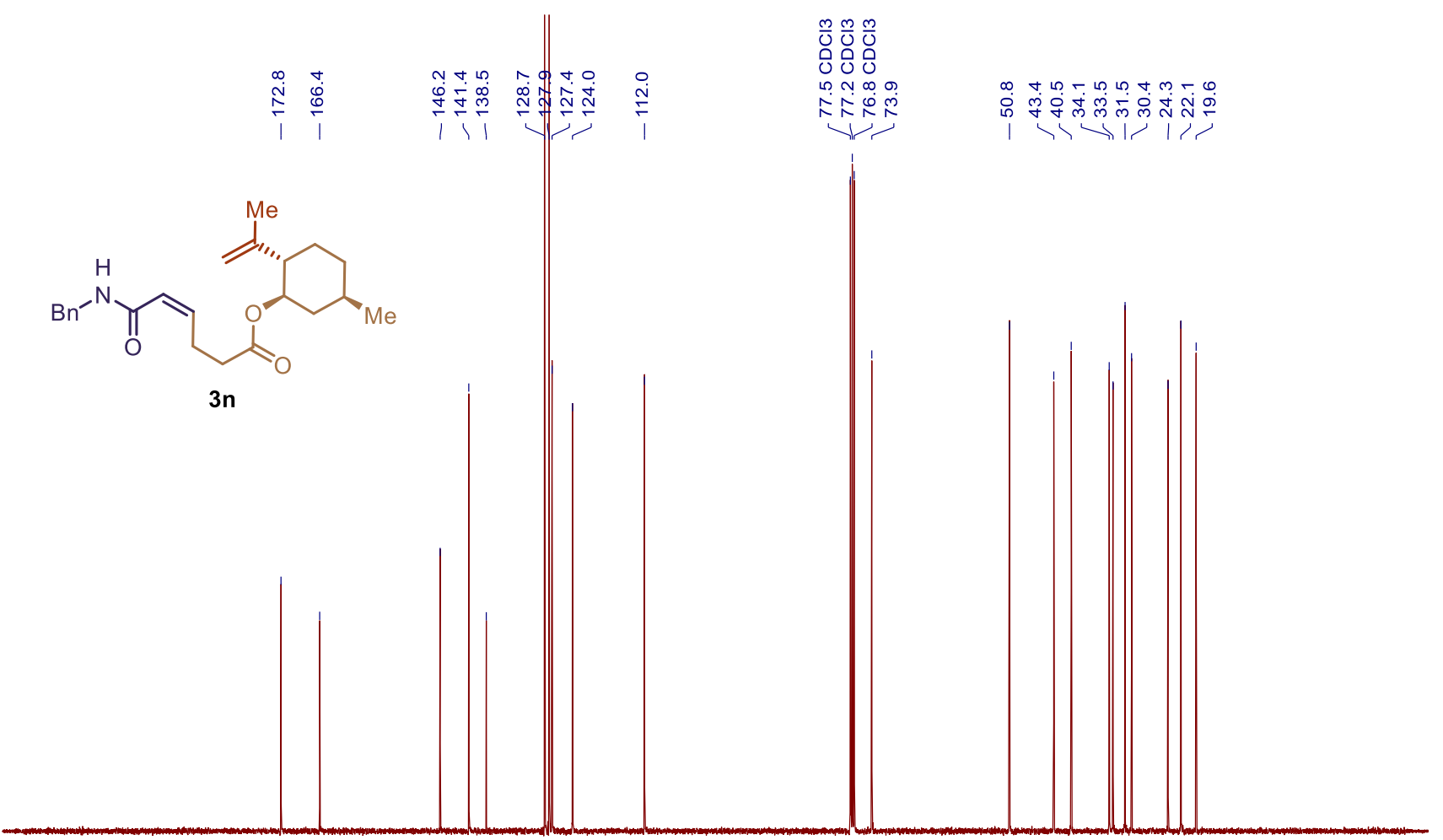

$\begin{array}{rllllllllllllllllllllllllllllllll}210 & 200 & 190 & 180 & 170 & 160 & 150 & 140 & 130 & 120 & 110 & 100 & 90 & 80 & 70 & 60 & 50 & 40 & 30 & 20 & 10 & 0 & -10\end{array}$ 
Figure S71 | ${ }^{1} \mathrm{H}$ NMR (400 MHz, $\left.\mathrm{CDCl}_{3}\right)$ spectrum of $3 \mathbf{3 o}$.

$\frac{m}{0}$

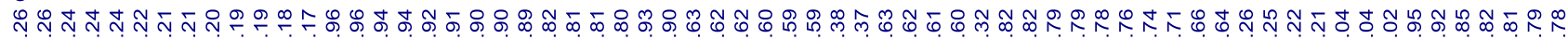

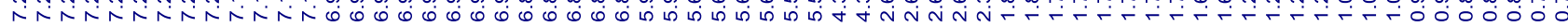
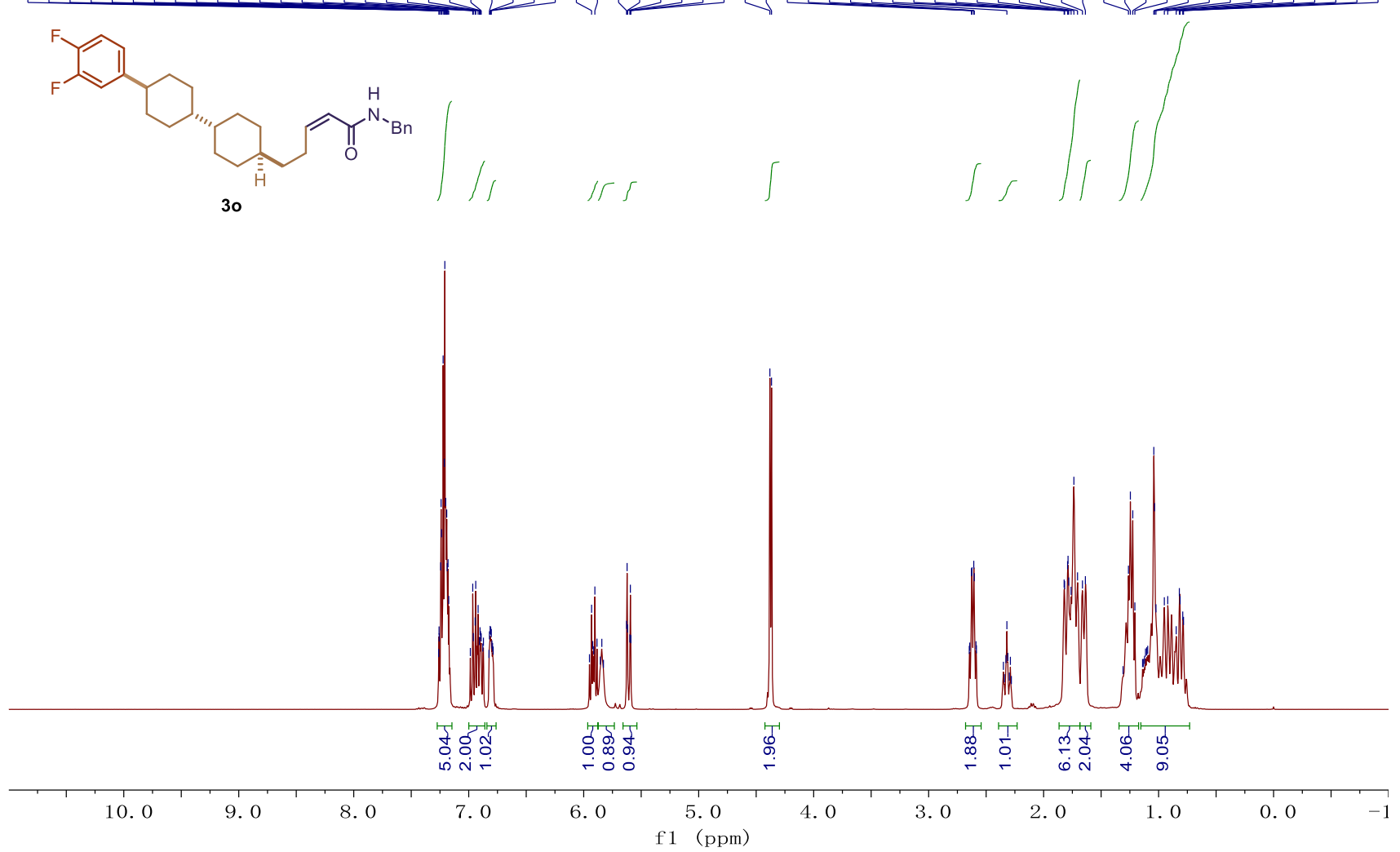

Figure S72 $\mid{ }^{13} \mathrm{C}$ NMR $\left(101 \mathrm{MHz}, \mathrm{CDCl}_{3}\right)$ spectrum of $\mathbf{3 o}$.

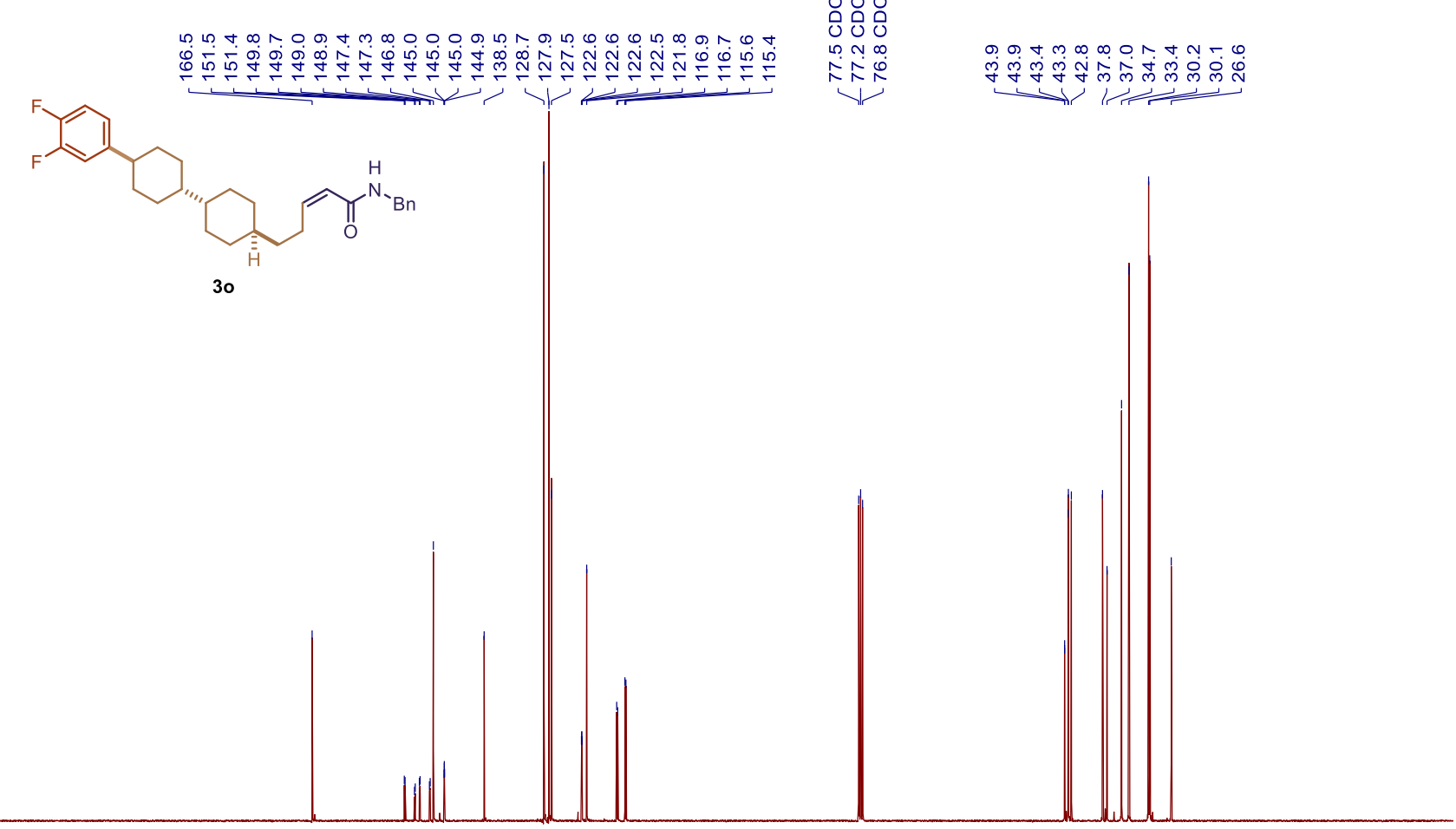

$\begin{array}{lllllllllllllllllllllll}210 & 200 & 190 & 180 & 170 & 160 & 150 & 140 & 130 & 120 & 110 & 100 & 90 & 80 & 70 & 60 & 50 & 40 & 30 & 20 & 10 & 0 & -10\end{array}$ 
Figure $\mathbf{S 7 3} \mid{ }^{19} \mathrm{~F}$ NMR (282 $\mathrm{MHz}, \mathrm{CDCl}_{3}$ ) spectrum of $3 \mathbf{3 o}$.

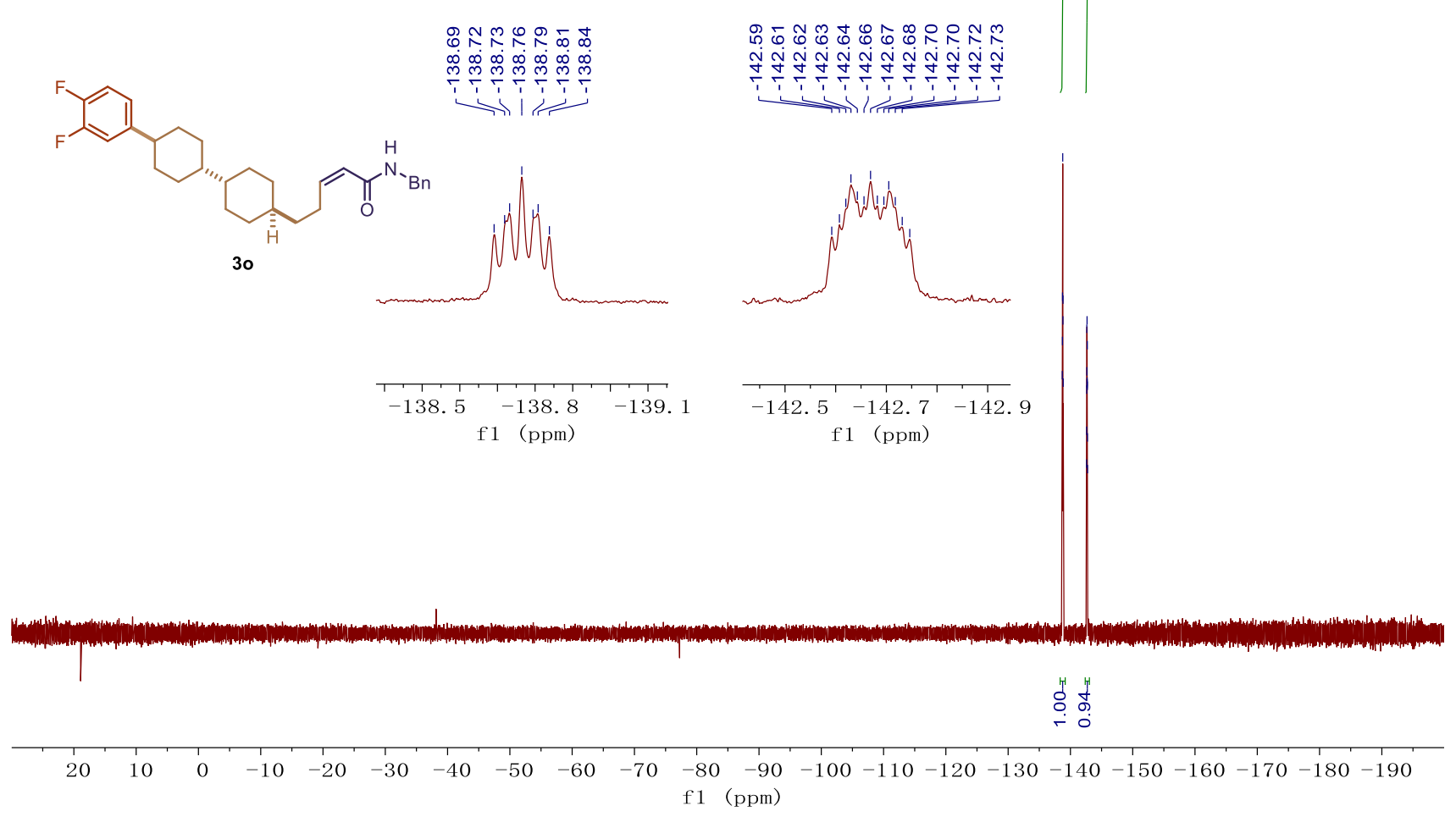


Figure S74 $\mid{ }^{1} \mathrm{H}$ NMR $\left(400 \mathrm{MHz}, \mathrm{CDCl}_{3}\right)$ spectrum of 3p.

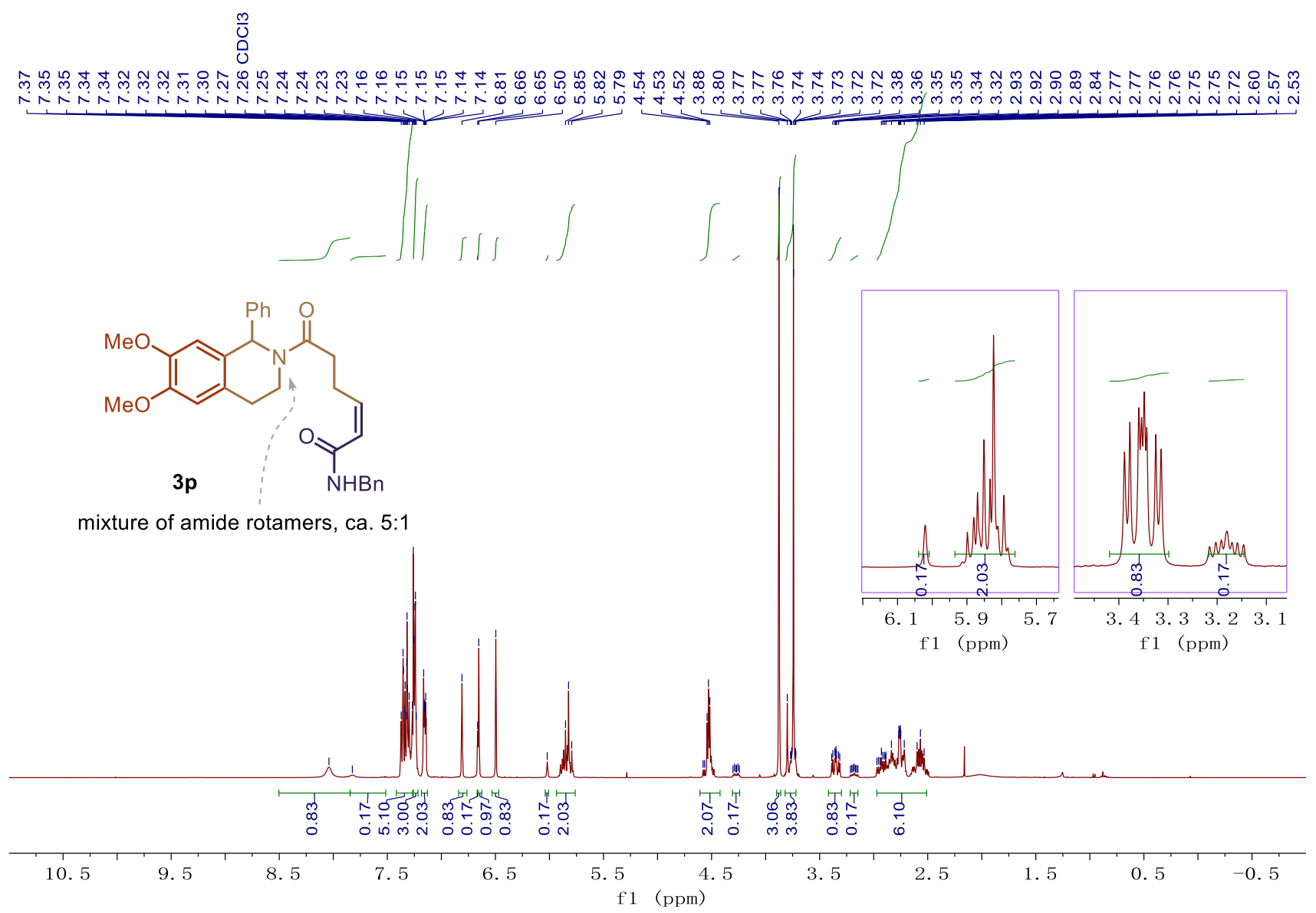

Figure S75 $\mid{ }^{13} \mathrm{C}$ NMR $\left(101 \mathrm{MHz}, \mathrm{CDCl}_{3}\right)$ spectrum of $\mathbf{3 p}$.

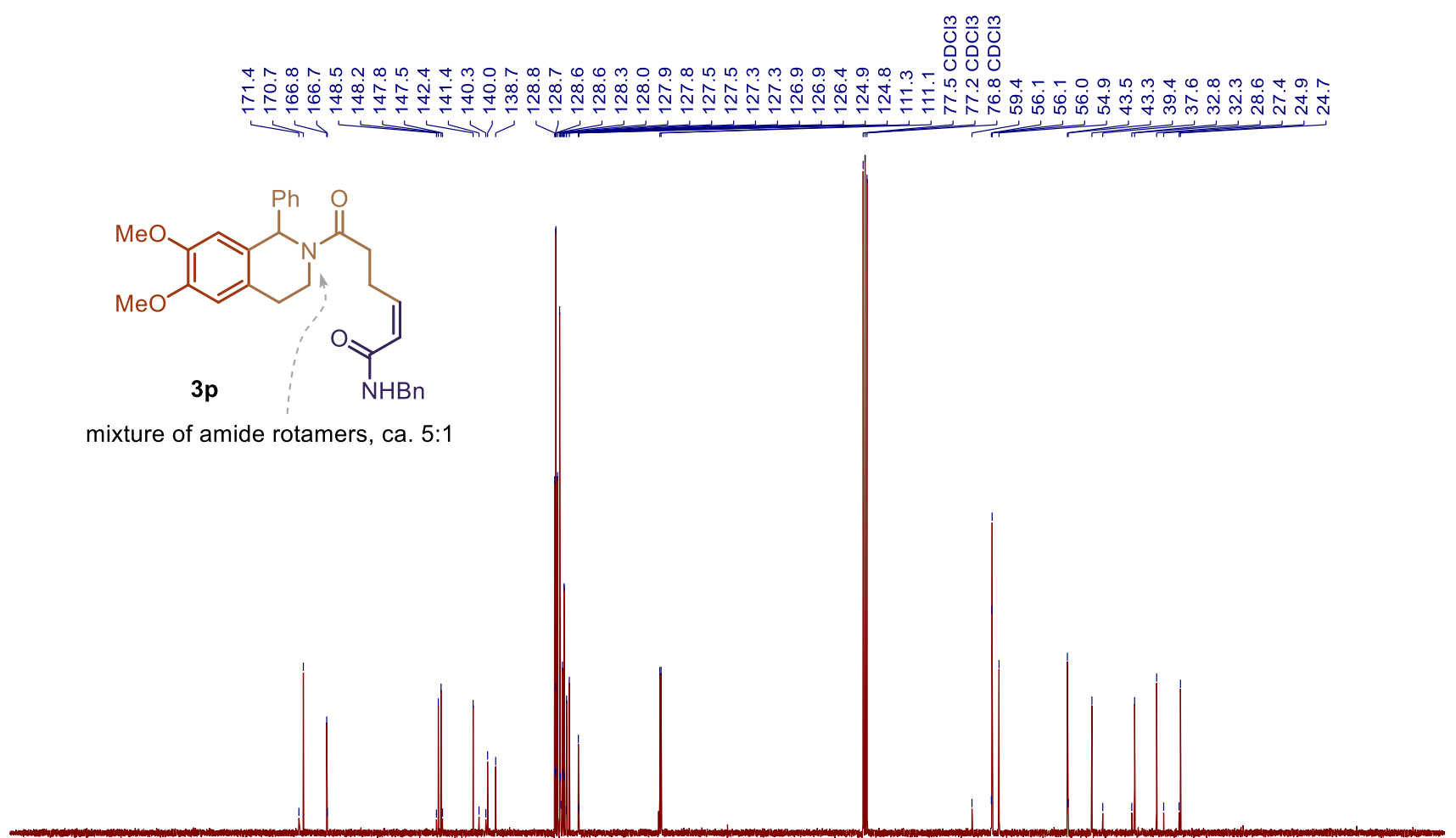

$\begin{array}{rllllllllllllllllllllllllllllllll}210 & 200 & 190 & 180 & 170 & 160 & 150 & 140 & 130 & 120 & 110 & 100 & 90 & 80 & 70 & 60 & 50 & 40 & 30 & 20 & 10 & 0 & -10\end{array}$ 
Figure $\mathbf{S 7 6}$ | ${ }^{1} \mathrm{H}$ NMR (400 MHz, $\left.\mathrm{CDCl}_{3}\right)$ spectrum of 3q.

品

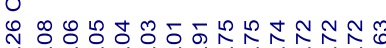

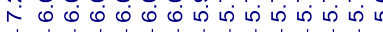

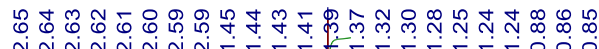
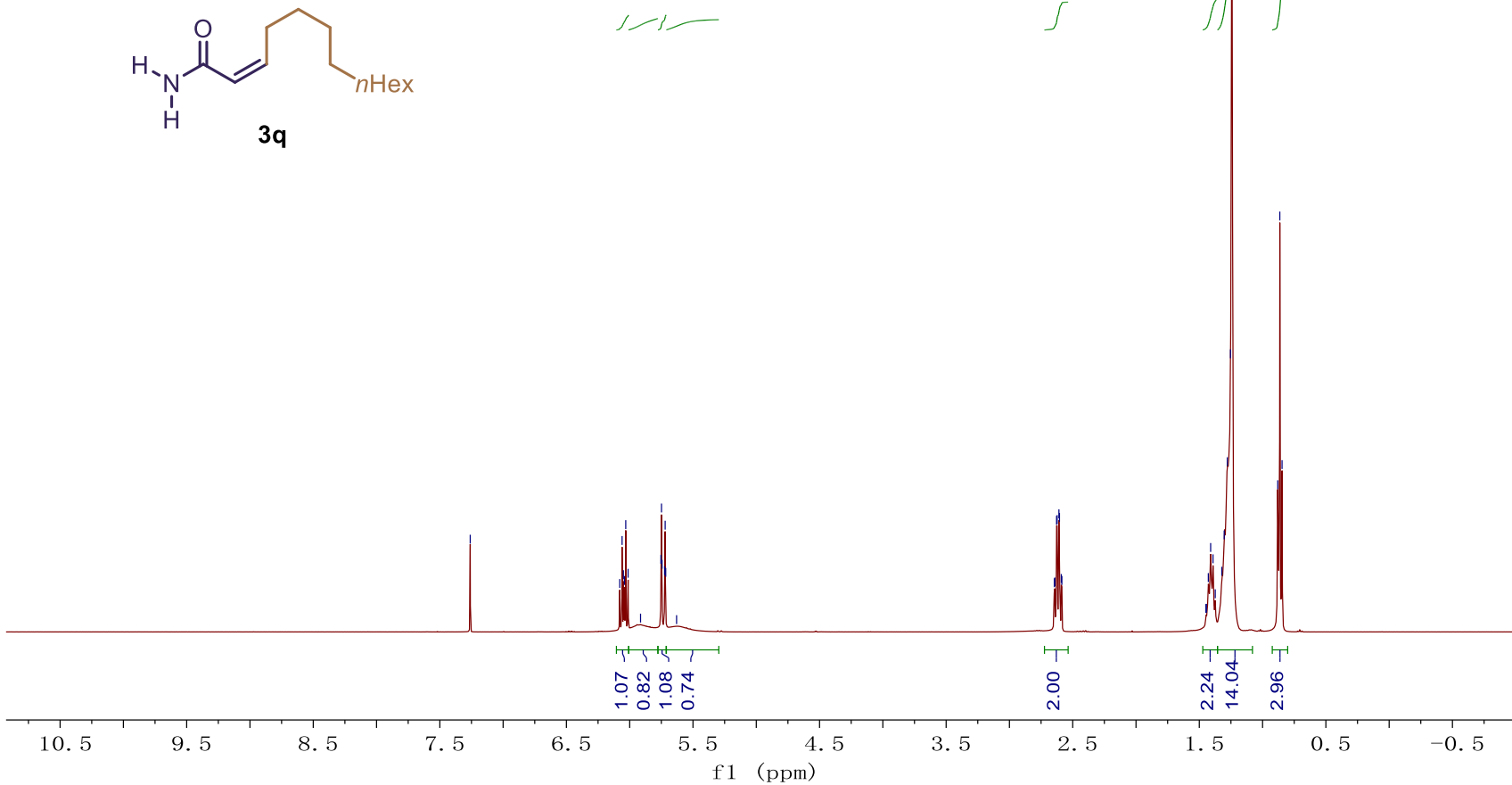

Figure $\mathbf{S 7 7} \mid{ }^{13} \mathrm{C}$ NMR $\left(101 \mathrm{MHz}, \mathrm{CDCl}_{3}\right)$ spectrum of $\mathbf{3 q}$.

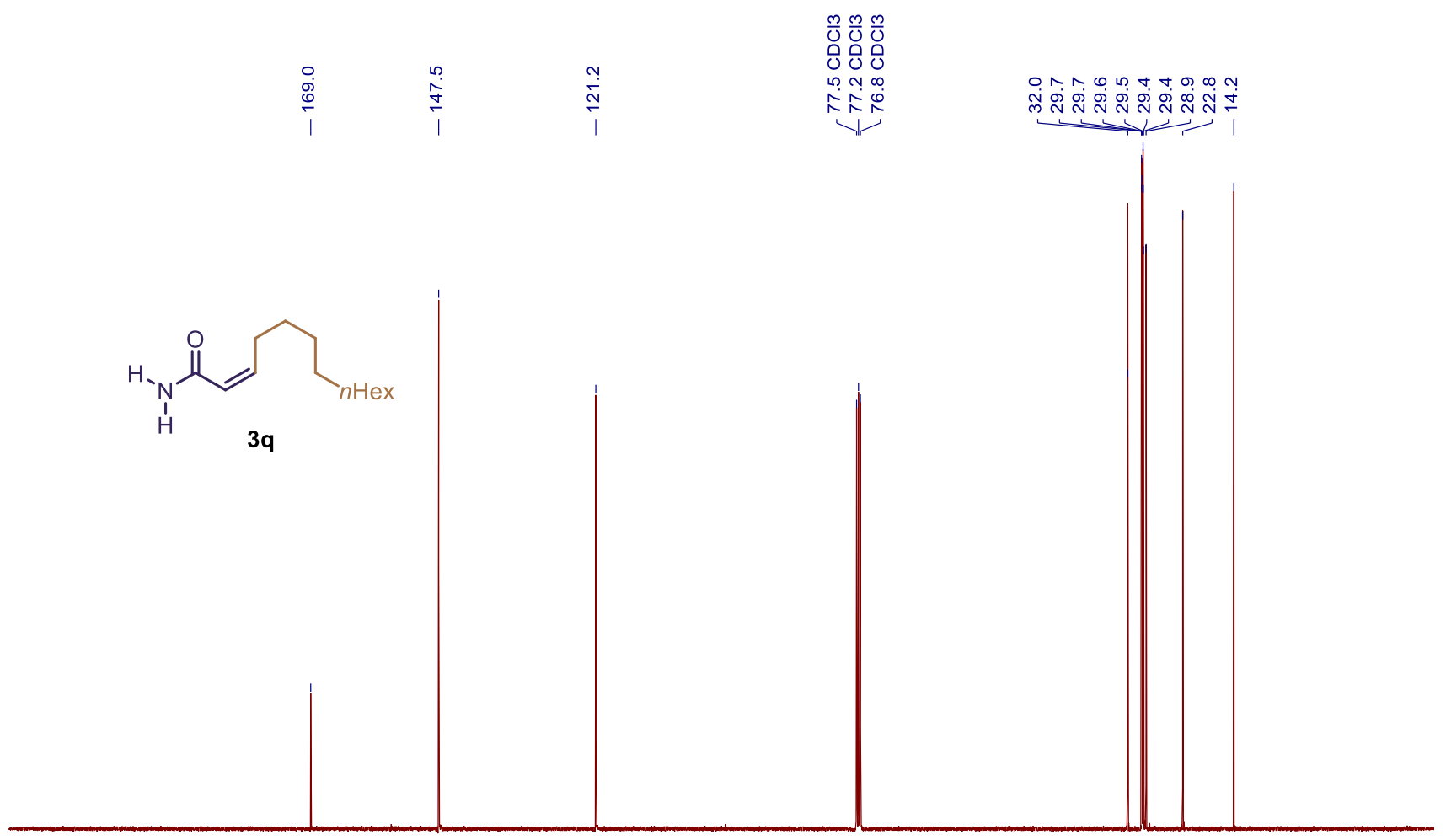

$\begin{array}{llllllllllllllllllllllll}210 & 200 & 190 & 180 & 170 & 160 & 150 & 140 & 130 & 120 & 110 & 100 & 90 & 80 & 70 & 60 & 50 & 40 & 30 & 20 & 10 & 0 & -10\end{array}$ 
Figure S78 $\mid{ }^{1} \mathrm{H}$ NMR (400 MHz, $\left.\mathrm{CDCl}_{3}\right)$ spectrum of $\mathbf{3 r}$.

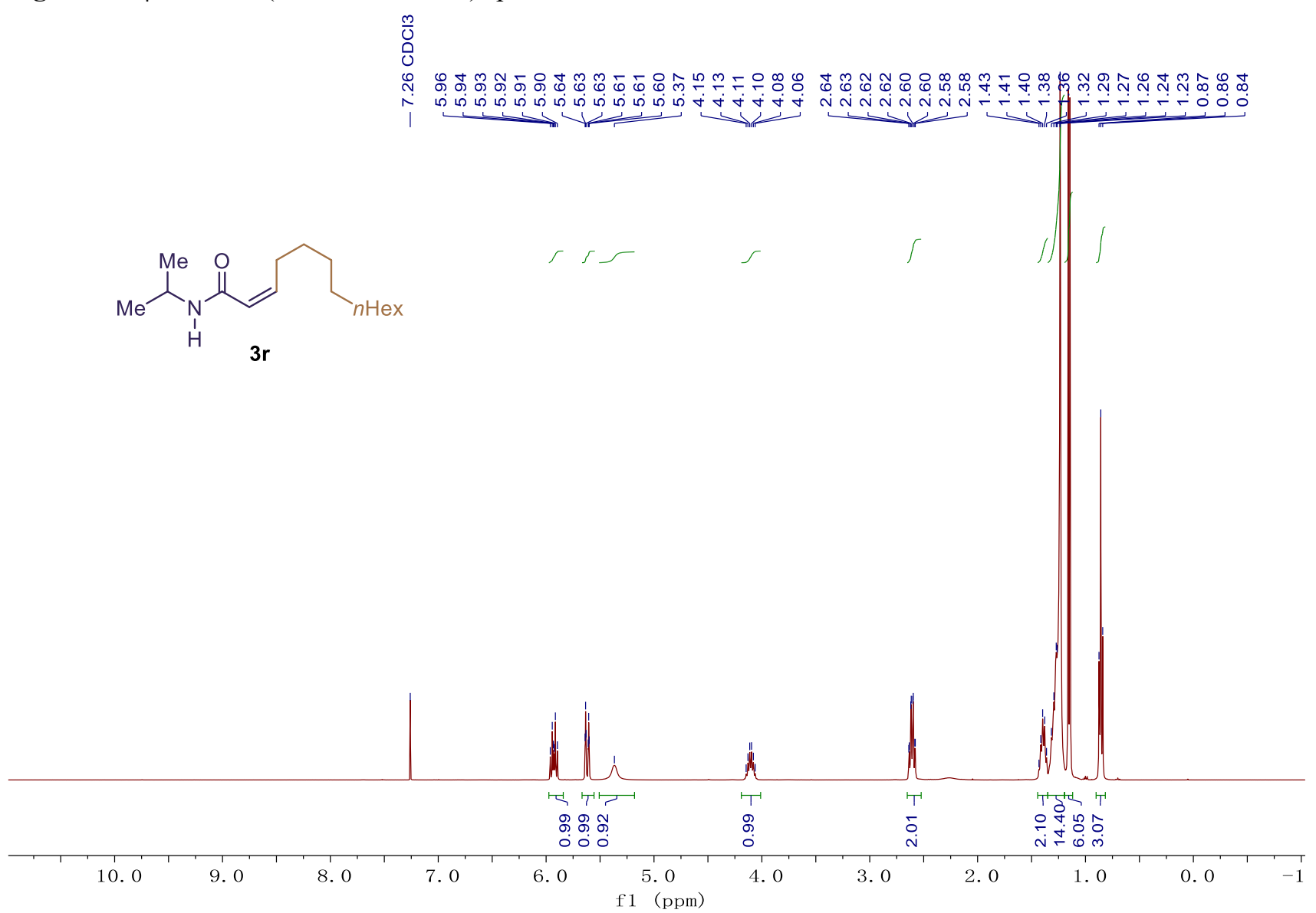

Figure S79| ${ }^{13} \mathrm{C}$ NMR $\left(101 \mathrm{MHz}, \mathrm{CDCl}_{3}\right)$ spectrum of $\mathbf{3 r}$.
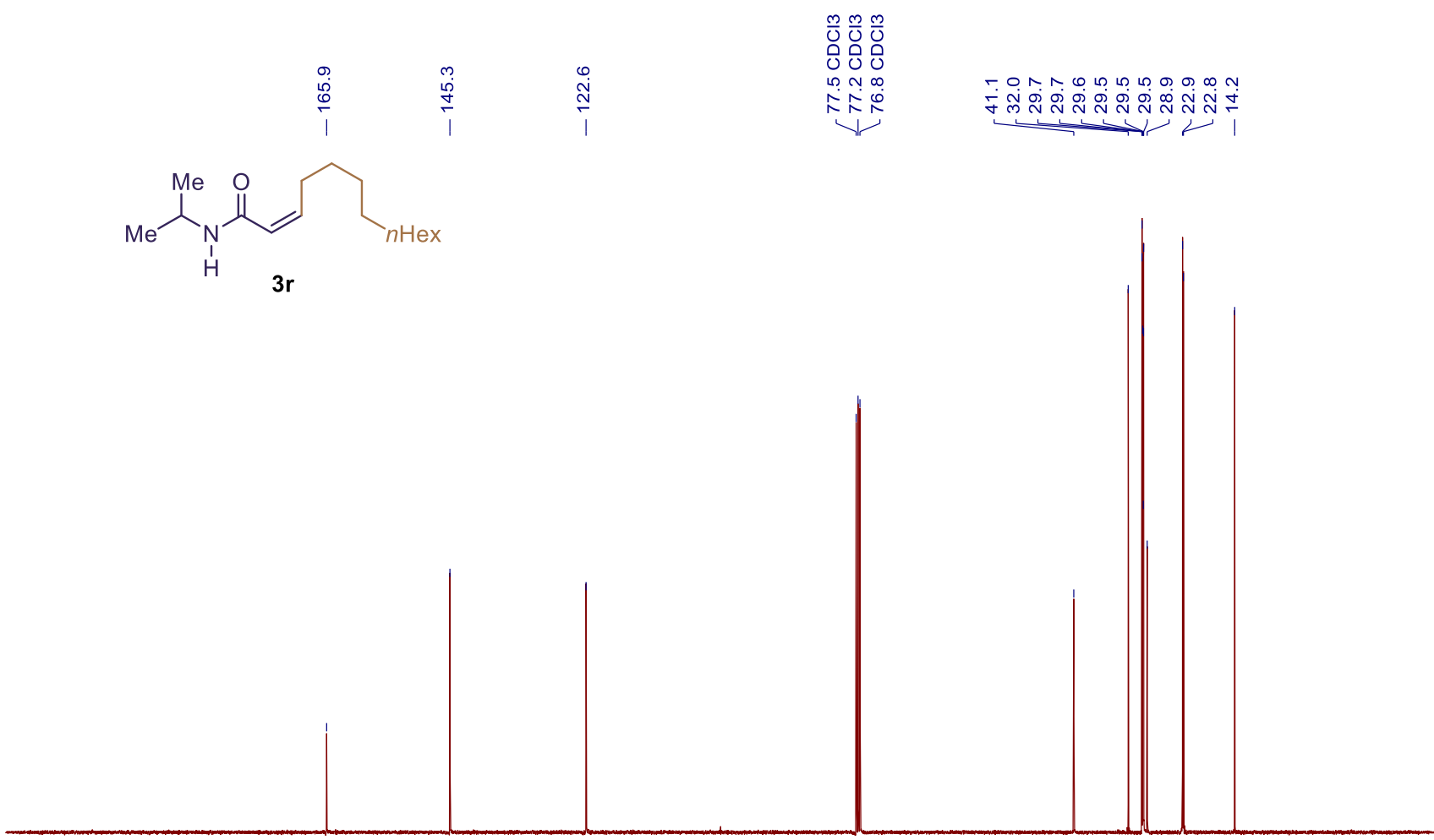

$\begin{array}{llllllllllllllllllllllll}210 & 200 & 190 & 180 & 170 & 160 & 150 & 140 & 130 & 120 & 110 & 100 & 90 & 80 & 70 & 60 & 50 & 40 & 30 & 20 & 10 & 0 & -10\end{array}$ 
Figure $\mathbf{S 8 0} \mid{ }^{1} \mathrm{H}$ NMR $\left(400 \mathrm{MHz}, \mathrm{CDCl}_{3}\right)$ spectrum of $\mathbf{3 s}$.
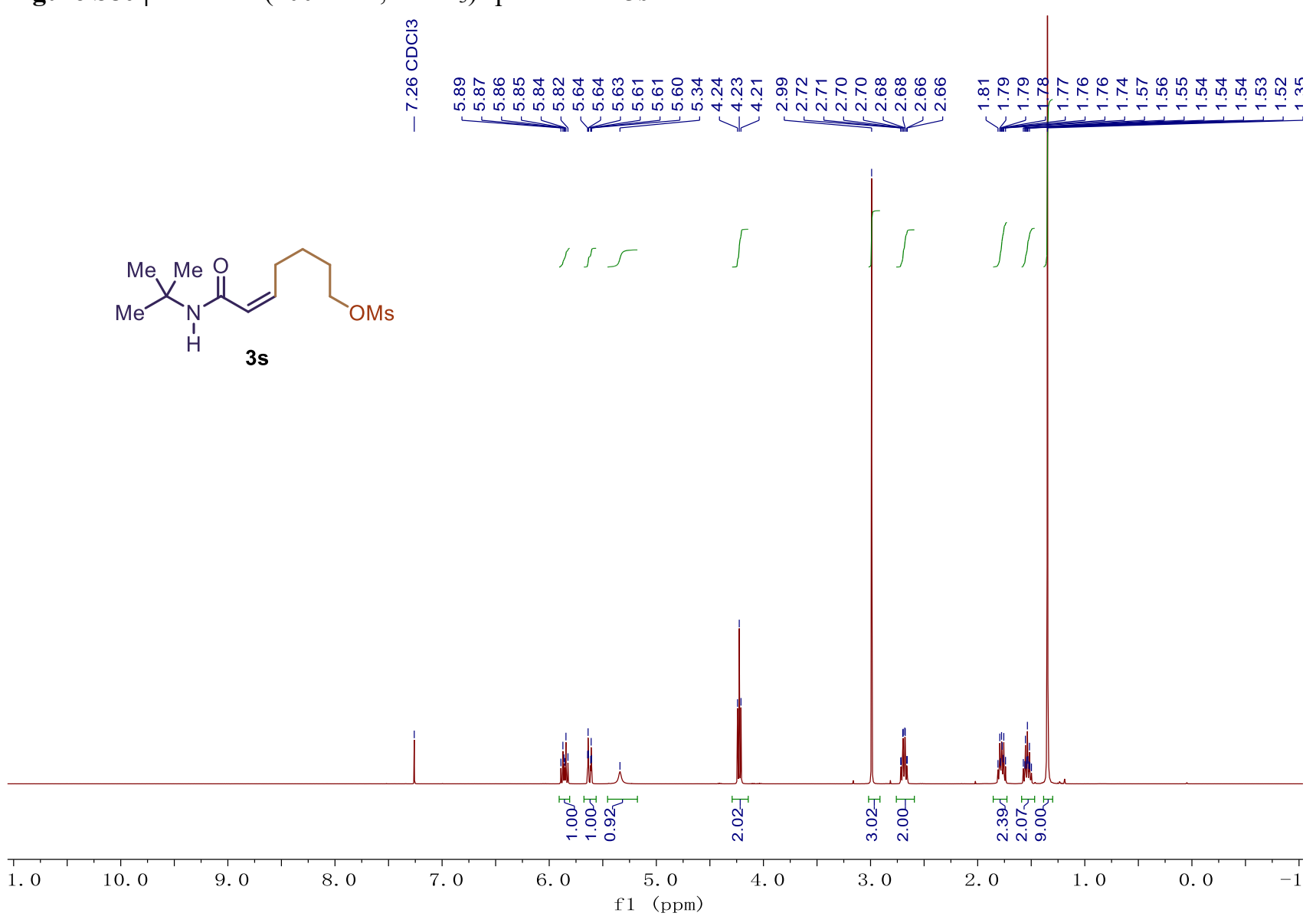

Figure $\mathbf{S 8 1} \mid{ }^{13} \mathrm{C}$ NMR $\left(101 \mathrm{MHz}, \mathrm{CDCl}_{3}\right)$ spectrum of $\mathbf{3 s}$.
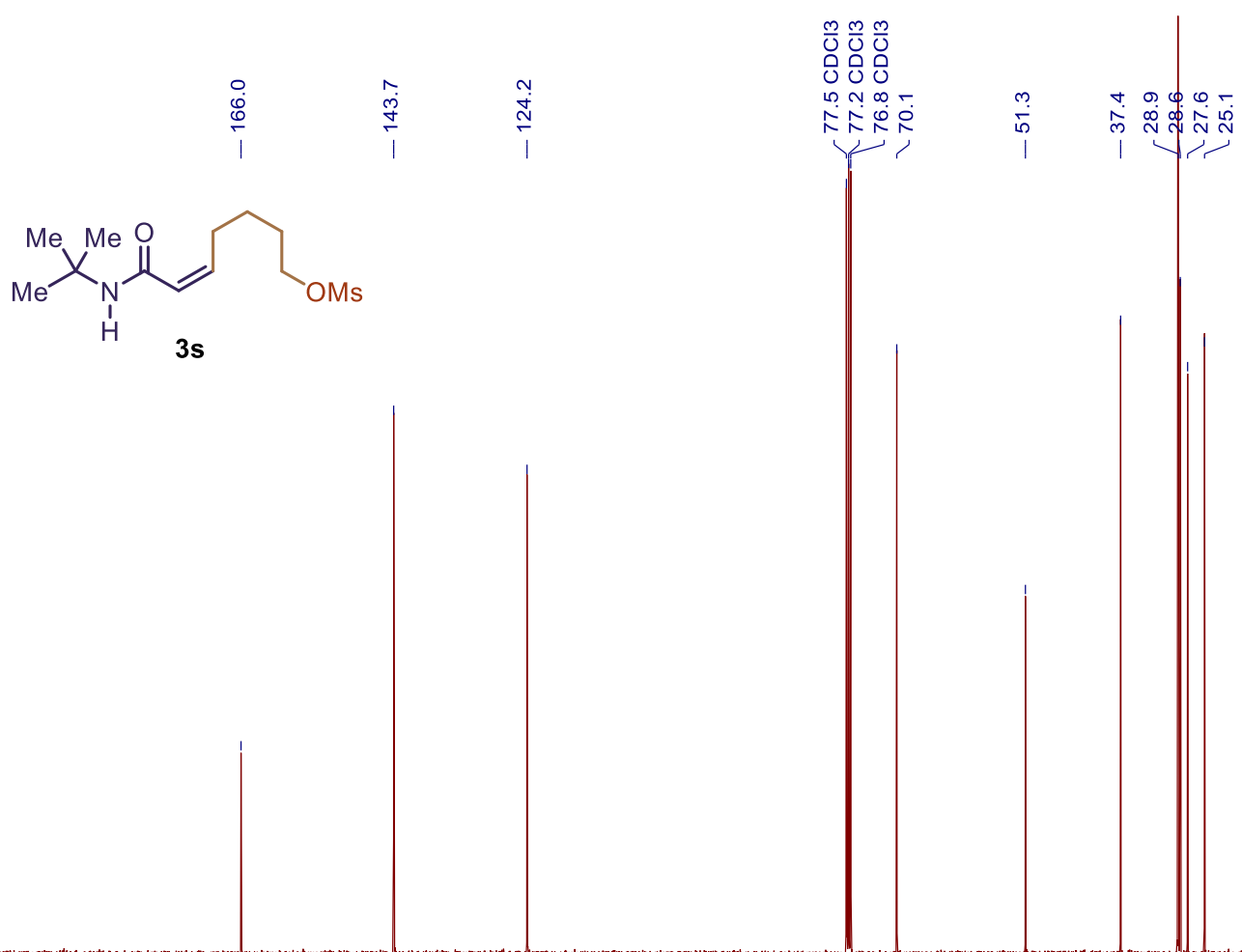

$\begin{array}{llllllllllllllllllllllll}1 & 1 & 200 & 190 & 180 & 170 & 160 & 150 & 140 & 130 & 120 & 110 & 100 & 90 & 80 & 70 & 60 & 50 & 40 & 30 & 20 & 10 & 0 & -10\end{array}$ 
Figure S82 $\mid{ }^{1} \mathrm{H}$ NMR (400 MHz, $\mathrm{CDCl}_{3}$ ) spectrum of 3t.

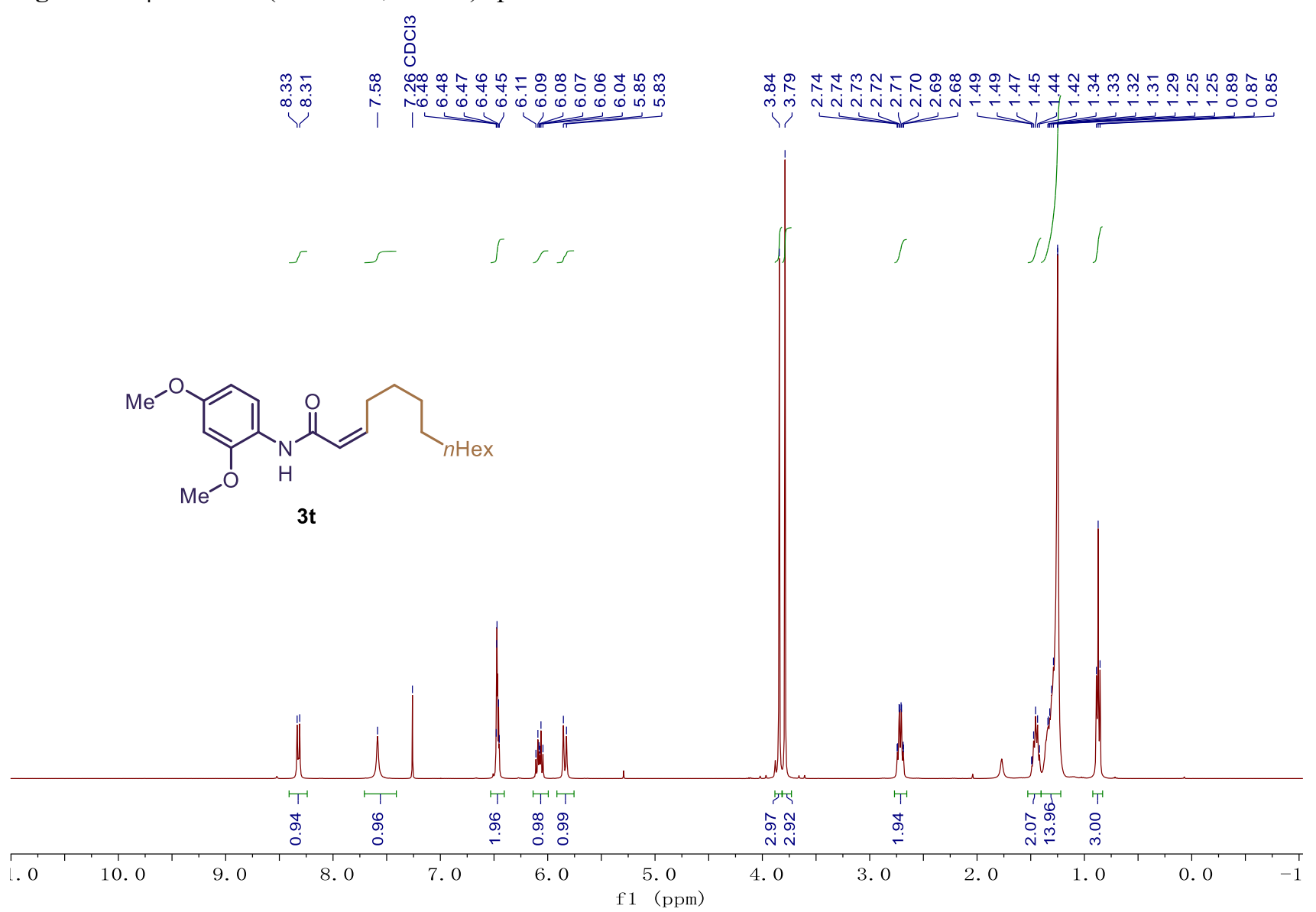

Figure S83 $\mid{ }^{13} \mathrm{C}$ NMR $\left(101 \mathrm{MHz}, \mathrm{CDCl}_{3}\right)$ spectrum of $\mathbf{3 t}$.

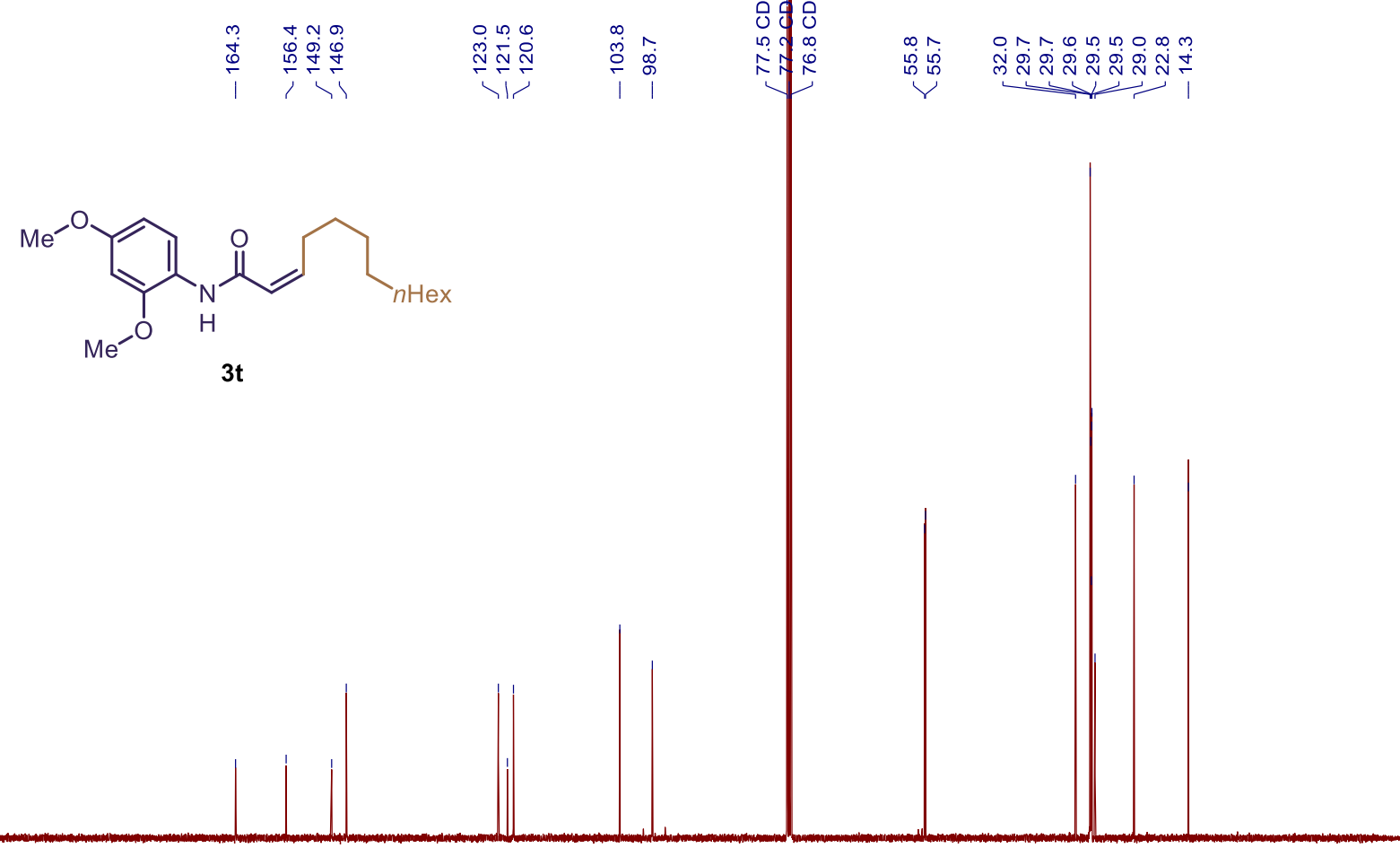

$\begin{array}{rllllllllllllllllllllllllllllll}210 & 200 & 190 & 180 & 170 & 160 & 150 & 140 & 130 & 120 & 110 & 100 & 90 & 80 & 70 & 60 & 50 & 40 & 30 & 20 & 10 & 0 & -10\end{array}$ 
Figure $\mathbf{S 8 4} \mid{ }^{1} \mathrm{H}$ NMR (400 MHz, $\left.\mathrm{CDCl}_{3}\right)$ spectrum of $\mathbf{3 u}$.

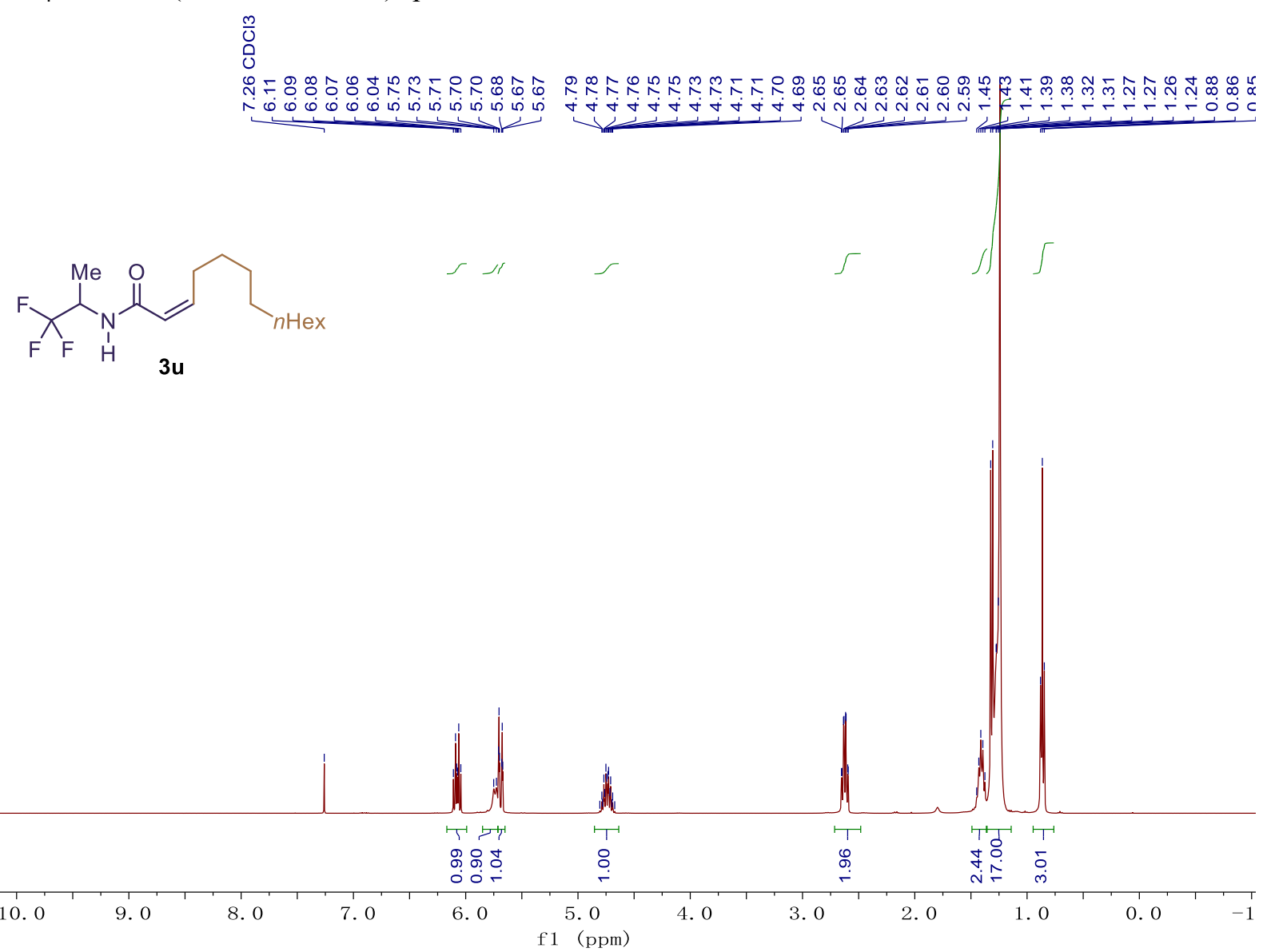

Figure $\mathbf{S 8 5} \mid{ }^{13} \mathrm{C}$ NMR $\left(101 \mathrm{MHz}, \mathrm{CDCl}_{3}\right)$ spectrum of $\mathbf{3 u}$.

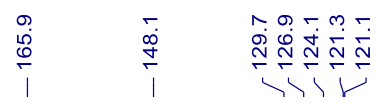
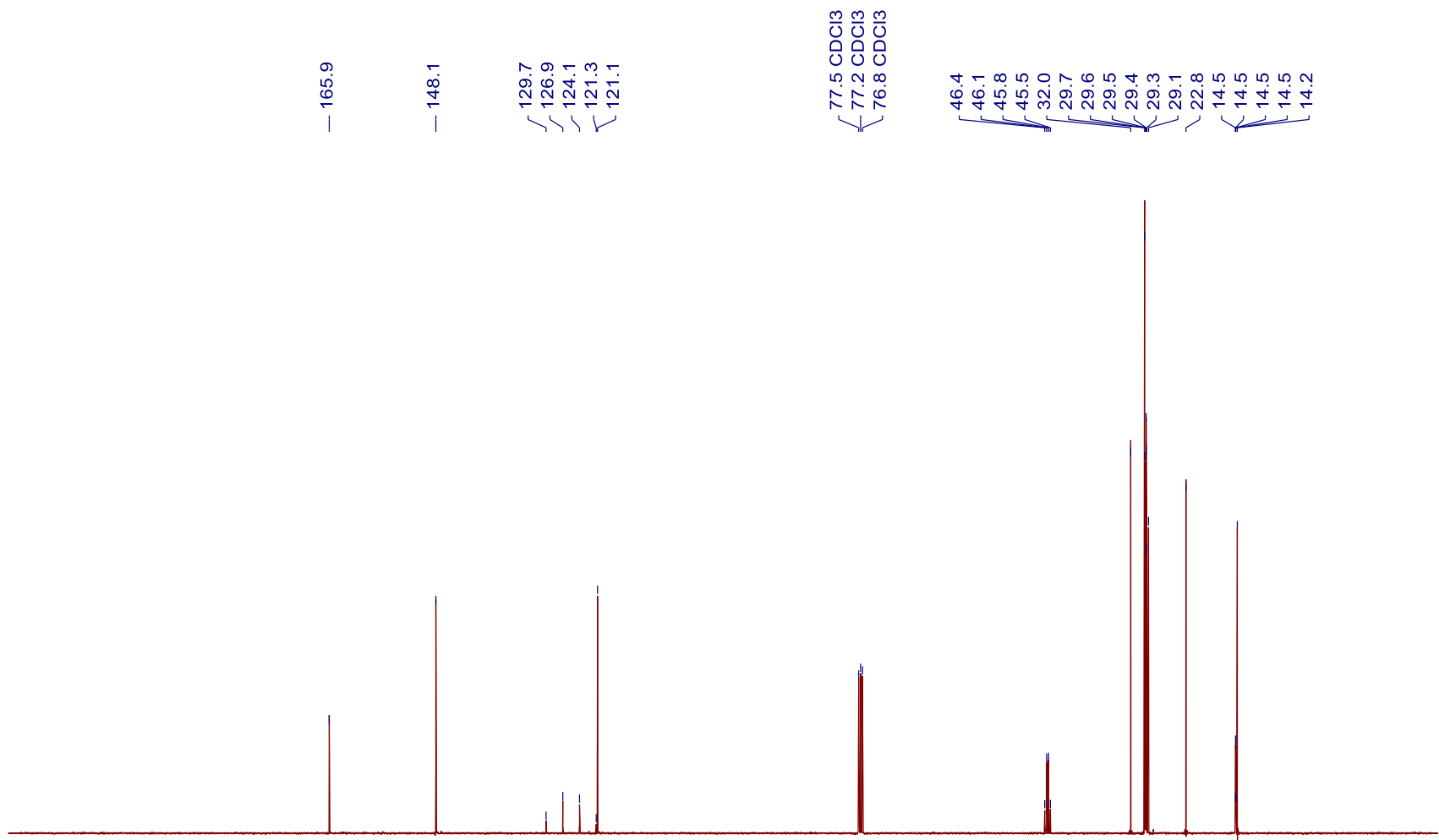

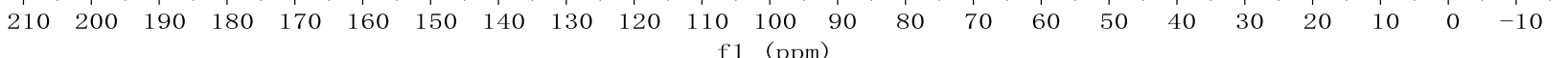


Figure S86 $\mid{ }^{19} \mathrm{~F}$ NMR $\left(282 \mathrm{MHz}, \mathrm{CDCl}_{3}\right)$ spectrum of $\mathbf{3 u}$.

$$
\text { 记 }
$$

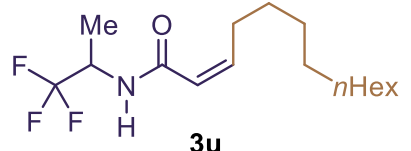

3u

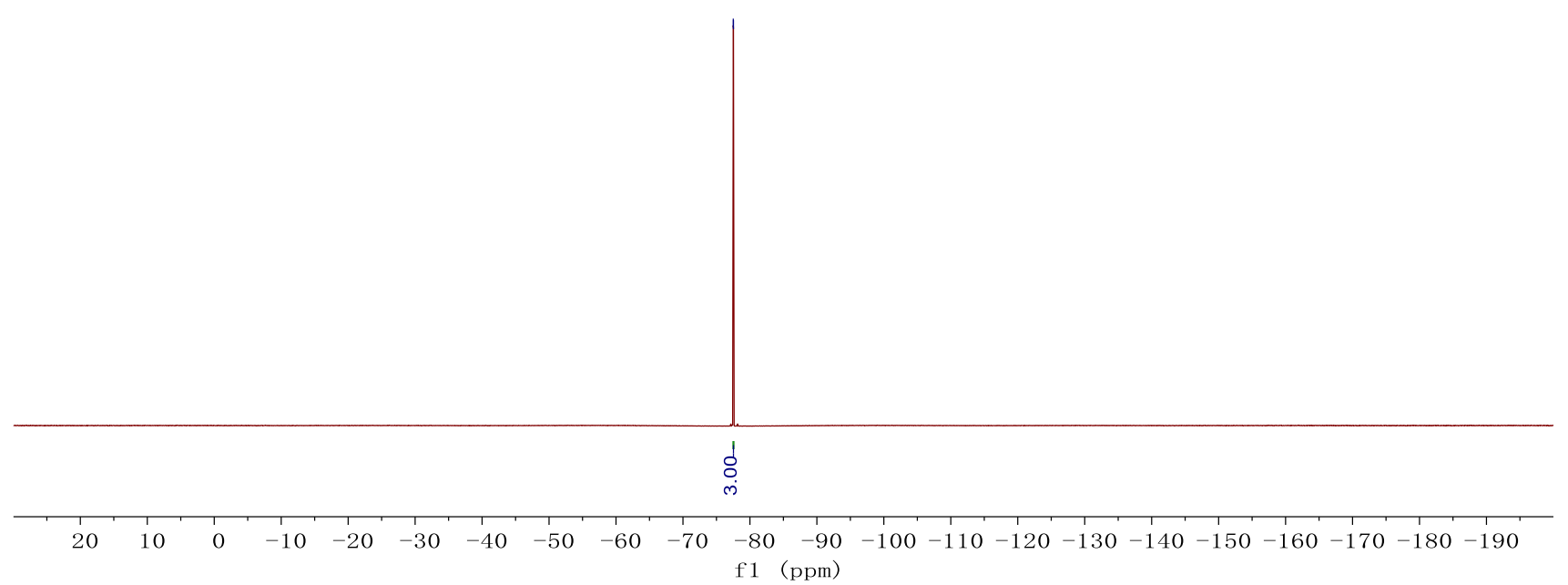


Figure S87 $\mid{ }^{1} \mathrm{H}$ NMR $\left(400 \mathrm{MHz}, \mathrm{CDCl}_{3}\right)$ spectrum of $\mathbf{3 v}$.

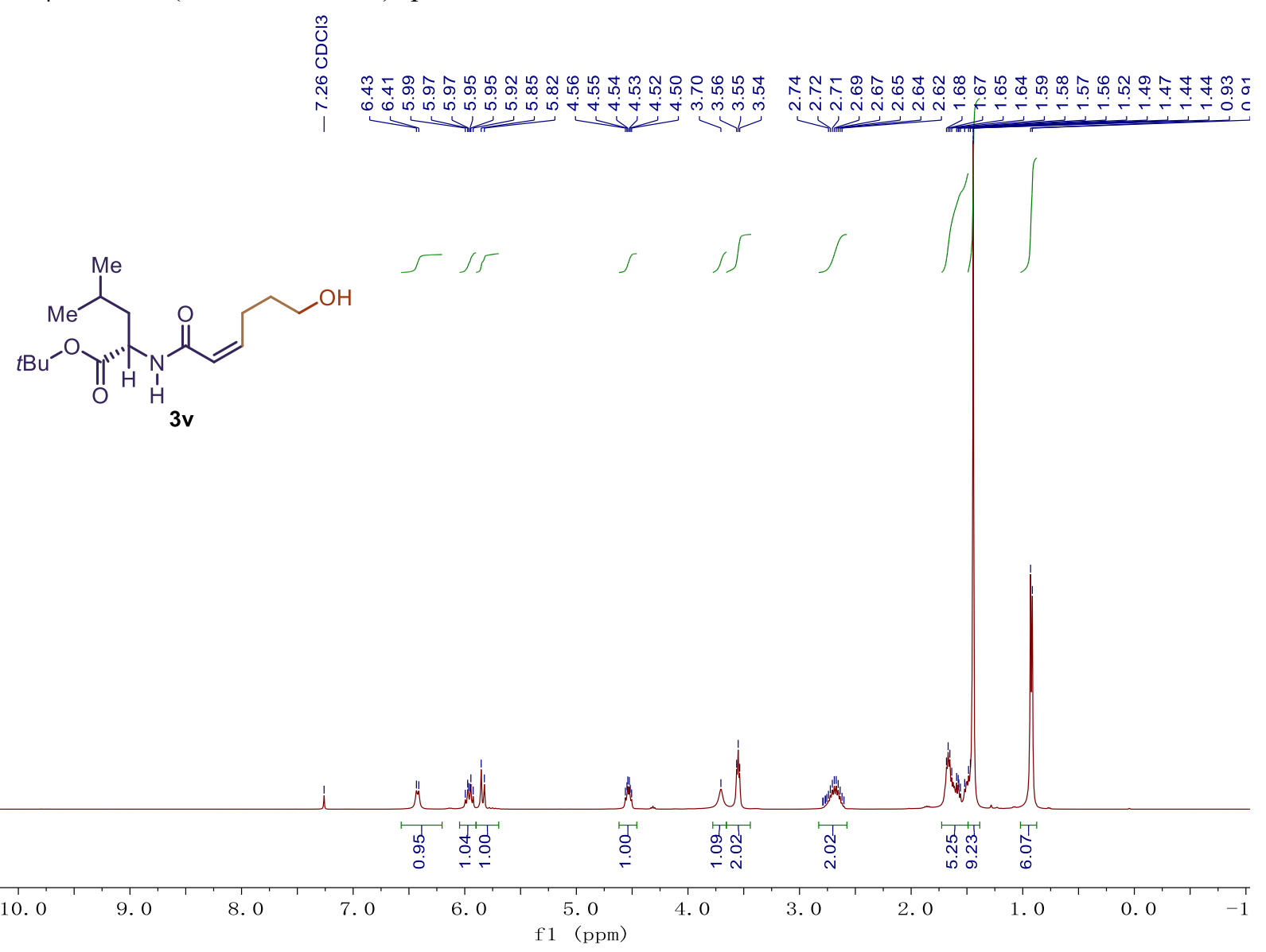

Figure S88 $\mid{ }^{13} \mathrm{C}$ NMR $\left(101 \mathrm{MHz}, \mathrm{CDCl}_{3}\right)$ spectrum of $\mathbf{3 v}$.

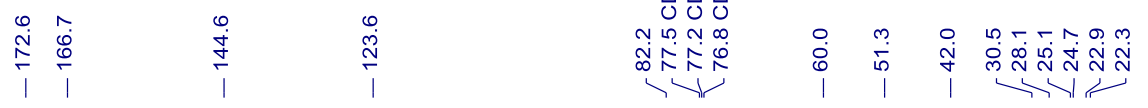<smiles>CC(C)COC(=O)C(CC(C)C)NC(=O)/C=C\CCCO</smiles>

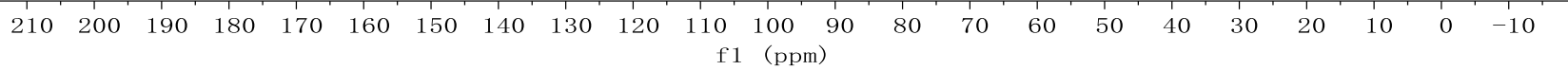


Figure S89 | ${ }^{1} \mathrm{H}$ NMR (400 MHz, $\left.\mathrm{CDCl}_{3}\right)$ spectrum of $\mathbf{3 w}$.

$\frac{m}{0}$

๑ุ̣

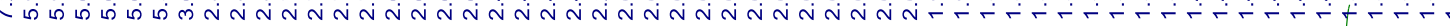<smiles>COC(=O)C1(C)CCC(NC(=O)/C=C\CCCCCCC2CO2)CC1</smiles>

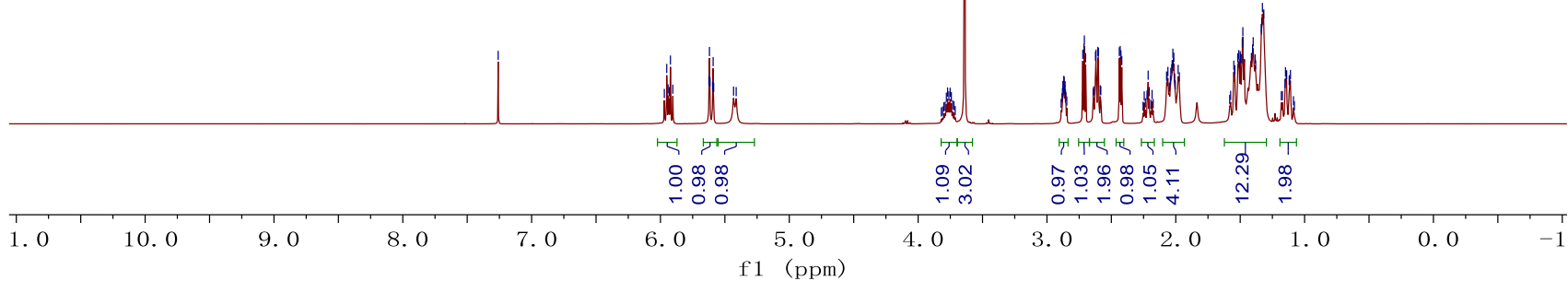

Figure S90 $\mid{ }^{13} \mathrm{C}$ NMR $\left(101 \mathrm{MHz}, \mathrm{CDCl}_{3}\right)$ spectrum of $\mathbf{3 w}$.

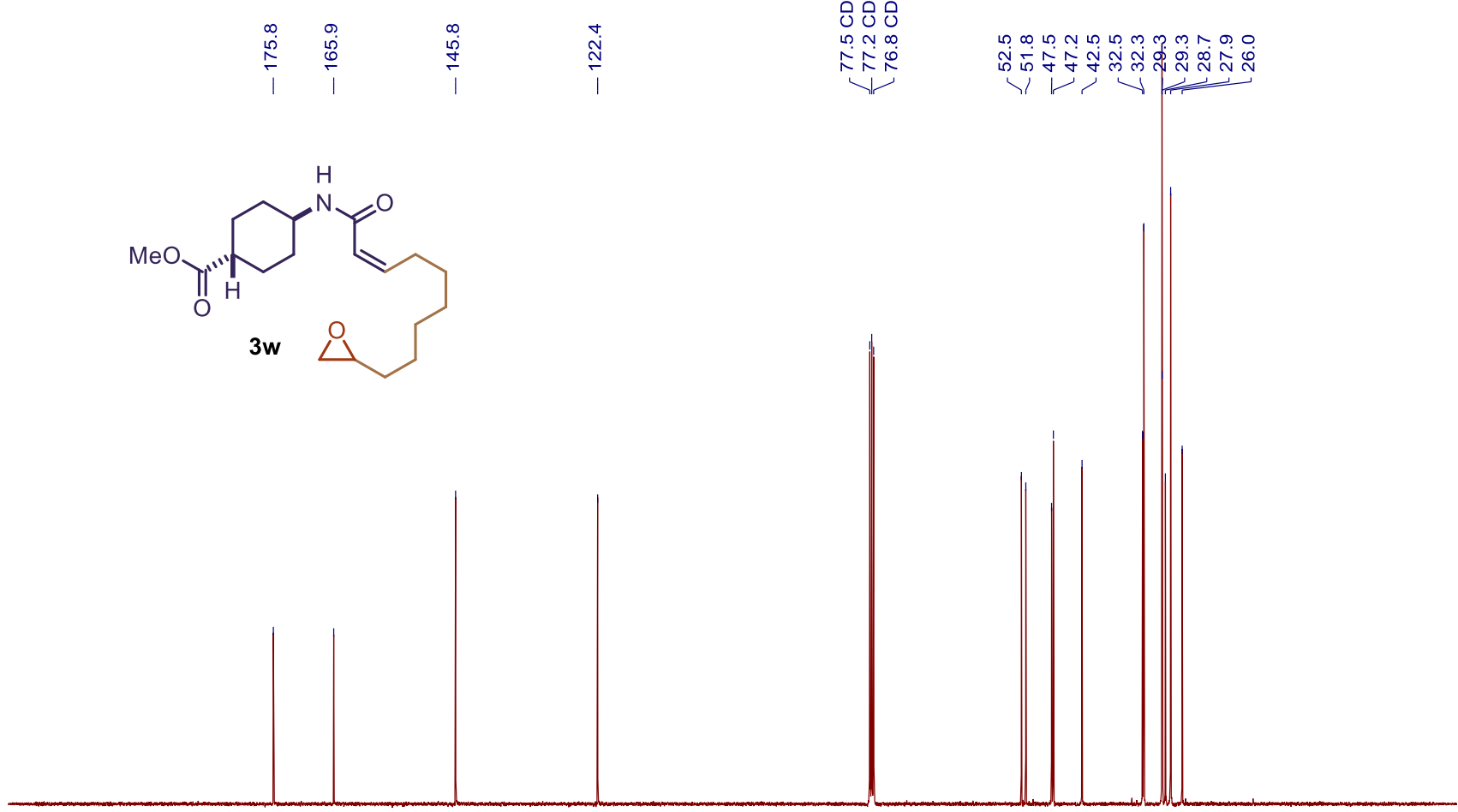

$\begin{array}{lllllllllllllllllllllllllll}210 & 200 & 190 & 180 & 170 & 160 & 150 & 140 & 130 & 120 & 110 & 100 & 90 & 80 & 70 & 60 & 50 & 40 & 30 & 20 & 10 & 0 & -10\end{array}$ 
Figure S91 | ${ }^{1} \mathrm{H}$ NMR $\left(400 \mathrm{MHz}, \mathrm{CDCl}_{3}\right)$ spectrum of $\mathbf{3 x}$.

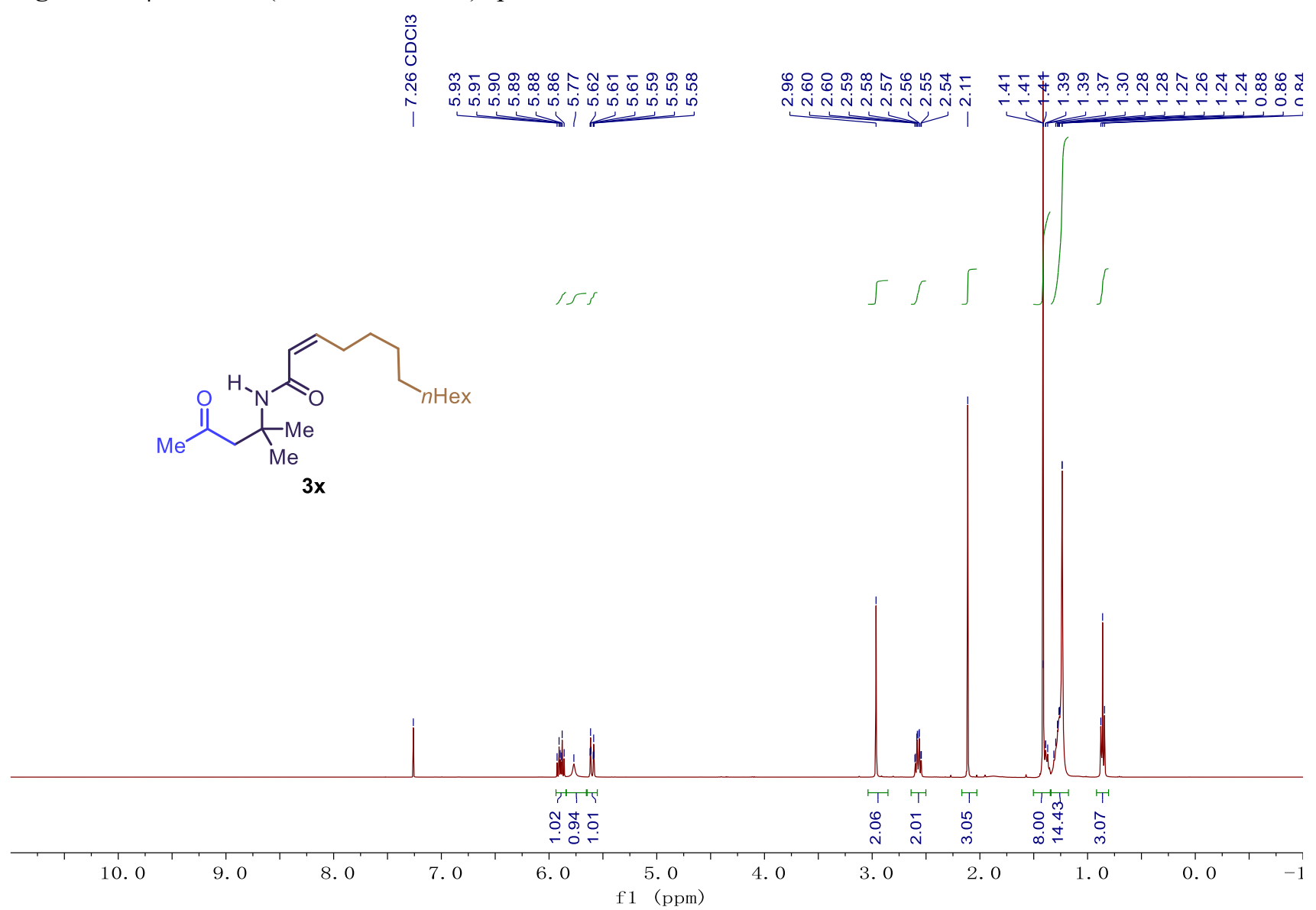

Figure S92 $\mid{ }^{13} \mathrm{C}$ NMR $\left(101 \mathrm{MHz}, \mathrm{CDCl}_{3}\right)$ spectrum of $\mathbf{3 x}$.

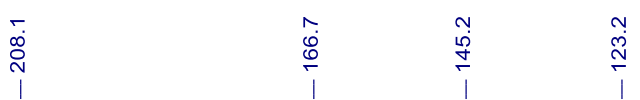
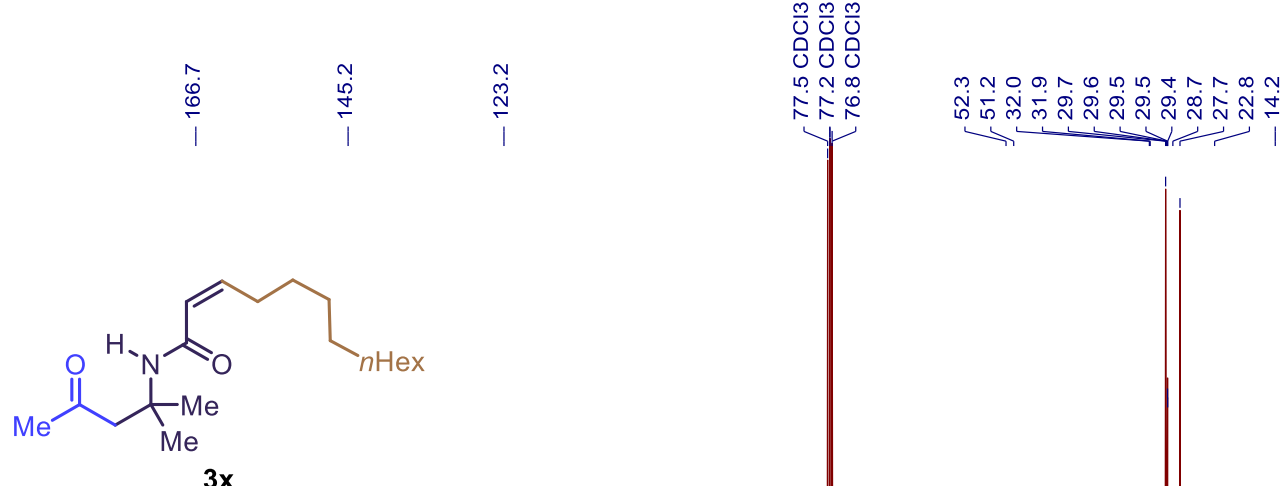

เก

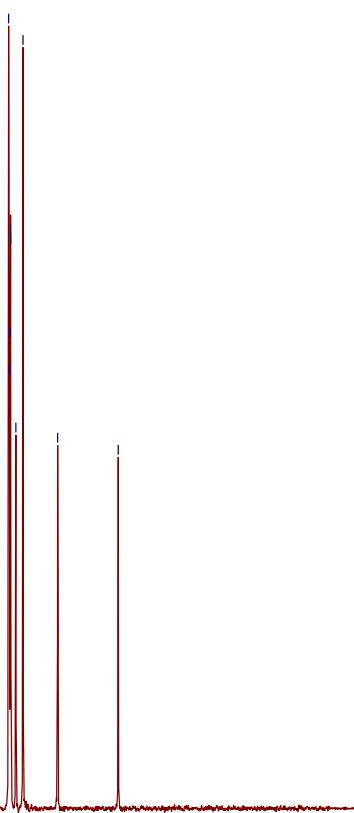

$\begin{array}{lllllllllllllllllllllllllll}210 & 200 & 190 & 180 & 170 & 160 & 150 & 140 & 130 & 120 & 110 & 100 & 90 & 80 & 70 & 60 & 50 & 40 & 30 & 20 & 10 & 0 & -10 & \end{array}$ 